\title{
14th Congress of the European Association for Clinical Pharmacology and Therapeutics (EACPT)
}

\author{
29 June - 2 July 2019 \\ City Conference Centre, Stockholm, Sweden
}

„Clinical pharmacology meeting tomorrows health care challanges“

\section{The Abstracts}


Abstract Comittee:

Ylva Böttiger, Markus Zeitlinger, Jamie Coleman, Michiel Agtmael, Gerard Rongen, Janne Backman, Jolanta Gulbinovic, Pierre Marquet, Caroline Samer, Achim Schmidtko, Simon Tabassome, Vangelis Manolopoulos, Arantxa Sancho, Aleksi Tornio, Emilio Sanz, Jelle Tichelaar, Susanna Wallerstedt, Erik Eliasson

This supplement was not sponsored by outside commercial interests. It is funded entirely by the publisher. 


\title{
Table of Contents
}

Advanced therapies

Outpatient parental antimicrobial treatment; an antibiotic stewardship tool

Comparison of biosimilar filgrastim utilization in 2018 in croatia with regards to other european countries

\begin{abstract}
Antimicrobials
Irrational use of antibiotics and clinical outcomes in children with infectious diseases

A link between antibiotic resistance and antibiotic consumption in greece, serbia and norway

Is selective decontamination of digestive tract justified in hospitalized infants

Co-trimoxazole induced hyperkalemia and potassium monitoring in hospitalized patients

Impact of voriconazole therapeutic drug monitoring (tdm) on toxicity avoidance in immunocompromised hematological patients

Interindividual variability in plasma levels of beta-lactam antibiotics in critically ill patients with or without continuous renal replacement therapy

Bacteremia in bone marrow transplantation.

Automated antimicrobial consumption data visualization for targeted stewardship

Adverse effects of bedaquiline in patients with extensively drug-resistant tuberculosis (xdr-tb)

The volume of ceftriaxone use and resistance to cephalosporins of enterobacteria - the theory of parallel damage by the example of a multidisciplinary hospital A randomized controlled study on impact of helicobacter pylori eradication in children with acute immune thrombocytopenia

Cushing's syndrome related to interaction between ritonavir or cobicistat and corticosteroid: a case control study in the french pharmacovigilance database
\end{abstract}

The comparison of urinary kidney injury molecule 1 as a biomarker of renal function to creatinine in critically ill patients in two referral teaching hospitals

The effect of flucloxacillin on warfarin anticoagulation: a swedish register-based nationwide cohort study

Clinical effects of green tea components on prevention of influenza infection

Prevention and detection of healthcare-associated infections using the comprehensive risk management system

Improving antibiotic stewardship in neonates through means of a new biomarker, soluble cd14 subtype (scd14-st): a systematic review and meta-analysis Comparison of dosage strategies of meropenem vs. Pseudomonas aeruginosa

Six versus four weeks of intravenous antibiotic treatment for staphylococcus aureus endocarditis: a pilot study

Posaconazole salvage therapy: the posifi study.

Cancer treatment

Preparation of an ibrutinib patch test

Real-life experience of eribulin in patients with advanced liposarcoma

Effectiveness and safety of nivolumab in geriatric patients

Effect of a single intraoperative dose of dexamethasone on blood glucose and lactate levels in brain tumor surgery

Survival of gastric and esophageal cancer patients with adjuvant and palliative chemotherapy - a retrospective analysis of a register-based patient cohort Medication safety of new oral antitumor agents: a randomized trial on the impact of a clinical pharmacological / pharmaceutical intervention on drugrelated problems and patient safety

Roles of myc-modulating long non-coding rna mincr in cell cycle regulation and apoptosis in non-small cell lung cancer

Effect of new preventive medicine on cisplatin-induced acute kidney injury

A bayesian approach for continual estimation of real-life effectiveness as new data emerge

Health and economic burden in direct costs of melanoma in moscow for 2016-2017

Assessment of antineoplastic drug approvals by european medicines agency (ema) and food and drug administration (fda) during 2018. Evaluation of possible differences and available evidence

Genetic polymorphisms of cyp450 and tolerability of tamoxifen therapy

Polymorphisms of cytochrome 450 and adverse events of tamoxifen therapy

Anticancer potential of nanogold conjugated toxin gnp-nn-32 from indian spectacled cobra venom

Investigation of the anti-tumour activity of azadirachta indica (neem) leaf extract on human ovarian cancer cells

Active therapeutic drug managing instead of passive therapeutic drug monitoring - towards a safer high-dose methotrexate regimen to adult patients with acute lymphatic leukaemia

Ribociclib induces macrocytosis in therapeutic doses: characterization of outcome

Analysis and improvement of clinical trial pharmacist's work in cancer center

Adverse reactions to drugs of special interest in a pediatric oncohematology service

Pulmonary toxicity after dasatinib treatment for chronic myeloid leukemia : a case report and the literature review

Post authorization safety profile in relation to off label use of rituximab

Successfully treat of severe hemorrhagic cystitis by intravesicle hyaluronic acid in a case of haploidentical stem cell transplant-a case report

Cardiovascular treatment

Mineralocorticoid receptor blockade attenuates disrupted glutathione-dependent antioxidant defense and elevated endoglin in the hearts of pregnant rats exposed to testosterone

Vasorelaxation of human saphenous vein induced by epicatechin 
Effectiveness and safety of alirocumab in a third level hospital

Methodological and reporting issues in pharmacoepidemiological research on drug safety. A review of studies underlying recent conclusions on associations between loop diuretics/beta-blockers and falls

Glucose control safety \& efficacy in type 2 diabetes, a systematic review and network meta-analysis (glucose dinet)

Absorption, distribution, metabolism, and excretion of selatogrel (act-246475), a p2y12 receptor antagonist for subcutaneous use

Additional value of a prescribing pharmacist treating patients with a vascular internist at the heart+vascular centre in the netherlands

Comparison of anti-fxa chromogenic assay for rivaroxaban used in north estonia medical centre with lc-ms $/ \mathrm{ms}$

A 1-year randomized trial of deferasirox and deferoxamine combination versus deferasirox for the treatment of iron overload in thalassemia major

Use and compliance of treatment with direct oral anticoagulants in non-valvular atrial fibrillation

Adherence to direct oral anticoagulants in patients with non-valvular atrial fibrillation cross-national comparison in six european countries

Physicians' and undergraduates' knowledge of drugs contraindicated for arterial hypertension treatment

Assessment of physicians' and senior medical students' knowledge of antihypertensive drugs contraindications in copd patients treatment

Poor adherence to renal function testing for dabigatran

Prescription patterns of lipid lowering agents among older patients in general practice: an analysis from a national database in the netherlands

Influence of abcb1, slcolb1, cyp3a5 and cyp3a4*22 genetic polymorphisms on antiplatelet effect of ticagrelor in patients with acute coronary syndrome

Can self-medication be a risk factor of inefficient pharmacotherapy in cardiovascular diseases?

A personalised, clinical pharmacological approach to the management of hypertension presenting acutely to secondary care

How to improve hypertension control? - evaluation of weak links in pharmacotherapy

Pharmacoepidemiological analysis of antithrombotic treatment of atrial fibrillation in the hospital in 2011-2012 and 2016-2017

The use of fall-risk-increasing drugs in comorbid patients with polypharmacy

Real-life safety of long-term treatment with direct oral anticoagulants

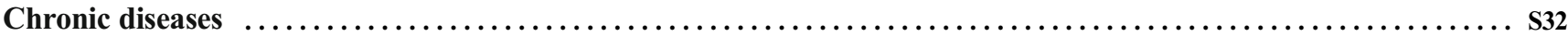

Sitagliptin ameliorates diabetic nephropathy by attenuating oxidative stress and apoptosis in a rat model of streptozotocin-induced type 1 diabetes

Polymorphic marker cyp2d6*4 and the response to propranolol in russian patients with liver cirrhosis

Efficacy and safety of different doses and durations of intravenous immunoglobulin treatment for immune thrombocytopenic purpura

Do patients with cystic fibrosis participating in clinical trials demonstrate placebo response? A meta-analysis.

Factors affecting medication non-adherence among patients with type 2 diabetes

Monoclonal antibody ocrelizumab for primary-progressive multiple sclerosis in adults: a systematic review

Anti-b-cell therapy ocrelizumab for relapsing multiple sclerosis in adults: a systematic review

Pharmacoepidemiological analysis of disease-modifying antirheumatic drugs adverse effects in rheumatoid arthritis

Pharmacological causes behind pulmonary disease

Antidiabetic drug therapy in patients with type 2 diabetes mellitus: a multicenter study in primary health care centers in greece

Priapism and atypical antipsychotics: case report and complete review

Case report: priapism due to antipsychotic drugs. Management proposal

Computerized decision support

Prioritising hospital patients at high-risk of medication harm: development and validation of a predictive risk model

A pharmacist-physician-based process to ensure quality assurance in standard order sets

Comparative efficacy and safety of treatments for secondary raynaud's phenomenon: systematic review and network meta-analysis of randomized trials The efficacy of a mobile clinical decision support application compared to usual care: a proof of concept study among prescribers in different stages of training

An algorithm for automatically assessing student answers to open questions in the pharmacotherapy e-learning web-application p-scribe

Pharros - a pilot project to create a standards-based computerized decision support system for therapeutic drug monitoring (tdm)

\section{Drug-drug interactions}

Influence of green tea on pharmacokinetics of fexofenadine in healthy volunteers

Pharmacokinetic interaction between ezetimibe/rosuvastatin and telmisartan in healthy male subjects

Effect of multiple oral administration of ds-8500a, a novel g protein-coupled receptor 119 agonist, on the pharmacokinetics of midazolam in healthy japanese subjects

The effect of multiple oral administration of esaxerenone on the pharmacokinetics of midazolam in healthy japanese males

Clinically significant drug-drug interactions in cardiovascular diseases: comparing the prevalence and prescribing patterns between inpatient and outpatient settings

Effect of ds-8500a, a novel g protein-coupled receptor 119 agonist, on the pharmacokinetics of atorvastatin in healthy japanese subjects

Evaluation of herbal medicines used by diabetes patients in nigeria for cyp p450 enzyme induction using human hepatocytes

Bradykinin-mediated angioedema associated with combination of angiotensin-converting enzyme and dipeptidyl peptidase iv inhibitors: a disproportionality analysis from the who database

Impact of the organic cation transporter 2 inhibitor cimetidine on the single-dose pharmacokinetics of the glucosylceramide synthase inhibitor lucerastat Therapeutic drug monitoring for lacosamide in japanese patients with epilepsy

Assessment of the pharmacokinetic interaction between fimasartan and linagliptin in healthy volunteers

The effect of simvastatin on warfarin anticoagulation: a swedish register-based nationwide cohort study.

Measurement of hepatic abcb1 and abcg2 transport activity with positron emission tomography 
Impact of the selective orexin-1 receptor antagonist act-539313 on the pharmacokinetics of the cyp3a4 probe drug midazolam and the endogenous cyp3a4 marker 6 $\beta$-hydroxycortisol

Queries about drug interactions to a drug information centre over a ten-year period - a descriptive analysis

Tolerability and blood pressure effects of a long-acting nitrate co-administered with vericiguat: results of the vericiguat isosorbide mononitrate interaction (visor) study

Placental drug transporter -mediated interactions and fetal malformations

Evidence of metabolic interaction between opioid and benzodiazepine: a study with oxycodone, diazepam and its derivative, diclazepam.

High-dose esomeprazole causes a strong and prolonged mechanism-based inhibition of cyp2c19 while only slightly inhibiting cyp3a 4 and inducing cypla2 activities in vivo

In vitro inhibition of twenty three anti-tuberculosis drugs on six ugt enzymes

Development and validation of a novel high-throughput double cocktail assay for in vitro evaluation of time-dependent inhibition of nine major human cytochrome p450 enzymes

Clinical impact of moderate and several potential drug- drug interactions in medical inpatients. Cohort study

Clinically relevant drug interactions; it's time for education

Drug regulation

Interchangeability of biologicals from a regulatory standpoint: european perspective and croatian experience

Medicines shortages in montenegrin hospitals during one-year period - a reason for new solutions for health authorities?

The journey of a centrally authorised medicine: step by step

Impact of pharmacist interventions on clinical outcome and cost avoidance in a university teaching hospital

Illegal use of anabolic androgenic steroids in sweden. A greater problem here than in the other nordic countries?

Sustainable access to innovative medicines in cancer therapy in Croatia

Experience in approving an eu-funded clinical trial with advanced therapy. Are we really ready for pan-european approval?

Antibiotic multiresistance: the reasons behind the phenomenon. Study on antibiotic knowledge in italy

Drug utilization

Rational use of methylprednisolone for treatment of immune mediated diseases: relationship between dosing, effectiveness and safety

An assessment of the knowledge of prescribers on pharmacovigilance in the south/ west region of Nigeria

Typical drug treatment in an older patient in primary care - a descriptive study applying indicators of prescribing quality as well as an overall medical perspective

Prescribing of newer antiepileptics, with the focus on pregabalin, using the defined daily dose methodology

Antidepressants and anxiolytics/hypnotics used by children and adolescents in four european countries. A real world data prevalence study

Assessing treatment adherence in adults with attention-deficit/hyperactivity disorder: a comparison of proportion of days covered for methylphenidate with or without antidepressant therapy

Indications related to antidepressant drug prescribing in the elderly population during 2010-2015 in two primary care databases: the netherlands and catalonia (spain)

Factors of importance for discontinuation of thiazides associated with hyponatremia in sweden - a population-based register study

Assessment of drug use management good practices in inpatient medical organizations in astana (kazakhstan)

A lc-ms method for the measurement of about 250 compounds of interest in toxicology with a fully-automated sample preparation

Increased risk of clozapine therapeutic failure during comedication with valproic acid - a retrospective drug monitoring study on 2143 patients

Preliminary study on influenza vaccination uptake in the active population

Comparison of biosimilar somatropin utilization in 2018 in croatia with regards to other european countries

The association between treatment with systemic prednisolone and antipsychotic treatment - a symmetry analysis

Off-label use of rituximab in patients with glomerulonephritis in a tertiary hospital: a retrospective observational study

Trends in use and misuse of opioids in the netherlands

Concomitant use of psychotropic drugs in attention deficit hyperactivity disorder (adhd)

Role of adverse drug reaction in transfers from psychiatric wards to emergency unit in geneva university hospitals

Use of psychoactive drugs in paediatric population in catalonia

Death due to suicide in barcelona: a case control study to identify main risk factors and opportunities for intervention.

Simple measures with significant outcomes- how evaluation and education can alter medication order and reduce costs

Investigation of off-label drug use in pediatric patients

Potentially inappropriate medications (pim) in the elderly. Project to create a spanish list (es-pia project)

Use of analgesic drugs among older people in greece consuming more than five drugs daily

Increased prescribing trends of psychotropic medicines in spanish paediatric population. Sensation, perception or reality?

Education

Patient information of magistral preparations using qr codes

Provision of teratology information service centre in nigeria: an opinion survey of doctors in obstetrics and gynecology specialty in lagos, nigeria Key learning outcomes for clinical pharmacology and therapeutics education in europe: a modified delphi study

"helpful to get time and opportunity to discuss drug treatment, that's what

$i$ think is the most important thing" - a qualitative study on key aspects to educate junior physicians in prescribing

Development of prescribing knowledge and skills of junior doctors in the first year after graduation 
Success rate of test questions concerning pharmacokinetics is lower compared to pharmacodynamics in clinical pharmacology teaching in a health and life sciences curriculum

The prescribing safety assessment in irish medical schools: progress to date and attitudes of newly qualified doctors to its implementation.

Fourth-year rational pharmacotherapy clerkship at koç university school of medicine

Clinical pharmacology in the undergraduate medical education: our experience in the context of the european curriculum

Do students want new methods or do we just think they do?

The junior-adverse drug event managers

Clinical and long term effects of a real life problem based pharmacovigilance intervention.

To create the personalized training program model for post-graduated year pharmacists to improve the capability of clinical skills

Clinical pharmacology in continuous medical education (cme) in slovakia: quest for quality and european compatibility

Four decades of clinical pharmacology as a medical specialty in slovakia (1979-2019)

The inter-professional student-led medication review program.

Clinical results of an inter-professional student-led medication review program

Majority dutch registrars in general practice find postgraduate training in prescribing insufficient

The impact of using virtual patients in pre-clinical pharmacology teaching

The attitude of clinical pharmacology and therapeutics educators towards sharing and creating open educational resources: an international survey

Flipped class teaching for clinical pharmacology and therapeutics

Study on expected participant enrollment in prospective interventional studies and analysis on formal sample size calculation

Phase i clinical trials evaluated by the research ethics committee of vall d'hebron university hospital in 2017 and 2018

Predictors of influenza vaccination among the elderly in greece

Sources and use of drug information among physicians in sweden - a descriptive study covering five counties in sweden

Effects of rational pharmacotherapy training on long term prescribing skills of medical students

General clinical pharmacology

Effectiveness of beclomethasone solution for management of gastrointestinal graft-versus-host disease in children

The pharmacotherapy team: a novel multidisciplinary strategy using participatory action research to improve appropriate in-hospital prescribing

Treprostinil iontophoresis in systemic sclerosis-related digital skin ulcers: a single ascending dose safety study

The therapeutic benefits of cannabis and cannabinoids evaluated by phase 3 randomized controlled clinical trials: a systematic review of recent literature

Influence of placebo / nocebo information in informed consent forms and decision to participate in a simulated clinical trial

A new device for direct quechers salts extraction: application to drugs of abuse

Systemic biobanking infrastructure initiative in slovakia - legal and ethical issues

Pregnancies during clinical trials: are we aware of what is actually going on?

Attitude and knowledge on generic drugs in adult outpatients that receive chronic pharmacotherapy

$\mathrm{H} 1$ antihistamines on minimal persistent inflammation in patients with allergic rhinitis

Geriatric treatment

Association of antidepressant use with risk of stroke and mortality in the elderly

Comorbidity and the risk of bleeding from the upper gastrointestinal tract to elderly patients, associated with the peculiarities of pharmacotherapy

The use of potentially inappropriate medications in older adults in quebec, canada: a population-based study

Persistence of potentially inappropriate medications use in older adults

Mortality associated with stopping statins in the oldest old - with and without ischemic heart disease

Analysis of the drug prescribing appropriateness to stopp/start criteria in elderly patients with type 2 diabetes mellitus in the endocrinological department

of a multi-speciality hospital

The structure of prescribing drugs in old and very old patients in a multi-speciality hospital in moscow (russia)

Impact of the anticholinergic burden on cognitive functions in very old patients

Impact of polypharmacy on cognitive functions in eldery patients with essential arterial hypertension and comorbities

Experimental and regulatory studies on adverse effects of some antidepressants in elderly

The importance of correct estimate of renal function for drug treatment in hospitalized geriatric patients: a prospective observational study

Effects of polyphenol-rich aroniamelanocarpa fruitjuice on bone mineral density and pain sensitivity threshold in ovariectomized rats

Intrinsic and extrinsic factors associated with falls, in older adults: a case-control study

The efficacy of an equivalent dose of ephedrine and phenylephrine in the prevention of post-spinal hypotension in senior adults

Health economy .

Ophthalmological dispensing patterns by pharmacies: a basis for health care and economic planning

Antihypertensive drugs: cost effectiveness analysis in Croatia

Misuse of medicines/substances

Adverse reaction to compounded preparations prescribed for weight loss: 2 cases of cerebral venous thrombosis 
High incidence of pharmacotherapeutic situations in the department of psychiatry of the tertiary hospital

Experimental binge drinking episode: are there gender differences in alcohol concentrations and pharmacological effects?

Medical prescriptions falsified by the patients before and after july 12th, 2017 when codeine, dextromethorphan, ethylmorphine and noscapine were scheduled in france

The 2018 state of the art on the cardiovascular risks of cannabis-based products: a systematic review

Cannabis-related cardiovascular outcomes observed in hospitalized inmates

Prescription drugs in fatal accidents - prescribed or not?

A pilot study: use of the adult adhd-self report scale in a south african patient population

The contribution of methadone and buprenorphine/naloxone maintenance treatment for opiate dependence to hiv prevention from 2009 to 2018 in taiwan

Nephropharmacology

The effects of co-administration of cholinergic and cannabinoid receptor ligands on different memory stages in mice

Acute renal injury in a colistin and tobramycin-treated patient with pseudomonas aeruginosa

Patient empowerment

Placebo effect and the incidence of anxiety and depression in patients with drug allergy

Quality of life in patients undergoing testing for drug allergy - with emphasis on placebo reactors

Pediatric treatment

Evaluation of efficacy and safety of human normal immunoglobulin after the change of administration route from intravenous to subcutaneous in pediatric patients

An artificial intelligence tool to find the best cyclosporine initial intravenous dosing regimen in pediatric hematopoietic stem cell transplantation

Switching from intravenous to oral cyclosporine after hematopoietic stem cell transplantation: an artificial intelligence tool

Anti-d or intravenous immunoglobulins for immune thrombocytopenia in children: systematic review and meta-analysis

An innovative ethosuximide granule formulation designed for paediatric use: comparative pharmacokinetics, safety, tolerability and palatability profile

vs syrup

Preparation of pediatric dosage forms in hospital pharmacy

Global trigger tool to measure drug safety in adolescents with acute psychotic episode: the first experience in child psychiatry

Investigation of nervous system drugs in paediatrics

The association between drug use and childhood overweight and obesity, in denmark. A case-control study

\section{Pharmacogenetics/omic}

Development and testing of a clinical decision support system for dosing in psychopharmacotherapy in patients with affective disorders based on the pharmacogenomic markers

Influence of abcb1, cyp3a4 and cyp3a5 genes polymorphisms on prothrombin time and rivaroxaban plasma concentration in patients after total hip or knee replacement surgery

Evaluating tacrolimus pharmacokinetic models in tunisian renal transplant patients

Population pharmacokinetic-pharmacodynamic modelling of metoprolol with regard to cyp2d6 genotypes in healthy korean volunteers

An impact of cyp3a4 genetic polymorphisms on the efficacy and safety of diazepam therapy in patients with alcohol use disorders

Influence of ace gene polymorphisms on angiotensin converting enzyme activity in thai population

A genome-wide association study of bisphosphonate-associated atypical femoral fracture

Effect of cyp3a5*3 polymorphism on anti-inflamatory therapy in children with bronchial asthma

Impact of fto genetic variability on psychological traits of patients with eating disorders

Influence of cyp450 genetic variability on the donor kidney biopsy score and consequences on clinical outcomes in renal transplant recipients

Effect of cyp3a5 and abcb1 polymorphisms on dose requirement and trough concentration of tacrolimus in renal transplant recipients

Implementing pre-emptive pharmacogenetic screening into clinical routine

Genotype and phenotype frequencies of isoniazid-metabolizing enzyme nat2 in yakutian and russian tuberculosis patients

Variants of fibroblast growth factor 21 gene affect dietary salt intake: potential implication on antihypertensive therapy

The polymorphisms frequencies of cytochrome $\mathrm{p} 450$ and abcb1 genes in russian patients

Variants of fibroblast growth factor 21 gene affect dietary salt intake

Crispr-cas9 enrichment and minion® nanopore sequencing: an innovative and effective tool for cyp3a4-cyp3a5 pharmacogenetics

Combinations of abcb1 and cyp 450 genetic polymorphisms as predictors of intolerance to benzodiazepine tranquilizer phenazepam

Abcb1 gene and benzodiazepine tranquilizer phenazepam's safety: haplotype analysis

Correlation between abcb1 gene polymorphisms, antiepileptic drug concentrations and the response to treatment

Pharmacogenetic predisposition for adverse reactions of tyrosin kinase inhibitors: a case report of a patient with chronic myeloid leukaemia

Rs5219 polymorphism in kcnj11 gene as a factor of response to metformin treatment

Pharmacokinetics/Pharmacodynamics

Pharmacokinetics of ertapenem in patients with urosepsis

Population pharmacokinetics and monte carlo simulations of imipenem to optimize dosage regimens in patients with severe infections during support with extracorporeal membrane oxygenation

Adaptation of alanine aminotransferase elevations with therapeutic doses of acetaminophen: an exploratory study in healthy subjects 
Pharmacokinetics of methylphenidate in plasma, exhaled breath, oral fluid and dried blood spots after a single oral dose of ritalin $20 \mathrm{mg}$

Safety, tolerability, and pharmacokinetics of tac-302 and food effects on tac-302 in healthy participants: randomized, double-blind, placebo-controlled, single-dose and multiple-dose studies

Estimated exposure to dabigatran etexilate influences efficacy and toxicity

Relationship between digestive function abnormality and blood levels of gastrointestinal peptides in patients receiving anticancer agents combined with aprepitant

Antidepressant-induced hyponatremia: a pharmacoepidemiological-pharmacodynamic analysis of suspected adverse drug reactions in the us-faers database

Targeting mitochondrial biogenesis to attenuate chronic low-grade inflammation in obesity

Pyrroloquinoline quinone counters the pcsk 9 induction associated limitations of atorvastatin: a pre-clinical study

Risk assessment in human using physiologically based pharmacokinetic model of diethyl phthalate and its major metabolite, monoethyl phthalate

Single-dose pharmacokinetics and tolerability of aprocitentan, a dual endothelin receptor antagonist, in subjects with severe renal function impairment

Naltrexone co-administration does not affect the pharmacokinetics of oxycodone

Pharmacokinetics and safety of evogliptin in patients with hepatic impairment

Effects of switching from racemic methadone to r-methadone on serum methadone concentrations and qtc intervals

Evaluation of perfluorohexanoic acid exposure using physiologically-based pharmacokinetic modeling and simulat

Use of a physiologically based pharmacokinetic linked pharmacodynamic (pbpk-pd) model to account for adjustments to dosing of s-warfarin in different populations

Quantification of anidulafungin and micafungin in wound secretion

Nonlinear pharmacokinetics and concentration-effect relationship of rituximab in anti-neutrophil cytoplasmic antibody associated vasculitis

A pharmacokinetic comparison between two formulations of fixed-dose combination of esomeprazole and sodium bicarbonate (ckd-381) and esomeprazole in healthy male subjects

The evaluation of prokinetic effect of low-dose sulpiride administration from the viewpoint of plasma brain-gut peptide levels in healthy human

Sex-by-formulation interaction in bioequivalence trials

Investigation on the existence of sex-by-formulation interaction in bioequivalence trials

Clopidogrel and gemfibrozil pronouncedly inhibit the cyp2c8-mediated 3-hydroxylation of desloratadine and increase desloratadine exposure in humans Population pharmacokinetics of cycloserine in patients with multidrug-resistant tuberculosis

Modeling of hysteresis phenomena in pharmacodynamics using nonlinear partial differential equations

Anal acoustic reflectometry can detect pharmacologically induced pressure changes in the anal sphincter: a randomized, double-blind, placebocontrolled crossover study with imipramine

Association between pain, variability and clinical efficacy in opioid analgesia

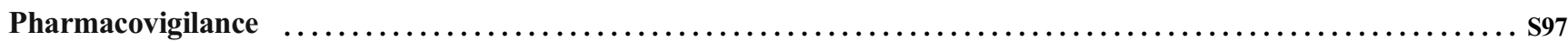

Mobile application for adverse drug reaction reporting by patients with relapsing remitting multiple sclerosis (vigipsep study): a national randomized controlled trial

The general practitioner's knowledge and attitude towards proton pump inhibitors adverse effects.

Analysis of adverse drug reactions of calcium channel blockers. Retrospective analysis of pharmacovigilance database

Incretin-based drugs and intestinal obstruction: analysis of the world health organization's adverse drug reactions database

Dress syndrome: analysis of 90 cases over 15 years

Association of liver cirrhosis with drug exposure: a case-control analysis

Patients knowledge and attitudes towards the yellow card scheme in greece

Case reports of non-arteritic anterior ischaemic optic neuropathy with phosphodiesterase type 5 inhibitors: a systematic review

Medication errors and near miss events in the finnish tertiary care hospitals

Heterogeneity between odds ratios and reporting odds ratios

Pulmonary arterial hypertension associated with protein kinase inhibitors: a pharmacovigilance-pharmacodynamic study

Observational, descriptive and retrospective study of the assessment of drug's adverse reactions in a spanish tertiary hospital

The effect of pharmacological antipyresis on mortality in patients with sepsis

Biosimilars

Drug-induced livery injury in patients with underlying chronic hepatic diseases

¿what about admission by adverse reactions in our hospital?

Medication errors

Severe hypersensitivity reactions associated with iron (iii) isomaltoside and ferric carboxymaltose. Analysis of eudravigilance database

Tramadol use in denmark, norway, and sweden: a population-based study

Accuracy of drug hepatotoxicity potential classifications in assessing severity related to drug-induced liver injury (dili)

Clozapine associated pneumonia. A disproportionate analysis of vigibase

Safety of immunomodulatory checkpoint inhibitors (icis): a critical look at available real-world evidence

The influence of sex hormones on drug-induced liver (dili) clinical presentation

Attitudes, knowledge and behaviors of pharmacists about adverse drug reactions reporting through yellow card

Pregnancy and infant outcomes after maternal exposure to rituximab

Real-world efficacy and safety of pharmacologic prophylaxis for clinically significant venous thromboembolism among non-surgical hospitalized patients

Psychopharmacology

Genetically determined gaba metabolism is associated with benzodiazepines' effectiveness in alcohol withdrawal syndrome

Physician specialty and antidepressant prescription: an analysis of the national e-prescription database of greece 
Nonadherence rates of antipsychotic drugs - a retrospective study based on blood sample analyses of 24,093 patients

Effect of short-term treatment with olanzapine and aripiprazole on prolactin in healthy subjects

Rare diseases

S107

Which opioids in case of mast cell activation disorders?

Use of sirólimus $0.4 \%$ ointment in vascular malformations

Therapeutic drug monitoring

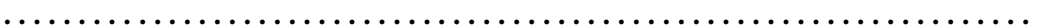

S108

High intra-patient variability in tacrolimus exposure is not associated with immune-mediated graft injury after liver transplantation

A validated lc-ms/ms method for simultaneous quantitation of tolvaptan and its monohydroxylate enantiomers in human plasma using a reversed-phase separation mode

Impact of endogenous serum immunoglobulin $g$ on serum level of denosumab in cancer patients

Pharmacological monitoring of therapeutic monoclonal antibodies: from the proof-of-concept of their quantifications by mass spectrometry to bedside personalized dosing 


\section{Advanced therapies}

\section{EACPT-1358}

Outpatient parental antimicrobial treatment; an antibiotic stewardship tool

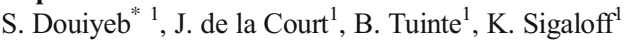

${ }^{1}$ Internal Medicine, Amsterdam VUmc, Amsterdam, Netherlands

\section{Advanced therapies, Antimicrobials, Therapeutic drug monitoring}

Introduction: Outpatient parental antimicrobial treatment (OPAT) is proven to be safe, cost-effective and enhances patients satisfaction by facilitating shorter hospital stay. The emergence of antimicrobial resistance limits available oral antibiotic options, consequently increasing the demand and complexity of OPAT applications. Consultation of an antibiotic stewardship team has the potential to optimize the choice of antimicrobial therapy and treatment monitoring of patients applicable for OPAT. We developed an antibiotic stewardship team, the OPAT-team, consisting of an infectious disease expert, microbiologist, pharmacologist and two physicians, which are responsible for intravenous catheter placement and coordinating and monitoring of patients discharged with OPAT.

Objectives: The aim of this study was to evaluate the effect of implementation of an antibiotic stewardship team in the organization and coordination of OPAT. We describe preliminary findings.

Methods: A mixed, single center observational study was conducted at the Amsterdam UMC, location VUmc. Patients were retrospectively identified from January 2016 until January 2019. Starting from February 2019 all patients which required outpatients parental antibiotic treatment, were subjected to the OPAT-team program. Inclusion criteria consisted of consenting adults with active infections which required antibiotic or antifungal intravenous therapy. Outcomes included the duration of hospitalization, complications during outpatient treatments and treatment alteration advised by an OPAT-team.

Results: Forty-four patients required outpatient antimicrobial therapy in 2016 versus 130 patients in 2018. A total of 7 patients were subjected to a consultation of the antibiotic stewardship team. The average duration in days between consultation of the OPAT team and placement of the PICC line was 1.2 days. The average duration from consultation of the OPAT-team until actual discharge was 3 days. A total of 2 complications were detected, consisting of an suboptimal antibiotic dosage and a possible oral switch, in which the OPAT-team intervened.

Summary / Conclusions: With the introduction of an expert OPAT team patients are able to receive an intravenous catheter on a short-term and are more likely to be closely monitored. This could lead to reduction of adverse drug events and hospital costs. More data will be available in Stockholm.

\section{Antimicrobials}

\section{EACPT-1053}

Irrational use of antibiotics and clinical outcomes in children with infectious diseases

S. Kasciuškevičiūte* ${ }^{*}$, 2 , E. Karinauske ${ }^{2}$, V. Galaune ${ }^{2}$, A. Usaite ${ }^{1}$, S. Stankeviciute $^{3}$, R. Maciulaitis ${ }^{2}$

${ }^{1}$ Physiology and Pharmacology Institute, ${ }^{2}$ Nephrology Department, Lithuanian University of Health Sciences, ${ }^{3}$ Hospital of Lithuanian University of Health Sciences, Kaunas, Lithuania

\section{Antimicrobials, Misuse of medicines/substances, Pediatric treatment}

Introduction: The prescription of antibiotics $(\mathrm{AB})$ in children is problematic. Even viral infections are treated with ABbecause doctors have a fear of misdiagnosis and complications. Also, there is pressure from the parents to prescribe $\mathrm{ABs}$
Objectives: To evaluate the rationality of antibiotic use and clinical outcomes in children.

Methods: This was a retrospective cohort study which included children born in 2014-2015 who were diagnosed with any infections for the first time in their lives and were prescribed antibiotics or symptomatic treatment. Data were obtained from medical records. All children were divided into three groups according to the treatment prescribed: symptomatic $(\mathrm{S})$, the narrow-spectrum $\mathrm{AB}(\mathrm{NS})$, broad-spectrum $\mathrm{AB}(\mathrm{BS})$. All medical records from the date of the first episode of the infection to the date of evaluation (follow-up period) was analyzed to determine clinical outcomes.

Results: 180 children (104 boys, 76 girls) were included in the study. The median age was 6 months. The median follow-up duration was 39 months. The most common infections were acute nasopharyngitis, pharyngitis, tonsillitis and bronchiolitis. 34 out of 180 children did not get any antibiotics and all these cases were judged as rational. 77 out of 180 children and 69 out of 180 children were prescribed narrow and broad spectrum antibiotic, respectively. Only 9 out of 77 and 2 out the 69 children were diagnosed with bacterial infections and the use of antibiotics were evaluated as rational. So, the rational use was determined in 45 out of the 180 children (25\%). 122 out of 180 children's $(67.8 \%)$ treatment was evaluated as "rationality is not known because the antibiotic was not indicated" and 5 out of $180(2.8 \%)$ was evaluated as "rationality is not known because antibiotic prescribed was broad spectrum". The BS children had more subsequent episodes of new infections (mean (SD)) during the follow up period compared to the NS and S children - 8.0 (5.2) vs. 4.5 (3.4) vs. 2.2 (2.4), respectively, p<0.05. However, the frequency (mean $\pm \mathrm{SD}$ ) of hospitalization due to those infections did not differ between NP $(0.5 \pm 0.7)$, NS $(0.58 \pm 1)$ and BS $(0.71 \pm 1.2)$ groups, $\mathrm{p}>0.05$. Summary / Conclusions: The use of broad spectrum antibiotics may be linked with increased risk of other infections developing. The rational use of antibiotics is expected to be from 3 to 100 percent in the investigated population.

\section{Oral Presentation}

\section{EACPT- 1080}

A link between antibiotic resistance and antibiotic consumption in greece, serbia and norway

A. Raskovic ${ }^{*}{ }^{1}$, N. Stilinović ${ }^{1}$, M. Ubavić ${ }^{2}$, M. Paut Kusturica ${ }^{1}$, N

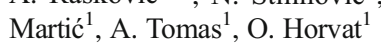

${ }^{1}$ Pharmacology, Toxicology and Clinical Pharmacology, School of Medicine, University of Novi Sad, ${ }^{2}$ Faculty of Pharmacy Novi Sad, Novi Sad, Serbia

\section{Antimicrobials}

Introduction: Emergence of antimicrobial resistance in microorganisms is a natural phenomenon; yet antimicrobial resistance selection has been driven by antimicrobial exposure in health care, agriculture, and the environment. Recent evidence comes from meta-analyses report positive associations between antimicrobial consumption and the development of resistance at both population and individual levels.

Objectives: The aim of this study was to measure the consumption of antimicrobial drugs in Greece, Serbia and Norway from 2012 to 2015 and to evaluate relationship between that consumption and resistance of selected bacterial strains in same countries.

Methods: This was a retrospective, observational, cross-sectional, population-based study of routinely collected data for consumption of antimicrobial drugs (from 2012 to 2015) and national antimicrobial resistance rates in Greece, Serbia and Norway (2014).

Results: The results for year 2015 have shown that Norway is the country with the lowest consumption of antimicrobial drugs, followed by Serbia and finally Greece. Results for trends in antibiotics use it can be noticed that both Greece and Serbia have increasing rates of consumption in the years 2012-2015, whereas Norway has a decreasing tendency. From the analysis of antibacterials subgroups it can be seen that beta lactam/ penicillins are the drugs of choice in all three countries. Furthermore, 
results from this study confirmed the relationship between community antimicrobial consumption and serious resistant infections in patients.

Summary / Conclusions: This study has emphasized the strength of relationship between community consumption rates and antimicrobial resistance rates in E. coli, Acinetobacter spp. and MRSA.

This work was supported by Ministry of Education, Science and Technological Development, Republic of Serbia (project No 41012).

\section{EACPT-1405}

Is selective decontamination of digestive tract justified in hospitalized infants

K. Demidenko ${ }^{1}$, K. Zagorodnikov ${ }^{*} 1$

${ }^{1}$ Almazov National Medical Research Centre, Saint Petersburg, Russian Federation

\section{Antimicrobials}

Introduction: Bacterial translocation (BT) has been discussed as one of the cause of generalized infection (GI). Children in their early postnatal age are considered to be at increased risk for this. Despite limited evidence of possible role of selective intestinal decontamination (SID) in reducing risk of GI, there is still controversy regarding its necessity.

Objectives: Our aim was to describe intestinal flora and prevalence of bacterial translocation (BT) in pediatric population of hospitalized patients under one year of age.

Methods: We analyzed all intestinal microbiology and sterile loci derived from hospitalized patients under 1 year of age at the neonatal pathology, surgery and intensive care units during last 3 years. The cases were intestinal microbiology was evaluated prior to and not later than 7 days before the sterile loci were included into analysis. BT was diagnosed when the microorganisms (MO) isolated from the blood sample were also isolated from the intestines at least 7 days earlier.

Results: out of 1274 cases 954 were eligible for analysis according to the criteria. Sterile loci were positive in $16 \%$ (151) of patients, designating presence of GI. We excluded 78 cases where positive cultures with either the same (25) or not the same (48) MO due to inability to establish causality. BT was identified in 3\% (34) of cases. Among all patients with generalized infection (73) it was caused by the MO previously isolated from intestines in $35 \%$ (25) patients. Klebsiella spp. was the most prevalent pathogen in overall study population (35\%) followed by Enterococcus spp (23\%), Enterobacter spp (13\%), CoNS (17\%) and S.aureus (10\%) in general population. Among those with BT equally common pathogens were Klebsiella spp, S.aureus, CoNS (20\% each), followed by Enterococcus spp (16\%). Among carriers of selected pathogens S.aureus was associated with infection in $7 \%$ of cases, CoNS - in 4\%; Klebsiella spp and Enterococci - in 2\%.

Summary / Conclusions: overall incidence of BT was rather low and can not justify SID in all hospitalized patients. It is valuable however to monitor intestinal flora because it may be related to generalized infection in $35 \%$ of cases, and may guide initial drug choice. Despite growing concern regarding infections caused by Klebsiella spp in hospitalized patients S.aureus remains the most virulent pathogen more frequently associated with infections through BT.

\section{Oral Presentation}

EACPT-1088

Co-trimoxazole induced hyperkalemia and potassium monitoring in hospitalized patients

M. Plantaz ${ }^{*}$, B. Veldman ${ }^{2}$, A. Esselink ${ }^{2}$, H. Fleuren ${ }^{1}$, C. Kramers ${ }^{1,2,3}$ ${ }^{1}$ Clinical Pharmacy, ${ }^{2}$ Internal Medicine, Canisius Wilhelmina Hospital, ${ }^{3}$ Clinical Pharmacology - Toxicology, Radboudumc, Nijmegen, Netherlands

\section{Antimicrobials, Pharmacovigilance}

Introduction: Co-trimoxazole is an antibiotic combination used for the treatment and prophylaxis of Pneumocystis jiroveci pneumonia, as well as for the treatment of other infections. Co-trimoxazole is known to increase serum potassium by blocking of the epithelial sodium channel in the distal nephron of the kidney, inhibiting sodium reabsorption and subsequently decreasing potassium excretion. For this reason, Dutch guidelines advise regular serum potassium monitoring in patients who are at a high risk for hyperkalemia.

Objectives: This study aims to determine average serum potassium rise after administration of intravenous co-trimoxazole in hospitalized patients, compared to intravenous ceftriaxone. We investigated also if serum potassium monitoring took place during intravenous co-trimoxazole therapy.

Methods: We collected and compared data from patients that received intravenous co-trimoxazole $(\mathrm{n}=66)$ and intravenous ceftriaxone $(\mathrm{n}=$ 132) in the Canisius Wilhelmina Hospital in the period of November 2008 - November 2017. We collected baseline potassium and followup potassium, if available. Additionally, we tested how often serum potassium was measured before and after antibiotic therapy.

Results: When compared to ceftriaxone, administration of intravenous co-trimoxazole was associated with a significant mean increase in serum potassium $(+0.55 \mathrm{mmol} / \mathrm{L}, 95 \%$ CI $0.29-0.80, \mathrm{p}<0.001)$. After correction for baseline serum potassium, renal function, the presence of haematological malignancies and usage of corticosteroids, this effect shrunk noticeably, but remained significant $(+0.28 \mathrm{mmol} / \mathrm{L}, 95 \% \mathrm{CI}$ $0.03-0.53, p=0.031$ ). Serum potassium measurements in the period of 48-120h after antibiotic initiation were significantly more frequent in patients using co-trimoxazole when compared to patients using ceftriaxone $(55 \%$ and $34 \%, p=0.006)$. However, $45 \%$ of patients using intravenous co-trimoxazole had no serum potassium measured in this period. From the 19 patients who used co-trimoxazole in combination with other potassium-increasing medication, $13(68 \%)$ had a serum potassium measurement in the period of 48-120h after antibiotic initiation.

Summary / Conclusions: Intravenous co-trimoxazole usage is associated with a significant mean increase in serum potassium, and therefore serum potassium monitoring is indicated, especially in high risk patients. However, serum potassium monitoring during co-trimoxazole therapy is often not performed.

\section{EACPT-1442}

Impact of voriconazole therapeutic drug monitoring (tdm) on toxicity avoidance in immunocompromised hematological patients

G. Vaca Recalde ${ }^{*}{ }^{1}$, R. Nogueiras Álvarez ${ }^{1}$, I. Mazón Maraña ${ }^{1}$, A. Illaro Uranga $^{2}$, L. Yáñez San Segundo ${ }^{3}$, N. Vega Gil ${ }^{1}$, A. Bautista Blázquez ${ }^{1}$, D. Z. Cuéllar Gómez ${ }^{1}$, P. Ortiz Petrosino ${ }^{1}$, M. B. Sánchez Santiago ${ }^{1}$

${ }^{1}$ Clinical Pharmacology, ${ }^{2}$ Hospital Pharmacy, ${ }^{3}$ Hematology, Hospital Universitario Marqués de Valdecilla, Santander, Spain

\section{Antimicrobials, Therapeutic drug monitoring}

Introduction: Voriconazole is a triazole antifungal developed for the prophylaxis or treatment of life-threatening fungal infections in immunocompromised patients, and has a nonlinear pharmacokinetic profile with a wide inter and intraindividual variety. This variability could be due to gender, age, genotypic variation, interactions, and liver dysfunction. TDM may be indicated to guide dosing resulting in better outcomes or fewer side effects.

Objectives: An association between serum trough levels of voriconazole, and adverse events has been reported. We aimed to analyse the impact of TDM in guiding voriconazole dosing and on toxicicy avoidance, in immunocompromised patients at the Haematology Service of our hospital. Methods: Medical Charts from 180 haematological patients who received voriconazol from May 2012 to January 2019 (identified in the electronic prescription tool), were reviewed for: indication of voriconazole, use of TDM to guide treatment, presence of toxicity, neurotoxicity, interactions, other triggering factors for toxicity. Serum voriconazole trough levels had been measured using a validated highperformance liquid chromatography method, 5 days after initiation or earlier if presence of toxicity. Desired target levels: $1.0-5 \mathrm{mg} / \mathrm{L}$ 
Results: 79 patients were under active treatment due to fungal infection (30 invasive fungal infections, and 49 other types) and 101 patients were under prophylaxis for IFI. Of the patients with active treatment only 31 (39\%) went under TDM. 12 patients (15\%) had toxic levels at first measurement with clinical symptoms except one (neurotoxicity and hepatotoxicity: 8 and 3 cases respectibily). Toxicity disappeared after dose adjustment in 8 cases, the rest had other uncontrolled causes. Of the patients under prophylaxis, 14 (14\%) went under TDM; Two had toxic levels at first measurement with clinical symptoms of neurotoxicity that stopped after adjustment. In patients not controlled with TDM (135/180), 23(17.03\%) suffered Symptoms compatible with toxicity (neurological in 12, hepatic in 9, 2 other). In all these cases, no voriconazole associated toxicity was suspected, neither confirmed.

Summary / Conclusions: TDM seems to be a good diagnostic tool in patients with clinic suspicion of voriconazole toxicity, although data suggest that there may be an underdetection of the toxicity that delays the dose adjustment due to underuse of this tool.

\section{Oral Presentation \\ EACPT-1453}

Interindividual variability in plasma levels of beta-lactam antibiotics in critically ill patients with or without continuous renal replacement therapy

E. Eliasson ${ }^{*}{ }^{1}$, C. Giske ${ }^{2}$, J. Petersson ${ }^{3}$

${ }^{1}$ Laboratory Medicine, Clinical Pharmacology, ${ }^{2}$ Laboratory Medicine, Clinical Microbiology, ${ }^{3}$ Physiology and Pharmacology, Section for Anesthesiology and Intensive Care, Karolinska Institutet and Karolnska University Hospital, Stockholm, Sweden

\section{Antimicrobials, Intensive care treatment, Pharmacokinetics/ phar- macodynamics, Therapeutic drug monitoring}

Introduction: Serious infections within intensive care are associated with a 30\% mortality. One contributory factor may be suboptimal use of antibiotics with a need for better individualisation of dose regimens.

Objectives: Characterisation of meropenem and piperacillin pharmacokinetics was carried out in critically ill patients with $(n=135)$ or without $(n=39)$ continuous renal replacement therapy, CRRT.

Methods: Patients were subject to sampling in the mid- or end of the dose interval after recommended standard doses of the beta-lactam antibiotics. Plasma concentrations of piperacillin and meropenem were determined by a quality-accredited LC-MS/MS method. Concentrations were compared to the upper break-point of $S$-classified Pseudomonas aeruginosa (MIC 16 and $2 \mathrm{mg} / \mathrm{L}$, respectively) after correction for protein binding of piperacillin. Results: At standard doses of piperacillin (4 g t.i.d.) or meropenem ( $1 \mathrm{~g}$ t.i.d.) there was striking inter-patient variability in plasma exposure levels that correlated surprisingly poorly to established markers of kidney function or CRRT flow. In patients without CRRT, $80 \%$ of patient met fT $>50 \%$ with regards to the pseudomonas cut-off, but only $40 \%$ of patients reached $\mathrm{fT}>\mathrm{MIC} 100 \%$. The corresponding data for meropenem was $89 \%$ and $42 \%$. As expected, antibiotic exposure levels were higher in patients with ongoing CRRT, but still a significant fraction did not reach fT $>$ MIC $100 \%$ for piperacillin (22\%) and meropenem $(6,5 \%)$.

Summary / Conclusions: Substantial inter-individual variability in plasma levels without any good correlation to kidney function estimates, indicates that therapeutic drug monitoring may be required to secure sufficiently high plasma levels of beta-lactam antibiotics in the critically ill. Four daily doses may prove to be a more efficient start of therapy before the MIC of the infective agent is known.

\section{EACPT-1147}

Automated antimicrobial consumption data visualization for targeted stewardship

J. De La Court ${ }^{*}$, J. Prehn ${ }^{1}$, T. Groot ${ }^{1}$, A. M. Kaiser ${ }^{1}$, A. Budding ${ }^{1}$, R. de jong $^{1}$, K. Sigaloff ${ }^{2}$
${ }^{1}$ Medical microbiology and infection prevention. Department of infectious diseases, ${ }^{2}$ Internal medicine, infectious diseases, Amsterdam UMC, Amsterdam, Netherlands

\section{Antimicrobials, Computerized decision support}

Introduction: Monitoring antimicrobial consumption is the cornerstone of antimicrobial stewardship and is historically done by cost and timeexpensive point prevalence surveys.

Objectives: Creating an automatically updated visualization of antimicrobial consumption data can facilitate antibiotic stewardship programs with minimal additional effort of the user.

Methods: Using the electronic health record system, a database was constructed that refreshed automatically every 48 hours. Antimicrobial consumption data was based on the ATC classification system. Every antimicrobial administration recorded was included. Days of therapy, defined as administration of a single agent at least once that day, was used as the numerator. To assess total treatment duration, uninterrupted days of therapy with the same agents for the same patient were added. For the denominator, admission days during which a given patient was admitted to a given department, were calculated. This allowed visualization of days of therapy versus admission days. Data validation was performed using historical point prevalence data and checking individual patient records. Results: Visualizations were presented as dynamic web based graphs, available for all medical microbiologists, infectious disease specialists, infection prevention experts and antibiotic stewardship team members. Different graphs were plotted: 1 . The distribution of antimicrobial consumption over different departments 2. Antimicrobial consumption over time. The latter visualisation was used to assess compliance to our recently updated surgical prophylaxis protocol (cefuroxim was replaced by cefazolin) 3. Treatment duration, allowing the detection of prolonged uninterrupted antibiotic treatment. 4. Use of last-resort antibiotics, stratified by. All data are automatically updated and can be consulted every day. In comparison with the point prevalence surveys, this automated visualization of data increased the frequency in which antimicrobial consumption data could be evaluated from once a year to every day.

Summary / Conclusions: Dynamic web based visualization of antimicrobial consumption data allows us to assess changes in antimicrobial usage and differences between departments on a daily basis. With this, the effect of our antibiotic stewardship programs can be continuously monitored. This allows immediate response to intra-hospital antimicrobial consumption changes, in a cost and time-effective manner.

\section{EACPT-1153}

Adverse effects of bedaquiline in patients with extensively drugresistant tuberculosis (xdr-tb)

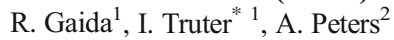

${ }^{1}$ Drug Utilization Research Unit (DURU), Department of Pharmacy, Nelson Mandela University, ${ }^{2}$ Eastern Cape Department of Health, Department of Health, Port Elizabeth, South Africa

\section{Antimicrobials}

Introduction: The World Health Organisation (WHO) Guidelines recommend that, due to the resistance patterns of extensively drug-resistant tuberculosis (XDR-TB) and its unique mechanism of action, bedaquiline be included in the regimen. While the results of clinical trials have shown bedaquiline to be beneficial, it also carries the risk of adverse effects, some potentially life-threatening.

Objectives: The study aimed to determine the incidence of adverse effects caused by bedaquiline in patients diagnosed with XDR-TB. The subsequent management of these adverse effects were also analysed.

Methods: Medical records of 30 patients aged 18 years or older living with XDR-TB who were prescribed bedaquiline in combination with a background regimen at a public sector drug-resistant (DR)-TB hospital in the Eastern Cape, South Africa were reviewed during September 2016. 
Results: Female patients constituted $66.67 \%(n=20)$ of the sample. Nearly half $(46.67 \%, \mathrm{n}=14)$ of the patients were living with HIV and $42.86 \%$ of them were female. Adverse effects were recorded for 26 patients $(86.67 \%)$. QTc prolongation was the most commonly experienced adverse effect and was recorded for 12 patients $(40.00 \%)$ with two of them experiencing persistent QTc prolongation. The QTcF was calculated using Fridericia's formula. Half of these patients required no clinical intervention as the prolongation was not deemed to be clinically significant. The first line of management employed by clinicians at the study site was to omit subsequent doses of bedaquiline. The number of omitted doses varied between patients based on the actual QTcF value ranging from one, to a maximum of three, skipped doses while patients were monitored using ECGs. Skin rashes ( $33.33 \%$ of patients) caused by bedaquiline therapy were managed using combinations of a systemic antihistamine (chlorpheniramine), topical steroid therapy (betamethasone cream) and a topical moisturiser (aqueous cream). Hyperlactataemia was observed in $10(33.33 \%)$ patients. There were no treatment discontinuations or deaths.

Summary / Conclusions: All patients completed the 24 weeks of bedaquiline treatment, indicating that the adverse effects did not require discontinuation of the drug. However, when pharmacological intervention is required for the management of adverse effects care should be taken to ensure that there is minimal interaction with other TB drugs and a low risk of further adverse effects.

\section{Oral Presentation}

\section{EACPT-1226}

Cushing's syndrome related to interaction between ritonavir or cobicistat and corticosteroid: a case control study in the french pharmacovigilance database

Sophie FEDRIZZI ${ }^{*}$, P. Besnier ${ }^{2}$, L. Demessine ${ }^{1}$, M. Biour ${ }^{3}$, D. HilaireBuys $^{4}$, C. de Canecaude ${ }^{5}$, S. Fedrizzi ${ }^{2}$, J.-J. Parienti ${ }^{6}$

${ }^{1}$ Pharmacovigilance Clinical Research, ${ }^{2}$ Centre Regional de Pharmacovigilance, University Hospital Center of Caen Normandy, Caen, ${ }^{3}$ Centre Regional de Pharmacovigilance, APHP, Paris, ${ }^{4}$ Centre Regional de Pharmacovigilance, CHU Montpellier, Montpellier, ${ }^{5}$ Centre Regional de Pharmacovigilance, CHU Toulouse, Toulouse, ${ }^{6} \mathrm{Clinical}$ Research, University Hospital Center of Caen Normandy, Caen, France

\section{Antimicrobials, Drug-drug interactions, Pharmacovigilance}

Introduction: People living with HIV (PLWH) are prone to being exposed to drug-drug interactions (DDI). In a previous work based on French Nationwide Healthcare Database, we found that among PLWH aged more than 65 years old, $8 \%$ were concerned by the combination of a booster with inhaled corticosteroids. The DDI between boosters and corticosteroids is known since 1999, and Liverpool website recommends to "Do not co-administer" boosters and corticosteroids that are metabolized substrates of hepatic CYP3A4. The DDI leads to an increase in exogenous corticosteroid plasma concentration that can result in steroid accumulation, adrenal suppression, and iatrogenic Cushing's syndrome (CS).

Objectives: To explore this signal, we aimed to conduct an analysis of $\mathrm{CS}$, as an adverse reaction due to the corticosteroid toxicity, in the French Pharmacovigilance Database (FPVD).

Methods: We conducted a retrospective case control study describing all CS recorded in the FPVD between 1996 (Norvir® market authorization) and 2018. We defined cases as CS involving booster and controls as CS without booster. Data concerning booster, seriousness, evolution and date of CS, corticosteroid and its route of administration and the duration of the DDI were described.

Results: Among 139 CS included, 34 (25\%) involved a booster (cases): ritonavir (31) between 2001 and 2015 or cobicistat (3) between 2016 and 2018, and 105 involved a corticosteroid alone (controls). Among cases and controls, 30/34 (88\%) and 62/105 (59\%) were serious, respectively, corresponding to a statistically significant association (Odds Ratio $=5.1$, 95\% Confidence Interval [1.6 to 21.2], $\mathrm{p}=0.002)$. More cases $(6 / 16,37 \%)$ than controls $(7 / 33,21 \%)$ had a not resolved outcome although this difference was not statistically significant $(\mathrm{p}=0.304)$. The most commonly involved corticosteroid was inhaled fluticasone (26), secondly injected triamcinolone (3) and thirdly inhaled budesonide (2). Prior to CS, duration of exposure to combination was 9 months (range [14 days -3 years]). Summary / Conclusions: In our study, ritonavir was first involved in the DDI and then cobicistat. This is coherent with the prescriptions' trend and cobicistat approval in Europe only in 2013. Clinicians are reminded to be vigilant: DDI with ritonavir is well-known but DDI with cobicistat is still not mentioned by the manufacturer, only "precaution" is mentioned.

\section{Oral Presentation}

EACPT-1242

The effect of flucloxacillin on warfarin anticoagulation: a swedish register-based nationwide cohort study

B. Mannheimer ${ }^{1}$, T. Bjerregaard Stage ${ }^{2}$, A. Pottegård ${ }^{2}$, J. Lindh $^{*} 3$

${ }^{1}$ Clinical Science and Education at Södersjukhuset, Karolinska Institutet, Stockholm, Sweden, ${ }^{2}$ Department of Public Health, University of Southern Denmark, Odense, Denmark, ${ }^{3}$ Clinical Pharmacology, Karolinska University Hospital, Stockholm, Sweden

\section{Antimicrobials, Cardiovascular treatment, Drug-drug interactions}

Introduction: Limited evidence indicate that co-dispensing flucloxacillin to patients already on warfarin may result in decreased warfarin efficacy.

Objectives: To investigate the effect of flucloxacillin on warfarin anticoagulation.

Methods: We performed a cohort study in 6015 warfarin-treated patients exposed to flucloxacillin for 10 days and 213 exposed for 30 days or more. To assess the potential for confounding by indication, we also analysed individuals receiving phenoxymethylpenicillin. INR values and warfarin doses were calculated day-by-day during co-treatment. Data were obtained from two large Swedish warfarin registers and from the Swedish Prescribed Drug Register.

Results: Following initiation of flucloxacillin with a planned treatment duration of 10 days and $\geq 30$ days, the mean INR decreased from 2.36 (95\% confidence interval $2.34 ; 2.37)$ to $2.20(95 \%$ $2.19 ; 2.22)$ and from $2.24(95 \%$ CI $2.16 ; 2.33)$ to $1.97(95 \% \mathrm{CI}$ $1.90 ; 2.04)$ respectively. As a consequence, the proportion of patients with a subtherapeutic INR $(<2)$ increased from $22 \%$ in the week preceding flucloxacillin initiation to $35 \%$ in the third week of co-treatment in individuals with 10 days treatment. The corresponding figures in patients with 30 days treatment was $34 \%$ and $63 \%$. In individuals initiating phenoxymethylpenicillin, INR levels did not decrease and even increased slightly from 2.49 (95\% CI $2.48 ; 2.50)$ at baseline to $2.52(95 \%$ CI $2.51 ; 2.53)$.

Summary / Conclusions: The risk of a subtherapeutic warfarin effect increased markedly during co-treatment with flucloxacillin and the interaction was most pronounced during long-term antibiotic treatment. To avoid thromboembolic complications, the initiation of flucloxacillin should be accompanied by closer INR monitoring, in particular for individuals receiving longer than usual flucloxacillin treatment.

\section{EACPT-1260}

Clinical effects of green tea components on prevention of influenza infection

H. Yamada* ${ }^{1}$, D. Furushima ${ }^{1}$

${ }^{1}$ Drug Evaluation \& Informatics, University of Shizuoka, Shizuoka, Japan

\section{Antimicrobials, Other}

Introduction: Influenza is an acute, highly infectious respiratory illness caused by the influenza virus; when aggravated, the 
infection may be life-threatening, making prevention very important. In basic experimental studies, green tea catechins have been reported to inhibit influenza virus adsorption and suppress replication. In addition, green tea contains theanine and vitamin $\mathrm{C}$, which enhance immunity against viral infections, thus leading to the hypothesis that green tea components may clinically prevent influenza infection.

Objectives: To investigate the clinical evidence of the effects of green tea components on the prevention of influenza infection.

Methods: We conducted a comprehensive worldwide literature search and critically evaluated the published studies, including our previous studies, on the clinical effects of green tea components on influenza prevention.

Results: Seven clinical studies, including 5 randomized controlled studies, were found from PubMed or manual search. Among them, only 3 studies showed significant effects of green tea components on influenza prevention. An epidemiological study showed that regular consumption ( 1 to 5 cups/day) of green tea significantly decreased the incidence of influenza infection. A prospective study on gargling with green tea catechin extracts (at a concentration of $20 \mathrm{mg} / \mathrm{dL}$ ) and a randomized controlled study on the consumption of catechins ( $378 \mathrm{mg} /$ day) and theanine $(210 \mathrm{mg} /$ day $)$ showed preventive effects against influenza infection.

Summary / Conclusions: Although the antiviral activity of green tea components has been demonstrated in basic studies, there is limited clinical evidence to support it. Further studies are needed to confirm the clinical efficacy of green tea components in influenza prevention.

\section{EACPT-1318}

Prevention and detection of healthcare-associated infections using the comprehensive risk management system

O. Konova* ${ }^{*}$, S. Payushchik ${ }^{1}$, E. Kleymenova ${ }^{1}$

${ }^{1}$ Department of Clinical Pharmacology and Therapy, Federal State Budgetary Educational Institution of Further Professional Education "Russian Medical Academy of Continuous Professional Education" of the Ministry of Healthcare of the Russian Federation, Moscow, Russian Federation

\section{Antimicrobials, Computerized decision support, Intensive care treatment}

Introduction: Centers for Disease Control (CDC) data on healthcare-associated infections showed that although significant progress has been made in preventing some healthcare-associated infection (HAI) types, there is much more work to be done. On any given day, about one in 31 hospital patients has at least one healthcare-associated infection and $11 \%$ of persons with no symptoms carry an unusual resistance germ.

Objectives: The study was aimed to prevent HAIs and identify already developed HAIs as early as possible via the comprehensive risk management system (combination of Global Trigger Tool method, decisionsupport system, and standard operating procedures (SOPs) for HAI early detection and prevention.

Methods: A combination of 2 triggers (positive blood, stool, or urine culture for multidrug-resistant strains; fever $>37.5^{\circ} \mathrm{C}$ presenting later 3 days after surgery or 2 days after hospital admission), the diagnostic guideline-based (CDC) decision support-system, and 3 SOPs was used to prevent HAI development and identify already developed HAIs as early as possible in Multidisciplinary Medical Center of the Central Bank of the Russian Federation in 2017-2018.

The SOP for ventilator-associated pneumonias prevention included an elevation of head of bed $\left(30^{\circ}-45^{\circ}\right)$, daily sedation and assessment of readiness to extubate, early patient activation, and care of the endotracheal tube.
The SOP for central line-associated bloodstream infections regulated the installation of a central venous catheter, subsequent care for it, and its removal.

The SOP for catheter associated urinary tract infection regulated strict indications for catheter insertion, aseptic technique for its insertion, adherence to care recommendations, daily monitoring of the need for catheterization, and timely catheter removal.

Results: Chi-square test has shown that the risk management system implementation lead to the statistically significant decrease in HAI incidence $(4.8$ per 1000 patients treated vs. 2.8 per 1000 patients treated, $\chi 2=$ 4.77, the two-sided P-value 0.029).

Summary / Conclusions: The study showed a statistically significant reduction in HAI incidence in 2018, however, further research is needed to validate the comprehensive risk management system and clarify the contribution of its individual elements, with an assessment of the time interval from trigger detection to the onset of antibiotic therapy.

\section{EACPT-1327}

Improving antibiotic stewardship in neonates through means of a new biomarker, soluble cd14 subtype (scd14-st): a systematic review and meta-analysis

I. Van Maldeghem ${ }^{*}$, D. Visser ${ }^{2}$, C. Nusman ${ }^{2}$

${ }^{1}$ Pharmacotherapy, ${ }^{2}$ Pediatrics, Amsterdam UMC, Amsterdam, Netherlands

\section{Antimicrobials, Pediatric treatment}

Introduction: Early diagnosis of bacterial sepsis in neonates is hampered by non-specific symptoms and the lack of rapid responding laboratory measures. Therefore, neonates with risk factors for infection are treated with antibiotics empirically. In Europe, the incidence of early onset sepsis (EOS) is estimated to be around $0.05-0.1 \%$, while almost $8 \%$ of the newborns is treated for EOS. To avoid unnecessary treatment of noninfected neonates, an early, sensitive and specific laboratory test would be helpful to decide whether or not to start antibiotics. The biomarker soluble CD14 subtype (sCD14-ST) seems promising in the diagnostic process of neonatal sepsis. In order to evaluate the diagnostic accuracy of sCD14-ST in EOS we assessed this systematic review and metaanalysis.

Objectives: To avoid unnecessary treatment of non-infected neonates, an early, sensitive and specific laboratory test would be helpful to guide clinicians to decide whether or not to start antibiotics. The biomarker soluble CD14 subtype (sCD14-ST) seems promising in the diagnostic process of neonatal sepsis. In order to evaluate the diagnostic accuracy of sCD14-ST in early onset sepsis (EOS) we assessed this systematic review and meta-analysis.

Methods: A systemic literature search was conducted in six international databases. All selected studies where assessed on their methodological quality and the risk of bias using the QUADAS2 tool. MetaDiSc 1.4 software was used to perform the analyses of the meta-analysis.

Results: Twelve articles were included in the systematic review and 10 in the meta-analysis. There was a high risk of bias on patient selection, index test and/or flow and timing. The overall quality of the included studies was moderate.

At sepsis onset a consequently higher level of sCD14-ST was found in septic neonates compared to healthy controls. In the first 24 hours after sepsis onset a significant increase in pooled means of plasma $\mathrm{sCD} 14-\mathrm{ST}$ levels was seen in EOS $(\mathrm{t}(71.6)=7.3, \mathrm{p}<.0001)$. Optimal cut-off values ranged from 305 to $672 \mathrm{ng} / 1$ for EOS cases versus healthy controls. The pooled sensitivity was $81 \%$ (95\%CI: 0.76 $0.85)$, the pooled specificity was $86 \%(0.81-0.89)$ with an AUC of 0.9412 (SE 0.1178).

Summary / Conclusions: $\mathrm{sCD} 14-\mathrm{ST}$ is a promising and rapidresponding diagnostic biomarker for EOS and therefore helpful in reducing unnecessary antibiotic treatment in neonates. 
Oral Presentation

EACPT-1340

Comparison of dosage strategies of meropenem vs. Pseudomonas aeruginosa

A. Nussbaumer-Proell ${ }^{*}{ }^{1}$, L. Schmidt ${ }^{1}$, M. Zeitlinger ${ }^{1}$

${ }^{1}$ Clinical Pharmacology, Medical University of Vienna, Vienna, Austria

\section{Antimicrobials, Pharmacokinetics/ pharmacodynamics}

Introduction: In antimicrobial treatment the dose must be sufficient for bacterial-killing, while toxicity must be prevented, and the risk of antimicrobial resistance should be kept low.

Objectives: As already shown in animal studies time for which meropenem levels exceed the minimum inhibitory concentration (MIC) correlates best with bacterial killing. $40 \% \mathrm{~T}>\mathrm{MIC}$ is usually associated with optimal killing. However, it is unknown if the distribution of percentage $\mathrm{T}>\mathrm{MIC}$ through a treatment day is also important. Therefore, we set out to investigate the antibiotic activity of meropenem, precisely if the $40 \% \mathrm{~T}>\mathrm{MIC}$ is achieved in one single long period (representing single dose), two $20 \%$ periods (dosing bid) or $3 \times 13.3 \%$ (dosing t.i.d.) thereby keeping the overall period of T>MIC constant.

Methods: Time kill curves (TKC) with P. aeruginosa-ATTC-27853 were done over 24 hours at $37^{\circ} \mathrm{C}$ in Mueller Hinton Broth (MHB) with concentrations from $0.5 x M I C$ up to $16 x$ MIC. To simulate periods over and under MIC cultures were centrifuged after the respective period to remove antibiotic by discarding supernatant and refilling the tubes with fresh MHB. Double and triple dosing involved a subsequent further addition of antibiotic. Complementary growth controls (GC) with and without centrifugation steps were done.

Results: Growth assays revealed no impact of centrifugation on bacterial growth. TKC showed strongest regrowth for the single dose treatment, whereas twice and triple dosage did not differ after 24 hours. Table shows the ratio of AUC in colony forming units $(\mathrm{CFU}) / \mathrm{ml} * \mathrm{~h}$ of single dosage (set to $100 \%$ ) compared to AUC of twice and triple dosage. Bacterial killing at MICx0.5 was equal for all dosage regimens, whereas for MICx1 up to MICx16 the AUC was consistently lowest for the triple dosage setting.

Summary / Conclusions: We confirm a positive effect of multiple antibiotic dosage compared to single dose for tested concentrations of meropenem

\begin{tabular}{llc}
\hline $\begin{array}{l}\text { P. aeruginosa } \\
\text { Meropenem } \\
(\text { MIC (1mg/L)) }\end{array}$ & $\begin{array}{c}\text { \% AUC twice } \\
\text { dosage }\end{array}$ & $\begin{array}{c}\text { \% AUC 3 } \\
\text { dosages }\end{array}$ \\
MICx16 & 69,28 & 61,98 \\
MICx4 & 84,61 & 74,17 \\
MICx2 & 95,42 & 93,07 \\
MICx1 & 97,12 & 92,87 \\
MICx0,5 & 102,84 & 101,68 \\
\hline
\end{tabular}

despite keeping the T>MIC target constant. Clearly a more efficient eradication of bacteria was seen for triple dosage of MICx 4 and above. Therefore, our data confirms clinical recommendations for meropenem, as treatment with concentrations of at least $\mathrm{MICx} 4$ are advised.

\section{Oral Presentation}

\section{EACPT-1419}

Six versus four weeks of intravenous antibiotic treatment for staphylococcus aureus endocarditis: a pilot study

S. Douiyeb ${ }^{*} 1$, K. Sigaloff ${ }^{2}$, A. Samson ${ }^{3}$, J. de la Court ${ }^{1}$, T. van der Vaart ${ }^{1}$, W. Baig ${ }^{4}$, J. Wu ${ }^{4}$, R. Gillot ${ }^{4}$, S. Kelly ${ }^{5}$, S. el Kadi ${ }^{6}$, J. Sandoe ${ }^{4}$

${ }^{1}$ Internal medicine, Amsterdam UMC , ${ }^{2}$ Internal medicine, Amsterdam VUmc, Amsterdam, Netherlands, ${ }^{3}$ Hull University Teaching Hospitals
NHS Trust, Hull , ${ }^{4}$ Leeds Teaching Hospital NHS Trust, Leeds, United Kingdom, ${ }^{5}$ Internal medicine, Leeds Teaching Hospital NHS Trust, Leeds, ${ }^{6}$ Internal medicine, Amsterdam UMC , Amsterdam, United Kingdom

\section{Antimicrobials, Cardiovascular treatment}

Introduction: Staphylococcus aureus (SA) is a major cause of nativevalve infective endocarditis (NVE). There is limited evidence to guide the duration of treatment of SA-NVE which is reflected in different recommendations in guidelines; European Society guidelines (ESC) advise 4-6 weeks, while the British guidelines suggest 4. Unnecessary long-term exposure to antibiotics should be avoided, since it increases the risk of adverse drug events, health-care associated infections and antibiotic resistance.

Objectives: The aim of this project was to assess the feasibility of comparing the outcomes of patients receiving a 4-week versus a 6-week course of antibiotic treatment for SA-NVE; we describe preliminary findings.

Methods: A multi-center retrospective pilot study was conducted at 4 hospitals: the Amsterdam University Medical Centers (VUmc and AMC hospitals), the Leeds Teaching Hospitals NHS Trust and Hull \& East Yorkshire Hospitals Trust. Between 2011 and December 2018 patients with a definite methicillin-sensitive SANVE, according to the modified Duke criteria, were included. Exclusion criteria were: prosthetic valve or device, right sided endocarditis, MRSA or extra cardiac complications (cardiac, cerebral, spinal abscess). Demographics, treatment and outcomes variables were collected. Clinical cure was compared between patients treated for four weeks ( $28+/-5$ days) or six weeks ( $42+/-5$ days).

Results: 42 patients were included in the pilot. Twelve versus 30 patients were identified in the 4-week and 6-weeks treatment groups, respectively. Ten patients (76.9\%) achieved clinical cure in the 4-week group versus 21 patients $(80.8 \%)$ in the 6-week group (see Table).

Summary / Conclusions: We identified patients who received 4 weeks of antibiotic treatment for S. aureus NVE. We confirmed that it is feasible to collect data and compare clinical outcomes between 4 and 6 weeks of therapy. The results of this study are expected contribute to more appropriate use of the available antistaphylococcal antibiotics.

\section{EACPT-1406}

Posaconazole salvage therapy: the posifi study.

J. Fortun $^{1}$, F. Gioia ${ }^{1}$, P. Puerta-Alcalde ${ }^{2}$, C. Gudiol ${ }^{3}$, E. Diago* ${ }^{4}$, J. J. Castón $^{5}$, P. Muñoz ${ }^{6}$, C. Cardozo ${ }^{2}$, M. Enzenhofer ${ }^{3}$, A. Ramos ${ }^{7}$, A. Frutos $^{5}$, M. Machado ${ }^{6}$, C. García-Vidal ${ }^{2}$, R. Parody ${ }^{8}$, P. Martín-Dávila ${ }^{1}$ ${ }^{1}$ Infectious Diseases , Hospital Ramón y Cajal, Madrid, ${ }^{2}$ Infectious Diseases, Hospital Clínic, Barcelona, ${ }^{3}$ Infectious Diseases , Hospital Universitari Bellvitge, L'Hospitalet de Llobregat, Barcelona, ${ }^{4}$ Clinical Pharmacology, Hospital Universitario Puerta de Hierro, Majadahonda, Madrid, ${ }^{5}$ Infectious Diseases, hospital Universitario Reina Sofía, Córdoba, ${ }^{6}$ Infectious Diseases, Hospital General Universitario Gregorio Marañón, Madrid, ${ }^{7}$ Infectious Diseases , Hospital Universitario Puerta de Hierro, Majadahonda, Madrid, ${ }^{8}$ Hematología, Institut Català d'Oncologia, Hospital Duran y Reynals, L'Hospitalet de Llobregat, Barcelona, Spain

\section{Antimicrobials}

Introduction: Posaconazole (PCZ) is used mainly for the prevention of invasive fungal infection (IFI); its role as a rescue therapy remains to be determined and, at present, it is controversial which azole is the best in this clinical setting.

Objectives: To analyse the safety and efficacy of PCZ in its different forms of administration in thetreatment (non-prophylactic) of invasive aspergillosis, zygomycosis, and other IFIscaused by mould and yeast in Spain (Posifi study). 
Methods: A multicentre observational study to analyse the efficacy and safety of PCZ in different forms of administration in the (nonprophylactic) treatment of IFI in Spain.

Results: Over an eight-year-period, 67 patients were included in this study. Patients with haematologic-oncologic diseases and solid organ transplants recipients $31(46.3 \%)$ and $20(29.8 \%)$ of the sample population, respectively. PCZ was administered as salvage therapy (intolerant or refractory to a previous antifungal agent) in 65/67 (97\%); of these, it was used against Aspergillosis 46 (68.6\%), Zygomycosis 9 (13.4\%), other moulds $6(8.9 \%)$ and other yeasts $2(3 \%)$. Median treatment duration was 43 days for previous antifungal therapy and 75 days for PCZ therapy. Oral solution was associated with low serum levels $(<0.7 \mathrm{mg} / \mathrm{L})$ in $63 \%$ of available patients.

Main study results can be found in Table 1 .

Table 1. Mortality attributable to invasive fungal infection at 3 and 12 months.

\section{MORTALITY ATTRIBUTABLE TO IFI}

\begin{tabular}{lll} 
& 3 months & 12 months \\
Aspergillosis; N (46) & $10(21.8 \%)$ & $11(24.0 \%)$ \\
Zygomicosis N (9) & $2(22.2 \%)$ & $2(22.2 \%)$ \\
Rest of IFI N (13) & $1(7.7 \%)$ & $2(15.4) \%$ \\
\hline
\end{tabular}

In spite of the prolonged use of PCZ, suspension by toxicity was only observed in $4(6 \%)$ and $5(7.5 \%)$ of patients at three months and 12 months respectively, mainly with grade III/IV elevations of liver function test (LFTs).

Summary / Conclusions: In this complicated scenario, the response achieved with PCZ was similar to that reported for other first-line antifungals under more favourable circumstances. PCZ salvage therapy, especially oral tablets, can be an effective alternative option for patients with IFI who cannot tolerate or do not respond to other antifungal therapies.

\section{Cancer treatment}

\section{EACPT-127}

Preparation of an ibrutinib patch test

R. Seisdedos Elcuaz ${ }^{*}$, B. Fernández Rubio ${ }^{1}$, E. Prado Mel ${ }^{1}$

${ }^{1}$ PHARMACY, HOSPITAL UNIVERSITARIO VIRGEN DEL ROCÍO, SEVILLA, Spain

\section{Cancer treatment, Drug utilization, Pharmacovigilance}

Introduction: Patch testing is one of the most profitable diagnostic tools for the evaluation of patients with suspected drug allergies. Unfortunately, only a few drugs with standardized material for patch test are available and sometimes there is no bibliography on this topic.

Objectives: The aim of this study was to create an Ibrutinib patch test in order to confirm an allergic reaction.

Methods: Literature research was conducted using the main biomedical databases, such as PubMed, Embase and Scopus. "Ibrutinib" and "patch test" were used as keywords.

Results: Due to Ibrutinib's water insolubility, prick test could not be selected. Instead, a drug patch was determined as test procedure. There were no available data on the procedure and concentration to develop the patch. According to literature, drugs in their commercialized form not described in studies or reviews should be tested at $30 \% \mathrm{w} / \mathrm{w}$-regarding to the total content- or less in case of prior severe skin symptoms. A test of the capsule cover separately is also recommended.

A patch test of Ibrutinib was designed using Imbruvica® $140 \mathrm{mg}$ capsules and petrolatum as lipophilic vehicle. All the elaboration was carried out in a containment ventilated enclosure and with the corresponding personal protection equipment. Components were mixed in a mortar obtaining an Imbruvica's concentration of 30\%. Thus, Ibrutinib's final concentration was $12,61 \%$. Concentration was selected by consensus with the allergist. Around $1 \mathrm{~g}$ of the final product was applied on the skin using an epicutaneous patch for ninety-six hours. The reading of the patch was negative.

Summary / Conclusions: Patch test is an essential in vivo test procedure to confirm drug allergies. In our test, the result was negative, which means that the effectiveness of the patch test remains unclear. According to literature, patch test concentration refers to the total content of the starting drug in the vehicle however the concentration with respect to the active principle is also correlated with the recommendations of the guidelines $(10 \%)$. Therefore further studies should be carried out to rule out a false negative.

\section{EACPT-1010}

Real-life experience of eribulin in patients with advanced liposarcoma H. Rodriguez Ramallo ${ }^{1}$, R. Jiménez Galán ${ }^{1}$, N. Báez Gutierrez ${ }^{1}$, S. Flores Moreno $^{1}$, L. Abdelkader Martin ${ }^{1}$, M. D. Vega Coca ${ }^{1}$, R. Seisdedos Elcuaz* 1

${ }^{1}$ Farmacia Hospitalaria, Hospital, Universitario Virgen del Rocío, Sevilla, Spain

\section{Cancer treatment}

Introduction: Eribulin was approved in April 2016 by European Medicines Agency (EMA) for treatment of patients with unresectable or metastatic liposarcoma who have progressed with prior anthracyclinecontaining treatment.

Real-life experience of eribulin on advanced liposarcoma is limited due to the low incidence of metastatic disease and the relative recent approbation by EMA.

Objectives: To evaluate effectiveness and safety of eribulin in real-world patients with advanced liposarcoma.

Methods: Observational retrospective and single-centre study that included patients who received treatment with eribulin for advanced liposarcoma from June 2016 to August-2018. Variables collected were: gender, age, basal Eastern Cooperative Oncology Group performance status (ECOG), prior lines of treatment, number of cycles, progression and death date, adverse events (AE), treatment discontinuation and dose reductions.

Response rate was assessed by computed tomography. Progression free survival (PFS) was measured from time of beginning treatment with eribulin to date of first progression or death from any cause. PFS was calculated by Kaplan-Meier analysis. Clinical data were obtained from clinic electronic history and corporate prescription software.

Results: We included 13 patients, 9 men and 4 women with a mean age of 59.7 years range (40.6-80.7). Seven patients had ECOG 0, four patients ECOG 1 and two patients ECOG 2. Eribulin was provided as second, third and fifth line chemotherapy in seven, two and one patients, respectively. The remaining three patients received it as first line due to contraindication to anthracyclines. Median number of cycles was 3 (range 1-9).

Complete response was not achieved in any patient; partial response and stable disease were recorded in one and four patients, respectively. The remaining patients showed progression disease. Median PFS was 2 months (95\% CI 0.95-3.04). AE occurred in 6 patients. Most frequent AE were gastrointestinal (30.8\%), neurotoxicity (15.4\%) and neutropenia $(7.7 \%)$. No patient discontinued treatment by toxicity and no dose reductions were necessary.

Summary / Conclusions: The effectiveness of eribulin on real-world patients was modest. PFS values were lower than published in the literature. Eribulin showed an acceptable toxicity profile; more than half of patients showed no AE and no patient required dose reduction. Studies with larger sample size are needed to determine real-life effectiveness of eribulin. 
EACPT-1016

Effectiveness and safety of nivolumab in geriatric patients

N. Báez Gutiérrez ${ }^{1}$, S. Flores Moreno ${ }^{1}$, H. Rodríguez Ramallo ${ }^{2}$, L. Abdelkader Martín ${ }^{1}$, M. D. Vega Coca ${ }^{1}$ R. Seisdedos Elcuaz* ${ }^{1}$

${ }^{1}$ Hospital Pharmacy, Hospital Universitario Virgen del Rocío, Seville, ${ }^{2}$ Hospital Pharmacy, Hospital Universitario Virgen del Rocío, Sevilla, Spain

\section{Cancer treatment}

Introduction: Elderly patients represent most of cancers diagnosed and deaths by age group. However, this subgroup of patients is underrepresented in clinical trials
Objectives: To evaluate the effectiveness and safety of Nivolumab treatment in geriatric patients

Methods: Retrospective study of patients $>65$ years in treatment with nivolumab during the years 2016-2017, in a tertiary level hospital.

Data collected were demographics and median of cycles administered (mCA).

Effectiveness was assessed by means of median duration of treatment $(\mathrm{mDT})$ and median overall survival (mOS).

Safety was evaluated based on the adverse effects (AEs) incidence, graduation and necessity of delay/discontinue treatment.

Data was obtained from the prescription program Farmis ${ }^{\circledR}$ and from clinical charts.

Results: 38 patients were analysed. The effectiveness data obtained are shown in the following table:

Table 1. Data collected

\begin{tabular}{llllllllll} 
& $\mathrm{n} .{ }^{\circ}$ patients & Age (years) & mCA & mDT (months) & OS (months) & IAEs (\%) & Grade 3-4 & Delays (\%) & Interruption (\%) \\
NSCLC & 23 & 74 & 11.52 & 11.09 & 9.59 & 95,65 & 17.4 & 60.87 & 4.34 \\
Melanoma & 7 & 75.9 & 10 & 5.3 & 10.6 & 100 & 14.28 & 14.28 & 14.28 \\
Renal cancer & 8 & 71.13 & 9.25 & 10.94 & 23.75 & 87.5 & 25 & 75 & 12.5 \\
\hline
\end{tabular}

- Non-small cell lung cancer (NSCLC): Most common AEs: asthenia $69.56 \%$, dyspnea $43.48 \%$, and anorexia $43.48 \%$. Immunorelated AE's: $17.4 \%$ gastrointestinal and $13.04 \%$ respiratory alterations.

- Melanoma: Most common AEs: asthenia $85.71 \%$, rash $42.85 \%$ and anorexia $42.85 \%$. Immunorelated AE's: $28.57 \%$ hyperbilirubinemia and $14.24 \%$ diarrhoea.

- Renal cancer: Most common AEs: asthenia 62.5\%, cough 50\%, rash $37.5 \%$, pruritus $37.5 \%$. Inmunorelated AE's: $12.5 \%$ gastrointestinal and $12.5 \% \%$ nephrotoxicity.

Summary / Conclusions: The efficacy of nivolumab in geriatric patients is similar to that reported in clinical trials in terms of response duration and OS in the case of NSCLC. In the case of melanoma and renal cancer, the assessment of the OS is not very useful due to the high percentage of censored data.

The observed AEs are all previously described, although the incidence is higher in our population than in the trials. The incidence of AEs per organ varies with respect to the literature, with the most frequent being asthenia, anorexia and dyspnea in geriatric patients. Due to the limited number of patients and the reallife prevalence of this subgroup of patients, it is desirable to perform additional prospective studies.

\section{EACPT-1044}

Effect of a single intraoperative dose of dexamethasone on blood glucose and lactate levels in brain tumor surgery

G. Tsaousi ${ }^{1}$, J. Pezikoglou ${ }^{1}$, J. Dalakakis ${ }^{1}$, C. Pourzitaki ${ }^{*}{ }^{1}$, D. Kouvelas ${ }^{1}$

${ }^{1}$ Aristotle University of Thessaloniki, Greece, Thessaloniki, Greece

\section{Cancer treatment, Therapeutic drug monitoring, Other}

Introduction: Dexamethasone, a first-line treatment regimen for brain tumor-induced edema, is known to augment blood glucose levels, which in turn might mediate exacerbation of brain injury. Yet, the effect of a single intraoperative dose of dexamethasone on the blood glucose and lactate concentration has not been elucidated.

Objectives: To quantify the magnitude of the blood glucose response to a single intraoperative dose of dexamethasone, with a view to optimize glucose levels management in patients undergoing brain tumor surgery. Methods: Prospective, single centre, cohort study enrolling 249 patients, scheduled to undergo elective craniotomy for brain tumor surgery, lasting at least 4 hours, who were given a single dose of dexamethasone $(0.15-0.2$ $\mathrm{mg} / \mathrm{kg}$ ) after anesthesia induction. Both arterial blood glucose and lactate concentrations were recorded immediately before (baseline) and 4 hours after treatment intraoperatively. This group of patients was matched (2:1), according to patients' demographics and glucose blood sampling, with a surgical population of 109 patients undergoing craniotomy for non-tumor pathologies, in whom no dexamethasone was given. Paired T-test, Spearman correlation and multivariate linear regression were used for data analysis.

Results: Both groups were comparable regarding their demographics and surgical characteristics. Significant between groups differences were detected for both blood glucose and lactate levels (Table) between baseline and 4 hours post-treatment $(\mathrm{p}<0.001)$. History of diabetes, increased dose of corticosteroids and use of inotropes were identified as the most powerful determinants of augmentation of glucose concentration $\left[\mathrm{F}(3,245)=38.450, \mathrm{p}<0.001, \mathrm{R}^{2}=0.366\right.$

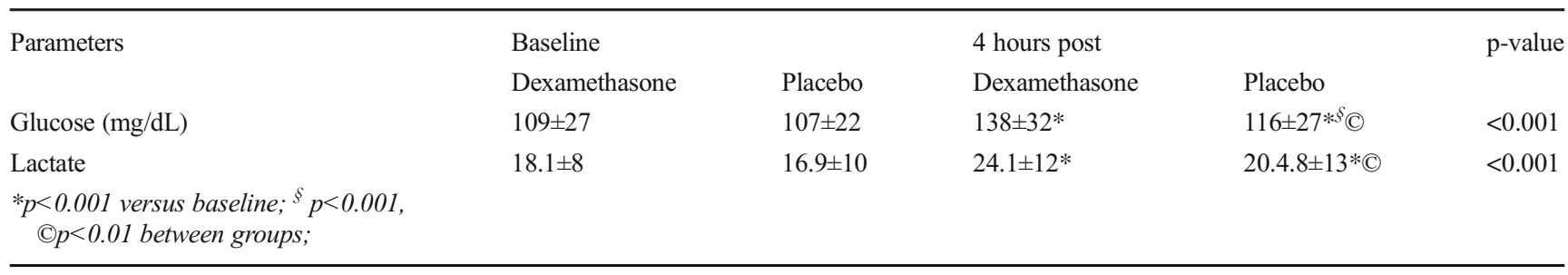


Summary / Conclusions: Although a single-dose of dexamethasone induced a dose-related augmentation of serum glucose and lactate levels 4 hours post administration, this effect seems not to be of paramount clinical importance as the concentrations of both parameters were maintained within relatively safe range. History of diabetes, hemodynamic compromise and increased dose of dexamethasone impose a more stringent approach in terms of corticosteroids use in brain tumor surgery.

\section{EACPT-1060}

Survival of gastric and esophageal cancer patients with adjuvant and palliative chemotherapy - a retrospective analysis of a register-based patient cohort

I. Ekheden $^{*} 1$, W. $\mathrm{Ye}^{1}$

${ }^{1}$ Medical Epidemiology and Biostatistics, Karolinska Institutet, Stockholm, Sweden

\section{Cancer treatment}

Introduction: Studies of the survival of esophageal and gastric cancer patients treated with chemotherapy outside of clinical trials are rare.

Objectives: Our aim was to compare the effectiveness of chemotherapy on the survival of esophageal and gastric cancer patients in the clinical setting. Methods: We used information about chemotherapy in all esophageal and gastric cancer patients diagnosed in a low-risk Western population of Stockholm and Gotland County during 2008-2013. We identified a cohort of 563 patients treated with curative intention and 575 patients treated with palliative intention. Information about demographics, treatment and survival were collected from registers. We used Cox proportional hazards regression models to estimate Hazard ratios (HRs) with 95 $\%$ confidence intervals (CIs) adjusting for the potential confounding factors: age, sex, tumor stage, comorbidity, marital status, education, income and country of birth.

Results: We observed a higher hazard for death among patients with esophageal squamous cell carcinoma who were treated with curative intention regimens other than 5 Fluorouracil/Cisplatin/Carboplatin/ Oxaliplatin compared with patients with the same cancer treated with 5Fluorouracil and Cisplatin corresponding to a HR of 3.32; 95\% CI 1.0710.28. Similarly, we observed a higher hazard for death among patients with gastric adenocarcinoma who were treated with curative intention regimens other than Epirubicin/Capecitabine/Oxaliplatin/5-Fluorouracil, compared with patients treated with Epirubicin, Oxaliplatin and Capecitabine (HR 3.15; 95\% CI 1.40-7.11).

Summary / Conclusions: The choice of chemotherapy might predict survival for esophageal squamous cell carcinoma and gastric adenocarcinoma patients in a low-risk Western population.

\section{Oral Presentation \\ EACPT-1065}

Medication safety of new oral antitumor agents: a randomized trial on the impact of a clinical pharmacological / pharmaceutical intervention on drug-related problems and patient safety

K. Schlichtig ${ }^{1}$, P. Dürr ${ }^{2}$, F. Dörje ${ }^{2}$, C. AMBORA Study Investigators ${ }^{3}$, M. F. Fromm ${ }^{*} 1$

${ }^{1}$ Clinical Pharmacology and Clinical Toxicology, Friedrich-AlexanderUniversity Erlangen-Nuremberg, ${ }^{2}$ Pharmacy, University Hospital Erlangen, ${ }^{3}$ Comprehensive Cancer Center (CCC) Erlangen-EMN, Erlangen, Germany

\section{Cancer treatment}

Introduction: More than 50 new oral antitumor agents have been approved in recent years. In addition to the convenient way of administration, these drugs brought clinical benefits in a number of indications. However, new risks have emerged partially threatening treatment success (e.g. adherence, impact of nutrition, new adverse drug reactions, drug interactions).
Objectives: To study the impact of a clinical pharmacological / pharmaceutical intervention on drug-related problems and patient safety.

Methods: Patients can be included in this randomized study, if they are scheduled for start of treatment with a new oral antitumor agent independent of the tumor entity. The duration of the study is 12 weeks for each patient. Patients are randomized (1:1) to the control group (C) with treatment according to the standards of the respective participating clinic or to the intervention group (I). In I clinical pharmacologists / pharmacists are involved in patient education and in detection and resolution of drug related problems (DRPs). Primary endpoints are the number of DRPs related to the oral antitumor agent and patient satisfaction determined with the TSQM questionnaire (category 'convenience').

Results: This is an interim analysis after inclusion of the first 100 patients. DRPs were reduced from $7.5 \pm 4.0$ in $\mathrm{C}$ to $5.9 \pm 3.9 \mathrm{DRPs}$ per patient in $\mathrm{I}(\mathrm{p}=0.066)$. Side effects decreased from $7.2 \pm 4.1$ in $\mathrm{C}$ to $5.9 \pm 3.8$ DRPs per patient in $\mathrm{I}(\mathrm{p}=0.098)$. Treatment satisfaction increased from $79.1 \pm 16.8$ in $\mathrm{C}$ to $94.1 \pm 10.1$ in $\mathrm{I}(\mathrm{p}=0.0003)$. Interestingly, severe side effects ( $\geq$ grade 3 ) were considerably reduced in I compared to $\mathrm{C}(0.7 \pm \mathrm{SD}$ vs $1.3 \pm \mathrm{SD}, \mathrm{p}=0.076)$. Discontinuation of treatment with the antitumor agent was twice as high in C compared to I ( 16 vs $8, \mathrm{p}=0.133$ ).

Summary / Conclusions: The data of this interim analysis point to a reduction of drug related problems in patients with intensive clinical pharmacological / pharmaceutical care. Patients' satisfaction increases. The data indicate that severe side effects and treatment discontinuations decline due to this intervention.

\section{EACPT-1067}

Roles of myc-modulating long non-coding rna mincr in cell cycle regulation and apoptosis in non-small cell lung cancer

S. Chen ${ }^{1}$, D. Shang ${ }^{2}$, Z. Zhao ${ }^{1}$, Z. Tu ${ }^{3}$, H. Liu $^{*}{ }^{2}$

${ }^{1}$ Affiliated Hospital, ${ }^{2}$ School of Pharmacy, ${ }^{3}$ Institute of Life Sciences, Jiangsu University, Zhenjiang, China

\section{Cancer treatment}

Introduction: Non-small cell lung cancer (NSCLC) is one of the leading causes of cancer death in the world. Long non-coding RNAs (lncRNAs) have been demonstrated to modulate cancer initiation and progression through a variety of molecular mechanisms.

Objectives: We sought to investigate the clinical significance, roles, and potential mechanisms of MYC-induced long non-coding RNA (MINCR) in NSCLC.

Methods: Expression levels of MINCR was first identified using The Cancer Genome Atlas (TCGA) datasets, further confirmed with specimens from NSCLC patients and cell lines using qRTPCR. Overexpression and knockdown of MINCR were performed in NSCLC cell lines through MINCR overexpression vectors and synthesized siRNAs, respectively. The roles of MINCR in NSCLC cell lines, such as cell proliferation, cell cycle arrest, and apoptosis, were identified by MTT, flow cytometry, and Western blot. The modulation of MINCR-regulated genes, including c-Myc and its downstream cyclins and cyclin dependent kinases (CDKs), as well as apoptosis-associated genes, was analyzed using Western blot.

Results: MINCR was aberrantly overexpressed in two major subtypes of NSCLC patients, lung adenocarcinoma (LUAD) and lung squamous carcinoma (LUSC), from TCGA datasets, and was also significantly up-regulated in our collected specimens from NSCLC patients and NSCLC cell lines. Knocking down of MINCR greatly inhibited the growth of NSCLC cell lines PC9 and A549. In addition, silencing of MINCR induced cell cycle arrest and apoptosis. Furthermore, silencing of MINCR reduced the expression levels of oncogene c-Myc and its downstream cyclin A, cyclin D, $\mathrm{Cdk} 4$, and $\mathrm{Cdk} 2$, as well as apoptosis-associated Bcl-2, while significantly increased the expression levels of cleaved PARP-1. 
In the meantime, overexpression of MINCR remarkably enhanced cell proliferation of PC9 cells and activated c-Myc and its downstream cyclins and CDKs.

Summary / Conclusions: MINCR is aberrantly overexpressed in NSCLC cell lines and tissues. Silencing of MINCR greatly inhibited the growth of NSCLC cell lines and induced the cell cycle arrest and apoptosis in NSCLC cell lines through reducing the expression levels of oncogene c-Myc and its downstream cyclins and CDKs. The results indicate that this IncRNA may serve as a potential therapeutic target for the treatment of NSCLC.

\section{EACPT-1114}

Effect of new preventive medicine on cisplatin-induced acute kidney injury

M. Goda ${ }^{*}{ }^{1,2}$, K. Saike ${ }^{1}$, M. Kanda ${ }^{2}$, T. Niimura ${ }^{1}$, K. Takechi ${ }^{3}$, M. $\mathrm{Chuma}^{3}$, Y. Izawa-Ishizawa ${ }^{4}$, Y. Zamami ${ }^{1,2}, \mathrm{~K}$. Ishizawa ${ }^{1,2}$

${ }^{1}$ Department of Clinical Pharmacology and Therapeutics, Tokushima University Graduate School of Biomedical Sciences, ${ }^{2}$ Department of Pharmacy, ${ }^{3}$ Clinical Trial Center for Developmental Therapeutics, Tokushima University Hospital, ${ }^{4}$ AWA Support Center , Tokushima University, Tokushima, Japan

\section{Cancer treatment}

Introduction: Cisplatin-induced acute kidney injury (AKI) is well known, and the nephrotoxicity of cisplatin restricts its clinical application. Currently, there are no drugs are recommended for the prevention of cisplatin-induced AKI. Forced hydration and diuresis may partially prevent nephrotoxicity of cisplatin, but it is still difficult to entirely prevent kidney injury. Thus, establishment of a new preventive method against cisplatin-induced $\mathrm{AKI}$ is required.

Objectives: Therefore, in this study, we attempted to identify candidates that could be used as preventive drugs against cisplatin-induced AKI, using big data analysis, and to verify the effectiveness of the candidate drugs.

Methods: Using FAERS (FDA Adverse Event Reporting System, largescale side effect case report database) and LINCS (gene expression database), existing drugs that might counteract cisplatin-induced AKI were extracted. In addition, in order to establish cisplatin-induced AKI animal model, C57BL/6 mice (male, 10-12 weeks old) were intraperitoneally administered with either cisplatin $(15 \mathrm{mg} / \mathrm{kg}$ ) or saline (control). Renal function was determined by evaluation of serum creatinine and blood urea nitrogen levels. Histological damage in the cortex of HE-stained kidney sections was examined by evaluating the percentage of tubules that displayed tubular necrosis, cast formation, and tubular dilation. The effect of preventive drugs on cisplatin-induced nephropathy was evaluated.

Results: The drug X was extracted as a candidate drug which exhibited the protective effect against cisplatin-induced kidney injury as revealed by FAERS and LINCS analysis. It was revealed that administration of drug X significantly suppressed cisplatin-induced AKI.

Summary / Conclusions: The results of this study indicated that the drug $\mathrm{X}$ selected after the analysis of FAERS and LINCS could act as a potential preventive drug for cisplatin-induced AKI.

\section{Oral Presentation}

EACPT-1142

A bayesian approach for continual estimation of real-life effectiveness as new data emerge

B. S. Kaas-Hansen ${ }^{*} 1$, S. E. Andersen ${ }^{1}$

${ }^{1}$ Clinical Pharmacological Unit, Zealand University Hospital, Roskilde, Denmark

\section{Cancer treatment, Health economy}

Introduction: Effectiveness reflects how well an intervention works in clinical use, as opposed to the efficacy in idealised populations, e.g., in randomised controlled trials (RCTs). Evidence from RCTs favouring a new drug may not apply to clinical practice where procedures and patients are less standardised. Bayesian models combine prior knowledge with new data to yield updated knowledge; enable down-stream decision analysis; and yield full posterior distributions with intuitive interpretations, unlike intangible significance tests of point estimates.

Objectives: To devise a hierarchical Bayesian model to synthesise reallife effectiveness of an intervention from digitised survival curves, using phase-II and -III RCT efficacy estimates as informative priors.

Methods: Having conducted a systematic literature review, we extracted survival curves from a Japanese phase-II RCT, a European phase-III RCT and eight observational real-life studies (6 published, 2 unpublished) on the anticancer drug TAS-102 used against metastatic colorectal cancer. We built a hierarchical Bayesian model to fit the extracted data to the log-logistic quantile function with three parameters: scale, shape and Gaussian error. This shrinks study-specific parameter estimates towards hyperparameters, reflecting the underlying survival profile. Each study's errors were weighted proportional to the inverse root of their size. We fit one model to the RCTs (efficacy model) to devise informative priors for the effectiveness model using data from the observational studies. We tried two hyperprior configurations in the efficacy model to gauge its robustness.

Results: Compared to the efficacy models, median overall survival (OS) decreased by 1.6 (95\% CrI: $-4.0 ; 0.8)$ months to 6.2 (95\% CrI: $5.4 ; 7.2)$ months and by 0.7 (95\% CrI: $-1.9 ; 0.5)$ months to 6.8 (95\% CrI: $6.1 ; 7.5)$ months in the effectiveness models with vague and restrictive hyperpriors. The probability of a lower median OS was $91 \%$ and $88 \%$ with the two hyperpriors.

Summary / Conclusions: This study indicates that the OS gain of TAS102 outside the context of clinical trials is inferior to the efficacy in RCTs conducted before market entry. Combining phase-II and -III efficacy data with real-life patient data, we produced updated estimates of the real-life benefit of a new drug. This approach could both facilitate clinical decision-making and guide regulatory decisions on, i.a., standard care and reimbursement.

\section{EACPT-1156}

Health and economic burden in direct costs of melanoma in moscow for 2016-2017

V. Krysanova $^{* 1,2}$, K. Poliakova ${ }^{1}$, T. Ermolaeva ${ }^{1}$, M. Davydovskaya ${ }^{1}$, K. Kokushkin $^{1}$

${ }^{1}$ SBI "Clinical Trials and Healthcare Technology Assessment ScientificResearch Centre of Moscow Department of Healthcare", ${ }^{2}$ Chair of Clinical Pharmacology and Introduction to Internal Medicine, I.M. Sechenov First Moscow State Medical University (Sechenov University), Moscow, Russian Federation

\section{Cancer treatment, Health economy}

Introduction: Melanoma care presents a significant economic burden in the population, particularly in late-stage disease. Skin cancer (including melanoma) poses a significant health threat as the first most commonly diagnosed cancer in Russia. The age-standardized melanoma incidence rate using the world standard population as a reference was estimated at 4.45 per 100000 leading to about 10'400 new melanoma cases in Russia for the year 2016. A steep rise in the melanoma incidence rate, which was one of the highest of all cancers between 2006 and 2016, was noted. The incidence of melanoma rose by $2.03 \%$ per year between 2006 and 2016 . Objectives: The aim of the study was to assess the preferential drug coverage for patients with melanoma (MEL) in Moscow in 2016-2017.

Methods: To conduct retrospective analysis of drugs utilization and cost in Moscow for period 2016-2017 for patients with MEL analytical model, relying on depersonalized data from regular database of reimbursed drug prescription of the Moscow City Health Department, has been developed. To evaluate the costs of drug therapy differentiation was conducted chemotherapy (CT), immunotherapy (IT) and targeted therapy (TT). The 10 most high-cost medications for MEL were analyzed. 
Results: About 10'270 patient's prescriptions for period 2016-2017 were analyzed. The 10 most high-cost medications for MEL in 2016 were vemurafenib, dabrafenib, trametinib, temozolomide, interferon $\alpha-2 b$, tumor necrosis factor $\alpha-1$, pazopanib, paclitaxel, vandetanib, fotemustin; in 2017 - ipilimumab, vemurafenib, dabrafenib, nivolumab, trametinib, pembrolizumab, cobimetinib, temozolomide, interferon $\alpha-2 b$, fotemustin. For MEL in 2017 the main share of costs was TT (55\%), $2^{\text {nd }}$ place - IT $(42 \%), 3^{\text {rd }}$ place - CT $(1.5 \%)$. Compared with 2016 , the costs of CT and TT significantly decreased and of IT increased. The structure of drugs prescription has changed, because clinical practice has included new TT and IT drugs (ipilimumab, nivolumab, pembrolizumab, cobimetinib). The most high-cost drug was vemurafenib in 2016, ipilimumab in 2017.

Summary / Conclusions: Data on the economic burden of melanoma in Russia are scarce in the literature, only one study was identified - Ignatyeva, et al., 2014. Our study is the first attempt to assess the economic burden of melanoma at the regional level. The impact of melanoma on drug expenditures for cancer was significant compared with other localization.

\section{Oral Presentation \\ EACPT-1323}

Assessment of antineoplastic drug approvals by european medicines agency (ema) and food and drug administration (fda) during 2018. Evaluation of possible differences and available evidence

P. Alcubilla-Prats ${ }^{*}$, M. Marfä ${ }^{1}$, M. V. de Dios ${ }^{1}$, G. Calvo ${ }^{1}$

${ }^{1}$ Clinical Pharmacology, Hospital Clínic of Barcelona, Barcelona, Spain

\section{Cancer treatment, Drug regulation}

Introduction: The field of oncology is currently undergoing a revolution in terms of new therapies made available for cancer treatments. However, it is not clear if new treatments and indications are synchronically approved in Europe and United States, and which is the quality of the evidence provided for efficacy at the time of approval.

Objectives: Perform a descriptive comparison between anticancer drug approvals (either new therapies or approved therapies for new indications) by EMA and FDA during 2018, to detect differences among agencies. We also revised available efficacy evidence for each treatment at the time of approval.

Methods: Revision of public reports and summaries of product characteristics on antineoplastic agent approvals by EMA and FDA from January 2018 to December 2018. Data on exact indication, special assessment program, and type of evidence from pivotal clinical trials was extracted for analysis.

Results: From January to December 2018, both agencies approved 23 new therapies for 28 indications and 5 already available drugs for 20 new indications. Only 12 new therapies were approved by both agencies. 11 drugs were approved by FDA and not by EMA. Of those, 9 are being revised by EMA. Among all therapies approved by both $(\mathrm{N}=21), 15$ were for same indication, 2 presented slight differences and 4 presented significant indication differences. $86 \%$ of therapies and indications approved by FDA were in a special assessment program (Fast-track, accelerated approval, breakthrough therapy or priority review) but only $22 \%$ of those approved by EMA (accelerated assessment, PRIME or conditional marketing authorization). Primary efficacy endpoint for approval was Overall Survival in $18 \%$, Progression-free-survival in $28 \%$ and overall response rate in $54 \%$. Pivotal trials were single-arm in $33 \%$ of approvals and randomized trials in $55 \%$

Summary / Conclusions: It seems that new anticancer therapies are available first in the US, probably due to differences on evaluation calendars and specific timings. We noticed a widespread utilization of special assessment programs by the FDA. When drug was approved by both agencies, significant differences in approved indications were uncommon. Overall, proof of evidence at the time of approval was mainly based on surrogate endpoints, with a significant proportion of uncontrolled single-arm studies.

\section{EACPT-1173}

Genetic polymorphisms of cyp450 and tolerability of tamoxifen therapy

A. Ignatova ${ }^{1}$, J. Zacharenkova ${ }^{2}$, I. Dudina ${ }^{2}$, M. Savelyeva* 3

${ }^{1}$ I.M. Sechenov First Moscow State Medical University of the Ministry of Health of the Russian Federation (Sechenov University), ${ }^{2}$ Sechenov University, ${ }^{3}$ Russian Medical Academy of Postgraduate Education, Moscow, Russian Federation

Cancer treatment, Pharmacogenetics/omics, Pharmacokinetics/ pharmacodynamics

Introduction: Effects of genetic polymorphisms of CYP450 enzymes on the tamoxifen (TX) efficiency and tolerability in the management of breast cancer are issues of current interest. The long-term TX adverse events (AEs) occur in 35 to $60 \%$. The frequency of AEs is associated with a higher plasma level of endoxifen, the active metabolite of TX, which concentration depends on allelic variants of CYP450.

Objectives: The aim of our study is to determine whether polymorphisms of CYP450 affect frequency of AEs during TX therapy. We used examples of two patients who have luminal A breast cancer with similar prognostic features but different genetic profiles.

Methods: Specially designed questionnaires were used to determine disease course characteristics, comorbidities, previous treatment, to analyze the AEs and assess their relation to the TX therapy. For the data processing, we used Excel. Specimens of the buccal epithelium (double sampling) were obtained. The genetic analysis was performed using real-time PCR.

Results: In patient A (64 years) mutations of the CYP2D6 $(* 1 / * 4)$, CYP3A5 $(* 3 / * 3)$ and CYP2C9 $(* 2 / * 3)$ genes were identified, patient B (53 years) haven't polymorphisms of these genes. Both women had stage Ia cancer, radical surgery and radiotherapy were previously performed. Within 1 month after the TX therapy initiation, prominent AEs (bone pain, weakness, dizziness, hot flashes, night sweats) occurred in patient $\mathrm{B}$ and required change of the medication. Patient $\mathrm{A}$ has been received TX for 19 months; she didn't notice any changes in her general condition. The differences of the genetic profile in these two patients with similar clinical characteristics may partially explain the different response to TX therapy. We hypothesize that AEs of TX are associated with the genetic polymorphisms of the CYP450 system. On the one hand, the presence of prominent AEs may indicate a high active metabolites concentration and the effectiveness of the therapy, but this may also alter the compliance and require adjustment (patient $\mathrm{B}$ ). On the other hand, the complete absence of AEs may indicate a lack of pharmacological activity of the drug due to the presence of clinically significant mutations in the CYP450 genes (patient A).

Summary / Conclusions: Applications of CYP450 testing in the clinical setting is expected to contribute to the personalized and effective drug prescription.

\section{EACPT- 1175}

Polymorphisms of cytochrome p450 and adverse events of tamoxifen therapy

A. Ignatova ${ }^{1}$, J. Zacharenkova ${ }^{2}$, I. Dudina ${ }^{2}$, M. Savelyeva* 3

${ }^{1}$ I.M. Sechenov First Moscow State Medical University of the Ministry of Health of the Russian Federation (Sechenov University), ${ }^{2}$ Sechenov University, ${ }^{3}$ Russian Medical Academy of Postgraduate Education, Moscow, Russian Federation

Cancer treatment, Pharmacogenetics/omics, Pharmacokinetics/ pharmacodynamics

Introduction: Tamoxifen (TX) is a selective modulator of estrogen receptors. Nowadays, it is widely used for ER-positive female breast cancer treatment. TX is a prodrug which is metabolized by cytochrome P450 to 
active forms. There is an emerging body of evidence linking high variability in the CYP genes to individual response, effectiveness and adverse events of TX therapy.

Objectives: To evaluate $\mathrm{P} 450$ polymorphisms influence on incidence of TX adverse events and identification of probable risk factors for such events in Russian women with resectable luminal breast cancer.

Methods: 27 patients with resectable luminal breast cancer after radical treatment were given the questionnaire in order to evaluate severity of TX adverse events and to reveal probable risk factors. Then samples of buccal epithelium were taken for genetic analysis of CYP2D6*4, CYP3A $5 * 3$, CYP3A4*17, CYP2C9*2,3, CYP2C19*2,3 gene mutations. The genetic analysis was performed using real-time PCR.

Results: TX adverse effects was observed in $92,6 \%(\mathrm{n}=25)$ : flushes $77.8 \%$, asthenia $55.6 \%$, body weight gain $51.8 \%$, ostealgia $48.1 \%$, alopecia $40 \%$, dyspepsia $18.5 \%$, vaginal hemorrhage $14.8 \%$. Hence, $29.6 \%$ $(\mathrm{n}=7)$ had a combination of four TX adverse effects, both $25,9 \%$ and $11,1 \%$ patients had one or a combination of three or five adverse effects. We hypothesized that heterozygotes with G1846A mutation in CYP2D6 rs 3892097 had a tendency to reduction of TX adverse events incidence in comparison with wild type homozygotes: flushes $57,1 \%$ vs. $85 \%$ ( $p>0.05$ ), body weight gain $42.8 \%$ vs. $55 \%$ ( $p>0.05$ ), ostealgia $28.6 \%$ vs. $55 \%$ ( $p>0.05)$, dyspepsia $14.3 \%$ vs. $20 \%$ ( $>0.05)$. In contrast, heterozygotes with G681A mutation in CYP2C19 rs 4244285 in comparison with wild type homozygotes have tendency to exacerbation of adverse events: flushes $87.5 \%$ vs. $73.6 \%$ ( $>00.05$ ), ostealgia $62.5 \%$ vs. $42.1 \%$ ( $>0.05$ ), body weight gain $62.5 \%$ vs. $47.4 \%$ ( $>>0.05$ ), dyspepsia $25 \%$ vs. $15.8 \%$ (p>0.05), asthenia $62.5 \%$ vs. $52.6 \%$ (p>0.05).

Summary / Conclusions: We suppose that patients with G1846A mutation in CYP2D6 associated with low concentrations of low metabolites more rarely have TX adverse events. Absence of adverse events might indirectly evidence the tamoxifen therapy ineffectiveness. We hypothesize that postmenopause, breast cancer II-III stage, long-term TX therapy and BMI $>25$ are the risk factors of adverse effects. Applications of CYP450 testing in clinics are expected to contribute to the personolized and effective drug perscription.

\section{Oral Presentation \\ EACPT-1265}

Anticancer potential of nanogold conjugated toxin gnp-nn-32 from indian spectacled cobra venom

S. Attarde ${ }^{*}$, S. Pandit ${ }^{2}$

${ }^{1}$ Evolutionary Venomics Lab, Centre for Ecological Sciences, Indian Institute of Science, Bangalore, ${ }^{2}$ Department of Zoology, Savitribai Phule Pune University, Pune, India

\section{Cancer treatment}

Introduction: Cancer, despite the all-out efforts from developed countries still causes one in five deaths. Surgery, chemotherapy and radiotherapy provide inadequate protection and instead affect normal cells along with cancer cells. The search for cancer cure from natural products (plants and animals) have been practised for over a century and the use of purified chemicals to treat cancer continues.

Objectives: To evaluate anticancer potential of nanogold conjugated GNP-NN-32 toxin from Indian spectacled cobra venom against human breast cancer cell lines.

Methods: Characterization of GNP-NN-32 was done by UV-Vis spectroscopy, Dynamic Light Scattering (DLS), Transmission Electron Microscopy (TEM), Atomic Force Microscopy (AFM) and Zeta Potential analysis. We have performed MTT assay, Neutral red uptake assay, Anti-proliferation assay and Detection of apoptosis by flow cytometric analysis on human breast cancer cell lines after treatment with NN32 and GNP-NN-32.

Results: NN-32 and GNP-NN-32 showed significant cytotoxicity against breast cancer cell lines (MCF-7 and MDA-MB-231) in both dose and time-dependent manner. The increased efficacy of GNP-NN-32 over NN32 was suggested by lower $\mathrm{IC}_{50}$ values. NN-32 and GNP-NN-32 showed an anti-proliferative effect on both MCF-7 and MDA-MB-231 cell lines. Neutral Red uptake assay showed a significant decrease in lysosomal activity in a dose-dependent manner. NN-32 and GNP-NN-32 induced apoptosis in both MCF-7 and MDA-MB-231 cells after treatment. Conjugated toxin GNP-NN-32 showed higher apoptotic activity towards both MCF-7 and MDA-MB-231 cells than that of the NN-32 alone. CFSE Cell Proliferation study suggested that NN-32 and GNP-NN-32 arrested cell division in both MCF-7 and MDA-MB-231 cell lines thereby inhibiting proliferation of these cancer cells.

Summary / Conclusions: NN-32 and GNP-NN-32 showed significant cytotoxicity against MCF-7 and MDA-MB-231 in both dose and timedependent manner and considerably less towards normal breast cells (MCF-10A). NN-32 and GNP-NN-32 induced apoptosis and arresting cell cycle on MCF-7 and MDA-MB-231 cells. Detailed chemical characterization and its cytotoxic property need to be analysed, which may prove a new dimension of anti-cancer potential of GNP-NN-32 that will accelerate its biomedical application in the near future.

\section{EACPT- 1275}

Investigation of the anti-tumour activity of azadirachta indica (neem) leaf extract on human ovarian cancer cells

L. Jno.Baptiste ${ }^{*} 1$, K. Hemming ${ }^{1}$, F. Javid ${ }^{1}$

${ }^{1}$ School of Applied Sciences, University of Huddersfield, Huddersfield, United Kingdom

\section{Cancer treatment}

Introduction: Ovarian cancer, the fifth leading cause of cancer death among women in Western Europe and United States has the highest mortality rate of all gynaecologic cancers (Kamath et al., 2009). The use of natural treatment is gaining more importance due to terrible side effects of current treatments.

Neem has been claimed to cure (Gunadharini et al., 2011) and prevent cancer (Harish Kumar et al., 2009) such as colon and lung by the production of antioxidant and carcinogen-detoxifying enzymes. (Paul et al., 2011).

Objectives: To investigate the ability of Neem extract to selectively inhibit the proliferation of human ovarian cancer cells in-vitro.

Methods: Phytochemicals were extracted by decoction for 30 minutes and maceration for 48 hours with varying solvents (deionised water, absolute ethanol, phosphate buffered saline (PBS), dimethyl sulfoxide (DMSO) or ethyl acetate (EA)). Filtered solvents were evaporated then extracts concentrated. Working solutions of $10 \%, 20 \%, 40 \%, 60 \%, 80 \%$ and $100 \%$ were used in treating CP70, A2780 and ARPE-19 cells followed by incubation for four days then the MTT assay. Experiment was conducted in triplicates.

Results: The extract of NE at $60 \%, 80 \%$ and $100 \%$ appeared to significantly $(\mathrm{p}<0.05)$ reduce the $\%$ cell viability of A2780 cells which were similar to cytotoxicity afforded by cis-plantin at $1 \mathrm{mM}$. NEA and NDMSO caused a reduction in cell growth at $10 \%$ and $40 \%$ above percentages respectively.

\begin{tabular}{llll}
\hline Cell Line & Extract & & \\
& NE & NEA & NDMSO \\
CP70 & 1.2 & 2.7 & 0.98 \\
A2780 & 0.82 & 2.3 & 1.2 \\
\hline
\end{tabular}

The extracts of NE at $40 \%, 60 \%, 80 \%, 100 \%$ showed significant $(\mathrm{p}<0.01)$ reduction in the cell viability of $\mathrm{CP} 70$ cells which were similar to cytotoxicity afforded by cis-plantin at $1 \mathrm{mM}$. NEA and NDMSO caused a reduction in cell growth when used at $10 \%$ and $20 \%$ above percentages respectively. 
Selectivity of NE48, NEA and NDMSO towards CP70 and A2780 cell lines.

Selectivity greater than one indicates good selectivity.

Summary / Conclusions: NE, NDMSO and NEA extracts showed cytotoxicity towards the cancerous cell lines. In the future, more replicates need to be conducted and another cell viability assay should be conducted with the MTT to further confirm results. In addition, the active components in these extracts need to be identified and the mechanism of action of these extracts in inducing cytotoxicity should be studied.

\section{EACPT-1315}

Active therapeutic drug managing instead of passive therapeutic drug monitoring - towards a safer high-dose methotrexate regimen to adult patients with acute lymphatic leukaemia

A.-K. Hamberg ${ }^{*}{ }^{1}$, J. Schubert ${ }^{1}$, E. B. Lennmyr ${ }^{2}$, H. Hallböök ${ }^{2}$

${ }^{1}$ Dept of Medical Sciences, Section of Clinical Pharmacology, ${ }^{2}$ Dept of Medical Sciences, Section of Haematology, Uppsala University Hospital, Uppsala, Sweden

Cancer treatment, Pharmacokinetics/ pharmacodynamics, Therapeutic drug monitoring

Introduction: A 24-hour infusion of high-dose methotrexate (HDMTX) is often used in the treatment of acute lymphatic leukaemia (ALL). The pharmacokinetics of HDMTX is known to be highly variable, both within and between patients [Evans, 1998]. This makes fixed dose regimens less likely to be effective and safe throughout treatment. MTX concentration at the end of infusion is commonly used to guide rescue therapy. Studies in children have shown that if MTX levels are measured early during the infusion; the infusion rate can be adjusted based on pharmacokinetic (PK) principles, reducing the risk of toxicity (Evans, 1998]. The protocol at our hospital use a fixed or empirically adjusted dose of HDMTX to adult patients.

Objectives: To develop a simple bedside algorithm for HDMTX to adult ALL-patients, with a reduced risk of delayed excretion (DE) and acute kidney injury (AKI) without compromising efficacy.

Methods: We combined a dose algorithm developed for children [Foster, 2019] with i) retrospectively collected treatment data from adult patients, with MTX-samples at hours 2, 6 and 23, ii) knowledge on MTX PK, and iii) clinical judgement of an experienced haematologist.

Results: In a cohort of 13 adult patients, the current protocol reached a steady-state concentration within, above and below the target range suggested for children with ALL $\left(\mathrm{C}_{\mathrm{ss}} 50-80 \mu \mathrm{M}\right)$ in $43 \%, 21 \%$ and $36 \%$ of 63 infusions. $\mathrm{DE}\left(\mathrm{C}_{42 \mathrm{~h}}>1 \mu \mathrm{M}\right)$ occurred in $33 \%$ of the infusions, with $11 \%$ having a creatinine increase $\geq 1.5 \times$ baseline, and one requiring glucarpidas. These findings indicate that adults could benefit from more tailored HDMTX. The published algorithm for children is based on MTX-levels at hours 2 and 6. Since almost all adjustments in children were made from the 6-hour sample, and no critical information was gained from the 2-hour sample in our cohort, we recommend MTXsampling at hour 6 accompanied by a creatine measurement as an early marker of AKI. Whether adjustment of the infusion rate is warranted depends on both MTX- and creatinine-levels. We also stress the importance of ensuring adequate hydration and maintaining urine $\mathrm{pH} \geq 7$ during the infusion due to poor solubility of MTX at acidic $\mathrm{pH}$.

Summary / Conclusions: We suggest a simple bedside algorithm for HDMTX to adult ALL-patients with monitoring of MTX and creatinine at hour 6 . The algorithm will be prospectively evaluated.

\section{EACPT-1324}

Ribociclib induces macrocytosis in therapeutic doses: characterization of outcome

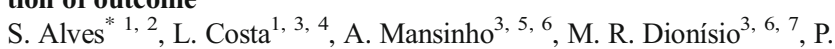
Andrade ${ }^{7,8}$

${ }^{1}$ Instituto de Medicina Molecular, ${ }^{2}$ Laboratório Farmacologia Clínica e Terapêtica, Faculdade de Medicina, Universidade de Lisboa, Lisboa, ${ }^{3}$ Centro Investigação Clínica HSM CHLN, Hospital Santa Maria, 1649-035
Lisboa, ${ }^{4}$ Serviço de Oncologia, Serviço de Oncologia, Hospital de Santa Maria, Centro Hospitalar Lisboa Norte, ${ }^{5}$ Instituto de Medicina Molecular, Instituto de Medicina Molecular, Faculdade de Medicina, Universidade de Lisboa, ${ }^{6}$ Serviço de Oncologia, Hospital Santa Maria, Centro Hospitalar Lisboa Norte, ${ }^{7}$ Instituto de Medicina Molecular, Faculdade Medicina, Universidade de Lisboa, Lisboa, ${ }^{8}$ Hospital Santa Maria, 1649-035 Lisboa, Portugal

\section{Cancer treatment}

Introduction: Ribociclib is a small-molecule that selectively inhibits cyclin-dependent kinases 4 and 6, blocking the phosphorylation of retinoblastoma protein and inducing of G1 phase arrest hence having an important role in the regulation and prevention of cell-cycle progression. The project OncoDynamics aims to explore possible correlations between therapeutic doses of ribociclib for treatment of breast cancer and other therapies with several analytic biomarkers and clinical data. Adverse advents of CDK 4/6 inhibitors are mostly hematologic being neutropenia the most frequent followed by anemia and thrombocytopenia as it is the case of palbociclib which has been recently described as associated with macrocytic, dysplastic anemia.

Objectives: This study aims to characterize the outcome of macrocytosis evident in our patients with ribociclib in therapeutic doses for treatment of breast cancer.

Methods: This study is integrated in the OncoDynamics project and is a prospective, unicentric cohort study with patients treated for metastatic breast cancer with ribociclic plus letrozole, each patient registered as digital case report form and evaluated data of mean corpuscular volume (MCV), hemoglobin ( $\mathrm{Hg}$ ) and red cell distribution width (RCDW) for descriptive analyses.

Results: Of the 7 patients with breast cancer treated with ribociclib $(14,8 \%)$ with a follow up period ranging from 3 to 18 months, 4 patients $(57,1 \%)$ had a sustained rise of the mean corpuscular volume ( $\mathrm{MCV}>97 \mathrm{fL}$ ), $\mathrm{Hb}$ ranging from 8,8 to $13,5 \mathrm{~g} / \mathrm{dL}$ and RCDW from 12,7 to $20,9 \mathrm{CV} \%$.

Summary / Conclusions: We found this macrocytosis to be related with treatment with ribociclic in therapeutic doses and in literature the relation between CDK 4/6 inhibitors and macrocytosis has been described in relation to palbociclib. Clinical evolution of these patients is under evaluation. We aim to extend this study to more patients in the context of breast cancer and other types of cancer.

This study had the approval from the Ethics Commission in 2017.

\section{EACPT-1334}

Analysis and improvement of clinical trial pharmacist's work in cancer center

H. Choo ${ }^{*}$, S. Chung ${ }^{1}$, J. Kim ${ }^{1}$

${ }^{1}$ Department of Pharmacy, Samsung Medical Center, Seoul, Korea, Republic Of

\section{Cancer treatment}

Introduction: Samsung Medical Center is the leading hospital in Korea and has established the Samsung Comprehensive Cancer Center (SCCC) for cancer treatment first in nation. Every year, 530,000 outpatients visit the cancer center. SCCC has opened a separate pharmacy for cancer clinical trials, which is the largest part of the clinical trials. With the recent spread of clinical trials for new anticancer drugs, the importance of investigational product (IP) management is growing.

Objectives: To analyze performance data of Clinical Trial Pharmacists $(\mathrm{CTPH})$ and find efficient management methods.

Methods: We analyzed the current data on ongoing clinical trials at SCCC's Clinical Trial Pharmacy (CTP) and the number of prescriptions for the month of February 2019. We also examined the time spent on each task of the CTPH and reviewed the efficiency plan.

Results: As of February 2019, 400 clinical trials were conducted at the SCCC CTP. Sponsor Initiated Trials (SIT) is $300(75 \%)$, Investigator Initiated Trials (IIT) and Emergency Investigational New Drug (e-IND) 
are $100(25 \%)$. Research on lung cancer was the most common (20.3\%). Twenty clinical trials were pharmacist-unblinded trials that required active intervention of СТPH. According to the analysis of the number of prescriptions for the month of February, there were 1109 doses (70\%) of IV admixture, and 477 (30\%) doses of oral and external IP. By types of cancer drugs, 499 (31.5\%) cases for immunotherapy and 447 (28.2\%) cases for targeted therapy were prescribed. In the entire course of clinical trials, CTPH was in charge of IV admixture preparation and patient counseling (43.7\%), IP management (56.3\%). The tasks that can be done efficiently by using the computerized management system were IP disposal, periodic reporting, and prescription reviewing.

Summary / Conclusions: In the course of clinical trials on cancer, CTPH was performing various IP management tasks as well as IV anticancer preparation. As clinical trials of various designs that require CTPH expertise are underway, computerized pharmacy management system should be improved to perform safe and efficient clinical trials management.

\section{EACPT-1344}

Adverse reactions to drugs of special interest in a pediatric oncohematology service

K. Amaro Hosey ${ }^{*}{ }^{1,2}$, I. Danés Carreras ${ }^{1,2}$, L. Vendrell Bosch ${ }^{1,2}$, G. Cereza García ${ }^{3}$, B. Renedo Miró ${ }^{4}$, L. Alonso García ${ }^{5}$, J. L. Dapena Díaz L. Gros Subías ${ }^{5}$, E. Diogène Fadini ${ }^{1,2}$

${ }^{1}$ Clinical Pharmacology Service, Vall d'Hebron University Hospital, ${ }^{2}$ Department of Pharmacology, Therapeutics and Toxicology, Universitat Autònoma de Barcelona, ${ }^{3}$ Fundació Institut Català de Farmacologia, ${ }^{4}$ Pharmacy Service, Vall d'Hebron University Hospital., ${ }^{5}$ Pediatric Oncology and Hematology Service, Vall d'Hebron University Hospital, Barcelona, Spain

\section{Cancer treatment, Pediatric treatment, Pharmacovigilance}

Introduction: Drugs used in oncological diseases are frequently related to adverse drug reactions (ADRs). Some drugs with an especially concerning toxicity profile are used off-label in children and toxicity may be increased.

Objectives: To evaluate the frequency and characteristics of ADRs to selected drugs in the Pediatric Oncohematology Service, in order to know their impact in real practice in our setting, and to identify opportunities to improve detection of ADRs and patient care.

Methods: An observational, longitudinal and prospective study has been carried out on a pediatric Oncohematology Service of a tertiary hospital. A list of drugs of special interest in pharmacovigilance (clofarabine, nelarabine, tioguanine, defibrotide, Erwinia asparaginase, E. Coli asparaginase, pegaspargase, crizotinib, imatinib, dasatinib, nilotinib, infliximab, rituximab, bevacizumab, eltrombopag and romiplostim) was agreed. During 2017, patients exposed to one or more of these drugs were identified and followed-up for at least 6 months each. Characteristics of ADRs, including causality and possible preventability, have been evaluated. Results: 72 patients have been treated with at least one study drug, and 159 ADRs episodes involving at least one of these drugs have been identified, with a total of 293 ADRs. Most episodes required hospitalization $(57 ; 36 \%)$ or happened in inpatients $(50 ; 31 \%)$, and $91 \%$ were severe. Blood disorders were the most frequent ADRs $(96 ; 33 \%)$, related to tioguanine (42) and pegaspargase (39) mainly, followed by infections $(86 ; 29 \%)$ related to tioguanine (32), pegaspargase (27), Erwinia asparaginase (14) and rituximab (13), and digestive $(23 ; 8 \%)$ and metabolic disorders $(22 ; 8 \%)$ related to pegaspargase (11 and 21, respectively). Two ADRs were unknown. Most ADRs were dose-dependent or expectable ( $>90 \%)$. Controversial additional measures of prevention, other than those already used, were identified. Additionally, 179 ADRs episodes involving other drugs not included in our list were detected, out of which $82 \%$ were severe.

Summary / Conclusions: ADRs are frequent in pediatric oncohematological patients, mainly blood disorders and infectious diseases. Most of them are expected or dose-dependent. Controversial additional measures of prevention other than those already used will be discussed. Only two ADRs were considered unknown. Patient monitoring has allowed us to detect ADRs related to other drugs also used in this population.

\section{EACPT-1389}

Pulmonary toxicity after dasatinib treatment for chronic myeloid leukemia : a case report and the literature review

J.-J. Hou ${ }^{*}$, Y.-L. Wang ${ }^{1}$

${ }^{1}$ Pharmacy Department, Kaohsiung Medical University Hospital, Kaohsiung, Taiwan, Province of China

\section{Cancer treatment, Pharmacovigilance}

Introduction: Dasatinib is a novel multi-targeted tyrosine kinase inhibitor approved for treatment of BCR-ABL positive chronic myeloid leukemia (CML) and Philadelphia chromosome positive acute lymphoblastic leukemia (ALL) after imatinib failure. Use of dasatinib is frequently complicated by pleural and pulmonary complication. Between 10 and 35 percent of patients treated with dasatinib in clinical trials developed pleural effusions. The mean time to onset of the pleural effusion was 11 months (range 3.6 to 18.6 months). Only the presence of concomitant pulmonary disease and higher initial daily dose (140 versus $100 \mathrm{mg})$ were significant risk factors. Altering the dasatinib regimen from $70 \mathrm{mg}$ twice daily to $100 \mathrm{mg}$ daily reduces the risk of pleural effusion, without affecting antitumor efficacy.

Objectives: The purpose is to present a case report related to pulmonary toxicity after using lower daily dose of dasatinib for chronic myeloid leukemia.

Methods: This is a 48 years old male of chronic myeloid leukemia in dasatinib $100 \mathrm{mg} / \mathrm{d}$ treatment for 6 years. Recently, he is diagnosed with parapneumonic effusion related to induce by dasatinib.

Results: This case had used dasatinib in low dose for 6 years, but dasatinib indused pulmonary toxicity recently. The treatments related to dasatinib causing pleural effusions also were discussed in this study.

Summary / Conclusions: Not only be careful pulmonary toxicity when using dasatinib at the first year, but also should notified the longterm toxicity when using in the low daily doses of dasatinib.

\section{Oral Presentation}

EACPT- 1440

Post authorization safety profile in relation to off label use of rituximab

A. Aldea ${ }^{1}$, P. Diez Pellicer ${ }^{2}$, J. Mateus ${ }^{3}$, E. J. Sanz ${ }^{*}$

${ }^{1}$ Clinical Pharmacology. IMIM. , ${ }^{2}$ Clinical Pharmacology, Hospital del Mar, ${ }^{3}$ Integrative Pharmacology and systems neuroscience research group, IMIM, Barcelona, ${ }^{4}$ Dept. of Physical Medicine and Pharmacology, Department of Pharmacology, La Laguna, Spain

\section{Cancer treatment, Chronic diseases, Pharmacovigilance}

Introduction: Off label use of rituximab to treat non-authorized conditions is high and the need for safety data may not be met.

Objectives: To analyze the Individual Clinical Safety Report (ICSR) reported to the global WHO database for Pharmacovigilance in order to assess the safety profile when off label use of rituximab is co-reported.

Methods: Vigilyze ${ }^{\circledR}$ was used to elicit all the ICSR with Rituximab [substance] and "off label uses and intentional product misuses/use issues" [HLGT] co- reported. Other Preferred terms co-reported are related to MEDRA Terms. Dataset date: 2019-03-10

Results: From 67195 ICSRs with rituximab, 2265 ICSRs were related to off label use. The ICSR with the association has increased 32\% from 2008 to 2018 , mostly last 4 years and still rising up. One thousand six hundred and fifty ICSRs were serious and 373 were fatal cases (some recorded as a term and others only as an outcome). The total reactions included in 2265 reports were 220380 reactions and death was involved as a co-reported reaction term in relation to the off label use in 227 ICSR (10\%). Other 
frequent reactions were "Infusion relation reaction", "fatigue" and "drug ineffective", $188(8,3 \%), 178$ (7.9\%) and $145(6,4 \%)$ respectively. "Drug ineffective" term was reported in 2471 ICRS with rituximab but only 145 ICSRs were co-reported with "off label use". Most suspected concomitant drugs or interactions were with prednisone, cyclophosphamide, doxorubicin, vincristine and mycophenolic acid. IC and IC025 values related to rituximab and "off label use" were greater than 1 (IC:1.52 and IC025:1.46). Summary / Conclusions: Post authorization safety profile in relation to off label use of rituximab seems to be similar than the safety profile described to the authorized indications. "Drug ineffective" was one of the most co-reported terms but it seems to be more reported in ICSR with rituximab used in normal conditions. More safety prospective studies would be performed for several off label indications.

\section{EACPT-1458}

Successfully treat of severe hemorrhagic cystitis by intravesicle hyaluronic acid in a case of haploidentical stem cell transplant-a case report

\section{C.-I. Yang ${ }^{*}{ }^{1}$, H.-H. Hsiao ${ }^{1}$}

${ }^{1}$ Department of Hematology and Oncology, Kaohsiung Medical University Hospital, Kaohsiung, Taiwan, Province of China

\section{Cancer treatment, Pharmacovigilance}

Introduction: Hemorrhagic cystitis(HC) is one of common complications following Hematopoietic stem cell transplantation (HSCT). Its incidence has been reported from 7-68\% with a median time to onset of 30 days after transplantation. The risk factors include viral infection,cyclophosphamide and busulfan usage,pelvic irradiation,older age at transplantation, allogeneic HSCT and Graft-versus-host disease(GVHD). The most common treatments for HC were: hyper-hydration, bladder catheter drainage with/without irrigation, platelet transfusion, hyperbaric oxygen therapy. However, there is no better therapeutic strategy of hemorrhagic cystitis after haploidentical transplantation.

Objectives: Here we presented a case of hemorrhagic cystitis after haploidentical transplantation with successful therapy by hyaluronic acide (HA).

Methods: A53- year-old male with history of acute myeloid leukemia in 2nd remission status received haploidentical HSCT with a standard conditioning regimen with FB4-PTCy( Cyclophosphamide $(29 \mathrm{mg} / \mathrm{kg}$ ), Fludarabine $(120 \mathrm{mg} / \mathrm{kg})$ and Busulphan $(12.8 \mathrm{mg} / \mathrm{kg})$ and thymoglobulin $(5 \mathrm{mg} / \mathrm{kg})$ and post-transplantation cyclophosphamide (PTCy)), Cyclosporine A and MMF for GVHD prophylaxis were prescribed but he experienced a Grade IV hemorrhagic cystitis with grade 2 of aGVHD. The gross hematuria and painful voiding pain began on 21 days after transplant with grade 2 of skin GVHD. There was a limit effect after hydration and irrigation by a saline solution. However, gross hematuria with much large clot formation were still noted, requiring irrigated, PRBC and platelet transfusion on a regular basis, and tranexamic acid treatment. Due to persisted gross hematuria without improvement by previous treatments for more than 100 days, Intravesical HA at a dose of $40 \mathrm{mg}$ in a $50 \mathrm{ml}$ Solution through Foley catheter irrigated (claimed for $1 \mathrm{~h}$ and the drained) was start on the Day 127, Day 132, Day 139. Afterward, he became symptom-free and urine turned back to a normal appearance after second dose HA.

Results: Severe HC often required prolonged and expensive hospitalization and occasionally resulted in death without optimal therapies. Several reviews revealed the promising experience of Hyaluronic acid(HA) treatment in interstitial cystitis.

Summary / Conclusions: Our case revealed that intravesical HA therapy could help in severe hemorrhagic cystitis after stem cell transplant with promising effects.

\section{EACPT-1006}

Vasorelaxation of human saphenous vein induced by epicatechin A. Novakovic ${ }^{*}{ }^{1}$, M. Marinko ${ }^{1}$, G. Jankovic ${ }^{1}$, I. Stojanovic ${ }^{2,}{ }^{3}$, P. Milojevic ${ }^{2,3}$, D. Nenezic ${ }^{2,3}$, V. Kanjuh ${ }^{4}$, Q. Yang ${ }^{5,6}$, G.-W. $\mathrm{He}^{5}$
${ }^{1}$ Department of Pharmacology, Faculty of Pharmacy, University of Belgrade, ${ }^{2}$ Institute for Cardiovascular Diseases "Dedinje", ${ }^{3}$ Faculty of Medicine, University of Belgrade, ${ }^{4}$ Academy of Sciences and Arts, Belgrade, Serbia, ${ }^{5}$ TEDA International Cardiovascular Hospital, Medical College, Nankai University, Tianjin, China, ${ }^{6}$ Department of Surgery, The Chinese University of Hong Kong, Hong Kong, Hong Kong

\section{Cardiovascular treatment, Other}

Introduction: Epicatechin is a major bioactive constituent of flavanolrich food, which gained increase attention after an inverse relationship between cardiovascular risk and consumption of flavanols has been suggested. In a number of biological studies it was shown that vasodilator, as well as antiproliferative, antiaggregation and hypolipemic effects of (-)-epicatechin contribute to its cardioprotective action. The exact mechanisms by which epicatechin causes vasodilation are not entirely clear.

Objectives: We aimed to investigate relaxant effect of epicatechin on the isolated human saphenous vein (HSV) and to define the mechanisms underlying this vasorelaxation.

Methods: Discarded segments of HSV were collected from patients undergoing coronary artery bypass grafting and studied in organ baths.

Results: Epicatechin induced a concentration-dependent relaxation of HSV pre-contracted by phenylephrine. Among $\mathrm{K}^{+}$channel blockers, 4-aminopyridine (4-AP), margatoxin and iberiotoxin significantly inhibited relaxation of HSV, while glibenclamide considerably reduced effects of the high concentrations of epicatechin. Additionally, epicatechin relaxed contraction induced by $80 \mathrm{mM} \mathrm{K}^{+}$, whereas in the presence of nifedipine produced partial relaxation of $\mathrm{HSV}$ rings pre-contracted by phenylephrine. In $\mathrm{Ca}^{2+}$ free solution, epicatechin relaxed contraction induced by phenylephrine, but had no effect on contraction induced by caffeine. Thapsigargin significantly reduced relaxation of HSV produced by epicatechin.

Summary / Conclusions: Our results demonstrate that epicatechin produces endothelium-independent relaxation of isolated HSV rings. Vasorelaxation to epicatechin probably involves activation of 4-AP- and margatoxin-sensitive $\mathrm{K}_{\mathrm{V}}$ channels, $\mathrm{BK}_{\mathrm{Ca}}$ channels, and at least partly, $\mathrm{K}_{\mathrm{ATP}}$ channels. In addition, not only the inhibition of extracellular $\mathrm{Ca}^{2+}$ influx, but regulation of the intracellular $\mathrm{Ca}^{2+}$ release, via inositoltrisphosphate receptors and reuptake into sarcoplasmic reticulum, via stimulation of $\mathrm{Ca}^{2+}$-ATPase, as well, most likely participate in epicatechin-induced relaxation of HSV.

\section{EACPT-1015}

Effectiveness and safety of alirocumab in a third level hospital N. Báez Gutiérrez ${ }^{1}$, C. Romero Lara ${ }^{1}$, H. Rodríguez Ramallo ${ }^{1}$, M. D. Santos Rubio ${ }^{1}$ R. Seisdedos Elcuaz ${ }^{*}$

${ }^{1}$ Hospital Pharmacy, Hospital Universitario Virgen del Rocío, Seville, Spain

\section{Cardiovascular treatment}

Introduction: Alirocumab is a fully human immunoglobulin G1 monoclonal antibody directed against proprotein convertase subtilisin/kexin type 9 (PCSK9) approved for the treatment of hypercholesterolemia in high-risk patients.

Objectives: To analyze de use and to evaluate the effectiveness and tolerance of alirocumab in patients refractory to conventional lipidlowering treatments in a third level hospital.

Methods: Retrospective observational study of patients treated with alirocumab during the year 2017. To asses effectiveness, the following variables were collected: demographics (sex and age), indication, objective and basal LDL levels, LDL levels at a month, 3, 6, 9 and 12 months after starting treatment, $\%$ of reduction with respect to basal LDL levels. To assess safety adverse effects were 
collected.The information was obtained from the corporate prescription program Athos Prisma ${ }^{\circledR}$ and from the Diraya ${ }^{\circledR}$ clinical station. Results: 75 patients were treated the mean age was $59.6(40-82)$ years and a $57.3 \%$ were women. The indications for treatment were: $72 \%$ heterozygous familial hypercholesterolemia: $16 \%$ stable atheromatous cardiovascular disease and $12 \%$ allergy or intolerance to statins.

- 73 patients started with a dose of $75 \mathrm{mg}$. $32.9 \%$ of these patients required an increase to $150 \mathrm{mg}$ due to therapeutic failure. Of the remaining $67.1 \%$ a $90 \%$ reached objective levels. The mean baseline LDLlevel was $131.8 \mathrm{mg} / \mathrm{dl}$, obtaining a reduction of $51 \%$ after one month of treatment. - Patients who required an increase of dose obtained a reduction of $52.65 \%$ a month after the dose change.

- Patients who started with $150 \mathrm{mg}$ had a baseline LDL level average of $304.5 \mathrm{mg} / \mathrm{dl}$, resulting in a reduction of $88 \%$ after a month of treatment.

The adherence was $100 \%$, and no medication-related problems were observed

Summary / Conclusions: Our small series confirms Alirocumab is an effective, safe and welltolerated treatment for reducing LDL levels when others lipid lowering treatments are contraindicated.

Almost a $100 \%$ of patients reached target LDL levels, either at the beginning or after the dose increase of Alirocumab.

\section{Oral Presentation}

\section{EACPT-1074}

Methodological and reporting issues in pharmacoepidemiological research on drug safety. A review of studies underlying recent conclusions on associations between loop diuretics/beta-blockers and falls S. Wallerstedt ${ }^{*}$, M. Hoffmann ${ }^{2}$

${ }^{1}$ Pharmacology, Sahlgrenska Academy, Sahlgrenska University Hospital, Göteborg, ${ }^{2}$ The NEPI foundation - Swedish Network for Pharmacoepidemiology, Linköping, Sweden

\section{Cardiovascular treatment, Pharmacovigilance}

Introduction: Three systematic reviews with meta-analyses, published in 1999, 2009, and 2018, report associations between cardiovascular drugs and falls. These aggregated data may have contributed to recommendations against cardiovascular drugs because of fall-risk properties in indicators of prescribing quality in the elderly. However, cardiovascular drugs also have established beneficial effects, and a closer look at the evidence underlying conclusions on cardiovascular drugs and falls would be valuable.

Objectives: To review studies underlying statistically significant metaanalyses in a current systematic review investigating cardiovascular drugs and falls.

Methods: All studies contributing data to recent conclusions that loop diuretics and beta-blockers are associated with falls were reviewed. Study characteristics were extracted, and methodological as well as reporting issues assessed.

Results: A total of nine studies, five of which published in 2015, were included in the review. The studies included 498 to 321,995 individuals, and six studies reported results where the association between loop diuretics/beta-blockers and falls were statistically significant. In the five studies with a cohort design, characteristics between exposed and unexposed was either not reported $(n=3)$ or differed substantially regarding morbidity $(n=2)$. In the four studies with a case-control design, morbidity characteristics between fallers and non-fallers were either not reported $(n=2)$, differed $(n=1)$, or reported according to the diseases matched for $(n=1)$. Confounding by indication was explicitly mentioned and discussed in two out of nine studies. In none of the abstract conclusions, precautions were made regarding causality. Three studies provided clinical advice for prescribers highlighting that the identified increased fallrisk should be an important aspect for decision-making. One of these also mentioned the benefit-risk assessment, taking into account the patient's current health condition, which shall accompany all drug treatment.
Summary / Conclusions: This review illustrates pitfalls in pharmacoepidemiological research which may be concealed in metaanalyses. Researchers and physicians need to be aware of these when designing studies and interpreting results not to draw hasty conclusions.

\section{EACPT-1090}

Glucose control safety \& efficacy in type 2 diabetes, a systematic review and network meta-analysis (glucose dinet)

G. Grenet ${ }^{*}$, S. Ribault ${ }^{2}$, G. B. Nguyen ${ }^{2}$, F. Glais ${ }^{2}$, A. Metge ${ }^{2}$, T. Linet $^{2}$, B. Kassai-Koupai ${ }^{1}$, C. Cornu ${ }^{1}$, T. Bejan-Angoulvant ${ }^{3}$, S. Erpeldinger ${ }^{4}$, R. Boussageon $^{4}$, A. Gouraud ${ }^{1}$, F. Bonnet ${ }^{5}$, M. Cucherat ${ }^{1}$, P. Moulin ${ }^{6}$, F. Gueyffier $^{1}$

${ }^{1}$ Pharmacotoxicology, University Hospital of Lyon, ${ }^{2}$ Université de Lyon, F-69000, Lyon, ${ }^{3}$ Service de Pharmacologie Médicale, CHRU de Tours, Tours, ${ }^{4}$ Département de médecine générale, Université Lyon 1, Lyon, ${ }^{5} \mathrm{CHU}$ Rennes, Université de Rennes 1 , Rennes, ${ }^{6}$ Fédération d'endocrinologie, maladies métaboliques, diabète et nutrition, INSERM UMR 1060 CARMEN Hospices Civils de Lyon, Université Lyon 1, Lyon, France

\section{Cardiovascular treatment, Chronic diseases}

Introduction: The last consensus on type 2 diabetes (T2D) recommends SGLT-2 inhibitors or GLP-1 agonists for patients with clinical cardiovascular (CV) disease. Last studies suggested beneficial effects of SGLT-2 inhibitors or GLP-1 agonists compared to DPP-4 inhibitors, in secondary $\mathrm{CV}$ prevention. Recently, a potential benefice of SGLT-2 inhibitors in primary CV prevention also has been suggested. However, no comparison of all the new and the old hypoglycemic drugs is available on CV outcomes.

Objectives: We aimed to compare the effects of old and new hypoglycemic drugs in T2D, on major adverse cardiovascular events (MACE) and mortality.

Methods: We conducted a systematic review and network meta-analysis. Randomized trials assessing contemporary hypoglycemic drugs on mortality or MACE in patients with T2D, were searched for in Medline, the Cochrane Central Register of Controlled Trials (CENTRAL), and ClinicalTrials.gov. Each drug was analyzed according to its therapeutic class. A random Bayesian network meta-analysis model was used. The primary outcomes were overall mortality, cardiovascular mortality, and MACE. Severe adverse events and severe hypoglycemia were also recorded.

Results: 175,966 patients in 34 trials from 1970 to 2018 were included. 17 trials included a majority of patients with previous cardiovascular history, 16 trials a majority of patients without. Compared to control, SGLT-2 inhibitors were associated with a decreased risk of overall mortality (OR $=0.84$ [95\% CrI: 0.74; 0.95]), SGLT-2 inhibitors and GLP-1 agonists with a decreased risk of MACE (OR $=0.89$ [95\% CrI: 0.81; $0.98]$ and $\mathrm{OR}=0.88$ [95\% CrI: 0.81;0.95], respectively). Compared to DPP-4 inhibitors, SGLT-2 inhibitors were associated with a decreased risk of overall mortality ( $\mathrm{OR}=0.82$ [95\% CrI: $0.69 ; 0.98]$ ), GLP-1 agonists with a decreased risk of MACE (OR $=0.88[95 \%$ CrI: $0.79 ; 0.99])$. Insulin and sulfonylureas were associated with an increased risk of severe hypoglycemia. In the trials including a majority of patients without previous $\mathrm{CV}$ history, the comparisons of SGLT-2 inhibitors, metformin and control did not showed significant differences on primary outcomes.

Summary / Conclusions: SGLT-2 inhibitors and GLP-1 agonists have the most beneficial effects, especially in T2D patients with previous CV diseases. Direct comparisons of SGLT-2 inhibitors, GLP-1 agonists and metformin are needed, notably in primary CV prevention.

\section{EACPT- 1120}

Absorption, distribution, metabolism, and excretion of selatogrel (act-246475), a p2y12 receptor antagonist for subcutaneous use 
M. Ufer ${ }^{*}$, J.-J. van Lier $^{2}$, H. Fischer ${ }^{3}$, J. Dingemanse ${ }^{4}$

${ }^{1}$ Department of Clinical Pharmacology, Idorsia Pharmaceuticals Ltd., Allschwil, Switzerland, ${ }^{2}$ PRA Health Sciences, Groningen, Netherlands, ${ }^{3}$ AA\&M Labor für Analytik und Metabolismusforschung Service GmbH, Bergheim, Germany, ${ }^{4}$ Dept. of Clinical Pharmacology, Idorsia Pharmac., Allschwil, Switzerland

\section{Cardiovascular treatment, Pharmacokinetics/ pharmacodynamics}

Introduction: Selatogrel (ACT-246475) is a P2Y12 receptor antagonist for subcutaneous (s.c.) use with rapid onset of action. It is in clinical development for treatment of acute myocardial infarction. Preclinical studies indicated that selatogrel is not a substrate of cytochrome P450 enzymes and mainly excreted in feces.

Objectives: To determine routes of elimination and to identify/quantify the metabolites of selatogrel in humans.

Methods: A single-center, open-label, single-dose human ADME study was performed in six healthy male subjects. A single s.c. dose of $16 \mathrm{mg}$ ${ }^{14} \mathrm{C}$ radiolabeled selatogrel (incl. $100 \mu \mathrm{Ci}$ ) was administered. Blood sampling and urine/feces collection were performed for at least $72 \mathrm{~h}$ post dosing until cumulative radioactivity recovery was $\geq 85 \%$ or daily radioactive excretion was $\leq 0.5 \%$ on two consecutive days. Radioactivity in urine and feces was quantified by liquid scintillation counting. Metabolite profiling/quantification was performed on pooled samples by HPLC-MS technology. Selatogrel was quantified in plasma by a validated HPLCMS/MS assay. Adverse event, ECG, vital sign, and clinical laboratory data were recorded for safety/tolerability evaluation.

Results: Geometric mean total recovery of ${ }^{14} \mathrm{C}$-radioactivity was $94.8 \%$ (range: $86.5-97.7 \%$ ). In feces, $92.4 \%$ of the ${ }^{14} \mathrm{C}$-radioactivity was recovered, whereas $2.4 \%$ was recovered in urine.

Metabolic profiling data indicated parent compound as most abundant in plasma (87.9\% of total radioactivity), urine $(64.1 \%)$, and feces $(86.6 \%)$. The most abundant metabolite in plasma was the glucuronide M21 $(8.5 \%)$. In urine, M21 (14.7\%) and an unknown metabolite (5.8\%) were most abundant, while in feces a mono-oxidized metabolite (A1) was most abundant $(6.2 \%)$. All other metabolites in plasma or excreta were identified at low abundance only $(<5 \%)$.

Median $t_{\max }$ and $t_{1 / 2}$ of selatogrel were $0.75 \mathrm{~h}$ and $4.7 \mathrm{~h}$, respectively. It was safe and well tolerated based on adverse events, ECG, vital signs, and laboratory data.

Summary / Conclusions: Selatogrel was almost exclusively eliminated in feces. No major metabolites were identified in plasma and none of the metabolic pathways contributed to a relevant extent to the overall elimination of selatogrel, i.e., by more than $25 \%$ as defined per regulatory guidance.

\section{EACPT-1149}

Additional value of a prescribing pharmacist treating patients with a vascular internist at the heart+vascular centre in the netherlands L. Van De Ven ${ }^{*}$, F. Vanmolkot ${ }^{2}$, A.-M. Scheepers-Hoeks ${ }^{1}$

${ }^{1}$ Clinical Pharmacy \& Toxicology, ${ }^{2}$ Internal Medicine, MUMC+, Maastricht, Netherlands

\section{Cardiovascular treatment, Chronic diseases, Drug-drug interactions}

Introduction: In the Netherlands prescribing is restricted to physicians. Other countries as the UK or Australia have implemented prescribing pharmacists in some form with success.

Objectives: To evaluate if a prescribing pharmacist does improve patient satisfaction, improves efficacy for doctors and gives additional value as drug expert at the Heart+Vascular Centre.

Methods: A pre-intervention study was performed between MayDecember 2018. During this period a vascular internist and a pharmacist together did consultations at the Maastricht Heart+Vascular Centre. Patients with hypertension and drug related problems were identified. Where hypertension was diagnosed by the internist, the pharmacist evaluated adherence, side effects, previous experiences with drugs, drug- interactions and nocebo effect. The internist and pharmacist discussed all data. Thereafter treatment policy was determined. The pharmacist prescribed a limited range of drugs. Patients' and doctors' satisfaction was measured.

Results: Patients' satisfaction was measured by a telephone questionnaire $(n=11)$ and the average score was $8.7(1-10) .80 \%$ of the patients experienced major improvement towards usual care. Physician satisfaction was measured by written questionnaire $(n=3)$. Positive experiences were extra attention for side-effects, adherence and label substitution. Less time for diagnosis and less time to report in the patients file was scored negative. Summary / Conclusions: This pre-intervention study provides insights that can be used for pharmacists and physicians when assessing drug related problems in patients with hypertension. All patients experienced added value of a prescribing pharmacist treating patients together with a vascular internist. Although some physicians had some restraint, the majority believed it was beneficial. In a larger intervention study we will aim on measurements for achieving blood pressure target.

\section{EACPT-1151}

Comparison of anti-FXa chromogenic assay for rivaroxaban used in North Estonia Medical Centre with LC-MS/MS

J. Arjakse ${ }^{*}{ }^{1}$, R. Malmström ${ }^{2,3}$, M. Allvee ${ }^{4}$, L. Gustafsson ${ }^{3}$, H. Ilumets ${ }^{5}$, J. Kostjuk ${ }^{5}$, M. Märk ${ }^{5}$, M. Pikta ${ }^{6,7}$, A. Pohanka ${ }^{3,8}$, Y. Rönquist ${ }^{3}$, T. Marandi ${ }^{9}, 10$

${ }^{1}$ Hospital Pharmacy, North Estonia Medical Centre, Tallinn, Estonia, ${ }^{2}$ Medicine Solna, Karolinska Institute, ${ }^{3}$ Department of Clinical Pharmacology, Karolinska University Hospital, Stockholm, Sweden, ${ }^{4}$ Department of Science and Development, ${ }^{5}$ Centre of General Internal Medicine, ${ }^{6}$ Laboratory, North Estonia Medical Centre, ${ }^{7}$ Department of Health Technologies, Tallinn University of Technology, Tallinn, Estonia, ${ }^{8}$ Department of Laboratory Medicine, Karolinska Institute, Stockholm, Sweden, ${ }^{9}$ Therapy Quality Division and Centre of Cardiology, North Estonia Medical Centre, Tallinn, ${ }^{10}$ Institute of Clinical Medicine, Department of Cardiology, University of Tartu, Tartu, Estonia

Cardiovascular treatment, Pharmacokinetics/ pharmacodynamics, Therapeutic drug monitoring

Introduction: During the treatment with rivaroxaban (RXN), determination of RXN exposure could be important in urgent situations like bleeding, need for surgical procedure or care of perioperative patient. The reference method for detecting RXN plasma concentration (P-RXN) is high-performance liquid chromatography tandem massspectrophotometry (LC-MS/MS), but this methodology is not available in most laboratories. In 2015 anti-FXa chromogenic assay for RXN as easily usable method was introduced in North Estonia Medical Centre (NEMC) laboratory.

Objectives: To determine the correlation between anti-FXa chromogenic assay results (analysed in NEMC) and P-RXN (analysed at Karolinska University Hospital) and to describe P-RXN values in patients with atrial fibrillation (AF) and venous thromboembolism (VTE).

Methods: RXN naïve patients, 14 women and 29 men (mean age 57.6 and 59.3 years, respectively), were included from March 2015 to November 2017 after routine decision of treating physician to start RXN. Among these patients were 10 with AF and 33 with VTE. Doses of RXN were $20 \mathrm{mg}$ once daily (OD) for AF and $15 \mathrm{mg}$ twice daily for first 3 weeks, then $20 \mathrm{mg}$ OD for VTE. Demographic data, vital signs, data on utilisation of medicines, adverse events and blood samples were collected at 4 visits (days $0,7,30$ and 180). Anti-FXa before and 2-4 hours after RXN dosing was measured by STA-Liquid anti-FX reagent using commercial RXN calibrator and control; STA-R Evolution, Diagnostica Stago, France. P-RXN trough and peak were measured by LC-MS/MS. Data was analysed by MS Excel. Comparison between chromogenic anti-FXa assay and LC-MS/MS methods was done and coefficient of determination $\left(\mathrm{R}^{2}\right)$ was calculated for trough, peak and all samples together. 
Results: Total 330 samples were analysed (165 for trough and 165 for peak). P-RXN trough $(\min ; \max (\mathrm{ng} / \mathrm{mL}))$ at different visits were: $\mathrm{AF}$ patients: Day $0(<2 ; 63)$; Day $7(5.7 ; 440)$, Day $30(8.8 ; 150)$ and Day 180 $(<2 ; 240)$ SVT patients: Day $0(<2 ; 26)$; Day 7 (20; 240), Day $30(7.8$; $110)$ and Day $180(<2 ; 84)$. Values of $R^{2}$ for $R X N$ trough and peak were 0.893 and 0.949 respectively. For all samples together $\mathrm{R}^{2}$ was 0.967 .

Summary / Conclusions: Good correlation between two methods indicates that anti-FXa chromogenic assay can be used for RXN exposure detection. However, variability of RXN plasma concentrations needs further analysis in context of clinical efficacy and events in population studied.

\section{Oral Presentation}

\section{EACPT-1190}

Use and compliance of treatment with direct oral anticoagulants in non-valvular atrial fibrillation

M. Giner-Soriano* 1, 2, 3 , A. Gomez-Lumbreras ${ }^{2,3}$, J. Cortes ${ }^{4}, \mathrm{R}$. Morros ${ }^{1,2,3}$

${ }^{1}$ Institut Català de la Salut, Barcelona, ${ }^{2}$ Universitat Autònoma de Barcelona, Bellaterra (Cerdanyola del Vallès), ${ }^{3}$ Fundació Institut Universitari per a la recerca a l'Atenció Primària de Salut Jordi Gol i Gurina (IDIAPJGol), ${ }^{4}$ Departament d'Estadística i Investigació Operativa, Universitat Politècnica de Catalunya, Barcelona, Spain

\section{Cardiovascular treatment, Drug utilization}

Introduction: Direct oral anticoagulants (DOAC) and vitamin K antagonists (VKA) are used for stroke prevention in non-valvular atrial fibrillation (NVAF).

Objectives: To describe oral anticoagulants (OAC) use in NVAF in Primary Care (PC). To describe doses prescribed, adherence and persistence to DOAC.

Methods: Population-based cohort study in SIDIAP (Information System for Research in PC), which captures data from PC electronic health records of 5.8 million people from Catalonia, Spain. We included all NVAF adults with a new prescription of OAC in 2014-2017.

Variables: socio-demographics, stroke and bleeding risks, comorbidities and comedications at baseline for all NVAF patients. Doses prescribed and their adequacy according to the recommendation in the summaries of products characteristics, persistence (measured as percentage of patients not discontinuing treatment during first year), and adherence (measured as Medication Possession Ratio, MPR) to DOAC in patients starting in 2014-2016.

Results: 77,723 patients initiated OAC in 2014-2017; 69,376 (89.3\%) were naïve to OAC treatment, $51.7 \%$ were men, with a mean age of 73.6 . VKA were prescribed in $68.6 \%$ of patients, apixaban in $11.7 \%$, dabigatran in $7 \%$, edoxaban in $1.3 \%$ and rivaroxaban in $11.4 \%$. Hypertension $(69.8 \%)$ and diabetes $(33 \%)$ were the most frequent comorbidities. $\mathrm{CHA}_{2} \mathrm{DS}_{2}$-VASc was $\geq 2$ in $85.1 \%$ of patients.

Doses prescribed were available in 12,438 DOAC patients. For apixaban, $67.5 \%$ of patients with $2.5 \mathrm{mg} / 12 \mathrm{~h}$ dosages and $5.7 \%$ of $5 \mathrm{mg} / 12 \mathrm{~h}$ were incorrect. For dabigatran, $43.4 \%$ of patients with $110 \mathrm{mg} / 12 \mathrm{~h}$ and $9.5 \%$ of $150 \mathrm{mg} / 12 \mathrm{~h}$ were incorrect. For rivaroxaban, $64.7 \%$ of patients with $15 \mathrm{mg} /$ day and $5.7 \%$ of $20 \mathrm{mg}$ /day were incorrect. Edoxaban doses were not available.

\begin{tabular}{lllll}
\hline & Apixaban & Dabigatran & Edoxaban & Rivaroxaban \\
N,\% (2014-2016) & 5,492 & 3,505 & 185 & 5,716 \\
Discontinued 1st month & $730,13.3 \%$ & $627,17.9 \%$ & $11,5.9 \%$ & $1016,17.8 \%$ \\
Persistent 1st year & $3281,59.7 \%$ & $1712,48.8 \%$ & $90,48.6 \%$ & $3127,54.7 \%$ \\
$\begin{array}{l}\text { Good adherence } \\
\quad \text { (MPR } \geq 80 \%)\end{array}$ & $1922,58.6 \%$ & $867,50.6 \%$ & $63,70 \%$ & $2405,76.9 \%$ \\
$\quad$ in persistent & & & & \\
\hline
\end{tabular}

Summary / Conclusions: Apixaban and rivaroxaban were the most prescribed DOAC. Doses prescribed were not adequate in $34.9 \%$ of apixaban patients, $27.6 \%$ for dabigatran and $21.8 \%$ for rivaroxaban.

The highest rate of persistence was for apixaban, followed by rivaroxaban, dabigatran and edoxaban. Adherence to DOAC was higher for rivaroxaban, then edoxaban, apixaban and dabigatran.

\section{Oral Presentation}

\section{EACPT-1251}

Adherence to direct oral anticoagulants in patients with non-valvular atrial fibrillation cross-national comparison in six european countries M. Sabaté Gallego ${ }^{*}{ }^{1,2,3}$, L. Ibáñez Mora ${ }^{2,3,4}$, X. Vidal Guitart ${ }^{1,2,3}$, E. Ballarin Alins ${ }^{2,3}$, M. Rottenkolber ${ }^{5}$, S. Schmiedl ${ }^{6,}$, A. Heeke ${ }^{8}$, C. Huerta $^{9}$, E. Martin Merino ${ }^{9}$, D. Montero ${ }^{10}$, L. M. León-Muñoz ${ }^{9}$, C.

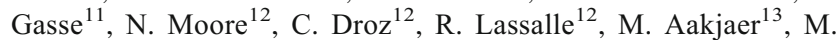
Andersen $^{14}$, M. De Briun ${ }^{15}$, R. Groenwold ${ }^{16}$, R. van den Ham ${ }^{17}$, P. Souverein $^{18}$, O. Klungel ${ }^{17,19}, \mathrm{H}$. Gardarsdottir ${ }^{20,21}$

${ }^{1}$ Department of Pharmacology, Toxicology and Therapeutics, Universitat Autònoma de Barcelona, ${ }^{2}$ Fundació Institut Català de Farmacologia, ${ }^{3}$ Clinical Pharmacology, Hospital Universitari Vall d'Hebron, ${ }^{4}$ Department of Pharmacology, Toxicology and Therapeutics, Universitat Autónoma de Barcelona, Barcelona, Spain, ${ }^{5}$ Diabetes Research Group, Medizinische Klinik und Poliklinik IV, Klinikum der Universität München, Munich, ${ }^{6}$ Philipp Klee-Institute for Clinical Pharmacology, Helios University Hospital Wuppertal, Wuppertal, ${ }^{7}$ Clinical Pharmacology, Faculty of Health, Witten/Herdecke University, Witten, ${ }^{8}$ AOK NORDWEST, Dortmund, Germany, ${ }^{9}$ Pharmacoepidemiology and Pharmacovigilance Division, Spanish Agency of Medicines and Medical Devices (AEMPS), ${ }^{10}$ Pharmacoepidemiology and Pharmacovigilance Division, Spanish Agency of Medicines ans Medical Devices (AEMPS), Madrid, Spain, ${ }^{11}$ Arhaus University, Aarhaus, Denmark, ${ }^{12}$ Bordeaux PharmacoEpi, Université de Bordeaux, Bordeaux, France, ${ }^{13}$ Drug Design and Pharmacology, University of Copenhagen, ${ }^{14}$ Drug Design and Pharmacology, University od Copenhagen, ${ }^{15}$ Pharmacy, University of Copenhagen, Copenhagen, Denmark, ${ }^{16}$ Clinical Epidemiology, Leiden University Medical Center, Leiden, ${ }^{17}$ Division of Pharmacoepidemiology and Clinical Pharmacology, ${ }^{18}$ Division of Pharmacoepidemiology and Clinical Pharmcology, Utrecht University, Utrecht, ${ }^{19}$ Julius Center, University Medical Center Utrecht, Utecht, ${ }^{20}$ Division of Pharmacoepidemiology andClinical Pharmacology, Utrecht University,

${ }^{21}$ Julius Center, University Medical Center Utrecht, Utrecht, Netherlands

\section{Cardiovascular treatment}

Introduction: Since 2011 direct oral anticoagulant drugs (DOACs) have been approved for the prevention of stroke in patients with non-valvular atrial fibrillation (NVAF). Different studies showed that treatment adherence with DOACs is higher than with VKA, but the comparisons are scarce and results are contradictory.

Objectives: To describe the adherence to three DOAC compounds using information from eight European databases (Mondriaan, Danish National Registers, Bavarian, AOK Nordwest, BIFAP, SIDIAP, CPRD and EGB) representing six European countries (The Netherlands, Denmark, Germany, Spain, United Kingdom and France).

Methods: Retrospective cohort study of new users (aged $\geq 18$ years) of DOACs (dabigatran, rivaroxaban, apixaban) with a diagnosis of NVAF identified from eight electronic health care databases (2008-2015). Switching and discontinuation percentages and probability of continuation, using the the Kaplan-Meier method, were estimated for all DOACs and for each individual DOAC at 3, 6 and 12 months.

Results: For all DOAC Mondriaan was the database with the lowest proportions of switchers $(2.4 \%)$, EGB showed the highest proportions of switchers (13.1\%) at 12 months. Regarding discontinuers CPRD had the lowest percentage (16\%) and the Bavarian database the highest (63.9\%). Discontinuers where most often patients using dabigatran (23.2\% CPRD- 
64.6\% Mondriaan), except in the Bavarian database where apixaban had the highest proportion $(69.5 \%)$ and in the AOK Nordwest where rivaroxaban had the highest proportion $(40.2 \%)$, followed by rivaroxaban (15.5\% CPRD-50.2\% Mondrian) and apixaban (12.3\% CPRD-46.3\% Mondriaan). Apixaban showed the highest probability of continuation at 12 months (range: 0.6-0.8), except in Mondriaan (rivaroxaban 0.43) and in AOK Nordwest (dabigatran 0.64). Dabigatran had the lowest probability of continuation at 12 months (range: 0.2-0.7), except in AOK Nordwest (apixaban 0.46).

Summary / Conclusions: Dabigatran was the DOAC with the highest proportion of discontinuers and switchers and apixaban had the lowest one, in most databases. More research is needed to evaluate whether low discontinuation rates for apixaban translate into lower rates of cerebrovascular outcomes and bleeding episodes. This study was funded by the European Medicines Agency (EMA/2015/27/PH; EU PAS Register No: 16014).

\section{EACPT-1241}

Physicians' and undergraduates' knowledge of drugs contraindicated for arterial hypertension treatment

R. Bontsevich ${ }^{1}$, A. Chernykh ${ }^{1}$, A. Leonova ${ }^{1}$, O. Kompaniets ${ }^{2}$, Y. Vovk ${ }^{*}$ ${ }^{1}$, G. Batisheva ${ }^{3}$, E. Miliutina ${ }^{1}$, N. Goncharova ${ }^{3}$, I. Elizarova ${ }^{3}$, O. Osipova $^{1}$, T. Pokrovskaia ${ }^{1}$, E. Kosmacheva ${ }^{2}$

${ }^{1}$ Belgorod State National Research University, Belgorod, ${ }^{2}$ Kuban State Medical University, Krasnodar, ${ }^{3}$ Voronezh State Medical University, Voronezh, Russian Federation

\section{Cardiovascular treatment, Chronic diseases, Education}

Introduction: The relevance of work is dictated by the fact that arterial hypertension is often associated with other diseases and every physician and senior student should be aware of the contraindications to antihypertensive therapy (AH) in the most common nosologies - aV-block 2nd and 3rd degree, WPW-syndrome, gout, COPD and pregnancy.

Objectives: to analyze physicians' and medical undergraduates' knowledge of main contraindications to $\mathrm{AH}$ treatment.

Methods: During the multicenter study devoted to the assessment of physicians' knowledge in treatment of AH (PHYSTARH), 296 people from 5 cities (Belgorod, Voronezh, Dnepr, Krasnodar, Maikop) were surveyed: $44,9 \%$ - 6 th grade students, $55,1 \%$ - physicians and interns of the therapeutic study course. We created an original questionnaire for the study. Multiple-questions on absolute contraindications to antihypertensive drugs prescription in different groups of patients were analyzed. Questions' analytics were the following: Patients groups: pregnant women, COPD patients, patients with aV-block 2nd and 3rd degree, WPW syndrome patients. List of AH-drugs: Enalapril, Verapamil, Bisoprolol, Hypothiazd and Torasemide. Possible answers: "right answer", "1 wrong answer", "2 or more wrong answers", "wrong answer". The question on COPD suggested only two variants of the answer: "true / false".

Results: Drugs Contraindicated in pregnancy: answered correctly $9.5 \%$, with 1 mistake $-50.3 \%$, with 2 or more mistakes $-22.6 \%$, answered wrongly $-17.6 \%$. Gout: answered correctly $-27.8 \%$, with 1 mistake $-39 \%$, with 2 or more mistakes $-2.8 \%$, answered wrongly $-30.4 \%$. Drugs Contraindicated in aV-block - 2nd and 3rd degree patients: Correct answers $-41.7 \%$, with 1 mistake $-44 \%$, with 2 or more mistakes $-0.3 \%$, answered wrongly - $14 \%$. Drugs Contraindicated in WPW-syndrome patients: Correct answers $39 \%$, with 1 mistake $-21 \%$, with 2 or more mistakes $-2.5 \%$, answered wrongly $-37.5 \%$. Drugs Contraindicated in COPD patients: true $-26 \%$, false $-74 \%$.

Summary / Conclusions: Despite the availability of antihypertensive treatment related clinical guidelines and sufficient number of information sources, the level of incorrect answers reaches a significant level in certain nosologies. The physicians' and undergraduates' knowledge of absolute contraindications for drugs prescription in mentioned patients' states remains unsatisfactory and requires further continuous improvement.
EACPT-1263

Assessment of physicians' and senior medical students' knowledge of antihypertensive drugs contraindications in copd patients treatment A. Leonova ${ }^{1}$, R. Bontsevich ${ }^{1}$, A. Chernykh ${ }^{1}$, O. Kompaniets ${ }^{2}$, Y. Vovk ${ }^{1}$, G. Batisheva ${ }^{3}$, E. Miliutina ${ }^{1}$, N. Goncharova ${ }^{3}$, I. Elizarova ${ }^{3}$, O. Osipova $^{1}$, T. Pokrovskaia ${ }^{1}$, E. Kosmacheva ${ }^{2}$

${ }^{1}$ Belgorod State National Research University, Belgorod, ${ }^{2}$ Kuban State Medical University, Krasnodar, ${ }^{3}$ Voronezh State Medical University, Voronezh, Russian Federation

Cardiovascular treatment, Chronic diseases, Education

Introduction: The relevance of work is that most physicians and medical undergraduates face certain difficulties in treatment of hypertension in patients with chronic obstructive pulmonary disease (COPD), which is connected with false perceptions about absolute contraindications to antihypertensive medications used for COPD treatment.

Objectives: to assess the knowledge of physicians and medical undergraduates in $\mathrm{AH}$ treatment during COPD.

Methods: During the multicenter research on physicians' and undergraduates' knowledge in AH treatment (PHYSTARH) we surveyed 296 people from 5 cities (Belgorod, Voronezh, Dnepr, Krasnodar, Maikop) - 45\% of senior students, $55 \%$ of physicians and interns. The list of AH-drugs analyzed in the relevant survey's question was the following: Enalapril, Verapamil, Bisoprolol, Hypothiazd and Torasemide. Results we split into two groups depending on their answers provided: "true" or "false".

Results: Answers given by the respondents distributed as follows: correct $-26 \%$ (physicians $-31.3 \%$, undergraduates $-19.5 \%$, $\mathrm{p}<0.001$ ), wrong $74 \%$. The following $\mathrm{AH}$ medications were mistakenly denoted as prohibited by the respondents: "Bisoprolol" - 58,1\%, "Enalapril" 18.7\%, "Verapamil" - 5,35\%, "Hypothiazid" - 3.3\%, "Torasemid" 3\%. Summary / Conclusions: Despite the availability of AH-treatment related scientific literature, respondents demonstrated lack of knowledge in this area. The level of correct answers reached $26 \%$ onlys. A half of the respondents believe that beta-blockers (BB) is an absolute contraindication for COPD, however, BB do not increase but, on the contrary, lower exacerbations and mortality risks in chronic obstructive pulmonary disease.

\section{Oral Presentation}

\section{EACPT-1291}

Poor adherence to renal function testing for dabigatran

B. Simpson ${ }^{*}$, 1, D. Reith ${ }^{2}$, N. Medlicott ${ }^{1}$, A. Smith ${ }^{1}$

${ }^{1}$ School of Pharmacy, ${ }^{2}$ Dunedin School of Medicine, University of Otago, Dunedin, New Zealand

\section{Cardiovascular treatment, Pharmacovigilance}

Introduction: Dabigatran has become widely used in New Zealand (NZ), with $51 \%$ of patients receiving oral anticoagulation now being treated with it. With approximately $80 \%$ of dabigatran being renally excreted, it is important to ensure that appropriately timed renal function testing is undertaken. Audits of NZ patients have shown that between $63-90 \%$ of patients receiving dabigatran had and annual renal function test. Such audits require a renal function test anytime in the 1 year following initiation. Clinical guidance by the Sponsor recommends that patients undergo renal function assessment yearly or more often if clinically indicated. Therefore, there may be misrepresentation of tests being undertaken at clinically appropriate times.

Objectives: To investigate if clinically appropriate renal function testing is being undertaken in NZ primary care for non-valvular atrial fibrillation (NVAF) patients prescribed dabigatran etexilate.

Methods: All NZ prescription data for dabigatran and, where available, NVAF patients primary care health data were extracted from national administrative databases for the period 01 July 2011 to 31 December 2015. Descriptive statistics were undertaken to determine demographics and if recommended protocols for renal function testing were adhered to. The 
proportion of patients who had tests were assessed with the $95 \%$ confidence interval (95\% CI, Clopper-Pearson method). To ascertain if testing was being undertaken at clinically appropriate times, we estimated Kaplan-Meier survival curves at baseline and to the time to second test. Patient covariates of gender, age, ethnicity, geographic location was investigated to determine if there was effect on testing adherence. Differences between covariates were compared using the log-rank test $\chi^{2}$ statistic.

Results: There were 1948 NVAF patients identified that had been dispensed dabigatran with $1752(89.9 \%$ [95\%CI; 88.5\%>91.2\%) having a renal function test at baseline. There were $1276(65.5 \%)$ patients who received one or more years supply of dabigatran and of these 332 (26.0\% [95\% CI; 23.6-28.5]) had a serum creatinine test 1 year after initiation (i.e. within 30 days of one-year post treatment initiation date). Kaplan-Meier curves further confirmed poor adherence to testing and that there was no significant difference between covariates.

Summary / Conclusions: There is an apparent lack of adherence to clinically appropriate renal function testing in NVAF patients being treated with dabigatran.

\section{EACPT-1303}

Prescription patterns of lipid lowering agents among older patients in general practice: an analysis from a national database in the netherlands

E. Kleipool ${ }^{*} 1$, M. Nielen ${ }^{2}$, M. Peters ${ }^{3}$, M. Muller ${ }^{4}$

${ }^{1}$ Internal/ geriatric medicine, VU University Medical center, Amsterdam, ${ }^{2}$ NIVEL institue, Utrecht, ${ }^{3}$ Amsterdam UMC, location VUmc, ${ }^{4}$ VU University Medical center, Amsterdam, Netherlands

\section{Cardiovascular treatment, Geriatric treatment}

Introduction: Dutch cardiovascular risk management guidelines state almost every older adult ( $\geq 70$ years) is eligible for a lipid lowering drug (LLD). However, life expectancy, frailty or comorbidities may influence this treatment decision.

Objectives: Investigate how many older adults, according to age, frailty (Drubbel frailty index), and comorbidities were prescribed LLDs.

Methods: Data of 244,328 adults $\geq 70 \mathrm{yrs}$ from electronic health records of 415 Dutch general practices (GP) from 2011-2015 were used. Number of LLD prescriptions in patients with $(n=55,309)$ and without $(n=189,019)$ cardiovascular disease $(C V D)$ was evaluated according to age, frailty and comorbidities.

Results: $69 \%$ of adults $\geq 70$ yrs with CVD and $36 \%$ without CVD were prescribed LLD. LLD prescriptions decreased with age; with CVD: $78 \%$ aged $70-74 \mathrm{yrs}$ and $29 \%$ aged $\geq 90 \mathrm{yrs}$ were prescribed LLD, without CVD: $37 \%$ aged $70-74 y$ rs, and $12 \%$ aged $\geq 90 \mathrm{yrs}$. In patients with CVD and within each age group, percentage of LLD prescriptions was 20 percent point(pp) higher in frail compared to non-frail. In patients without CVD, frail patients LLD prescriptions were $11 \mathrm{pp}$ higher in adults aged $70-74 \mathrm{yrs}$ and $40 \mathrm{pp}$ higher in adults aged $\geq 90 \mathrm{yrs}$. Similar trends were seen in the analyses with number of comorbidities.

Summary / Conclusions: In an older population, LLD prescriptions decreased with age but -contrary to our expectations- LLD prescriptions increased with higher frailty levels.

\section{EACPT-1412}

Can self-medication be a risk factor of inefficient pharmacotherapy in cardiovascular diseases?

B. Erika ${ }^{1}$, A. Brassai ${ }^{*} 1$, A. Kovacs ${ }^{2}$, E. Dho-Nagy ${ }^{1}$, Z. Kollo $^{1}$, F. Cori ${ }^{1}$, M. L. Garau ${ }^{1}$, L. Mathe ${ }^{2}$

${ }^{1}$ Pharmacology, ${ }^{2}$ UMFST Tg-Mures, Tirgu Mures, Romania

\section{Cardiovascular treatment, Drug utilization}

Introduction: Cardiovascular diseases represent one of the most important illnesses that physicians have to deal with and the pharmacological therapy is aimed to help the patient by improving the quality of life.

Objectives: The objective of the present study was to evaluate the relationship between the different types of the administered pharmacotherapy in certain cardiovascular diseases in aim to observe possible interactions or misuses which can lead to inefficient treatment.

Methods: We have performed a non-interventional, cross-sectional retrospective pharmacoepidemiological study regarding the pharmacotherapy of 301 adult cardiovascular patients ambulatory treatment (mainly rural, elderly population).

Results: The study revealed an elderly population with the average age of 66.32 years. The statistical analysis revealed that the most important drug associations were: Angiotensin Converting Enzyme Inhibitors +Diuretics (38.5\%), Non-steroidal Anti-inflammatory Drugs + Diuretics (17.9\%), Angiotensin Receptor Blockers + Diuretics $(10.0 \%)$, Angiotensin Receptor Blockers + Non-steroidal Anti-inflammatory Drugs (3.7\%). The occurrence of polypragmasy, defined as more than five drugs used simultaneously - was very high. In the studied population the frequency of the appearance of hyperkalemia was also high: $82.1 \%$. Analyzing the cause of it, we have observed that in about half of the cases it is related to the used pharmacological therapy.

Summary / Conclusions: The study revealed that even if a patient's treatment is well established, a lack of further monitoring and healthcare education and use of OTC medicines can lead to uncontrolled disease and even harmful interactions like hyperpotassemia.

\section{Oral Presentation}

\section{EACPT-1418}

A personalised, clinical pharmacological approach to the management of hypertension presenting acutely to secondary care A. Janmohamed ${ }^{*} 1$, N. Chapman ${ }^{1}$

${ }^{1}$ St Mary's Hospital, Imperial College Healthcare NHS Trust, London, United Kingdom

\section{Cardiovascular treatment}

Introduction: Hypertension (high blood pressure [BP]) is a common acute presentation to secondary care. Patients with hypertension are often admitted unnecessarily and poorly managed acutely (from inadequate treatment to over-rapid BP reduction, risking treatment-associated complications). Even very high BPs often do not warrant aggressive emergency treatment and, if uncomplicated, can be investigated and managed in an outpatient/ambulatory setting.

Objectives: Using a patient-centred approach to develop a novel pathway to improve management of patients presenting acutely to secondary care with very high $B P$.

Methods: Patients referred from the Emergency Department (ED), Ambulatory Emergency Care (AEC), General Practitioners (GPs), speciality clinics and those already admitted with high BP were reviewed by the clinical pharmacology team. Medications were rationalised to achieve BP control (taking into account drug tolerability, adverse effects and patient choice) and relevant investigations were performed. Care was transferred to outpatient clinic or GPs once BP was improving. Where possible, hospital admissions were avoided and inpatients discharged promptly after treatment initiation for early review in AEC.

Results: 110 patients (45 inpatients, 37 from ED/AEC, 28 from GPs/speciality clinics) were reviewed over 1 year. Mean age was $48 \pm 14$ years, $51 \%$ were female and $42 \%$ had previously undiagnosed hypertension. 102 patients were subsequently seen in AEC (median 3 reviews [IQR 2.8-6]). Median initial BP was 179/ $101 \mathrm{mmHg}$ [IQR 161-200/92-116] and median BP reduction between first encounter and transfer of care was $43 / 17 \mathrm{mmHg}$ [IQR 23-66/8-34; $<<0.0001]$. Median number of drugs increased from 1 [IQR 0-2] to 3 [IQR 2-4]. Drugs were amended according to 
guidelines and clinical response, and discontinued as appropriate. Overall, antihypertensive drugs were changed in $90 \%$, discontinued in $25 \%$ and rationalised (e.g. simplifying regimens to improve adherence) in 18\%; 48 admissions were avoided and early discharge facilitated in 39 inpatients.

Summary / Conclusions: Significant BP reductions were achieved, improving care, avoiding admissions, facilitating early discharge and reducing bed occupancy. A patient-centred, personalised medicine approach promotes appropriate prescribing in BP control. Ongoing work aims to develop algorithms for the acute treatment of hypertension to improve consistency of care and outcomes.

\section{EACPT-1424}

How to improve hypertension control? - evaluation of weak links in pharmacotherapy

E. A. Dho Nagy ${ }^{*}$, E. G. Ban ${ }^{1}$, Z. Kollo ${ }^{1}$, F. Cori ${ }^{1}$, M. L. Garau ${ }^{1}$, A. Brassai ${ }^{1}$

${ }^{1}$ UMFST Tg-Mures, Tg-Mures, Romania

\section{Cardiovascular treatment}

\section{Introduction:}

Cardiovascular diseases represent one of the most important cause of death in Europe, it corresponds to $37 \%$ of all deaths. In Romania the prevalence is considerably higher with a rate of $59 \%$. The prevalence of hypertension, the main risk factor for cardiovascular diseases, is $40 \%$ in some areas.

Objectives:

Based on these data, our objectives are to determine in a rural population the prevalence of hypertension, awareness rate and to provide evidence that in the everyday medical practice the pharmacotherapy is not at the level deemed by the current medical guidelines and there is a significant lack of healthcare education.

\section{Methods:}

Two consecutive cross-sectional observational pharmacoepidemiological studies were conducted in a small and relatively closed rural population in Maros county. The first one in 2005 involving 112 individuals and the second one in 2018 with 30 individuals. In these studies only the adult population was included.

Hypertension was defined as systolic blood pressure of at least $140 \mathrm{mmHg}$ and/or a diastolic blood pressure at least $90 \mathrm{mmHg}$ and the parameters were assessed by means of questionnaire interview and blood pressure measurements during two study visits.

Results:

In the first study $57 \%$ of volunteers were females and $43 \%$ males, from the analysed population $66 \%$ presented hypertension, $75 \%$ of them were aware of their condition, which indicates a high awareness rate, while $54 \%$ were treated with antihypertensive therapy. The most used drug group was the Angiotensin Converting Enzyme Inhibitors (ACE inhibitors) with $25 \%$, followed by Calcium Channel Blockers (CCB), than Beta-blockers $(\mathrm{Bb})$ and diuretics.

In the second study $64 \%$ of the individuals that have participated had hypertension, but only in $10 \%$ of the cases the antihypertensive treatment was efficient. Beta-blockers and ACE inhibitors were the most used drug groups followed by CCBs and diuretics in (only $13 \%$ of the cases).

Summary / Conclusions:

These data are sensibly different from the latest guidelines for hypertension treatment, such as the JNC8, which recommends as first-line treatments 4 classes of medications: thiazide-type diuretics, CCBs, ACE inhibitors, and ARBs.

In our geographical area the frequency of high blood pressure is very increased, the majority of the adults are suffering from this disease. Despite clear and readily available hypertension treatment guidelines blood pressure control rates remain low.
EACPT-1431

The use of fall-risk-increasing drugs in comorbid patients with polypharmacy

O. Konova* ${ }^{1}$, E. Ilina ${ }^{1}$, V. Shalygin ${ }^{1}$, D. Ivashchenko ${ }^{1}$, M. Saveljeva ${ }^{1}$, I. Sinitsina $^{1}$, S. Gorbatenckova ${ }^{1}$, S. Puzin ${ }^{2}$, O. Bogova ${ }^{2}$, D. Sychev ${ }^{1}$

${ }^{1}$ Department of Clinical Pharmacology and Therapy, ${ }^{2}$ Department of Geriatrics and Medical and Social Expertise, Federal State Budgetary Educational Institution of Further Professional Education "Russian Medical Academy of Continuous Professional Education" of the Ministry of Healthcare of the Russian Federation, Moscow, Russian Federation

Cardiovascular treatment, Drug-drug interactions, Geriatric treatment, Misuse of medicines/substances

Introduction: Drug-related falls, considered to be one of the most serious complications of pharmacotherapy in the elderly, have modifiable risk factors - polypharmacy and the fall-risk-increasing drugs (FRIDs) administration.

Objectives: The study was aimed to assess the relationship between FRIDs combinations and the development of drug-induced falls in elderly patients. Methods: During the study, 966 case histories of patients older than 75 hospitalized with comorbid conditions (neurological and cardiovascular diseases) in 2011-2016 were analyzed. Medication Administration Records (MAR) and pairwise descriptive statistics were evaluated using the contingency tables to analyze the relationship between all the identified combinations of FRIDs and the development of falls.

Results: Out of 242 FRID combinations, 25 combinations of two subgroups (cardiological and psychotropic) were reliably associated with falls ( $p<0.05)$. An increased risk of falling was detected with a combination of neuroleptics and hypnotics $(p=0.02)$, as well as with a combination of neuroleptics and $\mathrm{N}$-anticholinergics $(\mathrm{p}=0.016)$. However, it is not possible to calculate the odds ratio for these variables, since in this group, $100 \%$ of patients experienced a fall.

The cardiological drug combinations associated with falls in elderly patients were as follows: calcium channel blockers (CCBs) with thiazide (-like) diuretics $(\mathrm{OR}=10.48 ; 95 \% \mathrm{CI}=1.3-82)$, $\mathrm{CCBs}$ with nitrates $(\mathrm{OR}=2.51$; 95\% CI=1.1-6.0), and CCBs and $\mathrm{ACE}$ inhibitors $(\mathrm{OR}=2.21<95 \% \mathrm{CI}=1.1-4.4)$ The combinations of cardiological and psychotropic drugs associated with falls in elderly patients were as follows: hypnotics with both alpha-blockers $(\mathrm{OR}=22.89,95 \% \mathrm{CI}=2.7-191.8)$ and beta-blockers $(\mathrm{OR}=5.09,95 \% \mathrm{CI}=1.8$ 14.7); hypnotics with $\mathrm{ACE}$ inhibitors $(\mathrm{OR}=4.1,95 \% \mathrm{CI}=1.3-12.8)$; betahistine with both $\mathrm{ACE}$ inhibitors $(\mathrm{OR}=3.01,95 \% \mathrm{CI}=1.2-7.5)$ and loop diuretics $(\mathrm{OR}=4.24,95 \% \mathrm{CI}=1.1-16.7) ; \mathrm{N}$-anticholinergics and beta-blockers $(\mathrm{OR}=7.58,95 \% \mathrm{CI}=2.1-26.8)$; antidepressants with thiazide (-like) diuretics $(\mathrm{OR}=6.5,95 \% \mathrm{CI}=1.3-32.8)$; neuroleptics and ARA II $(\mathrm{OR}=13.63,95 \% \mathrm{CI}=1.7-109.9)$.

Summary / Conclusions: The combination of CCBs with thiazide diuretics, nitrates or ACE inhibitors may lead to serious complications. Combinations of ophthalmic beta-blockers with nitrates, neuroleptics, benzodiazepines and antidepressants as well as combinations of neuroleptics with ARA II, hypnotics or $\mathrm{N}$-anticholinergics significantly increase the risk of falls in elderly patients.

\section{Oral Presentation}

\section{EACPT-1438}

Real-life safety of long-term treatment with direct oral anticoagulants K. Urbánek ${ }^{*} 1$, R. Blažková ${ }^{1}$, P. Štrbová ${ }^{1}$

${ }^{1}$ Department of Pharmacology, Faculty of Medicine and Dentistry, Palacký University in Olomouc, Olomouc, Czech Republic

\section{Cardiovascular treatment, Drug utilization}

Introduction: Direct oral anticoagulants (DOACs) replace warfarin or LMWHs in several indications. While, clinical trials are timelimited, DOACs administration in atrial fibrillation (AF) may be much longer in real life. For the stroke prevention in AF, dabigatran has been used in our country since 2011 and rivaroxaban since the second half of 2012 . 
Objectives: The aim of the study was to evaluate the safety of DOACs for more than 3 years of use in the first patients treated for $\mathrm{AF}$ and compare the results of dabigatran and rivaroxaban treatment.

Methods: The study was conducted as real-life, single-centre, retrospective analysis performed in University Hospital in Olomouc. Data on patients with non-valvular AF, in which dabigatran treatment was started from 2011 to 2012 and rivaroxaban treatment from 2012 to 2014, were processed. Drug prescription data were obtained from the database of the Department of Pharmacology, clinical data from the hospital information system. Indication criteria, dosage, treatment failure, discontinuation rate and adverse events were analysed.

Results: A complete data about the treatment were found for 121 patients treated by dabigatran; 49 were treated with $300 \mathrm{mg}$ daily dose and 72 with a reduced dose of $220 \mathrm{mg}$. The initial dosing was later reduced in two patients and increased in another two. The treatment was interrupted or discontinued in 79 patients $(65 \%)$, only 47 patients (39\%) remained on treatment for 3 years or more. Adverse events occurred in 28 patients (20.1\%): 14 cases of bleeding, 6 cases of dyspepsia, 7 anaemia, 1 increased sweating. Treatment failed in 5 patients $(4.13 \%)$.

Similarly, complete follow-up data were found in 53 patients treated by rivaroxaban; 44 patients were treated with $20 \mathrm{mg}$ daily, in 4 patients with $15 \mathrm{mg}$, in 3 others the dose was reduced during the treatment, 2 patients received (off-label) dose of $10 \mathrm{mg}$. 26 patients $(49 \%)$ were treated 3 years or longer, 20 patients (38\%) discontinued the treatment. Adverse reactions were reported in 3 patients (5.7\%): 2 cases of anaemia and one case headache and general weakness. Treatment failed in 2 cases (3.7\%).

Summary / Conclusions: The results show high efficiency and safety of the DOACs in AF. A tendency towards better persistence and fewer side effects during treatment with rivaroxaban was found. Moreover, the use of full recommended dose was in dabigatran substantially lower than in published clinical studies.

\section{EACPT-1042}

Polymorphic marker cyp2 $26 * 4$ and the response to propranolol in russian patients with liver cirrhosis

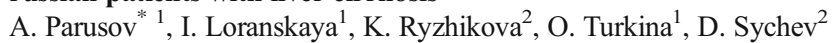
${ }^{1}$ Gastroenterology, ${ }^{2}$ Clinical pharmacology and Therapy, Russian Medical Academy of Continuous Professional Education, Moscow, Russian Federation

\section{Chronic diseases, Pharmacogenetics/omics}

Introduction: The problem of variceal bleeding preventing - one of the liver cirrhosis complication, is very actual in clinical practice. For this purpose, non-selective $\beta$-blockers are used, which can reduce the first bleeding risk by $30-40 \%$. Propranolol is a widely used non-selective $\beta$ blocker. The drug is prescribed in a dose that reduces the pulse rate by $25 \%$ or up to 55 beats per minute at the initial low pulse. However, in some cases, $\beta$-blockers do not have any clinical effect. In this regard, it is necessary to determine the predictors of a positive hemodynamic response. Many studies have shown that CYP2D6 plays an important role in the $\beta$-blockers metabolism, mainly due to the reaction of 4-hydroxylation.

Objectives: Determination of the CYP2D6 gene polymorphism influence on the propranolol hemodynamic effect in the portal hypertension syndrome of the Russian population patients.

Methods: Currently, the study involved 20 patients with liver cirrhosis of various etiologies and Child-Pugh classes aged from 28 to 70 years. Patients were prescribed propranolol in a daily dose of $30 \mathrm{mg}$. The Dopplerosonography method was used to determine the linear blood flow velocity in the portal vein before treatment and during therapy after 14 days. In addition, patients were determined by CYP2D6*4 genotype (G1846A) by real-time PCR. The response criterion is an increase in the portal vein linear blood flow velocity during therapy. Statistical data processing was carried out using STATISTICA 10.

Results: According to the results of genotyping, 5 GA heterozygotes and 15 GG homozygotes were detected, 11 of which responded to treatment $(73 \%)$. All 100\% heterozygous carriers showed a positive response to therapy.

\begin{tabular}{llll}
\hline & Responders & Nonresponders & $p$ \\
Gender (M/F) & $13 / 3$ & $4 / 0$ & 0,35 \\
Age (year) & $49,1 \pm 14,2$ & $54,5 \pm 15,1$ & 0,4 \\
Liver cirrhosis etiology & $9 / 4 / 3$ & $2 / 1 / 1$ & 0,96 \\
$\quad$ (alcoholic/viral/mixed) & & & \\
Child-Pugh (B/C) & $8 / 7$ & $1 / 3$ & 0,36 \\
Genotype (GG/GA) & $11 / 5$ & $4 / 0$ & 0,19 \\
\hline
\end{tabular}

Summary / Conclusions: According to the interim study results, it is impossible to conclude that the CYP2D6 gene polymorphism influence on the propranolol hemodynamic effect is significant statistical.

However, we assume that an increase in the sample size will lead to a trend towards the reliability of this dependence.

\section{EACPT-1058}

Efficacy and safety of different doses and durations of intravenous immunoglobulin treatment for immune thrombocytopenic purpura O. Lavon ${ }^{*}{ }^{1,2}$, R. Noy ${ }^{1}$

${ }^{1}$ Rappaport Faculty of Medicine, Technion, ${ }^{2}$ Clinical Pharmacology and Toxicology, Carmel Medical Center, Haifa, Israel

Chronic diseases, Drug utilization

Introduction: Immune Thrombocytopenic Purpura (ITP) is an acquired syndrome, either idiopathic or secondary, which causes destruction of platelets by auto-antibodies. Intravenous immunoglobulins (IVIG) are considered first line treatment for ITP. Current common IVIG treatment regimens - dosing and duration - are based on limited research.

Objectives: To evaluate efficacy and safety of different doses and durations of IVIG treatment in ITP patients.

Methods: A retrospective chart review of IVIG-treated ITP patients was conducted. Demographic and clinical data were collected and subjected to comprehensive statistical analysis including $\mathrm{Xi}^{2}$ test, Mann-Whitney test, Kruskal Wallis test, and uni- and multi-variant regression.

Results: 103 records were included in the final analysis. Average platelets count was 7,600 per microliter (range 2,000-26,000) upon admission and 64,000 per microliter (range 55,500-270,000) on discharge. Average IVIG dose was $0.4 \mathrm{gr} / \mathrm{kg} /$ day (range $0.1-0.91$ ); total average accumulated dose was $1.2 \mathrm{gr} / \mathrm{kg}$ (range 0.21-3), given during 4.23 days in average (range 1-10). There was no correlation between demographic or clinical parameters and the extent of platelets count improvement. The was no significant difference between IVIG dose regimens regarding the extent of platelets count improvement. Adverse drug reactions, mainly mild, were recorded in $18 \%$ of patients.

Summary / Conclusions: In the presented study, there was no advantage to higher IVIG doses or longer duration regarding improvement of platelet count. The prevalence of adverse drug reactions was similar to previously reported data. It is recommended to use IVIG low-dose regimen (up to $0.4-0.5 \mathrm{gr} / \mathrm{kg} / \mathrm{day}$ ) and examine daily each patient for the need for additional treatment.

\section{Oral Presentation}

\section{EACPT-1087}

Do patients with cystic fibrosis participating in clinical trials demonstrate placebo response? A meta-analysis.

J. Coton $^{* 1,2}$, H.-H. Le ${ }^{1}$, P. Janiaud ${ }^{1}$, M. Cucherat ${ }^{3}$, B. Kassai-Koupai ${ }^{4}$, F. Gueyffier $^{1}$, P. Reix ${ }^{2}$

${ }^{1}$ UMR 5558 CNRS, Equipe EMET, Université Claude Bernard, Lyon 1, Lyon, ${ }^{2}$ Centre de ressources et de compétences de la mucoviscidose, Hospices Civils de Lyon, Bron, ${ }^{3}$ Department of Clinical Pharmacology, Université Claude Bernard, Lyon 1, Lyon, ${ }^{4}$ EPICIME-Clinical Investigation Center, INSERM CIC1407/UMR5558, Bron, France 


\section{Chronic diseases, Rare diseases, Other}

Introduction: Patients' and families' expectation that a cure for cystic fibrosis (CF) will be found is high. In other debilitating conditions, high expectation has been shown to drive a strong placebo response (PR).

Objectives: Therefore, our goal was to evaluate PR on objective continuous outcomes (FEV1, BMI) and the CF Questionnaire RevisedRespiratory Domain (CFQR-RD), considered as particularly relevant in $\mathrm{CF}$, monitored during randomised clinical trials (RCTs).

Methods: We conducted a meta-analysis after a systematic review of the literature carried out to identify RCTs with FEV1, CFQR-RD and BMI as outcome measures. The standardised mean difference (SMD) was calculated to estimate the PR. A meta-regression analysis was conducted to assess other contributing factors on PR such as study design, trial duration, patient age and disease severity.

Results: Out of 289 RCTs found in the search, we identified 61 articles (published from 1987 to 2017) with respectively 59, 17 and 9 reporting FEV1, CFQR-RD and BMI at the start and at the end of the RCTs. No significant PR was found on FEV1 or CFQR-RD. However, a small but significant PR was found on BMI (SMD, 0.09 (95\% CI $(0.01 ; 0.17)$; $\mathrm{p}=0.03$ ).

Summary / Conclusions: To our knowledge, this is the first metaanalysis to assess PR in patients with CF investigated in RCTs. The research question behind this work may have implications on the interpretation of the therapeutic effect of past, ongoing and future RCTs for both clinicians and $\mathrm{CF}$ researchers.

\section{EACPT-1094}

Factors affecting medication non-adherence among patients with type 2 diabetes

O. Horvat ${ }^{*}{ }^{1}$, J. Popržen ${ }^{1}$, T. Halgato ${ }^{1}$, A. Tomas ${ }^{1}$, M. Paut Kusturica ${ }^{1}$, Z. Tomić $^{1}$, A. Sabo ${ }^{1}$

${ }^{1}$ Department of Pharmacology Toxicology and Clinical Pharmacology, Faculty of Medicine, Novi Sad, Serbia

\section{Chronic diseases}

Introduction: Non-adherence to medication among diabetic patients develops the short-term risks and the long-term complications as well as declines the quality of life.

Objectives: The study was conducted to assess non-adherence to medication and to identify factors that are asssociated with non-adherence in type 2 diabetes mellitus (T2DM) patients.

Methods: We conducted a retrospective chart review of 323 patients with T2DM attending the primary health care center of the Foča municipality in eastern part of Bosnia and Herzegovina. Adherence was measured using a validated survey form for medication adherence consisting of seven questions (4-point Likert scale). The SF-36v2 Health Survey (SF$36 \mathrm{v} 2$ ) was used to measure patients' quality of life.

Results: Half of the patients $(51,5 \%)$ treated pharmacologically were non-adherent. Results from the logistic regression demonstrated that gender $(B=-1.251 ; p<0.001)$, Physical Component Summary (PCS) scores $(\mathrm{B}=-0.042 ; \mathrm{p}=0.007)$ Menthal Component Summary $(\mathrm{MCS})$ scores $(\mathrm{B}=-$ $0.038 ; \mathrm{p}=0.007$ ) were the strongest predictors of poor adherence.

Summary / Conclusions: The findings suggest that male patients and patients reporting low PCS and MCS scores are less likely to be adherent. This information may be used to target interventions at TD2M patients who are likely to be non-adherent with their medication regimens. This work was supported by the Ministry of Science and Technological Development, Serbia (project No. 41012).

\section{EACPT-1107}

Monoclonal antibody ocrelizumab for primary-progressive multiple sclerosis in adults: a systematic review D. Klabukova ${ }^{*}{ }^{1}$, M. Davydovskaya ${ }^{2,3}$, T. Ermolaeva ${ }^{1}$, M. HolowniaVoloskova $^{1}$, K. Polyakova ${ }^{1}$, A. Fisun ${ }^{3}$
${ }^{1}$ Department of Drug Provision and Pharmacoeconomic Analysis, State Budgetary Institution of Moscow City "Clinical Trials and Healthcare Technology Assessment Scientific-Research Center of Moscow Department of Healthcare", ${ }^{2}$ Department of Neurology, Neurosurgery and Medical Genetics, Pirogov Russian National Research Medical University, ${ }^{3}$ Scientific and Clinical Department, State Budgetary Institution of Moscow City "Clinical Trials and Healthcare Technology Assessment Scientific-Research Center of Moscow Department of Healthcare", Moscow, Russian Federation

\section{Chronic diseases}

Introduction: Until recently, none of the available disease-modifying therapies (DMTs) demonstrated significant and reliable efficacy in patients with primary progressive multiple sclerosis (PPMS). Promising results in treatment patients with PPMS were demonstrated for the antiB-cell monoclonal antibody ocrelizumab.

Objectives: The study objective was to review the published evidence on the efficacy and safety of ocrelizumab in adult patients with PPMS.

Methods: Search was carried out in Embase, PubMed and Cochrane databases using keywords "ocrelizumab" and "multiple sclerosis" among the studies published in English from May 2008 to May 2018. This systematic review was conducted according to the PRISMA guidelines using specified $\mathrm{PICO}(\mathrm{S})$ criteria. The evidence levels of the studies were also determined.

Results: A total of 747 studies were identified from all databases. From 41 selected studies only 3 were included in the final literature review. One additional article was identified from citation reference lists. Therapy with ocrelizumab compared with placebo characterized by a decrease in the rate of confirmed disease progression $(\mathrm{p}=0.032)$, a significant reduction in the $\mathrm{T} 2$ lesion volume $(\mathrm{p}<0.001)$, faster performance of the 25 -foot walk $(\mathrm{p}=0.04)$, and an improvement in the change in brain volume $(\mathrm{p}=0.02)$. There was no significant difference between groups in the SF-36 physical component summary score. Compared with other monoclonal antibodies and immunotherapeutic DMTs, treatment with ocrelizumab had a significant slowdown in the disease progression of PPMS. Ocrelizumab safety profile is standard for immunosuppressive therapy. The most common adverse events were mild to moderate reactions associated with the drug infusion, as well as nasopharyngitis, upper tract respiratory and urinary tract infections, headaches. No cases of progressive multifocal leukoencephalopathy were noted. One case of a serious opportunistic infection (systemic infection with Pasteurella) was confirmed. Life years and quality-adjusted life years (QALYs) for patients receiving ocrelizumab were 16.11 and 3.33, compared to 15.61 and 2.75 for patients receiving best supportive care, respectively.

Summary / Conclusions: Ocrelizumab has proven its efficacy for the previously non-curable type of multiple sclerosis, PPMS, with risk profile acceptable with respect to clinical benefits.

\section{EACPT- 1140}

Anti-b-cell therapy ocrelizumab for relapsing multiple sclerosis in adults: a systematic review

D. Klabukova ${ }^{*}{ }^{1}$, M. Davydovskaya ${ }^{2,}{ }^{3}$, T. Ermolaeva ${ }^{1}$, M. HolowniaVoloskova $^{1}$, K. Polyakova ${ }^{1}$, A. Fisun ${ }^{3}$

${ }^{1}$ Department of Drug Provision and Pharmacoeconomic Analysis, State Budgetary Institution of Moscow City "Clinical Trials and Healthcare Technology Assessment Scientific-Research Center of Moscow Department of Healthcare", ${ }^{2}$ Department of Neurology, Neurosurgery and Medical Genetics, Pirogov Russian National Research Medical University, ${ }^{3}$ Scientific and Clinical Department, State Budgetary Institution of Moscow City "Clinical Trials and Healthcare Technology Assessment Scientific-Research Center of Moscow Department of Healthcare", Moscow, Russian Federation 


\section{Chronic diseases}

Introduction: More than 15 disease-modifying therapies (DMTs) for multiple sclerosis (MS) have been registered in the EU. Among them, monoclonal antibodies, such as ocrelizumab, are distinguished due to their significant efficacy. However, the problem of choosing a highly effective and safe drug for relapsing forms of multiple sclerosis such as relapsing-remitting and secondary progressive MS, remains relevant due to its polymorphic clinical manifestations.

Objectives: The study objective was to analyze the published evidence on the efficacy and safety of ocrelizumab in adult patients with relapsing forms of MS.

Methods: The search was conducted following PRISMA guidelines in the databases Embase, PubMed, Cochrane via keywords "ocrelizumab" and "multiple sclerosis". Studies with full text available, containing determined PICO(S) criteria, published in English from May 2008 to May 2018 were included. The evidence levels of the studies were determined.

Results: Total of 747 studies were identified. From 41 selected studies 6 were included in the final literature review. One additional article was identified from citation reference lists. Ocrelizumab was significantly more effective than interferon $\beta$-1a in reducing the annualized relapse rate and disability progression at 96 weeks. There was a reduction in the risk of new or increasing MRI T2 lesion and loss of brain volume. Patients receiving ocrelizumab were more likely to achieve NEDA (no evidence of disease activity) status. The most common adverse events of ocrelizumab therapy were infusion reactions, nasopharyngitis, upper tract respiratory and urinary tract infections, headaches. No cases of progressive multifocal leukoencephalopathy (PML) have been reported so far. For patients receiving ocrelizumab the average annual discontinuation probability was $5.0 \%$. Life years and quality-adjusted life years (QALYs) were 22.93 and 10.94, second only to alemtuzumab when compared to other DMTs. Ocrelizumab is more effective than first-line DMTs, including for the population of patients with aggressive (highly active) form of MS. It has similar efficacy in comparison with the second-line DMTs, but more favorable safety profile.

Summary / Conclusions: Ocrelizumab may be considered as the main treatment alternative in a subgroup of patients with highly active MS and in patients with a high risk for PML. An additional assessment of developing rare adverse events risk is required.

\section{EACPT-1310}

Pharmacoepidemiological analysis of disease-modifying antirheumatic drugs adverse effects in rheumatoid arthritis

O. Reshetko ${ }^{*}$, A. Levitan ${ }^{1}$

${ }^{1}$ Pharmacology, Saratov State Medical University, Saratov, Russian Federation

\section{Chronic diseases}

Introduction: Rheumatic diseases is one of human pathology severe forms. Rheumatoid arthritis (RA) is the most common nosology in this group of diseases. Disease-modifying antirheumatic drugs (DMARDs) are the main group of drugs used to RA treatment. However, adverse events (AEs) may limit these medications prescriptions.

Objectives: To study AEs developing structure and frequency during DMARDs therapy of RA.

Methods: An open-label retrospective cohort pharmacoepidemiological study has conducted in 544 patients with RA. Inclusion criteria: patients age more than 18 years, RA (according to classification criteria ACR/ EULAR (2010)) duration more than 6, DMARDs prescription duration more than 1 month. Outpatient charts have analyzed. This is a complete sample of patients, according to the inclusion criteria, who consistently came for medical care in the outpatient clinics of Saratov city from January 2018 to October 2018.
Results: AEs have occurred in 234 (from 544) patients (43\%) with RA that taking DMARDs. RA duration was from 6 month to 17 years (4,6 $6 \pm 2,1$ years) in patients with AEs and tended to increase compared

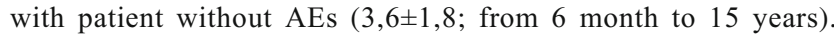
Dyspeptic disorders have registered in 17 patients, who taking sulfasalazine, and in 8, who taking leflunomide. All the other AEs had caused by the monotherapy of methotrexate. Methotrexate average dose that led to AEs was $15 \pm 7,1 \mathrm{mg}$ per week. Elevated level of aspartate aminotransferase (AST) and alanine aminotransferase (ALT) have occur in 78 patients that taking methotrexate. 47 patients had dyspepsia, 51 - hemodyscrasia (thrombocytopenia have registered in 31 patient, 14 - anemia, 6 - leukopenia). Enanthesis have occurred in 13 patients, stomatitis - in 10, vision disorders - in 4, skin sores - in 3, interstitial lung disease in 2, alopecia - in 1 patients, who taking methotrexate. So more AEs were in digestive system. DMARDs therapy duration was $4,1 \pm 1,9$ years in patients with AEs and $3,1 \pm 1,4$ in patients without AEs.

Summary / Conclusions: AE had occur often during DNARD therapy. Increasing the duration of RA and exposure to DMARD contribute to the development trend of $\mathrm{AE}$.

\section{EACPT-1378}

Pharmacological causes behind pulmonary disease

Z. Köllö ${ }^{*}$, D. L. Tarnoki ${ }^{2}$, E. Dho-Nagy ${ }^{1}$, F. Cori ${ }^{1}$, M. L. Garau ${ }^{1}$, E. G. Ban $^{1}$, A. Brassai ${ }^{1}$

${ }^{1}$ Department of Pharmacology, University of Medicine, Pharmacy, Science and Technology of Targu Mures, Targu Mures, Romania, ${ }^{2}$ Department of Radiology, Semmelweis University, Budapest, Hungary

\section{Chronic diseases, Drug utilization}

Introduction: In both pharmacology and radiology, it is important to know which drug can affect the lungs. This disease is called druginduced lung disease, which in most cases manifests as pulmonary fibrosis. Recently, the number of drugs, that can induce lung disease is over 400 , including antiarrhytmic drugs, antibiotics, chemotherapeutic and immunosuppressive agents. We performed a survey about pulmonary changes on radiologic images, with the aim to undertake a retrospective study and to examine how the active substance called nintedanib influences the status of the pulmonary diseases. We observed and evaluated patients' data as well as their radiologic images, and used these results to make a conclusion.

Objectives: Our aim was to examine the effects of nintedanib on radiological images and to conclude how much does it influences the chance of recovering.

Methods: In our retrospective study, we gathered information from 16 patients, regarding their gender, age, and disease. On their CT images, we inspected the ground glass opacity, honeycombing, traction bronchiectasis, reticulation, and compared these results between the ones who are treated with nintedanib and the ones who are not. The statistical analysis was performed using Excel and GraphPad Prism softwares.

Results: : In patients, who have been treated with nintedanib, the bilateral ground glass opacity decreased with $66 \%$, the bilateral reticulation with $33 \%$, the left-sided traction bronchiectasis also with $33 \%$ and right-sided traction bronchiectasis with $50 \%$, comparing with the patient who have not recieved nintedanib. Furthermore, the nintedanib eases the high WBC and high C-reactive protein values.

Summary / Conclusions: Besides diseases and traumas, also drugs can induce lung diseases, that are visible on radiological images. These are called drug-induced lung diseases, that represent an important part in both in clinical pharmacology and in radiology, and has a great significance in diagnosis and follow-up.

\section{EACPT-1407}

Antidiabetic drug therapy in patients with type 2 diabetes mellitus: a multicenter study in primary health care centers in greece 
Z. Tsimtsiou ${ }^{1,2,3}$, A. Papaioannou ${ }^{1,4}$, S. Birka ${ }^{1,2}$, G. Papazisis ${ }^{*} 5$, E. Apostolidou $^{5}$, C. Pourzitaki ${ }^{5}$, D. Kouvelas ${ }^{5}$

${ }^{1}$ Research Committee of Greek Association of General Practitioners, ${ }^{2}$ Health Center of Evosmos, ${ }^{3}$ Department of Hygiene, SocialPreventative Medicine and Medical Statistics, School of Medicine, Aristotle University of Thessaloniki, Thessaloniki, ${ }^{4} \mathrm{Health}$ Center of N. Makri, Athens, ${ }^{5}$ Clinical Pharmacology, School of Medicine, Aristotle University of Thessaloniki, Thessaloniki, Greece

\section{Chronic diseases, Other}

Introduction: Managing type 2 diabetes is challenging, especially in the primary health care setting. Though newer oral antidiabetics and types of insulin are available, metformin remains the gold standard and drug of choice to treat patients with type 2 diabetes.

Objectives: To evaluate the utilization of different classes of antidiabetic agents in patients with Type 2 Diabetes Mellitus in Primary Health Care Centers.

Methods: Nine primary care practices from seven different prefectures in Greece participated in the study. 225 diabetic patients were recruited (response rate $84 \%$ ) and completed a pre-tested 51-item questionnaire. The mean age was $70.9 \pm 9.5$ years (min: 32 , max: 92 ) and $117(52 \%)$ of them were males.

Results: Glycemic control (HbA1c $<7 \%)$ was achieved in $50.7 \%$ of the patients. The monthly cost of antidiabetic treatment was on average 37.9 euros ( \pm 38.3 , min: 1.7, max: 222 euros) with a median of 2 active substances per patient (IQR 2, min:1-max:5). Regarding the class of antidiabetic drugs, $80.4 \%$ of the patients received metformin, $41.8 \%$ DPP-4 inhibitors, $24.9 \%$ sulfonylureas, $16.4 \%$ insulin, 9.3\% SGLT-2 inhibitors, $9.3 \%$ glitazones, $3.1 \%$ meglitinides and $1.8 \%$ of the patients received GLP-1 agonists. The monthly treatment cost was significantly lower in patients who achieved glycemic control compared with patients who failed (28.8 versus 50.1 euros, $\mathrm{p}<0.001$ ).

Summary / Conclusions: Management of type 2 Diabetes Mellitus patients was not associated with the use of higher cost therapies. Metformin and DPP-4 inhibitors are the predominant therapies for the treatment of diabetic patients in Primary Health Care, while injectable treatments are of limited use. Penetration of the newer SGLT-2 inhibitors is small, although they have recently shown benefit in primary and secondary prevention of cardiovascular outcomes. The need for continuing education of primary health care physicians and their familiarization with injectable and newer therapies is imperative.

\section{EACPT-1414}

Priapism and atypical antipsychotics: case report and complete review

I. Mazón Maraña $^{*}$ 1, E. García Rumayor ${ }^{2}$, M. Ibáñez Alario², L. Boada Antón $^{2}$, D. Z. Cuéllar Gómez ${ }^{1}$, M. Sánchez Santiago ${ }^{1}$, N. Vega Gil ${ }^{1}$, M. Vaca Recalde $^{1}$, R. Nogueiras Álvarez ${ }^{1}$, A. Bautista Blázquez ${ }^{1}$, P. D. Ortiz Petrosino ${ }^{1}$

${ }^{1}$ Clinical Pharmacology, ${ }^{2}$ Psychiatry, Marqués de Valdecilla University Hospital, Santander, Spain

\section{Chronic diseases, Drug-drug interactions, Pharmacokinetics/ phar- macodynamics, Psychopharmacology, Rare diseases}

Introduction: Priapism is a urological emergency defined by potentially painful, uncontrolled and prolonged erection in absence of sexual stimulation. About $50 \%$ of drug related priapism is due to antipsychotics. The mechanism is not clear, although $\alpha$-adrenergic blocking effect is thought to be the cause. It has been reported with nearly all the atypical antipsychotic drugs (AAD), however, it is a rare adverse event (AE) and therefore, underappreciated.

Objectives: Starting from a case occurred in our center of a 39-year-old male with recurrent priapism, a systematic search was made of all the reports of drug related priapism associated with AAD. Statistical review was done to study the potential association between type of AAD, dose, time elapsed, interactions and pharmacology management.

Methods: A search was performed in Pubmed, Cinahl, Cochrane Library, Medline and Clinical Key through MeSH terms. The MeSH terms included multiple combinations of: Case, Priapism, Atypical Antipsychotic and each of the AAD known separately. The result was 126 articles, 96 after being validated. Subsequently, descriptive statistics were performed with SPSS taking into account: country, year of publication, age of the patients, antipsychotic and its dose, time elapsed, possible interactions, smoking, previous episodes, therapeutic changes and recurrences.

Results: 109 cases from 1992 to 2018. Patients' mean age was $34.6 \pm$ 12.98 years. The most associated drugs were risperidone $(37.6 \%)$, olanzapine $(17.4 \%)$, quetiapine $(16.5 \%)$ and clozapine $(11.9 \%)$. Dosages and time to AE showed a wide variability. In the $62,8 \%$ of the cases no dose changes, interactions or other triggering factor were found. $22 \%$ of the patients had already had a previous episode, $16.5 \%$ had a recurrence. After the AE, AAD was switched in $36.6 \%$ of cases; treatment was stopped in $32.1 \%$.

Summary / Conclusions: Priapism is a rare but serious AE. Mechanism of action is not well known. It seems to be a correlation between affinity for $\alpha$-receptors and incidence but priapism has been described for nearly all AAD and no relationship with dosages or treatment time has been established. In our case, after testing 6 AAD a washout period was required to avoid recurrences and subsequently the patient was retreated with a previous AAD without new episodes. However, in the cases reviewed only $20 \%$ of patients suffered recurrences, which could lead to a genetic susceptibility, unfortunately this has never been studied.

\section{EACPT-1444}

Case report: priapism due to antipsychotic drugs. Management proposal

I. Mazón Maraña* ${ }^{1}$, L. Boada Antón², D. Z. Cuéllar Gómez¹, E. García Rumayor $^{2}$, M. Ibáñez Alario ${ }^{2}$, M. Sánchez Santiago ${ }^{1}$, N. Vega Gil ${ }^{1}$, M. Vaca Recalde $^{1}$, R. Nogueiras Álvarez ${ }^{1}$, A. Bautista Blázquez ${ }^{1}$, P. D. Ortiz Petrosino ${ }^{1}$

${ }^{1}$ Clinical Pharmacology, ${ }^{2}$ Psychiatry, Marqués de Valdecilla University Hospital, Santander, Spain

Chronic diseases, Drug utilization, Drug-drug interactions, Psychopharmacology, Rare diseases

Introduction: Priapism is defined as a persistent and painful erection that lasts longer than 3 hours and that is not related to sexual stimuli. It is a urological emergency that may lead to multiple complications, some of them as serious and drastic as surgery, even penectomy in some cases.

Objectives: Around 25 to $40 \%$ of priapism events are related to the use of multiple pharmacological families, and of those, around $50 \%$ are related to the use of antipsychotics (AP). Given all said above, the aim of the study is to gather all available information about the management of recurrent priapism related to AP in patients that need those drugs.

Methods: We present a clinical case of a 33 years old male with a diagnosis of Autistic Spectrum Disorder treated with Clozapine and Risperidone for altered behaviour with two consecutive episodes of priapism. Related to the case, we conducted a bibliographic review about the current knowledge on the management of recurrent priapism secondary to the use of AP in psychiatric patients.

Results: The exact mechanism underlying AP related priapism is unknown. It is more likely a multifactorial etiology, although the most accepted theory is in relation to $\alpha$-adrenergic receptor affinity that most AP have in different degrees, blocking said receptors in the corpora cavernosa of the penis. Besides, the effect is idiosyncratic, seeming to not be related to dosage or time elapse with 
the drug. Among the management options, the most frequently chosen one is lowering the dose or completely interrupt its administration during the acute phase. Afterwards, in most cases physicians switch to an AP with less affinity to $\alpha$-adrenergic receptors, such as amisulpride. It's also discussed in the bibliography, in order to lessen adverse effects, the concomitant use of Clozapine and Fluvoxamine. Given the fact that Fluvoxamine is a CYP1A2 inhibitor (cytochrome responsible for the oxidation of Clozapine and its transformation to Norclozapine), it could diminish the adverse effects, priapism in the case at hand, maximizing its efficacy.

Summary / Conclusions: Priapism is a rare, but potentially severe adverse effect frequently caused by AP. Usual recommendations suggest switching AP to another one with less affinity to $\alpha$-adrenergic receptors such as amisulpride, or the concomitant use of fluvoxamine plus clozapine.

Computerized decision support

\section{Oral Presentation}

EACPT-1132

Prioritising hospital patients at high-risk of medication harm: development and validation of a predictive risk model

N. Falconer ${ }^{*} 1$, M. Barras ${ }^{1}$, A. Abdel-Hafez ${ }^{2}$, S. Radburn ${ }^{2}$, N. Cottrell ${ }^{1}$

${ }^{1}$ School of Pharmacy, University of Queensland, ${ }^{2}$ Clinical Informatics,

Princess Alexandra Hospital, Brisbane, Australia

\section{Computerized decision support, Pharmacovigilance}

Introduction: Inpatient medication harm affects approximately $7 \%$ of patients. At least half of these events are thought to be preventable. Timely clinician review can mitigate this harm. However, in increasingly busy hospitals, with high patient throughput, and limited resources, there is a need to prioritise high-risk patients. Current prioritisation methods can include many risk factors and are not well evaluated. A model that can assist with early identification of patients high-risk would be useful for targeted clinical interventions.

Objectives: To develop and validate a predictive risk model, to identify adult inpatients at risk of medication harm.

Methods: We conducted a retrospective cohort study of general medical and geriatric inpatients sequentially admitted over six months to a quaternary hospital in Brisbane, Australia. Classification of medication harm was undertaken using the Hallas criteria to establish causality. A total of 83 variables, were extracted from the hospital's digital databases. Univariable preselection was undertaken to identify variables, with pvalues $\leq 0.15$, for inclusion in multivariable logistic regression. Backwards elimination, was used for model development. The final model was validated using ten-fold cross validation.

Results: This study included 1982 patients (mean age of 70 years). A total of 137 (7\%) of patients experienced one or more events whilst hospitalised. Causality assessment classified $30 \%$ of events as definite, $43 \%$ as probable, and $27 \%$ as possible. The final model included: length of stay, hospital re-admission within 12 months, patients admitted with venous thromboemobolism, administration of $\geq 8$ medications at admission, Serum sodium $<126 \mathrm{mmol} / \mathrm{L}$, INR $>3$, use of anti-psychotic, use of antiarrhythmic, and use of immunosuppressant medications. Cross validation of the model produced an area under the receiver operative characteristic curve of 0.70 (95\% CI: 0.65-0.75). The model was well calibrated with a Hosmer-Lemeshow Goodness of Fit test, $\mathrm{p}=0.21$, and Brier score of 0.059 .

Summary / Conclusions: We have developed a model with clinically informative predictors, which demonstrated reasonable performance. Our study identified novel variables, including low serum sodium and raised INR, not previously reported in the literature. Future steps include external validation of the model and impact testing to evaluate clinical utility.
EACPT-1236

A pharmacist-physician-based process to ensure quality assurance in standard order sets

L. Ring Eriksson ${ }^{* 1}$, A. Helldén ${ }^{1,2}$, H. Lövborg ${ }^{1,2}$

${ }^{1}$ Department of Clinical Pharmacology, County Council of Östergötland/ Region Östergötland, ${ }^{2}$ Division of Drug Research, Department of Medical and Health Sciences, Faculty of Health Sciences, Linköping University, Linköping, Sweden

\section{Computerized decision support}

Introduction: The physicians in the County Council of Östergötland, Sweden, have been encouraged to use digital standard order sets (SOSs) for drugs since 2010. The intentions with the SOSs are e.g. to minimize variation through standardized formatting of orders and integrating best practice for specific conditions.

Objectives: The process to ensure the accuracy of the SOSs has not been described.

Methods: New requests and changes in the 2700 available SOSs in the Cambio COSMIC healthcare information system are triggered by e.g. new or changed guidelines, non-conformance reports of medication errors, updated deals for procured drugs and work-arounds due to defects in the system. Three full time pharmacists have authorization to the template administration tool where the SOSs are managed. The county council drug therapeutic committee designates physicians responsible for the different medical areas in which the SOSs are grouped.

Each month approximately 40 new requests or changes in the SOSs are registered. The requests are evaluated by a pharmacist or the responsible physician resulting in a refusal or approval of the request. Adjustments are carried out in cooperation between pharmacist and physician. For major changes that require adjustments in the documentation of the drug treatment process, e.g. in prescribing or administration, chief physician is consulted. Almost all areas are reviewed annually to make sure that the SOSs are upto-date.

A guideline for creation and adjustment of SOSs includes steps to ensure that the sets are complete and following authority regulations. All new SOSs and major changes in available sets are reviewed by one physician and two pharmacists before publishing.

Results: A flowchart presenting the above mentioned process will be presented.

When prescribing, the physician search SOSs within a well fitted selection since only the SOSs relevant to the unit where the physician is logged in, are shown. It is possible to extend the search result to all SOSs in the county council and also to prescribe without order sets.

Summary / Conclusions: The pharmacist-physician-based process includes steps to ensure that the SOSs are accurate and following national and regional guidelines. The high inflow of requests indicates that the physicians use SOSs and know where to turn when they have new needs.

\section{EACPT-1286}

Comparative efficacy and safety of treatments for secondary raynaud's phenomenon: systematic review and network metaanalysis of randomized trials

C. Khouri* 1, 1, 2, 2, 3, 3 , M. lepelley ${ }^{2}$, S. Bailly ${ }^{4}$, S. Blaise ${ }^{1,5}$, L. Trinquart $^{6}$, A. L. Herrick ${ }^{7,8}$, M. Matucci-Cerinic ${ }^{9}$, Y. Allanore ${ }^{10,11}$, J.L. Cracowski ${ }^{1,3}$, M. Roustit ${ }^{1,3}$

${ }^{1}$ UMR 1042-HP2, University Grenoble Alpes, ${ }^{2}$ Pharmacovigilance, ${ }^{3}$ Clinical Pharmacology Department INSERM CIC1406, Grenoble Aples University Hospital, ${ }^{4}$ UMR 1042-HP2, ${ }^{5}$ Medecine Vasculaire, Grenoble Alpes University Hospital, Grenoble, France, ${ }^{6}$ Department of Biostatistics, Boston University School of Public Health, Boston, United States, ${ }^{7}$ Centre for Musculoskeletal Research, Manchester University NHS Foundation Trust, Manchester Academic Health Science Centre, Manchester, ${ }^{8}$ NIHR Manchester Musculoskeletal Biomedical Research Centre, Central Manchester NHS Foundation Trust, Manchester 
Academic Health Science Centre, Manchester, United Kingdom, ${ }^{9}$ Department of Rheumatology AVC, Department of BioMedicine, Division of Rheumatology AOUC, Department of Medicine \& Denothecentre, , University of Florence, Firenze, Italy, ${ }^{10}$ INSERM U1016 UMR8104 Cochin Institute, Paris Descartes University, Sorbonne Paris CiteParis, ${ }^{11}$ Rheumatology A department, Paris Descartes University, Cochin Hospital, Paris, France

\section{Computerized decision support, Other}

Introduction: Several pharmacological treatments are currently available in secondary Raynaud's phenomenon (RP) but there is no current evidence demonstrating the superiority of one treatment over the other, owing to the lack of direct/indirect comparisons.

Objectives: To assess and compare the benefit and harms of all pharmacological treatments available in secondary RP.

Methods: We conducted an umbrella research strategy, we thus searched for reviews or meta-analyses published in MEDLINE and the Cochrane systematic review database up to January 2017 to identify all trials included in these studies. We also searched for all randomized controlled trials (RCTs) published between January 2015 and September 2018. Double blinded RCTs comparing two or more pharmacological treatments or placebo with more than $50 \%$ of secondary RP patients were selected. Pairwise meta-analyses and Bayesian network meta-analysis were used to synthesize data.

The following efficacy outcomes were used: 1. Daily frequency; 2. Severity; and 3. Daily cumulative duration of attacks. Tolerability (proportion of serious adverse event) and acceptability (all-cause discontinuation)were used as safety outcomes.

Results: A total of 56 double-blind RCTs including 3827 patients, among whom $92 \%$ had secondary RP, comparing 14 drug classes and placebo were included. PDE5i were the only class statistically more effective than placebo for the three efficacy outcomes, with a low to high level of evidence. CCBs were also superior to placebo on the frequency of attacks, with a low level of evidence, and an effect size close to that of PDE5i. Besides CCBs and PDE5i, two classes were superior to placebo on the severity of RP: SSRI, and oral $\mathrm{PGI}_{2}$ analogs. Yet, the level of evidence was low to very low for these drugs. Only PDE5i and oral $\mathrm{PGI}_{2}$ analogs had a lower acceptability and tolerability than placebo; CCBs also showed significantly worse tolerability than placebo. Summary / Conclusions: Despite moderate efficacy and level of evidence, CCBs and PDE5i are the best option when a pharmacological treatment is indicated. New therapeutic strategies, including non-pharmacological interventions, have to be further developed in SSc-related RP.

\section{Oral Presentation}

EACPT-1345

The efficacy of a mobile clinical decision support application compared to usual care: a proof of concept study among prescribers in different stages of training

M. J. Bakkum ${ }^{* 1,2}$, J. Tichelaar ${ }^{1,2,3}$, K. C. Sigaloff ${ }^{1,2}$, M. C. Richir ${ }^{1,2}$, M. A. van Agtmael ${ }^{1,2,3}$

${ }^{1}$ Department of Internal Medicine, section Pharmacotherapy, Amsterdam UMC, Vrije Universiteit Amsterdam, ${ }^{2}$ Research and Expertise Centre in Pharmacotherapy Education (RECIPE), Amsterdam, Netherlands, ${ }^{3}$ European Association for Clinical Pharmacology and Therapeutics (EACPT) Education Working group, Frankfurt, Germany

\section{Computerized decision support, Education}

Introduction: Current clinical practice guideline (CPG) adherence rates are as low as 30 to $70 \%$. Clinical decision support systems have been shown to reduce unwanted deviations and improve adherence rates. In order to improve the familiarity and usability of CPGs, we have created a platform that can be used to translate CPGs into mobile clinical decision support applications (MCDSA). These MCDSA are based on a transparent decision tree model that is shown as a series of multiple-choice questions. The MCDSA can be used anytime and anywhere on internet browsers of mobile and desktop devices. Additionally, the MCDSA provides important background information to educate it's user. In this study, the Dutch guideline for gastric prophylaxis was directly compared to the corresponding MCDSA.

Objectives: The aim of this study was to compare the effects of the MCDSA for gastric prophylaxis to usual care as proof of concept for the efficacy of MCDSAs in general.

Methods: A cross sectional examination was performed in medical specialists (consultants), junior doctors, medical students and physician assistant or nurse practitioner (PA/ANP) trainees. They were asked to complete 5 cases with clinical judgement and/or CPG and 5 subsequent cases using the MCDSA. All guideline-adherent answers were scored correct. Results: In the pilot study 4 junior doctors and 5 medical students were included. Together, using clinical judgement, they answered $44 \%$ of cases correctly. Using the guideline, $66 \%$ of cases were answered correctly and with the MCDSA 94\%. The MCDSA was significantly better (difference $50 \%, \mathrm{p}=<0.01$ ) than clinical judgement and guideline use (difference $28 \%, \mathrm{p}=<0.01$ ). The pilot study was insufficiently powered to detect such differences within the different populations.

Summary / Conclusions: Using the platform, guidelines may be easily translated into mobile clinical decision support applications that yield improved guideline adherence rates as compared to usual care on a case-based examination. Whether these applications are as effective in a real-life prescribing environment should be subject of further investigation. The complete results in 25 medical students, 25 PA/ANP trainees, 25 junior doctors and 25 medical specialists will be available at EACPT Stockholm 2019. Secondary outcomes include the required time to complete cases and user experience with the application.

\section{Oral Presentation}

\section{EACPT-1436}

An algorithm for automatically assessing student answers to open questions in the pharmacotherapy

e-learning web-application p-scribe

A. van Doorn ${ }^{*}$, T. van Dongen ${ }^{2}$, E. van Dijk ${ }^{3}$, H. Baarsma ${ }^{4}$

${ }^{1}$ Clinical Pharmacy and Pharmacology, University Medical Center Groningen, ${ }^{2} \mathrm{Fac}$. of Science and Engineering, Artificial Intelligence, ${ }^{3} \mathrm{Fac}$, of Sciences and Engineering, ${ }^{4} \mathrm{Fac}$. of Sciences and Engineering, Dep. of Molecular Pharmacology, Groningen, Netherlands

\section{Computerized decision support, Education}

Introduction: Assessing open questions (OQs) is a time-consuming task for teachers. The Dutch national pharmacotherapy e-learning web-application "P-scribe" based on the WHO-6Step patient treatment model is case oriented. Due to the fact that P-scribe is a mandatory component in medical and pharmacy curricula, teachers must review hundreds of OQs each year. We have developed an Artificial Intelligence Algorithm (AI/A) that can automatically assess students' answers to OQs in P-scribe.

Objectives: In this pilot study we present a $1^{\text {st }}$ version of such an approach with an $\mathrm{AI} / \mathrm{A}$ that can automatically classify student responses to OQs in the categories: "G = Good", "S = Sufficient" and "I = Insufficient".

Methods: Three different P-scribe cases (A, B, C) with their respective number of OQs $(7,5,21)$ were offered to all $3^{\text {rd }}$ year undergraduate pharmacy students in the course year 2017-18.The student participation (number of students and total given answers) were (case A: 54, 378; case B: 37,185 ; case C: 15, 315), The measured, collected and downloaded data were used as datasets for testing the AI/A. The cases were assessed by two different pharmacy teachers (experts) to evaluate. The students' answers were classified on their likeliness to an expert answer. The AI/A first pre-processes both answers (student, expert), and it compares the two answers using approximate or fuzzy string matching to account for close similarities. A synonym dictionary is used to explain possible synonyms that still have to match. The score based on matching is then classified into one of the three categories. Because the possible number of matches depends on the length of the expert response, this length is divided by [1-15] to create ranges in which the different classifications $\mathbf{G}, \mathbf{S}$ and $\mathbf{I}$ are made. 
Results: The effect of expert answers on the AI/A assessment of a case $1^{\text {st }}$ tested on ternary split $\mathbf{G}, \mathbf{S}$ and $\mathbf{I}$, and $2^{\text {nd }}$ on binary split where $\mathbf{G}$, and $\mathbf{S}$ were taken as one group are shown in table 1 .

\begin{tabular}{lll}
\hline $\begin{array}{l}\text { Expert nr \& assessed } \\
\text { Case }\end{array}$ & $\begin{array}{c}\text { \% correct G / S / I } \\
\text { matching in } \\
\text { ternary split } \\
\text { mode }\end{array}$ & $\begin{array}{c}\% \text { correct GS / I } \\
\text { matching in } \\
\text { binary split } \\
\text { mode }\end{array}$ \\
Expert 1 \& Case A & $61.9 \%$ & $77.7 \%$ \\
Expert 1 \& Case B & $68.1 \%$ & $84.3 \%$ \\
Expert 1 \& Case C & $60.3 \%$ & $49.2 \%$ \\
Expert 2 \& Case A & $37 \%$ & $53.4 \%$ \\
Expert 2 \& Case B & $64.9 \%$ & $80 \%$ \\
\hline
\end{tabular}

Table 1. Effect of expert answers on the AI/Algorithm assessment of a case in terms of the percentage (\%) of the correct answers in binary or ternary split mode.

Summary / Conclusions: This pilot study shows that the AI/A approach for automatically classifying student responses to OQs is a first step in the right direction to help teachers perform these assessment tasks more efficiently.

\section{Oral Presentation \\ EACPT-1446}

Pharros - a pilot project to create a standards-based computerized decision support system for therapeutic drug monitoring (tdm) Y. Böttiger $^{1}$, J. Axelsson ${ }^{*}$, R. Chen ${ }^{2,3}$, F. Bengtsson ${ }^{1}$, M. Hoffmann ${ }^{1}$

${ }^{1}$ Medical and Health Sciences, Linköping university, Linköping, ${ }^{2}$ Cambio Health Care Systems, ${ }^{3}$ Health Informatics Centre LIME, Karolinska Institutet, Stockholm, Sweden

\section{Computerized decision support}

Introduction: To interpret the result of a concentration analysis, information on the drug, its dosing schedule and time of sampling is crucial. Information on other medications taken by the patient, and patient characteristics, such as renal function, may also be useful in the interpretation. In an electronic health record, most of this information is available as structured data.

Objectives: The aim of the present pilot project is to build a prototype for a computerized decision support system (CDSS) that will collect structured data from the electronic health record to support an automated TDM evaluation. The project also aims to demonstrate a novel way to build a CDSS using open standards that has potential to be reusable across language and technical barriers.

Methods: Detailed requirements are gathered through a series of workshops. Both narrative requirement specification document and decision tree diagram are produced and validated by the clinician. Tacrolimus is selected to create the proof-of-concept (POC) application. Clinical models based on standards such as HL7 FHIR (Fast Healthcare Interoperability Resources), openEHR archetypes and GDL (Guideline Definition Language) are used to represent the clinical algorithms for analyzing the lab test results based the decision tree for Tacrolimus (Figure 1). Web standards such as HTML5, CSS and JavaScript are used to create the POC's user interface.

Results: A POC web-application, consists of standards-based clinical models and simple web user interface, is developed. The POC is deployed as cloud-based service and is available for demonstration and testing purpose. All clinical algorithms for Tacrolimus analysis are represented in openEHR GDL format which makes it easy to add support for additional languages and reference terminologies.

Summary / Conclusions: With automated retrieval and analysis of health record data relevant to the interpretation and clinical evaluation of a TDM result, there is a possibility to decrease the time needed to fill in referrals, and to increase both the quality and the quantity of information that is generated by the laboratory in connection to the concentration analysis. It is feasible to build an informatics standards-based CDSS to meet the requirements of clinical pharmacology test result analysis. Such CDSS may have a potential to be reused across the language and technical boundaries.

\section{Drug-drug interactions}

EACPT-1023

Influence of green tea on pharmacokinetics of fexofenadine in healthy volunteers

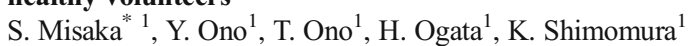

${ }^{1}$ Department of Bioregulation and Pharmacological Medicine, Fukushima Medical University, Fukushima, Japan

\section{Drug-drug interactions, Pharmacokinetics/ pharmacodynamics}

Introduction: Green tea has been reported to exert beneficial effects on health. Meanwhile, we have investigated drug transporter-mediated drug interaction between green tea and nadolol, a hydrophilic and nonmetabolized $\beta$-blocker, revealing that green tea catechins such as epigallocatechin gallate (EGCG) could reduce the plasma concentrations of nadolol by inhibiting its intestinal absorption. However, whether concomitant ingestion of green tea affects the pharmacokinetics of other hydrophilic drugs remains poorly understood.

Objectives: The aim of this study was to assess the influence of green tea catechin on the pharmacokinetics of fexofenadine, a hydrophilic H1 blocker, which is known to be a substrate of several intestinal drug transporters including P-glycoprotein and organic anion transporting polypeptides.

Methods: A randomized, open, single center, 2-phase crossover study was conducted in 10 healthy Japanese volunteers. After overnight fasting, subjects were administered fexofenadine $60 \mathrm{mg}$ with water $(150 \mathrm{~mL})$ or an aqueous solution of green tea extract (GTE). Sunphenon EGCG (Taiyo Kagaku, Tokyo, Japan; total catechin $97.4 \%$, EGCG 92.5\%, w/w) was used as GTE with EGCG dose equivalent to $300 \mathrm{mg}$ by dissolving in 150 $\mathrm{mL}$ of water before administration. Blood and urine were collected up to 24 hours after administration.

Results: The area under the plasma concentration-time curve (AUC) and maximum plasma concentration $\left(C_{\max }\right)$ of fexofenadine were significantly reduced by $71 \%(P<0.001)$ and $69 \%(P<0.01)$, respectively, in GTE phase. There were no differences in $T_{\max }$ and the elimination half-life $\left(T_{1 / 2}\right)$ and renal clearance of fexofenadine between phases. The decrease in fexofenadine AUC by GTE correlated with fexofenadine AUC in control $(r=-0.86, P=0.0015)$.

Summary / Conclusions: Coadministration of fexofenadine with green tea catechin resulted in a significant decrease in plasma concentrations of fexofenadine without changing its renal clearance, possibly by inhibiting the intestinal absorption of fexofenadine.

\section{EACPT-1128}

Pharmacokinetic interaction between ezetimibe/rosuvastatin and telmisartan in healthy male subjects

K. Y. Huh ${ }^{1}$, S. W. Lee ${ }^{1}$, S. B. Lee ${ }^{2}$, T. W. Lee ${ }^{2}$, S. Lee ${ }^{1}$, I.-J. Jang ${ }^{1}$

${ }^{1}$ Department of Clinical Pharmacology and Therapeutics, Seoul National University College of Medicine and Hospital, Seoul, ${ }^{2}$ Addpharma Inc., Gyeonggi-do, Korea, Republic Of

\section{Drug-drug interactions}

Introduction: Both dyslipidemia and hypertension are major risk factors for cardiovascular disease and combination therapy is commonplace. Although fixed-dose combination (FDC) of ezetimibe/rosuvastatin and telmisartan are frequently prescribed together, pharmacokinetic (PK) interaction among the three drugs has not been investigated.

Objectives: This study aimed to evaluate the PK interaction among ezetimibe/rosuvastatin and telmisartan in healthy male subjects. 
Methods: An open-labeled, randomized, 3-period, 6-sequence crossover study was conducted. Subjects orally received one of the following treatments once daily for 7 days in each period: the FDC of ezetimibe/ rosuvastatin $10 / 20 \mathrm{mg}$; telmisartan $80 \mathrm{mg}$; co-administration of the FDC of ezetimibe/rosuvastatin 10/20 mg with telmisartan $80 \mathrm{mg}$. PK sampling for free/total ezetimibe, rosuvastatin and telmisartan was conducted at pre-dose and up to 24 hours post-dose at steady-state in each period. PK parameters were calculated by the non-compartmental method and the geometric mean ratios (GMRs) of combination therapy to monotherapy and their $90 \%$ confidence intervals (CIs) for maximum plasma concentration $\left(\mathrm{C}_{\max , \mathrm{ss}}\right)$ and area under the time-concentration curve within a dosing interval ( $\mathrm{AUC}_{\mathrm{tau}, \mathrm{ss}}$ ) at steady-state were estimated. Tolerability was assessed by adverse events (AEs), vital signs, electrocardiograms, physical examinations and laboratory tests.

Results: A total of 36 healthy male subjects were enrolled and 31 subjects completed the study. GMRs $(90 \% \mathrm{CIs})$ for $\mathrm{C}_{\text {max,ss }}$ and $\mathrm{AUC}_{\text {tau,ss }}$ of free/total ezetimibe were $1.8458(1.5586-2.1861)$ and 1.1552 (1.0556$1.2642) / 1.1086(0.9949-1.2353)$ and 1.0136 (0.9524-1.0786), respectively. The corresponding values for rosuvastatin were 2.1334 (1.8755$2.4264)$ and 1.0883 (1.0282-1.1520), and for telmisartan were 1.1572 (1.0127-1.3222) and 1.2635 (1.1674-1.3675). Considering the flat pharmacokinetic-pharmacodynamic relationship of the drugs at the dose level studied, increases in systemic exposures of free ezetimibe, rosuvastatin and telmisartan after co-administration may not result in significant changes in clinical response. All AEs were mild in severity and no significant differences between combination therapy and monotherapy were noted.

Summary / Conclusions: The extent of PK interactions during coadministration of FDC of ezetimibe/rosuvastatin with telmisartan was not expected clinically significant and did not have impact on tolerability.

\section{EACPT-1129}

Effect of multiple oral administration of ds-8500a, a novel g proteincoupled receptor 119 agonist, on the pharmacokinetics of midazolam in healthy japanese subjects

K. Toyama ${ }^{1}$, H. Furuie ${ }^{2}$, M. Kato ${ }^{1}$, H. Ishizuka ${ }^{1}$

${ }^{1}$ Daiichi Sankyo Co., Ltd., Tokyo, ${ }^{2}$ Osaka Pharmacology Clinical Research Hospital, Osaka, Japan

\section{Drug-drug interactions, Pharmacokinetics/ pharmacodynamics}

Introduction: DS-8500a is a novel GPR119 agonist with anti-diabetic potential. A nonclinical study in human hepatocytes showed that DS8500a induces cytochrome P450 (CYP) 3A4; hence, it is clinically important to evaluate CYP3A-mediated drug-drug interaction (DDI) potential of DS-8500a. Midazolam is metabolized by CYP3A, and is associated with numerous DDIs. It is recommended as an index substrate for DDI testing and has been widely used as an indicator of CYP3A activity in humans. Objectives: This study investigated whether DS-8500a has CYP3Ainducing activity in healthy Japanese subjects.

Methods: This was a single-center, open-label, single-sequence study comprising two administration periods: period 1-single-dose of $2 \mathrm{mg}$ midazolam; and period 2 - $75 \mathrm{mg}$ DS-8500a once daily for 14 days, with the administration of $2 \mathrm{mg}$ single-dose midazolam on Day 14. Blood samples were collected at specified time intervals, and plasma concentrations of midazolam and DS-8500a were analyzed using validated liquid chromatography tandem-mass spectrometry methods. The pharmacokinetic parameters were determined by a non-compartment analysis. Primary pharmacokinetic endpoints were peak plasma concentration (Cmax) and area under the plasma concentration-time curve (AUC) of midazolam.

Results: Twenty-eight subjects (18 males and 10 females) were enrolled. One subject was withdrawn from the study due to a serious adverse event (asthma) that was unrelated to the study drug. Mean plasma midazolam concentration decreased after DS-8500a co- administration on Day 14. Ratios (two-sided $90 \%$ confidence interval) of geometric least squares means for Cmax, AUClast, and AUCinf of midazolam with to without DS-8500a were 1.024 $(0.930,1.127), 0.831(0.769,0.897)$, and $0.831(0.770,0.898)$, respectively. The mean half-life of midazolam was decreased by the co-administration of DS-8500a ( $3.22 \mathrm{~h}$ vs $2.82 \mathrm{~h})$. No safety concern was observed. No notable changes were found in the laboratory values, vital signs, or standard 12-lead ECG.

Summary / Conclusions: The repeated administration of $75 \mathrm{mg} /$ day DS8500 a led to a weak CYP3A-inducing activity, which might not be clinically significant.

\section{EACPT-1131}

The effect of multiple oral administration of esaxerenone on the pharmacokinetics of midazolam in healthy japanese males H. Furuie ${ }^{* 1}$, K. Toyama ${ }^{2}$, Y. Okuda ${ }^{2}$, K. Kuroda ${ }^{2}$, T. Shimizu ${ }^{2}$, M. Kato ${ }^{2}$, H. Ishizuka ${ }^{2}$

${ }^{1}$ Osaka Pharmacology Clinical Research Hospital, Osaka, ${ }^{2}$ Daiichi Sankyo Co. Ltd., Tokyo, Japan

Drug-drug interactions

Introduction: Esaxerenone is a novel oral, non-steroidal, and selective mineralocorticoid receptor blocker approved in Japan in January 2019 for hypertension treatment and is currently being developed for diabetic nephropathy treatment. In vitro studies demonstrated its cytochrome $\mathrm{P} 450$ (CYP) 3A-inducing and inhibiting potential; hence, it is clinically important to evaluate CYP3A-mediated drug-drug interaction (DDI) potential of esaxerenone. Midazolam is metabolized by CYP3A, and is associated with numerous DDIs. It is recommended as an index substrate for DDI testing and has been widely used as an indicator of CYP3A activity in humans.

Objectives: This study investigated the effect of esaxerenone on midazolam pharmacokinetics in healthy Japanese males.

Methods: This was a single-center, open-label, single-sequence study comprising two administration periods: period 1-single-dose of $2 \mathrm{mg}$ midazolam; and period $2-5 \mathrm{mg}$ /day esaxerenone for 14 days, with the administration of a single-dose of $2 \mathrm{mg}$ midazolam on Day 14. Blood samples were collected at specified time intervals, and plasma concentrations of midazolam and esaxerenone were analyzed using validated liquid chromatography tandem-mass spectrometry methods. The pharmacokinetic parameters were determined by a non-compartment analysis. Primary pharmacokinetic endpoints were peak plasma concentration (Cmax), area under the plasma concentration-time curve (AUC) from zero to the last quantifiable concentration (AUClast), and AUC from zero to infinity (AUCinf) of midazolam.

Results: The study enrolled male subjects ( $n=28$; mean age: 26.4 years). One subject was withdrawn due to a mild adverse event (increased hepatic enzyme levels), which resolved without intervention. Mean plasma midazolam concentration increased after esaxerenone co-administration on Day 14. Ratios (two-sided $90 \%$ confidence intervals) of geometric least squares means for Cmax, AUClast and AUCinf of midazolam with to without esaxerenone were 1.224 (1.116, 1.342), 1.201 (1.110, 1.300), and $1.201(1.112,1.297)$, respectively. The mean half-life of midazolam with or without esaxerenone was similar ( 2.86 h vs 2.63 h). No safety concern was observed.

Summary / Conclusions: The repeated administration of $5 \mathrm{mg}$ /day esaxerenone increased midazolam exposure by 1.2 -fold in healthy Japanese males; thus, the effect is not considered clinically important.

\section{EACPT-1139}

Clinically significant drug-drug interactions in cardiovascular diseases: comparing the prevalence and prescribing patterns between inpatient and outpatient settings

M. Kovačević ${ }^{*}{ }^{1}$, S. Vezmar Kovačević ${ }^{1}$, S. Radovanović ${ }^{2}$, P. Stevanović ${ }^{3}$, B. Miljković ${ }^{1}$

${ }^{1}$ Department of Pharmacokinetics and Clinical Pharmacy, University of Belgrade - Faculty of Pharmacy, ${ }^{2}$ University Hospital Medical Center 
Bežanijska kosa, University Hospital Center Dr Dragiša Mišović, ${ }^{3}$ University Hospital Medical Center Bežanijska kosa, University Hospital Center Dr Dragiša Mišović, University of Belgrade, School of Medicine, Belgrade, Serbia

\section{Drug-drug interactions}

Introduction: A drug-drug interaction (DDI) may result in deteriorated therapy efficacy or safety. There is a lack of data on prescribing patterns causing DDIs, as well as "the point of risk" during patient transfer between different settings, when DDIs may emerge.

Objectives: To assess the prevalence and prescribing patterns leading to DDIs identified at admission and during hospital stay, i.e. DDIs generated in outpatient/inpatient setting, respectively.

Methods: A retrospective observational study was conducted. Data were obtained from medical records. LexiInteract was used for DDIs screening. Clinically significant DDIs were considered X, D and C class. Descriptive and statistical analysis was performed using SPSS. A value $\mathrm{p}<0.05$ was considered statistically significant.

Results: Therapy was assessed for 213 patients, mean age $70 \pm 10$ years. Number of drugs/per patient increased from 6 (range 1-15) at admission to 8 (2-25) during hospitalisation. Number of clinically significant DDIs/per patient increased from 3 (range 0-23) to 6 (range 0-41). Prevalence of X DDIs (avoid combination) was not significantly increased: from $2.3 \%(n=5)$ to $3.8 \%(n=8)$. Patients had significantly higher exposure to D class (consider therapy modification): $27.7 \%(\mathrm{n}=59)$ to $41.3 \%$ ( $n=89$ ), and C class (monitor therapy): $83.6 \%(n=178)$ to $90.6 \%$ $(\mathrm{n}=193)$. Amiodarone, captopril and valsartan were significant predictors for the occurrence of clinically significant DDIs in the outpatient setting, whereas even 15 drugs were involved in DDI emergence during hospital stay: anticoagulants (acenocoumarol, warfarin), CVD drugs (amiodarone, digoxin, furosemide, ramipril, spironolactone), allopurinol, aminophylline, ciprofloxacin, diclofenac, ibuprofen, insulin, potassium chloride, and ranitidine.

Summary / Conclusions: CVD patients are highly exposed to clinically significant DDIs. Drugs used for gout, obstructive airway disease, pain, acid-related disorders, diabetes, as well as antibiotics, significantly increase the number and prevalence of DDIs in hospital. The close collaboration of health professionals with different specialties is necessary. DDI screening tools may be beneficial for prescribing optimal therapy.

\section{EACPT-1029}

Effect of ds-8500a, a novel g protein-coupled receptor 119 agonist, on the pharmacokinetics of atorvastatin in healthy japanese subjects Y. Maekawa ${ }^{1}$, H. Furuie ${ }^{2}$, M. Kato ${ }^{1}$, H. Ishizuka ${ }^{* 1}$

${ }^{1}$ Daiichi Sankyo Co., Ltd., Tokyo, ${ }^{2}$ Osaka Pharmacology Clinical Research Hospital, Osaka, Japan

Drug-drug interactions, Pharmacokinetics/ pharmacodynamic

Introduction: DS-8500a is a novel GPR119 agonist with anti-diabetic potential. It may inhibit some cytochrome P450 (CYP) isoforms and transporters, and induce CYP3A as observed in non-clinical studies. Therefore, it is clinically important to evaluate the drug-drug interaction potential of DS-8500a. DS-8500a can be used concomitantly with statins in clinical practice and both drugs have the potential for drug-drug interactions.

Objectives: This study investigated the effects of DS-8500a on the pharmacokinetics of atorvastatin in healthy Japanese subjects.

Methods: This was a single-center, open-label, single-sequence study comprising two administration periods: period 1 - single-dose of $10 \mathrm{mg}$ atorvastatin and period 2-75 mg DS-8500a once daily for 14 days, with the administration of a single-dose of $10 \mathrm{mg}$ atorvastatin on Day 14 . Blood samples were collected at specified time intervals, and plasma concentrations of atorvastatin and DS-8500a were analyzed using validated liquid chromatography tandem-mass spectrometry methods. The pharmacokinetic parameters were determined by a non-compartment analysis. Primary pharmacokinetic endpoints were peak plasma concentration (Cmax) and area under the plasma concentration-time curve (AUC) of atorvastatin.

Results: Twenty-eight subjects (20 males and 8 females) were enrolled. One subject discontinued because of a treatment-emergent adverse event, which was unrelated to the study medications and four subjects discontinued at their own request. Mean plasma atorvastatin concentration increased after DS-8500a co-administration on Day 14. Ratios (two-sided $90 \%$ confidence intervals) of geometric least squares means for Cmax and AUClast of atorvastatin with to without DS-8500a were $1.283(1.112,1.478)$ and 0.926 $(0.859,0.998)$, respectively. All treatment-emergent adverse events were of mild or moderate intensity and were unrelated to the study drugs, except for rash, which was considered related to DS-8500a. No notable changes were found in the laboratory values, vital signs, or standard 12-lead ECG.

Summary / Conclusions: The impact of DS-8500a on atorvastatin exposure was limited and may not be clinically relevant.

\section{EACPT- 1040}

Evaluation of herbal medicines used by diabetes patients in nigeria for cyp p450 enzyme induction using human hepatocytes

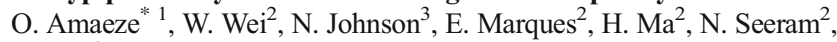
A. Slitt ${ }^{2}$

${ }^{1}$ Clinical Pharmacy and Biopharmacy, University of Lagos, Lagos, Lagos, Nigeria, ${ }^{2}$ Biomedical and Pharmaceutical Sciences, University of Rhode Island, Kingston, Rhode Island, ${ }^{3}$ Pharmacokinetics, Dynamics and Metabolism, Pfizer Inc., Groton, Connecticut, United States

\section{Drug-drug interactions}

Introduction: The induction of CYP P450 enzymes can lead to important drug interactions resulting in reduced therapeutic efficacy of a coadministered medication or an increased risk of reactive metaboliteinduced toxicity. The inductive effect of commonly consumed herbal medicines in Nigeria on CYP enzymes is largely unknown.

Objectives: This study was carried out to assess the inductive potential of five common Nigerian herbal medicines on the metabolic activities of CYP1A2, 2B6 and 3A4.

Methods: V. amygdalina, O. gratissimum, M. oleifera, A. indica leaves and $P$. nitida seeds were identified as being used frequently by type 2 diabetes patients in Nigeria. Crude methanol and aqueous extracts of these medicinal plants at 1,10 and $100 \mu \mathrm{g} / \mathrm{ml}$ were evaluated for their inductive effects using Cryostax 5-donor pool of cryopreserved human hepatocytes and specific CYP substrates. Regulatory recommended CYP inducers and $0.1 \%$ DMSO served as positive and vehicle controls respectively. The herbal extracts were assessed at non-toxic concentrations determined by a cell viability assay. CYP enzyme activity was determined by quantifying the formation of marker metabolites using LC-MS/MS methods. CYP mRNA levels were quantified using the QuantiGene 2.0 Plex assay and analyzed using a Bio-Rad Bio-Plex-200 platform. Fold induction of CYP1A2, 2B6 and 3A4 enzyme activities and mRNA levels were determined.

Results: The hepatocytes responded to prototypical inducers for the different enzymes with respect to mRNA gene expression and catalytic activity. The methanol extract of $O$. gratissimum caused a $>2$-fold induction in CYP2B6 mRNA gene expression. The herbal extracts did not have any inductive effect on CYP1A2 and 3A4 mRNA gene expression. At different concentrations $(1,10,100 \mu \mathrm{g} / \mathrm{ml})$, both the methanol and aqueous herbal extracts caused significant changes in CYP1A2, 2B6 and CYP3A4 catalytic activities which were not up to $20 \%$ response of the positive control inducers.

Summary / Conclusions: Using mRNA gene expression as the primary endpoint, the methanol $O$. gratissimum extract showed an inductive potential for CYP2B6, and can be described as a positive in vitro inducer. The other herbal extracts did not have a significant inductive effect on 
CYP1A2, 2B6 and 3A4 and are not likely to cause an enzyme inductionmediated herb-drug interaction.

\section{EACPT-1082}

Bradykinin-mediated angioedema associated with combination of angiotensin-converting enzyme and dipeptidyl peptidase iv inhibitors: a disproportionality analysis from the who database M. Lepelley ${ }^{1}$, C. Khouri* ${ }^{*}$, M. Mallaret ${ }^{1}$, L. Bouillet $^{2}$

${ }^{1}$ Pharmacovigilance, ${ }^{2}$ Centre de référence des angioedèmes bradykiniques, CHU Grenoble-Alpes, Grenoble, France

\section{Drug-drug interactions}

Introduction: Bradykinin-mediated angioedema (BMA) is a rare but potentially lethal adverse drug reaction of angiotensin-converting enzyme inhibitors (ACEi). Dipeptidyl peptidase IV inhibitors (DDP4i) are also involved in the pathogenesis of BMA. Concurrent use of vildagliptin and ACEi was associated with an increased risk of BMA. There is no data concerning the risk of BMA with others DPP4i alone, nor in combination with ACEi.

Objectives: We hypothesized that the concomitant use of ACEi and DPP$\mathrm{i}$ is associated with an increased risk of reports of angioedema compared to DPP-4i or ACEi alone.

Methods: We performed a disproportionality analysis using data from the WHO pharmacovigilance database by a case-noncase study, until the $14 / 12 / 2017$. We extracted all individual cases safety reports (ICSRs) included in the high level term "angioedemas", according to the Medical Dictionary for Regulatory Activities classification. Drug class exposure was "ACEi" and "DPP4i", suspect or concomitant, using the ATC classification. We calculated the reporting odds ratio (ROR) of angioedema with ACEi alone, DPP4i alone and combination of ACEi and DPP4i. A signal was considered to be significant if the lower bound of the confidence interval is superior or equal to 1 . According to the interaction additive model, a ROR value for coexposure that exceeds the sum of the RORs estimated for each individual class of drug support a potential drug-drug interaction (DDI).

Results: ACEi were associated with 22,827 ICSRs of angioedemas, DPP4i with 688 cases and combination of ACEi and DPP4i with 269 cases. The ROR was 6.67 (95\% CI 6.58-6.77) for ACEi alone, 0.44 (95\% CI 0.41-0.47) for DPP4i alone, and 27.64 (95\% CI 23.76-32.16) for combination of ACEi and DPP4i. No DDP4i was associated with a significant ROR of angioedema.

Summary / Conclusions: According to these results, combination of DPP4i and ACEi was associated with increased risk of report of angioedema, but not with DPP4i alone. This result is consistent with previous study and supports a pharmacodynamic interaction. Further studies are needed to confirm this potential risk with combination of ACEi and DPP4 $i$ and the absence of risk concerning DPP4 $i$ alone and occurrence of BMA.

\section{EACPT-1162}

Impact of the organic cation transporter 2 inhibitor cimetidine on the single-dose pharmacokinetics of the glucosylceramide synthase inhibitor lucerastat

M.-L. Boof ${ }^{*}$, M. Ufer ${ }^{1}$, A. Halabi ${ }^{2}$, J. Dingemanse $^{1}$

${ }^{1}$ Department of Clinical Pharmacology, Idorsia Pharmaceuticals Ltd, Allschwil, Switzerland, ${ }^{2}$ CRS Clinical Research Services Kiel GmbH, Kiel, Germany

\section{Drug-drug interactions, Pharmacokinetics/ pharmacodynamics}

Introduction: Lucerastat is an orally available glucosylceramide synthase inhibitor aimed at providing substrate reduction therapy for Fabry patients independent of genotype. In humans, the main route of elimination is through renal excretion of the unchanged parent compound. Since the proportion of lucerastat undergoing active renal secretion was estimated at $50 \%$, in vitro studies with renal transporters were performed. These studies suggested that lucerastat is a substrate of human organic cation transporter 2 (OCT2).

Objectives: To evaluate the influence of the OCT2 inhibitor cimetidine at steady state on the single-dose pharmacokinetics (PK) of lucerastat. The safety and tolerability of lucerastat administered alone and concomitantly with cimetidine were also evaluated.

Methods: A single-center, open-label, two-period, fixed-sequence study was performed to investigate the effect of cimetidine on the PK of lucerastat in 14 healthy male adults. Each subject received a single oral dose of $500 \mathrm{mg}$ lucerastat alone (on Day 1) or in combination (on Day 6) with $800 \mathrm{mg}$ twice-daily cimetidine administered for 7 days (Day 3 to Day 9). PK sampling of lucerastat was performed for $48 \mathrm{~h}$ (alone) and $96 \mathrm{~h}$ (in combination with cimetidine) post dose. PK sampling of cimetidine was performed from Day 3 to Day 9 prior to morning administration. Lucerastat and cimetidine were both quantified in plasma by a validated LC-MS/MS method. Adverse events, ECG, vital signs, and clinical laboratory tests were assessed for safety/tolerability evaluation.

Results: Exposure to lucerastat was slightly higher upon coadministration of cimetidine indicated by geometric mean $\mathrm{C}_{\max }$ and $\mathrm{AUC}_{0-\infty}$ ratios of 1.04 (90\% CI: 0.92-1.17) and 1.22 (1.16-1.27), respectively. Cimetidine delayed lucerastat tmax by $1 \mathrm{~h}$ but did not affect $\mathrm{t}_{1 / 2}$ as geometric mean ratio was $1.00(0.91-1.10)$ at cimetidine steady state. Lucerastat was safe and well tolerated when given alone and in combination with cimetidine. There were neither serious nor severe adverse events or any relevant changes of ECG, vital signs or laboratory data.

Summary / Conclusions: The study results indicate that the single-dose PK of lucerastat are not changed to a relevant extent by cimetidinemediated OCT2 inhibition, allowing the concomitant use of OCT2 inhibitors with lucerastat without any need for dose adjustment.

\section{EACPT-1165}

Therapeutic drug monitoring for lacosamide in japanese patients with epilepsy

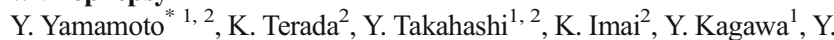
Inoue $^{2}$

${ }^{1}$ Clinical Pharmaceutics, Pharmaceutical Sciences, University of Shizuoka , ${ }^{2}$ Clinical Research, National Epilepsy Center, Shizuoka Institute of Epilepsy and Neurological Disorders, Shizuoka, Japan

\section{Drug-drug interactions}

Introduction: Lacosamide is a novel antiepileptic drug (AED) that was approved in 2016 for mono-and adjunctive treatment of focal epilepsy.

Objectives: The purpose of this study was to evaluate the influence of concomitant AEDs on serum lacosamide concentration.

Methods: We enrolled 430 patients (aged 1 years to 91 years) with epilepsy who were treated with lacosamide for epilepsy at our hospital between September 2016 and December 2018. A total of 1,557 samples were analyzed using routine therapeutic drug monitoring data, and the concentration-to-dose ratio (CD ratio) of lacosamide was compared among patients on various $\mathrm{AED}$ regimens. The study protocol was approved by the ethical committee of National Epilepsy Center (Shizuoka, Japan).

Results: 183 patients ( 725 samples) were taking enzyme-inducing AEDs, and the mean CD ratio of this group was $1.30 \pm 0.51 \mathrm{mg} / \mathrm{mL} / \mathrm{mg} / \mathrm{kg}$, which was significantly lower than that of 247 patients ( 832 samples) without enzyme-inducing AEDs $(1.87 \pm 0.77 \mathrm{mg} / \mathrm{mL} / \mathrm{mg} / \mathrm{kg}, \mathrm{p}<0.001)$. The mean $\mathrm{CD}$ ratio of lacosamide in patients concomitantly using phenytoin, phenobarbital, and carbamazepine was $1.30 \pm 0.47,1.33 \pm 0.52$, and $1.35 \pm 0.56$, respectively. In patients on multiple inducer regimens, the mean $\mathrm{CD}$ ratio of lacosamide was $1.17 \pm 0.38$.

Summary / Conclusions: Therapeutic drug monitoring is clinically useful to estimate the extent of drug interaction between lacosamide and concomitant AEDs. 


\section{EACPT-1237}

Assessment of the pharmacokinetic interaction between fimasartan and linagliptin in healthy volunteers

S. J. Seong ${ }^{1}$, W. Y. Kang ${ }^{1}$, B. Ohk ${ }^{1}$, B. K. Kim ${ }^{1}$, M.-R. Gwon ${ }^{1}$, S. Cho ${ }^{1}$, H. W. Lee ${ }^{1}$, Y.-R. Yoon ${ }^{1}$

${ }^{1}$ Kyungpook National University Hospital, Daegu, Korea, Republic Of

\section{Drug-drug interactions}

Introduction: Hypertension and type II diabetes are major risk factors for cardio-cerebrovascular disease. Combination therapy is often prescribed for the effective treatment of hypertension, diabetes, or dyslipidemia.

Objectives: The present study assessed the pharmacokinetic interactions between fimasartan, a selective inhibitor of angiotensin II type 1 receptor, and linagliptin, a dipeptidyl peptidase- 4 inhibitor after multiple oral administrations.

Methods: The study comprised of two separate parts (A and B). Each study was randomized, open-label, multiple-dose, two-period, and onesequence design. In part A, 24 subjects were given fimasartan $120 \mathrm{mg}$ once a day for 7 days during period I, and fimasartan with linagliptin $5 \mathrm{mg}$ once daily for subsequent 7 days during period II. In part B, participants took $5 \mathrm{mg}$ of linagliptin alone for 7 days (the first period), followed by concurrent administration of linagliptin and fimasartan for 7 days (the second period). Blood samples were drawn for up to 24 hours after last dosing and were analyzed by a validated liquid chromatography-tandem mass spectrometry. Safety was evaluated during the study based on adverse events (AEs), using vital signs, laboratory tests, 12-lead electrocardiogram, and subjects' reporting.

Results: The geometric mean ratio (GMR) and $90 \%$ confidence interval (CI) for the $\mathrm{C}_{\mathrm{max}, \mathrm{ss}}$ and $\mathrm{AUC}_{\text {tau,ss }}$ of fimasartan (with/without linagliptin) were 1.263 (0.9175-1.7396) and 1.174 (1.050-1.313), respectively. In part B of the study, the GMR and $90 \% \mathrm{CI}$ for the $\mathrm{C}_{\text {max,ss }}$ and $\mathrm{AUC}_{\text {tau,ss }}$ of linagliptin (with/without fimasartan) were $0.980(0.848-1.134)$ and 0.995 (0.932-1.062), respectively .

No serious AE was observed throughout the entire study period.

Summary / Conclusions: In our study, there are no clinically significant interactions between fimasartan $120 \mathrm{mg}$ and linagliptin $5 \mathrm{mg}$.

\section{EACPT-1239}

The effect of simvastatin on warfarin anticoagulation: a swedish register-based nationwide cohort study.

\section{L. Andersson ${ }^{*}$, B. Mannheimer ${ }^{2}$, J. D. Lindh ${ }^{1}$}

${ }^{1}$ Clinical Pharmackology, Laboratory Medicine, ${ }^{2}$ Department of Clinical Science and Education at Södersjukhuset, Karolinska Institutet, Stockholm, Sweden

\section{Drug-drug interactions}

Introduction: Simvastatin is one of the most used drugs and co-treatment with warfarin is common. Available data indicate that simvastatin use may slightly increase the anticoagulative effect of warfarin, but the evidence is scarce.

Objectives: The aim of this study was to investigate if, and to which extent, initiation of simvastatin therapy alters the anticoagulative effect of warfarin in a very large patient sample.

Methods: In a retrospective cohort, we investigated the change in warfarin dose during simvastatin therapy, by comparing warfarin doses before and after initiation of co-treatment with simvastatin. Data on warfarin doses and INR were collected from two large warfarin registers and simvastatin use was identified by using the Swedish Prescribed Drug Register. Mean INR values per week was calculated and the fraction of patients with INR values above 3 and 4 were summarized.

Results: In total 5637 individuals on stable warfarin treatment initiating simvastatin therapy were included in the study. INR increased from 2.43 at baseline to 2.58 four weeks after simvastatin initiation and did not stabilize until the last quarter of the year studied. The proportion of patients with an INR above 3 increased from $14.5 \%$ to $24.2 \%$. For INR above 4 , the proportion increased from $1.3 \%$ to $2.7 \%$. Warfarin doses were found to be $6.8 \%$ lower at the end of the study (337-364 days) compared to warfarin doses at baseline.

Summary / Conclusions: Initiation of simvastatin therapy was associated with a moderate increase in INR values. The fraction of patients with supratheraputic INR values increased markedly but still few patients had INR values above 4 . Even though the effect of this interaction is small, the high number of patients exposed to this combination will result in many patients reaching INR values associated with increased bleeding risk. Close monitoring of INR seems advisable when simvastatin therapy is started or withdrawn in patients on warfarin therapy.

\section{Oral Presentation}

EACPT-1255

Measurement of hepatic abcb1 and abcg2 transport activity with positron emission tomography

I. Hernández Lozano* ${ }^{1}$, B. Wulkersdorfer ${ }^{1}$, A. $\operatorname{Traxl}^{2}$, M. Bauer ${ }^{1}$, C. Philippe $^{3}$, B. Stieger ${ }^{4}$, C. Özvegy-Laczka ${ }^{5}$, G. Szakács ${ }^{5}$, T. Wanek ${ }^{2}$, M. Hacker $^{3}$, M. Zeitlinger ${ }^{1}$, O. Langer ${ }^{1,2,3}$

${ }^{1}$ Department of Clinical Pharmacology, Medical University of Vienna, Vienna, ${ }^{2}$ Preclinical Molecular Imaging, AIT Austrian Institute of Technology GmbH, Seibersdorf, ${ }^{3}$ Division of Nuclear Medicine, Department of Biomedical Imaging and Image-guided Therapy, Medical University of Vienna, Vienna, Austria, ${ }^{4}$ Department of Clinical Pharmacology and Toxicology, University Hospital Zurich, University of Zurich, Zurich, Switzerland, ${ }^{5}$ Institute of Enzymology, Research Centre for Natural Sciences, Hungarian Academy of Sciences, Budapest, Hungary

\section{Drug-drug interactions}

Introduction: $\mathrm{ABCB} 1$ and $\mathrm{ABCG} 2$ are two efflux transporters which mediate the hepatobiliary excretion of many drugs and their metabolites. Changes in hepatic $\mathrm{ABCB} 1$ and $\mathrm{ABCG} 2$ transport activity (caused e.g. by disease, drug-drug interactions or genetic polymorphisms) may lead to alterations in the plasma clearance of drugs, which may cause drug adverse effects.

Objectives: In this study we evaluated the suitability of positron emission tomography (PET) with the model $\mathrm{ABCB} 1 / \mathrm{ABCG} 2$ substrate $\left[{ }^{11} \mathrm{C}\right]$ tariquidar to measure hepatic $\mathrm{ABCB} 1 / \mathrm{ABCG} 2$ transport activity.

Methods: $\left[{ }^{11} \mathrm{C}\right]$ tariquidar PET scans were carried out in healthy volunteers and wild-type and transporter knockout mice $\left(A b c l a / b^{(-)}, A b c g 2^{(--)}\right.$ and $\left.A b c l a / b^{(--)} A b c g 2^{(--)}\right)$without and with i.v. treatment with unlabeled tariquidar. A 3-compartment pharmacokinetic model was used to determine the rate constants defining the hepatic kinetics of $\left[{ }^{11} \mathrm{C}\right]$ tariquidar. Furthermore, to assess a potential involvement of basolateral hepatocyte uptake transporters (SLCO1B1, SLCO1B3, SLCO2B1, SLC22A1 and SLC22A3) in the uptake of $\left[{ }^{11} \mathrm{C}\right]$ tariquidar from blood into the liver, in vitro uptake experiments were performed with $\left[{ }^{11} \mathrm{C}\right]$ tariquidar or $\left[{ }^{3} \mathrm{H}\right]$ tariquidar in different transporter overexpressing cell lines.

Results: In humans, no radiolabeled metabolites of $\left[{ }^{11} \mathrm{C}\right]$ tariquidar could be detected in plasma during the PET scan. The treatment with unlabeled tariquidar caused a significant $(\mathrm{p}<0.05)$ reduction in the rate constant describing the transfer of radioactivity from liver into bile $\left(k_{3}\right)$ suggesting saturation of $\mathrm{ABCB} 1 / \mathrm{ABCG} 2$ transport activity. In mice, treatment with unlabeled tariquidar significantly reduced $k_{3}$ in wild-type, $A b c 1 a / b^{(--)}$and $\mathrm{Abcg} 2^{(--)}$mice, but not in $\mathrm{Abcla} / \mathrm{b}^{(--)} \mathrm{Abcg} 2^{(--)}$mice. Moreover, in baseline scans, $k_{3}$ was significantly lower in $A b c l a / b^{(-)} A b c g 2^{(--)}$mice as compared to wild-type mice, but unaltered in $A b c l a / b^{(-/)}$and $A b c g 2^{(--)}$ mice. In vitro experiments indicated that tariquidar is not transported by any of the investigated uptake transporters.

Summary / Conclusions: These results suggest that $\left[{ }^{11} \mathrm{C}\right]$ tariquidar is a metabolically stable PET tracer which can be used to measure hepatic $\mathrm{ABCB} 1$ and $\mathrm{ABCG} 2$ transport activity without a confounding effect of basolateral hepatocyte uptake transporters. Our data point to a mutual functional compensation between $\mathrm{ABCB} 1$ and $\mathrm{ABCG} 2$ in the hepatobiliary excretion of dual substrate drugs. 


\section{EACPT-1278}

Impact of the selective orexin-1 receptor antagonist act-539313 on the pharmacokinetics of the cyp3a4 probe drug midazolam and the endogenous cyp3a4 marker $6 \beta$-hydroxycortisol

P. Kaufmann ${ }^{1}$, B. Berger ${ }^{1}$, A. Koch ${ }^{2}$, J. Dingemanse ${ }^{1}$

${ }^{1}$ Clinical Pharmacology, Idorsia Pharmaceuticals Ltd., Allschwil, Switzerland, ${ }^{2}$ Simbec Research Ltd., Pentrebach, United Kingdom

\section{Drug-drug interactions}

Introduction: ACT-539313 is a potent and selective orexin-1 receptor antagonist (SO1RA) in development for the treatment of anxiety disorders. CYP3A4 is the major cytochrome P450 enzyme involved in the metabolism of ACT-539313 in man.

Objectives: To investigate the effect of ACT-539313 on the pharmacokinetics $(\mathrm{PK})$ of midazolam and the urinary $6 \beta$-hydroxycortisol to cortisol ratio $(6 \beta-\mathrm{CR})$ and to, thereby, assess the CYP3A4 interaction potential of this SO1RA.

Methods: A single-center, open-label, fixed-sequence study was performed to investigate the effect of single-dose (on Day 2) and multipledose (on Day 11) twice daily (b.i.d.) administration of $200 \mathrm{mg}$ ACT539313 on the PK of $2 \mathrm{mg}$ midazolam in 20 healthy male adults. PK sampling of midazolam in plasma as well as urine collection to evaluate the $6 \beta-C R$ was performed over $24 \mathrm{~h}$ on Day 1 , Day 2, and Day 11 . Safety and tolerability were also assessed.

Results: Exposure to midazolam was higher during concomitant administration of single- as well as after repeated-dose ACT-539313 over 10 days compared to midazolam alone. In the presence of ACT-539313, the geometric mean $\left(\mathrm{GEO}_{\mathrm{m}}\right)(90 \% \mathrm{CI})$ of $\mathrm{C}_{\max }$ and $\mathrm{AUC}_{0-24}$ ratio increased by 1.18 -fold (1.06-1.31) and 1.79-fold (1.56-2.06) on Day 2, and by 2.13-fold (1.912.36) and 4.54-fold (3.95-5.22) on Day 11, respectively; the $t_{1 / 2}$ was prolonged 2.00-fold (1.79-2.23) on Day 2 and 2.11-fold (1.89-2.36) on Day 11 , while the median $\mathrm{t}_{\max }$ was unchanged at $0.50 \mathrm{~h}$. The $\mathrm{GEO}_{\mathrm{m}}(90 \%$ $\mathrm{CI}$ ) of the metabolic ratio (MR) of 1-hydroxymidazolam to midazolam in plasma showed a decrease to $0.60(0.53-0.68)$ on Day 2 and to $0.21(0.19$ 0.24) on Day 11. A similar outcome was also shown for urine, as the $\mathrm{GEO}_{\mathrm{m}}$ $(90 \% \mathrm{CI})$ of the $6 \beta-\mathrm{CR}$ showed a decrease to $0.78(0.70-0.88)$ on Day 2 and to $0.61(0.55-0.69)$ on Day 11 . The most commonly reported adverse events (AEs) included somnolence and headache. All AEs were transient and of mild intensity. No treatment-related effects on vital signs, clinical laboratory, and ECG were observed.

Summary / Conclusions: The observed corresponding decrease of both the endogenous ( $6 \beta-\mathrm{CR})$ and exogenous (midazolam MR) marker of CYP3A4 activity is indicative of a weak inhibition of CYP3A4 occurring after treatment with a single dose and a moderate inhibition occurring after repeated doses of $200 \mathrm{mg}$ b.i.d. ACT-539313.

\section{Oral Presentation}

EACPT-1292

Queries about drug interactions to a drug information centre over a ten-year period - a descriptive analysis

C. Tukukino* $1, \mathrm{~S}$. Wallerstedt ${ }^{2,3}$

${ }^{1}$ Clinical Pharmacology, Sahlgrenska University Hospital, ${ }^{2}$ Pharmacology, Sahlgrenska Academy, University of Gothenburg, ${ }^{3}$ HTA-centrum, Sahlgrenska University Hospital, Gothenburg, Sweden

\section{Drug-drug interactions}

Introduction: Drugs are an important treatment modality within health care, used to cure, prevent, relieve, and diagnose diseases. The availability of approved drugs is increasing, as is the number of people exposed to many drugs. This implies an increased risk for drug interactions. To the best of our knowledge, interactions queries posed to a drug information centre have not previously been characterized. Such analyses can provide important insights as they illustrate the need for interaction information in the clinical context.
Objectives: To describe health care professionals' drug interaction queries to a drug information centre over 10 years, focusing on drugs frequently queried about and the advice provided.

Methods: Drug interaction queries (2008-2017) to a drug information centre in Sweden were characterized. Advice provided was recorded in mutually exclusive groups: Avoid, Adjust dose, Separate intake, Vigilance, or No problem. For queries with Avoid, Adjust dose, or Separate intake advice, alerts were extracted from an interaction database (Janusmed).

Results: Of 4,335 queries to the centre during the study period, 589 (14\%) concerned interactions. Most were posed by physicians $(91 \%)$ and concerned a specific patient $(83 \%)$ before treatment initiation $(76 \%)$. Sertraline, warfarin, and methotrexate were the drugs most frequently queried about, whereas cyclophosphamide and rifampicine were most common in relation the number of exposed patients. Advice provided in $557(95 \%)$ replies comprised Avoid: $\mathrm{n}=86(15 \%)$, Adjust dose: $\mathrm{n}=$ $57(10 \%)$, Separate intake: $\mathrm{n}=17(3 \%)$, Vigilance: $\mathrm{n}=235(42 \%)$, or $N o$ problem: $\mathrm{n}=162$ (29\%). In all, $70(44 \%)$ of 160 queries with Avoid, Adjust dose, or Separate intake advice elicited the same level of alert on Janusmed.

Summary / Conclusions: Drug interaction queries often concern specific patients when the applicability of general, readily accessible recommendations may be limited. In seven out of ten replies, the drug information centre recommended an explicit drug treatment action, either regarding the prescribing per se or the conducting of an active follow-up.

\section{EACPT-1298}

Tolerability and blood pressure effects of a long-acting nitrate coadministered with vericiguat: results of the vericiguat isosorbide mononitrate interaction (visor) study

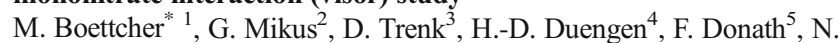
Werner $^{6}$, M. Karakas ${ }^{7}$, N. Besche ${ }^{8}$, D. Schulz-Burck ${ }^{9}$, M. Gerrits ${ }^{10}$, J. Hung $^{11}$, C. Becker ${ }^{1}$

${ }^{1}$ Clinical Pharmacology, Bayer AG, Wuppertal, ${ }^{2}$ Department of Clinical Pharmacology and Pharmacoepidemiology, University Hospital Heidelberg, Heidelberg, ${ }^{3}$ Clinics for Cardiology and Angiology II, Dept. Clin. Pharmacology, University Heart Center Freiburg-Bad Krozingen, Bad Krozingen, ${ }^{4}$ Cardiology, Charité, University Hospital Berlin, Berlin, ${ }^{5}$ SocraTec R\&D GmbH, Erfurt, ${ }^{6}$ Heart Center, Department of Medicine II, University Hospital Bonn, Bonn, ${ }^{7}$ General and Interventional Cardiology, University Heart Center Hamburg Eppendorf, Hamburg, ${ }^{8}$ Chrestos Concept GmbH \& Co. KG, Essen, ${ }^{9}$ Research \& Development, Pharmaceuticals, Bayer AG, Wuppertal, Germany, ${ }^{10}$ Merck \& Co., Inc. , Kenilworth, New Jersey, United States, ${ }^{11}$ Clinical Operations, Study Medical Experts, Bayer SA, São Paulo, Brazil

\section{Drug-drug interactions}

Introduction: Vericiguat is a novel stimulator of soluble guanylate cyclase for treatment of chronic heart failure (HF). Guidelines recommend short- and longacting nitrates for acute treatment and prophylaxis of angina in patients (pts) with coronary artery disease (CAD). Co-administration of the short-acting nitrate nitroglycerin (NTG) with vericiguat in dogs, healthy volunteers (EudraCT 2014-001235-36) and in CAD pts (NCT02617550) was well tolerated.

Objectives: The Vericiguat ISOsoRbide Mononitrate Interaction (VISOR) study (NCT03255512) aimed to investigate the pharmacodynamic (PD) drug-drug interaction (DDI) between co-administered isosorbide mononitrate (ISMN, extended-release) and vericiguat in pts with stable CAD $( \pm \mathrm{HF})$.

Methods: VISOR, a multicentre, double-blind, placebo (PBO)-controlled comparison study, investigated the co-administration of ISMN $60 \mathrm{mg}$ with vericiguat. Pts were randomised to vericiguat + ISMN $(\mathrm{n}=28)$ or PBO + ISMN ( $\mathrm{n}=13)$. Pts were administered ISMN (Q.D.) for 2 weeks followed by co-administration with $\mathrm{PBO} /$ vericiguat. Uptitration of $\mathrm{PBO} /$ vericiguat from 2.5 to 5 to $10 \mathrm{mg}$ (Q.D.) was performed in 2-week increments; pts on $\mathrm{PBO} /$ vericiguat $10 \mathrm{mg}+\mathrm{ISMN}$ were 
monitored for 2 weeks. The objectives were to evaluate PD DDIs (blood pressure [BP], heart rate [HR]), safety and tolerability.

Results: 35 pts completed treatment (PBO: $n=12$, vericiguat: $n=23$ ). Coadministration of vericiguat + ISMN led to mean baseline- and PBOadjusted reductions in systolic BP of $1.4-5.1 \mathrm{mmHg}$, diastolic BP of 0.4 $2.9 \mathrm{mmHg}$ and changes in HR of 0.0 to $1.8 \mathrm{bpm}$. These changes were not deemed clinically relevant. No consistent vericiguat dose-dependent PD effects were observed. Two discontinuations due to adverse events (AEs) occurred. They were related to ISMN (1) and to unstable angina (1), but not to $\mathrm{PBO}$ or vericiguat. The incidence of AEs was $92.3 \%$ (vericiguat + ISMN) and $66.7 \%$ (PBO + ISMN)

Summary / Conclusions: Based on the lack of symptoms associated with the changes in BP and HR in pts taking vericiguat + ISMN, these changes were not considered to be clinically relevant. This combination was well tolerated and is supported by the consistency of results from previous preclinical and human studies. Concomitant use of short- and long-acting nitrates with vericiguat is unlikely to cause clinically significant AEs beyond those known for NTG and ISMN.

\section{EACPT-1311}

Placental drug transporter -mediated interactions and fetal malformations

M. Ellfolk ${ }^{1}$, A. Tornio ${ }^{*}{ }^{2,}{ }^{3}$, M. Niemi ${ }^{2,3}$, A.-M. Lahesmaa-Korpinen ${ }^{4}$, M. K. Leinonen ${ }^{4}$ H. Malm ${ }^{1,2,3,5}$

${ }^{1}$ Teratology Information, Department of Emergency Medicine, ${ }^{2}$ Department of Clinical Pharmacology, University of Helsinki and Helsinki University Hospital, ${ }^{3}$ Individualized Drug Therapy Research Program, Faculty of Medicine, University of Helsinki, ${ }^{4}$ Information Services Department, Unit of Statistics and Registers, National Institute for Health and Welfare, Helsinki, ${ }^{5}$ Department of Child Psychiatry, University of Turku, Turku, Finland

\section{Drug-drug interactions}

Introduction: P-glycoprotein (P-gp) and breast cancer resistance protein (BCRP) are efflux transporters expressed in the placenta, limiting the degree of their substrates from reaching the fetus. Many drugs are substrates or inhibitors of these transporters. Polypharmacy may lead to inhibition of the transporters and increased fetal substrate exposure.

Objectives: Our aim was to investigate if concomitant prenatal exposure to several transporter substrates or inhibitors increases the rate of congenital anomalies compared to exposure to only one substrate or inhibitor.

Methods: The existing national 'Drugs and Pregnancy' database covering years 1996-2014 was utilized in this population-based birth cohort study. In the database, the Medical Birth Register, the Register on Induced Abortions, the Malformation register and the Register on Reimbursed Drug Purchases have been linked. The University of Washington Metabolism and Transport Drug Interaction Database was used to identify substrates and inhibitors of P-gp and BCRP.

We identified drug exposure to P-gp/BCRP substrates and inhibitors in early pregnancy. We categorized singleton pregnancies into groups of $\mathrm{P}-$ gp/BCRP polytherapy $(n=21,186), P-g p / B R C P$ monotherapy $(n=97,906)$, non-P-gp/BCRP polytherapy $(n=78,636)$, and those with no drug purchases (unexposed, $n=728,870$ ). Pregnancies exposed to known teratogens were excluded from study material. We investigated the association between the exposure groups and major congenital anomalies using logistic regression adjusting for several confounders. We also studied the P-gp and BCRP transporters separately.

Results: After adjustment, the risk of congenital anomalies was increased in the P-gp/BCRP polytherapy group (5.5\%) compared to the P-gp/BCRP monotherapy $(4.7 \%$, OR 1.12 ; $95 \%$ CI $1.05-1.20)$, compared to the non-Pgp/BCRP polytherapy (4.9\%, adjusted OR $1.14 ; 95 \%$ CI $1.06-1.23$ ), and compared to the unexposed group (4.1\%, OR 1.22 ; $95 \%$ CI 1.12-1.32). When the transporters were analysed separately, the results were similar for the P-gp group, but BCRP appeared not to have an independent effect.
Summary / Conclusions: Exposure to polytherapy with P-gp and/or BCRP substrates or inhibitors is associated with an increased risk for congenital anomalies. Placental drug transporter interactions may play a role in teratogenesis warranting future studies.

\section{Oral Presentation}

\section{EACPT- 1320}

Evidence of metabolic interaction between opioid and benzodiazepine: a study with oxycodone, diazepam and its derivative, diclazepam.

R. Lawson ${ }^{*} 1,2$, E. Zarrouk ${ }^{1,2}$, P. Griffeuille ${ }^{2}$, J. Javellaud ${ }^{1,2}$, N. Picard ${ }^{1,}$ ${ }^{2}$, P. Marquet ${ }^{1,2}$, F. Saint-Marcoux ${ }^{1,2}$, S. El Balkhi ${ }^{2}$

${ }^{1}$ Faculty of Pharmacy, University of Limoges, ${ }^{2}$ Inserm, IPPRIT, CHU Limoges (U1248), Centre de Biologie et de Recherche en Santé (CBRS), Limoges, France

Drug-drug interactions, Misuse of medicines/substances, Pharmacokinetics/ pharmacodynamics, Pharmacovigilance

Introduction: Overdose deaths and polydrug abuse frequently involve opioids and benzodiazepines (BZD). Efforts made to roll back this public health problem are inefficient due to the availability of designer drugs. Designer benzodiazepines (DBZD) are rapidly growing and commonly co-abused with opioids. Slight modification in the structure of BZD has a heavy impact on the pharmacokinetic profile and could also lead to important interactions especially in chronic intoxications. Additionally, little is known about metabolic interactions between opioid, BZD or DBZD.

Objectives: Our study aims to explore interactions in acute and chronic patterns between oxycodone (OXC), one of the most abused opioid, and diazepam (DZ) or, its derivative, diclazepam (DCZ), one of the most popular DBZD.

Methods: (i) To explore acute toxicity, concomitant single doses of OXC $(1.5 \mathrm{mg} / \mathrm{kg})$ with DZ $(5.0 \mathrm{mg} / \mathrm{kg})$ or with DZC $(0.5 \mathrm{mg} / \mathrm{kg})$ were administered to Swiss mice. Blood concentrations of the drugs and their metabolites: nordazepam, temazepam, oxazepam for DZ; lormetazepam, lorazepam for DCZ; noroxycodone (main metabolite) and oxymorphone (minor metabolite) for OXC were measured at $0.5,1.5$ and $3 \mathrm{~h}$ (LC-MSMS method). (ii) To mimic chronic intoxication with a DBZD, DCZ was introduced on day 10 to mice under OXC continuously infused. Drugs and metabolites were measured in blood and urines on day 15. For both protocols motor coordination and pain tests were performed.

Results: In acute condition, we observed that DZ and DCZ were respectively able to inhibit OXC metabolism. Noroxycodone/OXC ratio significantly decreased at $3 \mathrm{~h}$ from $19.29 \pm 8.02$ in presence of OXC to $11.07 \pm$ 7.14 in presence of $\mathrm{OXC}+\mathrm{DZ}$ and to $3.70 \pm 1.80$ in presence of $\mathrm{OXC}+$ DCZ ( $<<0.05$, ANOVA). OXC has no impact on both DZ and DCZ metabolism. In the chronic condition, we observed that DCZ enhanced the accumulation of oxymorphone (oxymorphone/OXC ratio with $\mathrm{DCZ}=$ $0.13 \pm 0.04$ vs 0 without DCZ).

Summary / Conclusions: We demonstrated that DZ and DCZ interact with the metabolism pathways of OXC. An acute dose of DZ or DCZ increases the accumulation of OXC in blood while in chronic condition, DCZ promotes the production of oxymorphone, rather than noroxycodone. This could be due to a metabolic shunt leading to a more potent and toxic metabolite. These results support that overdoses to opioids in the presence of a BZD may be partly due to metabolic interactions.

\section{EACPT-1362}

High-dose esomeprazole causes a strong and prolonged mechanismbased inhibition of cyp2c19 while only slightly inhibiting cyp3a4 and inducing cyp1 12 activities in vivo

T. Kaartinen ${ }^{*}{ }^{1,2}$, A. Tornio ${ }^{1,2}$, T. Launiainen ${ }^{1}$, T. Tapaninen ${ }^{1}$, M. $\mathrm{Niemi}^{1,2}$, J. Backman ${ }^{1,2}$

${ }^{1}$ Clinical Pharmacology, University of Helsinki and Helsinki University Hospital, ${ }^{2}$ Individualized Drug Therapy Research Program, Faculty of Medicine, University of Helsinki, Helsinki, Finland 
Drug-drug interactions, Pharmacokinetics/ pharmacodynamics

Introduction: Based on previous in vitro studies, esomeprazole, the Senantiomer of omeprazole is a mechanism-based inhibitor of CYP2C19 but in vivo data concerning its effects on CYP enzymes are limited.

Objectives: This study investigated the effects of high-dose esomeprazole on CYP1A2, 2C19 and 3A4 in healthy volunteers using pantoprazole, midazolam and caffeine as probe drugs, respectively. Moreover, this study characterized the suitability of R-pantoprazole as a sensitive in vivo index substrate for CYP2C19 activity.

Methods: In a 5-phase study, 10 healthy volunteers ingested $20 \mathrm{mg}$ pantoprazole, $50 \mathrm{mg}$ caffeine, and $0.5 \mathrm{mg}$ midazolam before and 1 , 25 , and 73 hours and $20 \mathrm{mg}$ pantoprazole 49 hours after the last dose of a 7-day pretreatment with $80 \mathrm{mg}$ esomeprazole twice daily. In each phase, blood samples were collected for 7 hours after ingestion of the study drugs. The plasma concentrations of the drugs and their metabolites were determined with liquid chromatography/ tandem mass spectrometry. The turnover half-life of CYP2C19 was estimated by measuring the recovery of the $\mathrm{CL} / \mathrm{F}$ of $\mathrm{R}$ pantoprazole.

Results: When administered 1 hour after the last dose of esomeprazole, the AUC of R-pantoprazole was increased 4.9-fold $(\mathrm{P}<0.001)$ and a significant but lower increase was observed even after 73 hours $(\mathrm{P}<0.05)$. However, individual increases in the AUC of Rpantoprazole were more than 5-fold in most normal and rapid CYP2C19 metabolizers suggesting a strong mechanism-based inhibition of CYP2C19. Esomeprazole increased the AUC of midazolam up to 1.4-fold $(\mathrm{P}<0.005)$ and the paraxanthine/caffeine ratio up to 1.2 -fold $(\mathrm{P}<0.05)$ indicating a slight inhibition of CYP3A4 and induction of CYP1A2, respectively. The estimate of the turnover half-life of CYP2C19 was approximately 46 hours.

Summary / Conclusions: In conclusion, high-dose omeprazole causes a strong and prolonged inhibition of CYP2C19 which lasts for at least 3 days and thus concomitant use of drugs metabolized by CYP2C19 should be considered cautiously. In addition, this result reinforced the previous findings that especially the R-enantiomer of pantoprazole is a sensitive marker substrate for CYP2C19 activity. The turnover estimate will be a useful tool for future in vitro-in vivo extrapolations and PBPK modelling.

\section{EACPT-1393}

In vitro inhibition of twenty three anti-tuberculosis drugs on six ugt enzymes

M.-J. Kim ${ }^{1,2}$, J.-G. Ha ${ }^{1}$, S.-E. Yoo ${ }^{1,2}$, H.-S. Kim ${ }^{*}$, D.-H. Kim ${ }^{1,2}$, J.-G. Shin ${ }^{1,2}$

${ }_{1}^{1} 1$ Department of Pharmacology and Clinical Pharmacology, PharmacoGenomics Research Center, ${ }^{2} 2$ Center for Personalized Precision Medicine of Tuberculosis, Inje University College of Medicine, Busan, Korea, Republic Of

\section{Drug-drug interactions}

Introduction: Anti-tuberculosis (anti-TB) drugs are well known to have drug-drug interaction potentials caused by cytochrome P450 enzymes. There is a lack of information on UDP-glucuronosyltransferase enzymes.

Objectives: The aim of this study was to evaluate the inhibitory potentials of twenty three anti-TB drugs on the six human hepatic UGT isozymes (UGT1A1, 1A3, 1A4, 1A6, 1A9, and 2B7) in vitro and to predict the potential for drug-drug interactions in vivo.

Methods: Inhibition of UGT isoform activities by anti-TB drugs was determined using in vitro UGT cocktail developed by us. Twenty three anti-TB drugs were simultaneously incubated and analyzed with six UGT isoform probe substrates.

Results: Clofazimine strongly inhibited UGT1A1-mediated estradiol-3glucuronidation. Rifabutin and rifapentine strongly inhibited UGT1A4- mediated trifluoperazine glucuronidation. The $I C_{50}$ value for clofazimine on UGT1A1 was $3.3 \mu \mathrm{M}$ and the $I C_{50}$ value of rifabutin and rifapentine on UGT1A4 were 2.6 and $6.5 \mu \mathrm{M}$, respectively. However, other anti-TB drugs did not show inhibitory effects on six UGT enzymes. Kinetic analysis revealed that clofazimine, rifabutin and rifapentine displayed competitive inhibition of UGT activities with $K_{i}$ values of 1.7, 1.5, and 5.5 $\mathrm{mM}$, respectively. Based on these values, the potential of in vivo inhibition of human hepatic UGT were predicted using the $R$-value $((1+(\mathrm{fu} \mathrm{x}$ $\left.[\mathrm{I}]_{\mathrm{in}, \max } / K_{i}\right)$ ). The $R$-values for the combination of $200 \mathrm{mg}$ clofazimine, $600 \mathrm{mg}$ rifabutin and $600 \mathrm{mg}$ rifapentine were estimated to be $1.01,5.58$ and 1.33 , respectively.

Summary / Conclusions: Our results suggest that rifabutin may cause clinically relevant drug interactions with co-administered drugs metabolized by UGT1A4.

Oral Presentation

\section{EACPT-1397}

Development and validation of a novel high-throughput double cocktail assay for in vitro evaluation of time-dependent inhibition of nine major human cytochrome p450 enzymes

H. Kahma ${ }^{1,2}$, L. Aurinsalo*1, 2, M. Neuvonen ${ }^{1,2}$, J. Viinamäki ${ }^{1,2}$, T. Launiainen $^{1,2}$, A. M. Filppula ${ }^{1,2}$, A. Tornio ${ }^{1,2}$, J. T. Backman ${ }^{1,2}$

${ }^{1}$ Individualized Drug Therapy Research Program, Faculty of Medicine, University of Helsinki, ${ }^{2}$ Department of Clinical Pharmacology, University of Helsinki and Helsinki University Hospital, Helsinki, Finland

Drug-drug interactions

Introduction: Inhibition of human cytochrome P450 (CYP) enzymes is a common cause of pharmacokinetic drug-drug interactions. In recent years, multiple cocktail methods have been developed to assess the inhibition of several CYP enzymes in a single assay. However, the majority of CYP cocktail assays have been developed for detecting direct inhibition, whereas few cocktails have been established for screening of the often more harmful time-dependent inhibition.

Objectives: The aim was to develop a reliable in vitro high-throughput cocktail assay with optimized incubation conditions for screening timedependent inhibition of the nine most important drug-metabolizing CYP enzymes.

Methods: The following probe substrates were selected: tacrine (CYP1A2), coumarin (CYP2A6), bupropion (CYP2B6), amodiaquine (CYP2C8), tolbutamide (CYP2C9), omeprazole (CYP2C19), dextromethorphan (CYP2D6), astemizole (CYP2J2), midazolam (CYP3A4) and omeprazole (CYP3A4). In vitro incubations with pooled human liver microsomes were conducted in 96-well plates using a Tecan Freedom Evo 150 automated liquid handler. Metabolite concentrations were quantified using UPLC-MS/MS.

Results: Based on preliminary interaction assays, the substrates were divided into two cocktails to minimize interactions between the substrates: $5 \mu \mathrm{M}$ tacrine, $50 \mu \mathrm{M}$ bupropion, $2 \mu \mathrm{M}$ amodiaquine, $100 \mu \mathrm{M}$ tolbutamide and $2 \mu \mathrm{M}$ midazolam in cocktail 1 , and $1 \mu \mathrm{M}$ coumarin, $0.3 \mu \mathrm{M}$ astemizole, $20 \mu \mathrm{M}$ omeprazole and $5 \mu \mathrm{M}$ dextromethorphan in cocktail 2. Two separate UPLC-MS/ MS quantitation methods were optimized for the cocktails with no interference between analytes. The metabolite formation of all probe substrates was linear with protein concentrations up to $0.1 \mathrm{mg} / \mathrm{ml}$ and with incubation times up to $5 \mathrm{~min}$. Substrate depletion was less than $20 \%$ for all analytes at 5 minutes. A preincubation time of 30 minutes, an incubation time of 5 minutes and a protein concentration of $0.05 \mathrm{mg} / \mathrm{ml}$ were selected for both cocktails. In further validation experiments, the CYP activities of the probe reactions were identical (difference $<30 \%$ ) in cocktail and single substrate assays. Summary / Conclusions: A rapid and sensitive in vitro double cocktail assay was developed and optimized for high-throughput screening of time-dependent inhibition of the nine most important drug-metabolizing CYP enzymes. 
EACPT-1455

Clinically relevant drug interactions; it's time for education R. Verbeek ${ }^{*} 1$, M. Richir ${ }^{1}$, J. Tichelaar $^{1}$, M. van Agtmael ${ }^{1}$

${ }^{1}$ Internal medicine, section pharmacotherapy, Amsterdam UMC, VUmc, Amsterdam, Netherlands

\section{Drug-drug interactions, Education}

Introduction: A large European cross-sectional study has shown that final-year students lack sufficient knowledge and confidence to perform their task as prescribers of medication. In particular, their knowledge of important contraindications and drug-drug interactions scored significantly worse than their knowledge in other areas of expertise such as mechanism of action and side effects. Also with regard to their skills, checking for contraindications and drug-drug interactions proved difficult for the final-year students. In a previous study of self-rated competence, it was shown that medical students felt insufficiently competent, especially in the area of checking for contraindications and drug-drug interactions. A possible explanation for this is that medical education and study material does not pay sufficient attention to important drug interactions and contraindications. The aim of this study was to create a list of the most relevant drug-drug interactions that every medical student should know. In addition we develop visual educational materials that every teacher in Europe can use in their education to teach these essential interactions.

Objectives:

Methods:

Results: .

Summary / Conclusions: .

\section{Drug regulation}

\section{EACPT-1186}

Interchangeability of biologicals from a regulatory standpoint: european perspective and croatian experience

D. Juricic Nahal ${ }^{*} 1$, S. Arapovic Dzakula ${ }^{1}$

${ }^{1}$ Agency for Medicinal Products and Medical Devices of Croatia, Zagreb, Croatia

\section{Drug regulation}

Introduction: Biosimilars are highly similar to their reference biologic medicines in terms of structure, efficacy, safety and immunogenicity. While scientific evaluation of biosimilars in EU is done by European Medicines Agency (EMA), interchangeability of biologicals is regulated at national level. The first recommendation issued by Croatian national competent authority (HALMED) in 2014 was rather conservative. In June 2018 the recommendation was updated to support interchangeability by switch under physician surveillance.

Objectives: To review recommendations on interchangeability of biologicals in EU countries and compare them to the updated Croatian recommendation.

To compare individual case safety reports (ICSR) reported for 3 biologic medicines before and after changing the recommendation in Croatia.

Methods: Relevant webpages of all EU member states were reviewed and available recommendations analysed.

VigiLyze database was searched for ICSRs to 3 biologic medicines and their biosimilars (infliximab, etanercept and adalimumab) from 1 January 2018 to 27 June 2018 (first period) and from 28 June 2018 to 31 December 2018 (second period) for Croatia. Details and completeness scores of ICSRs were compared.

Results: Available recommendations in EU are highly in line with the updated Croatian recommendation.

Number of ICSRs increased (infliximab: 20 vs 24 cases; etanercept 12 vs 14 cases; adalimumab 12 vs 20 cases) in the second period. Percentage of serious ADRs and completeness scores increased. MedDRA preferred terms (PT) were comparable for all serious ADRs in both periods.
Summary / Conclusions: Supporting interchangeability of biologicals prompted discussions in Croatian healthcare community regarding safety of the switch from originator to biosimilar and vice versa. It is thus unsurprising that the number of ICSRs to biologicals increased. Although the percentage of serious cases also increased, no significant differences in PTs were observed. Serious ADRs increased for reference medicines or biosimilar depending on the product, with no clear tendencies. Better quality of reports suggests vigilant surveillance of patients. In conclusion, despite enhanced public focus and increased ADR reporting, no significant differences were observed in the first 6 months after supporting interchangeability of biologicals in Croatia.

\section{EACPT-1281}

Medicines shortages in montenegrin hospitals during one-year period - a reason for new solutions for health authorities?

N. Duborija-Kovacevic ${ }^{*} 1$, T. Bochenek ${ }^{2}$, B. Asanin ${ }^{3}$, I. Huys ${ }^{4}$, M. Popovic $^{1}$, B. Sahmanovic ${ }^{1}$

${ }^{1}$ Department of Pharmacology and Clinical pharmacology, University of Montenegro, Medical Faculty, Podgorica, Montenegro, ${ }^{2}$ Jagiellonian University, Institute of Public Health, Department of Drug Management, Krakow, Poland, ${ }^{3}$ Department of Medical Ethics, University of Montenegro, Medical Faculty, Podgorica, Montenegro, ${ }^{4}$ Department of Pharmaceutical and Pharmacological sciences, Katholieke Universiteit Leuven, Leuven, Belgium

\section{Drug regulation}

Introduction: Medicines shortages (MS) in hospitals are a global increasing problem with potential serious consequences for patients, health-care practitioners and health system at all.

Objectives: The aim of this paper was to examine for the first time the type and dynamics of MS in Montenegrin hospitals over a one-year period and based on the obtained results, assess the need for new solutions in this area.

Methods: This research was carried out as a part of the two-year national scientific project on MS (DRUG-SHORT-MONT) that relies on a wider four-year European COST Action 15105 (2016-2020). After receiving the approval of the Ethics Committee of the Faculty of Medicine University of Montenegro, data on MS in 2018 were obtained through semi-structured interviews with the heads of pharmacies in all secondary and tertiary level state-owned general hospitals in Montenegro $(\mathrm{N}=8)$. The interviews were conducted in the period December 2018-February 2019 at the workplace of the respondents, after signing the Informed consent. Descriptive statistics was performed to process and show obtained results.

Results: The average lenght of working experience of interviewees was 16.4 years, all of them were females. All respondents faced with MS at their workplace in the last 12 months. Three quarters of the respondents said they were exposed to MS every month and more often during 2018. The half of them reported the average MS duration of 7-30 days. The most commonly affected by MS were medicines for blood and blood forming organs (ATC group B, led by enoxaparin injection), drugs for digestive tract and metabolism (ATC group A, led by bisacodil coated tablet and suppositoty), and the third place share anti-infectives for systemic use (ATC group J, led by benzylpenicillin injection) and drugs for nervous system (ATC group $\mathrm{N}$, led by petidin injection). The majority of reported drugs were contained in the national Essential list of drugs.

Summary/Conclusions: Given the apparent phenomenon of MS in Montenegrin hospitals and possible serious consequences, it is clear that new solutions at different levels (regulatory, educational, staffing, organizational) have to be carried out by health authorities.

This research was financially supported by the Ministry of Science of Montenegro. 
EACPT-1319

The journey of a centrally authorised medicine: step by step R. Salas ${ }^{*}$, E. González-Rojano ${ }^{1}$, L. Laredo ${ }^{1}$, A. Portolés ${ }^{1}$, E. Vargas ${ }^{1}$ ${ }^{1}$ Clinical Pharmacology, Hospital Clínico San Carlos, Madrid, Spain Drug regulation

Introduction: The European Medicines Agency (EMA) is responsible for the scientific evaluation of centralised marketing authorisation applications. The Committee for Medicinal Products for Human Use (CHMP) is in charge of the scientific evaluation of new medicines. The Agencia Española de Medicamentos y Productos Sanitarios (AEMPS) is the body within the Spanish Ministry of Health and Social Policy, which guarantees quality, safety, efficacy and accurate information on medicines and medical devices marketed in Spain.

Objectives: To describe and analyze the authorization procedure for the marketing of new medicines by the EMA and AEMPS in the period June 2018 and January 2019.

Methods: This study was a retrospective review of published data corresponding to the European regulatory authorization of centrally approved between June 2018 and January 2019. The Highlights from the Committee for Medicinal Product for Human Use published by the EMA and AEMPS CIMA were consulted. All sources and data consulted are public.

Results: From June 2018 to January 2019, the CHMP adopted a positive opinion for 60 medicines via the Centralised Procedure. Forty-seven medications have the date of issue of the marketing authorization and twenty medications have been authorized by the AEMPS. The average time since of the date of issue of the positive opinion until marketing authorization in the European Union was 59 days, and the average time since marketing authorization until the authorization by the AEMPS was 52 days. Thirteen medicines have been approved for orphan indications and nine medicines for biosimilar. Overall, $23.4 \%$ of medicine authorised were recommended for approval for haematology/haemostaseology and $22.95 \%$ were recommended for cancer.

Summary / Conclusions: The CHMP issued a positive opinion of $98.4 \%$ (60) of the evaluated medicines, of which $78.3 \%$ (47) have received marketing authorization. The most frequent therapeutic areas were haematology/ haemostaseology and cancer. At the moment of review, in Spain, the AEMPS has only registered the authorization of $33.3 \%$ (20) and only one medicine has price of financing.

\section{EACPT-1326}

Impact of pharmacist interventions on clinical outcome and cost avoidance in a university teaching hospital

J.-P. Jourdan* 1, 2 , A. Muzard ${ }^{3}$, I. Goyer ${ }^{2}$, V. Hamon Poupinel ${ }^{4}$, L. Peyro Saint Paul ${ }^{5}$, Y. Ollivier ${ }^{6}$, Y. Oulkhouir ${ }^{7}$, P. Henri ${ }^{8}$, J.-J. Parienti ${ }^{5}$, C. Breuil $^{2}$

${ }^{1}$ Centre d'Etudes et de Recherche sur le Médicament de Normandie, CERMN, Normandie Université, UNICAEN, ${ }^{2}$ Pharmacy, CHU de CAEN, ${ }^{3}$ Pharmacy, CAEN University teaching hospital, ${ }^{4}$ Medical Information department, ${ }^{5}$ Clinical Research and Innovation Division, ${ }^{6}$ Internal Medecine, ${ }^{7}$ Chest Medecine, ${ }^{8}$ Renal Disease Teaching Center, CHU de CAEN, CAEN, France

Drug regulation, Drug utilization, Drug-drug interactions, Health economy

Introduction: A significant number of clinical pharmacy services have shown to improve in-hospital medication safety and patient outcome. Prescription review and pharmacist interventions are a fundamental part of hospital clinical pharmacy activities. In a context of restricted financial resources, proving the economic and clinical impact of this activity seems necessary.

Objectives: The aim of this study was to assess the clinical impact on patient outcomes and economic benefit of prescription review by pharmacists. The study takes place in a 1624-bed tertiary French university teaching hospital.
Methods: It's a prospective single center study evaluating prescriptions for which a pharmacist intervention was issued over a 6-month period. The clinical impact of every pharmacist intervention was evaluated by a multidisciplinary experts committee. Economic benefit was evaluated from the public health care system spending standpoint.

Results: The Main outcome measure is the number of avoided hospitalization days and associated public health care system cost-avoidance. Prescription review and interventions by pharmacists prevented 73 intensive care unit hospitalization days, 74 continuous monitoring unit hospitalization days and 66 days of conventional hospitalization. $€ 252,294.00$ in public health expenditure were thus prevented. For every Euro invested in the prescription review activity, $€ 5.09$ of public health spending were potentially saved.

Summary / Conclusions: Our study shows that prescription review and clinical pharmacists' interventions had an impact on clinical outcomes which translated into prevented hospitalization days. Prescription optimization through pharmacist interventions allows significant health care cost savings which makes this service highly efficient.

\section{Oral Presentation \\ EACPT-1364}

Illegal use of anabolic androgenic steroids in sweden. A greater problem here than in the other nordic countries?

Y. Lood ${ }^{*}$, J. Ahlner ${ }^{1}$

${ }^{1}$ Department of Forensic Genetics and Forensic Toxicology, National Board of Forensic Medicine, Linköping, Sweden

\section{Drug regulation, Misuse of medicines/substances}

Introduction: Doping with anabolic androgenic steroids (AAS) have for a long time been a well-known problem in sports, but is now widespread in the society and has become a growing public health issue. Non-therapeutic use of AAS is in Sweden prohibited since 1999. In Sweden, testosterone is the only AAS used clinically, in treatment of male hypogonadism. There is no reliable data available on the prevalence of AAS misuse, at least 10,000 users and even up to 100,000 individuals have been estimated to have used AAS in Sweden, among a population of 10 million. In the USA, between 1 million and 3 million people are thought to have misused AAS. There has however been suggestions, that the prevalence of AAS use is higher in the Nordic countries compared to most other parts of the world. Interestingly the legislation is different in the five Nordic countries.

There is evidence connecting long-term AAS use with criminality and several physical and psychological disorders as well as mortality. AAS misusers administer supra-physiological doses of AAS, often in combination with other drugs of abuse. Despite this risk of serious adverse effects, AAS seem to be one of the least studied drugs of the world's major abused drugs.

Objectives: In this study we investigate the findings of AAS in doping cases in Sweden and compare the legislation of AAS between the Nordic countries. Methods: We retrieve information from the National Board of Forensic Medicine's database. All cases of suspected doping offence between 2010 and 2018 were identified $(\mathrm{N}=10735)$, and the AAS positive cases were then selected ( $\mathrm{N}=3636)$.

Results: We will present the toxicological findings in the forensic doping investigations in Sweden during the period 2010-2018. The situation and legislation of AAS use in the other Nordic countries will be discussed.

Summary / Conclusions: The use of doping agents is still legal in some of the Nordic countries. In Sweden a number of individuals are prosecuted each year for doping offenses.

\section{EACPT-1379}

Sustainable access to innovative medicines in cancer therapy in croatia

V. Erdeljic Turk ${ }^{*} 1$

${ }^{1}$ Division of Clinical Pharmacology, University Hospital Zagreb, Zagreb, Croatia 


\section{Drug regulation, Health economy}

Introduction: Novel immunotherapies as a new modality in addition to chemotherapy, hormonal therapies and targeted agents are creating new opportunities for patients but also an immense challenge for payers. With growing prices of novel oncologic therapies there is a strong perception that budgetary impact for payers will be unaffordable in the future. New cancer drugs are traded at an international market, and while the absolute price per unit is similar, the relative price is higher for countries with lower income.

Objectives: To propose mechanisms for ensuring an optimal, sustainable and value-based access to innovation in cancer therapy.

Methods: In this work, various currently employed and recommended mechanisms for sustainable access to innovative cancer medicines suitable for Croatia and similar countries will be addressed, including managed entry agreements, use of local HTA, different payment mechanisms, differential pricing, joint procurement agreements, and conditional reimbursement corrected based on clinical research or real-world evidence.

Results: Croatia has a mandatory health insurance system, with the Croatian Health Insurance Fund (CHIF) being the sole insurer and the main purchaser of health services. At $7.4 \%$ of GDP, Croatia's current health expenditure is much lower than the EU average of $9.9 \%$, translating to EUR 1241 per capita (adjusted for differences in purchasing power). With comparable incidence rates, mortality rates from lung, breast and colorectal cancer are among the highest in the EU. Indicative of poor efficiency, Croatia is among the EU Member States with the highest expenditure on medical goods (mainly pharmaceuticals) as a percentage of current health expenditure, amounting to $28.5 \%$, compared to an EU average of $18.5 \%$, with the highest expenditure on oncology drugs (21.5\%).

Summary / Conclusions: The annual budgetary increases of the CHIIFs fund for particularly expensive drugs covering innovative treatments, worth 122 mil Eur in 2017, on its own is not a sustainable strategy without an adaptation of health care policy and payer decision making processes. Currently, managed entry agreements are in place, but lack of dialogue among stakeholders, limited availability of HTA, lack of accelerated or conditional reimbursement possibilities, coupled with limited collection of real-world data through disease registries are the main obstacles for rational allocation of limited healthcare resources in Croatia.

\section{EACPT-1395}

Experience in approving an eu-funded clinical trial with advanced therapy. Are we really ready for pan-european approval?

C. Payares-Herrera ${ }^{1}$, A. Velasco-Iglesias ${ }^{2}$, N. Padilla-Eguiluz ${ }^{3}$, E. Gomez-Barrena $^{3}$, I. Salcedo de Diego ${ }^{1}$, A. Sancho-Lopez ${ }^{1}$, B. RuizAntoran $^{1}$, C. Avendano-Sola ${ }^{1}$

${ }^{1}$ Clinical Pharmacology, Hospital Universitario Puerta de Hierro,

${ }^{2}$ Spanish Clinical Research Network (SCReN), Instituto de Investigación Sanitaria Puerta de Hierro-Segovia de Arana, Majadahonda (Madrid), ${ }^{3}$ Servicio de Cirugía Ortopédica y Traumatología, Hospital Universitario La Paz, Madrid, Spain

\section{Drug regulation}

Introduction: Regulation EU536/2014aims to harmonise CTapproval by implementing 1authorisation procedure \&assessment leading to a 1 decision valid for all countries. Current experience shows major issues that must be resolved in order to avoid the failure of this initiative

Objectives: To report hurdles for conducting multistateCTs that must be resolved before the CT-Regulation is fully applied

Methods: Regulatory case study:EU-funded, advanced therapy, multi-state, randomised, controlled CT(ORTHOUNION,EudraCT2015-000431-32)

Previously, the same investigators participated in 3CTs using the same IMP, manufactured by the same Facilities

A VHP was selected to facilitate authorizations (France,Germany,Italy\&Spain), optimize efforts of the non-commercial sponsor and to keep a commonIMPD
Results: VHP:From submission(09/01/17) to opinion(22/03/17) took 72days.Grounds for non-acceptance were: Quality (control clarifications, validations); Clinical (refinement of trial population, study design);Statistics (sample size,non-inferiority margin, endpoints)

The outcome was a divergent opinion:

- France\&Spain:The CT was approvable, subject to submission of additional quality documents and updating the protocol; national approvals were granted $18 \& 25$ May,respectively

- Germany\&Italy:The same issues were unsolvable, none were grounds for refusal under CTRegulation

All issues were addressed before national re-submissions

Italy:From submission(05/09/17) to approval(28/02/18) took176days. Clarification on clinical/ design aspects was requested, none of which had been raised during the VHP

Germany:From submission(25/10/17) to approval(28/05/18) took215days.New quality and statistics issues were identified which required new validations

Summary / Conclusions: The VHP divergent opinion was based on reasons not contemplated in the CT Regulation. It led to a 12-month delay in the CT initiation and bureaucratic burden increase. Commercial Sponsors apply their resources to circumvent these hurdles, with the drawback of increased CTcosts. For non-commercial sponsors the burden could be overwhelming, resulting in investigators investing a lot of time and putting the $\mathrm{CT}$ at risk

Based on ORTHOUNION experience, there is still work to be done to eliminate discrepancies among National Authorities. They must be urgently addressed so as to avoid unfounded CT refusals once the Regulation is fully applied. Otherwise, this initiative would be bound for failure

\section{EACPT-1432}

Antibiotic multiresistance: the reasons behind the phenomenon. Study on antibiotic knowledge in italy

F. Cori ${ }^{1}$, M. L. Garau ${ }^{*}$, E. A. Dho-Nagy ${ }^{1}$, Z. Kollo ${ }^{1}$, A. Brassai ${ }^{1}$, E. Gyongyi Ban ${ }^{1}$, V. Paradisi ${ }^{2}$

${ }^{1}$ pharmacology, UMFST Tg-Mures, Targu Mures, Romania, ${ }^{2}$ SIMG , Catania, Italy

Drug regulation, Drug utilization, Education, Misuse of medicines/ substances

Introduction: Multi-resistant bacteria are presenting themselves as the modern challenge. To fight a disease, we, as future doctors, need to understand the etiology; to fight multi-resistance we need to find the causes.

Objectives: The main goal of our study: to understand the knowledge background of our nation and define a strategy for the organization of workshops and apps to point the importance of the rational use of antibiotics. Methods: We performed an observational, cross-sectional, epidemiological study from June to August of 2018. This prospective study, at this moment takes in considerations 266 individuals from Southern Italy, from different social and geographical backgrounds. Each person participated to the study voluntarily, without any compensation.

The questionnaire was presented on the native language of the person (i.e. Italian) and was divided in two parts: demographic variables and antibiotic use and knowledge. The maximum achievable grade in the second part is 10 and grades equal or above 8 were considered as adequate knowledge. The variables taken into account are: age, sex, educational level, employment, marital status, presence of an healthcare professional in the family nucleus and number of visits per year to the family doctor. The collected data were processed using the Chi Square test.

Results: The calculi revealed us the categories that are more prone to make mistakes about the pathologies needing antibiotic use and on posology. The highest average grade (i.e. 8.41) was achieved on the age group from 25 to 34 years while the lowest (i.e. 7.68) was reported on the group with secondary level education. The overall average was 8.18 with a standard deviation of 1.202. An interesting fact was that the people with no healthcare professionals in the family achieved a higher grade compared to those who were 
related to medical personnel; this will be a good starting point for increasing the awareness about the importance of preventive medicine.

Summary / Conclusions: In conclusion, the average grade achieved barely the adequate threshold; definitely not enough for reducing antibiotic overuse. The time frame at our disposal is decreasing, so we are planning the organization of talk-active workshops inside the hospital setting and outside with the development of an app containing the major indications of the antibiotics prescribed.

\section{Drug utilization}

\section{EACPT-1003}

Rational use of methylprednisolone for treatment of immune mediated diseases: relationship between dosing, effectiveness and safety V. Dominguez ${ }^{*}$, A. Danza ${ }^{1}$, I. Borgia $^{2}$, C. Amigo ${ }^{1}$, A. Baccelli $^{2}$

${ }^{1}$ Spanish Association Hospital, ${ }^{2}$ Faculty of Medicine, Montevideo, Uruguay

\section{Drug utilization}

Introduction: Methylprednisolone (MP) has high anti-inflammatory potency, but the precise doses and its benefit/risk balance are still not clear in autoimmune diseases treatment.

Objectives: To assess the pattern of use of MP pulses in clinical practice Methods: Retrospective descriptive study of all the patients who received MP pulses for autoimmune mediated disease treatment in three Uruguayan hospitals from 2013 to 2015. Age, gender, immune mediated disease, comorbidities, concomitant treatment, MP dosing, clinical response and adverse reactions were analyzed.

Results: 164 cases were included. Mean age was 48.4 (SD: 18); 118 $(72 \%)$ were female. The average dose of MP was $3.35 \mathrm{~g}$ (SD 1.4) and it was administrated in a mean of 4 days (SD: 1.56). 92 (56.1\%) had immune mediated neurologic disease, $72(44 \%)$ other immune mediated diseases. Complete response occurred in $77(47 \%)$, partial response in $62(37.8 \%)$ and non- response in $25(15.2 \%) .42(25.6 \%)$ presented adverse reactions, only 6 were severe. Fifteen $(68 \%)$ patients treated with MP pulses $\leq 1.5 \mathrm{~g}, 38$ (43.7\%) treated with pulses between 1.5-3 g and 24 $(43 \%)$ treated with pulses $>3 \mathrm{~g}$ had complete responses ( $>>0.05)$.

Adverse reactions were reported in $14(25 \%)$ cases who received $>3 \mathrm{~g}, 21$ (24\%) who received pulses of $1.5-3 \mathrm{~g}$ and $7(32 \%)$ with pulses of $<1.5 \mathrm{~g}$. $(\mathrm{p}>0.05)$

Summary / Conclusions: No relationship was found between MP pulse dose and clinical response or safety concerns in immune mediated diseases. Prospective studies should be performed to add evidence regarding this issue

\section{EACPT-1100}

Typical drug treatment in an older patient in primary care - a descriptive study applying indicators of prescribing quality as well as an overall medical perspective

N. Parodi Lopez ${ }^{*} 1,2$, S. M. Wallerstedt ${ }^{1,3}$

${ }^{1}$ Department of Pharmacology, Sahlgrenska Academy, University of Gothenburg, ${ }^{2}$ Närhälsan Kungshöjd Primary Care, ${ }^{3}$ HTA-centrum, Sahlgrenska University Hospital, Gothenburg, Sweden

Drug utilization, Geriatric treatment

Introduction: Quality of drug treatment has been extensively studied, primarily using indicators of prescribing quality. However, evidence on the typical patient to encounter is lacking.

Objectives: To describe the modes of drug treatment in older patients in primary care according to indicators of prescribing quality, and an overall medical assessment taking individual considerations into account.

Methods: All patients $\geq 65$ years of age, attending a primary health care centre in Region Västra Götaland for a non-urgent physician consultation in January 2016 were included. The drug treatment was screened for Potentially Inappropriate Medications (PIMs) and Potential Prescribing Omissions (PPOs) using three sets of indicators of prescribing quality
(STOPP/START, EU(7)-PIM, Swedish National Board of Health and Welfare). Thereafter, the treatment was assessed, by a physician taking the patient's condition into account, as either appropriate or suboptimal. Results: A total of 123 patients were analysed (48\% female, median age: 76 years). The patients had 0 to 14 chronic drugs; mode: 5 . Up to 17 PIMs/PPOs were identified in a single individual; mode: 0 PIMs $(n=45)$, 2 PPOs ( $n=36), 2$ PIMs/PPOs $(n=28)$. When an overall medical perspective was applied and individual factors like morbidity, life expectancy, and concurrent drug treatment taken into account, the mode was to have appropriate drug treatment $(\mathrm{n}=78,63 \%)$. In patients with appropriate drug treatment, up to 7 PIMs and 6 PPOs were identified in a single individual; mode: 0 PIMs $(n=44), 2$ PPOs ( $n=26)$. In those with suboptimal treatment up to 11 PIMs and 8 PPOs were identified; mode: 4 PIMs ( $n=11), 1 / 2$ PPOs ( $\mathrm{n}=10$ each). The PIM most likely to encounter was inappropriate prescribing of a proton pump inhibitor $(\mathrm{n}=71)$. The PPO most likely to encounter was presence of one or more drugs for which dosage should be adjusted to renal function without calculated eGFR at least once a year $(n=102)$, the majority of these patients having a creatinine value recorded over this period $(\mathrm{n}=98)$.

Summary / Conclusions: For older people attending primary care, the patient most likely to encounter will have 5 chronic drugs, 2 PIMs/PPOs, and appropriate drug treatment from an overall medical perspective. PIMs and PPOs are frequent also among those with appropriate treatment.

\section{EACPT-1158}

Prescribing of newer antiepileptics, with the focus on pregabalin, using the defined daily dose methodology

I. Truter ${ }^{*} 1$

${ }^{1}$ Drug Utilization Research Unit (DURU), Department of Pharmacy, Nelson Mandela University, Port Elizabeth, South Africa

\section{Drug utilization}

Introduction: Various antiepileptic medicines have been approved for indications other than epilepsy, such as mood disorders and neuropathic pain. Pregabalin is registered in South Africa for post-herpetic neuralgia and painful diabetic polyneuropathy in adults, and it is increasingly prescribed for neuropathic pain, generalised anxiety disorder and epilepsy.

Objectives: The aim of the study was to analyse the prescribing patterns and cost of the newer antiepileptics, with the focus on pregabalin.

Methods: A retrospective drug utilisation study was conducted on a South African medical insurance administrator database for 2017. The database contained 3898535 records for medicine, medical devices and procedures. All products in ATC subgroup N03AX were analysed.

Results: A total of 4983 prescriptions for antiepileptics in ATC subgroup N03AX was prescribed at a total cost of R398 856 claimed. Six active ingredients were prescribed. Lamotrigine $(39.76 \%)$ was the most often prescribed, followed by pregabalin $(31.71 \%)$. Pregabalin was available in three dosage strengths, under only one trade name (no generic equivalents were prescribed). The $75 \mathrm{mg}$ dosage strength was the most popular (61.27\% of prescriptions), followed by $25 \mathrm{mg}$ (25.52\%) and $150 \mathrm{mg}$ (12.21\%). The ages of patients ranged from 9 to 95 years. The 308 patients who were prescribed pregabalin, received on average only three prescriptions (average $=3.11 ; \mathrm{SD}=3.82$ ) for pregabalin during the year. The DDD for pregabalin is $300 \mathrm{mg}$ (oral). The average Prescribed Daily Dose (PDD) of pregabalin was 76.32 (SD=94.48) $\mathrm{mg}$. Nearly half $(44.05 \%)$ of the prescriptions for pregabalin were for less than 28 capsules. The average PDD for prescriptions with 28 or more capsules was 124.26 ( $\mathrm{SD}=102.42) \mathrm{mg}$, which is still less than half of the DDD. The average PDD for female patients was 118.32 ( $\mathrm{SD}=113.10) \mathrm{mg}$ and for male patients $132.33(\mathrm{SD}=85.30) \mathrm{mg}$. No diagnoses were available.

Summary / Conclusions: The study confirmed the importance of including diagnoses in databases. There was a wide variation in the dosages of pregabalin, with dosages on average a quarter of the DDD. In the absence of diagnoses, the variation in dosages and the seemingly low average PDD cannot be explained. It is assumed that pregabalin was prescribed for different indications and hence the variability in PDDs. 


\section{Oral Presentation}

\section{EACPT-1202}

Antidepressants and anxiolytics/hypnotics used by children and adolescents in four european countries. A real world data prevalence study

A. Gomez-Lumbreras ${ }^{*}{ }^{1,2}$, A. Garcia Sangenis ${ }^{2,3}$, O. Prat Vallverdú ${ }^{2,3}$, C. Vedia Urgell ${ }^{4,5}$, R. Morros Pedros ${ }^{2,4}$

${ }^{1}$ Medical School, Universitat de Girona, Girona, ${ }^{2}$ Medicines Research Unit, IDIAPJGol, ${ }^{3}$ Universitat Autònoma de Barcelona, Barcelona, Spain, ${ }^{4}$ Farmacologia, de Terapèutica i de Toxicologia, Universitat Autònoma de Barcelona, ${ }^{5}$ Unitat de farmacia SAP Barcelona Nord i Maresme, ICS, Barcelona, Spain

\section{Drug utilization, Pediatric treatment, Psychopharmacology}

Introduction: The use of psychotropic medication in children has been barely studied.

Objectives: To describe the use of antidepressants (AD) and anxiolytics/ hypnotics (AX) in children $\leq 19$ years from Catalonia, Denmark, Norway and Sweden in the last decade.

Methods: Population-based cohort study in 2008-2017 in children $\leq 19$ years. Data sources: Catalonia: Information System for Research in Primary Care (SIDIAP), with information on prescribed drugs in primary care and their invoice in $80 \%$ of the Catalan population. Danish Health Data Authority (Medstat.dk, Denmark). Norwegian Institute of Public Health (Norwegian Prescription Database). Sweden National Board of Health and Welfare (Socialstyrelsen) statistics database. Nordics databases covered 95-99\% of the country population and contain data on dispensed drugs. Exposure: at least one prescription of a drug from the next ATC classification groups: N05B, N05C and N06A. The prevalence of use of AD and AX by group and drug is estimated by users per 1000 children per year $(\% \circ)$ and by group of age $(0-4 ; 5-9 ; 10-14 ; 15-19$, until 18 years in Catalonia and Denmark) and sex. Results: Norway and Sweden rose AD consumption (4.2\%o and $7.2 \%$ in 2008 to $5.9 \%$ and $13.7 \%$ in 2017 respectively); opposite to Denmark and Catalonia (3.0\%o and $3.7 \%$ in 2008 to $2.2 \%$ and $2.1 \%$ in 2017 respectively). AD were most consumed by females from 15-19 years old. Sertraline was the drug more used in 2017 in all countries, and the drug with the highest increase.

Nordic countries increased AX use. In Catalonia it decreased, despite having the highest consumption. Prevalence AX use for 2008 and 2017 was, respectively: Sweden $(10.4 \% o$ and $33.3 \%$ ) , Norway $(12.0 \%$ and $20.7 \%$ ) , Denmark (2.5\%o and 6.2\%o) and Catalonia (41.6\%o and $28.1 \%$ o). In 2017, hydroxyzine was the most consumed AX drug in Catalonia, mainly in 0-4 years old group (both sexes), while in Nordic countries it was melatonin, especially females older than 14 years old. Summary / Conclusions: The use of $\mathrm{AD}$ and $\mathrm{AX}$ has risen in children in the last decade. The pattern of use differs from the Nordic countries to Catalonia, especially the AX group; melatonin is an OTC drug in Catalonia, with no reimbursement, so no information about its use is available in SIDIAP. Hydroxyzine has the indication for pruritus which could explain its use in children 0-4 in Catalonia (atopic, allergic reactions...).

\section{EACPT-1217}

Assessing treatment adherence in adults with attention-deficit/hyperactivity disorder: a comparison of proportion of days covered for methylphenidate with or without antidepressant therapy

\section{J. Regnart ${ }^{*}$, I. Truter ${ }^{1}$}

${ }^{1}$ Drug Utilization Research Unit (DURU), Department of Pharmacy, Nelson Mandela University, Port Elizabeth, South Africa

\section{Drug utilization, Psychopharmacology}

Introduction: Attention-Deficit/Hyperactivity Disorder (ADHD) is a neurodevelopmental condition presenting in childhood which can persist with diagnostically significant impairment into adulthood.
First-line treatment is pharmacological management, however, its success rate in adults is often poor. It is estimated that half of all patients fail to adhere to treatment guidelines or prematurely discontinue therapy. Suggested reasons vary from social stigma to drug tolerability and perceived efficacy. Assessment of treatment adherence may be challenging due to the potential to discontinuously use stimulants.

Depression may present co-morbidly in $30-50 \%$ of adults with ADHD, treatment of which with antidepressants should not be interrupted due to the possibility of abrupt withdrawal resulting in antidepressant discontinuation syndrome.

Objectives: To assess if methylphenidate therapy adherence is modified by concurrent or historical antidepressant therapy.

Methods: A retrospective, longitudinal pharmacoepidemiological study was conducted on community pharmacy dispensing records for 20122016 for all patients between the ages of 18 and 40 years who had any record of receiving a drug classified as per Monthly Index of Medical Specialities (MIMS) as "1.1.3" between January and December 2015. Patients endorsed with an ADHD-linked diagnostic code were identified and a Proportion of Days Covered (PDC) analysis done on their ADHD and/or depression treatment drugs.

Results: Fifty patients were identified, 23 women (46.0\%) and 27 men $(54.0 \%)$. Receipt of both methylphenidate and antidepressant therapy was identified in $42.0 \%$ of patients. The average PDC was higher for antidepressant therapy than for methylphenidate: $68.7 \%$ versus $57.8 \%$, with females generally showing better drug adherence than males at $63.1 \%$ versus $58.9 \%$. Higher PDC results were identified for methylphenidate therapy in patients of both genders wherein concurrent or historical antidepressant therapy was observed: $63.5 \%$ versus $53.6 \%$.

Summary / Conclusions: Pharmacological management of complex clinical presentations is challenging, especially where treatment adherence is a known problem. This study may help to identify factors associated with improved methylphenidate treatment adherence to help guide further studies to improve treatment recommendations.

\section{EACPT-1253}

Indications related to antidepressant drug prescribing in the elderly population during 2010-2015 in two primary care databases: the netherlands and catalonia (spain)

L. F. Camacho Arteaga ${ }^{*}$, H. Gardarsdottir ${ }^{2}$, P. Souverein ${ }^{2}$, L. van Dijk ${ }^{3}$, M. Sabaté ${ }^{1}, X$. Vidal ${ }^{1}$, E. Ballarín ${ }^{1}$, L. Ibañez ${ }^{1}$

${ }^{1}$ Clinical Pharmacology Department, Vall d'Hebron University Hospital, Barcelona, Spain, ${ }^{2}$ Division of Pharmacoepidemiology and Clinical Pharmacology, Utrecht Institute for Pharmaceutical Sciences , ${ }^{3}$ Netherlands Institute for Health Services Research, Utrecht, Netherlands

\section{Drug utilization}

Introduction: Antidepressant drug consumption in 18 European countries doubled between 2000 and 2012. Increase in the use of antidepressant drugs includes indications other than depression, some studies showed that prevalence of prescription is higher in the elder.

Objectives: To describe the indications related to new antidepressant drug prescriptions in two primary care databases from The Netherlands and Catalonia 2010-2015.

Methods: A retrospective descriptive study was conducted, including new antidepressant users (excluding IMAO inhibitors) aged $\geq 65$ years. Data was retrieved from the Dutch NIVEL Primary Care Database and SIDIAP (Information System for Research in Primary Care from Catalan Institute of Health) databases. Primary outcome was concurrent diagnoses for antidepressant prescribing as a proxy of indication, including: depression, anxiety, sleep disorders, migraine/headache, neuropathic pain, fibromyalgia, other mental and behavioral disorders or other diagnoses. Results were stratified by age and gender.

Results: A total of 16537 and 199168 new antidepressant users were identified in NIVEL-PCD and SIDIAP, respectively during the study period. Women 65-69 years old prevailed in both databases (22\% and $26.5 \%$, 
respectively). Depression was the most frequent active diagnosis in both databases during the study period ( $24 \%$ and $31 \%$, respectively). Tricyclic antidepressants were the most commonly prescribed (48.7\%) in NIVELPCD mainly for neuropathic pain and selective serotonin reuptake inhibitors in SIDIAP (63.1\%) mainly for depression. More details result below:

\begin{tabular}{lllll}
\hline & NIVEL(\%) & & SIDIAP(\%) \\
& 2010 & 2015 & 2010 & 2015 \\
Total users & 613 & 4715 & 33099 & 31444 \\
Indications & & & & \\
Depression & 28.5 & 21.9 & 27.7 & 32.9 \\
Anxiety & 12.7 & 11.2 & 14.2 & 25.6 \\
Sleep disorders & 12.4 & 8.1 & 23.5 & 28.5 \\
Neuropathic pain & 9.6 & 6.8 & 0.6 & 1.8 \\
Migraine/headache & 5.2 & 4.5 & 3 & 4.2 \\
$\begin{array}{l}\text { Fibromyalgia } \\
\text { Other mental and }\end{array} \quad 2$ & 3.6 & 0.8 & 1.3 \\
$\quad$ behavioral disorders & 3.9 & 2.5 & 3.4 & 6.8 \\
Other diagnoses, without & 42.6 & 50.9 & 48.6 & 29.5 \\
$\quad$ chosen diagnoses & & & & \\
\hline
\end{tabular}

Summary / Conclusions: Depression is the most common diagnosis related to antidepressant prescribing in both countries. Anxiety and Sleep disorders are other associated diagnoses which should be prescribed with caution in the elder.

\section{EACPT-1258}

Factors of importance for discontinuation of thiazides associated with hyponatremia in sweden - a population-based register study C. Fahlén Bergh ${ }^{*}{ }^{1}$, S. Toivanen ${ }^{2}$, K. Johnell ${ }^{3}$, J. Calissendorff ${ }^{4}, \mathrm{~J}$. Skov $^{5}$, H. Falhammar ${ }^{4}$, D. Nathanson ${ }^{4}$, J. D. Lindh ${ }^{6}$, B. Mannheimer ${ }^{1}$ ${ }^{1}$ Department of Clinical Science and Education, Södersjukhuset, Karolinska Institutet, ${ }^{2}$ Department of Public Health Sciences, Stockholm University, ${ }^{3}$ Department of Neurobiology, Care Sciences and Society, Aging Research Center, ${ }^{4}$ Department of Molecular Medicine and Surgery and Department of Endocrinology, Metabolism and Diabetes, ${ }^{5}$ Department of Molecular Medicine and Surgery, ${ }^{6}$ Department of Laboratory Medicine, Division of Clinical Pharmacology, Karolinska Institutet, Stockholm, Sweden

\section{Drug utilization}

Introduction: Hyponatremia is the most frequently encountered electrolyte disorder in health care. In a patient with clinically significant hyponatremia without other clear causes, thiazide treatment should be replaced with another drug. Data describing to which extent this is being done is scarce.

Objectives: The aim of this study was to investigate sociodemographic and socioeconomic factors that may be of importance for the withdrawal of thiazide diuretics in patients hospitalized due to hyponatremia.

Methods: The study population was sampled from a case-control study investiganting individuals hospitalized with a main diagnosis of hyponatremia. For every case, 4 matched controls were included. In the present study cases $(n=5204)$ and controls $(n=7425)$ that had been dispensed a thiazide diuretic prior to index date were identified and followed onward regarding further dispensations. To investigate the influence of socioeconomic and sociodemographic facotrs, multiple logistic regression was used. Results: The crude prevalence of thiazide withdrawal for cases and controls was $71.9 \%$ and $10.8 \%$ respectively. Thiazide diuretics were more often withdrawn in medium-sized towns (adjusted OR 1.52, 95\% CI 1.21-1.90) and rural areas (aOR 1.81, 95\% CI 1.40-2.34) compared to metropolitan areas, and less so among divorced (aOR $0.72,95 \%$ CI $0.53-0.97$. However, education, employment status, income, age, country of birth and gender did not influence withdrawal of thiazides among patients with hyponatremia.

Summary / Conclusions: Thiazide diuretics were discontinued in almost 3 out of 4 patients hospitalized due to hyponatremia. Thiazide diuretics were more often withdrawn in medium-sized towns and rural areas compared to metropolitan areas, and less so among divorced. However, the remaining investigated factors did not influence withdrawal.

\section{EACPT-1270}

Assessment of drug use management good practices in inpatient medical organizations in astana (kazakhstan)

G. Zhussupova $^{*}{ }^{1}$, S. Zhaldybaeva ${ }^{1}$, A. Ramazanova ${ }^{1}$, D. Utepova ${ }^{1}$

${ }^{1}$ RSE ON REM "RCHD" OF THE MOH RK, Astana, Kazakhstan

Drug utilization, Therapeutic drug monitoring

Introduction: More than $50 \%$ of the drugs in the world are prescribed, dispensed or sold irrationally, half of the patients don't use them properly, and a third of the world's population don't have access to the necessary medicines

Objectives: Assessment of the drug use management system in the medical organizations of Astana at the hospital level

Methods: Retrospective research method and taking into account World Health Organization (hereinafter - WHO) recommendations drug use management good practicesin health care organizationsevaluatingmethodology. Taking into account WHO recommendations drug use management good practices in health organizations evaluatingmethodology involves the use of 58 compliance indicators for the overall assessment and for the drug use stages: selection, purchase, distribution, use. The calculation and assessment of compliance indicators were made on a 5 -score scale by determining the percentage of the maximum points number - 290 (100\%):

90-100\%- excellent;

$75-89 \%$ - good;

$50-74 \%$ - satisfactorily;

$<50 \%$ - unsatisfactorily

Results: The obtained data showed an average value of drug use management effectiveness in the study objects, equal to $74.6 \%$ - "satisfactorily". Medical organizations' drug formulary includes the drugs with unproven clinical efficacy. There is a problem of access to reliable sources of drug information. As a result of the calculations of compliance indicators, the maximum number of points was 245 , the minimum was 174 , and the average number was 216.3 points, which corresponds to $74.6 \%$. According to the overall indicator "The presence of a policy that determines the algorithm of drug movement at all stages of their use" 3 of 6 study objects received maximum points due to developed by them standard operating procedures of the drug use management. Regarding the drug consumption assessment in medical organizations, there was revealed the absence of specially organized services for drug use monitoring

Summary / Conclusions: The most common types of irrational use of drugs are:polypragmasy, irrational use of antibacterial and injectable drugs, inconsistency of pharmacotherapy with clinical guidelines, selftreatment. The success of therapy and the rational use of medicines depends not only on the doctor, but also on the coordinated work of all parts of health organization, including pharmacy and nursing staff.

\section{EACPT- 1280}

A lc-ms method for the measurement of about 250 compounds of interest in toxicology with a fully-automated sample preparation T. Robin ${ }^{1}$, S. Moreau ${ }^{*}$, F. Saint-Marcoux ${ }^{1}$ ${ }^{1} \mathrm{CHU}$ of Limoges, Limoges, France, ${ }^{2}$ Shimadzu, Dusseldorf, Germany

\section{Drug utilization, Other}

Introduction: For screening of drugs two steps are needed: an analysis for identification and another for the measurement of detected compounds. At both stages, manual multiple-step extraction procedures are usually used before LC-MS analysis. 
Objectives: Our objectives are to propose a solution: using a fullyautomated extraction procedure directly coupled to the LC-MS system, with no human intervention; that simultaneously identifies and quantitates compounds of interest for toxicological application, based on an MRM spectrum mode (MRM-SpM) method.

Methods: The method was developed for 248 compounds. For each, the calibration curve was designed by taking into account its therapeutic range of concentration. Extraction procedure was fully-automated on a programmable liquid handler (CLAM-2000, Shimadzu). The method was validated according to the requirements of ISO 15189, and a robustness study was performed to evaluate how long acceptable accuracy could be provided by using historic calibration curve. Finally, 188 real samples were used to compare quantitative performances to those of validated quantitative methods routinely used in the Lab.

Results: Extracts were automatically prepared in 8 min using $50 \mu \mathrm{L}$ of sample, $100 \mu \mathrm{L}$ of acetonitrile and $10 \mu \mathrm{L}$ of deuterated internal standard. After mixing, samples were filtered into $\mathrm{LC}$ vials and $3 \mu \mathrm{L}$ was directly injected for analysis. Chromatographic separation was performed in $18 \mathrm{~min}$ using a Restek Biphenyl $2.7 \mu \mathrm{m} 2.1 \times 100 \mathrm{~mm}$ column. The MRM transitions were measured throughout the entire analysis period using MRM peak intensity to create an MRM-spectrum All the compounds were successfully validated for our predefined accuracy and CV acceptance criteria. Investigation of the system robustness by repeat quality control analysis demonstrated that samples could be quantified with an acceptable uncertainty using a calibration curve dating up to one month: accuracy variation was between 80 and $120 \%$ for $74 \%$ of the case at the limit of quantification (LOQ) and $92 \%$ of the case at 3 times the LOQ. The maximum CV was of $20.4 \%$ at the LOQ and $16.5 \%$ at 3 times the LOQ. The method was in good agreement with dedicated quantitative methods when applied to 188 patient samples): global $\mathrm{R}^{2}=0,85$; slope 0,81 .

Summary / Conclusions: A fully automated LC-MS/MS method was validated to identify and quantify 248 compounds. MRM spectrum increase confidence in compound identification.

\section{Oral Presentation}

\section{EACPT-1294}

Increased risk of clozapine therapeutic failure during comedication with valproic acid - a retrospective drug monitoring study on 2143 patients

L. Kyllesø $\varnothing^{*}{ }^{1}$, R. L. Smith ${ }^{1}$, K. B. Mathisen ${ }^{2}$, E. Molden ${ }^{1,2}$

${ }^{1}$ Center for Psychopharmacology, Diakonhjemmet hospital, ${ }^{2}$ Department of Pharmaceutical Biosciences, School of Pharmacy, University of Oslo, OSLO, Norway

Drug utilization, Psychopharmacology, Therapeutic drug monitoring

Introduction: Clozapine (CLZ) is superior to other antipsychotic agents in terms of clinical effect, but its use is limited by the risk of serious side effects, such as agranulocytosis and tonic-clonic seizures. Valproic acid (VPA) or lamotrigine (LAM) is often co-medicated with CLZ to prevent seizures or as add-on in treating schizoaffective disorders. It is known that VPA, in contrast to LAM, reduce the serum concentration of the active metabolite $N$-desmethylclozapine. However, the clinical impact of the interaction between VPA and CLZ is unknown.

Objectives: The aim of this study was to investigate if VPA comedication influences the risk of CLZ therapeutic failure, measured as switch in therapy from CLZ to other antipsychotic drugs (APs).

Methods: Therapeutic drug monitoring (TDM) records from CLZtreated patients co-medicated with VPA, LAM or no antiepileptics, were retrospectively collected from a routine service during the period 20052017. Switching from CLZ to other APs were identified from TDM data. CLZ therapeutic failure was defined as initiating TDM of at least one new AP within 12 months after terminating TDM of CLZ. Frequencies of therapeutic failure among VPA- vs LAM-treated patients were compared with patients absent of antiepileptic drugs as a reference group.
Results: In total, 2,143 CLZ users were included in the study. The population comprised a female minority $(38.8 \%)$ and a mean age of 45.8 years. Among the patients, 268 and 227 patients were labelled as VPA or LAM users, respectively, while 1648 were nonusers of antiepileptic drugs. Significantly more VPA users experienced CLZ therapeutic failure than patients absent of antiepileptics $(10.8 \%$ vs $5.4 \%, p=0.008)$, while there was no significant difference for LAM users experiencing CLZ failure $(7.6 \%$ vs $5.4 \%, \mathrm{p}=0.354)$.

Summary / Conclusions: This study suggests that comedication with VPA, but not LAM, increases the risk of CLZ therapeutic failure. The increased risk of CLZ termination and replacement with other APs during comedication with VPA, may potentially be related to the interaction of VPA reducing the serum concentration of the active $N$-desmethylclozapine metabolite.

\section{EACPT-1300}

Preliminary study on influenza vaccination uptake in the active population

G. F. Galistiani ${ }^{*}{ }^{1}$, M. Matuz ${ }^{1}$, M. Csatordai ${ }^{1}$, P. Doró ${ }^{1}$, K. Schváb ${ }^{1}$, R. Benkö $^{1}$

${ }^{1}$ Department of Clinical Pharmacy, University of Szeged, Szeged, Hungary

\section{Drug utilization}

Introduction: Influenza is a highly infectious viral disease and one of the most prevalent causes of respiratory infections. The most effective way to prevent the disease is to get influenza vaccination every year. Many studies have addressed the influenza vaccine uptake in targeted population (e.g. elderly). On the other hand, it is necessary to assess flu vaccine uptake in adult active population, since they are considered as high-transmitter group. Objectives: This study aims to invetigate determinants and barriers of influenza vaccination uptake in the active age population.

Methods: The study was an observational cross-sectional study design, using self-administered questionnaires through an online survey. Descriptive statistics were applied to describe all survey items and bivariate analysis was conducted to assess the possible determinants of vaccination uptake.

Results: Data of 1144 respondents was analyzed. The mean age of respondents was $27 \pm 5.56$ years, and 616 respondents had health and/or occupational risk factors. One-tenth $(10.23 \%)$ of respondents were vaccinated in the last season, and $19 \%$ of those who had any risk factors (health and/or occupational). Tertiary level of education was higher among vaccinated respondents compared to unvaccinated ones $(60.68 \%$ vs $43.72 \% ; p=0.002$ ). The mean knowledge score of vaccinated respondents was higher than unvaccinated ones (mean \pm SD: $84.63 \pm 9.81$ vs $70.50 \pm 14.74 ; p<0.0001$ ). The most frequent reason to get the influenza vaccination was self-protection $(94.02 \%)$, meanwhile not getting the flu frequently $(67.09 \%)$ was the most common reason for refusing the influenza vaccine. One-third of the respondents $(29.46 \%)$ stated that getting recommendation or opinion from family members influenced their decision to get or not to get seasonal influenza vaccination. On the other hand, health care professionals recommendation were less influential.

Summary / Conclusions: The results of this study show that the influenza vaccination uptake is associated with respondents' level of education, occupational risk factor, knowledge of influenza vaccination and recommendations influenced by family members. It can be concluded that interventions to improve the vaccination rates in active population for seasonal influenza should focus on health literacy or public health campaign using family-directed approaches.

\section{EACPT- 1336}

The association between treatment with systemic prednisolone and antipsychotic treatment - a symmetry analysis

T. Øhlenschlæger ${ }^{*} 1$, M. M. Hougaard Christensen ${ }^{1}$, J. Hallas ${ }^{1}$, D. Henriksen ${ }^{1}$

${ }^{1}$ Clinical Pharmacology, University of Southern Denmark, Odense, Odense C, Denmark 


\section{Drug utilization, Pharmacovigilance}

Introduction: It is well-established that treatment with systemic corticosteroids can cause psychiatric adverse events. However, this association has not been quantified among patients treated for obstructive airway diseases. Objectives: To quantify the strength of association between treatment with systemic prednisolone and initiation of antipsychotic treatment.

Methods: A prescription sequence symmetry analysis was employed on all Danish residents aged $\geq 18$ years with at least one filled prescription of treatment for obstructive airway diseases (ATC R03) from 2000-2016, who also filled their first ever prescription for prednisolone (ATC H02AB06) and antipsychotics (ATC N05A) within an interval of 14 days before or after the prednisolone treatment. Patients with a previous cancer diagnosis were excluded from the study. We calculated the crude sequence ratio (SR) and corresponding $95 \%$ confidence intervals $(95 \% \mathrm{CI})$. The SR is the ratio between subjects with prednisolone prescribed before antipsychotics and subjects with prednisolone prescribed after antipsychotics, which is a valid estimate of the incidence rate ratio. The attributable number of postprednisolone antipsychotic treatment was also calculated.

Results: During the study period, 316 persons were identified initiating antipsychotics within 14 days before and after their initial prednisolone prescription. Of the incident users of prednisolone and antipsychotics, 180 initiated prednisolone before and 136 initiated prednisolone after antipsychotic treatment, yielding a SR of $1.32(95 \% \mathrm{CI}$ 1.07-1.67). The attributable fraction was 44 (24.4\%). The SR of females and males was $1.45(1.08-2.02)$, and $1.20(0.89-1.67)$, respectively. The most pronounced association was found for the $25 \mathrm{mg}$ pack strength redeemed (SR 1.40 [95\%CI 1.07-1.88).

Summary / Conclusions: We found a weak association between the initiation of systemic prednisolone and subsequent treatment with antipsychotics among patients treated for obstructive airway disease. Compared to the baseline rate of antipsychotics, a $24 \%$ increase was induced secondary to prednisolone initiation.

\section{EACPT-1351}

Off-label use of rituximab in patients with glomerulonephritis in a tertiary hospital: a retrospective observational study C. Sans ${ }^{*} 1,2$, J. Bosch ${ }^{3,4}$, A. Agustí ${ }^{1,2}$, I. Danés ${ }^{1,2}$, I. Agraz ${ }^{5}$

${ }^{1}$ Clinical Pharmacology Service, Vall d'Hebron University Hospital, ${ }^{2}$ Department of Pharmacology, Therapeutics and Toxicology, Universitat Autònoma de Barcelona, ${ }^{3}$ Internal Medicine Service, Vall d'Hebron University Hospital, ${ }^{4}$ Department of Internal Medicine, Universitat Autònoma de Barcelona, ${ }^{5}$ Nephrology Service, Vall d'Hebron University Hospital, Barcelona, Spain

\section{Drug utilization, Nephropharmacology, Pharmacovigilance}

Introduction: Rituximab (RTX) is used off-label for the treatment of resistant glomerulonephritis (GN). However, more data on its efficacy and safety on these patients are needed.

Objectives: To assess the rate of response and tolerability of off-label use of RTX in patients with GN. The demographic characteristics of patients and other treatments were also assessed.

Methods: A retrospective observational study of requests of off-label use of RTX for patients with GN received at the Pharmacy Service of a tertiary hospital, between 2013 and 2015, was performed. Requests for idiopathic or secondary GN were included and patients were followed-up until December 2018. Each patient could have more than one request during the study period and follow-up. Information was obtained from medical records. Remission was classified as complete (CR), partial (PR) or no response (NR) according to the KDIGO criteria.

Results: A total of 56 requests for 34 patients were received. Median age was 55 (IQR 48-70) years old and $12(35.3 \%)$ were women. Median follow-up was 57 (IQR 51-67) months. Most frequent GN was Membranous GN (MGN) and CR or PR was achieved in $80.6 \%$ of RTX administrations. Tacrolimus was the most common concomitant and post-
RTX treatment. Most Membranoproliferative GN (MPGN) were related to hepatitis $\mathrm{C}$ virus (HCV) and type II cryoglobulinemia and the most frequent post-RTX treatment was antiviral therapy. Results of Minimalchange disease (MCD) and focal segmental glomerulosclerosis (FSGS) are presented in the table. Overall, 7 patients $(20.6 \%)$ had an adverse event (AE): 5 infusion-related reactions, 1 serum sickness and 1 other.

\begin{tabular}{lccll}
\hline Type of GN (n; \%) & $\begin{array}{l}\text { MGN } \\
(19 ;\end{array}$ & $\begin{array}{l}\text { MPGN } \\
(7 ;\end{array}$ & $\begin{array}{l}\text { MCD } \\
(6 ;\end{array}$ & $\begin{array}{l}\text { FSGS } \\
(2 ;\end{array}$ \\
& $55.8 \%)$ & $20.6 \%)$ & $17.7 \%)$ & $5.9 \%)$ \\
$\begin{array}{l}\text { Etiology (n; \%) } \\
\text { Idiopathic }\end{array}$ & $17 ; 89.5 \%$ & $1 ; 14.7 \%$ & $6 ; 100 \%$ & $1 ; 50 \%$ \\
Secondary & $2 ; 10.5 \%$ & $6 ; 85.7 \%$ & 0 & $1 ; 50 \%$ \\
Concomitant medication & $12 ; 63.2 \%$ & $4 ; 57.1 \%$ & $5 ; 83.3 \%$ & $1 ; 50 \%$ \\
$\quad(n ; \%)$ & & & & \\
Post-RTX therapy (n; \%) & $13 ; 68.4 \%$ & $5 ; 71.5 \%$ & $3 ; 50 \%$ & $2 ; 100 \%$ \\
RTX requests and admin. (n) & 36 & 8 & 9 & 2 \\
Response to RTX admin. (n; \%): & & & & \\
CR & $14 ; 38.9 \%$ & $3 ; 37.5 \%$ & $4 ; 44.4 \%$ & 0 \\
PR & $15 ; 41.7 \%$ & 0 & $3 ; 33.3 \%$ & 0 \\
NR & $7 ; 19.4 \%$ & $5 ; 62.5 \%$ & $1 ; 11.1 \%$ & $2 ; 100 \%$ \\
Unknown & 0 & 0 & $1 ; 11.1 \%$ & 0 \\
AE (n; \%) & $3 ; 15.8 \%$ & $1 ; 14.3 \%$ & $2 ; 33.3 \%$ & $1 ; 50 \%$ \\
\hline
\end{tabular}

Summary / Conclusions: Idiopathic MGN was the most common indication for off-label RTX, achieving CR or PR in most administrations. Most MPGN were HCV-related and most RTX bolus had NR. Infusionrelated reactions were the most common $\mathrm{AE}$.

\section{Oral Presentation}

EACPT-1354

Trends in use and misuse of opioids in the netherlands

G. Kalkman ${ }^{1}$, K. Kramers ${ }^{*}$ 1, 2 , R. van Dongen ${ }^{3,4}$, W. van den Brink ${ }^{5}$, A. Schellekens $6,7,8$

${ }^{1}$ Department of Clinical Pharmacy, Canisius-Wilhelmina Hospital, ${ }^{2}$ Department of Pharmacology and Toxicology, Radboud University Medical Center, ${ }^{3}$ Department of Pain Management and Palliative Care, Canisius-Wilhelmina Hospital, ${ }^{4}$ Department of Anaesthesiology and Pain Centre, Radboud University Medical Center, Nijmegen, ${ }^{5}$ Department of Psychiatry, Amsterdam University Medical Centers, Amsterdam, ${ }^{6}$ Department of Psychiatry, Radboud University Medical Center, ${ }^{7}$ Nijmegen Institute for Scientist-Practitioners in Addiction, ${ }^{8}$ Donders Institute for Brain, Cognition and Behaviour, Nijmegen, Netherlands

Drug utilization, Misuse of medicines/substances, Pharmacovigilance Introduction: The United States is currently facing a serious opioid-use epidemic that started with increased sales of oxycodone and eventually resulted in massive overdose mortality. In Europe, including the Netherlands, the medical use of opioids also increased over the past decade but no increase in opioid-related deaths has yet been observed.

Objectives: Given the situation in the United States, we evaluated trends in the number of opioid prescriptions, proxies for misuse and (fatal) overdoses in the Netherlands.

Methods: The number of prescription opioid users was based on healthcare reimbursement data from the Dutch National Health Care Institute. Opioid-related hospitalizations were obtained from the Dutch National Hospital Care Basic Registration. Information on opioid addiction treatment was obtained from the National Alcohol and Drugs Information System. Opioid related mortality was obtained from Statistics Netherlands. Each database covered all or most of the population of the Netherlands.

Results: Between 2008 and 2017 the overall number of prescription opioid users increased from 4.109 per 100.000 inhabitants to 7.489 per 100.000 inhabitants. This was mainly driven by the number of oxycodone users, which increased from 574 to 2.568 per 100.000 inhabitants in the same period. The number of hospitalizations due to opioid intoxication increased from 2,8 per 100.000 inhabitants in 2008 to 8,7 per 100.000 inhabitants in 
2017, and the number of patients in addiction care for opioid use disorders other than heroin and methadone/buprenorphine substitution therapy increased from 3,1 per 100.000 inhabitants in 2008 to 5,1 per 100.000 inhabitants in 2015. Finally, opioid related mortality increased from 0,24 per 100.000 inhabitants in 2008 to 0,65 per 100.000 inhabitants in 2017 .

Summary / Conclusions: Opioid prescribing increased substantially in the past decade and several proxies for misuse show an increasing trend. However, the Netherlands is not experiencing opioid misuse at the same scale as the United States, which has an opioid related mortality of 15 per 100.000 inhabitants (Scholl et al., 2018). Nevertheless, our focus should be to implement safe opioid prescribing guidelines to prevent further escalation of a potential public health threat.

\section{EACPT-1361}

Concomitant use of psychotropic drugs in attention deficit hyperactivity disorder (adhd)

L. Javaloyes Garachana* ${ }^{1}$, A. Sancho-López ${ }^{1}$, B. Ruiz-Antorán ${ }^{1}$, M. Palanca $^{2}$, G. Centeno Soto ${ }^{1}$, Á. Izquierdo ${ }^{2}$, P. del Sol ${ }^{2}$, C. AvendañoSolá ${ }^{1}$

${ }^{1}$ FARMACOLOGÍA CLÍNICA, ${ }^{2}$ Child and adolescent Psychiatric Unit, Hospital Universitario Puerta de Hierro, Majadahonda, Madrid, Spain

\section{Drug utilization, Pediatric treatment, Psychopharmacology}

Introduction: There is a perception that there is an increment in the number of paediatric patients who are receiving treatment with psychotropic drugs, particularly in ADHD patients

Objectives: Describe the profile of current drug therapy use in paediatric patients diagnosed of ADHD in specialized care setting and its evolution from 2011 to2017. Analyse concomitant medicines associated with stimulants for the treatment of ADHDwith/without psychiatric comorbidity

Methods: Retrospective,longitudinal drug utilization study. We included all patients with electronic medical records and a diagnosis of ADHD (ICD10:F90.0-F90.9) seen at the PaediatricNorthwestMentalHealthArea in Madrid between2011-2017

Results: Of the 7,078 patients seen in this period, 3,416patients diagnosed of ADHD were included. Of these, $44 \%$ had associated psychiatric-comorbidities. The longitudinal analysis showed a progressive increase in the percentage of patients diagnosed over the last years,from $28.4 \%$ in 2011 to $59.6 \%$ in 2017.The percentage of ADHD with psychiatric comorbidity remained stable

The overall percentage of treated patients increased from $74 \%$ in 2011 to $87 \%$ in 2017. There were no significant differences in relation to patients who had associated comorbidity(2017:86.2\% vs.86.9\%).The frequency of prescription for each pharmacological group is shown inTable 1.Stimulants were the most prescribed drugs. The prescription of other therapeutic groups was significantly higher in the group with psychiatric comorbidity $(\mathrm{p}<0.05)$
Summary / Conclusions: ADHD diagnosis has increased over time between2011-2017.Almost half of ADHDchildren have associated comorbidities. The percentage of patients with pharmacological treatment remained stable. It is remarkable that up to $10 \%$ of ADHDpatients without psychiatric comorbidities were also treated with other psychotropic medicines, which appears poorly justified

\section{EACPT-1377}

Role of adverse drug reaction in transfers from psychiatric wards to emergency unit in geneva university hospitals

V. Hede ${ }^{*} 1,2$, C. FROMENT ${ }^{1}$, J.-M. Aubry ${ }^{2}$, O. Rutschman ${ }^{3}$, J. Desmeules $^{4}$, M. Besson ${ }^{1,4}$

${ }^{1}$ Psychopharmacology Unit, clinical pharmacology and toxicology department, ${ }^{2}$ Service of psychiatric specialties, Psychiatry department, ${ }^{3}$ Emergency service, Department of Medicine, ${ }^{4}$ Clinical pharmacology and toxicology department, HUG - HÔPITAUX UNIVERSITAIRES DE GENÈVE, Geneva, Switzerland

Drug utilization, Drug-drug interactions, Misuse of medicines/substances, Pharmacovigilance, Psychopharmacology

Introduction: Epidemiological studies report that adverse drug reactions (ADR) are a major source of hospitalization with a prevalence of $10 \%$. Psychotropic drugs are listed in the top-ten of drugs leading to ADR requiring hospital admissions. In the psychiatric population the prevalence of ADR is elevated, estimated between 42 to $87 \%$ of exposed patients.

Objectives: To observe the incidence of ARD related transfers of psychiatric patients, from a psychiatric ward (PW) to the emergency unit (EU) of Geneva University hospitals during one year.

Methods: Observational retrospective study on patient's electronic file. Medical files of all PW to EU transferred patients during 2016 were analyzed. Demographic data, medical history and medication were collected. ADR imputability was independently assessed according to WHO criteria by a psychiatrist and a clinical pharmacologist. Preventability and severity were also quoted. The study was approved by the local ethical committee and according to the retrospective, non interventional design, a patient consent withdrawal was obtained.

Results: The agreement between the two observers for imputability was moderate to good $(\mathrm{k}: 0.662(\mathrm{p}=.000))$. The problematic cases were discussed and a consensus was reached. The agreement for preventability was good to very $\operatorname{good}(0.615(\mathrm{p}=.000)$ and $0.757(\mathrm{p}=.000)$ for preventable and clearly preventable respectively). Of 526 transfers, $42.6 \%(224)$ were related to an ADR; $18 \%$ were quoted certain, $15 \%$ probable and $67 \%$ possible. $71 \%$ of ADR were quoted preventable. Only $18 \%$ were diagnosed on admission to EU. Psychotropic drugs were the first class involved, mainly antipsychotics (54\% of ADR). Cardiac and neurological drugs followed. $54 \%$ of patients had more than 8 drugs. Off-label or

\begin{tabular}{|c|c|c|c|c|c|c|c|}
\hline$\%$ of patients without comorbidity treated & 2011 & 2012 & 2013 & 2014 & 2015 & 2016 & 2017 \\
\hline STIMULANTS* & 83.0 & 89.3 & 78.5 & 85.8 & 95.1 & 93.3 & 93.3 \\
\hline NO STIMULANTS** & 20.8 & 15.5 & 24.8 & 27.4 & 7.6 & 4.6 & 12.3 \\
\hline ANTIPSYCHOTICS & 9.4 & 6.8 & 7.7 & 2.8 & 8.0 & 10.5 & 7.6 \\
\hline HYPNOTICS/ANSIOLITICS & 1.9 & 2.9 & 2.9 & 0.5 & 1.5 & 2.9 & 1.7 \\
\hline ANTIDEPRESSIVES & 9.4 & 2.9 & 5.5 & 2.8 & 6.1 & 11.3 & 6.4 \\
\hline$\%$ of patients with comorbidity treated & 2011 & 2012 & 2013 & 2014 & 2015 & 2016 & 2017 \\
\hline STIMULANTS* & 70.9 & 72.6 & 63.5 & 80.4 & 82.5 & 81.4 & 84.4 \\
\hline NO STIMULANTS** & 25.5 & 22.6 & 24.6 & 30.1 & 6.6 & 8.8 & 19.7 \\
\hline ANTIPSYCHOTICS & 20.0 & 21.7 & 30.5 & 30.1 & 27.9 & 18.9 & 21.6 \\
\hline HYPNOTICS/ANSIOLITICS & 18.2 & 5.7 & 9.0 & 9.1 & 12.7 & 8.8 & 8.6 \\
\hline ANTIDEPRESSIVES & 20.8 & 25.1 & 31.5 & 45.4 & 30.9 & 29.0 & 17.7 \\
\hline
\end{tabular}


unjustified use was found in $74 \%$ of the cases, and drug-drug interactions in $71 \%$. Patient mean age was 59 years. Patients with ADR suffered mostly from dementia (24\%), depression (23\%) and psychotic disorder (22\%). Neurologic consequences represented $65 \%$ of all ADR. $30 \%$ needed hospitalization for somatic care.

Summary / Conclusions: This study underscores that half of the transfers from PW to EU are related to ADR and $70 \%$ are considered preventable. Besides, half of ADR were not recognized on admission to the emergency room. Further analysis will help identifying risk factors as a basis of preventing actions, but bedside teaching by a clinical pharmacologist may be one axis.

\section{EACPT-1387}

Use of psychoactive drugs in paediatric population in catalonia S. Pesiou 1 , M. Fradera ${ }^{1}$, X. Goldberg 2 , F. Torres ${ }^{3}$, R. Barcelo ${ }^{3}$, N. Cardoner $^{4}$, C. Pontes ${ }^{*} 1$

${ }^{1}$ Dept of Pharmacology, Therapeutics and Toxicology, Unitat Docent Parc Taulí, Facultat de Medicina. Universitat Autònoma de Barcelona, ${ }^{2}$ Psychiatry, Fundació Parc Taulí. Institut d'Investigació i Innovació Parc Taulí. Universitat Autònoma de Barcelona, Sabadell (Barcelona), ${ }^{3}$ Biostatistics unit, Facultat de Medicina. Universitat Autònoma de Barcelona, Bellaterra (Barcelona), ${ }^{4}$ Psychiatry, Hospital de Sabadell. Institut d'Investigació i Innovació Parc Taulí. Universitat Autònoma de Barcelona, Sabadell (Barcelona), Spain

\section{Drug utilization, Pediatric treatment, Psychopharmacology}

Introduction: In the region of Catalonia, the number of paediatric patients attended by mental healthcare centers has increased by $25 \%$ since 2008. Psychoactive drug use has been reported to increase globally, and there are concerns on progressively increasing use of psychoactive drugs in children, and on their potential effects on development and adulthood.

Objectives: The study was aimed to describe if prescriptions of psychoactive drugs to paediatric patients had increased in the studied period, and at the expense of which groups of drugs.

Methods: A retrospective observational quantitative study of the consumption of psychoactive drugs, including ATC codes N03A, N05, N06 and N07B, was done in the pediatric population in Catalonia between 2008 and 2017. Anonimysed data was obtained from the administrative registry of drug invoicing of the Catalan Health System, through the PADRIS program. The study was authorized by the Ethic's Committee of Corporació Sanitària Parc Taulí prior to any data extraction. Results: The prevalence of use of psychoactive drugs varied between $5.7 \%$ and $6.5 \%$, with no clear trend of increase along the years. Overall, $66 \%$ of dispensations corresponded to male subjects, but $48 \%$ of new dispensations were done to female patients. Anxiolytics accounted for the highest proportion of exposed subjects (3.3\% of all paediatric subjects, $60 \%$ of those exposed to any psychoactive drug) but in general with short exposures, and mostly due to hydroxyzine. Psychostimulants accounted for $45 \%$ of dispensations, referred to $27 \%$ of subjects exposed to any psychoactive drug, and led mostly by exposure to methylphenidate. Other relevant groups in terms of proportion of subjects exposed were antipsychotics, antiepileptic and antidepressant drugs (10\%, 8.5\% and $7.3 \%$ of all subjects exposed to psychoactive drugs).

Summary / Conclusions: Psychoactive drugs were dispensed to roughly $6 \%$ of paediatric subjects. Anxiolytics were used by $3.3 \%$ of the population with short exposures, but an $\mathrm{H} 1$ antihistamine widely used for treatment of allergic disorders was responsible for most of this exposure. Psychostimulants, and in particular methylphenidate, follow in magnitude of population exposed. It seems that there is no clear trend for sustained growth in overall exposure to psychoactive drugs in our setting.

\section{EACPT-1388}

Death due to suicide in barcelona: a case control study to identify main risk factors and opportunities for intervention.
M. Fradera ${ }^{1}$, R. Morros ${ }^{2}$, O. Prat ${ }^{2}$, C. Martín-Fumadó ${ }^{3}$, N. Cardoner ${ }^{4}$, D. Palao $^{4}$, V. Pérez-Solà ${ }^{5}$ C. Pontes ${ }^{* 1}$

${ }^{1}$ Dept of Pharmacology, Therapeutics and Toxicology, Unitat Docent Parc Taulí, Facultat de Medicina. Universitat Autònoma de Barcelona, Sabadell (Barcelona), ${ }^{2}$ Institut Universitari d'Investigació en Atenció Primària Jordi Gol, ${ }^{3}$, Institut de Medicina Legal de Catalunya, Barcelona, ${ }^{4}$ Psychiatry, Hospital de Sabadell. Institut d'Investigació i Innovació Parc Taulí. Universitat Autònoma de Barcelona, Sabadell (Barcelona), ${ }^{5}$ Psychiatry, Parc de Salut Mar. Universitat Autònoma de Barcelona., Barcelona, Spain

\section{Drug utilization, Pharmacovigilance, Psychopharmacology}

Introduction: Suicide is an increasingly relevant cause of death in many settings; in Spain causes more deaths than traffic accidents since 2008. Prevention requires to detect relevant and potentially modifiable risk factors.

Objectives: To describe and quantify the main risk factors for death from suicide in our setting.

Methods: A retrospective, population-based case-control study was done that identified subjects who died from suicide between 2010 and 2015 in the Barcelona province; any previous interaction with the public health system was retrieved from administrative databases; only subjects with electronic primary care health records in the SIDIAP database, that covers roughly $80 \%$ of Catalonian population, were included. Random sampling by density from SIDIAP database was used to create a control cohort (1:20) with similar age, sex and residence area. Available medical data for all subjects were retrieved and anonymised for analysis. A multivariable method was built using the random forest method to identify independent risk factors. The study was approved by the Ethic's Committee of IDIAP-Jordi Gol before any data extraction.

Results: 2,109 deaths were registered as due to suicide in the province of Barcelona between 2010 and 2015; 971 were registered in administrative databases and had electronic primary care medical records. Subjects were more frequently men $(74 \%)$ and aged ( $33.78 \%$ in people aged $>65$ years). Cases had more often psychiatric diagnosis ( $75.8 \%$ vs $42.3 \%$ ), prescriptions of psychoactive medications in the last year ( $65.1 \%$ vs $23.2 \%)$, more frequent healthcare, social work interactions with the public system, and sick leave during the year preceding death than controls. Previous suicidal attempt, schizophrenia and mania and bipolar disorders were strong risk factors. Exposure to medications also added risk, with a $10 \%$ increase of risk for each additional active principle of any type.

Summary / Conclusions: These results add information about suicide characteristics and risk factors and suggest that there are yet many opportunities for early risk detection and preventive interventions in our setting. In particular, it is sensible to further explore any potential risks associated to individual medications.

\section{EACPT-1409}

Simple measures with significant outcomes- how evaluation and education can alter medication order and reduce costs

E. Janefjord* ${ }^{1}$, A. Helldén ${ }^{1}$, Y. Böttiger ${ }^{1}$

${ }^{1}$ Clincal pharmacology, Linköping University Hospital, Linköping, Sweden

\section{Drug utilization, Education, Health economy}

Introduction: Physicians are on a tight schedule and do not have much time to consider rational drug use. Influenced by information by single drug companies, it is easy to keep old habits, without questioning efficiency. A clinical pharmacist from the Department of Clinical Pharmacology was introduced in February 2016 at the Department of Emergency and Elective Abdominal Surgery (DEEAS), to implement pharmaceutical knowledge at the department.

Objectives: This study was conducted to assess if simple measures can reduce costs by altered medication order. 
Methods: The prescription and use of drugs was evaluated statistically. It was observed that the DEEAS did not prescribe the recommended substance of strong opioids; they used oxycodone/naloxone, and mainly used ondansetron oral disintegrating tablets for nausea and vomiting at high costs. However, the physicians view on their medication order did not agree with the high use of disintegrating tablets. A survey answered by the nurses showed that the overall knowledge of antiemetic drugs and formulations were poor. By a closer look it was revealed that the nurses administered disintegrating tablets even though normal tablets were ordered, because that was their only oral option in stock. The pharmacist provided the physicians and nurses with education, gave the physicians an opioid conversion chart, and implemented new protocols and patient group directions in line with national and regional guidelines. The drug stock was updated and oxycodone/naloxone and disintegrating tablets were removed. Moreover, the medical record system was updated with correct standard order sets to support the changes and help the physicians. The results of the interventions were statistically evaluated after five months and presented to the health care team.

Results: The simple interventions lead to reduced costs and more effective treatment. The cost/DDD of prolonged release tablets of strong opioids were reduced with $44 \%$ in two years (2016-2018). The total costs of antiemetic drugs were reduced by $80 \%$, jun-oct 2018 compare to the same period 2017. The healthcare team was more educated in differences between drug formulations and uptake.

Summary / Conclusions: Simple measures can pursue interventions that optimize drug use and reduce costs if they are implemented at all levels and confirmed with the whole health care team.

\section{EACPT-1411}

Investigation of drug utilization in patients with dyslipidemia D. Bayram ${ }^{1}$, O. C. Sel ${ }^{1}$, V. Aydin ${ }^{2}$, A. S. Fak ${ }^{2}$, A. Altikardes ${ }^{2}$, A. Akici ${ }^{2}$ ${ }^{1}$ Medical Pharmacology, ${ }^{2}$ Hypertension and Atherosclerosis Research Center, Marmara University, Istanbul, Turkey

Drug utilization

Introduction: Substantial evidence exists regarding reduction of cardiovascular diseases and related mortality with the use of lipid-modifying drugs as part of the primary or secondary cardiovascular protection.

Objectives: The aim of this study was to describe drug utilization pattern in dyslipidemia patients who applied to a specialized heart center.

Methods: We retrospectively collected medical records of the patients applied to the Istanbul Medical Center of Turkish Heart Foundation from January 2016 till July 2018. Also considering that applying patients could have other comorbidities, the drugs included in the treatment of patients who were ever diagnosed with dyslipidemia were evaluated at ATC-1, ATC-3, and ATC-5 level as well as by their original/generic status.

Results: Among the total of 11,085 patients who had diagnosis data, $19.4 \%$ had dyslipidemia. A total of 1673 patients had drug information and the mean number of applications per person was $1.6 \pm 1.7$ (mean age: $63,4 \pm 12,7$ years; women: $52.7 \%$ ). It was found that these patients used 9488 medications in total, where the number of drugs per patient was 5.7 \pm 8.0 . When the drugs were examined at the ATC-5 level, the most common ten drugs were acetylsalicylic acid $(15.3 \%)$, atorvastatin $(11.7 \%)$, metoprolol $(8.9 \%)$, rosuvastatin $(5.8 \%)$, metformin $(5.2 \%)$, amlodipine (3.6\%), levothyroxine (3.4\%), clopidogrel (3.1\%), valsartan + hydrochlorothiazide (1.7\%), and ramipril (1.7\%). At the ATC-3 level, "antithrombotic agents" (19.2\%), "lipid-modifying drugs" (18.5\%), and "beta-blockers" (13.4\%) constitute the three most commonly used groups. ATC-1 classification showed mostly "cardiovascular system" $(59.6 \%)$ drugs, followed by "blood and blood-forming organs" (19.4\%) and "digestive system and metabolism" $(11.0 \%)$ drugs. It was found that $22.3 \%$ of total drugs were generic.

Summary / Conclusions: It appears that patients with dyslipidemia predominantly use drugs other than lipid-modifying drugs, where the latter constitutes only about one-fifth of the all drugs used. This suggests the need for designating comprehensive studies on the details of the surplus of comorbid disease burden requiring pharmacological treatment with dyslipidemia patients. Considering recent data raising concerns on the efficacy of aspirin on primary prevention in cardiovascular diseases, more attention would be neccessary to select patients who might benefit from aspirin therapy in the primary setting.

\section{EACPT-1416}

Investigation of off-label drug use in pediatric patients N. Akici ${ }^{1}$, N. I. Kirmizi ${ }^{2}$, V. Aydin ${ }^{2}$, B. Bayar ${ }^{3}$, A. Akici ${ }^{*} 2$

${ }^{1}$ Department of Pediatrics, Haydarpasa Numune Training Hospital, ${ }^{2}$ Department of Pharmacology, Marmara University Faculty of Medicine, Istanbul, ${ }^{3}$ Turkish Medicines and Medical Devices Agency, Ministry of Health, Ankara, Turkey

\section{Drug utilization, Pediatric treatment}

Introduction: The use of drugs outside the purpose for which they are approved, including different indications, doses, administration forms, age groups, or special populations etc. is called "off-label drug use" (OLDU). Across all age groups, children are exposed to such practice more often.

Objectives: This study aimed to investigate OLDU applications for pediatric patients in Turkey.

Methods: This retrospective study analyzed medical records of OLDU applications approved by the Turkish Medicines and Medical Devices Agency for pediatric patients ( $<18$ years old) during year 2015. Several classifications of these off-labelled drugs were described. Furthermore, prescribed patients were stratified by age groups as "infants" ( $<2$ years old), "children" (2-11 years old), and "adolescents" ( $\geq 12$ years old), where the drugs were compared at Anatomical Therapeutic Chemical (ATC)-1 level. Results: A total of 7896 OLDU applications was approved for pediatric patients during the study period. Boys constituted $52.9 \%$ of all applications, and the mean age was $9.3 \pm 5.2$ years. The age group for which OLDU was most commonly applied was children (52.7\%), followed by adolescents $(36.1 \%)$ and infants $(11.2 \%)$. Most commonly applied drugs were eculizumab (6.5\%), mycophenolic acid (5.6\%), and canakinumab (4.4\%). Among the top 15 ranked drugs in OLDU applications, it was found that $53.3 \%$ were biotechnological drugs and $13.3 \%$ were unlicensed in Turkey. The comparison of the age groups by their prescribed drugs at ATC-1 level showed "L-antineoplastic and immunomodulating agents" as the most common group in all "infants", "children", and "adolescents" groups $(28.2 \%, 45.5 \%$ and, $54.9 \%$ respectively; $\mathrm{p}<0.05$ for all pairwise comparisons). In addition, these age groups significantly differ from each other in terms of other ATC-1 drug categories $(\mathrm{p}<0.05)$, except "B-blood and blood forming organs" and "H-systemic hormonal preparations. excl. sex hormones and insulins".

Summary / Conclusions: Regardless of the age group, pediatric OLDU appears to be most commonly practiced for antineoplastic and immunomodulating agents, prescription of which further exhibits an age-dependent increase. Linkage of these drugs to critical illnesses during childhood may be important for relevant stakeholders focusing on providing easier pediatric access to drugs.

\section{EACPT-1422}

Potentially inappropriate medications (pim) in the elderly. Project to create a spanish list (es-pia project)

C. M. Rodríguez Jiménez ${ }^{1}$, M. González-Colaço Harmand ${ }^{2}$, M. GarcíaSáiz $^{1}$, A. Aldea-Perona ${ }^{3}$, P. B. Masiero-Aparicio ${ }^{*}{ }^{1}$, C. Grillo-Grillo ${ }^{1}$, C. Boada del Campo-Fernández ${ }^{1}$, A. Orellana-Mobilli ${ }^{1}$, E. FernándezQuintana $^{4}$, M. García Sánchez- Colomer ${ }^{4}$

${ }^{1}$ Clinical Pharmacology , ${ }^{2}$ Domiciliary Hospitalization , Complejo Hospitalario Universitario de Canarias, La Laguna, ${ }^{3}$ Clinical Pharmacology, IMIM: Institut Hospital del Mar d'Investigacions Mèdiques, Barcelona, ${ }^{4}$ Pharmacovigilance, Complejo Hospitalario Universitario de Canarias, La Laguna, Spain 


\section{Drug utilization, Geriatric treatment}

Introduction: In the last decades, different criteria have been developed for detecting inappropriate prescription in elderly. In Spain, translations and adaptations of international lists are available but it would be necessary a national list which could cope with the peculiarities of our health system, existing pharmaceutical market and prescription habits.

Objectives: Establish a list of potentially inappropriate medications (PIM) in elderly, reasons for inappropriate prescription and supporting evidence.

Methods: Delphi-method was used: 1.Formulation of the problem, 2.Selection of a coordinator team of 12 multidisciplinary experts, 3. Creation of a questionnaire of IM based on the review of publications, 4.Summary of Characteristics of each drug, and safety warnings of Spanish Medicines Agency, 5.Recruitment of 25 independent experts, 6.Mailing of two rounds of questionnaires. In each round, the experts were asked to give their agreement with statements proposed using a 10-point Likert-scale. Elaboration a final IM prescription list adapted to the Spanish pharmacopoeia, and applicable in people over 65 years

Results: 1462 SPC were reviewed. 160 sentences, in form of statements (eg. "Antiepileptics in the elderly increase risk of confusion, syncope, ataxia or alterations in psychomotor function"), was reviewed by the coordinator team and proposed in the first round questionnaire. A high degree of agreement was reached in 108 sentences (106 accepted; 2 rejected), 22 had low agreement, and 32 medium agreement. These 32 sentences passed to a second round, and in all of them, high agreement was reached. Finally, 138 sentences were accepted. Response rate was $100 \%$ in both questionnaires.

Summary / Conclusions: This project has allowed to develop a list of PIM in the elderly, supported by high degree of agreement in a large multidisciplinary team. The list will be published since it can be used as a tool to support prescription.

\section{EACPT-1430}

Use of analgesic drugs among older people in greece consuming more than five drugs daily

P. Papaioannidou ${ }^{* 1}$, M. Michailidou

${ }^{1}$ 1st Department of Pharmacology, School of Medicine, ARISTOTLE

UNIVERSITY OF THESSALONIKI, Thessaloniki, Greece

\section{Drug utilization}

Introduction: The use of some medicines, like analgesics, cannot be recorded in Greece, as most of them are not included in prescriptions, because they are not reimbursed by the National Health System (NHS).

Objectives: The aim of this study was to explore the use of analgesic drugs in older patients ( $>65$ years) receiving five or more than five drugs daily in the community of Thessaloniki, Greece.
Methods: The sample was collected using the new Electronic Health Records that have been applied in the last years in Greece. All prescriptions concerning patients older than 65 years and dispensed during one month in a pharmacy store of western Thessaloniki were used. In order to include non prescribed medications, we kept a record of medications bought by the patients without prescription, and we also asked the patients directly about the use of other medications. The statistical package SPSS was used for statistical analysis.

Results: Polypharmacy ( $\geq 5$ medications daily) was observed in $55 \%$ of patients older than 65 years. Analgesics were used by $70 \%$ of these patients. The most commonly used categories of analgesics were: paracetamol (78\%), other non-steroid antiinflammatory drugs (35\%), opioids (17\%), combinations with muscle relaxants $(7 \%)$, corticosteroids $(4 \%)$. Although analgesics were second in the frequency of usage (after cardiovascular drugs), most of them were not included in prescriptions, as they are not reimbursed by the NHS.

Summary / Conclusions: Analgesics are commonly used by older people in Greece but usually they are bought without prescription. In order to record actual use of analgesics , apart from the Electronic Health Records, it is necessary to collect data directly from the patients.

\section{EACPT-1434}

Increased prescribing trends of psychotropic medicines in spanish paediatric population. Sensation, perception or reality?

A. Sancho-López ${ }^{*}$ 1, L. Javaloyes Garachana ${ }^{1}$, 1, B. Ruiz-Antorán ${ }^{1}$, M. Palanca $^{2}$, G. Centeno Soto ${ }^{1}$, Á. Izquierdo ${ }^{2}$, P. del Sol ${ }^{2}$, C. AvendañoSolá$^{1}$, E. Diago Sempere ${ }^{1}$

${ }^{1}$ FARMACOLOGÍA CLÍNICA, ${ }^{2}$ Child and adolescent Psychiatric Unit, Hospital Universitario Puerta de Hierro, Majadahonda, Madrid, Spain

\section{Drug utilization, Pediatric treatment, Psychopharmacology}

Introduction: There is a perception that there is an increment in the number of paediatric patients who are receiving treatment with psychotropic medicines. Until now, updated data in the Spanish paediatric population about psychotropic drug use is limited.

Objectives: To describe the profile of psychiatric medications use in the paediatric population in specialized care setting and its evolution from 2010 to 2017

Methods: Observational, longitudinal, retrospective drug utilization study. All patients with electronic medical records and attended at the Paediatric Northwest Mental Health Area of Madrid between 2010 and 2017 were included. The exposure to the psychotropic drugs was considered per patient-year(s)

Results: A total of 7,078 patients were included, $64 \%$ males, mean age $11.4+4.1$. The longitudinal analysis showed a progressive increase in the percentage of patients receiving treatment with some psychotropic medicine, from $41.6 \%$ in 2010 to $72.5 \%$ in 2017 (Table 1). By age subgroups, this increment was observed across all groups: $0-6$ years: $3.4 \%$ to $12.9 \%$; $6-12$ years: $46.8 \%$ to $66.2 \% ; 12-18$ years: $60.7 \%$ to $83 \%$.

\begin{tabular}{|c|c|c|c|c|c|}
\hline & $\begin{array}{l}\% \text { of patients } \\
\text { treated with any } \\
\text { psychotropic-medication }\end{array}$ & ANTIPSYCHOTICS & ANXIOLYTICS /HYPNOTICS & ANTIDEPRESSANTS & STIMULANT \\
\hline 2010 & $41.6 \%$ & $8.9 \%$ & $25.0 \%$ & $15.2 \%$ & $66.1 \%$ \\
\hline 2011 & $46.0 \%$ & $27.5 \%$ & $26.2 \%$ & $23.6 \%$ & $50.2 \%$ \\
\hline 2012 & $53.1 \%$ & $19.3 \%$ & $25.5 \%$ & $33.9 \%$ & $50.5 \%$ \\
\hline 2013 & $53.3 \%$ & $21.4 \%$ & $18.1 \%$ & $32.7 \%$ & $57.9 \%$ \\
\hline 2014 & $66.9 \%$ & $21.0 \%$ & $14.7 \%$ & $32.9 \%$ & $64.0 \%$ \\
\hline 2015 & $69.8 \%$ & $25.2 \%$ & $17.4 \%$ & $40.1 \%$ & $62.4 \%$ \\
\hline 2016 & $72.3 \%$ & $23.3 \%$ & $17.6 \%$ & $36.1 \%$ & $64.3 \%$ \\
\hline 2017 & $72.5 \%$ & $18.9 \%$ & $12.0 \%$ & $28.8 \%$ & $71.5 \%$ \\
\hline
\end{tabular}


In relation to the concomitant medications, the percentage of patients with more than one psychotropic medication from different therapeutic groups increased from $14 \%$ in 2010 to $30 \%$ in 2017.

Up to $49 \%$ of treated patients received additional psychological treatment and this percentage remained stable over the years.

Summary / Conclusions: The use of psychotropic medicines in the paediatric population attended at Psychiatry Units has increased in recent years. There is an increase in stimulants and antidepressants use, possibly related to a broadening of the scope of indications. Only half of children treated pharmacologically received concomitant psychological intervention, which is far below our expectations

\section{Education}

\section{EACPT-129}

Patient information of magistral preparations using qr codes R. Seisdedos ${ }^{* 1}$, B. Fernández Rubio ${ }^{1}$, M. Alonso Moreno ${ }^{1}$, E. Prado $\mathrm{Mel}^{1}$

${ }^{1}$ PHARMACY, HOSPITAL UNIVERSITARIO VIRGEN DEL ROCÍO, SEVILLA, Spain

Education, Patient empowerment

Introduction: One of the main roles of the pharmacist is to provide pharmacotherapy information to the patient. Oral information needs to be strengthen using written sources. However, delivered information forms get sometimes lost or are not available at any time in front of the information contained in the labeling, which will always accompany the medication. QR code (quick response code) is a bar code in two dimensions that can store many information and allows access to a Web page, a social network or multimedia resources. Using web pages and carrying out simple procedures, everybody is able to generate a QR code, which can be recognized through the camera of a smart mobile phone.

Objectives: Provide extra information to magistral preparations through QR codes.

Methods: A google drive account was created in order to store the patient information forms in pdf format. Files were uploaded using the option "share via link", or what is the same, the QR code (enabling only "see" option and not "edit" or "comment"). Available literature regarding on the creation of QR codes attractive to users was reviewed. From a web generator of QR codes, the images with the code that were inserted in the labels of the magistral preparations were created.

Results: A customized QR code design was obtained using different colors and images that served as a claim to the patient. The code was inserted in the magistral preparation label, redesigning it to contain all the necessary information on a $105 \times 37 \mathrm{~mm}$ label. For each new treatment, the patient was given the information in paper format and the medication containing the QR code on the label.

Summary / Conclusions: Access to content using QR codes stands out for its immediacy, accessibility, possibility of constant updating, efficiency and low cost. Through this tool, the information is reinforced to the patient in an effective and simple way and it is ensured that the patient has the information available during the entire duration of the treatment. In the future, a visit analysis could determine its usefulness and thus design improvement strategies such as the creation of a propietary content platform. Currently, the use of the QR code in health is still incipient and nevertheless there are numerous opportunities for application both for use by patients and professionals.

\section{EACPT-1071}

Key learning outcomes for clinical pharmacology and therapeutics education in europe: a modified delphi study D. Brinkman ${ }^{1}$, J. Tichelaar ${ }^{1}$, W. Mokkink ${ }^{1}$, T. Christiaens ${ }^{2}$, R. Likic ${ }^{3}$, R. Maciulaitis ${ }^{4}$, J. $\operatorname{Costa}^{5}$, E. Sanz ${ }^{6}$, S. Maxwell ${ }^{7}$, M. Richir ${ }^{1}$, M. van Agtmael $^{1}$
${ }^{1}$ Amsterdam University Medical Centers, Amsterdam, Netherlands, ${ }^{2}$ Ghent University, Ghent, Belgium, ${ }^{3}$ University of Zagreb, Zagreb, Croatia, ${ }^{4}$ Lithuanian University of Health Sciences, Kaunas, Lithuania, ${ }^{5}$ University of Lisbon, Lisbon, Portugal, ${ }^{6}$ University of La Laguna, Tenerife, Spain, ${ }^{7}$ University of Edinburgh, Edinburgh, United Kingdom

\section{Education}

Introduction: Recent studies showed a general lack of essential prescribing compentencies among final-year medical students in Europa and showed that clinical pharmacology and therapeutics (CPT) education during the undegraduate medical curriculum in Europe is mainly based on traditional learning methods (e.g., lectures, written exams) (Brinkman et al. 2017a; Brinkman et al. 2017b). These results suggest that there is considerable room to improve CPT education in many European medical schools.

Objectives: As there are currently no uniform requirements for undergraduate CPT education in European countries, the aim of this study was to achieve consensus on key learning outcomes for undergraduate CPT education in Europe

Methods: We used a modified Delphi method consisting of three questionnaire rounds and a face to-face panel meeting. 129 experts from 27 European countries were asked to rate 307 learning outcomes (233 knowledge, 65 skills, 9 attitudes) on a 5-point scale ( $1=$ very unimportant, $5=$ very important); an outcome was included if $\geq 80 \%$ of the respondents awarded it a score of 4 or 5 . Panelists could also change the wording of outcomes and add outcomes if they felt these were missing.

Results: Between January and July 2017, 92 experts (71\%) from 25 European countries completed all three questionnaire rounds, and 33 experts from 17 European countries attended the face-to-face panel meeting. 232 learning outcomes from the original list, 15 newly suggested and 5 rephrased outcomes were included, giving a total of 252 outcomes. Many of the agreed outcomes were related to knowledge of basic and clinical pharmacology, and skills and attitudes about pharmacotherapy. Summary / Conclusions: This study identified 252 learning outcomes that should be included in undergraduate CPT curricula to ensure that European graduates are able to prescribe safely and effectively. These outcomes could also be useful elsewhere. We have developed a blueprint of a European core curriculum describing when and how the learning outcomes might be acquired.

\section{EACPT-1077}

"helpful to get time and opportunity to discuss drug treatment, that's what $i$ think is the most important thing" - a qualitative study on key aspects to educate junior physicians in prescribing

J. Lönnbro* ${ }^{1,2}$, S. M. Wallerstedt ${ }^{2,3}$

${ }^{1}$ Department of Medicine, Sahlgrenska University Hospital, ${ }^{2}$ Department of Pharmacology, Institute of Neuroscience and Physiology, ${ }^{3}$ HTA-centrum, Sahlgrenska University Hospital, Gothenburg, Sweden

\section{Education, Professional development}

Introduction: Prescribing skills are prerequisites for rational use of medicines and education is important to acquire these skills.

Objectives: The aim of the study was to explore what aspects junior physicians find important when being taught the art of prescribing.

Methods: Written feedback from 34 interns, participating in an educational session as part of a randomized controlled study in a university hospital, formed the qualitative data on which a manifest content analysis was performed. Meaning units were extracted based on the overall research question "Educating junior physicians in the art of prescribing: what aspects do they find important?", categorized, and emergent themes identified.

Results: Five different themes emerged. The first theme, clinical relevance, was exemplified by the categories valuable for the healthcare and perceived relevance for one's own work, whereas the second theme, applicable content, included categories like clinical advice, practical tips in the electronic 
medical record system, and tools that facilitate. The third and fourth themes, reality-based teaching and creative discussions, were exemplified by the categories patient cases and feedback; and discussion-based teaching and permissive and open atmosphere, respectively. In the last theme, effective structure, we found the categories clear structure, small group teaching, allocated time for discussion, and home assignment for practice.

Summary / Conclusions: Creative discussions, effectively structured in small group sessions, with clinically relevant and applicable reality-based content built on patient cases and feedback, are key aspect to educate junior physicians in the art of prescribing.

\section{Oral Presentation}

\section{EACPT-1089}

Development of prescribing knowledge and skills of junior doctors in the first year after graduation

E. Donker ${ }^{*}$, D. Brinkman ${ }^{1}$, A. Maassen van den Brink ${ }^{2}$, B. Janssen ${ }^{3}$, W. $\mathrm{Knol}^{4}$, G. Dumont ${ }^{5}$, P. Jorens ${ }^{6}$, A. Dupont ${ }^{7}$, T. Christiaens ${ }^{8}$, J. Smeden ${ }^{9}$, I. De waard-Siebinga ${ }^{10}$, L. Peeters ${ }^{11}$, R. Goorden ${ }^{12}$, M. Hessel ${ }^{13}, \mathrm{M}$. Richir $^{1}$, M. Van Agtmael ${ }^{1}$, K. Kramers ${ }^{14}$, J. Tichelaar

${ }^{1}$ Internal medicine, Amsterdam University Medical Center, location VUmc, Amsterdam, ${ }^{2}$ Internal medicine, Erasmus Medical Center, Rotterdam, ${ }^{3}$ Pharmacology and Toxicology, Maastricht University, Maastricht, ${ }^{4}$ Geriatric medicine, University Medical Center Utrecht, Utrecht, ${ }^{5}$ Clinical pharmacology and pharmacotherapeutics, Amsterdams University Medical Center, location AMC, Amsterdam, Netherlands, ${ }^{6}$ Critical Care Medicine, Antwerp University Hospital, Antwerp, ${ }^{7}$ Pharmacotherapy, Free University of Brussels, Brussels, ${ }^{8}$ Clinical pharmacology, Ghent University, Ghent, Belgium, ${ }^{9}$ Education, Foundation Center for Human Drug Research, Leiden, ${ }^{10}$ University Medical Center Groningen, Groningen, ${ }^{11}$ Clinical pharmacology, Erasmus Medical Center, Rotterdam, ${ }^{12}$ Radboud University Medical Center, Nijmegen, ${ }^{13}$ Leiden University Medical Center, Leiden,

${ }^{14}$ Pharmacology-Toxicology and Internal Medicine, Radboud University Medical Center, Nijmegen, Netherlands

\section{Education}

Introduction: Poor prescribing has negative effects on patient safety and healthcare costs. Medical students in Europe lack prescribing knowledge and skills at graduation (Brinkman et al., 2017, Brinkman et al., 2018), probably due to inadequate education in clinical pharmacology and therapeutics during the undergraduate medical curriculum (Brinkman et al., 2017). However, little is known about the development of prescribing knowledge and skills of junior doctors after graduation. This is important to develop relevant educational interventions after graduation.

Objectives: Investigating how the prescribing knowledge of junior doctors in the Netherlands and Flanders develops in the first year after graduation.

Methods: This is a prospective cohort study among medical graduates from 11 medical schools in the Netherlands and Flanders. In total, 1.506 medical students graduating between July 2016 and March 2018 were invited to participate. They were asked to complete an online assessment at three different time points: $\mathrm{T} 0=$ around graduation, $\mathrm{T} 1=$ six months after graduation, $\mathrm{T} 2=$ one year after graduation. Each assessment contained of 35 multiple choice questions extracted from the Dutch Pharmacotherapy Assessment database (Kramers et al, 2017). The primary outcome is the total percentage of correctly answered questions.

Results: In total, $556(36 \%)$ medical students agreed to participate of which 326 (58\%) completed all three rounds. Overall, prescribing knowledge increased significantly from $69 \%$ (SD 13,0 ) to $77 \%$ (SD 11,4) in the first six months and then decreased to $71 \%$ (SD 13,8) at the end of the year $(\mathrm{p}<0.001)$. For $5 / 7$ topics (anticoagulants, cardiovascular drugs, antidiabetics, psychotropics and basic pharmacokinetics and drugs calculation) there was a significant increase in prescribing knowledge during the first six months $(\mathrm{p}<0.001)$. However, except for basic pharmacokinetics and drugs calculation, this knowledge decreased during the second six months $(\mathrm{p}<0.001)$. For both antibiotics and analgesics, the prescribing knowledge increased significantly after one year (both $\mathrm{p}<0.001$ ).

Summary / Conclusions: There is an increase in prescribing knowledge of junior doctors in the first six months after graduation but then, except for antibiotics and analgesics, declines in the ensuing six months. This study indicates that for specific topics educational interventions after graduation are needed to improve prescribing knowledge.

\section{EACPT-1096}

Success rate of test questions concerning pharmacokinetics is lower compared to pharmacodynamics in clinical pharmacology teaching in a health and life sciences curriculum

M. Wilhelmus ${ }^{*}$, B. Drukarch ${ }^{1}$

${ }^{1}$ Anatomy and Neurosciences, VU University Medical Center, Amsterdam, Netherlands

\section{Education}

Introduction: Pharmacokinetics (PK) and pharmacodynamics (PD) are backbone topics in our clinical pharmacology course for second year Health and Life Sciences students at the Vrije Universiteit Amsterdam (VU). PK/PD requires sufficient insight to grasp its content and be able to use the knowledge within a different context. Student evaluations and personal teacher experience indicate $\mathrm{PK} / \mathrm{PD}$, and more specifically the $\mathrm{PK}$, as the most difficult part of the course. Students especially seem to struggle with the basic PK/PD components that require insight. So far, objective analysis of these experiences is lacking to motivate improvement of course content with respect to PK/PD.

Objectives: To determine success rate of PK and PD test questions, subdivided in 'knows' and 'knows how' questions, of 3 sequential yearly cohorts of enrolled students in our course.

Methods: Success rate analysis of PK/PD multiple-choice (mc)-questions, subdivided into 'knows' and 'knows how', was performed in tests of the 'pharmacology' course at bachelor level from 3 cohorts (total $n=$ 377) of second year Health and Life Sciences students of the VU. Tests consist of a total of 60 mc-questions, $n=20$ on PK and $n=17.3 \pm 1.5$ on PD. Difficulty and distinctiveness index was performed. Normal distribution of success rate of all question groups, between cohorts was determined. Success rate was analyzed on question and student level.

Results: Success rate of PK/PD questions, as well as the 'knows' and 'knows how' questions, was equally distributed between cohorts. Difficulty index demonstrated a 'medium' difficulty level and no significant difference in $p$-values between cohorts. Distinctiveness index of the questions was scored as 'sufficient'. We found an overall reduction $(\mathrm{p}<$ 0.001) in success rate in PK mc-questions compared to PD. In addition, a reduction $(p<0.001)$ in success rate of mc-questions was found between overall 'knows' and 'knows how' PK/PD-questions.

Summary / Conclusions: Success rate of PK test questions is lower compared to PD and 'knows how' test questions on PK/PD are most challenging, confirming student and teacher experience. These data will be used to guide improvements in our course, i.e. reinforcing time and effort on PK, and increasing deep learning in PK/PD by strengthening the role of active learning tools in our course.

\section{EACPT-1185}

The prescribing safety assessment in irish medical schools: progress to date and attitudes of newly qualified doctors to its implementation. H. Gallagher ${ }^{*}{ }^{1}$, M. Coyne ${ }^{1}$, N. Tighe ${ }^{2}$, S. Donnelly ${ }^{2}$, P. Murray ${ }^{1}$

${ }^{1}$ Clinical Pharmacology, School of Medicine, ${ }^{2}$ School of Medicine, University College Dublin, Dublin 4, Ireland

\section{Education, Professional development}

Introduction: The Prescribing Safety Assessment (PSA) is an online, validated examination devised by the British Pharmacological Society 
and Medical Schools Council to assess prescribing skills and safety. It is taken by medical students in all UK Medical Schools prior to graduation and has also been introduced in Ireland and Malta. In University College Dublin (UCD) all final year medical students take the exam.

Objectives: The main aim of this study was to investigate attitudes towards the PSA among newly qualified doctors in Ireland.

Methods: We surveyed intern doctors working in two large teaching hospitals attached to the UCD School of Medicine. All respondents were in the third of their four intern rotations in their first year of clinical practice and had passed the PSA exam before medical school graduation. Ethics permission was granted from the UCD Human Research Ethics Committee. Data were analysed and results are presented.

Results: Interim data analysis $(n=25)$ indicated that compared to other assessments done during medical school, $72 \%$ of doctors considered the PSA to be very relevant or extremely relevant to their future clinical practice. Forty-eight percent of doctors agreed, or strongly agreed, that their degree curriculum prepared them to sit the PSA, while $20 \%$ neither agreed nor disagreed with this statement. Regarding prescribing confidence, $88 \%$ reported feeling nervous or very nervous about prescribing when they commenced work as a doctor but the same proportion (88\%) agreed or strongly agreed that they now feel confident to prescribe medicines. Fortyeight percent of respondents reported seeking prescribing advice from more senior colleagues most or all of the time when they commenced clinical practice, but this reduced to $8 \%$ seeking such advice most of the time with 9 months experience. Sixty-eight percent agreed that the PSA helped to develop their basic competency as an intern doctor, while $76 \%$ reported that it increased their awareness of practicing medicine safely.

Summary / Conclusions: The PSA has had a positive influence on awareness of prescribing safety among intern doctors in Ireland. Efforts to adapt the medical curriculum so that doctors feel more prepared to prescribe upon graduation will continue. However, our survey suggests that doctors who have passed the PSA quickly acquire confidence in prescribing, once they start to work in clinical practice.

\section{EACPT-1203}

Fourth-year rational pharmacotherapy clerkship at Koç University School of Medicine

S. E. Gulmez ${ }^{*}$, G. Ozcan ${ }^{1}$, H. S. Orer ${ }^{1}$

${ }^{1}$ Medical Pharmacology, Koç University School of Medicine, ISTANBUL, Turkey

\section{Education}

Introduction: Pharmacotherapy teaching in many medical schools is often limited to the simple transfer of knowledge on drugs, lacking the acquisition of essential patient management skills such as choosing a drug and managing the treatment. A Rational Pharmacotherapy (RPM) clerkship, designed after Groningen University's rational pharmacotherapy programme, has been incorporated in the $4^{\text {th }}$-year curriculum of the Koc University School of Medicine (KUSOM) to improve the prescription skills of the medical students.

Objectives: To develop analytical prescription skills through a series of problem-based learning (PBL) sessions, and to build a personal formulary, named the $\mathrm{P}$ (personal)-drug list for a common condition.

Methods: First, moderated by the pharmacology faculty, students worked in groups to choose P-drugs for a specified indication based on efficacy, safety, suitability, and cost. Second, they plan the patients' treatment using the list compiled. At the end of the clerkship, formative assessment was made by objective structured clinical examination (OSCE) with standardized patients at the KUSOM Simulation Centre. Each student encountered sequentially with three hypertension cases who had different therapeutic endpoints. A designated faculty took charge of a particular OSCE case and assessed all 30 students to assure the objectivity. Pre- and post-training surveys were conducted to assess the self-efficacy levels, and the students were encouraged to give feedback throughout the course. Results: OSCE results provided with a detailed individual assessment of students' prescribing skills. The overall success rate was $80 \%$. Every student received post-OSCE feedback on the critical issues of his/her prescribing practice. The survey showed a significant increase in selfefficacy measured at different tasks as the training progressed.

Summary / Conclusions: KUSOM is a young, 10-year old institution accredited by the National Board in 2018. The implementation of RPM clerkship was a success as students showed appreciation despite the exhausting and stressful OSCE process. OSCE, combined with the selfefficacy surveys, provided with a more comprehensive assessment of the students' progress. Our future perspective includes the follow up of the clerkship with different skill keeper activities destined to promote the rational use of drugs in the upcoming, i.e., $5^{\text {th }}$ and $6^{\text {th }}$, years of medical education.

\section{EACPT-1214}

Clinical pharmacology in the undergraduate medical education: our experience in the context of the european curriculum

H. Glasova ${ }^{* 1,2}$, J. Glasa ${ }^{1,2,3}$

${ }^{1}$ Slovak Society of Clinical Pharmacology, o.b. Slovak Medical Association, ${ }^{2}$ Institute of Pharmacology and Clinical Pharmacology, ${ }^{3}$ Institute of Health Care Ethics, Slovak Medical University Bratislava, Bratislava, Slovakia

\section{Education}

Introduction: Clinical Pharmacology $(\mathrm{CP})$ as a self-standing medical speciality has a long-standing tradition in Slovakia, being established in 1970ies according to the WHO recommendations. $\mathrm{CP}$ founders' generation understood $\mathrm{CP}$ as an interdisciplinary field of inquiry into clinical effects and safety of medicaments and their rigorous evaluation.

Objectives: They expected it to be built creatively upon both the utmost understanding of human pharmacology and upon that of clinical medicine. The latter has been mostly internal or paediatric medicine, including their (sub) specialities, but other disciplines were also involved (e.g. neurology, psychiatry, toxicology, anesthesiology). This has been mostly the case with regard to the individual careers of physicians, building up their competency in CP on top of that of their original speciality. It has also been understood that for a competent rational prescriber the "initiation" in $\mathrm{CP}$ as soon as possible in his/her medical career is of vital importance, ideally already within the undergraduate studies.

Methods: Therefore, the "traditional" teaching of pharmacology to the students of medicine at some faculties of medicine in Slovakia has changed. From mostly pharmacology and pharmaceuticals considerations towards a more problem-oriented education, with an introduction to the principles of rational prescribing, and connecting more closely teaching of pharmacotherapy with clinical education and training. Since the very beginning, active participation of clinicians have been sought and generously provided.

Results: This approach resulted in positive feedback from students and was positively rated also by teachers. Interestingly, the same trends have been confirmed and clearly supported by the results of the recent EACPT based exercise (Brinkman DJ et al., Clin Pharmacol Ther. 2018 Aug; 104(2): 317-325). The presentation will detail more than a decade long experience of our Institute. It had the advantage to develop anew the comprehensive program in (medical) pharmacology within the newly established program in general medicine for both Slovakian and international students.

Summary / Conclusions: We believe, the approach exploiting the natural connection of teaching (medical) pharmacology and prescribing competencies with the clinical knowledge and skills education should clearly be recommended.

\section{Oral Presentation}

\section{EACPT-1252}

Do students want new methods or do we just think they do?

O. Lapatto-Reiniluoto* ${ }^{* 1,2}$

${ }^{1}$ Helsinki University Hospital, HUS, ${ }^{2}$ Clinical Pharmacology, Helsinki University, Helsinki, Finland 


\section{Education}

Introduction: Introduction:In the Finnish medical curriculum modern teaching methods are in use but we don't know what kind of learning is preferred; new technology or more old-fashioned style.

Objectives: Objectives:Evaluate students' opinions about teaching and assessments

Methods: Methods: $6^{\text {th }}$ year medical students (106) were asked to fill in a questionnaire which was focused on the clinical pharmacology course including 12 sessions with either modern teaching methods or an oldfashioned lecture, depending on the teacher.

Results: Results:Assessment: $46 \%$ of the students (49/106) would prefer a written examination after the course like now. $29 \%$ (29) want to be questioned by a fellow student after every session and $22 \%$ (23) didn't want to be tested at all. 5 students suggested filling an electronic examination.

Learning: The majority $(76,72 \%)$ selected to attend all 12 lectures. 22 (21\%) students thought one or two main lectures would be enough. Only 5 students would skip all contact learning. 1 student suggested even more contact learning.

Digital material: 95 students (90\%) found the amount of digital material to be sufficient while 4 students thought it was too much. 6 students preferred more digital material.

Three main points: The issues mentioned ranged from a specific DDI to larger entities like polypharmacy with elderly. Only few students mentioned issues that were given in digital format during the course (e.g. youtube videos).

Summary / Conclusions: Conclusion:The students appreciated enthusiastic teachers and the teaching method was not the main point. Most students preferred a combination of lectures and virtual learning. This is understandable considering the amount of knowledge that should be learned. Yet, it is more stimulating to vary the method than to use a single style.

To our surprise, almost half of the students wanted a written examination. They justified it by saying that it is the best method to make oneself to study.

Most often mentioned issues from one lecture were the main points but also some details were often mentioned. This demonstrated the teacher how easily different stories and jokes will stay in memory.

\section{EACPT-1289}

The junior-adverse drug event managers

M. Reumerman ${ }^{*} 1,2$, J. Tichelaar ${ }^{1,2}$, M. Richir ${ }^{1,2}$, M. van Agtmael ${ }^{1,2}$

${ }^{1}$ Section pharmacotherapy, department of Internal Medicine, Amsterdam UMC, ${ }^{2}$ RECIPE (Research \& Expertise Center In Pharmacotherapy Education), Amsterdam, Netherlands

\section{Education, Pharmacovigilance}

Introduction: Adverse drug event managers have been successfully implemented in numerous hospitals in Denmark and have shown to ease the pressure for healthcare professionals (HCP) and increase the number of Individual Case Safety Reports (ICSR). Since screening for possible ADRs and reporting ICSR could be of great value for educating our future $\mathrm{HCP}$ on pharmacovigilance we started a junior-adverse drug event mangers (J-ADEMs) team

Objectives: This study aims to investigate the feasibility of a J-ADEMs team and evaluate the clinical and educational value of this intervention. Methods: The J-ADEMS program was set up as a prospective longitudinal intervention study to detect and report ADRs on and during hospital admission. The J-ADEM team consists of 1 st -6 th year medical students and function as a passive and active service. The team can be contacted by phone or email but also actively screen the internal medicine ward for ADRs. When an ADR is detected the J-ADEMs report the ADR to the Netherlands pharmacovigilance centre Lareb, answer all follow-up questions and update the electronic patient record. After the report all patients and HCP evaluate the J-ADEM team.
Results: From October 2017 to January 2018, 35 patients with 116 problem list symptoms were analyzed by the J-ADEMs team. Most patients (27) were actively screened by the J-ADEMs team while 8 signals of ADRs were received from HCP. Of the 116 problem list symptoms, 25 ADRs were reported to the pharmacovigilance centre of which 20 were classified as serious ADRs. This result showed a $300 \%$ increase in the number of ICSR in our hospital by managing only one ward. Most reports were on electrolyte disorders (11) or hematological disorders (6) and were related to diuretics (6) and acetylsalicylic acid (4). Patient satisfaction was 7.9 (1-10, min-max) and $87 \%$ of patients found it (extremely) relevant that an ICSR was made when the ADR caused the hospital admission. All $\mathrm{HCP}$ agreed the symptoms were related to the medicine and stated indifference (14) and ignorance (7) as the most important reasons they had not reported the serious ADR themselves.

Summary / Conclusions: The J-ADEMs team is an highly innovative healthcare improvement for hospitals. Not only does this team vastly increase the number of ICSR, it also has the opportunity to increase pharmacovigilance awareness in current and future HCP. Further plans are to expand the service to other wards, evaluate the educational value and analyze the quality of the students ICSR.

\section{EACPT-1306}

Clinical and long term effects of a real life problem based pharmacovigilance intervention.

M. Reumerman ${ }^{* 1,2}$, J. Tichelaar ${ }^{1,2}$, R. van Eekeren $^{3}$, M. Richir ${ }^{1,2}$, M. van Agtmael ${ }^{1,2}$

${ }^{1}$ Section pharmacotherapy, department of Internal Medicine, Amsterdam UMC, ${ }^{2}$ RECIPE (Research \& Expertise Center In Pharmacotherapy Education), Amsterdam, ${ }^{3}$ Netherlands pharmacovigilance centre Lareb, 's Hertogenbosch, Netherlands

\section{Education, Pharmacovigilance}

Introduction: The adverse drug reaction (ADR) reporting rate, especially under (specialist) nurses, is high (0.0008 reports year/nurse) and hinders optimal ADR monitoring. Since (specialist oncology) nurses (SONs) administer most drugs they could play a major role in reporting Individual case Safety Reports (ICSRs). Only several interventions in healthcare professionals have proven effective in increasing knowledge, however failed to produce clinical and durable effects.

Objectives: This study aims to investigate the clinical and long-term effects of a real life problem based pharmacovigilance oriented educational intervention in SONs.

Methods: A case-control intervention study in the three postgraduate schools who offer the course "prescribing qualification" for SONs was initiated. We incorporated a pharmacovigilance introduction lecture, ICSR assignment and group discussion on the self-reported ADRs in one of the postgraduate schools. Clinical value was assessed by analyzing the quantity and quality of reported ICSRs to the Netherlands pharmacovigilance centre Lareb, up till two years after the course. SONs competences on ADR-reporting were evaluated using three equestionnaires with one year interval up till two years after the course. Results: Eighty five SONs were included in the study ( $81 \%$ of all SONs in the three postgraduate schools) of which 68 were in the intervention group and 17 in the control group. Sixty-four ADRs were reported during the course and thus far twelve ICSRs were completed in the follow-up period. In the entire follow-up period no ICSR were reported by the control group. This result showed a 10 time increase when compared to Dutch physicians or 220 time increase when compared nurses. Based on the ClinDoc tool, $93 \%$ of the reports were well documented and found to be relevant for the Netherlands pharmacovigilance centre Lareb. SONs in the intervention group also showed a continuous significantly higher competence score up till two years after the course compared to the control group.

Summary / Conclusions: This is the first study that shows a significant and clinically relevant increase in the quantity of reported ADRs after a single educational intervention. The SONs not only reported more high 
quality ICSR, they also show a sustainable long-term increase in pharmacovigilance competences. Further research is needed to analyze the additional value of this intervention in other healthcare professionals.

\section{EACPT-1332}

Clinical pharmacology in continuous medical education (cme) in slovakia: quest for quality and european compatibility

J. Glasa $^{* 1,2,3,4}$, H. Glasova ${ }^{1,3}$, K. Soboňová ${ }^{5}$

${ }^{1}$ Institute of Pharmacology and Clinical Pharmacology, ${ }^{2}$ Institute of Health Care Ethics, Slovak Medical University in Bratislava, ${ }^{3}$ Slovak Society of Clinical Pharmacology, b.o. Slovak Medical Association , ${ }^{4}$ Accreditation Council of Slovakia for Continuous Medical Education, Bratislava, ${ }^{5}$ Department of Clinical Pharmacology, Faculty Hospital in Nové Zámky, Nové Zámky, Slovakia

\section{Education}

Introduction: Clinical Pharmacology $(\mathrm{CP})$ is a fully recognized medical specialty within the health care system in Slovakia (SK; since 1979). A physician obtains the specialization degree in $\mathrm{CP}$ after completing, at a minimum, 4-years training period.

Objectives: Its first part comprises of a 2-years training in internal medicine (IM) ("internist stem"), which includes brief training in other disciplines (e.g. surgery, obstetrics - gynaecology, intensive care, general practice). It is concluded by a state board exam ("Preliminary Examination in IM"). The second part consists of 2years training in CP. Both parts must be completed in compliance with the state-accredited program. After passing the State Board Exam in CP, the CP full specialization degree is awarded. Then, $\mathrm{CP}$-ist is able to practice independently (open $\mathrm{CP}$ office or become head of a CP department).

Methods: Already during his/her specialization studies, a future CPist is expected to take part in CME activities in $\mathrm{CP}$ and in related disciplines (CPD system is just being developed in SK to complement the present $\mathrm{CME}$ activities). After specialization, $\mathrm{CP}$-ist enters the compulsory CME system to earn the prescribed number of CME credits to keep his/her qualification valid. The CME credits are automatically collected via an internet portal and numbers earned checked in 5-years intervals by the Slovak Medical Chamber (SMC). CME activities may be organised by hospitals, specialised education companies (focusing on $\mathrm{CME} / \mathrm{CPD}$ ), and specialised SMC sections, but most of them are set up by the Medical Societies of the Slovak Medical Association (SMA). Slovak Society of Clinical Pharmacology (SSCP) is an SMA (and a founding EACPT) member. The quality of the credited CME activities is closely monitored by the Accreditation Council of Slovakia for CME (ACS CME). The SSCP and ACS CME delegate took part in the founding session of the UEMS-EACCME Section of CP (2016).

Results: SSCP, on her part, organizes a majority of regular CMEs in CP (on annual basis): national CP conference, GCP workshop, 'new medicines' conference, and lecture series on 'innovative medical therapies'. Besides CP-ists, these activities are popular among, and well attended also by doctors of other specialties, especially those "stemming" from internal medicine, pharmacists, and by medical students.

Summary / Conclusions: Thus, the CP message is spread to broader CME audiences.

\section{EACPT-1333}

Four decades of clinical pharmacology as a medical specialty in slovakia (1979-2019)

J. Glasa* 1, 2, 3, J. Holomáńn ${ }^{1,3}$, H. Glasová ${ }^{1,3}$, K. Soboňová ${ }^{1,4}$

${ }^{1}$ Slovak Society of Clinical Pharmacology, b.o. Slovak Medical Association , ${ }^{2}$ Institute of Health Care Ethics, ${ }^{3}$ Institute of Pharmacology and Clinical Pharmacology, Slovak Medical University in Bratislava, Bratislava, ${ }^{4}$ Department of Clinical Pharmacology, Faculty Hospital in Nové Zámky, Nové Zámky, Slovakia

\section{Education, Professional development, Other}

Introduction: Clinical Pharmacology $(\mathrm{CP})$, as a medical speciality to be integrated into the health care provision $(\mathrm{HCP})$, has been officially established in Slovakia (SK) by acceptance of its conceptual document (...conception") by the SK Ministry of Health in 1979.

Objectives: It followed similar international developments and was a marked success of long-term efforts by a handful of devoted professionals stemming from various specialities of internal medicine (IM). Thus, SK's $\mathrm{CP}$ was understood from its beginning as a predominantly clinical, patient care-oriented discipline.

Methods: In an interdisciplinary manner, it helped to keep SK's medicine abreast with international developments of 1980-ies/1990-ies and to launch its modernisation after the fall of the 'Iron Curtain' in $\mathrm{CE}$ Europe. The areas developed as priorities in SK's CP first decades include (incl.): implementation of $\mathrm{CP}$ into the SK's HCP system, both in the hospital and in outpatient settings; clinical use of Therapeutic Drug Monitoring (TDM), incl. development of original pharmacokinetics PC programs; implementation of rational pharmacotherapy (RPT) in HCP; RPT in renal/hepatic insufficiency; RPT in neonatal, paediatric, and geriatric care; establishment of national medicinal drug policies and legislation (Law 140/1998 Coll. - the first SK's law on drugs), incl. implementation of pharmaco-economics; methodology development and help in establishing the national diagnostic and therapeutic standards, incl. national guidelines; implementation of contemporary clinical trials (CTs) methodology, incl. development of original guidelines for CTs in different medical specialities, and integration of SK's CTs capacities into existing international frameworks.

Results: Building up CP HCP capacities within the SK's health care system (HCS) went in parallel with establishment of an integrated, state-accredited system of physicians' postgraduate education and training in CP (1993 - recognition of CP as a subspecialty of IM or paediatrics, 2004 - as an independent medical speciality, 2007 -CP's prescribing authorisation extended to that of a fully qualified internist), supplemented by CME system for CP specialists.

Summary / Conclusions: Despite some recent setbacks and competence struggles (esp. with 'clinical' pharmacy), SK's CP is poised to develop its already well-established position within the SK's HCS, and hopefully flourish in the difficult decades ahead.

\section{Oral Presentation}

\section{EACPT-1383}

The inter-professional student-led medication review program. M. Reumerman ${ }^{*}{ }^{1,2}$, M. Richir ${ }^{1,2}$, P. Domela Nieuwenhuis ${ }^{1}, \mathrm{H}$. Daelmans $^{3}$, H. Springer ${ }^{4}$, M. Muller ${ }^{5}$, M. van Agtmael ${ }^{1,2}$, J. Tichelaar $^{1,2}$ ${ }^{1}$ Section pharmacotherapy, department of Internal Medicine, Amsterdam UMC, ${ }^{2}$ RECIPE (Research \& Expertise Center In Pharmacotherapy Education), ${ }^{3}$ VUmc School of Medical Sciences, ${ }^{4}$ Hogeschool Inholland, ${ }^{5}$ Geriatric medicine, department of Internal Medicine, Amsterdam UMC, Amsterdam, Netherlands

\section{Education, Geriatric treatment, Professional development}

Introduction: Rational prescribing and evaluating medication safety is a fundamental skill for every prescribing healthcare professional. However, most graduated healthcare professionals make frequent prescribing errors. Inter-professional collaboration between physician, specialist nurses and pharmacists could improve medication safety.

Objectives: To prepare students for their role in medication evaluation we started an context based inter-professional student-led medication review program (ISP).

Methods: The ISP was integrated as part of the memory clinic within the center for geriatric medicine Amsterdam. Every week a varying team of bachelor and master medical, pharmacy, physician assistants or nurse practitioners students evaluated the medication of patients who visited this outpatient clinic. After taking the medication history and performing a 
medication review the student team formed their medication advice. After a short meeting with a clinical pharmacologist, students pitched their medication advice in a multidisciplinary meeting and updated the electronic patient system. After the ISP pilot, the program was evaluated using a digital survey and all medication advices where analyzed on number of (applied) advices. Results: During the pilot 31 students participated in project. Most students had never participated in an inter-professional setting, had never taken a medication history or had conducted a medication review in a real patient. During the 24 visits the students reviewed 155 medications and formed 62 medication advices. In total 51 advices $(82 \%)$ were directly applied in clinical practice. Medication advice were predominantly given on vitamin supplementation, gastric protection and antihypertensive medication. Students valued the ISPpilot 7.7 (1-10 $\mathrm{min} / \mathrm{max})$, found it educational $4.5(1-5 \mathrm{~min} / \mathrm{max})$, and thought it would contribute to their future inter-professional relationships. Additionally most students found getting insight into the different perspectives and qualities of their inter-professional colleagues most interesting.

Summary / Conclusions: The ISP is an innovative healthcare improvement for hospitals. Most formulated advices were directly incorporated and could prevent future medication related harm. The ISP also offers students a first opportunity to work in an inter-professional manner and get insight into the perspectives and qualities of their future colleagues. An intervention study to analyze the patient outcomes has started.

\section{EACPT-1385}

Clinical results of an inter-professional student-led medication review program

M. Reumerman ${ }^{1,2}$, T. van den Beukel ${ }^{* 2,3}$, J. Tichelaar ${ }^{1,2}$, R. Sultan ${ }^{2}$, H. Daelmans $^{4}$, H. Springer ${ }^{5}$, M. Muller ${ }^{3}$, M. Richir ${ }^{1,2}$, M. van Agtmael ${ }^{1,2}$

${ }^{1}$ RECIPE (Research \& Expertise Center In Pharmacotherapy Education), ${ }^{2}$ Section pharmacotherapy, department of Internal Medicine, ${ }^{3}$ Geriatric medicine, department of Internal Medicine, Amsterdam UMC, ${ }^{4} \mathrm{VUmc}$ School of Medical Sciences, ${ }^{5}$ Inholland Hogeschool, Amsterdam, Netherlands

\section{Education, Geriatric treatment, Professional development}

Introduction: Medicine is becoming increasingly specialized with specific referral questions and clearly defined outpatient clinic goals. An inter-professional student-led medication review program (ISP) team reviewing medication in geriatric patients has shown promising results. Besides getting trained in practising medication evaluation this team could increase medication safety.

Objectives: We analyzed the added value of an ISP-team on top of standard care in regard to adverse drug reactions (ADRs), potentially inappropriate prescriptions (PIPs) and potential prescribing omissions (PPO). Methods: We performed a randomized controlled trial where a varying team of healthcare students (ISP-team) review two of the four patients visiting the memory clinic. After a medication review the students discuss their findings in a multidisciplinary meeting and update the electronic patient system. After each patients visit, medication advices of the control group (physician without ISP-team) and intervention group (physician with ISP-team) are compared to the START-STOPP criteria. A followup medication overview is requested from the local pharmacist to analyze applied changes after one month.

Results: So far 37 patients (23 control and 14 intervention) have been analyzed. ADRs were more frequently reported by the ISP $(36 \%)$ than by the physician (19\%). PIPs and PPOs were more often correctly addressed in the correspondence letter in the intervention group (PIPs 50\%, PPOs 79\%) than in the control group (PIPs 40\%, PPOs 60\%). In $14 \%$ and $36 \%$ the physician revised their advice regarding PIPs and PPOs because of the ISP-advice. These advices were related to stopping acetylsalicylic acid and tamsulosin and starting vitamin D and statin treatment. During the outpatient clinic visit $13 \%$ of PIPs and $83 \%$ of PPOs were directly changed, instead of giving the advice to the general practitioner. One month after the correspondence letter $63 \%$ of PIPs and $92 \%$ PPOs were corrected.
Summary / Conclusions: These preliminary results show that the addition of an ISP-team on top of standard care is effective in increasing the number of ADRs and reducing the number of PIPs and PPOs in the outpatient clinic correspondence letters. Most advices have actually reached the patient and could prevent future medication related harm. Since the study is still in progress we will continue including patients and show 3-month follow-up data regarding treatment satisfaction and $\mathrm{ADR}$ outcome.

\section{EACPT-1391}

Majority dutch registrars in general practice find postgraduate training in prescribing insufficient

R. Van Unen ${ }^{*}$, J. Tichelaar ${ }^{1}$, M. Richir ${ }^{1}$, M. van Agtmael ${ }^{1}$

${ }^{1}$ Internal Medicine section pharmacotherapy, Amsterdam University Medical Center, Amsterdam, Netherlands

\section{Education}

Introduction: A possible solution for reducing prescribing errors is pharmacotherapy education. Learning to prescribe already begins during the medical study but is really formed and reinforced during the registrar period. However, there are strong indications that junior doctors experience a lack in postgraduate pharmacotherapy education. It's unclear if and in which area a deficit is experienced.

Objectives: This study aims to gain insight in the views of final year Dutch registrars in general practice regarding their postgraduate pharmacotherapy teaching program.

Methods: Seven (of eight) Dutch universities providing a postgraduate teaching program in general practice participated in this study. All 607 final (3rd) year registrars in general practice received an email that contained a link to an internet-based questionnaire. The questionnaire contained 2 sections: (1) opinion on pharmacotherapy education and (2) an inventory of desired teaching methods. Reminders were send after 2 weeks.

Results: 259 (43\%) registrars completed the questionnaire. $85 \%$ of the registrars were of the opinion that in general the amount of pharmacotherapy education during the registrars period is too little or far too little. The areas where this shortage particularly is experienced are (1) assessing interactions and contraindications $(85 \%)$, (2) choosing a rational drug treatment $(55 \%)$ and (3) how to deal with (possible) side-effects $(54 \%)$. The top 3 desired teaching methods are structured case-based discussions under the direction of an experienced general practitioners and pharmacist $(61 \%)$, direct feedback from pharmacist on detected prescribing errors $(57 \%)$ and e-learning modules (53\%).

Summary / Conclusions: The vast majority of registrars in general practice in the Netherlands have a need for more pharmacotherapy education during their registrar period. In our opinion blended learning methods, such as combinations of case based-discussions and e-learning programs can play an important role to bridge this gap. The role of clinical pharmacologists must be increased in the postgraduate pharmacotherapy education of registrars in general practice in the Netherlands, Also further research is needed to gain insight in the pharmacotherapeutic skills of registrars in general practice.

\section{Oral Presentation}

\section{EACPT-1338}

The impact of using virtual patients in pre-clinical pharmacology teaching

P. Nicolaou ${ }^{*}{ }^{1}$, M. El Saifi ${ }^{1}$, P. McCrorie ${ }^{1}$, S. Nicolaou ${ }^{2}$

${ }^{1}$ Medical School, ${ }^{2}$ Department of Life and Health Sciences, University of Nicosia, Nicosia, Cyprus

\section{Education}

Introduction: Traditionally, medical pharmacology has been taught in isolation of other pre-clinical disciplines, using lecture-based learning. While didactic teaching may promote passive learning, educators have advocated its merits in conveying consistent information. Virtual patients (VPs) have recently been 
used to promote active learning. VPs are interactive computer-based patient scenarios, where the learner can choose how to proceed in the care of a VP. Interestingly, VPs are generally used in clinical years to cultivate the skills needed by a junior doctor. Conversely, the use of VPs in pre-clinical disciplines, including pharmacology, is not well-described in the literature.

Objectives: To investigate the potential beneficial effects of using VPs, as compared to problem solving, using case-based discussion (CBD) and single best answer (SBA) questions, in pharmacology tutorials, in pre-clinical years. Methods: All year 3 medical students ( $n=31)$, in an undergraduate 6-year medical programme at the University of Nicosia, were invited to participate in this naturalistic, prospective study. The study was carried out during the Systematic Pharmacology I course, delivered weekly, using lectures ( 3 hours), followed by a one-hour tutorial. Students were taught during the first 4 tutorials using $\mathrm{CBD} / \mathrm{SBAs}$ before their midterm exam and VPs for the next 6 tutorials leading up to the final exam. The impact of VPs was assessed by: 1) performance in the midterm vs the final examination and 2) student satisfaction and perceptions, using a validated, modified questionnaire (Sobocan \& Klemenc-Ketis, 2017). The study was approved by the Cyprus Bioethics committee.

Results: There was significant improvement in student performance in the final $(\mathrm{KR} 20=0.75)$, as compared to the midterm $(\mathrm{KR} 20=0.68)$ examination (Midterm: 57.6\%; Final: 62.8\%; $\mathrm{p}=0.04$ ). Students rated facilitation of learning, using a 5-point Likert scale, to be higher in VP tutorials, as compared to CBD/SBAs (Control: 3.40 ; VPs: 3.83 ; $\mathrm{p}=0.07$ ). A noharm effect of VPs was noted on the remaining questionnaire domains: acquisition/maintenance of knowledge, authenticity of learning, disadvantages of learning. Qualitative feedback from faculty and students indicated that VPs were well-received.

Summary / Conclusions: VPs may be effectively integrated in pre-clinical pharmacology teaching, with potential benefits on pharmacological knowledge and facilitation of learning, which merit further investigation.

\section{Oral Presentation \\ EACPT-1350}

The attitude of clinical pharmacology and therapeutics educators towards sharing and creating open educational resources: an international survey

M. J. Bakkum ${ }^{*}{ }^{1,2}$, J. Tichelaar ${ }^{1,2,3}$, M. C. Richir ${ }^{1,2}$, M. A. van Agtmael $^{1,2,3}$

${ }^{1}$ Department of Internal Medicine, section Pharmacotherapy, Amsterdam UMC, Vrije Universiteit Amsterdam, ${ }^{2}$ Research and Expertise Centre in Pharmacotherapy Education (RECIPE), Amsterdam, Netherlands, ${ }^{3}$ European Association for Clinical Pharmacology and Therapeutics (EACPT) Education Working group, Frankfurt, Germany

\section{Education}

Introduction: It was recently shown that European final year medical students feel unprepared for the prescribing task ahead of them and, indeed, lack the knowledge and skills to safely prescribe medicine. In addition, remarkable differences were found in the level and quantity of clinical pharmacology and therapeutics (CPT) education between different countries. Therefore, improvement and harmonization of European CPT education is urgently required. The EACPT education working group has established eleven recommendations to harmonize and modernize European prescribing education. One of the most promising recommendations is for schools to utilize more online learning resources and share these on a national or international level. The Amsterdam UMC (VUmc) and Research and Expertise Centre in Pharmacotherapy Education (RECIPE) have conducted a systematic literature review on the current use and effects of digital learning resources (DLRs) in CPT education. It shows that DLRs are commonly used and effective in teaching the knowledge, skills and attitudes required for safe and effective prescribing. However, the included DLRs were mostly produced and used locally, very few were openly available to other universities or actively shared. In addition to being essential in collaboratively improving European CPT education, openly sharing educational resources is known to increase cost-effectivity, quality and timely improvement.

Objectives: We hypothesize that the quality of European prescribing education will benefit from actively and openly sharing learning resources. The aim of this study is to assess the attitude and readiness of European CPT educators towards sharing existing learning resources and creating new ones and identify the potential barriers towards doing so. We aim to establish the wishes (e.g. for content, structure) and requirements for an online sharing platform and identify learning resources that may be shared on such a platform.

Methods: An online survey was sent out to all known principal CPT educators at medical schools in Europe.

Results: Results of this fourth international EACPT education working group study will be available at EACPT 2019 Stockholm.

Summary / Conclusions: The results will be used to create an international platform for sharing educational CPT resources.

\section{Oral Presentation}

\section{EACPT-1357}

Flipped class teaching for clinical pharmacology and therapeutics E. J. Sanz ${ }^{*}$, E. Puerta del Castillo ${ }^{1}$, M. Garcia-Saiz ${ }^{1}$, C. RodriguezJimenez ${ }^{1}$, C. Boada ${ }^{1}$

${ }^{1}$ Physical Medicine and Pharmacology, Univeristy of La Laguna, La Laguna, Spain

\section{Education}

Introduction: Traditional lectures and Multiple Choice Questions exams are the most common way of teachin Clinical Pharmacology and Therapeutics across Europe. The proposed method, applicable to large class rooms ( $>100$ students) allow theachers to direct the learning of the students. Students have to have an active participation both prior and during the lectures, increasing attendance, attention, retention and knowledge.

Objectives: To explore the possiblity of incoeporating the Flipped classroom techniques in the education of last year pre-graduated students for the teaching of Clinical Pharmacology and skills to prescribe correctly. Methods: Students have to watch a video (shorter than 30 minutes) with the theoretical content of the lecture, that includes several questions embedded on it. They get scores depending if they watch the video BEFORE the lecture and the scores of the questions of the video. The procedure is control by an automatic package included in the MOODLE plataform of the Virtual Campus and/ or using the aplication "Edpuzzle".

Lectures are devoted to active discussion in the class room with students around practical cases related to the topic. Students must participate answering questions on their mobile devices, by using the application "Wooclap". The discussion among the students is fostered during the lectures, and several rounds of questions are use, depending on the answers of the students.

Results: More than 150 students have participated during the last three academic years (2016-9) in this type of education. The participation on these activities was granted with up to $20 \%$ of the final scores. Mean scores in the subject increased between 20 and 30\% during the three academic years, as compare with previous years with standard academic lectures. The satifaction of the students was much higher with more than $90 \%$ scoring over 8 points in the scale of satisfaction.

Time devoted to the preparation of the educational Materia increased in more than $50 \%$ from the standard time devoted to the preparation of the lectures during the first year.

Summary / Conclusions: The introduction of Flipped class room activities in an, otherwise classical education environment, is feasible, produce better results in terms of academic results and satisfaction, both for teachers and students. However the time devoted to the preparation of the educational materials and the control of students increase in more than $50 \%$, specially the first year of introduction. 
EACPT-1392

Study on expected participant enrollment in prospective interventional studies and analysis on formal sample size calculation M. Marfá* ${ }^{1}$, P. Alcubilla ${ }^{1}$, M. V. De Dios ${ }^{1}$, B. Gómez ${ }^{2}$, G. Calvo ${ }^{1}$

${ }^{1}$ Clinical Pharmacology, ${ }^{2}$ Pharmacy department, Hospital Clínic i Provincial de Barcelona, Barcelona, Spain

\section{Education, Patient empowerment, Other}

Introduction: Hospital Clínic of Barcelona Research Ethics Committee (HCB-REC) approves around 900 studies per year. In the prospective and interventional studies, patients undergo complementary explorations or receive a different treatment from the usual clinical practice, which in turn could imply a potential added risk or burden. In this regard, it is important to assess study sample size based on expected differences in outcome variables, in order to avoid too many (or too less) patients being submitted to potential risks and obtain reliable results.

Objectives: Quantify the estimated patient enrollment in any prospective interventional study in a 18-month period at Hospital Clínic de Barcelona (HCB). Quantification of patients enrolled in prospective studies with or without formal sample size calculation, as a surrogate for robust study design.

Methods: We reviewed study protocols of all prospective interventional studies approved by HCB-REC or by another REC with HCB patients from January 2017 to July 2018. Information about type of study, number of participating centres, estimated sample size, sample size calculation and reasons provided in case of not calculating it, were extracted for analysis.

Results: From a total of 1.427 studies approved during this period, 875 were prospective and interventional. Assuming that every participating centre contributes equally to patient enrollment, the overall estimation is of 46.735 participants from HCB. This amount represents a 10,38\% from the 450.000 patients visited during this time period in HCB. We observed $369(43,20 \%)$ studies involving 25.168 patients $(53,8 \%$ from 46.735 at HCB) without a formal sample size calculation, $186(50,41 \%)$ were pilot/ feasibility studies $(\mathrm{N}=13.303)$, and in $183(49,59 \%)$ reason for not calculating sample size was not provided $(\mathrm{N}=11.865)$.

Summary / Conclusions: - The ratio of patient enrollment in prospective interventional studies in our centre is high. However, it could be understandable given we have analyzed the data in a tertiary hospital where clinical research investment is much higher than in other centres.

- We have realised that more than half of patients are enrolled in studies without formal sample size calculation. These studies give difficult interpretation results and more probability of bias, putting in doubt the balance between risk and benefit of our patients.

\section{EACPT-1403}

Phase i clinical trials evaluated by the research ethics committee of vall d'hebron university hospital in 2017 and 2018

L. M. Leguízamo Martínez ${ }^{*}$, A. Rodríguez ${ }^{2}$

${ }^{1}$ Clinical Pharmacology Service, Vall d'Hebron University Hospital,

${ }^{2}$ Clinical Pharmacology Service, Vall d'Hebron Research Institute (VHIR), Barcelona, Spain

\section{Education, Professional development, Other}

Introduction: In the last years the number of phase I clinical trials (CT) submitted for evaluation to the Research Ethics Committee (REC) of the Vall d'Hebron University Hospital (HUVH, Barcelona, Spain) have increased. Objectives: To describe phase I CT submitted from January 2017 to December 2018 to the REC of HUVH.

Methods: The main source of information used for the description of the CT was the evaluation reports made by the members of the Clinical Pharmacology Service. The documentation of the Part I of the CT application was also used.
Results: From 2017 to 2018, 222 CT were submitted for evaluation. $54(24 \%)$ of all CT evaluated were phase I CT: 29 phase I and 25 phase I/II. $22(41 \%)$ were first-in-human $94.4 \%$ were multicenter and international and all had commercial promotion. $54.7 \%$ evaluated the administration of a single drug, $37 \%$ two drugs, $5.6 \%$ three drugs and $2.7 \% \geq$ four drugs. $51.8 \%$ of the drugs were administered in monotherapy, $35.2 \%$ in combination and $12.6 \%$ in monotherapy and in combination. $18.6 \%$ of the drugs were authorized. The most frequent pharmacological groups were L01XC (monoclonal antibody: 29.63\%), L01XE (protein Kinase inhibitors: $14.8 \%$ ) and L01XX (other antineoplastic agents: $14.8 \%$ ). The most involved Clinical Management Units were Medical Oncology $(72.2 \%)$ and Hematology (14.8\%). The median of the sample size of the CT was 133 (range from 20 to 460). $87 \%$ of the patients included were adults and $96.3 \%$ of both sexes. Advanced solid tumors were the most studied pathology $(33 \%)$. The main objective of the CT was efficacy and safety $(66.7 \%)$ and only safety $(33.3 \%) .88 .9 \%$ had a design with dose escalation (classic design of $3+3(68,8 \%)$ and Bayesian design (29.2\%). 39\% had an adaptive design (6 in 2017 and 15 in 2018) and the most frequent type of adaptation was the treatment arm selection $(25.9 \%) .12(22 \%)$ of all trials met the definition of complex CT : 3 basket in 2017, 1 platform and 8 basket in 2018 . Summary / Conclusions: All Phase I CT submitted to the REC of HUVH had commercial sponsors. Majority of CT were from Oncology Department. The most frequent evaluated pathology and pharmacological group were advanced solid tumors and monoclonal antibodies, respectly. Most of patients were adults. The complexity of the CT increased between 2017 and 2018.

\section{EACPT-1421}

Predictors of influenza vaccination among the elderly in greece C. Pourzitaki ${ }^{*}{ }^{1}$, I. Dardalas ${ }^{1}$, G. Manomenidis ${ }^{2}$, F. Malliou ${ }^{1}$, G. Papazisis $^{1}$, D. Kouvelas ${ }^{1}$, T. Bellali ${ }^{3}$

${ }^{1}$ Department of Clinical Pharmacology, Faculty of Medicine, School of Health Sciences, Aristotle University of Thessaloniki, Greece, Thessaloniki, ${ }^{2}$ Nurse Infection Prevention and Control Specialist, General Hospital of Ptolemaida, Ptolemaida, ${ }^{3}$ Faculty of Nursing, "Alexander" Technological Educational Institute of Thessaloniki, Thessaloniki, Greece

Education, Geriatric treatment, Patient empowerment

Introduction: The elderly are vulnerable to influenza and its side-effects. Protection against the influenza virus and its transmission in this high-risk group of the population can be achieved by the influenza vaccine.

Objectives: To understand and analyze the attitudes, knowledge and behavior of people over the age of 60 on influenza vaccination and suggest measures to increase the vaccination uptake.

Methods: The survey included people over the age of 60 who can receive an influenza vaccine. A self-referral questionnaire was used with questions about their demographic characteristics, health status and two questionnaires, the first based on the Theory of Planned Behavior and the second on the MoVac-flu and MovAd scale. The analysis of the data was performed with the statistical program IBM SPSS.

Results: Study population included 318 participants with mean age of 70.7 years. About $56,6 \%$ of the sample had received a flu vaccine this year while $50,8 \%$ received it in the previous years. Behavioral, normative and control beliefs were correlated with promotion of the vaccine uptake and increased intention score was associated with increased probability of vaccination. Higher age and more frequent visits to the doctor were found to be vaccination promoters.

Summary / Conclusions: As a small proportion of those over 60 years old have been vaccinated with the influenza vaccine, while Greece counts more than 40 deaths in the middle of the influenza season, this finding is worrying about the impact of a future outbreak and indicates the need for more, better and evidence based information from the healthcare 
professionals to the elderly, to achieve greater vaccination coverage of the community.

\section{EACPT-1445}

Sources and use of drug information among physicians in sweden - a descriptive study covering five counties in sweden

P. Seidel ${ }^{*}$, B. Rolander ${ }^{2}$, S. Hägg ${ }^{3}$, A. Kling ${ }^{1}$

${ }^{1}$ Department of Pharmacology and Clinical Neurosciences, Umeå,

${ }^{2}$ Futurum - Academy for Healthcare, Jönköping, ${ }^{3}$ Department of drug research, Institution of Medicine and Health, Linköping, Sweden

\section{Education}

Introduction: Rational and cost-effective prescription of drugs requires up-to-date and readily accessible drug information. Few academic studies published have examined whether the drug information produced is compatible with the drug information requested by the physicians.

Objectives: The aim with this descriptive study was to examine what kind of information about drugs physicians in Sweden ask for, what kind of drug information they get and what sources of drug information they use.

Methods: A web-based questionnaire was sent by e-mail to 12011 physicians in five regions in Sweden (Västerbotten, Västernorrland, Östergötland, Jönköping and Västra Götaland). The first part contained nine questions about what kind of drug information physicians search for, what sources of information they use, what kind of information they get at their work and who give the information. The second part of the questionnaire contained eleven demographic questions.

Results: Of the 12011 who got the questionnaire $32,9 \%$ returned an answer. After excluding 14 responders that didn't belong to the target group (i. e. appeared to not be physicians), we had $32,8 \%$ responders. About $85 \%$ of the physicians reported that they search for information about drugs frequently (at least every week). A total of $75 \%$ search for information about pharmacological treatments, $62 \%$ about adverse effects and $45 \%$ about drug interactions. The group of drugs that most prescribers searched information about within the last six months was antibiotics $(83,6 \%)$, analgetics $(69 \%)$, anticoagulants $(67 \%)$, cardiovascular drugs (59\%) and antidepressants (59\%). The source of information prescribers use the most is the Swedish Pharmacopeia Drug Information (FASS), $93 \%$ had used it frequently within the last six months.

Summary / Conclusions: The need for useful and reliable information among physicians is huge and an important question with implications for management of drugs and patients' safety (e. g. adverse effects and drug interactions).

\section{General clinical pharmacology}

\section{EACPT-1011}

Effectiveness of beclomethasone solution for management of gastrointestinal graft-versus-host disease in children

H. Rodriguez Ramallo* ${ }^{1}$, N. Báez Gutierrez ${ }^{1}$, M. Ladrón de Guevara García $^{1}$

${ }^{1}$ Farmacia Hospitalaria, Hospital Universitario Virgen del Rocío, Sevilla, Spain

\section{Other}

Introduction: Gastrointestinal graft-versus-host disease (GI-GVHD) remains a major cause of morbidity and mortality after allogeneic hematopoietic stem cell transplantation in children; treatment with prolonged high-dose systemic corticosteroids (SDS) has limited success and considerable toxicity. Beclomethasone dipropionate (BDP) is a potent topically active steroid which has shown efficacy treating GI-GVHD. Data published about BDP effectiveness on GI-GVHD is limited, especially on children.

Objectives: To evaluate the effectiveness of formulated BDP $0.5 \mathrm{mg} / \mathrm{ml}$ olive oil solution for the treatment of GI-GVHD in children.
Methods: Retrospective observational study of children treated with BDP solution within 2013- 2017.

Data collected: demographics, diagnosis previous to allogenic transplantation, GI-GVHD confirmed by biopsy, dose and posology, treatment duration, concomitant use of SDS, partial/total withdraw of SDS using BDP, response to treatment, survival at 200 days after transplantation. BDP was formulated as a $0,5 \mathrm{mg} / \mathrm{ml}$ olive oil solution at our center.

The information was obtained from the corporate prescription program Athos Prisma ${ }^{\circledR}$.

Results: Clinical data was obtained for 22 patients, $(80 \%$ males, average age 10 years range (1-17)); 2 patients were excluded because the absence of data. Diagnosis: Acute lymphoid leukemia 10 patients, acute myeloid leukemia 4 patients, myelodysplastic syndrome 2 patients, no-hodking lymphoma 2 patients, hodking lymphoma and refractory immunodeficiency one patient each.

GI-GVHD was confirmed on $50 \%$ of patients by biopsy, the rest were treated based on symptomatology.

Patients were treated with $2 \mathrm{mg}$ of BDP administered every $6 \mathrm{~h}(45 \%), 8 \mathrm{~h}(45 \%), 12 \mathrm{~h}(10 \%)$.

Median BDP treatment duration: 97.5 days (range 29-274).

$70 \%$ of patients were treated with SDS + BDP (55\% stopped requiring SDS, $5 \%$ reduced the dose required) $40 \%$ were treated with BDP as monotherapy.

Complete response ratio: $70 \%$, partial response ratio: $0 \%$.

Survival at 200 days after transplantation ratio: $90.9 \%$

Summary / Conclusions:

Real-life BDP effectiveness for treatment GI-GVHD in children as monotherapy and in combination with SDS was acceptable and similar to the published data, studies with a larger sample size are needed to determine real life BDP effectiveness. BDP also showed reasonable results at replacing completely SDS. A prolonged BDP therapy could be a feasible alternative to SDS.

\section{Oral Presentation}

\section{EACPT-1036}

The pharmacotherapy team: a novel multidisciplinary strategy using participatory action research to improve appropriate in-hospital prescribing

R. F. Mahomedradja ${ }^{*}{ }^{1}$, J. K. Bekema ${ }^{1}$, D. J. Brinkman ${ }^{1}$, K. C. Sigaloff ${ }^{1}$, M. A. Kuijvenhoven ${ }^{2}$, M. A. van Agtmael ${ }^{1}$

${ }^{1}$ Section Pharmacotherapy, dept. Internal Medicine, ${ }^{2}$ Clinical Pharmacology and Pharmacy, Amsterdam UMC - location VUmc, Amsterdam, Netherlands

\section{Other}

Introduction: Inappropriate prescribing leads to medication-related harm, increase in hospital admissions and healthcare costs. Due to the complexity of the prescribing process, improvements are more likely to be effective if being multidisciplinary, multifaceted and tailor-made. Therefore Participatory Action Research (PAR), a research approach that involves relevant stakeholders to improve complex problems by tailormade interventions, could be useful.

Objectives: To explore if a multidisciplinary team using PAR is effective in reducing in-hospital prescribing errors (PEs).

Methods: A prospective pre- and post-intervention study was performed between June 2015 and April 2018 involving 12 clinical wards of Amsterdam UMC - location VUmc. A multidisciplinary 'pharmacotherapy team' was compiled, consisting of two physicians/clinical pharmacologists, a hospital pharmacist, an internist and a quality consultant. The team identified relevant stakeholders (specialists, junior doctors and nurses) per ward and coordinated a 10-month intervention. Interventions focused on organizational (e.g. redesigning working process), disciplinal (e.g. improving guideline accessibility) and individual 
aspects (e.g. education). Medications orders (MOs) of patients admitted to these wards were screened for PEs using a structured medication review. Identified PEs were categorized by the team according to the NCCMERP classification.

Results: 273 patients with 2683 MOs were included in the pre- and 178 patients with 2233 MOs in the post-intervention period. The total of MOs containing $\geq 1 \mathrm{PE}$ decreased from $13.6 \%$ (SD 17.6) during pre-intervention period to $12.5 \%$ (SD 14.8) versus the post-intervention period, although this difference was not statistically significant $(\mathrm{p}=0.69)$. There were differences in reduction between medical and surgical wards ranging from $1.0-6.0 \%$. During the pre- and post-intervention period, incorrect dosage (respectively $38.3 \%$ and $53.8 \%$ of all PEs) and unknown indication (respectively $32.9 \%$ and $31.7 \%$ of all PEs) were the most common PEs.

Summary / Conclusions: A multidisciplinary team using PAR was not effective in overall reduction of in-hospital PEs, although there were differences in reduction between wards. Critical success factors were dedicated on-ward stakeholders. Specific focus on high risk patients or high risk medications in future studies might possibly have more impact.

\section{Oral Presentation}

\section{EACPT-1098}

Treprostinil iontophoresis in systemic sclerosis-related digital skin ulcers: a single ascending dose safety study

A. Guigui* ${ }^{*}$, M. Roustit ${ }^{1}$, C. Cracowski ${ }^{1}$, M. Beau-guillaumot ${ }^{1}, \mathrm{~S}$. Blaise $^{2}$, J.-L. Cracowski ${ }^{1}$

${ }^{1}$ Grenoble Alpes University, Inserm HP2, CIC 1406, Grenoble Alpes University Hospital , ${ }^{2}$ Grenoble Alpes University, Inserm HP2, Vascular medicine department, Grenoble Alpes University Hospital, grenoble, France

\section{Other}

Introduction: Digital skin ulcers are a severe complication of systemic sclerosis (SSc). The first-line treatment is intravenous iloprost, but it induces dose-limiting adverse events. Local administration of treprostinil through skin iontophoresis may be a safe alternative.

Objectives: We conducted a two-step, randomized, placebo-controlled single ascending dose study in healthy volunteers and patients with SSc-related digital ulcer (SSc-DU). We further explored the effect of the procedure on skin blood flow.

Methods: In a first group of healthy subjects, treprostinil and placebo iontophoresis were performed at 3 different locations (i.e. 6 skin sites): the sole of the foot, the anterior side of the leg, and the fingers. We used a $1 \mathrm{mg} . \mathrm{mL}^{-1}$ hydrogel of treprostinil, with cathodal current at 0.033 $\mathrm{mA} . \mathrm{cm}^{-2}$ during 20 minutes on the leg, and 120 minutes on the feet and fingers (then reduced to 60 minutes). We then randomly treated SSc-DUs in a 3/1 ratio with treprostinil and placebo, respectively. Iontophoresis was performed during 60 minutes. We used currents between 0.004 and $0.016 \mathrm{~mA} \cdot \mathrm{cm}^{-2}$, and concentrations from 0.1 to $1 \mathrm{mg} \cdot \mathrm{mL}^{-1}$. All adverse events were recorded and graded, while skin microvascular blood flux was recorded with laser speckle contrast imaging and expressed as cutaneous vascular conductance (CVC).

Results: Among the 12 healthy volunteers, we observed local adverse effects: skin pain, burns, erythema and pruritus. They were graded 1 or 2 on the 5-point CTCAE scale, and occurred at the active or passive, treprostinil or placebo sites. Treprostinil iontophoresis significantly increased skin blood flow on the leg (AUC0-4h of CVC was 88460 \pm 6436 vs $12730 \pm 3397 \%$ BL.min, respectively; $p=0.002)$ and on the sole of the foot (AUC0-3h at $20124 \pm 6119$ vs $3142 \pm 3036 \%$ BL.min; $p=0.01$ ) with a trend on the finger (AUC0-3h was $10773 \pm 4888$ vs $5402 \pm 4343$ \%BL.min; $\mathrm{p}=0.093$ ). Among five patients with SSc-UD, two resolutive local adverse effects graded $1 / 5$ were reported ( 1 pruritus and 1 edema), on active electrodes with placebo.

No systemic adverse effect was observed.
Summary / Conclusions: Iontophoresis of treprostinil was well tolerated, with no systemic adverse effect and minor, local adverse effects, related to the iontophoresis procedure. This study permits to determine that treprostinil at $1 \mathrm{mg} \cdot \mathrm{mL}^{-1}$ with current up to $0.016 \mathrm{~mA} . \mathrm{cm}^{-2}$ is safe to be used in the next study, i.e. repeated iontophoresis SSc-related ulcers.

\section{Oral Presentation}

EACPT-1198

The therapeutic benefits of cannabis and cannabinoids evaluated by phase 3 randomized controlled clinical trials: a systematic review of recent literature

E. Jouanjus ${ }^{* 1,2}$, P. Barreiros ${ }^{2}$, M. Lapeyre-Mestre ${ }^{1,2}$

${ }^{1}$ Centre d'Addictovigilance, Service de Pharmacologie Médicale et Clinique, Centre Hospitalier Universitaire de Toulouse, ${ }^{2}$ Inserm UMR 1027, Equipe de Pharmacoépidémiologie, Université Toulouse III Paul Sabatier, Toulouse, France

\section{Other}

Introduction: Several cannabinoid-based pharmaceutical specialities are marketed in the world to treat pain, multiple sclerosis, or nausea/vomiting. In recent years, politics and health deciders from a growing number of countries have legalised the use of cannabis for medical purposes, while others are considering similar moves. Nevertheless, the real therapeutic benefits of cannabis and cannabinoids remain subject to controversy.

Objectives: The objective was to examine the published evidence on the potential therapeutic benefits related to the use of cannabis-based products evaluated in clinical trials.

Methods: A systematic review of trials and related articles published between 2012/01/01 and 2018/07/01 was performed per the international PRISMA (Preferred Reporting Items for Systematic Reviews and MetaAnalyses) Statement. Any phase 3, randomized, controlled, clinical trial which evaluated the efficacy of cannabis or natural or synthetic cannabinoids was eligible for inclusion. There was no restriction on the indication. The inclusion process was based on a search algorithm and performed in a blinded standardized manner, and included a qualitative assessment based on the international recommendations (CONSORT Statement) to exclude the clinical trials inadequately reported.

Results: Among the 812 clinical trials and related articles initially identified in the literature search, 41 remained after performing the inclusion procedure. Finally, the qualitative assessment retained 22 of these latter. All of them were placebo-controlled. They evaluated the efficacy of natural or synthetic cannabinoids in multiple sclerosis $(n=8)$, neuropathic pain $(n=6)$, cancer relatedpain $(n=5)$, epilepsy $(n=2)$ or post-operative nausea/vomiting $(n=1)$. Overall, $8(36,4 \%)$ of them evidenced a statistically significant superiority of cannabinoids on the main outcome measure.

Summary / Conclusions: Although cannabis and cannabinoids are perceived by the public as attractive therapeutic options to treat diverse pathologies, the level of available evidence appears limited and does not provide a definite answer. Also, the number of adverse reactions is high among subjects treated with cannabinoids than in those exposed to placebo.

\section{Oral Presentation}

EACPT-1210

Influence of placebo / nocebo information in informed consent forms and decision to participate in a simulated clinical trial

M. Farré ${ }^{*}$, C. Pérez-Mañá ${ }^{1}$, E. Papaseit ${ }^{1}$

${ }^{1}$ Clinical Pharmacology Unit, Hospital Universitari Germans Trias i Pujol (IGTP) and Universitat Autònoma de Barcelona, Badalona, Spain

\section{Other}

Introduction: In human research, obtention of informed consent is an essential part to fulfil ethical and good clinical practice regulations. As part of this process, participants must be provided with adequate 
information to facilitate the decisions to accept or not accept participation. However, the extent of the information and terminology about placebo and nocebo effects used in the forms can be confusing for the subjects and it not be fully understood.

Objectives: To assess whether the information provided about placebo and nocebo effect in the informed consent influences the decision of the subjects to participate in a placebo-controlled clinical trial.

Methods: We conducted the survey study in 1346 subject. Most of them students of a health degree $(n=560)$ and non-health degree $(n=608)$. We designed a simulated clinical trial, with two informed consent forms (A and B) and a structured questionnaire. The two consent forms were identical, except in the definition of placebo / nocebo and their effects (A less information; B more information about placebo / nocebo)). Participants were randomly assigned into two groups (A or B). In addition, subjects completed a questionnaire concerning their decision to participate; reasons to taking part in the survey and knowledge about placebo were evaluated.

Results: We found a relationship between the decision to participate in a clinical trial of placebo and the information offered about placebo in the informed consent forms (A 63,9\% and B 54,4\%, [A1] p<0.001).

Summary / Conclusions: The amount of information provided about placebo / nocebo in informed consent forms can influence the decision to participate in clinical trials.

Knowledgements: To Astrid Hernández and Rafel Balada, Funded in part by grants from Fundació Grifols, Instituto de Salud Carlos III-ISCIII (Juan Rodés JR16/00020 to E. Papaseit), and Suport Grups de Recerca AGAUR Gencat (2017 SGR 316).

EACPT-1282

A new device for direct quechers salts extraction: application to drugs of abuse

T. Robin ${ }^{1}$, S. Moreau ${ }^{*}$, F. Saint-Marcoux ${ }^{1}$

${ }^{1} \mathrm{CHU}$ of Limoges, Limoges, France, ${ }^{2}$ Shimadzu, Dusseldorf, Germany

\section{Other}

Introduction: Extraction procedures using QuEChERS consist of manually adding salts to the analyzed matrix, previously mixed in a polar solvent (acetonitrile, ACN). This approach is largely employed for pesticides residues but also for multiples classes of drugs.

Objectives: In this study, we explored the performance of a QuEChERS extraction based on a new pre-packed kit containing $100 \mathrm{mg}$ of salts (Micro Volume QuEChERS; MVQ) and compared it to a previously validated procedure, routinely used in a clinical lab (Dulaurent $\mathrm{S}$ et al. Anal Bioanal Chem 2016). The example of an LC-MS method for the analysis of 44 Drugs Of Abuse (DOA) using this new device is presented. Methods: For the new procedure using MVQ, extraction consisted in introducing $200 \mu \mathrm{L}$ of water and $300 \mu \mathrm{L}$ of acetonitrile into device. Then $100 \mu \mathrm{L}$ of matrix and $20 \mu \mathrm{L}$ of an internal standards solution before mixing. After centrifugation for $10 \mathrm{~min}$, the supernatant was injected into the LC-MS system (LCMS 8060, Shimadzu). Chromatographic separation was performed using a column Raptor biphenyl, $2.7 \mu \mathrm{m}$, $100 \times 2.1 \mathrm{~mm}$ I.D. (Restek, Lisses, France). The results of this new procedure were compared with those of the method previously validated: 18 real samples (14 urines and 4 oral fluids) from patients tested for DOA consumption were analysed. Repeatability was also explored using 2 different blood Internal quality controls containing 16 molecules.

Results: Following the new extraction procedure, the preparation of a classical batch that includes 6 calibration standards, 2 internal quality controls, and 10 patient samples required less than $30 \mathrm{~min}$ (compared to $45 \mathrm{~min}$ for current procedure). Among the real 18 samples, 79 molecules were detected by the two procedures. A regression analysis for concentrations measured from 3 to $500 \mu \mathrm{g} / \mathrm{L}$ (LOQ and ULOQ of the method, respectively) reported a $\mathrm{R}^{2}$ greater than 0.98 . For the 16 molecules of the ICQ, the mean CV value was $3.7 \%(1,4$ to $10,9 \%)$ and the mean bias value was $3.1 \%$. Using the example of the preparation of samples for measurements of DOA, this preliminary study suggests that the MVQ kit has the same performance as the manual sample preparations based on QuEChERS salts.

Summary / Conclusions: The new device is a ready-to-use solution which obviously shortens the time required for extraction and reduces possible human errors.

\section{EACPT-1305}

Systemic biobanking infrastructure initiative in slovakia - legal and ethical issues

J. Glasa ${ }^{*} 1,2,3$, I. Kvietiková ${ }^{4}$, D. Kollár ${ }^{4}$, P. Čvapek ${ }^{4}$, H. Glasová ${ }^{5}$, M. Antošová $^{6}$, D. Pella ${ }^{7}$

${ }^{1}$ Institute of ClinicalPharmacology \& Institute of Health Care Ethics, Slovak Medical University in Bratislava, ${ }^{2}$ Slovak Society of Clinical Pharmacology, b.o. Slovak Medical Association, ${ }^{3}$ Ethics Committee (National), ${ }^{4}$ Institute for Research and Development, Ministry of Health, ${ }^{5}$ Institute of Pharmacology and Clinical Pharmacology, Slovak Medical University in Bratislava, Bratislava, ${ }^{6}$ Office of European and National Projects, Jessenius Faculty of Medicine, Comenius University, Martin, ${ }^{7}$ Office of Dean , Pavol Jozef Šafárik University in Košice, Košice, Slovakia

\section{Other}

Introduction: Adequate biobanking does belong nowadays to the sine qua non pre-requisites for successful integration of any country's biomedical research institutions into international research collaborations. This also holds for high-quality clinical pharmacology research projects.

Objectives: In Slovakia, the country, which, since 1990-ies, has undergone a deep, complex transformation, including its health care and biomedical (and other) research system capacities, development of human cell and tissue banking for health care provision purposes mirrored closely similar developments in 'Western' Europe. Historic collections of human biological specimens have also been growing at the hospital and university clinical pathology departments, as elsewhere. More recently, these were joined by collections originated from prospective population studies and, mostly international, drug clinical trials. These collections, usually kept in a very good, orderly shape, and, more recently, being furnished with relevant health information and provided with necessary ethical and legal 'tags', already constitute an invaluable national resource. Methods: However, up till recently, the research use of these, mostly local banking structures have suffered from lack of adequate funding, coordination, standardization of operating procedures and other shortcomings. Moreover, the necessary national legislation, reflecting recent EU developments (e.g. Reg. 536/2014, Reg. 679/2016) as applicable to the biobanking sector, has still been missing. The non-existence of a modern, robust biobanking facility has also been hampering the Slovakian researchers' participation in international research.

Results: Thus, the Slovak Ministry of Health, together with other national stakeholders and with the support of international partners (e.g. BBMRIERIC, ECRIN via recently established Slovacrin) has launched a multistakeholder initiative aiming to achieve a necessary structural and organizational breakthrough and to guarantee sustainable biobanking infrastructure in Slovakia. At present, the necessary legislative pre-requisites are being completed, while the construction of an up-to-date, population and disease-specific based biobanking facilities in central and eastern Slovakia (cities Martin and Košice, respectively) is foreseen to be established. Summary / Conclusions: A detailed account on the ethical and legal aspects of these developments will be given in the presentation.

\section{EACPT-1341}

Pregnancies during clinical trials: are we aware of what is actually going on?

C. Sans ${ }^{*}{ }^{1,2}$, L. F. Camacho ${ }^{2,3}$, F. Rodríguez Cairoli ${ }^{4}$, E. Cucurull ${ }^{3}$

${ }^{1}$ Clinical Pharmacology service, Vall d'Hebron University Hospital,

${ }^{2}$ Department of Pharmacology, Therapeutics and Toxicology, 
Universitat Autònoma de Barcelona, ${ }^{3}$ Research Ethics Committee, Vall d'Hebron Research Institute, Barcelona, Spain, ${ }^{4}$ Hospital de Clínicas José San Martín, Universidad de Buenos Aires, Buenos Aires, Argentina

\section{Other}

Introduction: Inclusion of women of childbearing potential (WOCBP) in clinical trials (CT) as a vulnerable group due to the potential risk of drug pregnancy exposure has been a bioethical topic debate. Currently in the EU, expedited reporting is required in congenital anomalies, late foetal death, spontaneous abortions or adverse reactions in the newborn. In most cases, complete information on healthy pregnancies and newborns is provided in the post-authorization period.

Objectives: To describe pregnancy information included in the CT documents related to investigational medical products (IMP) provided by sponsors to a REC.

Methods: A descriptive study of the CT that could involve WOCBP evaluated in one year (2013) by the REC of a tertiary hospital in Spain was performed. Primary outcome was all information about pregnancies related to the IMP. Data was obtained from CT protocols, DSUR and Safety and Final Reports.

Results: We found 89 CT with WOCBP (3 phase I, 37 phase II, 43 phase III and 6 phase IV). Most frequent studied diseases in CT were neoplasms $(17 ; 19.1 \%)$, endocrine diseases $(13 ; 14.6 \%)$ and diseases of the nervous system $(10 ; 11.2 \%)$. IMPs (total 72) were grouped by product class: chemical $(46 ; 63.9 \%)$, biotechnological $(22 ; 30.5 \%)$ and biological $(4 ; 5.5 \%) .84$ pregnancies related to the IMP were found. The expedited reports (36) were $80.5 \%$ spontaneous abortions, $13.8 \%$ late foetal death, $2.7 \%$ congenital malformation, and $2.7 \%$ adverse reaction in newborns. Other outcomes reported (48) were $64.6 \%$ Unknown outcomes, $20.8 \%$ induced abortions and $14.6 \%$ healthy newborns.

Summary / Conclusions: We found that outcomes of pregnancies during $\mathrm{CT}$ are often unknown and spontaneous abortions are the most common expedited report. Since information on healthy pregnancies is as important as the unhealthy ones, notification of all pregnancy outcomes during $\mathrm{CT}$ could be helpful for patients and physicians to make evidence based decisions.

\section{EACPT-1381}

Attitude and knowledge on generic drugs in adult outpatients that receive chronic pharmacotherapy

S. Mimica Matanović ${ }^{1,2}$, K. Šantić ${ }^{*}$, A. Santic ${ }^{4}$

${ }^{1}$ Department of Pharmacology, Faculty of Medicine, ${ }^{2}$ Unit for Clinical Pharmacology, ${ }^{3}$ Clinical hospital centre Osijek, Osijek, Croatia, ${ }^{4}$ Department of Psychiatry, Clinical hospital centre Osijek, Osijek, Croatia

\section{Other}

Introduction: In Croatia many patients and even healthcare professionals still have doubts if quality of generic drugs equals that of brand drugs. Patients often prefer to be treated with brand/original drugs, reporting lower adherence if generic drugs are prescribed.

Objectives: The aim of the study was to evaluate attitude on generic drugs in adult patients who receiving chronic pharmacotherapy and have at least one chronic disease. Also, basic knowledge on generic drugs and their safety was evaluated.

Methods: One hundred adult outpatients treated in primary care centres in Osijek, Croatia was included in our survey. The patients had at least one drug in chronic therapy and at least one chronic condition. All patients were interviewed by the same physician, using a questionnaiare that was composed for this survey and which included demographic data, patients' level of education, general knowledge on medications, specific knowledge on generic drugs and behavioral questions.
Results: Median age of study participants was 58 and median number of drugs was 3. Among them, $43 \%$ was not familiar with the term ...generic" but on the other hand $90 \%$ understood the term ...copy drug". Seventy percent (70\%) of patients had an opinion that the single advantage of generic drugs was their price and $45 \%$ thought that brand drugs had better efficacy than generic drugs. Nineteen percent (19\%) thought that original drugs were safer than generics and 5\% thought that generic drugs could be harmful for human health . Seventy one percent $(71 \%)$ was of opinion that the patient himself should influence doctor's choice to prescribe brand or generic drug. Surprisingly, in our survey we found no statistically significant influence of sex or level of education to attitudes on generic drugs.

Summary / Conclusions: High percentage of patients in our survey showed lack of understanding of basic characteristics of generic drugs and they also expressed doubts on their efficacy and safety. Surprisingly, high percentage of patients thought that they should influence doctor's choice to prescribe generic or brand drug. Our findings suggest that contrary to many other countries where patients are well educated on this isue, strong efforts should be put to educate patients in Croatia, as lack of trust to generic drugs could be a major obstacle to drug adherence.

\section{EACPT- 1420}

H1 antihistamines on minimal persistent inflammation in patients with allergic rhinitis

I. C. Bocsan * 1, A. Muntean ${ }^{2}$, O. Sabin ${ }^{2}$, D. Deleanu ${ }^{2}$, A. Buzoianu ${ }^{1}$

${ }^{1}$ Clinical Pharmacology, ${ }^{2}$ Iuiu Hatieganu University of Medicine and Pharmacy, Cluj Napoca, Cluj Napoca, Romania

\section{Other}

Introduction: Allergic rhinitis (AR) is a risk factor for asthma occurrence. This relationship could be explain by the migration of inflammatory cells from the upper airway in the lower one.

Objectives: The aim of this study is to evaluate the minimal persistent inflammation in the lungs using fractional exhaled nitric oxide (FeNO) in patients with AR and the effect of AH1 in reducing this inflammation. Methods: Seventy nine patients with persistent allergic rhinitis were included in the study. Clinical evaluation was done based on the presence and severity of AR symptoms and quality of life was measured using the visual analogue scale (VAS). Subclinical inflammation in the lung was evaluated using FeNO measurement (NIOXMINOß). After the initial evaluation, patients were randomly divided into two groups, 39 patients were treated with levocetirizine $5 \mathrm{mg}$ /day and 40 patients received desloratadine $5 \mathrm{mg}$ /day. Patients were evaluated baseline and after one month of treatment with $\mathrm{H} 1$-antihistamines.

Results: Fifty-six patients (70.9\%) had moderate-severe AR, with a total symptom score (TSS) of over 6. Sleep quality was impaired; VAS was negatively correlated with TSS $(\mathrm{R}=-0.458, \mathrm{p}=0.000)$. Patients with AR had elevated FeNO (median value 27). AH1 improved TSS (median values 8 vs. $0, p<0.001$ ) and VAS (median values 4.6 vs. $9.8, \mathrm{p}<0.001$ ) and reduced FeNO (median values 27 vs. 16, p<0.001) after 1 month of treatment. Desloratadine was more efficient in the reduction of FeNO levels ( 38 vs. 14$)$ compared to levocetirizine ( 23 vs. 17.5$)(\mathrm{p}=0.05)$.

Summary / Conclusions: Patients with persistent AR have elevated FeNO levels. Levocetirizine and desloratadine improve symptoms and increase quality of life in patients with persistent AR. Levocetirizine and desloratadine as a continuous therapy determine a reduction of FeNO levels, limiting the progression of allergic inflammation to the lower airway.

\section{Geriatric treatment}

\section{Oral Presentation}

EACPT-1084

Association of antidepressant use with risk of stroke and mortality in the elderly

B. I. On Seker ${ }^{*}{ }^{1}$, X. Vidal ${ }^{2}$, U. Berger ${ }^{1}$, M. Sabaté ${ }^{2}$, E. Ballarín ${ }^{2}$, L. Ibáñez ${ }^{2}$ 
${ }^{1}$ Institute for Medical Information Processing, Biometry and Epidemiology, Ludwig-Maximilians University, Munich, Germany, ${ }^{2}$ Clinical Pharmacology Service, Vall d'Hebron University Hospital Department of Pharmacology Therapeutics and Toxicology, Fundació Institut Català de Farmacologia, Barcelona, Spain

\section{Geriatric treatment, Psychopharmacology}

Introduction: Current evidence on antidepressant-related stroke or mortality risk is inconsistent. Elderly have the highest exposure to antidepressants but are usually excluded from randomized controlled trials.

Objectives: We aimed to quantify the association of antidepressants with stroke and mortality risks in the elderly population.

Methods: Persons aged over 64 years old and registered in Information System for Research in Primary Care (SIDIAP) from Spain, during 20102015 comprised the study population (recruited $n=1,068,117$ ). Timevarying antidepressant exposure was categorized into current-use, recent-use (two months following the end of current-use), past-use (nine months following the end of recent-use), and non-use (reference) episodes. The effect of antidepressant exposure, combined and by antidepressant class, on the composite outcome (first of stroke or death) was analyzed with the Cox proportional hazards model. We adjusted for potential confounding factors, including mental health disorders, in the analysis by including them as co-variables. Hazard ratios (HR) were reported with $95 \%$ confidence interval.

Results: During follow-up, 212,621 persons (19.9\%) had antidepressant reimbursements and there were 204,281 (19.13\%) composite outcome events, 180,008 (16.85\%) deaths, and 34,652 (3.24\%) stroke events. Composite outcome risk significantly increased during antidepressant current-use (HR 1.04; 1.02-1.06), recent-use (HR 3.34; 3.27-3.41), and past-use (HR 2.06; 2.02-2.10) compared to non-use (see Table 1 ). Only the class of Other Antidepressants - excluding TCA and SSRI - increased the composite outcome risk (HR 1.21; 1.18-1.25) during current-use. Recent-use (HR: 5.87; 5.51-6.26) and past-use (HR: 3.17; 2.96-3.40) episodes of multiple antidepressant groups had the highest risk for the composite outcome. Antidepressant current-use was associated with increased stroke (HR 1.56; $1.50-1.61$ ), but decreased mortality risk (HR 0.93; 0.91-0.94). During recentuse and past-use, the individual risks of stroke and mortality were significantly increased compared to non-use of antidepressants.

Summary / Conclusions: Antidepressant use was associated with increased stroke or mortality risk in elderly. Considering their potential risks, antidepressants should be used with caution in this population.

\section{EACPT-1206}

Comorbidity and the risk of bleeding from the upper gastrointestinal tract to elderly patients, associated with the peculiarities of pharmacotherapy

I. Iurina ${ }^{*}$, N. Bondarenko ${ }^{2}$, A. Karandov ${ }^{3}$, S. Sarmanaev ${ }^{4}$

${ }^{1}$ Academy of postgraduate education Federal scientific clinical center FMBA of Russia, KB № 85 FMBA of Russia, ${ }^{2}$ KB № 85 FMBA of Russia, ${ }^{3}$ City clinical hospital. V. V. Vinogradov of Moscow, ${ }^{4}$ Academy of postgraduate education Federal scientific clinical center FMBA of Russia, Moskow, Russian Federation

\section{Geriatric treatment}

Introduction: Currently, elderly patients ( $>65$ years) are the fastest growing group in the population (V. G. Cicirelli, 2002; Werner C. A., 2010). That causes close attention to it, both in clinical studies and in the study of real clinical practice of pharmacotherapy of various diseases, taking into account the comorbid background.

Objectives: comparative assessment of the risk of bleeding from the upper gastrointestinal tract by patients younger than 65 years and older; study of the effect of comorbidity and pharmacotherapy on the risk of recurrence of bleeding.
Methods: a retrospective analysis of 130 medical records of inpatients who underwent gastroscopy to diagnose the source of bleeding and (or) hemostasis in 2018. As the outcomes of the intervention were taken: cases of bleeding and relapses in patients of different age groups associated with the peculiarities of pharmacotherapy of comorbid diseases (lack of regular monitoring of $\mathrm{MNO}$ in patients taking warfarin, drug interaction with drugs of other pharmacological groups, which can also affect the development of bleeding).

Quantitative risk assessment was carried out using key indicators (relative risk reduction (COR), absolute risk reduction (CAR), odds ratio (OR), number need to treat (NNT)).

Results: in the analysis of 130 medical records of patients who underwent gastroscopy for the incident or existing bleeding. Patients under the age of 65 years-group I $(n=69)$, patients over 65 years - group II $(n=61)$. The comorbid background in all patients of group II was presented by oncological and cardiovascular diseases (55\% and $45 \%)$, in group I-comorbid background was represented by single (4\%) cases of chronic viral hepatitis $\langle\mathrm{B} »$ and $\langle\mathrm{C} »$ and liver cirrhosis. The causes of bleeding most often $(88 \%)$ were ulcers of the stomach and duodenum, from the enlarged veins of the esophagus (12\%).

The risk of bleeding and recurrence was significantly higher in group II than in group I $(\mathrm{CAR}=18,7 \%, \mathrm{CI}=95 \%, \mathrm{p}<0,005, \mathrm{OR}=0,19, \mathrm{NNT}=5$; $\mathrm{CAR}=17,9 \%, \mathrm{p}=0,02 ; \mathrm{OR}=0,31 ; \mathrm{NNT}=6$, respectively).

Summary / Conclusions: an objective assessment of the risk of bleeding and recurrence in patients associated with the peculiarities of pharmacotherapy, showed that at the age of 65 years they are significantly higher due to the presence of multiple comorbidities, which not only during treatment, but also in itself can increase the risk (cardiovascular and cancer).

\section{Oral Presentation}

\section{EACPT-1218}

The use of potentially inappropriate medications in older adults in quebec, canada: a population-based study

B. Roux ${ }^{* 1,2}$, C. Sirois ${ }^{3,4}$, M. Simard ${ }^{3}$, M.-È. Gagnon ${ }^{3}$, M.-L. Laroche ${ }^{1,2}$ ${ }^{1}$ INSERM UMR 1248, University of Limoges, ${ }^{2}$ Centre of Pharmacovigilance and Pharmacoepidemiology, University Hospital of Limoges, Limoges, France, ${ }^{3} \mathrm{INSPQ},{ }^{4}$ Department of preventive and social medicine, University of Laval, Quebec, Canada

\section{Geriatric treatment}

Introduction: The prescription of potentially inappropriate medications (PIMs) in older adults is associated with negative health outcomes and a significant cost burden for health care organizations, making their use a major public health concern worldwide.

Objectives: To assess the prevalence and incidence of potentially inappropriate medication (PIM) use in community-dwelling older adults $\geq 66$ years in Quebec, Canada.

Methods: A retrospective population-based cohort study was conducted using the Quebec Integrated Chronic Disease Surveillance System (QICDSS) databases. The QICDSS includes data on drug claims for community-dwelling older adults insured by the public drug insurance plan ( $90 \%$ of people aged $\geq 65$ years). Individuals aged $\geq 66$ years who were continuously insured with the public drug plan between April 1 ${ }^{\text {st }}, 2013$ and March 31 ${ }^{\text {st }}, 2015$ were included for the measure of prevalence $(n=1,057,024)$. Among this population, individuals who initiated a PIM (i.e. did not use any PIM in the previous year) were identified for the measure of incidence. PIMs were defined using the 2015 Beers criteria.

Results: Around half of the older adults $(48.7 \%$; $n=515,168)$ were prescribed at least one PIM. Of these, $62.9 \%$ were women, their mean age was 75.6 years (SD 7.2) and their mean number of medications was 11.5 (SD 6.1). The most prevalent PIMs were benzodiazepines (26.8\%), protonpump inhibitors (20.5\%), first- and second- generation antipsychotics $(5.4 \%)$ and antidepressants including tricyclics and paroxetine (5.1\%). Among users of PIMs, $15.7 \%(n=80,674)$ initiated a PIM during the study period, yielding 
an annual incidence of $7.6 \% ; 55.6 \%$ were women, their mean age was 74.7 years (SD 7.0) and their mean number of medications was 10.3 (SD 5.7). The PIMs most frequently initiated among incident users were benzodiazepines $(34.1 \%)$, proton-pump inhibitors (33.5\%), skeletal muscle relaxants $(10.4 \%)$, first- and second- generation antipsychotics $(7.1 \%)$ and firstgeneration antihistamines (5.5\%).

Summary / Conclusions: Almost half of community-dwelling older adults use a PIM. From a public health perspective, it is imperative to reduce the use of PIMs, by both limiting their initiation and by promoting their deprescribing at large scale.

\section{EACPT-1219}

Persistence of potentially inappropriate medications use in older adults

B. Roux ${ }^{* 1,2}$, M.-L. Laroche ${ }^{1,2}$, M.-È. Gagnon ${ }^{3}$, M. Simard ${ }^{3}$, C. Sirois ${ }^{3,4}$ ${ }^{1}$ INSERM UMR 1248, University of Limoges, ${ }^{2}$ Centre of Pharmacovigilance and Pharmacoepidemiology, University Hospital of Limoges, Limoges, France, ${ }^{3}$ INSPQ, ${ }^{4}$ Department of Social and Preventive Medicine, University of Laval, Quebec, Canada

\section{Geriatric treatment}

Introduction: The use of potentially inappropriate medications (PIMs) is associated with negative health outcomes. Although previous studies have assessed the trend of PIM use over time, none has determined whether PIMs are used continuously in the same individuals.

Objectives: To assess one-year persistence of PIM use and to identify factors associated with such persistence in community-dwelling older adults.

Methods: We conducted a retrospective population-based cohort study of community-dwelling older adults aged $\geq 66$ years in Quebec, Canada, using the Quebec Integrated Chronic Disease Surveillance System. To be included, individuals had to be continuously insured with the public drug plan between 01/04/2013 and 31/03/2015. We defined PIMs according to the 2015 Beers Criteria's list (drugs that should be avoided). Individuals who initiated a PIM between 01/04/2014 and 31/03/2015 and who had at least one-year follow-up after the first PIM dispensing were included. Persistence of PIM use at one year was defined as a continuous treatment with any PIM, with no interruption longer than 60 days between prescriptions refills. Similarly, we measured one-year persistence of specific PIM use in subcohorts of individuals who had initiated those specific PIMs. Multivariate robust Poisson regressions were performed to identify factors associated with one-year persistence. Results were reported as rate ratios (RR) with their 95\% confidence intervals.

Results: Among the 75844 older individuals who initiated a PIM, $25.1 \%(24.8 \%-25.4 \%)$ had a persistent use of at least one PIM over a one-year period. The median time to first PIM discontinuation was 31 days (IQR:21-92) in non-persistent individuals. The probability of persisting at one-year was higher for those who initiated peripheral alpha-blockers (50.5\%), antipsychotics (43.9\%), long-duration sulfonylureas $(40.2 \%)$ and proton pump inhibitors $(36.0 \%)$. Factors significantly associated with persistence of PIM use included being older (86y, RR:1.82;1.76-1,89), being a man $(1.13 ; 1.11-1.15)$, and having a higher number of medications and chronic diseases (ranges 1.06-1.34/1.18-1.62), specifically the presence of Alzheimer's disease and related disorders $(1.81 ; 1.74-1.89)$.

Summary / Conclusions: A quarter of older adults initiating a PIM is persistently exposed to at least one PIM the whole following year. There is a need to further explore if persistent use of PIM leads to a higher risk of adverse events than sporadic use.

\section{Oral Presentation}

EACPT-1264

Mortality associated with stopping statins in the oldest old - with and without ischemic heart disease

M. Ioffe ${ }^{1}$, A. Kremer ${ }^{1}$, I. Nachimov ${ }^{2}$, M. Swartzon ${ }^{3}$, D. Justo ${ }^{*} 1$
${ }^{1}$ Geriatrics, Sheba Medical Center, Ramat-Gan, ${ }^{2}$ Geriatrics, Dorot Medical Center, Natanya, Israel, ${ }^{3}$ Florida International University, Miami, United States

\section{Geriatric treatment}

Introduction: Some physicians are reluctant to stop statins in the oldest old - especially if they have history of ischemic heart disease (IHD).

Objectives: To study the association between stopping statins and 1-year mortality in the general population of the oldest old with or without IHD. Methods: A historical prospective study. Included were all consecutive patients $(n=369)$ aged 80 years or more (mean age 87.8 years) hospitalized in a single Geriatrics department during one year. The study group included 140 patients in whom statins were stopped upon admission (statin stoppers). The control group included 229 patients who did not use statins in the first place (statin non-users). All-cause 1-year mortality rates were studied in both groups following propensity score matching and in IHD patients separately.

Results: Overall, 110 (29.8\%) patients died during the year following admission: $38(27.1 \%)$ statin stoppers and $72(31.4 \%)$ statin non-users $(p=0.498)$. Cox regression analysis showed no association between stopping statins and 1-year mortality in the crude analysis (HR $0.976,95 \% \mathrm{CI}$ 0.651-1.463, $\mathrm{p}=0.907$ ) and following propensity score matching (HR $1.067,95 \%$ CI $0.674-1.689, \mathrm{p}=0.782$ ). Among 108 IHD patients, 38 (35.2\%) patients died during the year following admission: $18(27.7 \%)$ statin stoppers and $20(46.5 \%)$ statin non-users $(\mathrm{p}=0.059)$. Cox regression analysis showed a nearly significant association between stopping statins in IHD patients and lower 1-year mortality (HR 0.524, 95\%CI 0.259 $1.060, \mathrm{p}=0.072$ ).

Summary / Conclusions: Stopping statins in the general population of the oldest old is safe, and may be even associated with a favorable prognosis in IHD patients.

\section{EACPT- 1290}

Analysis of the drug prescribing appropriateness to stopp/start criteria in elderly patients with type 2 diabetes mellitus in the endocrinological department of a multi-speciality hospital

V. De ${ }^{*} 1$, A. Kochetkov ${ }^{2}$, O. Ostroumova ${ }^{1,2}$, A. Starodubova ${ }^{3}$, M. Chernyaeva $^{4}$, N. Voevodina ${ }^{5}$, M. Chachiashvili ${ }^{5}$

${ }^{1}$ A.I. Yevdokimov Moscow State University of Medicine and Dentistry, ${ }^{2}$ Russian Clinical and Research Center of Gerontology, ${ }^{3}$ Federal Research Centre of Nutrition, Biotechnology and Food Safety, ${ }^{4}$ Veterans Hospital No. 2, ${ }^{5}$ E.O. Mukhin Municipal Clinical Hospital, Moscow, Russian Federation

\section{Geriatric treatment}

Introduction: The rapid increase in the incidence of type 2 diabetes mellitus (T2DM) worldwide is associated with an increase in life expectancy and urbanization-related lifestyle changes. With age advance the number of chronic diseases and amount of prescribed drugs are elevated. Pharmacotherapy optimization using STOPP/START criteria will improve the quality of drug prescribing, reduce the probability of druginduced side effects, decrease the duration of hospitalization, improve the prognosis and life quality in elderly patients.

Objectives: To assess the appropriateness of prescribed drugs to STOPP/ START criteria in elderly patients with T2DM at the endocrinology department of a multi-speciality hospital.

Methods: The medical records of patients $\geq 65$ years old with T2DM hospitalized in the endocrinology department of the E.O. Mukhin Municipal Clinical Hospital (Moscow, Russia) were analyzed. The study included data from 230 patients (mean age 75.0 \pm 6.5 years). Drug prescriptions were evaluated according to STOPP/START criteria.

Results: According to the STOPP/START criteria, we revealed 62 potentially inappropriate drugs in the prescription lists of 87 patients $(37,8 \%)$. 
The most frequent identified STOPP criteria were: «Glibenclamide or chlorpropamide with T2DM» $(46.8 \%)$; «Non-steroidal antiinflammatory drug (NSAID) with history of peptic ulcer disease or gastro-intestinal bleeding, unless with concurrent histamine $\mathrm{H} 2$ receptor antagonist, proton pump inhibitors or misoprostol» $(6.5 \%)$ and «NSAID with estimated glomerular filtration rate $20-50 \mathrm{ml} / \mathrm{min}$ ute» $(6.5 \%)$. The most frequent START criteria were: «Statin therapy with a documented history of coronary, cerebral or peripheral vascular disease, where the patient's functional status remains independent for activities of daily living and life expectancy is $>5$ years» $(28,7 \%)$; «Aspirin with a documented history of atherosclerotic coronary disease in patients with sinus rhythm» $(18,0 \%)$; «Clopidogrel with a documented history of cerebral or peripheral vascular disease» $(15.5 \%)$.

Summary / Conclusions: In patients $\geq 65$ years old with T2DM potentially not recommended drugs are often administrated and there are no prescriptions for some appropriate drugs required in certain clinical settings.

\section{EACPT-1329}

The structure of prescribing drugs in old and very old patients in a multi-speciality hospital in moscow (russia)

M. Cherniaeva* 1,2, O. Ostroumova ${ }^{3,4}$, A. Morozov $^{2}$, M. Kulikova ${ }^{3}$, A. Kochetkov $^{4}$, D. Sychev ${ }^{5}$

${ }^{1}$ Central State Medical Academy of Department of Presidential Affairs, ${ }^{2}$ Veterans Hospital No. $2,{ }^{3}$ A.I. Yevdokimov Moscow State University of Medicine and Dentistry, ${ }^{4}$ Russian Clinical and Research Center of Gerontology, ${ }^{5}$ Russian Medical Academy of Continuous Professional Education, Moscow, Russian Federation

\section{Geriatric treatment}

Introduction: Polypharmacy is an obligate component of the population ever-increasing life expectancy, as it is a consequence of the growing number of age-related diseases. Some studies had proved that polypharmacy along with multimorbidity, has a serious impact on people's somatic health as well as plays a key role in the development of variety drug-induced side effects.

Objectives: To analyze pharmacotherapy in old and very old patients regarding to the presence of polypharmacy and evaluate the structure of prescribing medications in these individuals.

Methods: We examined 416 people $\geq 65$ years old (mean age 79.9 years, $28 \%$ men).

Results: The mean number of drugs taken per one patient was 5.1, the mean Charlson comorbidity index score was 6.1. Most of the patients took simultaneously $\geq 5$ drugs $(26 \%)$. We divided the patients into 2 groups depending on age: the first one included individuals $65-79$ years old $(n=185$, mean age 71.5 years, $31 \%$ of men), the second one comprised people $\geq 80$ years ( $n=231$, average age 86.7 years, $25 \%$ men). In the first group, the average number of prescribed drugs was $4.9(0-12), \geq 5$ drugs were taken by $58 \%$ of patients, the average Charlson comorbidity index score was 4.9 (2-14). The most frequently used drugs were: diuretics $(61.6 \%)$, aspirin $(56.2 \%)$, angiotensin-converting-enzyme (ACE) inhibitors (48.1\%), beta-blockers [BB] (47.6\%), metabolic drugs and antioxidants $(38.4 \%)$. In the second group, the average number of prescribed drugs was 5.3 (2-13), $\geq 5$ drugs simultaneously were taken by $58 \%$ of patients, the average Charlson comorbidity index score was 7.0 (4-18). The most frequently administered drugs were: diuretics (74.5\%), ACE inhibitors (60.2\%), BB $(58.9 \%)$, metabolic drugs and antioxidants $(55 \%)$.

Summary / Conclusions: The amount of prescribed drugs and the comorbidity index score increases with age incrementing despite the fact that qualitative proportion of most frequently used medications remain the same. This results indicates the necessity of more precision drug administration by physicians in order to preserve the quality of life in elderly patients.
EACPT-1330

Impact of the anticholinergic burden on cognitive functions in very old patients

M. Cherniaeva ${ }^{*}{ }^{1,2}$, M. Kulikova $^{3}$, O. Ostroumova ${ }^{3,4}$, O. Golovina ${ }^{2,5}$, A. Kochetkov ${ }^{4}$, D. Sychev ${ }^{5}$

${ }^{1}$ Central State Medical Academy of Department of Presidential Affairs, ${ }^{2}$ Veterans Hospital No. $2,{ }^{3}$ A.I. Yevdokimov Moscow State University of Medicine and Dentistry, ${ }^{4}$ Russian Clinical and Research Center of Gerontology, ${ }^{5}$ Russian Medical Academy of Continuous Professional Education, Moscow, Russian Federation

\section{Geriatric treatment}

Introduction: A lot of frequently used medications have a varying degree of anticholinergic activity, which can cause anticholinergic side effects, including a decrease of cognitive functions. Due to fact that polypharmacy is very common phenomenon among older adults, it is very important to access the effect of anticholinergic burden on cognition in geriatric patients ( $\geq 80$ years old).

Objectives: To investigate the possible association between cognitive functions and administration of drugs with anticholinergic activity and their number in patients $\geq 80$ years old with essential arterial hypertension. Methods: We examined 74 patients $\geq 80$ years old (median age 86 years [ 82 88], 23\% men) in the therapeutic department of a multi-speciality hospital. Cognitive functions were assessed using MMSE, MoCA, WAIS IV, Verbal fluency tests (phonemic and semantic categories), Boston naming test, DSST, Word List Test and Adas-cog. Anticholinergic burden was determined using anticholinergic cognitive burden scale (ACB).

Results: The patients were divided into 2 groups according to the presence (group 1, $\mathrm{n}=49$, median age 86 [82-88], 16.3\% men) or absence (group 2, $n=25$, median age 85 [81 -88], 36\% men) on the prescription list medications with anticholinergic activity. In group 1 as opposed to group 2, there were a significantly lower MMSE score (median 21 [18-23] and 22.5 [20-26], respectively, $\mathrm{p}=0.040$ ) and a significantly less score in the Boston naming test (median 27 [25-30] and median 30 [28-31], respectively, $\mathrm{p}=0.014)$. The Adas-cog total score in group 1 was significantly higher (median 16.7 [12-19.3]) than in group 2 (median 12.7 [11-14.6]), $\mathrm{p}=0.030$. We also compared patients into groups according to the absence of anticholinergic burden (group A, $n=25$, median age 85 [81-88], 36\% men) or its minimal presence - 1 point of the ACB score (group B, $n=30$, median 86.5 age [83-89], 16.7\% men). The Adas-cog total score in group B patients was significantly higher than in group A (median 17.7 [13.320.7] and 12,7 [11-14,6], respectively, $\mathrm{p}=0.024)$.

Summary / Conclusions: There is a decrease in the global cognition in presence of anticholinergic burden. Patients, taking drugs with anticholinergic activity, have a lower cognitive status compared to individuals without administration of similar medications.

\section{EACPT-1331}

Impact of polypharmacy on cognitive functions in eldery patients with essential arterial hypertension and comorbities

M. Kulikova ${ }^{1}$, M. Cherniaeva ${ }^{* 2,3}$, O. Ostroumova ${ }^{1,4}$, A. Kochetkov ${ }^{4}$, D. Sychev ${ }^{5}$

${ }^{1}$ A.I. Yevdokimov Moscow State University of Medicine and Dentistry, ${ }^{2}$ Central State Medical Academy of Department of Presidential Affairs, ${ }^{3}$ Veterans Hospital No. 2, ${ }^{4}$ Russian Clinical and Research Center of Gerontology, ${ }^{5}$ Russian Medical Academy of Continuous Professional Education, Moscow, Russian Federation

\section{Geriatric treatment}

Introduction: The increase of life expectancy, growing number of elderly persons, and at the same time amplification in amount of chronic diseases results in excessive prescription of drugs, named as polypharmacy, which is the main cause of drug-induced side effects. It is known that decrease in cognitive functions is associated with advanced 
age and with the presence of vascular risk factors, while the impact of polypharmacy is unclear.

Objectives: To assess the cognitive functions in patients $\geq 80$ years old with essential arterial hypertension (EAH) in relation to comorbidity index score and polypharmacy.

Methods: We examined 58 patients $\geq 80$ years old (median age 86 years [8288], $29.3 \%$ men). Duration of EAH is more than 10 years, mean arterial pressure - 141.6/78.3 $\mathrm{mm} \mathrm{Hg}$, concomitant diabetes mellitus - in 50\% of patients, history of myocardial infarction - in $12.1 \%$. Cognitive functions were assessed using MMSE, MoCA, WAIS IV, Verbal fluency tests, Strup's test, Boston naming test, TMT, DSST, Word List Test and Adas-cog. Results: According to the median value of the Charlson comorbidity index $(\mathrm{CCI})$ score (median $=6$ ) patients were divided into 2 groups: group 1 - CCI score $\leq$ median $(n=33$, median age 84 [82-86], 30.3\% men), group 2 - CCI score $>$ median ( $n=25$, median 88 [85-91], $28 \%$ men). There were no significant differences in the cognitive tests results in these groups. Depending on the number of prescribed medications, patients were divided into 2 groups: the first one included patients taking $<5$ drugs $(n=17$, median 84 age [82-88], 35.3\% men) and the second one comprised patients taking $\geq 5$ drugs $(n=41$, median 86 age [83-89], 26.8\% men). In group 2, compared to group 1, there were found significantly lower MMSE scores (median 24 [1926] and 21 [19-23], respectively, $p=0.042$ ) as well as significantly lower WAIS IV scores (median 8 [7-9] and 7 [6-9], respectively, $\mathrm{p}=0.030$ ). Patients in the second group compared the first one called less words during immediate recall (median no. words 18 [16-19] and 14 [12-19], respectively, $\mathrm{p}=0.016$ ) and delayed recall (median no. words 5 [4-6] and 3 [1-6], respectively, $\mathrm{p}=0.038$ ).

Summary / Conclusions: In patients with EAH $\geq 80$ years old polypharmacy is associated with a decrease in cognitive functions (the global cognition, shortand long-term memory).

\section{EACPT-1380}

Experimental and regulatory studies on adverse effects of some antidepressants in elderly

K. Koleva ${ }^{1}$, S. Marchev ${ }^{*}{ }^{1,2}$, R. Nikolov ${ }^{1}$, L. Todorova ${ }^{1,3}$, S. Surcheva ${ }^{1}$, M. Vlaskovska ${ }^{1}$

${ }^{1}$ Department of Pharmacology and Toxicology, Medical University Sofia, Faculty of Medicine, Sofia, ${ }^{2}$ Department of Pharmacology and Clinical Pharmacology and Therapeutics, Medical University Prof. Dr. Paraskev Stoyanov, Varna, ${ }^{3}$ Bulgarian Drug Agency, Sofia, Bulgaria

\section{Geriatric treatment}

Introduction: Elderly population will continue to be the fastest growing age group in the next years and represent the largest user of medications, which commonly include antidepressants and analgesics. At the same time, the elderly are more prone to develop adverse drug effects such as serotonin syndrome (SS), a typical side effect of some antidepressants.

Objectives: To study the effect of some antidepressants - fluoxetine and tranylcypromine on body temperature as an equivalent of SS in rats. To investigate the effect of risperidone on the hyperthermic response, which is a life-threatening feature of the SS.

To review the Summaries of Product Characteristics (SPC) of centralized and nationally authorized antidepressants with regard to indications and adverse effects in the elderly.

Methods: Male Wistar rats have been used. Fluoxetine $(10 \mathrm{mg} / \mathrm{kg})$ and tranylcypromine $(3,5 \mathrm{mg} / \mathrm{kg})$ have been injected intraperitoneally. The change in body temperature has been measured by using thermistor probes (TX-8) inserted rectally and monitored in a multichannel recorder.

The SPC of some antidepressants were reviewed.

Results: It was found that fluoxetine and tranylcypromine significantly increased the body temperature of the rats. Pretreatment with risperidone resulted in a dosedependent alleviation of hyperthermia thus confirming the role of $5-\mathrm{HT}_{2}$ receptors. Studies of SPC of antidepressants revealed that there is a lack of information for the elderly population mainly in nationally authorized antidepressants before 2007 .
Summary / Conclusions: The present findings confirm the role of 5- $\mathrm{HT}_{2}$ receptors in antidepressants-induced hyperthermia which could reveal new approaches in the prevention of SS.

A proposal to the Bulgarian Drug Agency has been made to reassess and harmonize SPC of antidepressants with regard to indications and adverse effects in the elderly.

\section{Oral Presentation}

\section{EACPT-1396}

The importance of correct estimate of renal function for drug treatment in hospitalized geriatric patients: a prospective observational study

A. Helldén ${ }^{*}{ }^{1,2}$, U. Bergman ${ }^{2}$, I. Odar-Cederlöf ${ }^{2}$

${ }^{1}$ Division of Drug Research/Pharmacology, Linköping University Hospital, Linköping, Sweden, Department of Medical and Health Sciences, Linköping, ${ }^{2}$ Department of Laboratory Medicine, Division of Clinical Pharmacology, Karolinska Institutet, Stockholm, Sweden

\section{Geriatric treatment}

Introduction: Renal function in most subjects declines from the age of about 40 . The number of elderly is increasing, especially the very old requiring hospital care. Thus, many elderly hospitalized patients may have low renal function. In addition, age-related diseases such as atherosclerosis, heart failure, diabetes, hypertension, and infections may further decrease the renal function. When prescribing drugs to elderly it is important to know the actual renal clearance, especially in in-patients. The Cockroft \& Gault (C\&G) formula has for long been used for estimation of the creatinine clearance. Newer estimation formulas based on creatinine $(\mathrm{Cr})$ and/or cystatin $\mathrm{C}$ (cyst $\mathrm{C}$ ) have been introduced, such as the eGFR MDRD formula, CAPA formula, or the Lund Malmoe revised (LM rev), or the mean value of the two latter. MDRD is currently used at our hospital, but since LM rev mean $\left(\mathrm{GFR}_{\mathrm{CC}+\text { creat }}\right)$ is increasingly used at other hospitals, a change to this eGFR formula is discussed. We investigated which of the formulas that correlated best to iohexol clearance (iohexcl), the reference standard used in Sweden, in hospitalized geriatric patients

Objectives: To compare the absolute clearance in $\mathrm{mL} / \mathrm{min}$ of iohexcl to the MDRD, C\&G and $\mathrm{GFR}_{\mathrm{CC}+\text { creat }}$ formula.

Methods: This was a prospective quality improvement study including $108 \geq 75$ years old hospitalized patients in a university geriatric department. Iohexcl was performed by 3-4-points sampling. $\mathrm{Cr}$ and cyst $\mathrm{C}$ was collected after one week at the ward. Mean values $\pm \mathrm{SD}$ and significant difference were calculated. Agreement between the methods was tested by Bland \& Altman analysis.

Results: Mean iohexcl, C\&G, MDRD, and $\mathrm{GFR}_{\mathrm{CC}+\text { creat }}$ were $36.7 \pm$ $15.2,39.3 \pm 16.0(\mathrm{p}=0.21), 53.8 \pm 20.8(\mathrm{p}<0.0001)$, and $43.4 \pm 15.2$ $(\mathrm{p}=0.001) \mathrm{mL} / \mathrm{min}$, respectively. Bias for $\mathrm{C} \& \mathrm{G}, \mathrm{MDRD}$, and $\mathrm{GFR}_{\mathrm{CC}+}$ creat were 2,16 , and $6 \mathrm{~mL} / \mathrm{min}$, respectively.

Summary / Conclusions: Geriatric hospitalized patients may receive too high a dose of renally excreted drugs if absolute values of MDRD or $\mathrm{GFR}_{\mathrm{CC}+\mathrm{creat}}$ are used for estimation of renal function, while $\mathrm{C} \& \mathrm{G}$ still have a place when dosing drugs.

\section{EACPT-1401}

Effects of polyphenol-rich aroniamelanocarpa fruitjuice on bone mineral density and pain sensitivity threshold in ovariectomized rats S. Valcheva-Kuzmanova ${ }^{1}$, A. Georgieva ${ }^{1}$, M. Eftimov ${ }^{1}$, M. Todorova ${ }^{1}$, V. Kuzmanova ${ }^{2}$, A. Kuzmanov ${ }^{2}$, S. Marchev ${ }^{*}{ }^{2}$, K. Kuzmanov ${ }^{3}$, M. Vlaskovska $^{4}$

${ }^{1}$ Department of Pharmacology and Clinical Pharmacology and Therapeutics, ${ }^{2}$ Medical University Prof. Dr. ParaskevStoyanov, Varna, Bulgaria, ${ }^{3}$ Vivarium, Medical University Prof. Dr. ParaskevStoyanov, Varna, ${ }^{4}$ Department of Pharmacology and Toxicology, Medical University - Sofia, Sofia, Bulgaria 


\section{Geriatric treatment}

Introduction: Osteoporosis is a major disorder in menopause. Dietary interventions are an important tool for bone strengthening. Aroniamelanocarpa fruit juice (AMFJ), a rich source of polyphenols, has been demonstrated to possess a potent antioxidant activity and important metabolic functions. The most popular model of osteoporosis/osteopenia is generated in rodents by ovariectomy. Pain hypersensitivity developsin ovariectomy-induced osteoporosis.

Objectives: The aim of the present study was to investigate the effects of AMFJ on pain sensitivity threshold and bone mineral density (BMD) in ovariectomized(OVX) rats.

Methods: Female Wistar rats were divided into 4 groups, each of 14 animals: $\mathrm{SO}$ (sham-operated), OVX, OVX+AMFJ 5 and $\mathrm{OVX}+\mathrm{AMFJ}_{10}$. Beginning 2 weeks after the operation, $\mathrm{SO}$ and OVX groups were treated daily orally with distilled water $(10 \mathrm{ml} / \mathrm{kg})$ while $\mathrm{OVX}+\mathrm{AMFJ}_{5}$ and $\mathrm{OVX}+\mathrm{AMFJ}_{10}$ rats received AMFJ at doses of $5 \mathrm{ml} / \mathrm{kg}$ and $10 \mathrm{ml} / \mathrm{kg}$,respectively. The main polyphenolic substances in $1 \mathrm{ml}$ AMFJ were: total phenolics $(6.65 \mathrm{mg})$, total proanthocyanidins $(3.93 \mathrm{mg})$, chlorogenic acid $(0.69 \mathrm{mg})$ and neochlorogenic $\operatorname{acid}(0.84 \mathrm{mg})$. After 10 weeks of treatment, hot plate test was performed to assess thermal pain sensitivity. Femur BMD was measured by dual energy Xray absorptiometryusing a computer program for small subjects.

Results: In the hot plate test, the latency time of OVX rats was significantly shorter $(p<0.05)$ than that of SO rats. Hot-plate latency periodsof both OVX+ $\mathrm{AMFJ}_{5}$ and $\mathrm{OVX}+\mathrm{AMFJ}_{10}$ groupswere not significantly different from SO latency time and were significantly longer $(\mathrm{p}<0.001)$ compared to the results of OVX rats. Three months after ovariectomy, the femur BMD of OVXgroup was reduced compared to SO group. AMFJ dose-dependently prevented bone loss. Thus, BMD of OVX+AMFJ ${ }_{10}$ rats was significantly higher $(\mathrm{p}<0.05)$ than that of OVX rats.

Summary / Conclusions: Treatment of OVX rats with AMFJ antagonized OVX-induced decrease in BMD and prevented osteoporotic hyperalgesia to heat. Therefore, AMFJmight be beneficial for postmenopausal osteoporosisprevention.

\section{EACPT-1443}

Intrinsic and extrinsic factors associated with falls, in older adults: a case-control study

D. Mino-León ${ }^{*} 1$, C. Rios-Fraustro ${ }^{1}$, M. E. Galván-Plata ${ }^{2}$, D. GaliciaGomez $^{3}$, L. Giraldo-Rodriguez ${ }^{4}$, M. Agudelo-Botero ${ }^{5}$

${ }^{1}$ Unidad de Investigación en Epidemiología Clínica. Hospital de Especialidades CMN SXXI, IMSS, ${ }^{2}$ Coordinación de Investigación, Instituto Mexicano del Seguro Social, ${ }^{3}$ Facultad de Farmacia, Universidad Autónoma de Morelos, ${ }^{4}$ Investigación, Instituto Nacional de Geriatría, ${ }^{5}$ Centro de Investigación en Políticas, Población y Salud. Facultad de Medicina, Universidad Nacional Autónoma de México, México, Mexico

\section{Geriatric treatment}

Introduction: Falls among older adults are a global public health problem with multifactorial causes.

Objectives: Estimate the risk of falls associated with polypharmacy, potentially inappropriate prescriptions and with fall-risk-increasing drugs in older adults receiving medical care at the Mexican Social Security Institute.

Methods: A case-control design that included individuals of both sexes, aged $\geq 60$ years. The cases were patients who arrived at the emergency department of a specialized hospital and in whom the diagnosis of injury or fracture secondary to a fall was established; the controls were from the Family Medicine Unit of the Mexican Social Security Institute, where the cases received their primary care, and were matched by age and sex. Descriptive, bivariate (Chi-square and Student's T tests for independent samples) and multivariate (simple and fitted logistic regression models) analyses were performed.

Results: 342 patients (171 cases and 171 controls) were included. The mean age was $76.1 \pm 8.8$ years; $66 \%$ were women, and by self-report
$2.9 \%$ had no chronic disease. In the bivariate analysis, no differences were observed in polypharmacy; however, differences were observed for body mass index, cognitive impairment, the use of walking devices and dependence to perform basic and instrumental activities of daily living. The multivariate analyses (simple and fitted) revealed an association between the fall event and cognitive impairment and dependence to perform instrumental activities of daily living.

Summary / Conclusions: Older adults with cognitive impairment and dependence for instrumental activities of daily living are at increased risk for falls.

\section{EACPT-1456}

The efficacy of an equivalent dose of ephedrine and phenylephrine in the prevention of post-spinal hypotension in senior adults M. Zunic ${ }^{*} 1$, M. Kamenik ${ }^{2}$

${ }^{1}$ Pharmacology and toxicology, Medical faculty University of Maribor, Slovenia, EU, ${ }^{2}$ Department of Anesthesiology, Intensive Care and Pain Management, University clinical centre Maribor, Maribor, Slovenia

\section{Geriatric treatment}

Introduction: The study of Saravaan et al. demonstrates a potency ratio(PR) of 81.2 for equivalence between phenylephrine and ephedrine in prevention of hypotension after spinal anaesthesia for Caesarean section (CS), with mean dose used for phenylephrine was $496.45 \mathrm{mg}$ and for ephedrine $39.64 \mathrm{mg}$.

Objectives: In prospective, randomized, double-blind, and placebocontrolled study were included 90 patients older than 60 years scheduled for hip and knee orthopedic surgery under spinal anaesthesia.

Methods: Patients were allocated randomly to the E (ephedrine infusion), $\mathrm{P}$ (phenylephrine infusion) and $\mathrm{C}$ (control group) group. After subarachnoid injection of levobupivacaine $15 \mathrm{mg}$ the participants in group $\mathrm{E}$ got the infusion of ephedrine $20 \mathrm{mg}$, in group P phenylephrine $250 \mathrm{mcg}$, in C group only saline infusion. The observed parameters were measured and compared 15 minutes before and in 10, 20 and 30 minutes after SA: noninvasive systolic arterial pressure (SAP), mean arterial pressure (MAP), heart rate (HR). A hypotensive, hypertensive or bradycardic patients were defined as patients who developed at least one episode of hypertension, hypotension or bradycardia throughout the case and were treated according to the protocol.

Results: 14 developed complications. 7 patients from the $\mathrm{C}$ group had complications because of hemodynamic instability ( 5 patients got severe hypotension, 1 had bradycardia and 1 had hypotension with bradycardia). 2 patients from the $\mathrm{P}$ group had hypotension with bradycardia and 2 had hypotension episode alone. 2 patients from the E group developed hypotension, 1 patient developed bradycardia and hypotension. There were no significant differences between groups. The incidence of bradycardic and hypotensive events was not statistically different between the treatment groups ( $p=0.33$ ). Although the number of the bradycardic $(n=3)$ and the hypotensive $(\mathrm{n}=6)$ patients was higher in the group $\mathrm{C}$, the differences between the groups did not reach statistical significance $(\mathrm{p}=0.86$ and $\mathrm{p}=0.53$ ). There were no significant differences between the groups with respect to the number of patients receiving ephedrine $(\mathrm{p}=0.58)$ or phenylephrine $(\mathrm{p}=0.54)$ as the rescue drug.

Summary / Conclusions: This study demonstrates efficacy of the same PR for equivalence between phenylephrine and ephedrine as It was in a prevention of hypotension after spinal anaesthesia for CS, with a half mean dose used for the elderly.

Health economy

\section{EACPT-1170}

Ophthalmological dispensing patterns by pharmacies: a basis for health care and economic planning

I. Truter* 1

${ }^{1}$ Drug Utilization Research Unit (DURU), Department of Pharmacy, Nelson Mandela University, Port Elizabeth, South Africa 


\section{Health economy}

Introduction: Ophthalmic conditions and visual impairment pose a significant health burden on both individuals and society. Pharmacoepidemiological studies analyse trends in drug usage, and the results may give a crude estimate of the disease prevalence and estimate of drug expenditure. Primary eye care in South Africa is faced with multiple challenges, including the availability of resources. Pharmacists are able to provide basic eye screening services and dispense ophthalmological products.

Objectives: The study aimed to report on the dispensing patterns of ophthalmologicals in community pharmacies.

Methods: A retrospective cross-sectional pharmacoepidemiological study was conducted on community pharmacy dispensing records for 2015. All records for ATC group S01 (ophthalmologicals) were extracted, and analysed with Microsoft Access and Excel.

Results: A total of 578688 ophthalmological products (135 different trade names and dosage forms) were dispensed to 347438 patients at a total sales value of R69 061 132.43. Just more than half of the patients were female $(55.24 \%)$. The average age of patients was 46.30 $(\mathrm{SD}=15.58)$ years. Patients received on average $1.67(\mathrm{SD}=2.35)$ products during the year, at an average sales value of R198.77 $(\mathrm{SD}=\mathrm{R} 478.74)$ per product. Nearly half of the products were decongestants and antiallergics (S01GX10) (46.23\% of volume; $27.21 \%$ of cost), followed by antiglaucoma preparations and miotics $(23.07 \%$ of volume; $36.68 \%$ of cost). Most products were ophthalmic drops and eye ointments, and most products were Schedule 2 and Schedule 4. The 15 most frequently dispensed products accounted for $58.96 \%$ of prescription volume and $49.05 \%$ of sales value. The most often prescribed product was an eye drop containing antazoline hydrochloride $0.5 \mathrm{mg}$ and tetryzoline hydrochloride $0.4 \mathrm{mg}$. Decongestants and antiallergics (ATC code SGX10) showed a clear seasonal pattern with less products dispensed during the winter months.

Summary / Conclusions: Few studies report on the dispensing patterns of ophthalmologicals. Pharmacoepidemiological studies are a means to assist with health planning in specific therapeutic areas. It is an indirect way to measure pharmacies' involvement in managing eye conditions that require medication use. The results of this study also provide baseline information for further pharmacoeconomic investigations into ophthalmologicals.

\section{EACPT-1400}

Antihypertensive drugs: cost effectiveness analysis in croatia D. Vitezić* ${ }^{*}, 2$, M. Kučan ${ }^{3}$, I. Lulić ${ }^{4}$, M. Vitezić ${ }^{3}$, J. Mršić Pelčić ${ }^{1}$ ${ }^{1}$ University of Rijeka Medical School, ${ }^{2}$ Clinical Hospital Center Rijeka, ${ }^{3}$ Community Health Center Primorsko-goranska County, ${ }^{4}$ University of Rijeka Faculty of Engineering, Rijeka, Croatia

\section{Health economy}

Introduction: Several national and international guidelines for the management of arterial hypertension that have been published recently, but pharmacoeconomic evaluation is rarely included.

Objectives: The aim of this study is to assess the cost-effectiveness of major four groups of first-line antihypertensive drugs: diuretics (D), betablockers (B), angiotensin converting enzyme (ACE) inhibitor/ angiotensin-II receptor blockers (ARBs) and calcium channel blockers (C) alongside a "do nothing" comparator.

Methods: The Markov model was used, and the study included 5 health states (during each 1-year cycle of the model, a proportion of patients enter one of the qualifying event health states: myocardial infarction, unstable angina, stroke, heart failure or death). Transition probabilities data were collected from the literature. Cost values includes average cost of drugs used (for each intervention), average cost of treatment in general practice, and average costs of specific cardio-vascular rehabilitation in specialized hospitals (collected from Croatian Health Insurance Fund data). The study was conducted separately for 65-year old man and woman with initial probability of cardiovascular death risk of $2 \%$, and heart failure risk of $1 \%$. Results are presented as difference in cost and difference in QALY (quality-adjusted life year) of all investigated scenarios compared with no intervention.

Results: The incremental QALY and the savings for diuretics compared with no intervention for men was 0.76 and $886 €$ (for woman 0.93 QALY and $987 €$ ). Treatment of arterial hypertension with beta-blockers resulted in 0.40 in incremental QALY but with additional cost of $41 €$ (for woman 0.48 QALY and $148 €$ ).

Summary / Conclusions: Treating arterial hypertension with diuretics, calcium channel blockers and angiotensine-converting enzyme inhibitors/ angiotensin-II receptor blockers are cost effective comparing with nointervention scenario. The most cost-effective first-line treatment are diuretics. The scenario with beta-blockers resulted in the additional QALY compared with no intervention, but also additional costs, and, according to our results, this therapy is not recommended as first line treatment.

\section{Misuse of medicines/substances}

\section{EACPT-1066}

Adverse reaction to compounded preparations prescribed for weight loss: $\mathbf{2}$ cases of cerebral venous thrombosis

B. Mainoli* 1, 2, M. Carvalho Dias ${ }^{3}$, A. M. Anes ${ }^{1,4}$, P. Canhão ${ }^{3,4}$, J. Ferreira $^{2,3,4}$, M. M. Rosa ${ }^{1,2,3,4}$

${ }^{1}$ Unidade de Farmacovigilância de Lisboa, Sistema Nacional de Farmacovigilância, ${ }^{2}$ Laboratory of Clinical Pharmacology and Therapeutics, Faculdade de Medicina, Universidade de Lisboa, ${ }^{3}$ Neurology Department (Stroke Unit), Hospital de Santa Maria, Centro Hospitalar Universitario Lisboa Norte, ${ }^{4}$ Faculdade de Medicina, Universidade de Lisboa, Lisbon, Portugal

\section{Misuse of medicines/substances, Pharmacovigilance}

Introduction: Compounded preparations are pharmaceutical products prepared and distributed under the responsibility of a pharmacist and are intended to fulfil particular patient needs in situations in which proprietary medicines available do not meet the specific requirements. Special combinations are usually used in paediatrics, dermatology and oncology treatment; as in the presented cases, combinations could be prepared as cocktails containing several active substances prescribed for weight loss. Quality and safety of compounded medicines are not guaranteed, as these medicines are exempt from marketing authorization and not subjected to formal clinical studies of high scientific quality.

Objectives: We aim to bring attention to Cerebral Venous Thrombosis as suspected Adverse Drug Reaction to weight loss compounded preparations.

Methods: We report two cases of 48 and 51 years-old females presented at emergency department with headache and mild or absent neurological signs; a diagnosis of Cerebral Venous Thrombosis (CVT) was identified by CT scan and confirmed by MRI with angiography. The first patient had started taking a weight loss compounded preparation 8 months before which included: chlordiazepoxide, bupropion, topiramate, metformin, furosemide, orlistat, cascara sagrada, senna, phenolphthalein, chromium picolinate, and centella asiatica; oral contraceptive (ethinylestradiol $0.030 \mathrm{mg}+$ gestodene $0.075 \mathrm{mg}$ ) had been reintroduced 5 months before. The second patient had been treated with same oral contraceptive for the past 16 years, and in the last year before she had taken a similar compounded preparation prescribed for weight loss composed of: chlordiazepoxide, bupropion, topiramate, metformin, furosemide, Hoodia Gordonii, Cascara Sagrada, and orange extract.

Results: In both the presented cases there is suspicion of additive/ synergic interaction with oral contraceptives, increasing the risk of venous thromboembolism associated with the latter, possibly due to dehydration caused by the diuretics and laxatives contained in these 
preparations. Causality was assessed with global introspection method as Possible by the Pharmacovigilance Unit of Lisbon.

Summary / Conclusions: Further epidemiologic studies are needed to confirm a possible association of these kinds of products with the occurrence of CVT, as well as additional investigation to determine the exact pathophysiologic mechanism.

\section{EACPT-1161}

High incidence of pharmacotherapeutic situations in the department of psychiatry of the tertiary hospital

E. Karinauske ${ }^{*} 1,2$, S. Kasciuskeviciute ${ }^{1,2}$, V. Galaune ${ }^{1,2}$, A. Usaite ${ }^{1,2}$, V. Adomaitiene ${ }^{3}$, R. Maciulaitis ${ }^{1,2}$

${ }^{1}$ Department of Nephrology, ${ }^{2}$ Institute of Physiology and Pharmacology, ${ }^{3}$ Department of Psychiatry, Lithuanian University of Health Sciences, Kaunas, Lithuania

\section{Misuse of medicines/substances, Psychopharmacology}

Introduction: The role of clinical pharmacologist (CP) is not yet systematically developed in Lithuania. There is a need to analyze and identify clinically meaningful pharmacotherapeutic situations in the tertiary hospital. Objectives: To analyze and identify clinically meaningful pharmacotherapeutic situations in the tertiary hospital.

Methods: 33 consecutive patients by psychiatric ICD code (F01-F59) were included from the Department of psychiatry of tertiary hospital in December 2018. The prescribed pharmacotherapy was analyzed by diagnose, efficacy, safety, adverse events, contraindications, drug interactions, off label use, therapeutic drug monitoring. The results are presented as descriptive statistics.

Results: Total 156 prescriptions (33 patients: 12 women and 21 men) from the Department of psychiatry were analyzed. Median age was 44 $(18 ; 80) .29$ out of 33 patients $(87.9 \%)$ had at least one pharmacotherapeutic situation. 18 out of 33 patients $(54.5 \%)$ had efficacy, 25 out of 33 patients $(75,8 \%)$ had safety situations and 23 out of 33 patients $(69.7 \%)$ had drug-drug interactions, such as high risk of occurrence of serotoninergic syndrome $(5 / 23-21.74 \%)$, sedation of central nervous system (CNS) $(17 / 23-73.9 \%)$, prolongation of QTc interval $(6 / 23-26.1 \%)$. Adverse reactions occurred to 4 patients out of 33 $(12.1 \%)$, they were an allergic reaction, hyponatremia and signs of dyspepsia. Off label use was identified to 5 out of 33 patients (15.2\%), the use of trihexyphenidyl for prophylaxis of extrapyramidal symptoms.

Summary / Conclusions: There is a very high risk covering predominantly safety risk as well as efficacy issues and unexpectedly, rather low level of off label use and contraindications in psychiatric patients. There is unexplored use of safer combination of antipsychotics diminishing risk for serotoninergic syndrome, sedation of CNS and prolongation of QTc interval. The reasons why safer alternatives are not used are unknown and should be investigated further. This shows a need of CP to be in the team of specialists when prescribing medications.

\section{EACPT-1204}

Experimental binge drinking episode: are there gender differences in alcohol concentrations and pharmacological effects?

L. Poyatos ${ }^{1,2}$, C. Pérez-Mañá, ${ }^{2,3}$, S. Martin ${ }^{3}$, D. Fuster ${ }^{2,}{ }^{4}$, R. Muga ${ }^{2,5}$, A. M. Barriocanal ${ }^{1,2}$, S. Malumbres ${ }^{6}$, M. Farré ${ }^{2,3}$, E. Papaseit ${ }^{* 2,3}$

${ }^{1}$ Institut Germans Trias i Pujol (IGTP), Badalona, ${ }^{2}$ Universitat Autònoma de Barcelona (UAB), Barcelona, ${ }^{3}$ Clinical Pharmacology, ${ }^{4}$ Internal Medicine, Hospital Universitari Germans Trias i Pujol (HUGTiPIGTP), ${ }^{5}$ Internal Medicine, Hospital Universitari Germans Trias i Pujol (IGTP), ${ }^{6}$ Clinical Chemistry, Hospital Universitari Germans Trias i Pujol (HUGTiP), Badalona, Spain

Misuse of medicines/substances, Pharmacokinetics/ pharmacodynamics, Psychopharmacology

Introduction: Binge drinking (BD) is defined as a pattern of drinking that reach blood alcohol concentration (BAC) to $80 \mathrm{mg} / \mathrm{dL}$ in a short period of time ( 2 hours) that typically occurs after 4 drinks for women and 5 drinks for men.

Objectives: The aim of this study was to evaluate the gender differences after the administration of alcohol simulating a BD episode under experimental conditions.

Methods: Sixteen male and eight healthy female volunteers, with previous BD behavior participated as outpatients in one experimental session. All of them received an oral single dose of $70 \mathrm{~g}$ of alcohol, mixed with zero orange soda without bubbles (Trina ${ }^{\circledR}$ ) distributed in 6 alcoholic drink glasses (total volume $900 \mathrm{ml}$ ) over a 2-hour period (20 minutes for glass). The trial was single-blind and non-randomized. Study variables included: vital signs and subjective effects [Visual Analogue Scales (VAS) and Biphasic Alcohol Effects Scales (BAES)] and BAC measured along a 12 hours period.

Results: Preliminary results show that the administration of alcohol simulating a BD episode under experimental conditions produce the prototypical alcohol effects (VAS drunkenness, and BAES stimulant and sedative effects) and BAC $>80 \mathrm{mg} / \mathrm{dL}$. Both, BAC and effects peaks were observed at 2 hours. In comparison to males, females presented higher and long lasting $\mathrm{BAC}$ and displayed more intense negative effects (dizziness and headache). There were no serious or unexpected adverse events.

Summary / Conclusions: Females achieved higher concentrations of alcohol in comparation to males and presented more intense negative effects. These preliminary results indicate that females could be more vulnerable to alcohol's effects administrated under BD conditions than males.

Acknowledgements: Ministerio de Sanidad, Política Social e Igualdad (Plan Nacional Sobre Drogas, 2016I024). Instituto de Salud Carlos III (Red de Trastornos Adictivos ISCIII-FEDER RD16/0017/0003; Esther Papaseit is a Juan Rodes fellowship ISCIII JR16/0002) Suport Grups de Recerca AGAUR Gencat (2017 SGR 316).

\section{Oral Presentation}

\section{EACPT-1205}

Medical prescriptions falsified by the patients before and after july 12th, 2017 when codeine, dextromethorphan, ethylmorphine and noscapine were scheduled in france

E. Jouanjus ${ }^{*} 1$, 2, M. Lapeyre-Mestre ${ }^{1,2}$, T. French Addictovigilance Network ${ }^{3}$

${ }^{1}$ Inserm UMR 1027, Equipe de Pharmacoépidémiologie, Université Toulouse III Paul Sabatier, ${ }^{2}$ Centre d'Addictovigilance, Service de Pharmacologie Médicale et Clinique, Centre Hospitalier Universitaire de Toulouse, Toulouse, ${ }^{3}$ FAN, National, France

\section{Misuse of medicines/substances}

Introduction: Serious intoxications following recreational use of psychoactive medical drugs in adolescents and young adults, including 2 deaths in 2017, were identified. Based on this signal, the French Ministry of Health scheduled codeine, dextromethorphan, ethylmorphine and noscapine from nonprescription to prescription-only drugs on July 12th, 2017.

Objectives: To assess the impact of this new regulation on the diversion modalities of the concerned drugs by analysing the national OSIAP (Ordonnances suspectes indicateur d'abus possible) database.

Methods: All medical prescriptions with at least one mention of codeine, dextromethorphan, ethylmorphine, noscapine or pholcodine recorded between 2013 and 2017 were extracted from the OSIAP database, which records falsified medical prescriptions presented to community pharmacies at the national level. Annual rates of mentioning were estimated by dividing the number of mentions by that of falsified prescriptions recorded the year considered. A descriptive analysis compared the characteristics of these prescriptions recorded before and after July $12^{\text {th }}, 2017$.

Results: Overall, 95 mentions of the requested drugs were extracted. Codeine was the most frequent $(\mathrm{n}=82)$, followed by dextromethorphan $(n=6)$, pholcodine $(n=5)$, and the codeine/ethylmorphine combination 
$(n=2)$. There was no mention of noscapine. Except for codeine, the annual rates of mentioning varied between 0 and $0.3 \%$ over the study period, without significant variation. As far as codeine was concerned, a first increase was identified in $2016\left(0.7 \%, \mathrm{n}=9\right.$ mentions). From July $12^{\text {th }}$, 2017 , this increase reached $10.3 \%(n=62)$. Interestingly, the profile of subjects who presented these falsified prescriptions has evolved accordingly, with a higher proportion of males (after July $12^{\text {th }}, 2017: 85 \%$ versus before: $60 \%$ ) and a younger age (after: 23 years old versus before: 37 ).

Summary / Conclusions: These results corroborate previous findings from a pilot study in community pharmacies. Drug diversion remains despite the restricted access to codeine, dextromethorphan, ethylmorphine and noscapine. In particular, the recourse to falsified prescriptions has sharply increased concerning codeine whereas the other drugs seem to have not been impacted. Delivery of the concerned drugs, especially codeine, should be carefully surveyed.

\section{EACPT-1207}

The 2018 state of the art on the cardiovascular risks of cannabisbased products: a systematic review

E. Jouanjus ${ }^{* 1,2}$, A. Déguilhem ${ }^{1}$, M. Lapeyre-Mestre ${ }^{1,2}$

${ }^{1}$ Inserm UMR 1027, Equipe de Pharmacoépidémiologie, Université Toulouse III Paul Sabatier, ${ }^{2}$ Centre d'Addictovigilance, Service de Pharmacologie Médicale et Clinique, Centre Hospitalier Universitaire de Toulouse, Toulouse, France

\section{Misuse of medicines/substances, Other}

Introduction: In 2016, the World Health Organization (WHO) emphasized there was a need for a better understanding of the cardiovascular risks associated with cannabis use. The systematic review of the literature published between 2011 and 2016 evidenced an increased risk of cannabis-related ischemic strokes, while the evidence was lower regarding the risk of cardiac disorders.

Objectives: To update the analysis of the published evidence on the cardiovascular risks related to the use of cannabis-based products.

Methods: A systematic review of articles published between 2016/06/01 and 2018/08/01 was performed per the international PRISMA statement to update a previous analysis. Articles presenting data on humans exposed to cannabis-derived products and suffering from any cardiovascular pathology, affection or anomaly, without any distinction on age, gender or nationality, were eligible for inclusion. The inclusion process was based on the previously defined search algorithm and was performed in a blinded standardized manner.

Results: Among the 457 articles found in the literature search, 74 remained after performing the inclusion procedure. These were 45 case reports and 29 observational studies. The mean age of the 70 individuals who were the subject for case reports was 29.7 (Standard deviation $\mathrm{SD}=$ 13.2). They were more frequently men than women $(72.9 \%$ and $27.1 \%$, respectively) and mainly suffered from cardiac diseases ( $n=23$, including 12 acute coronary syndromes) and cerebral disorders ( $n=18$, including 9 hemorrhages, 7 ischemic strokes and 6 reversible cerebral vasoconstriction syndromes). Other cardiovascular disorders were also reported. Overall, the 29 included observational studies evidenced an increased cardiovascular risk among cannabis users. This risk mainly corresponded to ischemic strokes and acute coronary syndromes, whereas less data were available concerning the other cardiovascular disorders.

Summary / Conclusions: By contrast to our previous analysis, the potential association between use of cannabis-based products and cardiac disorders was investigated. They evidenced a significantly increased risk for acute coronary syndrome. The continuously extended use of cannabis-based products is a worrying cause for public health concern considering the likelihood of higher negative cardiovascular impact on the exposed population.

\section{EACPT-1209}

Cannabis-related cardiovascular outcomes observed in hospitalized inmates
M. Mongiatti ${ }^{1}$, P. Bayle ${ }^{1}$, A. Lagarrigue ${ }^{1}$, N. Telmon ${ }^{1}$, M. LapeyreMestre $^{2,3}$, E. Jouanjus ${ }^{*} 2,3$

${ }^{1}$ Unités hospitalière sécurisée interregionale (UHSI), Médecine légale et médecine pénitentiaire, Centre Hospitalier Universitaire de Toulouse, ${ }^{2}$ Inserm UMR 1027, Equipe de Pharmacoépidémiologie, Université Toulouse III Paul Sabatier, ${ }^{3}$ Centre d'Addictovigilance, Service de Pharmacologie Médicale et Clinique, Centre Hospitalier Universitaire de Toulouse, Toulouse, France

\section{Misuse of medicines/substances, Other}

Introduction: In prison, subjects may use cannabis and other psychoactive substances despite their detained condition. Although it seems legitimate to wonder about the potential health consequences of this use, the scientific literature lacks data on the health status of inmates. Inmates who require a longer than 48-hour hospitalisation are admitted in specific polyvalent short-stay units called UHSI (Unité hospitalière sécurisée interrégionale).

Objectives: To describe the cardiovascular outcomes related to cannabis use in inmates hospitalised in UHSI of Toulouse University Hospital between 2012 and 2016.

Methods: The UHSI located in Toulouse University Hospital receives inmates from all over the Occitanie region (Southern France). In this study, all hospitalisations in this UHSI between 2012 and 2016 potentially related to cannabis use were extracted from the national hospital database (PMSI, Programme de Médicalisation des Systèmes d'Information) using previously defined diagnosis codes indicative of possible cannabis abuse or addiction. Included patients were those hospitalised for an inaugural cardiovascular event or a deterioration of a pre-existing cardiovascular illness who declared having consumed cannabis while imprisoned.

Results: A total of 31 cardiovascular outcomes occurred in 30 cannabisusing hospitalised inmates. All were males, and the mean age was 43 years old. All of them used cannabis and tobacco, 5 used cocaine, and none used alcohol. Cannabis was used on a daily basis by $55 \%$ of patients, and $85 \%$ of them were exposed to more than 15 pack-year tobacco. The most frequent events identified were coronaropathy $(n=13)$, including 9 cases of acute coronary syndrome. They were followed by obliterating arteriopathy of the lower limb $(n=7)$, rhythmic cardiomyopathy $(n=4)$, venous thrombosis $(n=3)$, infectious cardiopathy $(n=2)$, and ischemic stroke $(\mathrm{n}=2)$.

Summary / Conclusions: To our knowledge, we provide the first series of serious cardiovascular outcomes described in the context of cannabis use in prison. A sharpened awareness of health providers involved in the care of inmates is crucial. This initial study was focused on the cases more likely to be associated to cannabis use based on the scientific literature, but further investigations should be conducted to evaluate more silent outcomes, together with disorders affecting systems different from the cardiovascular system.

\section{EACPT-1293}

Prescription drugs in fatal accidents - prescribed or not? A. Jönsson ${ }^{1}$, J. Ahlner ${ }^{*} 1$

${ }^{1}$ Department of Forensic Genetics and Forensic Toxicology, NATIONAL BOARD OF FORENSIC MEDICINE, Linköping, Sweden

\section{Misuse of medicines/substances}

Introduction: Impairment by ethanol and/or other psychoactive substances increases the risk of involvement in fatal accidents.

Objectives: The aim of this study was to describe prevalence of nonprescribed use of narcotic drugs among fatal accidents, split into roadaccidents, drownings, falls, burns and other accidents.

Methods: The study population consists of Swedish residents, who died due to accidents between 1/6/2006 and 30/6/2016, where a prescription drug was identified in blood during postmortem analyses. For the 
included population information was retrieved from The Cause of Death Register, the National Forensic Toxicology Database and the Swedish Prescribed Drug Register.

Results: Results show that, 6270 prescription drugs were identified among the 2709 included fatalities. The majority of the included individuals were men $(\mathrm{N}=1934,71 \%)$, with a median age of 62 years. The most prevalent drug-classes present were opioids $(\mathrm{N}=582)$ and sedative and hypnotics $(\mathrm{N}=534)$. For $50 \%$ of the prescription drugs identified a valid prescription for the drugs were identified within in 1 year prior to the death. The proportion of valid prescription was $45 \%$ for traffic fatalities of the substances identified postmortem the equivalent proportion was $52 \%$ for other accidents, $56 \%$ for falls, $58 \%$ for fire victims and $64 \%$ for drownings. Looking at different drug classes, for sedative and hypnotics the victims had a valid prescription in $66 \%$ of the cases, which is in contrast opioids, where only $29 \%$ had a valid prescription. However, within each class there was a huge variation between different substances.

Summary / Conclusions: This study shows that use of narcotic prescriptions drugs without a valid prescription was common among victims of fatal accidents in Sweden.

\section{EACPT-1349}

The contribution of methadone and buprenorphine/naloxone maintenance treatment for opiate dependence to hiv prevention from 2009 to 2018 in taiwan

H.-Y. Lee ${ }^{*} 1$, H.-C. Hsu ${ }^{1}$, C.-H. Liu ${ }^{2}$

${ }^{1}$ Pharmacy Department, Kaohsiung Municipal Kai-Syuan Psychiatric Hospital, ${ }^{2}$ Pharmacy Department, Kaohsiung Medical University Chung-Ho Hospital, Kaohsiung, Taiwan, Province of China

\section{Misuse of medicines/substances, Psychopharmacology}

Introduction: In Taiwan, the incidence of HIV/AIDS reached a height of 2,461 in 2005. Prior to 2003, the HIV incidence was mostly due to men having sex with men. However, the percentage of IDUs related to HIV increased from $34.7 \%$ in 2000 to $63.9 \%$ in 2004 , and the percentage of IDUs sharing needles increased from $4.0 \%$ in 2000 to $15 \%$ in 2004 . One year after the national pilot harm reduction program including a methadone maintenance treatment (MMT), the Taiwan Centers for Disease Control (TCDC) reported a dramatic $10 \%$ decrease in all new HIV seropositive cases, and a nationwide harm reduction program was subsequently implemented. Moreover, TCDC also supplied buprenorphine/ naloxone maintenance treatment (BNMT) in 2010.

Objectives: The purpose of this study is to represent the contribution of methadone and buprenorphine/naloxone maintenance treatment for opiate dependence to HIV incidences from 2010 to 2018 in Taiwan.

Methods: This observational study was designed to collect the change over time in the incidence of HIV and the numbers of participants in the maintenance treatments in Taiwan from 2010 to 2018. The HIV/ AIDS data were obtained from the national HIV/AIDS registry of the TCDC, in which physicians are requested by law to report identified cases of HIV infection and AIDS within $24 \mathrm{~h}$ of diagnosis. The numbers of participants in the maintenance treatments were obtained from Ministry of Health and Welfare.

Results: The total numbers of participants in maintenance treatments from 2010 to 2018 are from 11,892 persons to 8,182 persons. The percentage of participants in MMT and BNMT from 2010 to 2018 are from $99.66 \%$ to $93.28 \%$ and from $0.34 \%$ to $6.92 \%$, respectively. The patients in BNMT seems to increase in the recently year. Moreover, the HIV incidences among IDUs in Taiwan from 2010 to 2018 are from $6.51 \%$ to $2.21 \%$. Compared to the HIV incidence among IDUs in 2004 (63.9\%), the incidence sharply decrease to $2.21 \%$ in 2018 . During this 13 -year period, IDUs with HIV accounted for $18.64 \%$ of the total reported cases. Summary / Conclusions: It showed that MMT and BNMT sustained to reduce HIV cases related to IDUs from 2009 to 2018 in Taiwan. Although the interventions of maintenance treatments play important roles in HIV prevention, the participants joined in maintenance treatments decreasing yearly should still be notified.

\section{EACPT-1394}

A pilot study: use of the adult adhd-self report scale in a south african patient population

J. Regnart $^{*}{ }^{1}$, I. Truter ${ }^{1}$, Z. Zingela ${ }^{2,3}$, A. Meyer ${ }^{2,4,5,6}$

${ }^{1}$ Drug Utilization Research Unit (DURU), Department of Pharmacy, ${ }^{2}$ Psychology, Nelson Mandela University, Port Elizabeth, ${ }^{3}$ Psychiatry, Walter Sisulu University, Mthatha, ${ }^{4}$ Psychology, University of Limpopo, Sovenga, ${ }^{5}$ Behavioural Medicine, University of KwaZuluNatal, Pietermarizburg, ${ }^{6}$ Psychology, North-West University, Potchefstroom, South Africa

\section{Misuse of medicines/substances, Psychopharmacology}

Introduction: Attention-Deficit/Hyperactivity Disorder (ADHD) is a neurodevelopmental disorder which typically presents in childhood. This diagnosis may often be overlooked in adulthood, particularly in psychiatric populations. The Adult ADHD-Self Report scale (ASRS) is an internationally used and reliable screener, however studies investigating its use in African populations are limited.

Objectives: To investigate the application of the ASRS in a South African setting.

Methods: A convenience sample of acutely presenting psychiatric participants admitted for stabilisation was used in Port Elizabeth, South Africa. This population was identified as representing a developing or low- and middle-income country population. Fieldworkers administered the ASRS and collected information relating to demographics, differential diagnoses, substance use disorder (SUD) presence, substance consumption and prescribed medication relating to current or historical treatment of ADHD

Results: The study sample included 30 participants, with black people representing the majority of participants. ASRS completion revealed the rate of ADHD within the study population to be $43.3 \%$, a contrast to the initially presumed prevalence of $6.7 \%$ which was based on reported methylphenidate therapy. A difference in SUD prevalence was identified between subjects screening positively and negatively for ADHD with a greater tendency towards SUDs seen for ASRS-positive individuals. Significant differences were identified in relation to cannabis- and polysubstance use for ASRS-positive individuals.

Summary / Conclusions: Despite limitations related to the sample used and challenges in ASRS administration, investigation findings support recommendations for ADHD screening inclusion in acute inpatient settings in South Africa and ASRS translation into indigenous African languages.

\section{Nephropharmacology}

\section{EACPT-1119}

The effects of co-administration of cholinergic and cannabinoid receptor ligands on different memory stages in mice M. Kruk-Slomka ${ }^{1}$, A. Dzik ${ }^{1}$, G. Biala* ${ }^{1}$

${ }^{1}$ Chair and Department of Pharmacology and Pharmacodynamics, Medical University of Lublin, Faculty of Pharmacy with Medical Analytics Division, Lublin, Poland

\section{Nephropharmacology, Psychopharmacology}

Introduction: The endocannabinoid system (ECS), through the cannabinoid type $\mathrm{CB} 1$ and $\mathrm{CB} 2$ receptors, is involved in many physiological functions, including memory. Due to their properties, cannabinoid compounds, especially CB2 receptor ligands, appears to be a promising therapeutic target in the treatment of memory-related disorders.

The most known dysfunction of cognition (e.g. Alzheimer's disease, AD) is connected with cholinergic system. However, the interaction between 
cholinergic system and the ECS in the context of cognitive processes remains poorly understood.

Objectives: The aim of the study was to determine the influence of the selective CB2 receptor ligands on the different stages of memory processes in the context of the interactions with cholinergic system. We examined an impact of CB2 receptor agonist (JWH 133) and antagonist (AM 630) on the memory acquisition and consolidation processes modified by an acute administration of nicotine, a cholinergic receptor agonist, as well as scopolamine, a cholinergic receptor antagonist, in mice.

Methods: To assess and understand the memory-related effects we used the passive avoidance (PA) test commonly used to examine long-term emotional memory. The deficit in PA performance was expressed as the difference between retention and training latencies and is taken as an index of latency (IL).

Results: Co-administration of JWH $133(0.25 \mathrm{mg})$ or AM $630(0.25$ $\mathrm{mg} / \mathrm{kg})$ with the non-effective dose of nicotine $(0.05 \mathrm{mg} / \mathrm{kg})$ enhanced cognition in the PA test in mice. However, an acute injection of JWH 133 $(0.25 \mathrm{mg} / \mathrm{kg})$ or AM $630(0.25 \mathrm{mg} / \mathrm{kg})$ had no influence on memory enhancement induced by the effective dose of nicotine $(0.1 \mathrm{mg} / \mathrm{kg})$. In turn, co-administration of JWH $133(0.25 \mathrm{mg})$ or AM $630(0.25 \mathrm{mg} / \mathrm{kg})$ with the effective dose of scopolamine $(1 \mathrm{mg} / \mathrm{kg})$ attenuated the scopolamine-induced memory impairment in the PA test in mice.

Summary / Conclusions: Our experiments show that ECS, through CB2 receptors, participates in the modulation of memory processes, especially those in which cholinergic pathways are implicated. As such, our findings could be helpful in further research concerning the effective pharmacotherapy of diseases which are associated with cognitive impairments, especially referred to cholinergic system dysfunction, such as AD.

\section{Patient empowerment}

\section{Oral Presentation}

\section{EACPT-1343}

Placebo effect and the incidence of anxiety and depression in patients with drug allergy

I. Cegec ${ }^{*}$, V. Erdeljic Turk ${ }^{1}$, R. Likic ${ }^{1}$, I. Kraljickovic ${ }^{1}$, K. Makar Ausperger ${ }^{1}$, M. Radacic Aumiler ${ }^{1}$, I. Mercep ${ }^{1}$

${ }^{1}$ Unit for clinical pharmacology, University Hospital Zagreb, Zagreb, Croatia

\section{Patient empowerment}

Introduction: Drug hypersensitivity reactions represent up to one-third of all adverse drug reactions. Drug hypersensitivity testing is a complex process and it is subject to many subjective influences. Moreover, false positive reactions can occur due to psychological symptoms especially in terms of anxiety and depression.

Objectives: The aim of this investigation was to assess, based on the Hamilton's scale, the differences in the incidence of anxiety and depression between placebo reactors and non-reactors in patients with a history of drug hypersensitivity.

Methods: In assessing the causal link between the administration of the drug and hypersensitivity reactions in patients, the single blind provocation test is used as the "gold standard". During testing for drug hypersensitivity, it is necessary to use placebo since patients who have previously developed reactions to drugs are susceptible to the placebo effect. During the process of testing, according to the usual protocol, the patient's psychological condition was assessed using the Hamilton's scale for anxiety (HAM-A: 5 -point scale: $0=$ not present to $4=$ severe) and depression (HAM-D: 8 items are scored on a 5-point scale: $0=$ not present to $4=$ severe, 9 items are scored from 0-2).

Results: Based on placebo challenge results, from 87 patients (16 males and 71 females), 21 (24\%) were placebo reactors $(91 \%$ females). The average score on HAM-A was 14 (mild anxiety) for placebo reactors and 5(normal) for non-reactors. The average score on HAM-D scale was more than two times higher for placebo reactors than for non-reactors (9 (mild depression) versus 4 (normal)). Based on anamnestic data, placebo reactors reported having hypersensitivity reactions to a larger number of generically different drugs versus placebo non-reactors (average 4.6 vs 2.8, respectively). Furthermore, placebo reactors reacted on average to a larger number of different drug groups (2.4) vs non-reactors (1.6). Finally, the duration of hypersensitivity testing was longer in the placebo reactors (5.8 days on average) group in comparison to the non-reactors (4.4 days).

Summary / Conclusions: Better understanding of the reactions of patients with a history of hypersensitivity to drugs will lead to avoidance of misdiagnosing drug hypersensitivity and in turn averting unnecessary reduction of treatment options as well as complicating the treatment regimens.

\section{EACPT-1384}

Quality of life in patients undergoing testing for drug allergy - with emphasis on placebo reactors

V. Erdeljic Turk ${ }^{*} 1$, I. Čegec ${ }^{2}$, M. Radačić-Aumiler ${ }^{1}$, K. MakarAušperger ${ }^{1}$, R. Likicí ${ }^{1,3}$, I. Kraljičković ${ }^{1}$, I. Merćep ${ }^{1,3}$

${ }^{1}$ Division of Clinical Pharmacology, ${ }^{2}$ University Hospital Zagreb, ${ }^{3}$ Medical School Zagreb, Zagreb, Croatia

\section{Patient empowerment, Other}

Introduction: Drug allergies are drug-hypersensitivity reactions that are frequently seen in practice and affect quality of life considerably. Currently, skin testing followed by an oral challenge is considered the gold standard for diagnosing an immediate drug allergy. Generally, placebo should be considered during allergy testing to reduce false-positive results, especially in females and in patients with multiple drug allergies. Division of Clinical Pharmacology at the University Hospital Zagreb comprise the largest drug allergy center in Croatia providing services to more than 1.300 patients/year.

Objectives: The aim of this study is to investigate the effect of drug allergy on quality of life using the SF-36 health questionnaire, with emphasis on differences in percieved quality of life between placebo reactors and placebo non-reactors.

Methods: A total of 200 adult patients referred to our center for drug allergy testing were consecutively evaluated using the Short Form-36 (SF-36) questionnaire. SF-36 is a set of generic, coherent, and easily administered quality-of-life measures. These measures rely upon patient self-reporting and are now widely utilized for routine monitoring and assessment of care outcomes in adult patients. We included only patients tested with oral challenge tests. In addition, socio-demographic characteristics, medical history data, and outcomes of drug allergy testing were collected and evaluated.

Results: The results on SF-36 quality of life scale in patients diagnosed with drug allergy will be presented in total and with regard to their placebo reactor status (placebo reactors vs. placebo non-reactors) and medical history (eg, number of drug allergies, atopy, type of allergic reaction). In addition, other factors that may influence the quality of life in the evaluated patient population will be accounted for (eg, comorbidities, age, gender, education).

Summary / Conclusions: Conclusions on differences in quality of life in subsets of patients undergoing testing for drug allergy will be made based on previous research and explanations will be proposed.

\section{Pediatric treatment}

EACPT-1012

Evaluation of efficacy and safety of human normal immunoglobulin after the change of administration route from intravenous to subcutaneous in pediatric patients 
N. Báez Gutiérrez ${ }^{1}$, M. Moleon Ruiz ${ }^{1}, H$. Rodriguez Ramallo ${ }^{1}$, C. Alvarez Del Vayo ${ }^{1}$ R. Seisdedos Elcuaz

${ }^{1}$ Hospital Pharmacy, Hospital Universitario Virgen del Rocío, Seville, Spain

\section{Pediatric treatment}

Introduction: Compared with intravenous immunoglobulin (IVIg) treatment, subcutaneous immunoglobulin ( $\mathrm{SCIg}$ ) can more easily be selfadministered at home and has a lower incidence of adverse effects relating to peak immunoglobulin levels

Objectives: To evaluate the efficacy and safety in the administration of immunoglobulin ( $\mathrm{Ig}$ ) in pediatric patients with primary immunodeficiency (PID) after the change of administration route from IVIg to SCIg.

Methods: We conducted an observational and prospective study at a Tertiary Level Hospital. All pediatric patients with PID who had switched from IVIg to SCIg from May 2017 ato August 2018 were included. The data recorded were: diagnosis, duration of treatment with SCIg, age, sex and dosage regimen of IVIg and SCIg. Trough $\mathrm{IgG}$ plasma concentrations were recorded before and after the change of route. Likewise, reported serious bacterial infections and safety incidents were collected.

Results: We included 8 patients whose diagnoses were: 4 with agammaglobulinemia associated with chromosome $\mathrm{X}, 3$ with combined immunodeficiency and 1 with hypogammaglobulinemia. The mean duration of treatment was 9.2 months during the study period. The mean age of the patients, at the time of change of route, was 12 years (9-15), $100 \%$ males. The mean dose of IVIg was $500.5 \mathrm{mg} / \mathrm{kg} /$ month (214.3-1351) and of $\mathrm{SCIg}, 118.6 \mathrm{mg} / \mathrm{kg} /$ week (90.6-161.8); this represents an accumulated dose of $461.3 \mathrm{mg} / \mathrm{kg} / \mathrm{month}$ (362.4-647.3). The change from IVIg to SCIg was made at a dose of 1: 1 .

The mean trough $\mathrm{IgG}$ serum concentration before the change of route was $743.8 \mathrm{mg} / \mathrm{dL}$ (485.1-930.2); and after the change was $828.2 \mathrm{mg} / \mathrm{dL}$ (7031161.3). In all the patients except one, the trough concentrations increased with the change of route.

In $3(37.5 \%)$ patients, an increase of more than $10 \%$ in serum IgG concentrations was observed with the change of route. Only one patient had a severe bacterial infection (pertussis infection). Two patients presented local reactions at the injection site.

Summary / Conclusions: The SC administration of Ig allows achieving higher and maintained serum concentrations, allowing in our case a reduction of the dose in at least 3 of the treated patients.

The treatment with SCIg is an effective alternative to avoid infections and in general well tolerated in pediatric patients with PID.

\section{Oral Presentation}

EACPT-1061

An artificial intelligence tool to find the best cyclosporine initial intravenous dosing regimen in pediatric hematopoietic stem cell transplantation

V. Leclerc ${ }^{* 1,2}$, M. Tod ${ }^{1,2}$, M. Ducher ${ }^{1,2}$, N. Bleyzac ${ }^{1,2}$

${ }^{1}$ EMR3738 Ciblage thérapeutique en oncologie, Faculté de Médecine et de Maïeutique Lyon-Sud Charles Mérieux, Université Claude Bernard Lyon 1, 165 chemin du Grand Revoyet-BP 12, Oullins, ${ }^{2}$ Pharmacy Department, Hospices Civils de Lyon, Lyon, France

\section{Pediatric treatment}

Introduction: Allogeneic hematopoietic stem cell transplantation (HSCT) revolutionized the treatment of both malignant and non-malignant hematologic diseases. An immunosuppressive regimen is needed after HSCT, generally including cyclosporine A (CsA). However, this drug with narrow therapeutic index is difficult to handle for clinicians. Finding the best initial dosing regimen in pediatric patients is not only challenging because of the extremely wide inter-individual variability encountered in this population, but also essential to shorten time to reach therapeutic range which reduces the risk of occurrence and severity of both acute and chronic graft versus host disease. Objectives: The aim of this work was to design a decision tool to help clinicians finding the best initial CsA dosing regimen after pediatric HSCT by using a probabilistic artificial intelligence method (Bayesian network, BN).

Methods: We built a BN with data from a retrospective monocentric cohort of 149 pediatric patients undergoing HSCT from 2008 to 2016. We recorded anthropometric, clinical, and biological data. We used the WEKA software (version 3.8.2) to build the $\mathrm{BN}$ on train data and to perform a ten-fold crossvalidation. We generated with the Tree-Augmented Naïve (TAN) algorithm the network structure and used conditional probabilities to fill the probabilities tables. We assessed predictive performance with AUC-ROC, the percentage of misclassified patients, the true and false positive rates (TPR and FPR respectively). The final $\mathrm{BN}$ was then replicated in the Netica software (version 6.05), allowing an easy online use.

Results: We included 11 variables in the final model in addition to the target variable (the initial intravenous dosing regimen). On train data, the TAN-BN AUC-ROC was 0.99, the percentage of misclassification $4.03 \%$, the TPR 0.96, and the FPR 0.08. After a 10 -fold cross-validation the model had an AUC-ROC of 0.95, 10.07\% of misclassified patients, a TPR of 0.90 and a FPR of 0.19 .

Summary / Conclusions: We designed and cross-validated a Bayesian network showing interesting predictive capacity to find the best CsA initial dosing regimen after pediatric HSCT. This model should undergo an external validation before its use in clinical practice.

\section{EACPT-1062}

Switching from intravenous to oral cyclosporine after hematopoietic stem cell transplantation: an artificial intelligence tool V. Leclerc ${ }^{* 1,2}$, M. Tod ${ }^{1,2}$, N. Bleyzac ${ }^{1,2}$, M. Ducher ${ }^{1,2}$

${ }^{1}$ EMR 3738, Ciblage Thérapeutique en Oncologie, Faculté de Médecine et de Maïeutique Lyon-Sud Charles Mérieux, Université Claude Bernard Lyon 1, 165 chemin du Grand Revoyet-BP 12, Oullins, ${ }^{2}$ Pharmacy Department, Hospices Civils de Lyon, Lyon, France

\section{Pediatric treatment}

Introduction: Switching a drug from intravenous (IV) to oral route is often challenging for clinicians, particularly for drugs with narrow therapeutic index. This is the case in pediatric hematopoietic stem cell transplantation (HSCT) with immunosuppressant such as cyclosporine A (CsA), the most widely used one. This drug has a large interindividual variability towards bioavailability, justifying a global approach. Bayesian networks (BN) are classification and prediction probabilistic models, belonging to artificial intelligence (AI). They are of interest in medicine as they can deal with many variables, missing values, and provide an easy-to-understand graph allowing predictions thanks to inference algorithms.

Objectives: The purpose of this work was designing a decision tool to help clinicians finding the best oral CsA dosing regimen in pediatric HSCT patients when switching from IV route, using a probabilistic AI method (BN).

Methods: We built a BN with data from a retrospective monocentric cohort of 147 pediatric patients undergoing HSCT from 2008 to 2016. We recorded anthropometric, clinical, and biological data. We used the WEKA software (version 3.8.2) to build the $\mathrm{BN}$ on train data and to perform a ten-fold crossvalidation. We used the Tree-Augmented Naïve (TAN) algorithm for the network structure and conditional probabilities to fill the probabilities tables. We assessed predictive performance with AUC-ROC, the percentage of misclassified patients, the true and false positive rates (TPR and FPR respectively). The final BN was then replicated in the Netica software (version 6.05), allowing an easy online use.

Results: We included 16 variables in the final model in addition to the target variable (the oral dosing regimen at switching time). On train data the TAN-BN AUC-ROC was 0.97 , the percentage of misclassification $13.6 \%$, the TPR 0.86 , 
and the FPR 0.06. After a 10-fold cross-validation the model had an AUC-ROC of $0.85,30.6 \%$ of misclassified patients, a TPR of 0.69 and a FPR of 0.14 .

Summary / Conclusions: We designed and cross-validated a bayesian network showing promising predictive capacity to find the best oral CsA dosing regimen at time of changing administration route after pediatric HSCT. This model should undergo an external validation before its use in clinical practice.

\section{EACPT-1124}

Anti-d or intravenous immunoglobulins for immune thrombocytopenia in children: systematic review and meta-analysis

B. Lioger ${ }^{1}$, F. Maillot ${ }^{2}$, D. Ternant ${ }^{3}$, C. Passot ${ }^{4}$, G. Paintaud ${ }^{3}$, T. BejanAngoulvant ${ }^{*} 5$

${ }^{1}$ Service de Médecine Interne et Maladies Systémiques, APHP, Paris, ${ }^{2}$ Service de Médecine interne, ${ }^{3}$ Medical pharmacology department, CHRU de Tours; Université de Tours, Tours, ${ }^{4}$ Institut cancérologie de l'Ouest, Angers, ${ }^{5} \mathrm{CHRU}$ de Tours; Université de Tours, Tours, France

\section{Pediatric treatment}

Introduction: Immune thrombocytopenia (ITP) is characterized by a platelet count (PC) $<100 \times 10^{9} / \mathrm{L}$ related to an immune dysregulation. In children, ITP occurs mainly after an infectious episode and PC recovers frequently without specific treatment. Cutaneous manifestations are frequent, but intracranial hemorrhage is very rare. Intravenous (IVIG) and anti-D (anti-D) immunoglobulins are sometimes needed.

Objectives: To compare the efficacy and safety of IVIG and anti-D in pediatric ITP, since each treatment has its own strengths and weaknesses, and both were found to rapidly increase PC in small clinical studies.

Methods: We conducted a systematic review and meta-analysis (PRISMA guidelines) including all randomized controlled trials assessing the efficacy and safety of anti-D and IVIG in children with ITP. Primary outcomes were the proportion of children achieving PC response as defined in each study and bleeding response. Other safety outcomes included infusion reactions and hemolysis. We extracted aggregate data and reported results as risk ratios with $95 \%$ CI. We used fixed effect Mantel-Haenszel method for PC outcome. Statistical heterogeneity across trials was assessed with chi2 and I2 statistics. With regard to adverse reactions, we used a fixed effect Peto method.

Results: Eleven studies with 558 children were included. Anti-D was significantly inferior to IVIG at increasing PC, both for thresholds of $>20 \times 109 / \mathrm{L}$ at $24-72$ hours (response rate ratio for anti-D vs IVIG: $0.85,95 \%$ CI $0.78-0.94$ ) and $>50 \times 109 / \mathrm{L}$ at $24-72$ hours (response rate ratio for anti-D vs IVIG: $0.75,95 \%$ CI 0.61-0.92). Bleeding response was assessed in 4 studies, but some heterogeneity in reporting leads to unclear conclusion. General symptoms after anti-D infusion were less frequent than after IVIG (Peto OR 0.39, 95\% CI 0.25-0.62). Hemolysis was more frequent after anti-D. No death was reported in included studies. Overall, the quality of included studies was poor. High or unclear "risk of bias" issues were present in all studies, mainly because all studies were openlabeled with a high risk of performance bias.

Summary / Conclusions: Compared with anti-D, IVIG led to a better response in terms of PC and may be preferred as a first-line treatment of ITP in children with acute hemorrhagic symptoms. However, the clinical significance of IVIG superiority on PC remains unclear.

\section{Oral Presentation \\ EACPT-1196}

An innovative ethosuximide granule formulation designed for paediatric use: comparative pharmacokinetics, safety, tolerability and palatability profile vs syrup

L. Diezi ${ }^{1}$, K. Dao ${ }^{1}$, V. Jullien ${ }^{2}$, C. Roussel-Maupetit ${ }^{3}$, I. Burton ${ }^{4}$, P. André $^{1}$, C. Bardinet ${ }^{1}$, C. Csajka ${ }^{1}$, M. Manso ${ }^{3}$, C. Guittet ${ }^{3}$, F. BrunnerFerber $^{5}$, F. Vandenhende ${ }^{4}$, C. Chiron ${ }^{6}$, G. Pons ${ }^{7}$, L.-A. Granier ${ }^{8}$, T. Buclin $^{* 1}$

${ }^{1}$ Service of Clinical Pharmacology, UNIVERSITY HOSPITAL CENTER OF LAUSANNE (CHUV), Lausanne, Switzerland, ${ }^{2}$ Service de Pharmacologie, Hôpital Européen Georges Pompidou, Paris, ${ }^{3}$ Advicenne Pharma, Nîmes, France, ${ }^{4}$ ClinBay Sprl, Genappe, Belgium, ${ }^{5}$ Brunner-Naga Health Science Consulting, Pfaeffikon-ZH, Switzerland, ${ }^{6}$ Service de neuropédiatrie, Hôpital Necker Enfants Malades, ${ }^{7}$ Département de pharmacologie, Groupe Hospitalier Cochin SaintVincent-de-Paul, ${ }^{8}$ Advicenne Pharma, Paris, France

\section{Pediatric treatment}

Introduction: Ethosuximide is a first line therapy for treating childhood absence epilepsy, but is currently formulated as a syrup with unpleasant bitter taste and high sugar content, poorly adapted to paediatric patients. The aim of this European collaborative FP7 project named KIEKIDS was to develop an innovative sugar-free and tasteless ethosuximide formulation convenient for paediatric use.

Objectives: This dual Phase I study evaluated two granule formulations (A and B) based on Lipid MultiParticulate technology.

Methods: Panels A and B followed a randomised, placebo controlled, partly double-blind, 3-way cross-over study design, comparing placebo granules, ethosuximide granules $\mathrm{A}$ or $\mathrm{B}$, and the marketed syrup Zarontin ${ }^{\circledR}$ at a single dose of $10 \mathrm{mg} / \mathrm{kg}$. The pharmacokinetic profile and relative bioavailability of ethosuximide in plasma were compared, as well as palatability, safety and tolerability. Twelve healthy adult volunteers were enrolled (6 per panel). Ethosuximide concentrations were determined by highperformance liquid chromatography / UV detection. Visual analogue scales (VAS) were used to assess palatability, neurological and digestive tolerability.

Results: While granules A in Panel A proved not adequate due to bitter taste and adherence to container walls, granules B in Panel B presented an excellent palatability similar to placebo granules and were easy to dispense.

The relative bioavailability of granules A versus Zarontin®, based on dose-normalised $\mathrm{C}_{\max }$ and $\mathrm{AUC}_{0-\infty}$, was 93.7 [90\% CI: 76.3-115.1] and 96.1 [91.0-101.5], respectively. For granules B it was 87.6 [81.694.0] and 92.5 [88.5-96.6], respectively. Granules B displayed a delayed $\mathrm{T}_{\max }$ of $0.75 \mathrm{~h}[0.5-4.05]$ versus syrup $0.5 \mathrm{~h}[0.3-0.8]$.

VAS for tolerability revealed slight transient dizziness, sedation, and anxiety, more pronounced and more frequent after syrup than after both active granules. Other neurological and digestive VAS assessments were similar between treatments.

Summary / Conclusions: The innovative ethosuximide granule formulation B has achieved the target profile for paediatric use, being sugarfree, tasteless, bioequivalent within the $80-125 \%$ regulatory range, and well tolerated, while enabling graded adjustment to body weight.

\section{EACPT-1271}

Preparation of pediatric dosage forms in hospital pharmacy Ü. Erbey ${ }^{1}$, D. Kundakçı ${ }^{*}{ }^{1}$, A. Özyıldırım ${ }^{1}$, B. Aktaşl1 ${ }^{1}$, S. S. Vural ${ }^{1}$, M. Sayın $^{1}$, S. Arslan ${ }^{1}$, L. B. Ölçü ${ }^{2}$, B. Bozkurt ${ }^{2}$, D. Küçükler ${ }^{2}$, F. Küçükerenköy ${ }^{2}$, I. Bozkurt ${ }^{2}$

${ }^{1}$ Pharmacy, ${ }^{2}$ VKV. American Hospital, İstanbul, Turkey

\section{Pediatric treatment, Other}

Introduction: Medication errors are an important health problems in all hospitalized population that threatens patient safety and increases treatment costs. Studies have shown that 1.5 million people suffer from medication errors every year which can be prevented. The Joint Comission stated safety usage of medication as one of the national patient safety goals In 2015,

Pediatric populations are exposed to medication errors more often than adults. The lack of pediatric dosage forms of some medications or requirements of dose adjustmentare the subtantial challenges to deal with. Therefore, prevention of medication errors in pediatric patients has particular importance. 
Objectives: We believe that by preparing the pediatric dosage forms at the pharmacy,we will prevent drug administration errors

Methods: Between January 2018 to April 2018,1613 prescriptions which were written for Pediatric populations were examined in order to preparation of pediatric drug dosages in pharmacy. As a result of the survey, the pharmacy detected that 1743 parenteral doses, 2204 oral doses and 71 high-risk aerosol medications were ordered by physician and prepared by nurse at the ward. After the Pharmacy and Therapeutics Commitee's assesment of the process, whole parenteral pediatric drug dosages, oral dosage forms that requires dose adjustment from adult dosages,parenteral nutrition solutions and high risk inhaler medication dosages $\mathrm{s}$ have started to prepare/ reconstitute in the pharmacy department.

Table charts and information leaflets that include stability information of the drugs, storage conditions and dilution schemes of the certain drugs were prepared by pharmacy ready-to-administer team in order to guidance of the new process. Whole drug dosages within the scope of the pediatric patients have been prepared by ready-to-administer team in inpatient pharmacy, since May 2018

Results: Between May 2018 to January 2019,4168 parenteral drug dosages, 223 high-risk inhaler dosages and 169 total parenteral nutrition therapy prepared in pharmacy. While there were 13 medication administration errors were reported regarding to pediatrics unitafter the establisment of this new process 3 medication administration errors were reported to the quality improvement department in 2017.

Summary / Conclusions: The aim of the study is to contribute to patient safety by decreasing the number of advers drug reactions seen during the preparation, distribution and administration of pediatric units throughout the hospital

\section{Oral Presentation}

\section{EACPT-1276}

Global trigger tool to measure drug safety in adolescents with acute psychotic episode: the first experience in child psychiatry

D. Ivashchenko* ${ }^{1,2}$, N. Buromskaya ${ }^{3}$, L. Savchenko ${ }^{4}$, Y. Shevchenko ${ }^{5}$, D. Sychev ${ }^{6}$

${ }^{1}$ Child Medical Centre of Russian Federation President's Business Administration, ${ }^{2}$ Department of Personalized Medicine, Russian Medical Academy of Continuous Professional Education, Moscow, Russian Federation, ${ }^{3}$ G.E. Sukhareva Research Practical Centre of Children and Adolescents Mental Health, Moscow, -- ${ }^{4}$ Russian Medical Academy of Continuous Professional Education, Moscow, Russian Federation, ${ }^{5}$ Department of Child psychiatry and psychotherapy, ${ }^{6}$ Department of Clinical pharmacology and therapeutics, Russian Medical Academy of Continuous Professional Education, Moscow, Russian Federation

\section{Pediatric treatment, Pharmacovigilance, Psychopharmacology}

Introduction: Drug safety isthe priority in child psychiatry. Psychopharmacology is prescribing for a very long time. Global trigger tool (GTT) have not been used yet for children with mental disorders, although there are child versions - Pediatric All-Cause Harm Measurement Tool (PACHMT), Global Assessment of Pediatric Patient Safety Tool (GAPPS). Objectives: Our aim was to evaluate drug safety in adolescents with acute psychotic episode with adopted trigger list based on GTT, PACHMT and GAPPS.

Methods: 151 complete cases being selected for analysis. Special trigger list for psychiatric patients was developed, based on PACHMT, GAPPS and general (GTT). Each case was analyzed with recommended GTT algorithm. Moreover, Medical Appropriateness Index (MAI) was calculated for each case. Treatment safety parameters were calculated by trigger tool analysis. Statistical analysis included Pearson's Chi-square, Mann-Whitney, Kruskal-Walles and multinominal logistic regression.

Results: A total of 261 triggers were identified among 151 analyzed cases, 51 of which were associated with the adverse drug effect (ADE) (total positive prediction value $=19.54 \%$ ). ADE per 1000 bed-days was 4.73, ADE per 100 admissions - 33.77\%. Extrapyramidal reactions to antipsychotics $(58.8 \%)$ were the most common ADE, with a lower frequency - abrupt medication stop of one or more drugs due to ADE $(25.5 \%)$. The length of mental disorder was a significant risk factor of developing ADEs associated with medication stop $(\mathrm{OR}=1.187,95 \% \mathrm{CI}$ 1.061-1.328; $p=0.003)$. Significant predictors of extrapyramidal adverse reactions to antipsychotics were the age $(\mathrm{OR}=1.607,95 \% \mathrm{CI} 1.097-2.355$; $\mathrm{p}=0.015)$, MAI score $(\mathrm{OR}=2.876,95 \% \mathrm{CI} 1.497-5.523 ; \mathrm{p}=0.002)$ and total number of hospital admissions $(\mathrm{OR}=0.191,95 \% \mathrm{CI}$ 0.052-0.697; $\mathrm{p}=0.012$ ).

Summary / Conclusions: For the first time, a study of the PACHMT and GAPPS methods was conducted in a child psychiatric hospital. Significant risk factors for ADE are the patient's older age, duration of hospitalization, duration of the disease, and a higher MAI score.

The research was funded by Russian Science Fundation, project No 1875-00046

\section{EACPT-1413}

Investigation of nervous system drugs in paediatrics

N. I. Kirmizi ${ }^{1}$, N. Akici ${ }^{2}$, M. Aksoy ${ }^{3}$, F. İsli ${ }^{3}$, V. Aydin ${ }^{1}$, A. Akici ${ }^{1}$ ${ }^{1}$ Department of Pharmacology, Marmara University Faculty of Medicine, ${ }^{2}$ Department of Pediatrics, Haydarpasa Numune Training Hospital, Istanbul, ${ }^{3}$ Turkish Medicines and Medical Devices Agency, Ministry of Health, Ankara, Turkey

\section{Pediatric treatment, Psychopharmacology}

Introduction: Nervous system drugs were used in numerous indications. In paediatric patients, physicians from different specialties prescribe these drugs in line with various treatment protocols.

Objectives: In this study, we aimed to investigate prescriptions that included nervous system drugs and prescribed by paediatricians and child psychiatrists.

Methods: As registered in the Prescription Information System (PIS) of the Turkish Medicines and Medical Devices Agency, the prescriptions of paediatricians (general or subspecialties of paediatrics) and child psychiatrists was retrospectively analysed, provided that they were written to children $(<18$-year) in the year of 2016 . The drugs were classified according to ATC (Anatomical Therapeutic Chemical) system, where those included in "N-Nervous System" were evaluated in detail.

Results: A total of 33,243,029 prescriptions were found to be registered in PIS during the study period; $6.7 \%$ of which was prescribed by paediatricians and child psychiatrists $(n=2,230,276 ; 97.0 \%$ by paediatricians). These prescriptions contained 6,108,393 drug items and 653,573 (10.7\%) of them were in "N-Nervous System" group according to the ATC-1 code (13.6\% of drugs items by child psychiatrists). Stratification at ATC-2 level showed "N02-analgesics" (92.5\%), "N03-antiepileptics" (2.4\%), and "N01-anesthetics" $(2.4 \%)$ as the most commonly prescribed groups among paediatricians. Furthermore, most of the "N02-analgesics" were detected as paracetamol and paracetamol-containing combinations (97.6\%) among the 521,904 drugs items that was prescribed by paediatricians. Child psychiatrists were found to mostly prescribe "N06psychoanaleptics" (69.4\%), "N05-psycholeptics" (29.3\%), and "N03-antiepileptics" (1.1\%) groups. Analysis of all "N-Nervous System" drugs at ATC-5 level revealed most commonly prescribed drugs to be paracetamol-containing drugs $(78.0 \%)$, which were followed by methylphenidate $(5.4 \%)$, risperidone $(2.6 \%)$, lidocaine-containing local anaesthetics $(2.1 \%)$, and metamizole $(1.8 \%)$.

Summary / Conclusions: This was the first study to show preferences of paediatricians and child psychiatrists towards prescribing nervous system drugs in Turkey, based on PIS data. While it is expected that each group of physicians have different prescribing tendencies, it is remarkable to encounter relatively higher frequency of paracetamol-containing analgesics and lidocaine-containing anaesthetics, indicating the need for further comprehensive studies in this manner. 
EACPT-1425

The association between drug use and childhood overweight and obesity, in denmark. A case-control study

C. Gade ${ }^{1}$, T. B. Jensen ${ }^{1}$, K. P. Dalhoff ${ }^{1}$, H. R. Christensen ${ }^{1}$, J. C. Holm ${ }^{2}$, ${ }^{3}$, H. Holst ${ }^{1}$

${ }^{1}$ Department of Clinical Pharmacology, Copenhagen University Hospital, Bispebjerg and Frederiksberg, Copenhagen NV, ${ }^{2}$ Children's Obesity Clinic, Department of Paediatrics, Copenhagen University Hospital Holbaek, Holbaek, ${ }^{3}$ Novo Nordisk Foundation Centre for Basic Metabolic Research, Copenhagen University, Copenhagen, Denmark

\section{Pediatric treatment}

Introduction: The increase in frequency of childhood obesity is welldescribed. However, studies comparing medication use in overweight and obese children with nonobese children are few and often inconclusive. Some studies have documented differences of regular medications, e.g. that obese children and adolescents are more likely to use respiratory medication, explained by the association between childhood obesity and asthma.

Objectives: To investigate whether drug use is associated with overweight and obesity in children aged 2-11 years and adolescents aged 12-19 years, within a two-year period, in Denmark.

Methods: The study was designed as a case-control study. Information about height and weight status was gathered from The Danish Childhood Obesity Biobank and adherent registry. By use of unique identification numbers, the information was linked to The Danish National Prescription Registry and the Danish Fertility Database from Statistics Denmark (DST, https://www.dst.dk/en). Type of medication was described by the Anatomical Therapeutical Chemical (ATC) classification system. A Body Mass Index (BMI) standard deviation score (SDS) $<1.28$ and BMI SDS $\geq$ 1.28 classified nonobese and overweight/obese children, respectively.

Results: Totally 5977 children and adolescents were identified with valid identification numbers, height and weight measures. Both overweight/ obese children and adolescents had a significantly higher use of medications for musculoskeletal conditions (ATC group M) and drugs acting on the nervous systeme $(\mathrm{N})$ than their nonobese peers, also when adjusted for parent income and education level. Overweight /obese children aged 2-11 years had a more frequent use of cardiovascular system (C), dermatologicals (D), and antiinfectives for systemic use (J), also when adjusted for socioeconomic covariates. In addition, overweight/obese adolescents had a more frequent use of alimentary tract and metabolism group medications (A), and systemic hormonal preparations (H) as compared to nonobese adolescents. In general, medications for respiratory conditions (R) were frequently prescribed to all children independently of BMI SDS, but with higher frequency in the young children and a significant difference between overweight/obese and nonobese children.

Summary / Conclusions: Overweight/obese children in Denmark are more likely to use prescribed medications as compared to their nonobese peers.

\section{Pharmacogenetics/omic}

\section{Oral Presentation}

EACPT-1002

Development and testing of a clinical decision support system for dosing in psychopharmacotherapy in patients with affective disorders based on the pharmacogenomic markers

M. Zastrozhin ${ }^{* 1,2}$, D. Sychev ${ }^{1}$, A. Sorokin ${ }^{2}$, K. Ryzhikova ${ }^{1}$, E. Bryun ${ }^{2}$

${ }^{1}$ Russian Medical Academy of Continuous Professional Education of the Ministry of Health of the Russian Federation, ${ }^{2}$ Moscow Research and Practical Centre on Addictions, Moscow, Russian Federation

\section{Pharmacogenetics/omics,Psychopharmacology, Other}

Introduction: There are currently approximately two dozen pharmacogenomic clinical decision support systems (CDSS) used in psychiatry. Implementation of the clinical decision support systems capable of forming the recommendations on drug and dose selection according to the results of pharmacogenetic testing is an urgent task. Fulfillment of this task will allow increasing the efficacy of therapy and decreasing the risk of undesirable side effects.

Objectives: To study the effect of implementing the decision support system to optimize the drug dosage regimen, based on pharmacogenomic biomarkers, on the efficacy and safety of therapy for patients with affective disorders and comorbid alcohol addiction.

Methods: The study included 118 male patients (48 in the main group and 70 in the control group) with affective disorders and comorbid alcohol use disorder. To evaluate the efficacy and safety of therapy several international psychometric scales and rating scales to measure side effects were used. Genotyping was performed using real-time polymerase chain reaction with allele-specific hybridization. Pharmacogenetic test results were interpreted using free software PGX2 (www.pgx2.com).

Results: The statistically significant differences between the scores derived from all psychometric scales were revealed. For instance, the total score on Hamilton Rating Scale by day 9 was 14.5 [14.0; $15.0]$ for the main group and $20.0[18.0 ; 21.0](\mathrm{p}<0.001)$ for the control group; by day 16 it was $14.0[13.0 ; 15.0]$ for the main group and $14.0[13.0 ; 14.0](\mathrm{p}<0.001)$ for the control group. The UKU side effect rating scale (UKU) also revealed a statistically significant difference. The total score on UKU scale by day 9 was $4.0[4.0 ; 5.0]$ for the main group and $5.0[5.0 ; 6.0](\mathrm{p}<0.001)$ for the control group; by day 16 this difference grew significantly: $3.0[0.0 ; 4.2]$ for the main group and $9.0[7.0 ; 11.0](\mathrm{p}<0.001)$ for the control group.

Summary / Conclusions: Pharmacogenetic-guided personalization of the drug dose in patients with affective disorders and comorbid alcohol use disorder can reduce the risk of undesirable side effects and pharmacoresistance. It allows recommending the use of pharmacogenomic clinical decision support systems for optimizing drug dosage.

\section{EACPT-1033}

Influence of abcb1, cyp3a4 and cyp3a5 genes polymorphisms on prothrombin time and rivaroxaban plasma concentration in patients after total hip or knee replacement surgery

R. Minnigulov* 1, T. Morozova ${ }^{2}$, D. Sychev ${ }^{3}$, P. Bochkov ${ }^{4}$, K. Ryzhikova $^{5}$, A. Lychagin ${ }^{6}$, I. Yudina ${ }^{1}$

${ }^{1}$ Department of Clinical Pharmacology and Propaedeutics of Internal Diseases, ${ }^{2}$ Department of General Medical Practice, I.M. Sechenov First Moscow State Medical University (Sechenov University), ${ }^{3}$ Department of Clinical Pharmacology and Therapy, ${ }^{4}$ Department of Personalized Medicine, Research Center, ${ }^{5}$ Department of Molecular Biological Research, Research Center, Russian Medical Academy of Continuous Professional Education, ${ }^{6}$ Department of Traumatology, Orthopedics and Disaster Surgery, I.M. Sechenov First Moscow State Medical University (Sechenov University), Moscow, Russian Federation

\section{Pharmacogenetics/omics, Pharmacokinetics/ pharmacodynamics}

Introduction: The aim of our study was to investigate the impact of gene polymorphisms rs1045642, rs4148738, rs776746, rs35599367 on prothrombin time (PT) and $\mathrm{C}_{\mathrm{ss} \text { max }}$ of rivaroxaban $10 \mathrm{mg}$ OD in patients after total hip or knee replacement surgery.

Objectives: The present study enrolled 28 patients after total hip ( $\mathrm{n}=9$; $32,1 \%)$ or knee $(n=19 ; 67,9 \%)$ replacement surgery (mean age $57 \pm 11$ years; women $n=21(75,0 \%)$, men $n=7(25,0 \%)$.

Methods: Venous blood samples were taken on the day 5 after starting rivaroxaban therapy: 3 hours after taking the medication. Gene polymorphisms were genotyped by real-time polymerase chain reaction. PT test was performed using Tekhplastin test ${ }^{\circledR}$. $\mathrm{C}_{\mathrm{ss} \max }$ was determined using an Agilent 1200 Series HPLC System. Statistical analysis was performed 
using PASW Statistics 18 (Mann-Whitney U test, Pierson correlation coefficient).

Results: Genotype distribution of gene polymorphisms: rs $1045642 \mathrm{CC}$ $(33,3 \%)$, CT $(36,7 \%)$, TT $(30,0 \%)$; rs $4148738 \mathrm{CC}(30,0 \%)$, CT $(46,7 \%)$, TT (23,3\%); rs776746 AG (13,3\%), GG (86,7\%); rs35599367 CC (90,0\%), CT $(10,0 \%)$. Comparison $\mathrm{C}_{\mathrm{ss} \max }$ in patients with genotypes: rs $1045642 \mathrm{CC}$ vs CT ( $U=26,0, p=0,133)$; rs $1045642 \mathrm{CC}$ vs TT ( $U=37,0$, $p=0,796) ;$ rs 1045642 CT vs TT $(U=26,0, p=0,133) ;$ rs $4148738 \mathrm{CC}$ vs CT ( $U=34,0, p=0,169)$; rs4148738 CC vs TT ( $U=15,0, p=0,091)$; rs4148738 CT vs TT ( $U=38,0, p=0,773)$; rs776746 AG vs GG $(U=40,0, p=0,635)$; rs35599367 CC vs CT $(U=27,0, p=0,477)$. Comparison PT in patients with genotypes: rs1045642 CC vs CT $(U=32,0, p=0,305)$; rs $1045642 \mathrm{CC}$ vs TT ( $U=37,5, p=0,815)$; rs 1045642 CT vs TT ( 7,5 vs $12,78, U=20,0$, $p=0,041) ; \mathrm{rs} 4148738 \mathrm{CC}$ vs CT $(U=35,0, p=0,187)$; rs $4148738 \mathrm{CC}$ vs TT $(U=19,0, p=0,21) ;$ rs4148738 CT vs TT $(U=36,5, p=0,665)$; rs776746 AG vs GG $(U=47,0, p=0,963)$; rs $35599367 \mathrm{CC}$ vs CT $(U=31,5$, $p=0,687)$. There was statistically significant linear relationship between $\mathrm{PT}$ and $\mathrm{C}_{\mathrm{ss} \max }\left(\mathrm{R}^{2}=0,52, p=1,5 \times 10^{-5}\right)$.

Summary / Conclusions: We found that PT correlated strongly with rivaroxaban plasma concertation. This is associated with other scientific articles and indicates that PT can be used to monitor rivaroxaban's anticoagulant activity. It was statistically significant that patients with genotype rs1045642 TT of gene $A B C B 1$ have PT that is higher than in patients with rs1045642 CT. Future work is required to inclusion more patients in our research.

\section{EACPT-1037}

Evaluating tacrolimus pharmacokinetic models in tunisian renal transplant patients

I. Hannachi ${ }^{1,}{ }^{2}$, N. Ben Fredj ${ }^{1}$, Z. Chadli ${ }^{1}$, H. Ben Romdhane ${ }^{1}$, E. Kerkeni $^{1}$, M. Hammouda ${ }^{3}$, N. Ben Fadhl ${ }^{1}$, A. Chaabane ${ }^{1}$, Y. Touitou ${ }^{4}$, N. A. Boughattas ${ }^{*}$, K. Aouam ${ }^{1}$

${ }^{1}$ Pharmacology, Faculty of Medecine, Monastir, ${ }^{2}$ Faculty of Sciences, Bizerte, ${ }^{3}$ Nephrology, University Hospital of Monastir, Monastir, Tunisia, ${ }^{4}$ Fondation A. de Rothschild, Chronobiology Unit, Paris, France

\section{Pharmacogenetics/omics, Pharmacokinetics/ pharmacodynamics}

Introduction: The pharmacokinetics of Tacrolimus (Tac) is characterized by high interindividual variability, mainly explained by polymorphisms in CYP3A4 and CYP3A5 isoenzymes.

Objectives: is to develop a population pharmacokinetic model ( $\mathrm{Pk}$ pop) able to describe the evolution of Tac exposure as a function of posttransplant phases (PTP) in Tunisian renal transplant patients and to study the effect of $C Y P 3 A 4 * 1 B, C Y P 3 A 4 * 22$ and $C Y P 3 A 5 * 3$ polymorphisms on the pharmacokinetics of Tac in this population.

Methods: We performed a cross-sectional study including Tac trough concentrations $(\mathrm{C} 0)$ issued from Tunisian renal graft patient. The $\mathrm{Pk}$ pop study was performed using a nonparametric approach (Pmetrics). The exposure index used in the present $\mathrm{Pk}$ pop was the dosenormalized trough concentration $(\mathrm{C} 0 / \mathrm{D})$. The influence of covariates (age, weight, sex, hematocrit and $C Y P 3 A 4 * 1 B$ (rs2740574), CYP3A4*22 (rs35599367) and CYP3A5*3 (rs776746) polymorphisms was tested on the Pk pop parameters. The performance of the final model was assessed using an external dataset

Results: A total of 409 C0 (53 patients) were evaluated. The population was divided into a structural model group $(255 \mathrm{C} 0)$ and a validation group (154 C0). A one-compartment model (Vd: volume of distribution, CL: Clearance of Tac) was found to correctly describe the evolution of the $\mathrm{C} 0$ / $\mathrm{D}$ regardless of the PTP (the correlation between the predicted $\mathrm{C} 0 / \mathrm{D}$ and observed C0/D showed an $\left.\mathrm{r}^{2}: 0.70\right)$. The influence of covariates has shown that only the $C Y P 3 A 4 * 1 B$ and $C Y P 3 A 4 * 22$ polymorphisms were significantly associated with CL regardless of PTP $(\mathrm{p}=0.04$ and 0.02 , respectively). Only the $C Y P 3 A 4 * 22$ polymorphism influenced CL ( $\mathrm{p}=$ 0.02 ) during $\mathrm{P} 1(>3$ months). During P2, we found that both $C Y P 3 A 4 * 22$ and $C Y P 3 A 4 * 1 B$ polymorphisms were significantly associated with $\mathrm{CL}$
( $p=0.03$ for both covariates). The External validation of the covariatemodel showed a \%MPE and \% RMSE of 8 and 35 respectively, regardless of PTP. The assessment of the model precision during P1 showed that \% MPE and \% RMSE were 6 and 15, respectively. During P2, the model showed a respective \% MPE and \% RMSE of 19 and 35.

Summary / Conclusions: We developed a Pk pop model of Tac in Tunisian renal transplant patients taking into account the $C Y P 3 A 4 * 1 B$ and $C Y P 3 A 4 * 22$ polymorphisms, only covariates involved in the interindividual variability of CL of Tac. This model could be highly useful in proposing doses of Tac in this population at any post-transplant day.

\section{EACPT-1048}

Population pharmacokinetic-pharmacodynamic modelling of metoprolol with regard to cyp2d6 genotypes in healthy korean volunteers

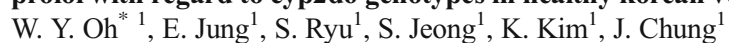

${ }^{1}$ National institute of Food and Drug Safety Evaluation, Cheongju, Korea, Republic Of

Pharmacogenetics/omics, Pharmacokinetics/ pharmacodynamics, Other

Introduction: Metoprolol, a selective $\beta 1$-adrenoreceptor blocking agent, is widely used in the treatment of hypertension and primarily metabolized by CYP2D6.

Objectives: The aim of this study was to develop a population pharmacokinetic-pharmacodynamic (PK-PD) model of metoprolol to evaluate the effect of CYP2D6 genotype on the PK-PD relationship in healthy Korean volunteers.

Methods: A total of 48 healthy male Korean volunteers were enrolled. The subjects were classified into 3 groups based on CYP2D6 genotype which was categorized as group A (at least one active alleles), group B (2 decreased alleles), and group $\mathrm{C}$ (one decreased allele with one inactive allele or two inactive alleles). All the subjects were administered a single oral dose of $100 \mathrm{mg}$ metoprolol. Blood samples were collected at $0,0.5$, $1,1.5,2,2.5,3,4,6,8,10,12$ and 24 hours after administration and heart rate (HR) and systolic blood pressure (SBP) were measured at $0,1,2,3,4$, $6,10,24$ hours post-dosing and the PK-PD analysis was performed using NONMEM® 7.3 .

Results: A two-compartment model with erlang-type absorption described the PK data adequately. The clearance was reduced and the bioavailability was increased in group $\mathrm{B}$ and $\mathrm{C}$ compared to those in group A. Sigmoid Emax model was used and circadian rhythm model described the HR and SBP changes within 24 hours was combined for the sequential PK-PD analysis. The HR and SBP in group B and C were 1.23 and 1.8 times higher than in group A, respectively.

Summary / Conclusions: In conclusion, we successfully built a PK-PD model of metoprolol including the genotype of CYP2D $6 * 5$ and $* 10$ as a covariate, which were frequently found in Korean compared to Caucasians. Compared to PK results, less difference between CYP2D6 genotype groups was observed in PD response. Considering that this study was performed with healthy volunteers and the difference in HR and SBP between the genotypic groups should be evaluated in view of clinical significance, further study may be necessary for suggesting the dose adjustment of metoprolol.

\section{EACPT-1093}

Influence of ace gene polymorphisms on angiotensin converting enzyme activity in thai population

A. Chaikan ${ }^{*} 1$, N. Malisorn ${ }^{1}$

${ }^{1}$ Pharmacology Division, Department of Preclinical Science, Faculty of Medicine, Thammasat University, Pathumthani, Thailand

\section{Pharmacogenetics/omics}

Introduction: Ethnic differences affected the occurrence of angiotensin-converting enzyme (ACE) inhibitor-induced cough. Higher incidence of cough due to ACE inhibitors was reported in 
Hong Kong Chinese (53\%) than in Caucasians (18\%). Forty-four percent of Thai patients taking imidapril experienced cough. The $A C E$ gene variation (rs4344) was associated with increased risk of ACE inhibitor-related to cough in female Spanish hypertensive patients. The $A C E$ polymorphism (rs4267385) was a protective SNP against ACE inhibitor-associated cough in Caucasians. Genomewide association studies (GWS) in Japanese population showed the relationships between ACE SNPs (rs4309, rs4311, rs4343, rs4353 and rs4362) and ACE level. However, GWS in Han Chinese found that only rs4343 on $A C E$ gene was associated with ACE activity.

Objectives: This present study aimed to investigate the relationships between $A C E$ polymorphisms (rs4309C $>\mathrm{T}$, rs $4311 \mathrm{C}>\mathrm{T}$, rs $4316 \mathrm{C}>\mathrm{T}$, rs $4343 \mathrm{~A}>\mathrm{G}, \mathrm{rs} 4344 \mathrm{~A}>\mathrm{G}, \mathrm{rs} 4353 \mathrm{~A}>\mathrm{G}, \mathrm{rs} 4362 \mathrm{C}>\mathrm{T}$ and $\mathrm{rs} 4267385 \mathrm{C}>\mathrm{T}$ ) and serum ACE activity in Thai population.

Methods: Genomic DNA was isolated from whole blood of 100 Thai healthy subjects. Real-time PCR was used to genotype eight $A C E$ SNPs. Measurements of ACE activity in serum was conducted through spectrophotometer. Median and interquartile range (IQR) were used to describe data. Differences in serum ACE activity between two genotypes were assessed by Mann-Whitney U test.

Results: Individuals homozygous for rs4309T, rs4316T and rs4353G variant alleles had lower ACE activity (median: 26, 26 and $24 \mathrm{U} / \mathrm{L}$ ) than individuals homozygous for $\mathrm{rs} 4309 \mathrm{C}$, rs $4316 \mathrm{C}$ and rs4353A wild-type alleles (median: 45, 52 and $48 \mathrm{U} / \mathrm{L})(p=0.004, p=0.011$ and $p=0.001)$, respectively. Whereas higher ACE activity were observed in subjects with homozygous for rs4311C $>\mathrm{T}$, rs $4343 \mathrm{~A}>\mathrm{G}, \mathrm{rs} 4344 \mathrm{~A}>\mathrm{G}$ and $\mathrm{rs} 4362 \mathrm{C}>\mathrm{T}$ mutation (median: 52, 52, 52 and $48 \mathrm{U} / \mathrm{L}$ ) compared to rs $4311 \mathrm{C}$, rs4343A, rs4344A and rs4362C homozygotes (median: 52, 52, 52 and $48 \mathrm{U} / \mathrm{L})(p=0.001, p=0.004, p=0.000$ and $p=0.002)$, respectively. However, there was no association between rs4267385C $>$ T SNP and ACE activity $(p=0.433)$.

Summary / Conclusions: The ACE polymorphisms influenced serum ACE activity in Thai subjects. The $A C E$ gene variation is a good predictor to perform studies of ACEI-induced cough in Thai population.

\section{EACPT-1163}

Impact of fto genetic variability on psychological traits of patients with eating disorders

L. M. González ${ }^{*} 1$, S. Mota-Zamorano ${ }^{2}$, D. Alburquerque ${ }^{3}$, A. GarciaHerráiz $^{4}$, I. Flores ${ }^{4}$, G. Gervasini ${ }^{1}$

${ }^{1}$ Department of Medical \& Surgical Therapeutics, Medical School, University of Extremadura, ${ }^{2}$ Department of Medical \& Surgical Therapeutics, Medical School, University of University, Badajoz, ${ }^{3}$ University General Hospital, Valencia, ${ }^{4}$ Eating Disorders Unit, Institute of Mental Disorders of Extremadura, Badajoz, Spain

\section{Pharmacogenetics/omics}

Introduction: Dopaminergic signaling plays a key role in eating disorders (ED), due to the connection between this neurotransmitter and food intake, novelty seeking, impulsivity, or reward mechanisms. This dopaminergic activity has been shown to be influenced by the fat mass and obesity associated (FTO) gene, a locus whose variability has been consistently associated with body mass index and obesity because of an altered reward processing.

Objectives: Our objective was to determine if genetic variants in the FTO gene were associated with the risk of EDs or with different psychopathological features observed in these patients.

Methods: The study group consisted of 122 healthy women and 169 unrelated consecutive female patients with anorexia $(\mathrm{AN}, \mathrm{n}=106)$ or bulimia (BN, n=63) nervosa. We analyzed 23 tag-single nucleotide polymorphisms (SNPs) in the coding sequence and adjacent regions of the FTO gene. Personality dimensions and general psychopathological symptoms were assessed by the EDI-2 and SCL-90R inventories. Single- marker association analyses were carried out by logistic regression models adjusted for age using the SNPassoc R package. The effect of allele combinations was determined by using a sliding-windows approach (PLINK v.1.09)

Results: None of the SNPs or haplotypes analyzed were associated with a greater risk of ED or correlated with anthropometric parameters. However, in the BN group, carriers of rs12927155 SNP scored lower in two SCL-90R scales, namely positive symptom distress index $(\mathrm{p}=0.0048)$ and global severity index $(\mathrm{p}=0.0379)$. Furthermore, statistically significant differences between carriers and non-carriers of this SNP were observed in the scores of several EDI-2 dimensions: ineffectiveness $(\mathrm{p}=0.0003)$, paranoid ideation $(\mathrm{p}=0.0015)$ and impulse regulation $(\mathrm{p}=0.0021)$.

The sliding-windows analysis showed a region between the rs 9921255 and rs12927155 polymorphisms containing several 3-SNP haplotypes that were consistently associated with ineffectiveness, paranoid ideation, impulse regulation, drive for thinness and maturity fears.

Summary / Conclusions: Our results suggest that variability in the FTO gene locus is associated with psychopathological symptoms and personality dimensions in $\mathrm{BN}$ patients.

\section{Oral Presentation}

\section{EACPT-1189}

Influence of cyp450 genetic variability on the donor kidney biopsy score and consequences on clinical outcomes in renal transplant recipients

S. Mota Zamorano* ${ }^{1}$, L. M. González ${ }^{1}$, E. Luna ${ }^{2}$, J. J. Fernandez ${ }^{3}$, A. Gomez $^{3}$, A. Nieto ${ }^{3}$, N. R. Robles ${ }^{4}$, G. Gervasini ${ }^{1}$

${ }^{1}$ Medical and Surgical Therapeutics, University of Extremadura, ${ }^{2}$ Service of Nephrology, Infanta Cristina University Hospital, ${ }^{3}$ Service of Anatomical Pathology, ${ }^{4}$ Service of Nephrology, Infanta Cristina University Hospital, Badajoz, Spain

\section{Pharmacogenetics/omics}

Introduction: Cytochrome P (CYP)-450 enzymes metabolize arachidonic acid to vasoactive eicosanoids such as epoxyeicosatrienoic acids (EETs) and 20-Hydroxyeicosatetraenoic acid (20-HETE).

Objectives: We aim to analyze the influence of polymorphisms in four CYP genes of the donor on the scores independently assigned by pathologists to kidney biopsies. Additionally, we examined whether this score had any influence on clinical outcomes in the renal transplant recipients. Methods: A cohort of 119 recipients and their corresponding 91 donors were genotyped for the CYP4F2 V433M, CYPY4A11 F433S, CYP2C8*3 and $C Y P 2 J 2 * 7$ polymorphisms. The association of the donors' SNPs with the biopsy scores and the result of clinical outcomes in recipients was evaluated by multivariate regression analysis adjusting by significant covariates.

Results: The presence of $C Y P 2 C 8 * 3$ was significantly associated with lower scores assigned to donor biopsies [OR=0.21 (0.05-0.83), $\mathrm{p}=0.012$ ]. In addition, the CYP4F 2 V433M variant also showed a statistical trend towards lower scores [OR=0.38 (0.15-0.99), $\mathrm{p}=0.043]$. Higher scores were related to an increased risk of acute rejection [OR=6.93 (1.57$30.60), p=0.011]$ and a worse creatinine clearance one year after grafting , patients with low scores displayed an average of $53.21 \pm 17.13$ vs. 46.11 $\pm 18.51 \mathrm{ml} / \mathrm{min}$ for recipients with high scores $(\mathrm{p}=0.052)$.

Summary / Conclusions: Our results show that biopsies from donors who are carriers of the $C Y P 2 C 8 * 3 \mathrm{SNP}$ displayed better scores than noncarriers did. In turn, this score is associated with acute rejection and creatinine clearance in renal transplant recipientes.

\section{EACPT-1194}

Effect of cyp3a5 and abcb1 polymorphisms on dose requirement and trough concentration of tacrolimus in renal transplant recipients E. Yildirim ${ }^{1}$, G. Sahin ${ }^{2}$, Z. Kaltus ${ }^{*}$, E. Colak ${ }^{3}$ 
${ }^{1}$ Medical Pharmacology, ${ }^{2}$ Nephrology, ${ }^{3}$ Biostatistics, Eskisehir Osmangazi Universitesi Faculty of Medicine, Eskisehir, Turkey

\section{Pharmacogenetics/omics}

Introduction: Tacrolimus, an immunosuppressive agent, has large interindividual variability. Cytochrome P450 (CYP)3A and P-glycoprotein $(\mathrm{ABCB} 1)$ play an important role in the absorption and metabolism of tacrolimus.

Objectives: To evaluate whether or not CYP3A5 and ABCB1 gene polymorphisms are associated with dose requirement and dose-adjusted concentration of tacrolimus in renal transplant patients.

Methods: CYP3A5 [*1/*1 (expressor); *1/*3 (expressor); *3/*3 (nonexpressor)] and ABCB1 (MDR1) polymorphisms were determined by allele-specific polymerase chain reaction in 67 adult Turkish renal transplant patients receiving tacrolimus. Tacrolimus dose $(\mathrm{mg} / \mathrm{kg})$ and trough concentration $\left(\mathrm{C}_{0}\right)$ of each patient were acquired from patient's file and dose-adjusted concentrations $\left(\mathrm{ng} / \mathrm{mL}\right.$ per $\mathrm{mg} / \mathrm{kg} / \mathrm{d}$ ) were calculated at $1^{\text {th }}$, $3^{\text {th }}, 6^{\text {th }}$ and $12^{\text {th }}$ months after transplantation. The correlated serum hematocrit, platelet, urea, creatinine and albumin were also determined.

Results: The allelic frequencies of CYP $3 \mathrm{~A} 5 * 1 / * 3$ and CYP $3 \mathrm{~A} 5 * 3 / * 3$ were $5.97 \%(n=4)$ and $94.03 \%(=63)$, respectively. There were no patients with the CYP3A5*1/*1 genotype. Tacrolimus dose $(\mathrm{mg} / \mathrm{kg} / \mathrm{d})$ was significantly lower in $* 3 / * 3$ genotype than $* 1 * 3$ genotype $\left(3^{\text {th }}, 6^{\text {th }}\right.$ months: $\mathrm{p} \leq 0.001 ; 12^{\text {th }}$ month: $\left.\mathrm{p} \leq 0.05\right)$. Dose-adjusted tacrolimus concentration was statistically higher in $* 3 * 3$ genotype than $* 1 * 3$ genotype at $3^{\text {th }}$ and $6^{\text {th }}$ months $(\mathrm{p} \leq 0.05)$. The allelic frequencies of MDR1 CC, CT and TT were $26.87 \%(n=18), 49.25 \%(n=33)$ and $23.88 \%(n=16)$, respectively. No statistically significant differences were detected between MDR1 C3435T polymorphism and tacrolimus concentration, dose and doseadjusted $\mathrm{C}_{0}$ in all months. There were also no statistically significant differences in all analyzed laboratory parameters.

Summary / Conclusions: CYP3A5*1/*3 polymorphisms were found to be associated with tacrolimus pharmacokinetics and dose requirements in renal transplant recipients. Pharmacogenetic methods could be used to help initial dose selection and to individualize immunosuppressive therapy.

This study was funded by Eskisehir Osmangazi University Scientific Research Projects Commission (Project number: 2018-2055)

\section{Oral Presentation}

EACPT-1243

Implementing pre-emptive pharmacogenetic screening into clinical routine

J. Forsström ${ }^{*} 1$

${ }^{1}$ Medical, Abomics, Turku, Finland

\section{Pharmacogenetics/omics}

Introduction: In recent years, increasing number of studies have shown the potential benefits and the cost-effectiveness of pre-emptive pharmacogenetic testing in patient care. However, implementing PGx testing into clinical routine has been slow due to many challenges.

Objectives: If PGx testing is used as a diagnostic test after an adverse drug reaction, it has little effect on health care costs. Patients may have their pharmacogenetic profile done, but a physician needs easy access to it in order to utilize it. Since many of the pharmacogenetically relevant drugs are commonly used drugs in primary care, awareness of pharmacogenetics should be raised among all physicians. Abomics aims at bringing PGx testing as a clinical routine by developing advanced decision support tools for physicians.

Methods: Abomics has created an advanced pharmacogenetic database for clinical setting, which summarizes clinically relevant pharmacogenetics. This GeneRx database is also used to provide easy to understand reports from pharmacogenetic test data. PGx test results are stored into
Abomics cloud service, which is integrated to electronic patient records (EPRs). Also, patients have access to their own electronic data.

Results: In collaboration with the largest electronic patient record vendor in the Nordic Countries (Tieto), Abomics has implemented an automated alerting system in Tieto's prescription module. This is so far the largest decision support integration to electronic prescription modules in Europe. Usage of GeneRx has shown a significant increase in pre-emptive testing of PGx panels. In Finland Oulu psychiatric departments have been increasingly active in PGx testing and it will be seen in the decreasing number of control visits to the clinic, better drug correspondent and improved quality of life (1).

Summary / Conclusions: Abomics has created a medical technology with ICT providers, that enables personalized medicine to be utilized in primary care setting. Easy-to-understand database on the clinically relevant pharmacogenetic variants and related drugs is available for all professionals. When the test results are available in a cloud service, the results can be used whenever new drugs are prescribed. The patients find the services relevant and easy to use.

REFERENCE

Gardner et al. The Potential Utility of Pharmacogenetic Testing in Psychiatry [Review Article]. Psychiatry journal. Volume 2014, Article ID 730956, 6 pages, http://dx.doi.org/10.1155/2014/730956

\section{EACPT-1247}

Genotype and phenotype frequencies of isoniazid-metabolizing enzyme nat2 in yakutian and russian tuberculosis patients

O. Suvorova* ${ }^{1,2}$, D. Sychev ${ }^{1}$, D. Ivashchenko ${ }^{1}$, N. Val ${ }^{3}$, A. Kravchenko $^{3}$, N. Krasnova ${ }^{4}$, Y. Chertovskikh ${ }^{5}$, Z. Rudykh ${ }^{5}$

${ }^{1}$ Russian Medical Academy of Continuous Professional Education of the Ministry of Health of the Russian Federation, ${ }^{2}$ Federal State Autonomous Educational Institution of Higher Education I.M. Sechenov First Moscow State Medical University of the Ministry of Health of the Russian Federation (Sechenov University), Moscow, ${ }^{3}$ «Scientific and Practical Centre of Phthisiology», ${ }^{4}$ Federal State Autonomous Educational Institution of Higher Education Ammosov North-Eastern Federal University, ${ }^{5}$ Centre of personalized medicine, Republican hospital № 3, Yakutsk, the Republic of Sakha (Yakutia), Russian Federation

\section{Pharmacogenetics/omics}

Introduction: Tuberculosis (TB) is one of the top 10 causes of death. Isoniazid is the most effective anti-TB drug, but it has significant side effects. INH is metabolized by N-acetyltransferase 2 (NAT2). Pharmacogenetic studies of the NAT2 polymorphisms prevalence among ethnic groups are necessary to estimate the risk of side effects in TB patients and to select the most effective and safe treatment course.

Objectives: The aim of our study was to establish the prevalence of the NAT2 gene polymorphic types among Yakutians and Russians living in the Republic of Sakha (Yakutia) and Russians living in Moscow region. Methods: 158 patients were examined: 50 - Yakutians, 41 Russians (Yakutia region), 67 - Russians (Moscow region), $5 \mathrm{ml}$ of venous blood from each patient was received for genotyping. 6 NAT2 polymorphisms were genotyped using real-time PCR: 3 slow (NAT2T341C*5, NAT2G590A*6, NAT2G857A*7) and 3 fast (NAT2C481T*11, NAT2A803G*12, NAT2C282T*13) types. The calculation of compliance with the Hardy-Weinberg equilibrium was performed as well as the statistical analysis using SPSS Statistics 20 software program for which the chi-square test was calculated.

Results: Statistically significant differences were revealed between Yakutians (Y) and Russians from Yakutia (RY) and from Moscow region $(\mathrm{RM})$ in the prevalence of NAT2 polymorphisms: NAT2*5 (Y - 32,00\%; RY - 80,50\%; RM - 61,20\%; $\mathrm{p}=0.0001)$; NAT2*11 ( Y - 34,00\%; RY $78,00 \%$; RM - 56,70\%; $=0.0001)$; NAT2*12 ( Y - 34,00\%; RY - 82,90\%; $\mathrm{RM}-58,20 \%$; $\mathrm{p}=0.0001)$, which are more common in Russians. Among 
other polymorphisms no significant differences were identified: NAT2 $* 13(\mathrm{p}=0.756) ; \mathrm{NAT} 2 * 6(\mathrm{p}=0.415) ; \mathrm{NAT} 2 * 7(\mathrm{p}=0.205)$. Statistically significant differences were also identified in the prevalence of the following phenotypes: rapid (Y - 26,00\%; RY - 0,00\%; RM $9,00 \% ; \mathrm{p}=0.0001)$ and slow $(\mathrm{Y}-20,00 \%$; RY - 63,40\%; RM - 55,20\%; $\mathrm{p}=0.0001)$ : rapid acetylators are more common in Yakutians.

Summary / Conclusions: A different prevalence of NAT2 polymorphisms and NAT2 phenotypes between Yakutians and Russians living in Yakutia and Moscow region has been revealed. Therefore, the use of isoniazid in Yakutians might be associated with the higher risk of insufficient response to the standard daily dose of INH. Expansion is required to increase the relevance of the results.

\section{EACPT-1272}

Variants of fibroblast growth factor 21 gene affect dietary salt intake M. M. Saber-Ayad ${ }^{* 1,2,3}$, S. Hammoudeh ${ }^{3,4}$, S. Manzoor ${ }^{3,4}$, H. Jabbar ${ }^{4}$, R. Wardeh ${ }^{4}$, A. Ashraf ${ }^{4}$, H. Radwan ${ }^{5}$, R. Hammoudi ${ }^{3,4}$

${ }^{1}$ Clinical Sciences, College of Medicine, University of Sharjah, Sharjah, United Arab Emirates, ${ }^{2}$ Cairo University, Cairo, Egypt, ${ }^{3}$ Sharjah Institute for Medical Research, University of Sharjah, ${ }^{4}$ College of Medicine, University of Sharjah, ${ }^{5}$ College of Health Sciences, University of Sharjah, Sharjah, United Arab Emirates

\section{Pharmacogenetics/omics}

Introduction: The hepatokine, fibroblast growth factor 21 (FGF21) is a peptide hormone secreted by the liver and many other tissues. Injection of FGF21 may lead to weight loss with improvement in insulin sensitivity, so it is considered a potentially attractive therapeutic target. However, recent data showed that serum FGF21 level is elevated in hypertension, atherosclerosis and coronary artery disease. There is an association of FGF21 rs838133 and increased consumption of sweets in Caucasians. Only few studies addressed the link between gene variants and food intake in the Middle East, where hypertension and obesity show high prevalence.

Objectives: To explore the potential effect of FGF21 rs838133 and rs838145 on the pattern of food intake in Emirati population.

Methods: Out of 215 healthy adult Emiratis recruited, only 150 subjects were considered after removing outliers and normalizing body mass index and other variables using a QQ-plot (mean age $30.84 \pm 8.9$ years, females $=59.3 \%$ ). Genotyping for FGF21 rs838133 and rs838145 was performed using DNA from saliva samples. Dietary intake was assessed by a sixty-one-item FFQ that estimated food and beverage intakes over the past year. The daily energy, macronutrient, and sodium intake were computed using food processor software.

Results: The mean BMI of the study population was $26.08 \mathrm{~kg} / \mathrm{m}^{2}$ Sodium intake was significantly higher in the FGF21 rs838145 GG group ( $p=0.008)$ compared to other genotypes. There was a trend of increased sodium intake in rs838133 AA group. rs838145 GG group also showed higher intake of vegetables $(\mathrm{p}=0.005)$ and vitamin $\mathrm{A}(\mathrm{p}=0.03)$. Intake of carbohydrate, fat, protein, vitamin $\mathrm{D}$ did not show significant difference among genotypes.

Summary / Conclusions: The current study points out an association between FGF 21 rs838145 G allele and high salt intake in Emirati population. Our results pave the way to a deeper understanding of geneenvironment interactions that may underlie frequent failure of antihypertensive treatment. Patients carrying specific gene polymorphisms may need particular attention and dietary modification.

\section{EACPT-1284}

The polymorphisms frequencies of cytochrome p450 and abcb1 genes in russian patients

A. Tarasenko ${ }^{*}{ }^{1,2}$, D. Sychev ${ }^{1}$, N. Denisenko ${ }^{1}$, K. Ryzhikova ${ }^{1}$, Z. Sozaeva $^{1}$, E. Grishina ${ }^{1}$

${ }^{1}$ Russian Medical Academy of Continuous Professional Education of the Ministry of Health of the Russian Federation, ${ }^{2}$ Sechenov University, Moscow, Russian Federation

\section{Pharmacogenetics/omics}

Introduction: Proton pump inhibitors (PPI) are metabolized in liver by the cytochrome P450 (CYP) system and its genes exhibit a clinically important genetic polymorphism which is related to pharmacokinetics of PPI. P-glycoprotein, encoded by $A B C B 1$ gene, is an ATP-dependent membrane transporter which defines the molecular permeability of mucosal cells. All of the above genes influence the effectiveness of antiulcer drug therapy.

Objectives: The aim of the study was to investigate the frequencies of CYP2C19, CYP3A4, CYP3A5, $A B C B 1$ polymorphisms in patients with peptic ulcers.

Methods: 39 Russian patients (mean age \pm SD was $48,2 \pm 16,9$ years, range 20-83 years, 21 males and 18 females) diagnosed with peptic ulcers were treated with omeprazole ( $20 \mathrm{mg} \times 2$ per day). Blood samples were collected using tubes containing EDTA and genotyping was performed by means of real-time PCR. All patients were evaluated for clinically important allelic variants of cytochrome P450 genes: CYP2C19*2 (G681A, rs4244285), CYP2C19*3 (G636A, rs4986893), CYP2C19*17 (C-806T, rs12248560), CYP3A4*22 (C>T intron 6, rs35599367), CYP3A5*3 $(\mathrm{A} 6986 \mathrm{G}, \mathrm{rs} 776746)$ and rs1045642 (C3435T), rs4148738 (C>T) of $A B C B 1$ gene.

Results: All of the observed genotype frequencies except one were consonant with the Hardy-Weinberg equilibrium $(C Y P 2 C 19 * 2: \mathrm{p}=0,6$; $C Y P 2 C 19 * 17: \mathrm{p}=0,8 ; A B C B 1(\mathrm{rs} 1045642): \mathrm{p}=0,06 ; A B C B 1$ (rs4148738): $\mathrm{p}=0,6 ; C Y P 3 A 4 * 22: \mathrm{p}=0,9 ; C Y P 3 A 5 * 3: \mathrm{p}=0,4)$. The $C Y P 2 C 19 * 3$ allele was absent in the sample which could be due to the ethnic patterns of prevalence, thus we did not evaluate its genotype frequencies. The frequency of genotypes in our study was generally consistent with the frequencies from other studies $(C Y P 2 C 19 * 2: \mathrm{p}=0,16$, Hilli J, 2007.; $C Y P 2 C 19 * 17$ : $\mathrm{p}=0,37$, Pedersen R.S., 2010; $C Y P 3 A 4 * 22: \mathrm{p}=0,10$, Sanchez Spitman A.B., 2017; CYP3A5*3: p=0,26, Hilli J., 2007), although we did not evaluate the $C Y P 2 C 19 * 3$. A statistically significant difference in the $A B C B 1$ rs 1045642 (C3435T) genotype frequencies ( $\mathrm{p}=0,02$, Gaikovitch E.A., 2003) was revealed. The mutant allele was identified more often in our sample twice $(C Y P 2 C 19 * 17: \mathrm{p}=0,02$; $A B C B 1$ rs $1045642(\mathrm{C} 3435 \mathrm{~T}): \mathrm{p}=0,002)$ and less often once $(C Y P 2 C 19 * 2: \mathrm{p}=0,03)$.

Summary / Conclusions: The allele frequencies in our sample were almost similar to that in European populations. Nevertheless, we observed a higher frequency of the CYP $C 19 * 17$ allele, which may cause the lack of effectiveness of antiulcer therapy.

\section{EACPT-1309}

Variants of fibroblast growth factor 21 gene affect dietary salt intake: potential implication on antihypertensive therapy

M. Saber-Ayad ${ }^{*} 1,2,3$, S. Hammoudeh ${ }^{1,4}$, S. Manzoor ${ }^{1}$, H. Jabbar ${ }^{1}$, R. Wardeh $^{1}$, A. Ashraf ${ }^{1}$, H. Radwan ${ }^{5}$, R. Hammoudi ${ }^{1}$

${ }^{1}$ College of Medicine, University of Sharjah, sharjah, United Arab Emirates, ${ }^{2}$ Cairo University, Cairo, Egypt, ${ }^{3} 2$ Research Institute for Medical and Health Sciences, ${ }^{4}$ Research Institute for Medical and Health Sciences, ${ }^{5}$ College of Health Sciences, University of Sharjah, sharjah, United Arab Emirates

\section{Pharmacogenetics/omics}

Introduction: The hepatokine, fibroblast growth factor 21 (FGF21) is a peptide hormone secreted by the liver and many other tissues. Injection of FGF21 may lead to weight loss with improvement in insulin sensitivity, so it is considered a potentially attractive therapeutic target. However, recent data showed that serum FGF21 level is elevated in hypertension, atherosclerosis and coronary artery disease. There is an association of FGF21 rs838133 and increased consumption of sweets in Caucasians. Only few studies addressed the link between gene variants and food intake in the Middle East, where both hypertension and obesity show high prevalence. 
Objectives: To explore the potential effect of FGF21 rs838133 and rs838145 on the pattern of food intake in Emirati population

Methods: Out of 215 healthy adult Emiratis recruited, only 150 subjects were considered after removing outliers and normalizing body mass index and other variables using a QQ-plot (mean age $30.84 \pm 8.9$ years, females $=59.3 \%$ ). Genotyping for FGF21 rs838133 and rs838145 was performed using DNA from saliva samples. Dietary intake was assessed by a sixty-one-item FFQ that estimated food and beverage intakes over the past year. The daily energy, macronutrient, and sodium intake were computed using food processor software.

Results: The mean BMI of the study population was $26.08 \mathrm{~kg} / \mathrm{m}^{2}$. Sodium intake was significantly higher in the FGF21 rs 838145 GG group ( $\mathrm{p}=0.008$ ) with a trend of increased sodium intake in rs838133 AA group. rs 838145 GG group also showed higher intake of vegetables $(\mathrm{p}=0.005)$ and vitamin $\mathrm{A}(\mathrm{p}=0.03)$ compared to other genotypes. Intake of carbohydrate, fat, protein, vitamin D did not show significant difference among genotypes.

Summary / Conclusions: The current study points out an association between FGF 21 rs838145 G allele and high salt intake in Emirati population. Our results pave the way to a deeper understanding of gene-food interaction that may underlie frequent failure of antihypertensive treatment; defining groups of patients who may show refractory hypertension due to their genetic variants.

\section{EACPT-1367}

Crispr-cas9 enrichment and minion ${ }^{\circledR}$ nanopore sequencing: an innovative and effective tool for cyp3a4-cyp3a5 pharmacogenetics T. Willeman ${ }^{1}$, F. Stanke-Labesque ${ }^{1,2}$, X. Fonrose ${ }^{1}$

${ }^{1}$ Laboratory of Pharmacology, Pharmacogenetics and Toxicology, CHU Grenoble Alpes, ${ }^{2}$ INSERM U1042, HP2, GRENOBLE, France

\section{Pharmacogenetics/omics}

Introduction: CYP3A4 and CYP3A5 contribute to the metabolism of approximately $50 \%$ of marketed drugs. Their genes are highly polymorphic, thereby responsible of an established variability in drug pharmacokinetics and response. Multiallelic genetic polymorphisms have identified in exonic or intronic regions, resulting in distinct pharmacogenetic phenotypes. Current sequencing methods are based on amplification sequencing of exome or small targeted region, leading to miss intronic variant, structural variant or rare variant. Long read sequencing of native DNA, using Oxford Nanopore MinION® sequencer, may be an answer to this problematic.

Objectives: Our aim was to develop a targeted gene sequencing method based on amplification-free CRISPR-Cas 9 enrichment followed by nanopore sequencing on MinION $®$ sequencer, designed to analyze the entire genomic region carrying CYP $3 A 4$ and CYP $3 A 5$ genes and promoters in a single process.

Methods: We designed two pairs of crRNA using CHOPCHOP v2 and CRISPR-Cas9 digestion lasted 30 minutes. Sequencing run lasted approximately 14 hours on a R9.4 flowcell and SQK-LSL109 kit. Data were acquired with MinKNOW and basecalled with guppy. Data analysis included mapping to the GRCh38 human reference genome, variant calling and average sequencing depth. Data visualization was performed with Integrative Genome Browser. To evaluate our protocol, one patient has been selected because of his $C Y P 3 A 5 * 1 / 3$ genotype obtained with Taqman assays.

Results: Genomic regions of interest were 36,633bp long for CYP3A5 and 30,549bp for CYP3A4. We generated 518.7 megabases with 43,664 reads $(80.5 \%$ with a Q-score $>7)$. Mean read length was $11,879 \mathrm{bp}$ with a read length $\mathrm{N} 50$ of $20,620 \mathrm{bp}$. The mean sequencing depth resulted in $20 \mathrm{X}$ for CYP3A5 and $70 \mathrm{X}$ for CYP3A4 regions. Sequencing depth was sufficient to determine genotypes. MinION ${ }^{\circledR}$ sequencing data were in accordance with Taqman assays $C Y P 3 A 5 * 3, * 6, * 7$ and $C Y P 3 A 4 * 22$.
Summary / Conclusions: This preliminary evaluation demonstrates that nanopore sequencing with a MinION® sequencer could be implemented to pharmacogenetics laboratories. Given its simple development, this method could be a multipurpose tool for next-generation pharmacogenomics since it avoids amplification bias and allows exhaustive genotyping characterization with haplotype phasing and detection of base modifications.

\section{EACPT-1369}

Combinations of abcb1 and cyp450 genetic polymorphisms as predictors of intolerance to benzodiazepine tranquilizer phenazepam O. Tereshchenko ${ }^{* 1,2}$, D. Ivashchenko ${ }^{1}$, I. Temirbulatov ${ }^{1,3}$, E. Grishina ${ }^{1}$, D. Sychev ${ }^{1}$

${ }^{1}$ Russian Medical Academy of Continuous Professional Education, ${ }^{2}$ Pirogov Russian National Research Medical University, ${ }^{3}$ I.M. Sechenov First Moscow State Medical University, Moscow, Russian Federation

\section{Pharmacogenetics/omics}

Introduction: Phenazepam is the widely used benzodiazepine tranquilizer in Russia. Cytochrome P450 isoenzymes CYP3A5, CYP2C9, CYP2C19 are involved into benzodiasepines' metabolism, possible role of P-glycoprotein also have been supposed.

Objectives: To analyze association of $A B C B 13435 C>T, 1236 C>T$, $2677 G>T, C Y P 3 A 5 * 3, C Y P 2 C 9 * 2, C Y P 2 C 9 * 3, C Y P 2 C 19 * 2$, $C Y P 2 D 6 * 4$ polymorphisms combinations with subjective tolerance to phenazepam.

Methods: 102 male patients with non-comlicated alcohol withdrawal syndrome (F10.3 by ICD-10) were involved into the study. Each participant was prescribed Phenazepam. UKU Side Effects Rating Sale was used to measure safety of pharmacotherapy at 6th day. $5 \mathrm{ml}$ of venous blood was collected from each participant for genotyping to detect $A B C B 13435 C>T, A B C B 1$ $1236 C>T, A B C B 12677 G>T, C Y P 3 A 5 * 3, C Y P 2 C 9 * 2, C Y P 2 C 9 * 3$, $C Y P 2 C 19 * 2, C Y P 2 D 6 * 4$ polymorphisms by real-time polymerase chain reaction. Haplotype analysis was made by SNPStats online-tool.

Results: Haplotype ABCB1 3435-1236-2677-CYP3A5*3 (T-T-T-G) was associated with increased severity of intolerance: $\mathrm{OR}=5,03 ; 95 \%$ CI 1.65 - 15.34; $\mathrm{p}=0,0056$; similar results were obtained for $A B C B 1$ 3435-1236-2677-CYP2C9*2 $(T-T-T-C)-\mathrm{OR}=3,61 ; 95 \%$ CI $1,31-$ 9,92; $\mathrm{p}=0,015$ and $A B C B 13435-1236-2677-C Y P 2 C 19 * 2(T-T-T-G)-$ $\mathrm{OR}=2,52 ; 95 \%$ CI $1,05-6,08 ; \mathrm{p}=0,042$.

For haplotype $A B C B 1$ 3435-1236-2677-CYP2D6*4 (T-T-T-G) deterioration of tolerance to phenazepam was observed as a tendency towards significance - OR $=2,37 ; 95 \%$ CI $1,01-5,54 ; \mathrm{p}=0,05$.

A protective effect was identified in $A B C B 1$ 3435-1236-2677$C Y P 2 C 9 * 3(C-C-G-A)$, this haplotype improved drug tolerance $(\mathrm{OR}=0,37 ; 95 \%$ CI $0,16-0,84 ; \mathrm{p}=0,019)$.

Summary / Conclusions: The study results confirmed that haplotypes $A B C B 1$ 3435-1236-2677-CYP3A5*3 (T-T-T-G), ABCB1 3435-12362677-CYP2C9*2 and $A B C B 1$ 3435-1236-2677-CYP2C19*2 (T-T-T$G)$ decreased phenazepam safety, $A B C B 1$ 3435-1236-2677-CYP2C9*3 $(C-C-G-A)$ showed protective effect.

Funding: Research was supported by the grant of Russian Foundation for Fundamental Research, project №18-315-00005.

\section{EACPT-1402}

Correlation between abcb1 gene polymorphisms, antiepileptic drug concentrations and the response to treatment O. Sabin ${ }^{*} 1$, C. Bocșan ${ }^{1}$, A. Buzoianu ${ }^{1}$

${ }^{1}$ Pharmacology, Toxicology and Clinical Pharmacology, Iuliu Hatieganu Medicine and Pharmacy University, Cluj Napoca, Romania

\section{Pharmacogenetics/omics}

Introduction: A possible molecular mechanism of clinically defined multidrug- resistant epilepsy involves drug efflux transporters such as $\mathrm{P}$ 
glycoprotein (P-gp), a member of the ATP-binding cassette subfamily B1 (ABCB1).

Objectives: We have investigated the prevalence of the C3435T, G 2677T/A, and T129C single-nucleotide polymorphisms in promoter region of $\mathrm{ABCB} 1$ gene, in Romanian epileptic patients and the association of different mutations with non-responder type and antiepileptic drugs concentrations.

Methods: 70 epileptic patients evaluated in the Neurology Department of Cluj County Hospital were included in the study. The response to treatment was assessed by reviewing the seizure diaries and the patients were classified as responders or non-responders. Antiepileptic drug (AED) plasmatic concentrations were measured and the patients were divided into 2 groups: first group with AED concentrations in therapeutic range and the second one with sub-optimal AED concentrations. Genotyping the DNA samples we have investigated MDR1 gene polymorphism by polymerase chain reaction (PCR). Results were expressed as genotype and allele frequencies per response group and compared between subgroups.

Results: 33 patients $(47.14 \%)$ were classified as responders, while the remaining 37 patients $(52.86 \%)$ were classified as non-responders. A comparison of responders and non-responders revealed no significant difference in genotype frequency for any of the three mutations studied. The CT heterozygote for ABCB1 T129C had a significant lower AED concentration ( $\mathrm{p}=0.041)$, with no significant difference for the others studied polymorphisms.

Summary / Conclusions: In our study, we found an association of CT variant in $\mathrm{ABCB} 1 \mathrm{C} 129 \mathrm{~T}$ with lower $\mathrm{AED}$ plasmatic concentrations and no association between $\mathrm{ABCB} 1$ variants and the drug responsiveness.

\section{EACPT-1423}

Pharmacogenetic predisposition for adverse reactions of tyrosin kinase inhibitors: a case report of a patient with chronic myeloid leukaemia

P. Mas ${ }^{* 1,2}$, L. Bielen ${ }^{1,3}$, D. Sertic ${ }^{1}$, N. Bozina ${ }^{1,3}$

${ }^{1}$ University Hospital Centre Zagreb, ${ }^{2}$ Agency for Medicinal Products and Medical Devices of Croatia, ${ }^{3}$ University of Zagreb School of Medicine, Zagreb, Croatia

\section{Pharmacogenetics/omics}

Introduction: Chronic myeloid leukaemia (CML) is a myeloproliferative disorder characterized by increased proliferation of the granulocytic cell line. Tyrosine kinase inhibitors (TKI) such as imatinib, nilotinib, dasatinib, bosutinib and ponatinib are used for treatment of CML. TKI are mainly metabolised with CYP3A4, while other CYP isoenzymes may present alternative metabolic pathways for some TKI. Polymorphisms of $\mathrm{P}$-gp/ABCB1 gene may alter systemic exposure to imatinib, dasatinib and nilotinib. Bosutinib is not a P-gp substrate, but it is a strong P-gp inhibitor. Objectives: We present a case together with pharmacogenetic analysis of a patient who was treated with various TKIs for CML and developed serious adverse drug reactions.

Methods: A blood sample was obtained and extracted DNA was analysed for CYP3A4 *22, CYP2D6 *2, *3, *4, *5, *6, *41, CYP2C19 *2, *17, CYP2C9 *2, *3, ABCG2 421 C>A, SLCO1B1 $521 \mathrm{~T}>\mathrm{C}, \mathrm{ABCC} 2-24 \mathrm{C}>\mathrm{T}$ and $\mathrm{ABCB} 13435 \mathrm{C}>\mathrm{T}$ variants using realtime PCR methods.

Results: 57-year old female patient with CML was started on imatinib and developed generalized urticaria with angioedema. Antiallergic therapy was given and therapy with imatinib temporarily discontinued. 2 month following gradual reintroduction of imatinib, the patient developed interstitial pneumonitis. Therapy with imatibin was permanently discontinued and nilotinib was introduced. 6 days after introduction, patient presented with acute pancreatitis.

Pharmacogenetic analysis was performed and revealed that the patient had, according to genotype predicted, normal enzyme activity of CYP3A4 and intermediate enzyme activity of CYP2C9, CYP2C19 and CYP2D6. According to genotype of transporters, weak activity for P-gp/
$\mathrm{ABCB} 1$ and OATP1B1 and intermediate for MRP2/ABCC2 could be predicted.

Due to disease progression, it was decided to start treatment with dasatinib. Patient achieved successful therapeutic response for 5 years when she presented with pulmonary toxicity. Therapy was then changed to bosutinib and the patient successfully continued treatment without new adverse reactions. Summary / Conclusions: Serious reactions to three different TKI can be at least partly explained by decreased transport activity of P-gp, ABCC2 and intermediate enzyme activity of CYP2C9, CYP2C19 and CYP2D6. Other concomitant therapy with CYP3A4 and P-gp inhibitors also had contributory role.

\section{EACPT-1448}

Rs5219 polymorphism in kenj11 gene as a factor of response to metformin treatment

K. Zagorodnikova ${ }^{*}$, P. Shorokhova ${ }^{2}$, V. Baranov ${ }^{2}$, N. Vorokhobina ${ }^{2}$

${ }^{1}$ clinical pharmacology, Almazov National Medical Research Center, ${ }^{2}$ endocrinology, North-western state medical university n.a.I.I.Mechnikov, St Petersburg, Russian Federation

\section{Pharmacogenetics/omics}

Introduction: KCNJ11 polymorphism is associated with certain types of diabetes and with response to sulfonureas, for which KCNJ11 is a pharmacological target. This polymorphism being responsible for overall increased insulin secretion may also play role in effectiveness of other glucose lowering medications.

Objectives: We evaluated association of rs5219 (C>T) polymorphism in KCNJ11 gene with individual susceptibility to metformin (MTF).

Methods: We prospectively recruited medication-naïve patients first diagnosed with type 2 diabetes mellitus, who had clinical indications to start treatment with metformin. They were evaluated at inclusion, 3 and 6 months of treatment. MTF dose adjustment followed clinical decision. The patients could also be switched to a drug combination or an alternative treatment. At each point we calculated body mass index (BMI), fasting glucose (FPG) and postprandial glucose (PPG), glycated hemoglobin $(\mathrm{HbA} 1 \mathrm{c})$, fasting C-peptide. All patients were genotyped for rs5219 (C>T) polymorphism in KCNJ11 gene.

Results: By the time of analysis we recruited 77 patients (30 male) aged

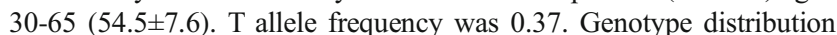
followed Hardy-Weinberg equilibrium. Starting dose of MTF was 1500$1700 \mathrm{mg}$ and did not differ between the genotype groups. FPG, PPG and $\mathrm{HbA} 1 \mathrm{c}$ were at comparable levels not statistically different. At 3 months of treatment 41 and $48 \%$ of $\mathrm{T}$ allele non-carriers and T-allele carriers respectively had increased MTF dose for clinical reasons (the resulting mean doses did not differ between the groups), and 20 and $21 \%$ respectively were switched to a drug combination. At 3 months BMI was lower in patients with CC genotype 2.6[1.4;4.1] \% compared to T allele carriers 2.6[1.4;4.1] \% ( $<<0.01)$. HbA1C decrease was $2.3[1.2 ; 3.1] \%$ in CC genotype and $1.5[0.7 ; 2.2] \%$ in T allele carriers $(\mathrm{p}<0.05)$. C-peptide levels were $2.42[2.10 ; 3.10] \mathrm{ng} / \mathrm{ml}$ vs $3.20[2.50 ; 4.20] \mathrm{ng} / \mathrm{ml}(\mathrm{p}<0.01)$.

Summary / Conclusions: We can conclude that $\mathrm{T}$ allele is associated with worse response to metformin treatment in medication naïve patients with type 2 diabetes and may be considered an additional prognostic marker.

\section{Pharmacokinetics/Pharmacodynamics}

\section{EACPT-1020}

Pharmacokinetics of ertapenem in patients with urosepsis N. Nopparatana ${ }^{*} 1$, S. Jaruratanasirikul ${ }^{1}$

${ }^{1}$ Department of Medicine, Faculty of Medicine, Prince of Songkla University, Hat Yai, Songkla, Thailand

\section{Pharmacokinetics/ pharmacodynamics}

Introduction: Pathophysiological changes in patients with sepsis resulting in altered pharmacokinetic (PK) patterns that may affect 
therapeutic plasma concentrations have been found with several antimicrobial agents. The emergence of extended-spectrum b-lactamase (ESBL)-producing Enterobacteriaceae has become a significant public health threat and ertapenem, a broad-spectrum carbapenem, has shown promising activity against this microorganism. This agent was licensed for clinical use in complicated intra-abdominal infections, complicated skin and skin-structure infections, acute pelvic infections, complicated urinary tract infections and community-acquired pneumonia.

The objective of the study was to determine the PK of ertapenem in patients with urosepsis.

Objectives: The objective of the study was to determine the PK of ertapenem in patients with urosepsis.

Methods: The study was conducted with 11 patients with urosepsis admitted to Songklanagarind Hospital. Ertapenem PK studies were carried out during administration of the $3^{\text {rd }}$ dose of a 30-min infusion of $1 \mathrm{~g}$ diluted in $100 \mathrm{~mL}$ of normal saline solution, delivered via infusion pump at a constant flow rate, once-daily. Non-compartment model PK parameters were determined by using the WinNonlin version 1.1 program (Scientific Consulting Inc.NC, USA).

Results: The mean age of the 7 male and 4 female patients was $66.28 \pm$ 21.01 year (range, 28 to 86 year), and their mean weight was $60.54 \pm$ $10.40 \mathrm{~kg}$ (range, 47.7 to $80 \mathrm{~kg}$ ). The PK parameters are shown in table 1 .

\begin{tabular}{lll}
\hline PK Parameter & Mean value & $\mathrm{SD}$ \\
$C_{\max }(\mathrm{mg} / \mathrm{L})$ & 21.23 & 11.36 \\
$C_{\min }(\mathrm{mg} / \mathrm{L})$ & 0.79 & 0.67 \\
$\mathrm{AUC}_{0-24}(\mathrm{mg} . \mathrm{h} / \mathrm{L})$ & 86.64 & 63.28 \\
$t_{1 / 2}(\mathrm{~h})$ & 7.70 & 2.89 \\
$V(\mathrm{~L})$ & 143.10 & 105.05 \\
$\mathrm{CL}(\mathrm{L} / \mathrm{h})$ & 15.39 & 8.90 \\
$k_{\mathrm{e}}\left(\mathrm{h}^{-1}\right)$ & 0.11 & 0.07 \\
\hline
\end{tabular}

Summary / Conclusions: The study found that the $V$ and CL of ertapenem in patients with urosepsis were greater than the values obtained from a previous study in healthy volunteers.

\section{EACPT-1030}

Adaptation of alanine aminotransferase elevations with therapeutic doses of acetaminophen: an exploratory study in healthy subjects M. Maeda ${ }^{*} 1$, R. Tanaka ${ }^{2}$, M. Aso ${ }^{3}$, Y. Sakamoto ${ }^{4}$, I. Song ${ }^{2}$, M. Ochiai ${ }^{2}$, Y. Saito ${ }^{5}$, K. Maekawa ${ }^{6}$, N. Arakawa ${ }^{5}$, Y. Ohno ${ }^{7}$, Y. Kumagai ${ }^{2}$

${ }^{1}$ Kitasato University Graduate School of Medical Sciences/ Kitasato University Hospital, ${ }^{2}$ Kitasato Clinical Research Center, Kitasato University School of medicine, The Kitasato Institute, Sagamihara, ${ }^{3}$ Solei Kawasaki Medical Center for the Handicapped, Kawasaki, ${ }^{4}$ Biobank Translational Research Support Section, National Cancer Center Hospital East, Kashiwa, ${ }^{5}$ Division of Medical Safety Science, National Institute of Health Sciences, Kawasaki, ${ }^{6}$ Faculty of Pharmaceutical Sciences, Doshisha Women's College of Liberal Arts, Kyoto, ${ }^{7}$ Kihara Memorial Yokohama Foundation for the Advancement of Life Sciences, Yokohama, Japan

\section{Pharmacokinetics/ pharmacodynamics}

Introduction: Acetaminophen (APAP) overdose is known cause ALT elevation. Recent studies have reported asymptomatic and self-limiting ALT elevation, known as adaptation, in several cases on therapeutic APAP doses. However, the mechanism of these ALT elevations has not been sufficiently understood.

Objectives: To investigate factors related to adaptation of ALT elevations with therapeutic APAP doses.

Methods: In a randomized, single-blind, placebo-controlled study, 242 healthy adults (161 males) were enrolled. Each subjects received either $3 \mathrm{~g}$
APAP daily $(n=202)$ or placebo $(n=40)$ for 28 days. All subjects were analyzed for genetic polymorphisms in cytochrome P450 2E1 (CYP2E1) and UGTIAl and had liver function monitoring with ALT, AST, ALP, LDH, GGTP, TBIL, DBIL, miR-122, and high mobility group box-1 (HMGB-1). Subjects with an increase in AST or ALT over 2 times the upper limit of normal (ULN) were withdrawn from the study. We defined subjects in whom peak ALT level was $>1 \times$ ULN as susceptibles, and subjects in whom ALT level remained at baseline or $<1 \times$ ULN for 28 days as tolerators. Among the susceptibles, subjects with self-limited ALT elevations were defined as adapters. The pharmacokinetics of APAP and its metabolites (glucuronide, sulfate, cysteine, mercapturate) were measured.

Results: In the APAP group, 66 subjects were susceptibles, 129 were tolerators. Adapters were observed 30 subjects out of 66 susceptibles. None developed liver injury. Twelve susceptibles were withdrawn from the study due to ALT elevation (withdrawn susceptibles). ALT elevations were correlated with miR122 significantly, but not with HMGB-1. No correlation was found between plasma APAP concentrations and ALT levels. The excretion rate of mercapturate in susceptibles was significantly higher than in tolerators $(\mathrm{p}=0.0182)$. In susceptibles, the frequency of subjects who were homozygotes or compound heterozygotes for $U G T 1 A 1 * 6$ or $U G T 1 A 1 * 28$ was significantly higher than in tolerators $(\mathrm{p}=0.0172)$. The frequency of subjects who had the genotype of CYP $2 E 1 * 1 / * 5 A$ was higher among withdrawal susceptibles than among other groups.

Summary / Conclusions: ALT elevations, which may be caused by preclinical damage of hepatocytes, was found after $3 \mathrm{~g}$ daily doses of APAP, but these elevations are considered to be transient and self-limiting. ALT elevations may be associated with the disposition of APAP metabolites and genetic factors.

\section{Oral Presentation}

EACPT-1052

Pharmacokinetics of methylphenidate in plasma, exhaled breath, oral fluid and dried blood spots after a single oral dose of ritalin 20 mg

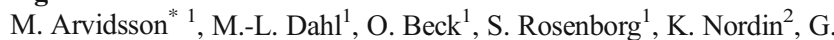
Lenk $^{3}$

${ }^{1}$ Department of Laboratory Medicine, Karolinska Institutet, ${ }^{2}$ Karolinska University Hospital, ${ }^{3}$ Department of Micro Nanosystems, KTH Royal Institute of Technology, Stockholm, Sweden

\section{Pharmacokinetics/ pharmacodynamics}

Introduction: Attention-deficit hyperactivity disorder (ADHD) is a common neurodevelopmental disorder. The first line pharmacological treatment for ADHD is stimulant medications, either methylphenidate or amphetamine. Methylphenidate has two centers of chirality and the threo enantiomers account for the central stimulant effect. Because of the inter-individual variability, methylphenidate dosage must be individually titrated. Urine is considered to be the gold standard for drugs of abuse testing. Oral fluid, exhaled breath and dried blood spot are some of the alternatives to drug testing matrices such as urine and venous blood.

Objectives: The primary aim of this study was to assess the correlations between methylphenidate concentrations in exhaled breath, oral fluid, dried blood spot and plasma. The secondary aim of this study was to validate a new method for enantio-selective determination of methylphenidate and ritalinic acid in plasma.

Methods: Twelve healthy volunteers received a single oral dose of methylphenidate (Ritalin $®$ capsules $20 \mathrm{mg}$ ) and repeated sampling of plasma, exhaled breath, oral fluid and whole blood in dried blood spots was done for 24 hours. Plasma was analyzed for enantiomers of methylphenidate and ritalinic acid with LC-MS/MS. Exhaled breath, oral fluid and dried blood spot were analyzed for methylphenidate and ritalinic acid with LCMS/MS 
Results: We identified one subject who displayed an unusual concentration-versus-time profile for $\mathrm{d}$ - and 1 - methylphenidate with a biphasic profile for 1-methylphenidate with an early peak at 1 hour and a second peak at 10 hours and with the highest dmethylphenidate exposure of all subjects. In all subjects, the concentration-time profile of methylphenidate in oral fluid roughly reflected that of methylphenidate in plasma. Methylphenidate could be detected at some time points in exhaled breath in all subjects, but there was no consistent concentrationtime pattern. The methylphenidate concentrations in DBS were up to 9.6 times higher (range 4.2-9.6) than in plasma

Summary / Conclusions: The enantio-selective determination of dand 1-methylphenidate in plasma is a valuable tool in analyzing interindividual differences in the metabolism of methylphenidate. Our data suggest that oral fluid may serve as an alternative matrix to monitor compliance.

\section{EACPT-1056}

Safety, tolerability, and pharmacokinetics of tac-302 and food effects on tac-302 in healthy participants: randomized, double-blind, placebo-controlled, single-dose and multiple-dose studies

E. Nishida ${ }^{*}$, S. Sesoko ${ }^{2}$, T. Okayama ${ }^{3}$, K. Miyoshi ${ }^{1}$

${ }^{1}$ Clinical Development II, Taiho Pharmaceutical Co., Ltd., Chiyoda-ku, Tokyo, ${ }^{2}$ Sosenkai Clinic Edogawa, Edogawa-ku, Tokyo, ${ }^{3}$ Pharmacokinetics Research Laboratories, Taiho Pharmaceutical Co., Ltd., Tsukuba, Ibaraki, Japan

\section{Pharmacokinetics/ pharmacodynamics}

Introduction: TAC-302 is one of the most potent cyclohexenoic longchain fatty alcohol derivative that promotes neurite outgrowth in cultured neurons. This neurotrophic activity of TAC-302 is expected to improve partially denervated bladder and lower urinary tract tissue, thereby restoring urinary function.

Objectives: We conducted two phase 1, randomized, placebo-controlled studies to confirm the safety and pharmacokinetics (PK) of single doses of TAC-302 and to assess the safety, tolerability, and PK of multiple doses of TAC-302 in healthy Japanese adult males. We also evaluated the effects of food on TAC-302 PK.

Methods: In a first-in-human single-dose study, TAC-302 was administered at doses of 100-1200 mg after an overnight fast. In addition, the effects of food on the PK of TAC-302 $400 \mathrm{mg}$ were studied. In the multiple-dose study, effects of meals (low-fat meal, ordinary meal, and high-fat meal) on the PK of single doses of TAC-302 (100, 200, or 400 $\mathrm{mg}$ ) and multiple doses of TAC-302 administered for 5 days (100, 200, and $400 \mathrm{mg}$ twice daily) in the fed state were evaluated.

Results: Sixty (single-dose study) and thirty-six (multiple-dose study) participants underwent randomization. No serious or severe adverse events (AEs) occurred, and none of the participants discontinued the study because of AEs. No differences were observed in $\mathrm{AE}$ incidences between the TAC-302 and placebo groups in either study. TAC-302 showed PK linearity up to doses of $1200 \mathrm{mg}$ in the fasting state and across the dose range of $100-400 \mathrm{mg}$ in the fed state, and no accumulation effect was observed. Geometric mean ratios for $\mathrm{C}_{\max }$ and $\mathrm{AUC}_{0-48 \mathrm{~h}}$ in the fed versus fasting states were 12.31 and 12.98 , respectively. Furthermore, the mean values of $\mathrm{C}_{\max }$ and $\mathrm{AUC}_{0-12 \mathrm{~h}}$ of TAC-302 $400 \mathrm{mg}$ after a high-fat meal were approximately 3 times greater than those after a low-fat meal. Summary / Conclusions: TAC-302 was safe and well-tolerated. Its PK was affected by meal and furthermore influenced by meal fat content. However, owing to its anticipated favorable safety profile and unique mechanism of action, TAC-302 may be a promising novel therapeutic agent for patients with refractory lower urinary tract dysfunction, particularly those with dysuria.

\section{EACPT-1057}

Estimated exposure to dabigatran etexilate influences efficacy and toxicity
B. Simpson ${ }^{*}{ }^{1}$, D. Reith ${ }^{2}$, N. Medlicott ${ }^{1}$, A. Smith ${ }^{1}$

${ }^{1}$ School of Pharmacy, ${ }^{2}$ Dunedin School of Medicine, University of Otago, Dunedin, New Zealand

Pharmacokinetics/ pharmacodynamics, Pharmacovigilance

Introduction: Dabigatran etexilate has become widely used for the prevention of stroke in patients with non-valvular atrial fibrillation (NVAF). Currently, there is limited information in 'real-world' patients relating to dabigatran etexilate exposure and response.

Objectives: To investigate the relationship between dabigatran etexilate exposure and adverse response in 'real-world' patients.

Methods: This retrospective cohort study used administrative health data for NVAF patients dispensed dabigatran etexilate between 1 July 2011 and 31 December 2015. Adverse outcomes of cerebrovascular accident (CVA), systemic embolism and haemorrhage were extracted. Simulated pharmacokinetic parameters were obtained using a previously published population pharmacokinetic model of dabigatran etexilate. Area under the curve (AUC), the exposure parameter, was derived using these simulations and dosing data. The exposure-response relationship was investigated by assessing the risk of adverse outcomes at AUC quartiles and compared using Poisson regression expressed as incidence rate ratios (IRR) (95\% CI) adjusted for known potential confounders.

Results: In total, 2660 NVAF patients had been dispensed dabigatran etexilate. For these patients there was a decreased risk of haemorrhage $(0.51,0.32-0.79)$ when dabigatran AUC was in the second quartile 1.70 $1.96 \mathrm{mg} * \mathrm{~h} / \mathrm{L}$ and thromboembolism or CVA $(0.34,0.16-0.76)$ was in the third quartile $1.97-2.26 \mathrm{mg} * \mathrm{~h} / \mathrm{L}$. An increased risk of haemorrhage (1.68, 1.18-2.38) was observed when AUC was in the fourth quartile 2.27$12.76 \mathrm{mg} * \mathrm{~h} / \mathrm{L}$.

Summary / Conclusions: An exposure response relationship for dabigatran etexilate was described, where the most effective response was observed when AUC was in the range of 1.70 to $2.26 \mathrm{mg} * \mathrm{~h} / \mathrm{L}$. Hence, it is feasible to develop guidance for optimal dosing to improve outcomes for patients with NVAF.

\section{EACPT-1068}

Relationship between digestive function abnormality and blood levels of gastrointestinal peptides in patients receiving anticancer agents combined with aprepitant

R. Tatsuta ${ }^{* 1}$, Y. Suzuki ${ }^{1}$, K. Suzuki ${ }^{2}$, Y. Sato ${ }^{1}$, T. Shibata ${ }^{2}$, H. Itoh ${ }^{1}$

${ }^{1}$ Department of Clinical Pharmacy, Oita University Hospital, ${ }^{2}$ Department of Gastroenterological and Pediatric Surgery, Oita University Faculty of Medicine, Oita, Japan

\section{Pharmacokinetics/ pharmacodynamics}

Introduction: Aprepitant acts specifically on neurokinin 1 receptor expressed in the vomiting center and inhibits binding of substance $\mathrm{P}$ (SP; an emetic inducer) to the receptor, thereby providing potent therapeutic effect on chemotherapy-induced nausea and vomiting (CINV). Although basic research using CINV animal models has reported that aprepitant ameliorated the loss of appetite caused by anticancer drug administration, the effect on cancer patients is unknown.

Objectives: In this study, we examined the relationship between gastrointestinal dysfunction (anorexia and CINV) and blood levels of gastrointestinal peptides in patients receiving anticancer agents combined with aprepitant.

Methods: Patients who received highly emetogenic chemotherapy including cisplatin for esophageal cancer concomitant with aprepitant or fosaprepitant between April 2017 and April 2018 were included. Aprepitant or fosaprepitant was administered before anticancer drug administration (day 1). The presence or absence of gastrointestinal symptoms including nausea and vomiting was investigated using a questionnaire for gastrointestinal symptom evaluation. Data of laboratory tests (including blood urea nitrogen, serum albumin), dietary intake, and use of other antiemetic agents on days 1 to 8 were also obtained. On days 1, 3, 5 , and 8 , blood was collected from patients, and SP, neuropeptide Y 
(NPY), motilin, ghrelin and leptin concentrations were measured by enzyme immunoassay.

Results: Ten patients were eligible. Among 10 patients, 1 had vomiting, 5 had nausea, and 8 experienced anorexia. Eight of 10 patients had no emetic episodes and did not require rescue medications (complete response; CR). The CR rate was higher than previous report. Blood leptin concentration increased significantly on days 3 and 5 compared with day 1 , but did not correlate with dietary intake on days 3 and 5 . On the other hand, decrease in dietary intake correlated weakly with increase in SP and decrease in NPY. Summary / Conclusions: In patients receiving anticancer therapy, reduced dietary increase was probably associated with suppression of appetite due to decreased NPY level as well as nausea and anorexia induced by increased SP level. Since the number of cases was as small, further investigation in a larger number of cases is necessary.

\section{EACPT-1086}

Antidepressant-induced hyponatremia: a pharmacoepidemiologicalpharmacodynamic analysis of suspected adverse drug reactions in the us-faers database

F. Mazhar ${ }^{*} 1$, M. Pozzi ${ }^{2}$, E. Clementi ${ }^{3}$, S. Radice ${ }^{1}$, C. Carnovale ${ }^{1}$

${ }^{1}$ CLINICAL PHARMACOLOGY UNIT, UNIVERSITY OF MILAN, Milano, ${ }^{2}$ Scientific Institute, IRCCS E. Medea, ${ }^{3}$ Scientific Institute, IRCCS E. Medea, Bosisio Parini LC, Italy

Pharmacokinetics/ pharmacodynamics, Pharmacovigilance, Psychopharmacology

Introduction: Hyponatraemia induced by antidepressant drugs is a rare but potentially life-threatening adverse reaction. Whether it is associated with all or only some antidepressant drugs is still unclear.

Objectives: To quantify the strength of association between the use of different a antidepressant drugs and hyponatraemia by using information reported to the Food and Drug Administration (FDA) Adverse Event Reporting System (FAERS). The secondary objective was to investigate the putative relationship between different antidepressant pharmacological targets and the risks of hyponatraemia induced by antidepressant drugs using the 'pharmacoepidemiological-pharmacodynamic' method Methods: A case-noncase analysis was used using spontaneous reports from the FAERS database to measure the risk of reporting between suspected antidepressant and hyponatraemia with all other ADRs [expressed as a reporting odds ratio (ROR) while adjusting for gender, age, and concomitant medications associated with hyponatremia]. We assessed to what extent the receptor binding properties of antidepressants could associate to the RORs of hyponatraemia/SIADH of antidepressants, building a linear regression model that included as independent variables the binding affinities (pKi) to the serotonin transporter (SERT), dopamine transporter, norepinephrine transporter, and 5- $\mathrm{HT}_{2} \mathrm{C}, 5-\mathrm{HT}_{2} \mathrm{~A}, 5-\mathrm{HT}_{1} \mathrm{~A}, \alpha_{1}$ and $\alpha_{2}$ adrenergic receptors]

Results: 2,233 reports were identified. The adjusted ROR for the association between antidepressant drugs use and hyponatraemia was of 1.91 [95\% CI 1.83, 2.00]. The association was strongest for mirtazapine, followed by selective serotonin reuptake inhibitors (SSRIs), and lowest with serotonin modulating antidepressant drugs. A significant linear correlation was found between the adjusted RORs for hyponatraemia/ SIADH and $\mathrm{pKi}$ for the adrenergic receptors $\alpha_{1}$ and $\alpha_{2}$.

Summary / Conclusions: Hyponatraemia/SIADH is reported at a disproportionately higher rate with classes of antidepressant drugs (NaSSAs and serotonin modulators) that are in general considered to have a better profile of tolerability in terms of hyponatraemia. The risk of hyponatraemia with mirtazapine appears to be greater than what was reported in the literature. Our PE-PD analysis indicates also that inhibition of SERT may not be involved in the hyponatraemia linked to the use of antidepressant drugs.

\section{EACPT-1091}

Targeting mitochondrial biogenesis to attenuate chronic low-grade inflammation in obesity
K. Devasani ${ }^{*}{ }^{1}$, A. Majumdar ${ }^{1}$

${ }^{1}$ Pharmacology, Bombay College of Pharmacy, Mumbai, India

\section{Pharmacokinetics/ pharmacodynamics}

Introduction: Statins are the cornerstone in the management of dyslipidaemia. However, their use is limited by side effects like myalgia and rhabdomyolysis. In obese states, abnormal mitochondrial function leads to lipid accumulation and insulin resistance, which in turn triggers chronic low-grade inflammation. Pyrroloquinoline quinone (PQQ) is a novel supplement involved in redox modulation and mitochondrial biogenesis in energy sensitive cells of skeletal muscle, liver and adipose tissue.

Objectives: Our objective was to decipher the impact of Pyrroloquinoline quinone (PQQ) per se and in combination with Atorvastatin (ATS) in a model of high fat, $10 \%$ fructose diet (HFFD) induced chronic low-grade inflammation, dyslipidaemia and mitochondrial dysfunction.

Methods: This study investigated the effect of ATS, PQQ per se and in combination on the low-grade inflammatory state in HFFD fed male SD rats. HFFD was given for 10 weeks followed by treatment for 5 weeks with either ATS 10 or $20 \mathrm{mg} / \mathrm{kg}$, PQQ 10 or $20 \mathrm{mg} / \mathrm{kg}$, p.o and PQQ along with ATS in respective doses. The effect of PQQ on blood glucose, lipid profile and serum insulin, CRP, TNF- $\alpha$, IL- $1 \beta$, IL-18, IL-6 was estimated. Gene and protein expression of PGC $1 \alpha$, SIRT1, TFAM, NLRP 3 and Caspase 1 was assessed. Histopathological examination of adipose tissue and liver was performed using $\mathrm{H}$ and $\mathrm{E}$ staining.

Results: Compared with the positive control, rats receiving PQQ and ATS revealed significant decrease in body weights, anthropometric parameter and adipose tissue. The rats receiving PQQ per se and the combination exhibited improved glucose tolerance, lipid profile, insulin indices and displayed lower serum levels of CRP, inflammatory cytokines TNF- $\alpha$, IL- $1 \beta$, IL-18 and IL- 6 along with a rise in adiponectin when compared to positive control. Treatment with PQQ and combination elevated the mitochondrial gene expressions of PGC $1 \alpha$, SIRT1, TFAM while subduing the inflammatory gene expressions of NLRP3 and Caspase 1. Histopathological examination revealed, increase in infiltration of inflammatory cells in the liver and adipose tissue of positive control rats which was not evident in the treated rats.

Summary / Conclusions: The study depicts the therapeutic promise of using atorvastatin, a popular drug used in hyperlipidaemia along with mitochondrial stimulator PQQ as a supplement to effectively combat chronic low-grade inflammation associated with obesity.

\section{EACPT-1099}

Pyrroloquinoline quinone counters the pesk 9 induction associated limitations of atorvastatin: a pre-clinical study A. Majumdar ${ }^{*} 1$, K. Devasani ${ }^{1}$

${ }^{1}$ Pharmacology, Bombay College of Pharmacy, Mumbai, India

\section{Pharmacokinetics/ pharmacodynamics}

Introduction: HMG-Co A reductase inhibitors like atorvastatin are known to activate expression of both low-density lipoprotein receptors (LDLRs) as well as proprotein convertase subtilisin kexin type 9 (PCSK 9) gene. However, on long term use PCSK 9 binds to LDLR and leads to their degradation rather than recycling. This phenomenon limits the efficacy of statin induced LDL-C reduction. Further, statins are known to cause mitochondrial dysfunction and reduce serum levels of Coenzyme Q10, which may be a mechanism for the statin induced myopathy. Pyrroloquinoline quinone (PQQ) is a novel supplement which promotes cellular redox balance and mitochondrial biogenesis.

Objectives: The study investigated the impact of PQQ on atorvastatin induced induction of liver PCSK 9 and its efficacy end points in a model of high fat, $10 \%$ fructose diet (HFFD) induced obesity in rats.

Methods: This study investigates the anti-PCSK9 and anti-obesity effect of PQQ in combination with atorvastatin per se and in combination in 
high fat, $10 \%$ fructose diet (HFFD) fed rats. HFFD was given for 10 weeks followed by randomisation of animals for the 5 weeks treatment schedule with either ATS 10 or $20 \mathrm{mg} / \mathrm{kg}$, PQQ 10 or $20 \mathrm{mg} / \mathrm{kg}$, p.o and PQQ along with ATS in respective doses p.o. The effect of PQQ, ATS per $s e$ and in combination on body weight, anthropometric parameters, lipid profile and gene expression of liver PCSK 9 and LDL-R was assessed.

Results: Compared with the positive control, rats receiving PQQ and ATS showed significant decrease in body weights, water intake, BMI, Lee's index, AC/TC ratio. Further, serum levels of triglycerides, total cholesterol, LDL, VLDL, non-HDL and atherogenic index were markedly lowered in these groups receiving both PQQ and ATS. Combined administration of PQQ and ATS also lead to decreased expression of PCSK9 while increasing expression of LDL-R.

Summary / Conclusions: The study generates interesting evidence on the role of mitochondrial cofactors or redox modulators like PQQ in countering the PCSK 9 induction associated clinical limitations of statins like atorvastatin. This needs to be investigated in clinical settings.

\section{EACPT-1108}

Risk assessment in human using physiologically based pharmacokinetic model of diethyl phthalate and its major metabolite, monoethyl phthalate

Y.-B. Lee ${ }^{*}{ }^{1}$, H.-Y. Cho ${ }^{2}$

${ }^{1}$ COLLEGE OF PHARMACY, CHONNAM NATIONAL UNIVERSITY, Gwangju, ${ }^{2}$ COLLEGE OF PHARMACY, CHA UNIVERSITY, Seongnam, Gyeonggi-Do, Korea, Republic Of

\section{Pharmacokinetics/ pharmacodynamics}

Introduction: Diethyl phthalate (DEP), which belongs to the group of phthalate with short alkyl chains, is a substance used in the production of objects frequently used in human life. Therefore, humans are widely exposed from the surrounding environment such as water, air, soil, and food. As previously reported in many studies, DEP is an endocrine disruptor with reproductive toxicity. In addition, monoethyl phthalate (MEP), a major metabolite in vivo of DEP, is a biomarker for DEP exposure assessment and is known as an endocrine disruptor reported to be reproductive toxicity similar to DEP. Therefore, toxicokinetic studies on DEP and MEP that have not yet been reported in detail are needed.

Objectives: The aim of this study is to develop and evaluate physiologically based pharmacokinetic (PBPK) model for DEP and MEP in rats, and to extend this to human risk assessment based on human exposure. Methods: We conducted this study in vivo after oral or intravenous administration of DEP in male $(0.1-10 \mathrm{mg} / \mathrm{kg}$ dose $)$ and female $(2 \mathrm{mg} / \mathrm{kg}$ dose) rats. The biological samples consisted of plasma, urine, and 11 different tissues. We analyzed the samples using UPLC-ESI-MS/MS method.

Results: For DEP, the tissue to plasma partition coefficient was the highest for kidney followed by liver. In contrast, for MEP, it was the highest for liver but was less than unity for all other tissues. The predicted rat plasma, urine, and tissue concentrations using a developed PBPK model fitted well with the observed values. We extrapolated the PBPK model in rats to a human PBPK model of DEP and MEP based on human physiological parameters. The reference dose of $0.92 \mathrm{mg} / \mathrm{kg} /$ day of DEP and external doses of $0.106 \mathrm{mg} / \mathrm{kg} /$ day (children), $0.015 \mathrm{mg} / \mathrm{kg} /$ day (adolescents), $9.450 \mu \mathrm{g} / \mathrm{kg} /$ day (adults), and $5.601 \mu \mathrm{g} / \mathrm{kg} / \mathrm{day}$ (elderly) of DEP for human risk assessment were estimated using Korean biomonitoring values.

Summary / Conclusions: Our study provides valuable insight into human health risk assessment regarding DEP exposure.

\section{EACPT-1115}

Single-dose pharmacokinetics and tolerability of aprocitentan, a dual endothelin receptor antagonist, in subjects with severe renal function impairment

P. N. Sidharta ${ }^{*}$, I. Ulč ${ }^{2}$ J. Dingemanse ${ }^{1}$
${ }^{1}$ Department of Clinical Pharmacology, Idorsia Pharmaceuticals Ltd, Allschwil, Switzerland, ${ }^{2}$ Cepha s.r.o., Pilsen, Czech Republic

\section{Pharmacokinetics/ pharmacodynamics}

Introduction: The orally active dual endothelin receptor antagonist aprocitentan targets a novel pathway in the treatment of hypertension and could be a key player in salt/volume-dependent hypertension. Its pharmacokinetic(PK) profile supports a once daily dosing strategy. Currently, doses of 12.5 and $25 \mathrm{mg}$ aprocitentan once daily are investigated in a Phase 3 study in subjects with difficult-to-control hypertension.

Objectives: As hypertensive patients may also experience concomitant renal disease, the objectives of this study were to evaluate PK and tolerability of aprocitentan in subjects with severe renal function impairment (SRFI) and compare these with matched healthy subjects.

Methods: In this open-label, single-centre, phase 1 study (NCT03165071) 8 subjects with SRFI (mean estimated glomerular filtration rate [eGFR] $21.9 \mathrm{~mL} / \mathrm{min} / 1.73 \mathrm{~m}^{2}$ ) and 8 healthy subjects (mean eGFR $94.9 \mathrm{~mL} / \mathrm{min} / 1.73 \mathrm{~m}^{2}$ ) received a single dose of $50 \mathrm{mg}$ aprocitentan followed by an observation period of up to 17 days. Plasma PK parameters of aprocitentan were derived by noncompartmental analysis of the plasma concentration-time profiles. Differences in PK parameters were explored using geometric mean ratios (GMR) and $90 \%$ confidence intervals (CI) with SRFI subjects as test and healthy subjects as reference group. Safety and tolerability evaluations included adverse events (AEs), electrocardiograms, vital signs, and clinical laboratory tests.

Results: All 16 subjects received aprocitentan and completed the study. The PK of aprocitentan were similar in SRFI and healthy subjects with maximum plasma concentrations reached at $7.6 \mathrm{~h}$ and $5.0 \mathrm{~h}$, respectively. Maximum plasma concentrations did not differ as indicated by a GMR (90\% CI) of 1.04 (0.85-1.28). Due to a slightly lower observed clearance in SRFI subjects, half-life was longer $(53.2 \mathrm{~h}$ compared to $47.4 \mathrm{~h}$ in healthy subjects), while exposure expressed as area under the curve $\left(\mathrm{AUC}_{0-\infty}\right)$ was $34 \%$ higher (GMR 90\% CI 1.13-1.58). There were no differences in plasma protein binding ( $>99 \%$ bound). Aprocitentan was well tolerated in subjects with SRFI with no notable difference compared to healthy subjects.

Summary / Conclusions: Based on the single-dose results, SRFI subjects with mild, moderate, or severe renal function can be included in clinical studies without need for dose adjustment.

\section{EACPT-1143}

Naltrexone co-administration does not affect the pharmacokinetics of oxycodone

E. Kleideiter ${ }^{*}{ }^{1}$, S. Buller ${ }^{1}$, R. Nemeth ${ }^{2}$, R. Terlinden ${ }^{3}$, J. Rengelshausen ${ }^{1}$ ${ }^{1}$ Data Sciences - Clinical Pharmacology, ${ }^{2}$ Data Sciences - Biostatistics, ${ }^{3}$ Preclinical Drug Development - Pharmacokinetics, Grünenthal GmbH, Aachen, Germany

\section{Pharmacokinetics/ pharmacodynamics}

Introduction: Oxycodone is a centrally acting $\mu$-opioid agonist which is used as a strong analgesic. Naltrexone, an antagonist at opioid receptors, is usually co-administered to healthy subjects in clinical trials evaluating the pharmacokinetics (PK) of oxycodone to minimize discrete opioid adverse effects like euphoria.Opioids are known to delay gastric emptying and intestinal transit in humans. As naltrexone is expected to antagonize these gastrointestinal opioid effects, it is sensible to investigate whether naltrexone co-administration may influence and confound the PK of oxycodone.

Objectives: To investigate the effect of naltrexone co-administration on the PK of oxycodone after administration of two different oxycodone prolonged-release (PR) tablets to healthy subjects under fasted conditions. 
Methods: A randomized, single dose, parallel-group, within groups crossover, clinical trial was conducted in 24 healthy male subjects.

In group I, 12 subjects received a new oxycodone PR tablet (test), containing $20 \mathrm{mg}$ oxycodone $\mathrm{HCl}$, with and without co-administration of naltrexone (treatment $\mathrm{T}+$ and treatment $\mathrm{T}$, respectively) under fasted conditions. In group II, 12 subjects received an Oxygesic $®$ PR tablet (reference), containing $20 \mathrm{mg}$ oxycodone $\mathrm{HCl}$, with and without naltrexone coadministration (treatment $\mathrm{R}+$ and treatment $\mathrm{R}$, respectively) under fasted conditions. $50 \mathrm{mg}$ of naltrexone $\mathrm{HCl}$ was given 1.5 hours prior to oxycodone administration.

The oxycodone PK parameters were investigated by means of analysis of variance. The point estimates and the shortest $90 \%$ confidence intervals (CIs) were calculated for the relevant ratios.

Results: The results showed no difference in the PK parameters $\mathrm{C}_{\max }$, $\mathrm{AUC}_{0-\mathrm{t}}$, and $\mathrm{AUC}$ for both the test and reference when oxycodone was administered with and without naltrexone. The treatment ratios $\mathrm{T}+/ \mathrm{T}$ and $\mathrm{R}+/ \mathrm{R}$ for $\mathrm{C}_{\max }, \mathrm{AUC}_{0-\mathrm{t}}$, and $\mathrm{AUC}$ were close to unity and the corresponding $90 \%$ CIs fell within the commonly accepted bioequivalence range of $80-125 \%$ (see Table 1).

Table 1

\begin{tabular}{|c|c|c|c|c|}
\hline & $\begin{array}{c}\text { Treatment } T+v s . \\
\text { treatment } T\end{array}$ & $\begin{array}{l}\text { Treatment } R+v s . \\
\text { treatment } R\end{array}$ & & \\
\hline Parameter & $\begin{array}{l}\text { Point estimate } \mathrm{T}+/ \mathrm{T} \\
\quad(\%)\end{array}$ & $90 \%$ CI (\%) & $\begin{array}{l}\text { Point estimateR+/R } \\
\quad(\%)\end{array}$ & $\begin{array}{r}90 \% \mathrm{CI} \\
(\%)\end{array}$ \\
\hline $\mathrm{C}_{\max }(\mathrm{ng} / \mathrm{mL})$ & 103 & $88-119$ & 104 & $97-112$ \\
\hline $\mathrm{AUC}_{0-\mathrm{t}}\left(\mathrm{ng} \mathrm{g}^{*} \mathrm{~h} / \mathrm{mL}\right)$ & 97 & $87-108$ & 95 & $88-102$ \\
\hline AUC (ng*h/mL) & 97 & $88-108$ & 94 & $87-100$ \\
\hline
\end{tabular}

Summary / Conclusions: There is no effect of pre-treatment with naltrexone on the PK of oxycodone under fasted conditions. A naltrexone block can be applied in oxycodone PK trials in healthy subjects to minimize opioid adverse effects.

\section{EACPT-1155}

Pharmacokinetics and safety of evogliptin in patients with hepatic impairment

T. Hong ${ }^{*}{ }^{1}$, B. H. Jin ${ }^{2}$, C. O. Kim ${ }^{1}$, B. W. Yoo ${ }^{1}$, M. S. Park ${ }^{1,3}$

${ }^{1}$ Department of Clinical Pharmacology, Severance Hospital, Yonsei University College of Medicine, ${ }^{2}$ Department of Pharmaceutical Medicine and Regulatory Sciences, Colleges of Medicine and Pharmacy, Yonsei University, ${ }^{3}$ Department of Pediatrics, Yonsei University College of Medicine, Seoul, Korea, Republic Of

\section{Pharmacokinetics/ pharmacodynamics}

Introduction: Evogliptin is a potent and selective dipeptidyl peptidase-4 inhibitor for the glycemic control of patients with type 2 diabetes mellitus. Objectives: We investigated the pharmacokinetic (PK) characteristics and safety of evogliptin in patients with mild or moderate hepatic impairment compared with healthy volunteers.

Methods: An open-label, parallel-group, and multi-center study was conducted in participants with mild $(n=6)$ or moderate $(n=6)$ hepatic impairment and in healthy control subjects $(n=12)$ matched to each patient for gender, age and body mass index. A single 5-mg dose of evogliptin was administered orally and serial blood samples were collected up to 120 hours to assess the PK characteristics of evogliptin and its metabolites (M7 and M8).

Results: Patients with mild hepatic impairment and matched healthy control showed similar maximum concentration $\left(\mathrm{C}_{\max }\right)$ and area under the time-concentration curve from 0 to 120 hours $\left(A U C_{\text {last }}\right)$; the geometric mean ratio (GMR) (90\% confidence interval (CI)) were $1.04(0.80$, $1.35)$ and $1.01(0.90,1.14)$, respectively. Exposure to evogliptin $\left(\mathrm{C}_{\max }\right.$ and $\left.\mathrm{AUC}_{\text {last }}\right)$ was increased about $40 \%$ in patients with moderate hepatic impairment; GMR $(90 \%$ CI) were $1.37(1.09,1.72)$ and $1.44(1.18,1.75)$, respectively. The metabolic ratios of M7 and M8 in patients with moderate hepatic impairment were lower than matched healthy control. Evogliptin was well tolerated by both patients and healthy subjects.

Summary / Conclusions: Although evogliptin exposure was increased in patients with moderate hepatic impairment, the increase is unlikely to adversely affect safety and efficacy, based on the exposure-response data accumulated in the previous clinical studies.

\section{Oral Presentation \\ EACPT-1168}

Effects of switching from racemic methadone to R-methadone on serum methadone concentrations and QTe intervals

S. M. Havig* 1, 2, R. M. Berg-Pedersen ${ }^{3}$, H.-M. Krabseth ${ }^{1,4}$, L. Dannevig-Müller ${ }^{1}$, H. K. Zare ${ }^{1}$, K. Gjesdal ${ }^{5}$, P. Krajci ${ }^{3} \wedge$, M. Stokke Opdal ${ }^{1,5}{ }^{\wedge}$

${ }^{1}$ Department of Pharmacology, ${ }^{2}$ Department of Forenscic Sciences (current), ${ }^{3}$ Department of Substance Use Disorder Treatment, Oslo University Hospital, Oslo, ${ }^{4}$ Department of Pharmacology (current), St Olavs Hospital, Trondheim, ${ }^{5}$ Institute of Clinical Medicine, University of Oslo, Oslo, Norway

\section{Pharmacokinetics/ pharmacodynamics, Therapeutic drug monitoring}

Introduction: Racemic methadone may prolong the QTc interval, which is associated with fatal arrhythmias. In vitro studies have shown that Rmethadone has less inhibitory effect than S-methadone on the voltagegated potassium channel current, and is thus thought to have less effect on the QTc interval.

Objectives: We hypothesized that switching from racemic to Rmethadone would reduce the methadone concentration and also its effect on the QTc interval. In order to detect a reduction in the QTc interval of $20 \mathrm{~ms}$ with a standard deviation of $15 \mathrm{~ms}$ and $90 \%$ power, we had to include 9 patients in our study.

Methods: The Regional Ethics Committee approved the study. Ten methadone maintenance therapy patients, stabilized on a racemic methadone dose for four weeks and with a QTc interval $\geq 450 \mathrm{~ms}$ read automatically, were included. Between 7-14 days after inclusion, patients came to the trial unit to measure serum methadone concentrations and record an ECG before (Cmin) and 3 hours after (Cmax) taking their racemic dose. The following day, the patient was switched to R-methadone and given half of the original dose. After another 14 and 28 days, the patient returned for additional serum measurements and ECG recordings. Daily methadone intake was supervised by the dispensing pharmacy or a nurse. Serum methadone concentrations were measured by UPLC-MSMS. A cardiologist read the ECGs and calculated QTc intervals using Bazett's formula. Results: The median (range) age was 53 (38-63) years and the racemic methadone dose was $80(40-120) \mathrm{mg}$. The initial Cmin serum methadone concentration was 993 (459-1451) nmol/L, falling to 528 (385-839) nmol/L (mean of day 14 and 28) after the switch. QTc intervals were on average $37 \mathrm{~ms}$ longer when read automatically than manually. Manual readings were used after inclusion. The mean (SD) QTc interval at Cmin was significantly reduced by 5 (7) $\mathrm{ms}$ after the switch ( $\mathrm{p}=0.039$ with paired t-test). The change in the QTc interval at Cmax of -4 (19) ms was not significant ( $\mathrm{p}=0.51$ with paired t-test). Scatterplots of racemic and R-methadone concentrations at Cmin and Cmax versus QTc intervals did not indicate a linear relationship $\left(\mathrm{R}^{2}=0.0175\right.$ and $\mathrm{R}^{2}=0.0146$ for racemic methadone and $\mathrm{R}^{2}=0.0269$ and $\mathrm{R}^{2}=0.0458$ for $\mathrm{R}$-methadone).

Summary / Conclusions: The mean QTc interval reduction of $5 \mathrm{~ms}$ at Cmin after converting from racemic to R-methadone was far less than 20 $\mathrm{ms}$, which would be considered clinically relevant.

\section{EACPT-1179}

Evaluation of perfluorohexanoic acid exposure using physiologicallybased pharmacokinetic modeling and simulation 
H.-Y. Cho ${ }^{*}$, G.-W. Choi ${ }^{1}$, Y.-B. Lee ${ }^{2}$

${ }^{1}$ College of Pharmacy, CHA University, Seongnam-si, ${ }^{2}$ College of Pharmacy, Chonnam National University, Gwangju, Korea, Republic Of

\section{Pharmacokinetics/ pharmacodynamics}

Introduction: Perfluorohexanoic acid (PFHxA) is one of the perfluoroalkyl acids (PFAAs) that are a group of synthetic chemicals. It is classified as short-chain PFAAs (i.e., perfluoroalkyl carboxylic acid with $<7$ perfluorinated carbons). Although short-chain PFAAs have more favorable environmental, health, safety, and kinetic profile than longchain PFAAs, several epidemiological studies of PFHxA have provided limited and/or weak evidence for a relationship between PFHxA exposure and specific adverse health effects. Therefore, the investigation of pharmacokinetics (PKs) and health risk assessment of PFHxA are needed.

Objectives: The purpose of this study is the development of physiologically-based pharmacokinetic (PBPK) model for PFHxA in rats for applying its human health risk assessment.

Methods: The rat PBPK model of PFHxA has been built based on in vivo PK data after an oral or intravenous administration of PFHxA in male (2-10 $\mathrm{mg} / \mathrm{kg}$ ) and female rats $(10 \mathrm{mg} / \mathrm{kg})$. The biological samples including plasma, liver, kidney, heart, lung, spleen, brain, gastrointestinal tract, muscle, adipose tissue, urine, and feces were analyzed by Ultra-Performance Liquid Chromatography coupled tandem mass spectrometry. The PK analysis of PFHxA was conducted by non-compartmental and compartmental analysis using WinNonlin ${ }^{\circledR}$ software (version 8.0, Pharsight ${ }^{\circledR}$, a Certara ${ }^{\mathrm{TM}}$ Company). The construction of PBPK model and parameter estimation were performed by Berkeley Madonna V 8.3.14 software (Berkeley, CA, USA). For the calculation of external and reference dose, the Korean biomonitoring values were applied.

Results: The key parameters of the PBPK model were estimated at 122.21 $\mu \mathrm{g} / \mathrm{h}$ of transport maximum, and $500.12 \mu \mathrm{g} / \mathrm{L}$ of transport affinity constant. The PBPK model of PFHxA in rats was extrapolated to a human PBPK model for projection of the PK profile of PFHxA in humans. The external and reference dose were predicted by the developed human PBPK model through various dosing scenarios. The external dose and point of departure of PFHxA were $2.99 \mu \mathrm{g} / \mathrm{kg} / \mathrm{day}$ and $5.30 \mathrm{mg} / \mathrm{kg} /$ day, respectively.

Summary / Conclusions: The developed PBPK model of PFHxA in rats fitted well the observation values and successfully extrapolated to the human situation. Our study provides valuable information about health risk assessment for PFHxA.

\section{EACPT- 1200}

Use of a physiologically based pharmacokinetic linked pharmacodynamic (pbpk-pd) model to account for adjustments to dosing of swarfarin in different populations

U. Ezuruike $^{* 1}$, H. Humphries ${ }^{1}$, K. Abduljalil ${ }^{1}$, S. Neuhoff ${ }^{1}$

${ }^{1}$ Certara UK (Simcyp Division), Sheffield, United Kingdom

\section{Pharmacokinetics/ pharmacodynamics}

Introduction: The PBPK approach aims to predict the exposure of a drug in the body, by integrating compound specific data with the physiology of the population of interest. Once a model has been verified in one population, it can be used to predict the exposure in other populations by accounting for demographic and genetic differences. Linking the predicted exposure via a pharmacodynamic (PD) model can aid dose adjustment. Objectives: To use PBPK-PD to predict the required dose of S-warfarin in different ethnic populations to maintain the international normalised ratio (INR) within the therapeutic range.

Methods: The default Simcyp ${ }^{\circledR}$ Simulator V18 library S-warfarin PBPK model was linked to a published PD model ${ }^{1}$. In brief, a minimal PBPK model with first order absorption and elimination via the different CYP2C9 allelic forms described the PK of S-warfarin. Total S-warfarin plasma concentrations were input into the PD model. The PD model was an indirect model wherein the synthesis of prothrombin (NPT) in plasma was assumed to be inhibited by the
Emax model. A non-linear model based on the percentage inhibition of baseline NPT described the time course of INR in response to the decrease in NPT concentration ${ }^{1}$.

Results: Based on the developed PBPK-PD model, a loading oral daily dose of $5 \mathrm{mg}$ S-warfarin for 5 days followed by a daily maintenance dose of $2.5 \mathrm{mg}$ resulted in a steady state INR response level of 2.57 in a 20 -year-old male Caucasian assumed to have the CYP2C9*1*1 allele and VKORC $1 * 1 * 2$ genotype. However, the same dose administered to age and genotypematched Japanese and Chinese males produced much higher INR levels of 3.22 and 3.39 respectively. Using the steady state $\mathrm{S}$-warfarin concentration, a reduced maintenance dose of $1.5 \mathrm{mg}$ was estimated, which produced comparable steady state INR response levels of 2.32 and 2.47 for the Japanese and Chinese respectively. Subsequently, the PBPK-PD model was used to explore the impact of changes in various system parameters like age, sex, weight and CYP2C9 genotype on the INR response level.

Summary / Conclusions: This study demonstrates the utility of a PBPKPD model in estimating dosage adjustments for S-warfarin in order to maintain recommended INR therapeutic ranges for different populations. Reference:

Ohara et al 2014 PLOS ONE Vol 9(8)

\section{EACPT-1211}

Quantification of anidulafungin and micafungin in wound secretion T. Gasperetti ${ }^{1}$, R. Welte ${ }^{1}$, I. Lorenz $^{2}$, P. Schellongowski ${ }^{3}$, K. Burgmann ${ }^{3}$, T. Staudinger ${ }^{3}$, M. Joannidis ${ }^{1}$, R. Bellmann ${ }^{*} 1$

${ }^{1}$ Internal Medicine I, ${ }^{2}$ Department of General and Surgical Intensive Care Medicine, Medical University of Innsbruck, Innsbruck, ${ }^{3}$ Department of Medicine I, Medical University of Vienna, Vienna, Austria

\section{Pharmacokinetics/ pharmacodynamics}

Introduction: Anidulafungin and micafungin are echinocandins recommended for the treatment of invasive Candida infections because they are highly effective in patients with candidaemia. However, their efficacy in soft tissue and wound infection is largely unknown. Achievement of fungicidal concentrations at the target site is crucial for clinical response.

Objectives: Assessment of anidulafungin and micafungin penetration into wound secretion of adult critically ill patients.

Methods: Wound secretion was collected via drainages inserted for therapeutic purpose. Samples were taken once or, when available, before as well as $1,4,8,12,18$, and 24 hours after start of infusion. Blood samples were drawn simultaneously. From patients treated with V.A.C. (Vacuum Assisted Closure) therapy, wound secretion was taken once when the collection canister was changed. In the canisters, wound secretion was adsorbed to a gel from which anidulafungin and micafungin had to be extracted. Anidulafungin and micafungin were quantified by high performance liquid chromatography (HPLC) and UV detection at $306 \mathrm{~nm}$ after prepurification by solid phase extraction.

Results: The concentrations of anidulafungin and micafungin were determined in wound secretion samples of fifteen patients. Multiple wound secretion samples were obtained from seven patients. Single samples were taken from four patients via drains and from four patients on V.A.C. therapy. Anidulafungin concentrations of $<0.01-1.94 \mathrm{mg} / \mathrm{L}$ were measured in wound secretion, whereas simultaneous anidulafungin plasma concentrations amounted to $0.91-9.41 \mathrm{mg} / \mathrm{L}$. The micafungin levels were $0.01-1.98 \mathrm{mg} / \mathrm{L}$ in wound secretion and $0.40-7.43 \mathrm{mg} / \mathrm{L}$ in the respective plasma samples. The time to the peak concentration (Tmax) in wound secretion was 8-24 hours for anidulafungin and $1-24$ hours for micafungin.

Summary / Conclusions: Both anidulafungin and micafungin reached lower concentrations in wound secretion than in plasma samples collected at the same time. Minimal inhibitory concentrations of some relevant Candida species might equal or even exceed anidulafungin and micafungin levels in wound secretion. 
EACPT-1235

Nonlinear pharmacokinetics and concentration-effect relationship of rituximab in anti-neutrophil cytoplasmic antibody associated vasculitis

G. Paintaud ${ }^{*}$, A. Bensalem ${ }^{2}$, D. Ternant ${ }^{1}$, N. Azzopardi ${ }^{2}$, V. GouilleuxGruart $^{3}$, T. Bejan-Angoulvant ${ }^{1}$, D. Cornec ${ }^{4}$, U. Specks ${ }^{5}$, D. Mulleman ${ }^{6}$ ${ }^{1}$ Medical Pharmacology, Tours University Hospital, ${ }^{2}$ EA 7501 GICC, Tours University, ${ }^{3}$ Immunology, Tours University Hospital, Tours, ${ }^{4}$ Rheumatology, Brest University Hospital, Brest, France, ${ }^{5}$ Pulmonary and Critical Care Medicine, Mayo Clinic, Rochester, United States, ${ }^{6}$ Rheumatology, Tours University Hospital, Tours, France

Pharmacokinetics/ pharmacodynamics

Introduction: Rituximab is approved in patients with antineutrophil cytoplasmic antibody (ANCA) associated vasculitis (AAV). Levels of antibodies to proteinase 3 (PR3-ANCA) or myeloperoxidase (MPO-ANCA) are correlated with disease activity, decrease with treatment, and may therefore be used as biomarkers of response.

Objectives: The objectives of this study were to investigate the pharmacokinetics of rituximab and the relationship between rituximab concentration and ANCA levels in AAV patients.

Methods: Ninety-two AAV patients from the RAVE trial (rituximab for ANCA- associated vasculitis) were assessed. Rituximab concentrations and levels of both MPO-ANCA and PR3-ANCA were measured over time. Pharmacokinetics of rituximab was described using a semi-mechanistic model that included a latent target antigen turnover and allowed the estimation of both target-mediated elimination and non-specific elimination. Concentration-ANCA relationship was described using semimechanistic Friberg models that included a blood compartment, where ANCA concentrations are measured, and a production compartment that was sensitive to rituximab treatment and to negative feedback by blood ANCA. These models included $0,1,2$ or 3 transit compartments. Pharmacokinetic and PK-PD parameters were estimated using nonlinear mixed-effects models with Monolix Suite 2018R2.

Results: A two-compartment model including target-mediated elimination best described pharmacokinetic data. A Friberg model with no transit compartment best described the concentration-autoantibody relationship. Mean (interindividual standard deviation) estimated systemic clearance and target-mediated elimination rate constant were $0.15 \mathrm{~L} /$ days $(7.7 \%)$ and, $20.10^{-6} \mathrm{nmol}^{-1}$ day $^{-1}$, respectively. Concentrations of rituximab leading to $50 \%$ decrease of ANCA input in patients with MPO-ANCA and PR3-ANCA were $37.5 \mathrm{mg} / \mathrm{L}(29.3 \%)$ and $21.1 \mathrm{mg} / \mathrm{L}(34.7 \%)$, respectively.

Summary / Conclusions: This study is the first to describe rituximab pharmacokinetics in AAV using population PK-PD modeling approach. A nonlinear target-mediated elimination of rituximab was detected. Concentration-ANCA levels relationship was well described by a validated Friberg model with no transit compartment. The potency of rituximab in depleting ANCA input was higher in patients with PR3-ANCA than in patients with MPO-ANCA.

\section{EACPT-1312}

The evaluation of prokinetic effect of low-dose sulpiride administration from the viewpoint of plasma brain-gut peptide levels in healthy human

F. Katagiri ${ }^{*}{ }^{1}$, K. Kimura ${ }^{1}$, R. Takayanagi ${ }^{1}$, Y. Yamada ${ }^{1}$

${ }^{1}$ Tokyo University of Pharmacy and Life Sciences, Hachioji, Japan

\section{Pharmacokinetics/ pharmacodynamics, Other}

Introduction: Sulpiride, a dopamine receptor antagonist, is prescribed for gastroduodenal ulcer at a low dose $(<150 \mathrm{mg})$, and for depression or schizophrenia at a high dose $(>150 \mathrm{mg})$ in
Japan. The pharmacological mechanism of its anti-ulcer effect is caused by improvement of mucosal microcirculation. Although the other dopamine receptor antagonists, such as metoclopramide and domperidone, are used as prokinetics, sulpiride has not been evaluated as a prokinetics.

Objectives: We studied the effect of sulpiride on the levels of plasma bioactive substance related to cytoprotection and gut-motility in healthy human.

Methods: Sulpilide (150 mg) or placebo was orally administered in four healthy male volunteers. The blood samples were taken repeatedly from 0 to 240 minutes after administrations, followed by the extracting procedure, and submitted to the highly sensitive enzyme immunoassays as previously developed. All values were expressed as mean and statistically evaluated.

Results: Single administration of sulpiride caused significant $(p<0.05)$ increases of plasma calcitonin gene-related peptide and vasoactive intestinal peptide, compared with placebo, which might be involved in the cytoprotective effect. Although sulpiride caused a drug concentration-dependent decrease of plasma gastrin levels, somatostatin levels were significantly increased compared with placebo. Furthermore, the increments of motilin release were positively correlated with the area under the drug concentration-time curve, which might indicate that sulpiride had the potential dosedependent prokinetic effect.

Summary / Conclusions: In this study, we revealed that the cytoprotective effect of sulpiride might depend on alteration of plasma bioactive peptide, and from the viewpoint of gut-regulatory peptide alteration, we indicated the potential of sulpiride as a prokinetics.

\section{EACPT-1316}

Sex-by-formulation interaction in bioequivalence trials

E. González-Rojano* ${ }^{1}$, R. Salas-Butrón ${ }^{1}$, A. Portolés ${ }^{1}$, E. Vargas ${ }^{1}$

${ }^{1}$ Clinical Pharmacology, Hospital Clínico San Carlos, Madrid, Spain

Pharmacokinetics/ pharmacodynamics

Introduction: The US-FDA recommends that if drug products are intended for use in both sexes, similar proportions of males and females should be recruited for bioequivalence studies (BE). In contrast, in the EU subjects can belong to either sex. Accordingly, studies conducted generally in men only are extrapolated to women under the assumption that a sex-by-formulation interaction ( $\mathrm{SxF}$ ) does not exist and, when observed, it is considered an artefact because limited data are suggestive of the existence of $\mathrm{SxF}$ in $\mathrm{BE}$ studies.

Objectives: The purpose of this work is to review pairs of BE studies conducted with the same batch and investigate if $\mathrm{SxF}$ is detected consistenly when the study is repeated.

Methods: All clinical trials performed in Hospital Clínico San Carlos, between 2000 and 2018 conducted with the same product have been reviewed. A total of $8 \mathrm{BE}$ studies were available for retrospective analysis ( 2 products of rivaroxaban, 1 product of lansoprazol and 1 containing levosulpiride). The SxF interaction includes: sequence, sex, sequence*sex, subject (sequence*sex), period, formulation and sex*formulation.

Results: Only in one of our studies with Rivaroxaban a SxF was detected, where the point estimate (and the $90 \% \mathrm{CI}$ ) of the interaction were $121.69 \%(107.4-138.33 \%, \mathrm{P}=0.02)$ for $\mathrm{Cmax}$ and $129.43 \%$ (108.38$154.57 \%, \mathrm{P}=0.02$ ) for AUCt (Table 1). However, these differences were not detected in the other study with the same product, where the results were $107.46 \%(85.48-135.09 \%, \mathrm{P}=0.51)$ for $\mathrm{Cmax}$ and $82.17 \%$ (62.82$107.48 \%, \mathrm{P}=0.18$ ) for AUCt.

The appropriate investigation on the existence of $\mathrm{SxF}$ should be based on the $90 \%$ confidence interval of the SxF, which represents the uncertainty around the adjusted ratio between the formulation effect in both sex groups.

In one of the studies the $90 \%$ CI of the interaction was outside the $20 \%$ acceptance range and not include the 100 value, which could be suggestive of SxF. However, the results of the other study do not support these 
conclusions because for Cmax the point estimate of the interaction is close to $100 \%$ and for AUC the interaction, if any, has the contrary direction.

Summary / Conclusions: Our results show that detected SxF interaction in one of the studies was an artifact because it is not detected consistently. If the $\mathrm{SxF}$ is observed repeatedly, it could not be assumed to be at random and regulatory changes regarding the demonstration of $\mathrm{BE}$ in both sexes would be necessary.

\section{EACPT-1317}

Investigation on the existence of sex-by-formulation interaction in bioequivalence trials

E. González-Rojano ${ }^{*}$, R. Salas-Butrón ${ }^{1}$, A. Portolés ${ }^{1}$, E. Vargas ${ }^{1}$

${ }^{1}$ Clinical Pharmacology, Hospital Clínico San Carlos, Madrid, Spain

\section{Pharmacokinetics/ pharmacodynamics}

Introduction: The US-FDA recommends that if a drug product is intended for use in both sexes, similar proportions of males and females should be recruited for bioequivalence studies. In contrast, in the EU subjects can belong to either sex. Accordingly, studies conducted generally in men only are extrapolated to women under the assumption that a sex-by-formulation interaction $(\mathrm{SxF})$ does not exist and, when observed, it is considered an artefact because limited data are suggestive of the existence of $\mathrm{SxF}$ in BE studies.

Objectives: The purpose of this work is to investigate if $\mathrm{SxF}$ is observed in BE studies performed in Clinical Trials Unit of Hospital Universitario Clínico San Carlos, Madrid between 2002 and 2007.

Methods: We have reviewed all clinical trials performed in this Unit. A total of 26 bioequivalence studies were available for retrospective analysis, which corresponds to 33 analyses, this meaning 66 datasets (33 each for AUCt and Cmax).

Results: The review of 26 studies shows that statistically significant $\mathrm{SxF}$ occur with a remarkable frequency ( 5 out of 26 studies, $19.2 \%$ ) (Table 1). In 3 datasets $(4.5 \%)$ a statistically significant $\mathrm{SxF}$ is detected, although the difference between males and females is lower than $15 \%$, which may be considered clinically not important. Only in 2 datasets (3.0\%), a statistically significant $\mathrm{SxF}$ was accompanied by a large difference in the point estimates of females and males.

The analyses based on the $90 \%$ confidence interval of the SxF and a $20 \%$ limit for defining a relevant interaction, by analogy with the $80-125 \%$ acceptance range for bioequivalence, show that in no case was the $90 \%$ CI of the interaction completely outside of the $20 \%$ limit, therefore, evidence of a relevant interaction has not been found. In all cases, except one, the studies lack the necessary precision for the interaction. Only in one dataset the estimation is precise and in that dataset a relevant interaction can be excluded because the $90 \% \mathrm{CI}$ is completely inside of the classical acceptance range 80-125\%.

Summary / Conclusions: A proper analysis of the SxF based on its $90 \%$ confidence interval is not able to confirm any case with an important interaction. Consequently, upon this study there is no evidence to require studies in both sex groups, combined or separately.

\section{EACPT-1353}

Clopidogrel and gemfibrozil pronouncedly inhibit the cyp2c8mediated 3-hydroxylation of desloratadine and increase desloratadine exposure in humans

A. Tornio ${ }^{1,2}$, M. Neuvonen ${ }^{1,2}$, M. K. Itkonen ${ }^{* 1,2}$, P. J. Neuvonen ${ }^{1,2}$, M. Niemi $^{1,2}$, J. T. Backman ${ }^{1,2}$

${ }^{1}$ Clinical pharmacology, University of Helsinki and Helsinki University Hospital, ${ }^{2}$ Individualized Drug Therapy Research Program, Faculty of Medicine, University of Helsinki, Helsinki, Finland

\section{Pharmacokinetics/ pharmacodynamics}

Introduction: A recent in vitro study proposed a critical role for CYP2C8 in the biotranformation of desloratadine, a $\mathrm{H} 1$ receptor inverse agonist. If
CYP2C8 proves as important in the metabolism of desloratadine in vivo, it could be applicable as a selective CYP2C8 index substrate in clinical drugdrug interaction studies. Moreover, both clopidogrel and gemfibrozil have glucuronide metabolites that have been identified as time-dependent inhibitors of CYP2C8, but their inhibition potencies have not been compared in a clinical setting.

Objectives: Our primary aim was to investigate the contribution of CYP2C8 in desloratadine metabolism in humans. Secondarily, we compared the magnitude of CYP2C8 inhibition caused by clinically relevant doses of gemfibrozil and clopidogrel.

Methods: In a three-phase, randomized, placebo-controlled crossover study, 11 healthy volunteers were administered either gemfibrozil (600 $\mathrm{mg}$ b.i.d. for five days) on days 1-5, clopidogrel on days 3-5 (300 $\mathrm{mg}$ on day 3, followed by $75 \mathrm{mg}$ on days 4-5), or placebo. On day 3 of each phase, a single $5 \mathrm{mg}$ dose of desloratadine was administered. The plasma concentrations of the drugs and their metabolites were determined with liquid chromatography/mass spectrometry.

Results: Clopidogrel augmented the area under the plasma concentration-time curve $\left(\mathrm{AUC}_{0-\infty}\right)$ and peak plasma concentration $\left(C_{\max }\right)$ of parent desloratadine to $280 \%\left(P=3 \cdot 10^{-7} ; 90 \%\right.$ CI 232 $338 \%)$ and $165 \%(P=0.0006 ; 90 \%$ CI $133-204 \%)$ of that during placebo, respectively. The corresponding increases by gemfibrozil were to $462 \%\left(P=4 \cdot 10^{-7} ; 90 \%\right.$ CI $\left.346-616 \%\right)$ and $174 \%$ $(P=0.0006 ; 90 \%$ CI $137-221 \%)$. Furthermore, the $3-$ hydroxydesloratadine:desloratadine $\mathrm{AUC}_{0-71 \mathrm{~h}}$ ratios were $21 \%$ $\left(P=7 \cdot 10^{-10} ; 90 \%\right.$ CI $\left.18-24 \%\right)$ and $1.7 \%\left(P=8 \cdot 10^{-11} ; 90 \%\right.$ CI $1.3-$ $2.4 \%)$ of placebo during the clopidogrel and gemfibrozil phases.

Summary / Conclusions: This study substantiates that CYP2C8 has a crucial role in the 3-hydroxylation of desloratadine in humans, making desloratadine a potential CYP2C8 index substrate. Furthermore, the results support the previous approximations that clinically relevant doses of clopidogrel lead to $70-80 \%$ CYP2C8 inhibition, while those of gemfibrozil almost abolish the enzyme activity.

\section{EACPT-1398}

Population pharmacokinetics of cycloserine in patients with multidrug-resistant tuberculosis

H.-S. Kim ${ }^{*}{ }^{1,2}$, Y.-K. Choi ${ }^{1,2}$, J.-L. Ghim ${ }^{2,3}$, J.-G. Shin ${ }^{1,2,} 3$

${ }^{1}$ Dep. of Pharmacology \& Pharmacogenomics Research Center, ${ }^{2}$ Center for Personalized Precision Medicine of Tuberculosis, Inje University College of Medicine, ${ }^{3}$ Dep. of Clinical Pharmacology, Inje University Busanpaik Hospital, Busan, Korea, Republic Of

\section{Pharmacokinetics/ pharmacodynamics}

Introduction: Cycloserine is a bacteriostatic anti-tuberculosis drug used in combination for the treatment of multidrug-resistant tuberculosis (MDR-TB). There is a lack of the information on pharmacokinetics (PK) of cycloserine Korean patients, which may result in inappropriate dosing leading to therapeutic failure or drug resistance.

Objectives: The objectives of this study was to estimate the population PK parameters of cycloserine, and to evaluate the recommended dosage regimens. Methods: A prospective PK study was performed in the patients taking second-line anti-TB drugs including cycloserine. Serial samples were obtained at 12 time points. Plasma concentrations were analyzed using liquid chromatography-tandem mass spectrometry (LC-MS/MS). Population PK models were developed using non-linear mixed effect modelling (NONMEM, Version 7.30; ICON Development Solutions, Ellicott City, MD, USA).

Results: Twenty-five Korean patients with MDR-TB aged 20 to 78 years included in this study. The mean body weight and creatinine clearance were $56.6 \mathrm{~kg}$ (range, 46-73) and $87.6 \mathrm{~mL} / \mathrm{min} / 1.73 \mathrm{~m}^{2}$ (range, 41.7 118.3), respectively. A one-compartment model with first-order absorption and elimination described PK of cycloserine. The estimated total 
body clearance and volume of distribution were $0.84 \mathrm{~L} / \mathrm{hr}$ and $22.8 \mathrm{~L}$, respectively. Clearance was decreased according to creatinine clearance which was explained using power model. The base model incorporating $\mathrm{CL}_{\mathrm{CR}}$ on $\mathrm{CL} / \mathrm{F}$ reduced the AIC level, improved visual inspections of goodness-of-fit plots, and decreased the objective function value. There was no other statistically significant covariate from demographic and clinical information.

Summary / Conclusions: The PK behaviors of cycloserine were well described by the developed population PK model. This model can be helpful to set therapeutic drug monitoring system for anti-TB drugs including cycloserine. Further studies will be needed to validate the suggested results.

\section{EACPT-1408}

Modeling of hysteresis phenomena in pharmacodynamics using nonlinear partial differential equations

A. Chind ${ }^{* 1}$

${ }^{1}$ Pharmacology, Kings College London, London, United Kingdom

\section{Pharmacokinetics/ pharmacodynamics}

Introduction: Nonlinear phenomena are well recognised in physics and chemistry. One example of a nonlinear phenomenon in pharmacology or toxicology is hysteresis of the Exposure-Response (ER) relationship to a drug (toxicant). Clockwise hysteresis occurs when a drug's effect decreases with time for a given exposure while anti-clockwise hysteresis involves an increase in measured response with time. While ordinary differential equations (ODEs) are used extensively in pharmacokinetic modeling, partial differential equations (PDEs) are rarely used in pharmacology. PDEs allow the modeling of a variable, say, $u$ that is dependent on two or more independent variables, usually time $(t)$ and space $(x)$ e.g. $u(x, y)$. The simplest example of a nonlinear hyperbolic PDE is the inviscid Burgers equation.

Objectives: To examine the value of a nonlinear PDE based on the the inviscid Burgers equation to help model nonlinear ER phenomena: (1) clockwise hysteresis and (2) anti-clockwise hysteresis.

Methods: We represent drug response as $R(E, t)$ where $E$ is the plasma concentration and $t$ is time. We solve the PDE using the method of characteristics, a qualitative method for finding solutions of hyperbolic PDEs. We modify the parameters of the PDE to help model (1) decreasing response to exposure with time and (2) increasing response to exposure with time.

Results: The plot of the ER curve, the solution of the nonlinear PDE representing the drug's ER relationship, has a gradient catastrophe and then becomes multi-valued, representing a weak solution of the underlying PDE. This multi-valued quality of the solution indicates that for the same plasma concentration $(E)$, there are two different values of response $(R)$ at different values of time. Both clockwise and anti-clockwise hysteresis responses are duplicated using this model.

Summary / Conclusions: Whereas traditionally, drug response is modeled only as a function of exposure, our approach using a PDE allows the explicit modeling of response as a function of both exposure and time, i.e $R(E, t)$. Our study has shown that with appropriate modifications, we can qualitatively model hysteresis phenomena found in pharmacodynamics using a nonlinear PDE based on the inviscid Burgers equation and the method of characteristics. The use of PDEs in pharmacological modeling has the potential to improve the understanding of pharmacology and help harmonize it with other quantitative sciences.

\section{EACPT-1441}

Anal acoustic reflectometry can detect pharmacologically induced pressure changes in the anal sphincter: a randomized, double-blind, placebo-controlled crossover study with imipramine

J. Kornholt ${ }^{*}$, D. Peick Sonne ${ }^{1}$, T. Riis ${ }^{1}$, J. Sonne ${ }^{1}$, N. Klarskov ${ }^{2}$

${ }^{1}$ Department of Clinical Pharmacology, University Hospital Bispebjerg and Frederiksberg, Copenhagen, ${ }^{2}$ Department of Gynecology and Obstetrics, Herlev and Gentofte Hospital, Herlev, Denmark

\section{Pharmacokinetics/ pharmacodynamics, Other}

Introduction: Drugs that increase the tone of the anal sphincter may be a viable strategy for pharmacological treatment of fecal incontinence. Conventional anorectal manometry can be used to assess drug induced pressure changes in the anal canal but the method is difficult to use and reproducibility is lacking. Anal acoustic reflectometry (AAR) is a new method to assess anal sphincter function. The anal opening pressure (AOP) measured with AAR correlates better with symptom severity and can distinguish subgroups of fecal incontinence better than anorectal manometry.

Objectives: We wanted to test whether AAR can detect pharmacologically induced pressure changes in the anal sphincter, and to describe the standard deviations associated with these measurements (for future power calculations). We tested imipramine against placebo, since tricyclic antidepressants previously have shown effect in fecal incontinence with associated increases in anal sphincter pressure.

Methods: In a randomized, double-blind, placebo-controlled crossover trial in 16 healthy women, we measured the AOP during rest and squeeze with AAR before and 1 hour after a single dose of $50 \mathrm{mg}$ imipramine or placebo. The washout period was minimum of 1 week. The study was approved by the local ethics committee, conducted according to the Good Clinical Practice guidelines, and registered on ClinicalTrials.gov and EudraCT before recruitment of subjects. Funding was provided by the clinical department.

Results: There were no dropouts and no serious adverse events. Imipramine compared to placebo increased AOP in the resting condition with 15.2 $\mathrm{cmH}_{2} \mathrm{O}$ (95\% confidence interval [CI]: 2.0 to $28.2 \mathrm{cmH}_{2} \mathrm{O}$ ), $\mathrm{P}=0.03$, and in the squeeze condition with $15.1 \mathrm{cmH}_{2} \mathrm{O}\left(95 \% \mathrm{CI}: 4.2\right.$ to $\left.26.0 \mathrm{cmH}_{2} \mathrm{O}\right), \mathrm{P}=$ 0.01. The within subject, within day standard deviation (SD) for placebo was $16.4 \mathrm{cmH}_{2} \mathrm{O}$ during rest. The within subject, between days SD for placebo was $16.4 \mathrm{cmH}_{2} \mathrm{O}$ during rest. The within subject $\mathrm{SD}$ for the effect of imipramine versus placebo was $15.8 \mathrm{cmH}_{2} \mathrm{O}$ during rest.

Summary / Conclusions: AAR can detect pharmacologically induced pressure changes in the anal sphincter, and might be used to screen drug candidates for the treatment of fecal incontinence. Single dose imipramine increased the AOP, but clinical trials in patients with fecal incontinence are needed to assess any potential clinical benefits.

\section{Pharmacovigilance}

\section{Oral Presentation}

\section{EACPT-1019}

Mobile application for adverse drug reaction reporting by patients with relapsing remitting multiple sclerosis (vigipsep study): a national randomized controlled trial

L. Peyro-Saint-Paul* ${ }^{*}$, S. Fedrizzi ${ }^{2}$, F. Montastruc ${ }^{3,4}$, F. Le Caignec ${ }^{1}$, D. Chevanne ${ }^{5}$, J.-J. Parienti ${ }^{6}$, G. Defer ${ }^{5}$

${ }^{1}$ Pharmacovigilance Clinical Research, ${ }^{2}$ Regional Pharmacovigilance Center, University Hospital Center of Caen Normandy, Caen, ${ }^{3}$ Regional Pharmacovigilance Centre of Midi-Pyrénées, ${ }^{4}$ Department of Clinical and Medical Pharmacology, Faculty of Medicine and Toulouse University Hospital, Toulouse, ${ }^{5}$ Neurology, ${ }^{6}$ DRCI, University Hospital Center of Caen Normandy, Caen, France

\section{Pharmacovigilance}

Introduction: The reporting of adverse drug reactions (ADRs) by patients represents an interesting challenge in the field of pharmacovigilance.

Objectives: The aim of the VigipSEP study was to validate a proof-of-concept that the use of a mobile application (App) compared to usual ways (by phone, mail or on the internet) increases ADRs reporting among patients with relapsing-remitting multiple sclerosis (RR-MS) receiving first-line disease-modifying drugs (DMD). 
Methods: The Vigip-SEP study (NCT03029897) was a multi-centric, open cluster-randomized controlled trial, using the App My eReport France ${ }^{\circledR}$ by RR-MS patients to report ADRs to French pharmacovigilance system. Patients who were initiated on, or switched to, a first-line DMD have been included. In the experimental arm, a neurologist introduced the patient to the App. In the control arm, the patient was informed of the existence of the App but was not introduced to its use and then encouraged to use the usual reporting procedures. Primary assessment criteria was defined as the average number of ADRs per patient and per center. The hypothesis was that the App compared to usual ways increases ADR reporting by 10 -fold.

Results: With the last visit of the last patient in April 2019, the statistical analysis was planned in May. The first results will be presented at the meeting EACPT 2019. Twenty-four centers (12 per arm: 6 MS academic expert centers, 3 general hospitals, 3 private practice neurologists) were randomized, with 161 patients enrolled. The mean age at the enrollment was 40 years old (range 20-79) with a mean delay for diagnosis of 6 years (range 0-31). At the time of the submission, 78 ADRs in 56 reports from VigipSEP patients of the two arms have been recorded in national pharmacovigilance database. Thirteen drug-event combinations (16.7\%) were unknown (not listed in the Summary Product Characteristics).

Summary / Conclusions: The increase in patients' reporting of ADR in a real-life setting would become very important for therapeutic management, especially for monitoring new approved DMDs for a better knowledge of safety profile.

\section{EACPT-1102}

The general practitioner's knowledge and attitude towards proton pump inhibitors adverse effects.

M.-L. Laroche ${ }^{*}$, K. Rudelle ${ }^{2}$

${ }^{1}$ Centre of Pharmacovigilance and Pharmacoepidemiology, University Hospital of Limoges, ${ }^{2}$ Family Medicine University Department, Faculty of Medicine , Limoges, France

\section{Pharmacovigilance}

Introduction: Proton pump inhibitors (PPIs) are frequently prescribed and generally considered as safe by general practitioners (GPs). However, more and more potential adverse drug reactions (ADRs) with PPIs are described. Objectives: To determine both the level of knowledge and the GP's attitude towards PPI ADRs in adult patients

Methods: We established a list of PPI ADRs based on study results weights according to the evidence level, the number of studies, who were identified by a litterature review, and the information provided by both the SPCs and the Martindale ${ }^{\circledR}$. We interviewed 2773 GPs in the NouvelleAquitaine region and 319 clinical assistant professors in 35 Family Medicine University Departments, using an online questionnaire. An ADR was considered as known if $70 \%$ or more GPs answered correctly. Results: 232 physicians participated and $38.4 \%$ have been confronted with PPI ADRs in the last 6 months, essentially digestive disorders. Consequently, the drug was discontinued ( $82 \%$ of cases) and the ADR was reported to pharmacovigilance $(2.3 \%)$. A list of 12 ADRs associated with PPI was established for the survey: neutropenia, thrombocytopenia, anemia, hypomagnesemia, increased liver enzymes, renal failure, pneumonitis, skin reactions, fracture, gastric polyp, microscopic colitis, gastrointestinal infection including clostridium difficile colitis. All these ADRs were unknown by GPs. Among the GPs aware of the PPI ADRs, the attitude was the permanent or temporary discontinuation of the drug. No difference in knowledge was found between university internship supervisor and non-university internship supervisor. $74.7 \%$ of GPs declared a need for information on PPI risks and $80.4 \%$ were willing to deprescribe PPIs.

Summary / Conclusions: This study indicates that PPIs are not as safe as usually hypothesized considering all the ADRs highlighted by the review; these ADRs are not necessarily reported in SPC. This survey shows that GPs were often unaware of PPI risks, or even underestimated them. Those who knew them had a suitable attitude in terms of stopping PPIs.
Physicians have expressed a need for better information and guidance for an adapted prescription of PPIs, which also involves knowing how to "deprescribe". Intervention perspectives seem relevant to improve the use of PPIs in primary care, while not forgetting to communicate about the risks of PPI use.

\section{EACPT-1164}

Dress syndrome: analysis of 90 cases over 15 years

A. Chaabane ${ }^{*}$, N. Ben Fadhl ${ }^{1}$, H. Ammar ${ }^{1}$, Z. Chadli ${ }^{1}$, H. Ben Romdhane $^{1}$, N. Boughttas ${ }^{1}$, N. Ben Fredj ${ }^{1}$, K. Aouam ${ }^{1}$

${ }^{1}$ Pharmacology, Faculty of Medicine of Monastir, Monastir, Tunisia

\section{Pharmacovigilance}

Introduction: Drug rash with eosinophilia and systemic symptoms (DRESS) is a severe and rare adverse drug reaction. Several drugs are known to induce DRESS. Anticonvulsants, sulfonamides, and allopurinol are the most related and described drugs in the literature.

Objectives: To study the clinical, biological and chronological DRESS feature, identify the implicated drugs.

Methods: We carried a cross-sectional study including all DRESS cases notified to the Pharmacovigilance Unit of Monastir (Tunisia) during 15 years (2004-2018).

Results: Our cohort of 90 patients had a median age of 56 years. Clinical examination revealed skin eruption and fever among all patients. Laboratory findings showed marked eosinophilia among all patients, hepatic cytolysis among 23 patients and creatinine serum level increase among 12 patients. An interstitial pulmonary syndrome was noted among five patients. After culprit-drug withdrawal, outcomes were favorable for all patients. The main implicated drugs were allopurinol $(n=23)$, carbamazépine $(n=21)$, cefotaxime $(n=10)$, sulfasalazine $(n=5)$, teicoplanine $(n=3)$, vancomycine $(n=2)$, oxacilline $(n=2)$, ciprofloxacine $(n=2)$, lamotrigine $(n=2)$, phenobarbital $(n=2)$ and captopril $(n=2)$. Other drugs were culprit in one case each. Skin tests were positive with carmabazepine, cefotaxime, lamotrigine, teicoplanine, captopril, ethambutol and antimoniate de meglumine and negative with the rest of drugs. Summary / Conclusions: Originally, our cohort revealed the involvement of some drugs unknown to induce DRESS and we highlight the contribution of skin tests in the management of DRESS.

\section{EACPT-1166}

Association of liver cirrhosis with drug exposure: a case-control analysis

Z. Chadli ${ }^{1}$, A. Chaabane ${ }^{*}{ }^{1}$, Z. Hlaoui ${ }^{1}$, W. Ben Mansour ${ }^{2}$, N. Ben Fredj ${ }^{1}$, N. Ben Fadhl ${ }^{1}$, H. Ben Romdhane ${ }^{1}$, N. Boughttas ${ }^{1}$, K. Aouam ${ }^{1}$

${ }^{1}$ Pharmacology, ${ }^{2}$ Faculty of Medicine of Monastir, Monastir, Tunisia

\section{Pharmacovigilance}

Introduction: Although the exact cause of cryptogenic cirrhosis is unknown, there are correlations that non-alcoholic steatohepatitis (NASH) plays a dominant role. To date, case-control and cohort studies have revealed numerous drugs that may cause Drug Induced Liver Disease (DILI). However no studies of this type have been published on cryptogenic liver cirrhosis so far

Objectives: Analyze the epidemiological and clinical characteristics of patients with cryptogenic cirrhosis and study the association of cryptogenic cirrhosis with drug exposure using a case-control approch.

Methods: The association between drugs and cryptogenic cirrhosis was assessed using a case/non-case method. Drugs were grouped according to the ATC (Anatomical, Therapeutic Chemical) classification system. Patients were defined as "cases" if they have developed cryptogenic cirrhosis. And patients with none liver injury were defined as "non-cases". Association between reactions and drugs was calculated using the reporting odds ratio (ROR) with $95 \%$ confidence intervals (CIs). A p 
value $<0.05$ was considered significant. A multivariate analysis was conducted using logistic regression.

Results: Results: The analysis was carried out on 64 patients with cryptogenic cirrhosis (cases) and 254 controls (non-cases). There were no significant differences in epidemiological characteristics (age, gender, weight) and lifestyle variables (tobacco, alcohol) between cases and non-cases groups. The univariate analysis revealed a significant association between diabetes, drugs (metformine, glibenclamide, méthyldopa, amiodarone, captopril, furosémide, nifédipine, propranolol et la levothyroxine) and cryptogenic liver cirrhosis. In a multivariate analysis, only metformine ( $\mathrm{ROR}=27.9$; 95\% IC 9.4 to 82.9 ), amiodarone ( $\mathrm{ROR}=7.7 ; 95 \%$ IC 2.4 to 24.4 ), methyldopa (ROR=5.2; 95\% IC 1.3 to 21.8$)$ and glibenclamide ( $\mathrm{ROR}=3 ; 95 \%$ IC 1.2 to 7.7 ) have a significant association with cryptogenic liver cirrhosis.

Summary / Conclusions: Our results confirm the risk observed with some drugs such as methyldopa and amiodarone in the occurrence of liver cirrhosis. Further studies including more patients and other risk factors are required in future, to validate these findings and to assess the role of metabolic syndrome in the occurrence of liver cirrhosis.

\section{EACPT-1184}

Patients knowledge and attitudes towards the yellow card scheme in greece

G. Papazisis ${ }^{*}{ }^{1}$, A. Pappa $^{2}$, D. Serefidou ${ }^{3}$, L. Spilioti ${ }^{3}$, G. Koutsouki ${ }^{3}$, N. Kostakis $^{3}$, V. Nikolopoulou ${ }^{4}$, A. Milonas ${ }^{4}$, C. Pourzitaki ${ }^{1}$, D. Kouvelas ${ }^{1}$ ${ }^{1}$ Clinical Pharmacology, School of Medicine, Aristotle University of Thessaloniki, Thessaloniki, ${ }^{2}$ EL.E.AN.A. - Hellenic League Against Rheumatism, Athens, ${ }^{3}$ School of Medicine, Aristotle University of Thessaloniki, Thessaloniki, ${ }^{4}$ Ministry of Health, Athens, Greece

\section{Pharmacovigilance}

Introduction: Although spontaneous adverse drug reactions (ADRs) reportingis a cornerstone of pharmacovigilance, under-reporting remains a major worldwide problem and unfortunately, only the minority of the reports are coming from patients. Studies estimating patients knowledge about the ADRs spontaneousreporting systemsand their attitudes towards reporting are lacking.

Objectives: The aim of ourstudy was to determine knowledge and attitudes of patients towards theADRs reporting system, the Yellow Card Scheme, in Greece.

Methods: A sample of patients completed an anonymous, self-administered, web-based survey assessing knowledge and attitudes towards the yellow card scheme in Greece. The questionnaire included 18 questions and was sent via the patient organization EL.E.AN.A. (Hellenic League Against Rheumatism), thus, most of the patients answering the survey were suffering from chronic rheumatic diseases.

Results: 305 patients completed the questionnaire. $85.2 \%$ were women with a mean age of 49.6 years (17-82). Atotal $67 \%$ of the patients reported that they hadexperienced a severe ADR, and this ADR resultedin treatment discontinuation in $58 \%$ of them. $26.6 \%$ required medical treatment for the ADR, while $13.5 \%$ required hospitalization because of the ADR. The ADR was considered as life-threatening by $8.2 \%$ of thepatients. The vast majority $(71 \%)$ of the affected patients had reported this ADR to their physician whereas only 5\% reported it to their pharmacist. Only $20 \%$ of the patients knew what the Yellow Card Scheme is, while 39\% of them was informed about it by their doctors, $32 \%$ from the internet and $19.3 \%$ by a relative or friend. Lastly, only $7 \%(\mathrm{n}=16)$ of the patients has submitted a Yellow Card to the Greek agency, and just $2 \%$ of them more than once. The majority of the patients stated that it was relative/extremely difficult to complete the form. Finally, $95 \%$ considers the official reporting scheme very important.

Summary / Conclusions: Although the majority of the patients had experienced severe ADRs,most of them were not aware of the YCSand the vast majority hasn't used this official way of reporting. It is important to educate and encourage patients to voluntary report ADRs but it is also important to strengthen physicians education in order to promote pharmacovigilance and safer use of medicines.

\section{EACPT-1199}

Case reports of non-arteritic anterior ischaemic optic neuropathy with phosphodiesterase type 5 inhibitors: a systematic review A. Penedones ${ }^{1,2}$, F. Batel Marques ${ }^{*} 1,2$

${ }^{1}$ Laboratory of Social Pharmacy and Public Health, School of Pharmacy, University of Coimbra, ${ }^{2}$ Association for Innovation and Biomedical Research on Light and Image (AIBILI), Coimbra, Portugal

\section{Pharmacovigilance}

Introduction: The development of non-arteritic anterior ischaemic optic neuropathy (NAION) has been described to phosphodiesterase type 5 (PDE5) inhibitors. Three safety alerts were issued based on spontaneous reports. There is a pharmacologic rationale for the association between NAION and PDE5 inhibitors. A previous systematic review and metaanalysis did not provide evidence on the association between NAION and PDE5 inhibitors exposure.

Objectives: The aim of this systematic review was to identify and characterize the cases reported in the literature describing such association. Methods: The MEDLINE, EMBASE, Cochrane Library, Google Scholar, Web of Science, and Open Grey databases were searched, since its inception until November, 2018. Data on all case reports describing the development of NAION associated with PDE5 inhibitors was extracted. Data were analysed using descriptive statistics.

Results: A total of 52 case reports were identified ( $90 \%$ males, median of age 54 years [ 7 months-76 years]). Twelve (23\%) patients did not have risk factors to develop NAION. Hypertension $(n=16 ; 31 \%)$ was the most described risk factor. Forty $(77 \%)$ patients were treated for erectile dysfunction. Sildenafil was the PDE5 inhibitor most reported ( $n=47 ; 90 \%)$. A regular administration of PDE5 inhibitors was observed in 25 (48\%) case reports. Among those, five patients have doubled or tripled the dose of PDE5 inhibitors. The majority of the cases reported the development of NAION only in one eye $(n=39 ; 75 \%)$.

Summary / Conclusions: A plausible mechanism can explain the development of NAION associated with PDE5 inhibitors exposure. A systematic review and meta-analysis of the available evidence did not support this association. The number of case reports identified in this systematic review was very low. In the light of these findings, it is not possible to obtain robust evidence. A close monitoring of PDE5 inhibitors is foreseen of great value.

\section{EACPT-1233}

Medication errors and near miss events in the finnish tertiary care hospitals

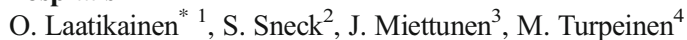

${ }^{1}$ Pharmacology and toxicology, University of Oulu, ${ }^{2}$ Oulu university hospital, ${ }^{3}$ center of life course health research and medical research center oulu, ${ }^{4}$ research unit of biomedicine and medical research center oulu, Oulu university hospital and university of Oulu, Oulu, Finland

\section{Pharmacovigilance}

Introduction: Medication errors and adverse drug events are leading causes of patient harm. As a result, voluntary reporting systems have become more common in health care all over the world. The value of voluntary global pharmacovigilance reporting systems is well recognized, but they are focused on adverse effects/events related to drug products instead of medication errors. The clinical validity and applicability of hospital-based voluntary reporting systems are far less established.

Objectives: Describe

- Medicine groups commonly involved in the reports

- The types of incidents, risk classification and patient outcome assessed in the reports 
Methods: The study was conducted retrospectively using 90352 tertiary care medication safety incident reports from 2007 to 2017. Information was gathered about the nature of the event, occupation of the reporter, patient outcome, risk category, and type of incident. Medicines involved were categorized by ATC classes using search terms created out of every brand name and active substance name currently on the Finnish market. Results: Majority of the reported 90352 events resulted in no harm or minor harm to the patient. In $4.5 \%$ of the reports, patient outcome was assessed moderate or significant. The most common error types were administration error, documenting error, and dispensing error. Medicines were identified from 83427 reports. Almost one third $(27 \%)$ of medicines were from ATC class $\mathrm{N}$ (Nervous system). Most common subcategories in these reports were analgesics and psycholeptics.

Majority of events caused no harm or minor harm to the patient.

Most common events were administration errors, dispensing errors, and documentation errors.

Summary / Conclusions: Voluntary reporting systems provide valuable information on medication errors occurring in hospitals. N (nervous system) class medicines were most often involved in the reports covering $27 \%$ of all reports made. Majority of incidents resulted in no harm or minor harm to the patient. $\mathrm{N}$ class medicines did not cause more severe outcomes than medication errors involving drugs from other ATC groups. Although the percentage of more serious events is low, the amount of patients annually affected by them is relatively high. Further research is needed to specify the medicine-specific risk and types of events typical to medicines most commonly involved in medication errors in healthcare units.

\section{EACPT-1268}

Heterogeneity between odds ratios and reporting odds ratios C. Khouri* ${ }^{*}, 2,3$, C. petit ${ }^{4}$, M. Tod ${ }^{5,6}$, M. lepelley ${ }^{1}$, B. Revol $^{1}$, M. Roustit $^{2,3}$, J.-L. Cracowski ${ }^{2,3}$

${ }^{1}$ Pharmacovigilance, ${ }^{2}$ Clinical Pharmacology Department INSERM CIC1406, Grenoble Aples University Hospital, ${ }^{3}$ UMR 1042-HP2, University Grenoble Alpes, ${ }^{4}$ Pharmacovigilance, Grenoble Alpes University Hospital, Grenoble, ${ }^{5}$ Pharmacie Hôpital de la Croix Rousse, Hospices Civils de Lyon, ${ }^{6}$ EMR 3738, Ciblage Thérapeutique en Oncologie,, Faculté de Médecine et de Maïeutique Lyon-Sud Charles Mérieux,Université Claude Bernard Lyon 1, lyon, France

\section{Pharmacovigilance}

Introduction: Disproportionality analyses (DAs) are widely used for safety signal detection in pharmacovigilance spontaneous reporting systems databases. While DAs are not intended to provide risk quantification, several studies recently suggested a correlation between the measures of disproportionality and adverse drug reactions (ADRs) risks.

Objectives: In this context, we tried to determine whether DAs correlate with the risk of ADRs, estimated from meta-analyses. We further aimed at testing the influence of different bias minimization strategies and correlations methods.

Methods: We searched all meta-analyses published in major medical journal (NEJM, JAMA, Plos Medicine, BMJ, JAMA Internal Medicine, Lancet and Annals of Internal Medicine). We extracted Odds Ratios (ORs) for specific ADR, used as a reference. We calculated the DAs for corresponding ADRs from the WHO pharmacovigilance database, expressed as Reporting Odds Ratio (RORs), and correlated RORs to ORs. We tested five bias minimization strategies (standardization on time on the market, region of reporting, therapeutic area, including concomitant reports, excluding competitors) and 2 correlation methods (with lower boundary of confidence intervals and robust to outliers). We also calculated the relative bias of ROR compared to OR and adapted the measures of vibration of effect to the disproportionality analyses. Lastly, we correlated the p-values in a regression analysis with the ADR characteristics.
Results: Among the retrieved 425 meta-analyses, only 5 assessed the risk of a specific ADR. Those 5 meta-analyses displayed a panel of 13 heterogeneous ADRs, ranging from dose-related ADR such as hypoglycemia with antidiabetics, to rare and unexpected ADR such as suicidal ideation with antidepressant. We found a significant correlation for 6 out of the 13 ADRs studied in the primary analysis but the relative bias of ROR against OR was extremely important. The presence of a protopathic bias was significantly associated with worst correlations. Importantly, this study highlighted the wide vibration of effect associated with DAs.

Summary / Conclusions: This study provides evidence that a correlation could exists between ORs and RORs for diverse ADR risks. In contrast, it also highlights the large vibration of effect associated with DAs, opening the door of selective reporting. These data emphasize the importance of protocol pre-registration and the presentation of results across different methods.

\section{Oral Presentation}

\section{EACPT-1285}

Pulmonary arterial hypertension associated with protein kinase inhibitors: a pharmacovigilance-pharmacodynamic study

C. Khouri ${ }^{*}$ 1, 2, 3 , L. Cornet ${ }^{4}$, M. Roustit ${ }^{2}, 3$, C. Guignabert ${ }^{5,6,7}$, M.-C.

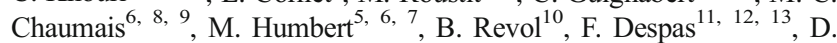
Montani $^{5,6,7}$, J.-L. Cracowski ${ }^{3,14}$

${ }^{1}$ Pharmacovigilance Unit, ${ }^{2}$ Clinical Pharmacology Department INSERM CIC1406, Grenoble Alpes University Hospital, ${ }^{3}$ UMR 1042-HP2, University Grenoble Alpes, ${ }^{4}$ Pharmacovigilance Unit, Grenoble Aples University Hospital, Grenoble, ${ }^{5}$ Service de Pneumologie, Hôpital Bicêtre, AP-HP, Le Kremlin-Bicêtre, ${ }^{6}$ Inserm UMR S 999, Hôpital Marie-Lannelongue, Le Plessis-Robinson, ${ }^{7}$ Université Paris-Sud, Faculté de Médecine, Université Paris-Saclay, ${ }^{8}$ Service de Pharmacie, Hôpital Bicêtre, AP-HP, ${ }^{9}$ Université Paris-Sud, Faculté de Pharmacie, Université Paris-Saclay, Le Kremlin-Bicêtre, ${ }^{10}$ Pharmacovigilance, Grenoble Aples University Hospital, Grenoble, ${ }^{11}$ Medical and clinical pharmacology unit, CHU Toulouse university hospital, ${ }^{12}$ INSERM UMR1027 (French National Institute of Health and Medical Research), University of Toulouse III Paul-Sabatier, ${ }^{13}$ INSERM CIC 1436, Toulouse clinical investigation center, Toulouse, ${ }^{14}$ Clinical Pharmacology Department INSERM CIC1406, Grenoble Aples University Hospital, Grenoble, France

\section{Pharmacovigilance}

Introduction: The pathophysiology of pulmonary arterial hypertension $(\mathrm{PAH})$ induced by protein kinase inhibitors (PKI) remains unclear.

Objectives: To gain knowledge into this rare and severe pathology we performed a study combining a pharmacovigilance approach and the pharmacodynamics properties of PKI.

Methods: A disproportionality analysis on the WHO pharmacovigilance database using the Reporting Odds Ratio (ROR) and 95\% confidence interval was first performed. Then, we identified the most relevant cellular targets of interest through a systematic literature review and correlated the pharmacovigilance signals with the affinity for the different PKI. We further performed a hierarchical cluster analysis to assess patterns of binding affinity. Results: A positive disproportionality signal was found for dasatinib, bosutinib, ponatinib, ruxolitinib and nilotinib. Five nonreceptor protein kinases significantly correlate with disproportionality signals: $\mathrm{c}-\mathrm{src}(\mathrm{r}=0.79$ and $\mathrm{p}=0.00027)$, c-yes $(\mathrm{r}=0.82$ and $\mathrm{p}=0.00015)$, Lck $(\mathrm{r}=0.81$ and $\mathrm{p}=0.00046)$, Lyn $(\mathrm{r}=0.80$ and $\mathrm{p}=0.00036)$, all belonging to the SRC protein kinase family; and TEC $(r=0.85$ and $p=0.00006)$. Kinases of the BMP signaling pathway also seem to play a role in the pathophysiology of PKI-induced PAH. Interestingly, dasatinib affinity profile seems different from that of other PKIs in the cluster analysis.

Summary / Conclusions: The study highlights potential role of SRC protein kinases family and TEC in PAH induced by PKI. This approach 
combining pharmacovigilance and pharmacodynamics data allowed us to generate some hypothesis about the pathophysiology of the disease, however the results have to be confirmed by further studies.

\section{EACPT-1299}

Observational, descriptive and retrospective study of the assessment of drug's adverse reactions in a spanish tertiary hospital C. Pérez Ingidua ${ }^{*} 1,2$, L. Laredo Velasco ${ }^{1,3}$, N. Rodríguez Galán ${ }^{1}$, A. Portolés Pérez ${ }^{1,2,3}$, C. M. Elvira Martínez ${ }^{4,5}$, E. Vargas Castrillón ${ }^{1,3}$ ${ }^{1}$ Clinical Pharmacology, Hospital Clínico San Carlos, ${ }^{2}$ Instituto de Investigación Sanitaria del Hospital Clínico San Carlos (IdISSC), ${ }^{3}$ Pharmacology, Universidad Complutense, ${ }^{4}$ Admitting Department, Hospital Clínico San Carlos, ${ }^{5}$ Universidad Complutense, Madrid, Spain

\section{Pharmacovigilance}

Introduction: Adverse drug reactions (ADRs) are one of the ten main causes of mortality in the world, as well as a cause of hospital admissions or prolongation of hospitalization. Previous studies carried out in our center, showed an incidence close to $10-11 \%$.

Objectives: To assess the incidence of adverse drug reactions that occurred in Clínico San Carlos Hospital between January to July 2018, as well as the causality, severity and preventability of such reactions.

Methods: An observational, descriptive and retrospective study was performed. A total of 281 patients hospitalized in the different hospital departments were included. Descriptive statistic was used to summarize and analyze the demographic characteristics of the subjects and of the ADRs. The approval of Clínico San Carlos Ethical Committee was obtained.

Results: An incidence of $8,9 \%$ ADRs was observed between January to July 2018 .

The average length of hospitalization was 5 days. In the same way, the average number of drugs per patient during hospitalization was 11 .

The proportion of patients with at least one ADR was $7,1 \%$, and the most frequently ADRs were gastrointestinal $(24 \%)$ and metabolic alterations $(16 \%)$. The main drugs involved were furosemide (12\%), risperidone $(12 \%)$, acenocoumarol $(8 \%)$ and acetylsalicylic acid (8\%).

According to the Venulet Criteria, $56 \%$ of the reactions were considered moderate intensity, $20 \%$ mild intensity and $24 \%$ severe intensity. However, according to the Criteria of the SEFV (Sistema Español de Farmacovigilancia), $44 \%$ of the ADRs were considered non-serious and $56 \%$ considered of serious intensity.

The causality of the ADRs according to the Method of Naranjo et al. showed $72 \%$ of the ADRs had a "likely" causality score, $16 \%$ "possible" causality score and $12 \%$ "certain" causality score. Using the method of Karch-Lasagna modified, one reaction (4\%) was considered "conditional / unclassified", $60 \%$ "likely", $12 \%$ as "possible" and $24 \%$ as "definitive". According to the Hallas et al. Method to analyze the preventability of the ADRs, 21 were considered "not preventable" and 4 "probably preventable". Summary / Conclusions: ADRs continue to be an important cause of morbidity in our center and a significant proportion of ADRs detected were possibly preventable $(16 \%)$

\section{EACPT-1304}

The effect of pharmacological antipyresis on mortality in patients with sepsis

R. Nannan Panday ${ }^{*}$, M. Schinkel ${ }^{1}$, N. Alam ${ }^{1}$, P. Nanayakkara ${ }^{1}$

${ }^{1}$ Section Acute Medicine, Department of Internal Medicine, Amsterdam UMC, Location VU University Medical Center, Amsterdam, Netherlands

\section{Pharmacovigilance}

Introduction: There are no accepted international guidelines on the use of paracetamol for fever control in sepsis. Fever is important for immune function and it therefore seems counterintuitive to administer antipyretic drugs in sepsis.

Objectives: The aim of this study is to determine whether antipyretic medication influences mortality in patients with sepsis.
Methods: We performed a sub-analysis of the Prehospital Antibiotics Against Sepsis (PHANTASi) trial to compare patients with sepsis who received paracetamol in the ambulance and patients who did not. The primary outcome was 28-day mortality. Outcomes were adjusted for sex, age and sepsis severity. In addition we compared the 28-day mortality for hypothermic and hyperthermic patients, to provide better insights into the effects of body temperature on mortality in sepsis.

Results: 2528 patients were included in the primary outcome analysis: 254 patients received paracetamol in the ambulance and 2274 did not. There was no significant difference in 28-day mortality between the paracetamol and non-paracetamol groups (OR: 0.63 [95\% CI 0.35 to 1.13]; $\mathrm{p}=0.12$ ). We also established that paracetamol administration caused a significantly larger decrease in temperature compared to placebo $\left(0.26^{\circ} \mathrm{C}(95 \% \mathrm{CI}[0.15-0.36] ; \mathrm{p}<0.001)\right.$. Furthermore, hyperthermic patients had lower mortality rates when compared to hypothermic patients (OR: 0.20 [95\% CI: 0.11-0.37]; p<0.001). When different cut-off values in temperature were examined, patients with higher body temperatures had consistently lower mortality rates than patients with lower body temperatures.

Summary / Conclusions: Our results suggest that administration of paracetamol does not influence mortality rates in patients with sepsis, regardless of the initial body temperature.

\section{EACPT- 1325}

Drug-induced livery injury in patients with underlying chronic hepatic diseases

I. Medina-Caliz ${ }^{1}$, J. Sanabria-Cabrera ${ }^{*}$, M. García-Cortés ${ }^{1}$, M. RoblesDiáz ${ }^{1}$, C. Stephens ${ }^{1}$, A. González-Jiménez ${ }^{1}$, C. Parra-Martínez ${ }^{1}$, L. Sanz-Villanueva $^{1}$, A. Rodriguez-Nicolás ${ }^{1}$, A. Ortega-Alonso ${ }^{1}$, M. Jiménez-Pérez ${ }^{2}$, R. González-Grande ${ }^{2}, \mathrm{H}$. Hallal ${ }^{3}$, M. Prieto ${ }^{4}$, I. Conde $^{5}$, C. Fernández ${ }^{6}$, A. Castiella ${ }^{7}$, G. Soriano ${ }^{8}$, A. Giráldez ${ }^{9}$, M. Cabello $^{1}$, R. J. Andrade ${ }^{1}$, M. I. Lucena ${ }^{1}$

${ }^{1}$ UGC Aparato Digestivo, Instituto de Investigación Biomédica de Málaga (IBIMA), Hospital Universitario Virgen de la Victoria, Universidad de Málaga, CIBERehd, ${ }^{2}$ UGC Aparato Digestivo, Complejo Hospitalario Regional de Málaga, Malaga, ${ }^{3}$ UGC Aparato Digestivo, Hospital General Universitario J.M. Morales Meseguer, Murcia, ${ }^{4}$ UGC Aparato Digestivo, Hospital Universitari I Politècnic la $\mathrm{Fe},{ }^{5} \mathrm{UGC}$ Aparato Digestivo, Hospital Universitari I Politècnic la Fe, Valencia, ${ }^{6}$ UGC Aparato Digestivo, Complejo Hospitalario de Especialidades Torrecárdenas, Almería, ${ }^{7}$ UGC Aparato Digestivo, Hospital Universitario Donostia-Donostia Unibertsitate Ospitalea, Donostia/San Sebastián, ${ }^{8}$ UGC Aparato Digestivo, Hospital de la Santa Creu I Sant Pau, Barcelona, ${ }^{9}$ UGC Aparato Digestivo, Hospital Universitario Virgen del Rocío, Sevilla, Spain

\section{Pharmacovigilance}

Introduction: DILI is a multifactorial disorder affected by pharmacologic, environmental and host risk factors.

Objectives: In this study, we aimed to determine the influence of underlying hepatic diseases on severity and outcome of the DILI episode.

Methods: We compared demographics, clinical and biochemical parameters in 843 DILI patients included in the Spanish DILI Registry according to the presence or absence of underlying liver diseases

Results: Fifty-three (6\%) DILI cases present underlying hepatic disease, mainly chronic viral hepatitis $(n=29)$, cirrhosis $(n=12)$, and nonalcoholic fatty liver disease $(n=7)$. Men were more frequent in DILI patients with underlying hepatic conditions compared with those with underlying conditions $(70 \%$ vs $51 \%, p=0.009)$. All other demographic and clinical characteristics analyzed were very similar in the two groups. Hepatocellular cases predominate in both groups ( $51 \%$ vs $58 \%, p=0.5$ ). In the liver profile at DILI onset, the mean value of ALT was lower in those with underlying hepatic disease ( $14 \mathrm{xULN}$ vs $19 \mathrm{xULN}, p=0.5$ ). A similar trend was found for ALP ( $2 \times U L N$ vs $2.2 \times U L N, p=0.5)$, although none of the differences reached statistical significance. There were differences in the CIOMS 
causality score with fewer cases of highly probable DILI (21\% vs $34 \%$ ) and many cases of probable DILI (61\% vs $51 \%$ ) and possible cases (18\% vs $15 \%)$ in individuals with previous hepatic disease. More severe and fatal cases were found among those with underlying hepatic disease, especially death due to acute liver failure was more frequent in this group (7.6\% vs $1.8 \%, p=0.02)$. Most frequent drug class in this group were antiinfectives (antituberculous drugs $(\mathrm{n}=11)$, amoxicillin-clavulanate $(\mathrm{n}=4))$ and statins (atorvastatin $(n=2)$, fluvastatin $(n=2)$ and rosuvastatin $(n=1)$ )

Summary / Conclusions: Fatal DILI cases due to acute liver failure were more frequent in cases with underlying liver diseases. The CIOMS score should take into consideration the differentiation between alternative causes and underlying hepatic diseases.

Funding: AEMPS, FEDER (PI-0310-2018, PI-0285-2016, PI-02742016, PI18-00901, PI18/01804. MRD holds a "Joan Rodes" (JR16/00015) and JSC is recipient of a "Rio-Hortega" (CM17/00243) research contract from the National Health System, ISCIII. SCReN and CIBERehd are funded by Instituto de Salud Carlos III. COST Action CA17112

\section{EACPT-1352}

¿what about admission by adverse reactions in our hospital?

D. M. Campodónico* ${ }^{1}$, D. Romero Palacian ${ }^{1}$, M. J. Hernandez Martinez ${ }^{1}$, G. Mejia Abril ${ }^{1}$, F. Abad Santos ${ }^{1}$

${ }^{1}$ Clinical Pharmacology, Hospital Universitario de La Princesa ( University Hospital of La Princesa ), Madrid, Spain

\section{Pharmacovigilance}

Introduction: As a Clinical Pharmacology Department, we consider clinically important the detection of adverse reactions that require urgent medical assistance, especially those that involve admission to hospital .

Objectives: To assess the prevalence of hospital admission related to adverse drug reactions (ADRs) in our third-level teaching hospital.

To analyse and describe the characteristics of patients who have experienced an adverse reaction to drugs requiring admission.

Methods: This is a cross-sectional study perform from January to December 2018. The information was collected from the electronic clinical records.

Patients who were urgently hospitalized entered the study, discarding the programmed hospital admissions.

A descriptive analysis of suspicious drugs, pharmacological group, sex, age, pharmacological group, and type of adverse reaction was performed.

Results: There were 11767 admissions in 2018 in our hospital and 8651 of them were urgent. An adverse reaction was the reason for admission in 189 patients (2.2\% of urgent admissions). 57,2\% (107) are women and 42.8\% (80) men. About adverse reactions and drugs involved we found:

Alterations of coagulation (Hemorrhages, haematoma, etc.) 62 (32.80\%): Platelet inhibitors $30.48 \%$, vitamin $\mathrm{K}$ antagonists $35.36 \%$, low molecular weight heparins (LMWH) $7.31 \%$, new oral anticoagulants $11 \%$. Others $15.85 \%$.

Hydro-electrolytic alterations 16 ( 8.47\%): Diuretics 40.75\%, ACE inhibitors/ ARAII: $14.81 \%$. antidepressants $3.71 \%$, anti-epileptic $11.11 \%$, other $26.62 \%$. Infections 16 (8.47\%): Antibiotics 68,42\%, immunosuppressive $26.31 \%$, antineoplastic agents $5.27 \%$

Hematological alterations (neutropenia, thrombocytopenia, eosinophilia...) 15 (7,94\%): antineoplastic agents; Inmunosuppressive $16.7 \%$. Other $16.67 \%$.

Toxicoderma (DRESS, Steven-Johnson, local reactions...) 14 (7,41\%): Antibiotics $23.52 \%$; analgesics: $17.64 \%$, Otrhers 58.82\%

Renal Failure 8 (4,23\%): Diuretics $27.27 \%$, antihypertensive $27.27 \%$, other $46 \%$.

Severe hepatotoxicity 7 (3,70\%) Antibiotics 60\%, other $40 \%$.

Summary / Conclusions: The adverse reactions justify more than $2 \%$ of urgent admissions. The reduction of ADR must be a priority objective in any health organization. So, it is necessary to establish systems that allow the prevention, early detection and treatment.
EACPT- 1356

Medication errors

E. Tärning ${ }^{*}{ }^{1}$, I. Jacobsson ${ }^{1}$, H. Lövborg ${ }^{1,2}$

${ }^{1}$ Department of Clinical Pharmacology,, County Council of Östergötland/ Region Östergötland,, ${ }^{2}$ Division of Drug Research,, Faculty of Health Sciences, Linköping University, Linköping, Sweden

\section{Pharmacovigilance}

Introduction: Drugs are the most common treatment in modern health and medical care. Most of the drug treatment is of course beneficial to the patient, but there are always risks or side effects that may cause injury to the patient and sometimes even death. A medication error is any error that occurs at any point in the medication use process. To report medication errors in healthcare, an IT based support is used.

Objectives: The aim of this study was to identify the causes of the medication errors from different departments and compile them and follow up on a regional level.

Methods: During 2018 there were 1372 reported medication errors. All reports were analyzed and categorized based on where in the medication process the error occurred. Categories were ordering, prescription, preparation, administration, and distribution.

Results: : The most frequent type of error was incorrect prescription (36\%), followed by incorrect administration (25\%). Distribution, preparation and ordering was reported in $16 \%, 14 \%$ and $4 \%$ respectively. Fortunately, most events caused no serious harm. One major problem is that there are several drug lists that do not match and therefore create confusion both to doctors and nurses that lead to wrong prescription and administration.

There were three common causes to the errors; lack of time and understaffing, lack of education and lack of communication. Regarding communication, shortcomings were seen intraprofessionally and interprofessionally as well as between departments and units. Examples of error types, causes and interventions to reduce specific errors will be presented.

Summary / Conclusions: The most common causes of medication errors are an incorrect or not up-to-date prescription and lack of follow-up. When several healthcare caregivers cooperate on a patient's drug treatment, it places high demands on clarity in communication and information transfer as well as adherence to certain routines., Drug-related injuries remain one of three priority areas within the county council. The increased knowledge of similar events provides opportunities to propose measures and improve routines on a regional level.

\section{EACPT- 1360}

Severe hypersensitivity reactions associated with iron (iii) isomaltoside and ferric carboxymaltose. Analysis of eudravigilance database

P. Manita ${ }^{*}{ }^{1}$, U. Lertxundi ${ }^{1}$, I. Palacios ${ }^{2}$, M. Cardenas ${ }^{3}$, M. Garcia ${ }^{4}$, C. Aguirre $^{4}$

${ }^{1}$ Pharmacy, Araba Mental Health Network, Vitoria-Gasteiz, ${ }^{2}$ Pharmacy, Galdakao-Usansolo Hospital, ${ }^{3}$ Pharmacy, Barrualde integrated Health Care Orgaization. Galdakao-Usansolo Hospital, ${ }^{4}$ Basque Pharmacovigilance Unit, Galdakao-Usansolo Hospital, Galdakao, Spain

\section{Pharmacovigilance}

Introduction: Hypersensitivity reactions (HSRs), especially severe anaphylaxis due to intravenous iron are a matter of concern. From January 2017 to June 2017, iron (III) isomaltoside was the only high dose intravenous iron available in the Basque Public Health Care System. In that period of time 691 patients were treated, resulting in a total of 24 HSRs reported to the Basque Pharmacovigilance Unit (BPU), 23 of which were considered severe. In three cases the result was cardiorespiratory arrest. On the contrary, no cases were reported for ferric carboxymaltose in the 
previous three year period when it was used (2014-2017), where a total of 1350 patients were treated.

Objectives: Considering the number and the severity of the HSRs reported to the BPU, we aimed to determine if severe HSRs were reported more frequently for iron (III) isomaltoside than for ferric carboxymaltose to the European Pharmacovilance Database (EudraVigilance).

Methods: Spontaneously reported severe HSRs were obtained from EudraVigilance using the MedDRA Preferred Terms "anaphylactic reaction", "anaphylactic shock", "anaphylactoid reaction" and "anaphylactoid shock" until $8^{\text {th }}$ of March 2019.

To assess the association between HSR cases and each iron product, we calculated the overall reporting odds ratio (ROR) comparing each high dose iron with all the rest of the drugs included in EudraVigilance and the relative ROR, by comparing each high dose iron with all the rest of available intravenous irons.

Results: A total of 202 HSRs were reported for iron (III) isomaltoside. The overall ROR (IC 95\%) was: 12.6 (11.04-14.5), while the relative ROR IC $(95 \%)$ vs all the rest of intravenous irons: 1.90 (1.64-2.21).

A total of 446 HSRs were reported for ferric carboxymaltose. The overall ROR (IC 95\%) was: 6.9 (6.2-7.6), while the relative ROR IC (95\%) vs all the rest of intravenous irons: 0.9 (0.8-1.03).

Summary / Conclusions: HSRs were frequently reported with both iron products in Eudravigilance. However, among all intravenous irons, the risk seems higher for iron (III) isomaltoside.

\section{EACPT-1372}

Tramadol use in Denmark, Norway, and Sweden: A populationbased study

A. B. Schelde ${ }^{*}$, A. M. S. Sørensen ${ }^{1}$, M. G. Hinds $\varnothing^{2}$, M. B. Christensen ${ }^{1}$, ${ }^{3,4}$, E. Jimenez-Solem ${ }^{1,5}$, R. Eriksson ${ }^{1,6}$

${ }^{1}$ Department of Clinical Pharmacology, Copenhagen University Hospital, Bispebjerg and Frederiksberg Hospital, ${ }^{2}$ Department of Endocrinology, Copenhagen University Hospital, Hvidovre Hospital, ${ }^{3}$ Department of Biomedical Sciences, University of Copenhagen, ${ }^{4}$ Clinical Metabolic Physiology, Steno Diabetes Copenhagen, Copenhagen University Hospital, Gentofte Hospital, ${ }^{5}$ Institute of Clinical Medicine, University of Copenhagen, ${ }^{6}$ Department of Disease Systems Biology, Novo Nordisk Foundation Center for Protein Research, Faculty of Health and Medical Sciences, University of Copenhagen, Copenhagen, Denmark

\section{Pharmacovigilance}

Introduction: Concern has been raised in Danish media regarding tramadol's addictive properties. To assess whether this is of public health concern, it is important to examine the prevalence of tramadol users and to analyze utilization patterns and trends.

Objectives: To examine the prevalence and characteristics of tramadol users in Denmark, Norway, and Sweden.

Methods: The cohort constitutes the entire population of Denmark, Norway, and Sweden between 2007 and 2015. Data was gathered from the national prescription databases to assess the prevalence of redeeming a prescription and sold defined daily doses (DDDs) of tramadol and other opioids.

Results: The prevalence of tramadol users increased in Denmark from 45 to 52 per 1,000 residents, and in Norway from 20 to 41 per 1,000 residents. In Sweden, the prevalence decreased from 36 to 17 per 1,000 residents. In comparison, the prevalence of other opioid users decreased in Denmark and Norway, and increased in Sweden. The average tramadol DDD per treated patient remained fairly constant in Norway, while it increased in Denmark and Sweden. In Denmark and Norway, women consistently received a higher DDD than men. In Sweden, both genders received close to the same DDDs. There was a general trend that the prevalence of tramadol users increased with age in all three countries. The amount of sold tramadol and other opioids combined per 1,000 residents was highest in Denmark.

Summary / Conclusions: The prescription patterns of tramadol and other opioids differed between the three countries between 2007 and 2015. The prevalence of tramadol users and amount of total redeemed opioids were highest in Denmark. The high prevalence of tramadol users could be of public health concern, and should be the basis for safety studies.

\section{EACPT-1366}

Accuracy of drug hepatotoxicity potential classifications in assessing severity related to drug-induced liver injury (dili)

L. Sanz-Villanueva ${ }^{1}$, C. Parra-Martinez ${ }^{1}$, I. Medina-Caliz ${ }^{1}$, J. SanabriaCabrera $^{*}{ }^{1}$, M. Robles-Diaz ${ }^{1}$, A. Ortega-Alonso ${ }^{1}$, A. Gonzalez-Jimenez ${ }^{2}$, J. Gasca ${ }^{1}$, R. Sanjuan-Jimenez ${ }^{1}$, M. Garcia-Cortes ${ }^{1}$, M. I. Lucena ${ }^{1}$, R. J. Andrade $^{1}$

${ }^{1}$ UGC Aparato Digestivo y Servicio de Farmacología Clínica, Instituto de Investigación Biomédica de Málaga (IBIMA), Hospital Universitario Virgen de la Victoria, Universidad de Málaga, CIBERehd, ${ }^{2}$ UGC Aparato Digestivo y Servicio de Farmacología Clínica, Instituto de Investigación Biomédica de Málaga (IBIMA), Hospital Universitario Virgen de la Victoria, Universidad de Málaga, , Málaga, Spain

\section{Pharmacovigilance}

Introduction: Hepatotoxicity potential is a key factor in the development of safertherapeutic treatments. In 2016, a new categorization (LiverTox) based on number of published DILI case reports (A: $\geq 50$ reports; B: $12-$ 49; C: 4-11; D: 1-3; E: 0) was presented (Björnsson \& Hoofnagle, 2016). Objectives: We aimed to compare this categorization with data obtainedfrom the Spanish DILI Registry to determine the accuracy of this categorization in assessing severity related to DILI.

Methods: We applied the LiverTox 5-catogory classification to 191 causative drugs from 867 cases enrolled in the Spanish DILI Registry. Two categories were formed, that one with causative drugs which includes well-known DILI cases (categories $\mathrm{A}+\mathrm{B}$ ) and other with the DILI potential drugs that was not adequately documented (categories $\mathrm{C}+\mathrm{D}$ ). We also collected information on drugs causing acute liver failure (ALF) in the Spanish DILI Registry and drug-labelling information from The Liver Toxicity Knowledge Base (LKTB).

Results: Of the 867 DILI cases included in the Spanish DILI Registry, 31 ALF cases were found caused by 19 different drugs, such as ibuprofen, antiTBC, carbamazepine (category A), levofloxacine (category B), orlistat, nefazodone (category $\mathrm{C}$ ), and bicalutamide (category D). Cases caused by the $\mathrm{C}+\mathrm{D}$ drugs presented higher severity with more ALF cases compared to cases caused by the A+B drugs ( $4.9 \%$ vs $3.2 \%, p=0.047$ ). Both the Spanish DILI Registry and the LiverTox categorization contained $17 \%$ of A drugs with black box warnings, $2.4 \%$ and $6.6 \%$ in category $\mathrm{B}, 7.7 \%$ and $6.3 \%$ in C, respectively; and $3.2 \%$ of the LiverTox D drugs.

Summary / Conclusions: Classification of drug hepatotoxicity potential based on numbers of published case reports can be misleading. Further elements such as DILI frequency, severity including number of cases progressing to ALF, and causality should also be considered together with information on liver safety regulatory measures in order to provide a better estimate of its hepatotoxicity potential.

Funding: AEMPS, FEDER (PI-0310-2018, PI18-00901, PI18-01804), SCREN, CIBERehd, COST Action CA-17112. Research contract from the Spanish National Health System, ISCIII (JR16/00015, CM17/00243).

\section{EACPT-1368}

Clozapine associated pneumonia. A disproportionate analysis of vigibase

E. J. Sanz ${ }^{*}{ }^{1,2}$, C. De las Cuevas ${ }^{3}$, A. Aldea ${ }^{4}$

${ }^{1}$ Clinical Pharmacology, University Hospital of the Canary Islands, ${ }^{2}$ Dept. of Physical Medicine and Pharmacology, ${ }^{3}$ Internal Medicine \& Psychiatry, Univeristy of La Laguna, La Laguna, ${ }^{4}$ Clinical Pharmacology, IMIM. Hospital del Mar, Barcelona, Spain

\section{Pharmacovigilance, Psychopharmacology}

Introduction: There are several reports on the association of (specially high doses of) clozapine and the ocurrence of Penumonia in those 
patients. We have used Vigibase (Uppsala Monitoring Centre) in order to analyze the apparent disproportionality of ICR (Individual Case Reports) spontaneously submitted to the WHO Database for Pharmacovigilance. Objectives: To analyze the ICR reported to the global WHO database for Pharmacovigillance in order to asses the disporportionality of reports of Pneumonia related to the use of Clozapine and other antipsychotics.

Methods: Vigilyze was used to elicit the association between drugs of the N05A (ATC classification) Antipsychotics, and each of its components, in relation to MEDRA Terms:Bacterial lower respiratory tract infections, Lower respiratory tract infections NEC and Respiratory tract infections NEC.

IC values (and IC025 and IC0005) were analyzed for all compounds whitin the ATC group N05A, and the Preferred Terms related to the MEDRA Terms.

Results: IC and IC025 values greater than 1 , were only achieved by the combinations: Clozapine-Lower respiratory tract infections (IC: 3,27; IC025: 3,21), Clozapine-Lung abscess (IC: 2,88; IC025: 2,45) and clozapinepneumonia (IC: 1,97; IC025: 1,93). Other associations of clozapine with respiratory problems with IC025 $>0$ were: Respiratory tract infections, tracheobronquitis, Pneumonia necrotising, Atypical pneumonia, and Pulmonary Sepsis.

Only for Pimavanserin-Pneumonia (IC025: 0,7 n:142), ProthipendylPneumonia (IC025: 0,7 n:142), Quetiapine-Bronchitis (IC025: 0,5 $\mathrm{n}: 297)$,Benperidol-Pneumonia (IC025: 0,5 n:8), Amisulpiride-Lower respiratory tract infections (IC025: 0,3 n:17), Rimonabant-Respiratory tract infection (IC025: 0,26 n:5) y clopenthixol (IC025: 0,02 n:5) IC025 values were over 0. All other associations between antipsychotics and respiratory problems were not reported higher than expected.

Summary / Conclusions: There is a disproportion in the number of ICR spontaneously submitted of the association of clozapine and Penumonia / Lower respiratory tract infections in relation with other substances of its own group and with other antipsychotics. According to the literature this might be related to the dysphagia, increased sialorrea and/or sedation associated with (specially high doses of ) clozapine

\section{EACPT-1373}

Safety of immunomodulatory checkpoint inhibitors (icis): a critical look at available real-world evidence

J. Sáez-Peñataro ${ }^{*}{ }^{1,2}$, P. Alcubilla-Prats ${ }^{1}$

${ }^{1}$ Clinical Pharmacology Department, Hospital Clínic. Barcelona, ${ }^{2}$ Univeristy of Barcelona, Barcelona, Spain

\section{Pharmacovigilance}

Introduction: The introduction of ICIs has generated an increasing interest as antitumor agents, as well as with the inherent onset of immune-related adverse events (irAEs). However, the quantity and quality of evidence out of the context of pivotal clinical trials remains poorly explored.

Objectives: Perform a systematic review on the quantity and quality of real-world evidence of the safety profile of ICIs.

Methods: Pubmed, Cochrane and LILACS databases, and google scholar were screened by two independent reviewers. Real-world and observational studies assessing the safety profile of ICIs, were included. Pivotal clinical trials and papers not addressing safety were excluded from the initial search. Cut-off date for data extraction was March $3^{\text {rd }}, 2019$. Discrepancies and final selection were performed by consensus between the two reviewers.

Results: 24.949 unique citations were identified. Of these, 136 were selected. Level of evidence was assessed with the classification of the Centre for Evidence-Based Medicine. Of the selected studies, 15 were assessed with a $2 b$ level of evidence, and 121 with a 4 level. Likewise, 15 were prospective studies, with a median of 53 patients and a median follow-up of 14 months, and 121 were retrospective studies.

Considering total number of treatments per study, melanoma was the most frequent indication (154), followed by lung (66) and urogenital cancer (21). The most frequent ICI evaluated was nivolumab (92), followed by ipilimumab (72) and pembrolizumab (64). Other ICIs were underrepresented: durvalumab (12), atezolizumab (14), avelumab (2) and tremelimumab (9).
Completeness of AE reporting: 69 studies presented a complete report of all detected AEs, whereas 71 described a targeted reporting towards a specific $\mathrm{AE}$. Subpopulations: 2 studies included HIV patients, 4 elderly patients, 11 pre-existing autoimmune disorders, 4 transplanted patients and 3 assessed the impact of treatment of irAEs with steroids in overall efficacy.

Summary / Conclusions: Some real-world evidence has been found. However, additional large prospective observational studies with a longer follow-up are needed. Interestingly, while ipilimumab was the first ICI to be approved, fewer studies have been conducted at a post-market setting compared to other more recent ICIs. Especial populations, underrepresented in pivotal clinical trials, remain an unanswered question at a real-world setting.

\section{EACPT- 1390}

The influence of sex hormones on drug-induced liver (dili) clinical presentation

J. Sanabria-Cabrera ${ }^{1}$, S. Stankeviciute ${ }^{*}{ }^{2}$, I. Medina-Caliz ${ }^{1}$, M. RoblesDíaz $^{1}$, A. Gonzalez Jimenez ${ }^{1}$, R. González-Grande ${ }^{3}$, M. Jiménez-Pérez ${ }^{3}$, C. Parra-Martínez ${ }^{1}$, R. J Andrade ${ }^{1}$, M. Isabel Lucena ${ }^{1}$

${ }^{1}$ UGC Aparato Digestivo y Servicio de Farmacología Clínica, Instituto de Investigación Biomédica de Málaga (IBIMA), Hospital Universitario Virgen de la Victoria, Universidad de Málaga, CIBERehd, SCReN, Malaga, Spain, ${ }^{2}$ Hospital of Lithuanian University of Health Sciences, Kaunas, Lithuania, ${ }^{3}$ UGC Aparato Digestivo, Instituto de Investigación Biomédica de Málaga (IBIMA), Hospital Universitario Regional de Málaga, Malaga, Spain

\section{Pharmacovigilance}

Introduction: Despite being relatively rare, drug-induced liver injury (DILI) is a serious condition due to the risk of acute liver failure (ALF). Host factors have been suggested to play a role in clinical phenotypes of DILI, with females having higher risk of developing ALF. Sex hormones are known to affect drug metabolism and transport, and could therefore influence DILI phenotypes. There is evidence that suggest sex hormones are associated with various inflammatory features and hepatic injury in DILI, although robust evidence is lacking.

Objectives: To assess possible sex hormonal influences on DILI clinical presentation and outcome

Methods: A cohort including 419 woman and 308 men from the Spanish DILI Registry diagnosed with DILI was analyzed. The female cohort was divided into two age classes: $18-50$ years (younger) and $>50$ years (older, a proxy of menopause). Groups were compared according to culprit drugs, clinical features and biochemical parameters

Results: Of the female DILI patients 118 were classified as younger and 202 as older and compared to 308 men (18-90 years). Central nervous system drugs were more frequently found as culprits in younger females compared to older females and males ( $28 \%$ vs $13 \%$ and $8.7 \%, p=0.001$, respectively), and cardiovascular drugs more frequently in older females and men compared to younger females ( $15 \%$ and $11 \%$ vs $5.4 \%$ vs, $p=0.012$, respectively). Antiinfectives were more frequently found to cause DILI in males compared to the two female cohorts ( $50 \%$ vs $38 \%$ and $33 \%, p=0.001$, respectively). Jaundice was more prevalent in older women and men compared to younger women ( $68.8 \%$ and $69.2 \%$ vs $53.4 \%, p=0.05$,respectively). Positive autoantibody titres were more prevalent in women than men (20\% and $29 \%$ vs $18 \%$, respectively). Hepatocellular pattern of injury was more prevalent in younger women compared to older women and men (79\% vs $58 \%$ and $55 \%$, respectively). The frequency of fatal/liver transplantation cases were higher in younger and older females compared to men $(4.7 \%$ and $5.1 \%$ vs $0.4 \%$, respectively)

Summary / Conclusions: Sex appears to influence the clinical presentation of jaundice and in particular severity and outcome in DILI. However, further research is needed in order to elucidate the exact roll that sex hormones play in DILI

\section{EACPT-1426}

Attitudes, knowledge and behaviors of pharmacists about adverse drug reactions reporting through yellow card 
C. Pourzitaki ${ }^{*}{ }^{1}$, P. Staikou ${ }^{1}$, E. Stamoula ${ }^{1}$, P. Rigopoulos ${ }^{1}$, G. Tsaousi ${ }^{2}$, G. Papazisis ${ }^{1}$, D. Kouvelas ${ }^{1}$, T. Bellali ${ }^{3}$

${ }^{1}$ Department of Clinical Pharmacology, Faculty of Medicine, School of Health Sciences, ${ }^{2}$ Clinic of Anesthesiology and Intensive Care Unit, Faculty of Medicine, School of Health Sciences, Aristotle University of Thessaloniki, Greece, ${ }^{3}$ Faculty of Nursing, "Alexander" Technological Educational Institute of Thessaloniki, Thessaloniki, Greece

\section{Pharmacovigilance}

Introduction: In Greece, healthcare providers and patients are responsible for reporting through Yellow Card, every time they suspect that a drug has caused an adverse drug reaction (ADR). However, the underreporting of adverse drug reactions remains a major problem.

Objectives: The aim of this study was to investigate the role of pharmacists in pharmacovigilance, by assessing their attitudes, knowledge and behaviors about Yellow Card reporting.

Methods: The study was conducted with voluntary questionnaire reply by pharmacists, of central and north Greece. The questionnaire consisted of four sections and was based on the Theory of Planned Behavior, including questions about demographic, practical and professional characteristics of participants and their knowledge and skills on pharmacovigilance. Moreover, the questionnaire was related to their intentions and attitudes about Yellow Card reporting and factors that make the process easy or difficult, were investigated.

Results: Study population included 120 pharmacists with 11.6 years mean professional time period. About $54.5 \%$ of the sample had never reported an adverse drug reaction while almost $41.5 \%$ didn't know when an ADR must be reported. Moreover, $75 \%$ of the pharmacists believed that only medical doctors are responsible for reporting ADR's and almost 62.6\% didn't know how a yellow card is completed and where it should be sent.

Summary / Conclusions: The goal of this study was to note pharmacists' compliance about the implementation of the Yellow Card institution, in order to provide relevant information and measures about the rational and safe use of drugs. However, only a small proportion of the pharmacists included in the study knew the necessity and benefits of reporting adverse drug reactions, indicating the need of training health professionals other than medical doctors in pharmacovigillance.

\section{EACPT-1437}

Pregnancy and infant outcomes after maternal exposure to rituximab K. Zagorodnikova ${ }^{1}$, K. Monosova* ${ }^{1}$, V. Ivanov ${ }^{2}$, A. Zaritskey ${ }^{2}$

${ }^{1}$ clinical pharmacology, ${ }^{2}$ Hematology-oncology, Almazov National Medical Research Center, St Petersburg, Russian Federation

Pharmacovigilance

Introduction: Rituximab (RTX) is increasingly used for diffuse large Bcell lymphoma (DLBCL) and systemic lupus erythematosus (SLE) including pregnant patients. RTX was not teratogenic in animals and in limited amount of data in humans. There have been concerns regarding its immunosuppressive effects. Whether it is a substantial obstacle in its wide use is still unclear.

Objectives: The aim was to describe our experience of use of RTX in pregnant patients with DLBCL or SLE

Methods: We analyzed case histories of pregnant patients who received RTX since 2013 at our center. Details of use and outcomes were derived from medical records.

Results: Eight pregnant women received RTX during the study period. Indications included DLBCL (n 6) and SLE (n 2). Gestational age at diagnosis was $\geq 16$ weeks; RTX was administered for $1-4$ cycles prior to delivery. Treatment regimens involved 3 weekly infusions of $375 \mathrm{mg} /$ $\mathrm{m}^{2}$ of RTX for DLBCL or 2 infusions 14-21 days apart of $1000 \mathrm{mg}$ of RTX for SLE. Concomitant medications for DLBCL included: cyclophosphamide, doxorubicin, vincristine, and prednisonelor dexamethasone, filgrastim, ampicillin/sulbactam; for SLE: intravenous IgG, hydroxychloroquine and acyclovir; for both groups: omeprazole, nadroparin, acetaminophen, chloropyramine. Among DLBCL patients four pregnancies resulted in preterm deliveries (24;34-36 weeks), one full-term baby ( 37 weeks), one stillbirth ( 29 weeks) - intrauterine infection was the cause of death. Two live born babies were small for gestational age. The SLE patients delivered at 28 and 38 weeks. The latter baby had severe hypoglycemia. One baby had malformation (atrial and ventricular septal defects). Four neonates had hematologic abnormalities (neutropenia, lymphocytopenia, thrombocytopenia/anemia). Six placentas had histological signs of infection. Three neonatal infections were reported: cytomegalovirus infection, necrotizing enterocolitis with pneumonia, and one unspecified infection.

Summary / Conclusions: The most common problems identified were preterm delivery $(6 / 8)$ and intrauterine infections. The former cannot be strictly linked to RTX use due to possible influence of underlying diseases and co-medications. High frequency of infections seems to be in possible relation to RTX due to its mechanism of action. Maternal and neonatal immunosuppression and subsequent infection may be a serious concern in pregnant RTX users.

\section{EACPT-1447}

Real-world efficacy and safety of pharmacologic prophylaxis for clinically significant venous thromboembolism among non-surgical hospitalized patients

O. Lavon ${ }^{*}$, Z. Tamir ${ }^{2}$

${ }^{1}$ Clinical Pharmacology and Toxicology, ${ }^{2}$ Carmel Medical Center, Haifa, Israel

\section{Pharmacovigilance}

Introduction: Venous Thromboembolism (VTE) is considered a leading safety concern during hospitalization. Pharmacologic prophylaxis is recommended for the mitigation of VTE.

Objectives: To evaluate the real-world efficacy and safety of pharmacologic prophylaxis for clinically significant VTE among non-surgical hospitalized patients.

Methods: A single center, large-scale retrospective computerized chart review study of hospitalized non-surgical patients during 26 months (April 1, 2014 to May 31, 2016). Outcomes included clinically diagnosed symptomatic VTE events during hospitalization and up to 90 days after discharge, any and major bleeding events, mortality during hospitalization and up to 90 days thereafter, and readmission up to 90 days after discharge. VTE diagnosis was independently validated. Risk for VTE was evaluated using the Padua Prediction Score

Results: 5,117 patient records were analyzed after screening and validation. $1,120(22 \%)$ patients were defined as high-risk, of which $277(24.7 \%)$ were prophylactically treated. Overall, 14 $(0.27 \%)$ VTE events were observed: 3 cases in the high-risk group $(0.27 \%)$ and $11(0.28 \%)$ in the low-risk group, without any statistically significant difference. Prophylactic treatment among the high-risk patients did not significantly improve VTE incidence: $1 / 277(0.36 \%)$ treated vs. $2 / 843(0.24 \%), \mathrm{p}=0.76$. There was no significant difference between the study groups regarding the rates of bleeding, mortality or readmission.

Summary / Conclusions: Based on the study results, clinically significant VTE incidence among non-surgical hospitalized patients is low. VTE pharmacologic prophylaxis in these patients, while relatively safe, does not offer any significant benefit.

\section{Psychopharmacology}

\section{EACPT-1399}

Physician specialty and antidepressant prescription: an analysis of the national e-prescription database of greece G. Papazisis $^{*}{ }^{1}$, S. Siafis ${ }^{1}$, E. Apostolidou ${ }^{1}$, D. Kouvelas ${ }^{1}$

${ }^{1}$ Clinical Pharmacology, School of Medicine, Aristotle University of Thessaloniki, Thessaloniki, Greece 


\section{Psychopharmacology, Other}

Introduction: Over-prescription of antidepressants is evident, yet a considerable number of patients might remain inadequately treated. Inappropriate prescription of antidepressants by specialties with limited experience on psychotropic drugs could contribute to this phenomenon. Objectives: Aim of our study was to investigate the prescribing patterns of all-categories antidepressants in Greece identifying the physician specialties that prescribe them.

Methods: Individual anonymized antidepressant prescriptions were extracted from the national e-prescription database of Greece (from 20172018). Antidepressants were classified as SSRI/non-SSRI and physician specialty as psychiatry/non-psychiatry. The relationship between physician specialty and class of antidepressants was examined with relative risk (RR) and a chi-squared test of independence. Analysis was conducted in $R$ version 3.5.1, alpha was set at 0.05 .

Results: In the examined period, 1048575 antidepressant prescriptions were extracted. The most frequently prescribed antidepressants were SSRIs (694853 prescriptions, 66.3\%). The SNRIs venlafaxine (126466 prescriptions, $12.1 \%$ ) and duloxetine (72214 prescriptions, $6.9 \%$ ), followed by mirtazapine (97726 prescriptions, $9.3 \%$ ) were also common. Interestingly, only a fifth of them $(220527,21 \%)$ were prescribed by psychiatrists, while the rest by general practitioners/internists, neurologists and other specialties (Table 1). Non-psychiatrists prescribed less frequently non-SSRIs than psychiatrists (RR: 0.762 ; 95\% CI: 0.758-0.767; chi-squared with Yatescorrection $=7589.594$, degrees of freedom $=1, p<0.001$ ). However, demographic characteristics and antidepressant indication were not examined as well as some prescriptions might not be covered by the database.

\begin{tabular}{ll}
\hline Specialties & $\%$ \\
General practitioner & 34.18 \\
Internal medicine & 22.31 \\
Psychiatry & 19.56 \\
Neurology & 12.08 \\
Physician (residents) & 6.34 \\
Cardiology & 1.83 \\
Neurology-Psychiatry & 1.34 \\
Rheumatology & 0.55 \\
Nephrology & 0.49 \\
Pulmonology & 0.34 \\
Oncology & 0.24 \\
Orthopedic surgery & 0.19 \\
Endocrinology & 0.18 \\
General Surgery & 0.16 \\
Gastroenterology & 0.13 \\
Anesthesiology & 0.13 \\
Child and adolescent psychiatry & 0.13 \\
\hline
\end{tabular}

Summary / Conclusions: Non-psychiatrists prescribed a large portion of antidepressants, yet more frequently SSRIs (which are usually considered as first-line treatment). Our results warrant further investigation of the current antidepressant prescription patterns in Greece.

\section{Oral Presentation}

EACPT-1415

Nonadherence rates of antipsychotic drugs - a retrospective study based on blood sample analyses of 24,093 patients

R. L. Smith" ${ }^{*}$, L. Kylles $\varnothing^{1}$, M. Tveito ${ }^{1}$, O. Andreassen ${ }^{2}$, E. Molden ${ }^{1,3}$
${ }^{1}$ Center for Psychopharmacology, Diakonhjemmet Hospital, ${ }^{2}$ NORMENT \& K. G. Jebsen Center for Psychosis Research, Institute of Clinical Medicine, University of Oslo and Oslo University Hospital, ${ }^{3}$ Department of Pharmaceutical Biosciences, School of Pharmacy, University of Oslo, OSLO, Norway

\section{Psychopharmacology, Therapeutic drug monitoring}

Introduction: Nonadherence is a major issue in antipsychotic drug treatment in terms of causing therapeutic failure.

Objectives: The aim of present study was to quantify and compare the definitive nonadherence rates of antipsychotic drugs by using blood detection profiles in patients prescribed the various agents.

Methods: In total, 23,920 patients $(127,198$ blood samples) who were prescribed quetiapine ( $\mathrm{n}=8549$ patients), olanzapine (8406), aripiprazole (4532), risperidone (3723), clozapine (2392), amisulpride (998), chlorprotixene (958), zuclopentixol (937), perphenazine (917), haloperidol (731), levomepromazine (597), flupentixol (443), or ziprasidone (435) as oral treatment were retrospectively included from a therapeutic drug monitoring (TDM) database at the Center for Psychopharmacology, Diakonhjemmet Hospital, Oslo, Norway. Nonadherence was defined as absence of the prescribed drug in the respective patient's blood sample submitted for TDM. The rate of nonadherence per blood samples was compared between the antipsychotic drugs and assessed in relation to patient characteristics.

Results: The overall rates of nonadherent blood samples (TDM measurements) and nonadherent patients were $1.7 \%$ and $6.9 \%$, respectively, in the whole study population. A lower nonadherence rate was found in clozapine-treated patients $(0.31 \%)$ vs. all other subgroups, where nonadherence rates in increasing order were as follows: zuclopentixol $(1.4 \%)$, risperidone $(1.5 \%)$, aripiprazole $(1.6 \%)$ ziprasidone $(1.6 \%)$, quetiapine $(1.9 \%)$, levomepromazine $(2.2 \%)$, flupentixol $(2.3 \%)$, amisulpride $(2.3 \%)$, olanzapine $(2.6 \%)$, haloperidol $(3.0 \%)$, perphenazine $(4.8 \%)$ and chlorprotixene $(6.9 \%)$. The state of being nonadherent was significantly associated with age and degree of polypharmacy $(\mathrm{p}<0.001)$.

Summary / Conclusions: The present study shows that the nonadherence rates differ significantly between antipsychotic drugs during oral treatment. Clozapine has the lowest nonadherence rate, which may contribute to its superiority in the treatment of schizophrenia compared to the other antipsychotic drugs.

\section{EACPT-1457}

Effect of short-term treatment with olanzapine and aripiprazole on prolactin in healthy subjects

G. Mejia ${ }^{1}$, S. Martín-Vilchez ${ }^{1}$, D. Romero ${ }^{1}$, D. Ochoa ${ }^{1}$, M. Roman ${ }^{1}$, D. Koller $^{1}$, M. Saiz-Rodríguez ${ }^{1}$, A. de Miguel $^{1}$, C. Belmonte ${ }^{1}$, F. AbadSantos $^{* 1}$

${ }^{1}$ Clinical Pharmacology, Hospital Universitario de la Princesa, Universidad Autónoma de Madrid, Madrid, Spain

\section{Psychopharmacology}

Introduction: Hyperprolactinemia mediated by antagonism of dopaminergic neurotransmission in the pituitary gland is a common adverse effect of first-generation antipsychotics. Second-generation antipsychotics display attenuated elevation of prolactin levels, although adverse events related to prolactin have also been reported with this group of drugs.

Objectives: To compare the effects on prolactin levels produced by two different second-generation antipsychotics (olanzapine, that is a dopamine $\mathrm{D}_{2}$ receptors antagonist, and aripiprazole that acts as a partial agonist at dopamine $\mathrm{D}_{2}$ receptors) administered as multiple doses to healthy volunteers. The differences between men and women were also evaluated. 
Methods: 21 healthy volunteers (12 men and 9 women, 19-43 years old) were enrolled in a randomized, crossover clinical trial. All subjects received oral treatment with olanzapine $5 \mathrm{mg} /$ day or aripiprazole $10 \mathrm{mg}$ /day at 9:00 on days 1 to 5, in a randomized order, with a washout period of 28 days. Prolactin concentrations were measured by chemiluminescence at 8:00-9:00 (before dosing) on days 1, 3, 5, 6 and 15, and at 14:00 on days 1 and 5. Data were compared by ANOVA or T-test. Study was approved by local Research Ethics Committee and all subjects signed an informed consent.

Results: As shown in table 1, olanzapine increased the prolactin levels, especially in women. On the contrary, aripiprazole slightly decreased the prolactin levels, mainly in men. Levels were normalized 10 days after stopping treatment. No subject showed adverse reactions related to hyperprolactinemia but a woman during olanzapine treatment.

Table 1. Mean of prolactin plasma concentrations $(\mathrm{ng} / \mathrm{mL})$

\begin{tabular}{|c|c|c|c|c|c|c|c|c|}
\hline & & At 8:00-9:00 (predose) & At $14: 00$ (5h postdose) & & & & & \\
\hline & $\operatorname{Sex}(n)$ & Day 1 & Day 3 & Day 5 & Day 6 & Day 15 & Day 1 & Day 5 \\
\hline Olanzapine & Men (12) & 12.2 & 15.5 & 24.4 & 22.6 & 13.1 & 28.1 & 27.6 \\
\hline Olanzapine & Women (9) & 15.4 & 25.6 & 34.2 & 39.2 & 13.6 & 45.0 & 41.1 \\
\hline Aripiprazole & Men (12) & 14.1 & 6.0 & 6.4 & 6.3 & 9.2 & 8.2 & 4.9 \\
\hline Aripiprazole & Women (9) & 13.8 & 11.2 & 10.9 & 11.2 & 14.4 & 16.1 & 9.2 \\
\hline
\end{tabular}

Summary / Conclusions: Olanzapine treatment increased prolactin levels in healthy subjects, what could be related to side effects in longterm treatment. On the contrary, short-term treatment with aripiprazole decreased prolactin levels.

This work is co-financed by H2020 Marie Sklodowska-Curie Innovative Training Network 721236 grant.

\section{Rare diseases}

\section{EACPT-1083}

Which opioids in case of mast cell activation disorders? M. Lepelley ${ }^{1}$, C. Khouri ${ }^{*}{ }^{1}$, P. Pralong ${ }^{2}$, J. Rossignol ${ }^{3}$, C. Gréco ${ }^{4}$, I. Boccon Gibod $^{5}$, L. Bouillet ${ }^{6}$

${ }^{1}$ Pharmacovigilance, ${ }^{2}$ Allergo-dermatologie, CHU Grenoble-Alpes, Grenoble, ${ }^{3}$ Centre de référence des Mastocytoses, ${ }^{4}$ Pain and Palliative Care Unit, CHU Necker, Paris, ${ }^{5}$ Médecine interne, ${ }^{6}$ Centre de référence des angioedèmes bradykiniques, CHU Grenoble-Alpes, Grenoble, France

\section{Rare diseases}

Introduction: Patients suffering from mast cell disorders can experience symptoms associated with sudden and massive mast cellmediators release, including urticaria. Mast cell mediators release may be triggered by drugs such as opioids. Mast cells activation differs depending on the opioid type: morphine, codeine and pethidine provoke histamine release, whereas fentanyl and its derivatives do not and are preferred in case of mast cell disorders. Tramadol and hydromorphone are supposed not to release histamine and could be proposed to mast cell disorderspatients. However robust data are still lacking to confirm this hypothesis.

Objectives: To evaluate whether tramadol and hydromorphone were associated with an increased urticaria reporting risk, compared to others opioids.

Methods: We performed a disproportionality analysis using data from the World Health Organization (WHO) pharmacovigilance database. Given the tight link between histamine and urticaria, we used this adverse drug reaction (ADR) as a proxy of the opioid ability to induce histamine release. We extracted all individual cases safety reports (ICSRs) including the term "urticaria", until April $5^{\text {th }} 2018$. We calculated the reporting odds ratio (ROR) of urticaria associated with tramadol, hydromorphone, fentanyl, morphine, codeine and pethidine. The cut-off for signal detection was defined as a lower boundary of the ROR $95 \%$ confidence interval $\geq$ 1 , and number of reports $\geq 3$.
Results: A total of 476442 ICSRs of urticaria were reported in the WHO pharmacovigilance database. According to the disproportionality analysis, morphine, codeine and pethidine were associated with an increased reporting of urticaria while tramadol, hydromorphone and fentanyl were not. Pethidine showed the highest disproportionality signal.

Summary / Conclusions: Despite some limitations since ADR, especially non-serious ADR including urticaria, are underreported, these results are consistent with previous literature given that urticaria was significantly less reported with fentanyl than with morphine and pethidine. Additionally, tramadol and hydromorphone could be interesting alternatives for patients suffering mast cell disorders and requiring analgesics. These results need to be confirmed by prospective controlled studies.

\section{EACPT- 1374}

Use of sirólimus $0.4 \%$ ointment in vascular malformations M. Mejias ${ }^{*}{ }^{1}$, M. Ladrón de Guevara ${ }^{1}$, M. Soriano ${ }^{1}$, R. Seisdedos ${ }^{1}$ ${ }^{1}$ Farmacia, Hospital Universitario Virgen del Rocío, Sevilla, Spain

\section{Rare diseases}

Introduction: Vascular malformations are anomalies that present a variable clinical range from asymptomatic spots to lesions which could endanger patient life. These lesions are benign and progressively worsen throughout the patient's life without disappearing. They are rare diseases so it is difficult to know which is the best treatment.

Objectives: To evaluate the effectiveness and safety of Sirólimus $0.4 \%$ topical ointment in vascular malformations.

Methods: Descriptive observational survey including patients diagnosed with vascular malformations who had been dispensed at least once the master formula of Sirólimus $0.4 \%$ ointment from the Pharmacy Service. The questionnaire included: gender, age, time of disease evolution, previous treatments, time in treatment with Sirólimus, improvement of lesions and adverse reactions related to its administration.

For a period of three months, patients who went to the Pharmacy service to collect Sirolimus were interviewed. The digital clinical history and dispensing program were also consulted.

Results: It was found that currently seven patients collect medication in our service:

-Six patients were interviewed (ages between 22 and 68 years old), four of them were women.

-Four were diagnosed with Schobinger stage II arteriovenous malformation, one with venous malformation and another with glomovenous malformation. 
-Five had received previous treatment with pulsed laser, electric scalpel or surgery. Patients with arteriovenous malformation are also treated with Propranolol 10-20 mg twice daily. Two patients discontinued propranolol, one due to tachycardia and another due to lack of effectiveness, continuing with Sirolimus $0.4 \%$

-All patients have been on Sirolimus for over a year, except for two patients whose treatment was discontinued, the first one for local irritation and the second one for bleeding not caused by the ointment.

-Three patients perceive improvement of the lesions, two determine that they do not improve, although one of them recognizes lack of adherence, and about the last one, we have no data because he abandoned the treatment.

Summary / Conclusions: Sirólimus $0.4 \%$ ointment is a therapeutic option for the treatment of asymptomatic lesions associated with vascular malformations. It appears to be effective and safe although more investigations are needed to assess whether the $0.4 \%$ concentration of Sirolimus is the most appropriate, which type of vascular malformation responds better, and how long the treatment will last.

\section{Therapeutic drug monitoring}

\section{EACPT-1055}

High intra-patient variability in tacrolimus exposure is not associated with immune-mediated graft injury after liver transplantation M. van der Veer ${ }^{*}{ }^{1}$, N. Nangrahary ${ }^{1}$, D. Hesselink ${ }^{1}$, N. Erler ${ }^{1}, H$. Metselaar $^{1}$, T. van Gelder ${ }^{1}$, S. Darwish Murad ${ }^{1}$

${ }^{1}$ Erasmus Medical Center Rotterdam, Rotterdam, Netherlands

\section{Therapeutic drug monitoring}

Introduction: A high intrapient variability in tacrolimus exposure is associated with impaired long-term clinical outcome after kidney transplantation. It remains to be determined if a high tacrolimus intrapatient variability is equally detrimental for liver transplant recipients.

Objectives: The objective of this study was to investigate the association between intrapatient variability in tacrolimus exposure and immunemediated graft injury after liver transplantation.

Methods: For 326 liver transplant recipients, transplanted between 2000 and 2015, tacrolimus intrapatient variability was calculated from at least 5 tacrolimus trough samples obtained between month 6 and 18 after liver transplantation and was expressed as the coefficient of variation. The primary composite endpoint consisted of immune-mediated graft injury (chronic rejection, biopsy proven late-acute rejection and suspected late-acute rejection) after month 6 post-liver transplantation. Secondary outcomes were the association between tacrolimus intrapatient variability on (1) loss of renal function per year of follow-up and (2) cytomegalovirus viremia after month 6 post-liver transplantation.

Results: Of the 326 included liver transplant recipients, 70 patients $(21.5 \%)$ reached the primary endpoint. Median tacrolimus coefficient of variation was $28 \%$. There was no significant difference in reaching the primary composite endpoint between the low and high tacrolimus variability group $(p=0.068)$. Tacrolimus intrapatient variability modeled as a continuous variable remained non-significantly associated with the risk of reaching the primary endpoint in a multivariable analysis. MELD-score pre-transplantation and the number of acute rejections were identified as independent predictors for immune-mediated graft injury $(\mathrm{p}=0.049$, $\mathrm{p}=0.016$ ). For the secondary endpoints, a higher tacrolimus variability in combination with a low kidney function at baseline (eGFR $<40 \mathrm{ml} / \mathrm{min}$ ) was associated with greater loss of renal function per year of follow-up $(\mathrm{p}=0.007)$. Tacrolimus variability was not associated with late cytomegalovirus viremia.

Summary / Conclusions: High intrapatient variability in tacrolimus exposure beyond month 6 post-liver transplantation was not found to be associated with immune-mediated graft injury.
EACPT-1111

A validated lc-ms/ms method for simultaneous quantitation of tolvaptan and its monohydroxylate enantiomers in human plasma using a reversed-phase separation mode

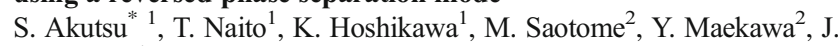
Kawakami $^{1}$

${ }^{1}$ Department of Hospital Pharmacy, ${ }^{2}$ Third Department of Internal Medicine, Hamamatsu University School of Medicine, Hamamatsu, Shizuoka, Japan

\section{Therapeutic drug monitoring}

Introduction: Tolvaptan, a nonpeptide vasopressin receptor V2 antagonist, has an asymmetric carbon and is converted to six monohydroxylates with optical and V2 receptor antagonistic activity, including 4S5R-diol, 4R5S-diol, 4R5R-diol, 4S5S-diol, 3R5R-diol, and 3S5S-diol forms, via CYP3A. However, no quantitative methods of 5R- and 5S-tolvaptan and their monohydroxylate enantiomers in human plasma has been published. Objectives: This study aimed to develop a validated liquid chromatography coupled to tandem mass spectrometry (LC-MS/MS) method for simultaneous quantitation of 5R- and 5S-tolvaptan and their monohydroxylate enantiomers in human plasma and to apply it to pharmacokinetic analyses. Methods: Deproteinized plasma specimens were separated using a 3- $\mu \mathrm{m}$ particle size chiral column with silica gel support coated on cellulose tris(3,5dimethylphenylcarbamate) as a chiral separator in a reversed-phase elution mode. The mobile phase consisted of a mixture of acetonitrile and $0.1 \%$ acetic acid in water and its flow rate was $0.3 \mathrm{~mL} / \mathrm{min}$ with a total run time of 20 minutes. The mass spectrometer was run in positive ion electrospray ionization mode. The present method was applied to the quantitation of plasma samples collected from heart failure patients treated with tolvaptan.

Results: The calibration curves of tolvaptan and its monohydroxylate enantiomers in human plasma were linear over the concentration ranges of $0.25-125$ and $0.025-12.5 \mathrm{ng} / \mathrm{mL}$, respectively. Their extraction recovery rates and matrix factors in human plasma were $87.3-112.9 \%$ and $87.1-118.5 \%$, respectively. The intra- and inter-day imprecisions and accuracies were $0.9-9.1 \%$ and $89.9-109.8 \%$ for all analytes, respectively. The plasma concentration ranges of 5R- and 5S-tolvaptan, 4S5R-diol, 4R5S-diol, 4R5R-diol, 4S5S-diol, 3R5R-diol, and 3S5S-diol forms were $0.61-128,1.25-354,0.033-12.7,0.081-36.0,0.041-8.17,0.78-50.1$, $0.028-5.58$ and $0.17-42.3 \mathrm{ng} / \mathrm{mL}$, respectively, in heart failure patients. The plasma concentrations of tolvaptan and its monohydroxylate enantiomers were measurable within the ranges of their calibration curves with dilution procedure.

Summary / Conclusions: The present method with an acceptable analytical performance can be helpful for characterizing the plasma concentrations of tolvaptan and its monohydroxylate enantiomers in heart failure patients.

\section{EACPT-1193}

Impact of endogenous serum immunoglobulin $g$ on serum level of denosumab in cancer patients

H. Shida ${ }^{*}$, T. Naito ${ }^{1}$, K. Shibata ${ }^{1}$, Y. Yamada ${ }^{2}$, J. Kawakami ${ }^{1}$

${ }^{1}$ Department of Hospital Pharmacy, ${ }^{2}$ Department of Clinical Oncology, Hamamatsu University School of Medicine, Hamamatsu, Shizuoka, Japan

\section{Therapeutic drug monitoring}

Introduction: Denosumab, a human monoclonal antibody targeting receptor activator of nuclear factor kappa-B ligand, is commonly used for the prevention of skeletal-related events in cancer patients with bone metastases. Our previous study reported that cancer patients showed a large variation in serum level of denosumab. Cancer patients with poor nutrient condition or glucocorticoid treatment often cause hypoproteinemia including hypoalbuminemia and hypogammaglobulinemia. To date, the associations between serum denosumab and endogenous serum albumin or immunoglobulin have not been fully evaluated in cancer patients. 
Objectives: The aim of this study was to examine the relationships between serum levels of denosumab and endogenous proteins in cancer patients.

\section{Oral Presentation}

EACPT-1266

Pharmacological monitoring of therapeutic monoclonal antibodies: from the proof-of-concept of their quantifications by mass spectrometry to bedside personalized dosing

T. Willeman ${ }^{1}$, J.-F. Jourdil ${ }^{1}$, E. Gautier-Veyret ${ }^{1}$, B. Bonaz ${ }^{2}$, F. StankeLabesque $^{* 1,3}$

${ }^{1}$ Pharmacology, Grenoble University Hospital-INSERM U1042, ${ }^{2}$ gastroenterology, Grenoble university hospital, ${ }^{3}$ INSERM U1042, Grenoble, France

\section{Therapeutic drug monitoring}

Introduction: The prescription of therapeutic monoclonal antibodies (mAbs) is increasing. Several studies suggested the interest of mAbs therapeutic drug monitoring (TDM) to improve clinical or biological response rate. Proofs of concept of mAbs quantification by liquid chromatography mass spectrometry (LC-MS/MS) emerged, but a multiplex, specific and reliable routine-suited quantification method is still needed to facilitate $\mathrm{mAbs}$ TDM.

Objectives: The first objective was to develop a multiplex and easy-toperform LC-MS/MS method for the quantification of adalimumab, infliximab, secukinumab, tocilizumab, trastuzumab, cetuximab and rituximab. The second objective was to determine, using this LC-MS/MS method, the therapeutic cut-off for the infliximab and adalimumab trough concentration $\left(\mathrm{C}_{\min }\right)$ in patients with inflammatory bowel disease (IBD). Methods: Plasma sample preparation was performed using protein-G purification and trypsin digestion. Proteotypic peptides were quantified by electrospray ionization LC-MS/MS. We also retrospectively measured infliximab and adalimumab residual concentrations (Cmin) respectively in 55 and 65 plasma samples from 2 independent cohorts of patients with inflammatory bowel disease, and determined infliximab and adalimumab Cmin cut-offs associated with biological remission (defined as a plasma CRP level $<5 \mathrm{mg} / \mathrm{L}$ and fecal calprotectin level $<150 \mu \mathrm{g} / \mathrm{g}$ stool).

Results: LC-MS/MS calibration curves were linear over the range of 1-100 $\mathrm{mg} / \mathrm{L}$, except for rituximab $(5-100 \mathrm{mg} / \mathrm{L})$. This method was reproducible, repeatable and accurate (coefficient of variation and bias $<20 \%$ ) with no cross contamination. Compared to patients without biological response, infliximab and adalimumab median concentrations were higher in patients with biological remission ((infliximab: $8.8 \mathrm{mg} / \mathrm{L}$ vs $5.4 \mathrm{mg} / \mathrm{L} ; \mathrm{p}=0.038$ ); adalimumab: $11.3 \mathrm{mg} / \mathrm{L}$ vs $9.5 \mathrm{mg} / \mathrm{L} ; \mathrm{p}=0.0198)$. Cut-off concentration set to $6.2 \mathrm{mg} / \mathrm{L}$ for infliximab (sensitivity $=75 \%$; specificity $=61 \%$ ) and set to $8.0 \mathrm{mg} / \mathrm{L}$ for adalimumab (sensitivity: $74.1 \%$, specificity: $57.9 \%$ ) discriminated patients with or without biological remission.

Summary / Conclusions: LC-MS/MS measures of infliximab or adalimumab $\mathrm{Cmin}$ and the determination of new thresholds of $\mathrm{Cmin}$ associated with biological remission are new steps towards infliximab or adalimumab treatment personalization in patients with IBD.

\section{EACPT-1267}

Immunosuppressant therapy with tacrolimus and cyclosporine in lung transplant recipients and cytomegalovirus infection: a retrospective review

R. Nogueiras Álvarez ${ }^{*}{ }^{1}$, I. Mazón Maraña ${ }^{1}$, N. Vega Gil ${ }^{1}$, G. Vaca Recalde $^{1}$, P. Ortiz Petrosino ${ }^{1}$, V. M. Mora Cuesta ${ }^{2}$, A. Bautista Blázquez ${ }^{1}$, D. Z. Cuéllar Gómez ${ }^{1}$, B. Sanchez Santiago ${ }^{1}$

${ }^{1}$ Farmacología Clínica, ${ }^{2}$ Neumología, Hospital Universitario Marqués de Valdecilla, Santander, Spain

\section{Therapeutic drug monitoring}

Introduction: Immunosuppression has improved graft survival but leaves patient susceptible to infections. Cytomegalovirus (CMV) infection is a frequent complication after transplant and major cause of morbidity-mortality. Lung transplant recipients (LTR) are particularly at risk given the intense immunosuppression required

Objectives: A retrospective review of LTR from 01/01/11 to 31/12/18 was done to explore potential association between development of CMV infection and blood concentration of calcineurin inhibitor within previous 90 days. Other factors such as demographics, donor/recipient CMV serostatus, basiliximab induction and lymphocytes count were also studied

Methods: 339 LTR patients were included. Those with diagnosis of CMV infection determined by virus quantitative PCR were considered cases and those who remained negative for $\mathrm{CMV}$, controls. According to time from transplant to first positive viral load quantification in each case, a control for equivalent post-transplant time interval was selected. We reviewed blood levels on same day first positive CMV viral load was detected and also the last 3 levels prior to CMV positive result. In controls, same data from same timepoints were recorded. Blood concentration of immunosuppressant was measured in Clinical Pharmacology lab by Chemiluminescent Microparticle Immunoassay.

Results: We found $128 \mathrm{CMV}$ infection cases. Median time from transplant to first positive CMV viral load was 391.5 days. 132 subjects were finally selected as controls. $\mathrm{N}=260,64 \%$ males and $64.3 \%$ double-lung. Diffuse intestitial lung disease (DILD) was most frequent $(47.8 \%)$ reason, 91.9\% received tacrolimus, $8.1 \%$ cyclosporin. Drug blood levels at selected timepoints showed no statistically significant difference between cases-controls. However, use of cyclosporine appeared to be associated to CMV infection $(\mathrm{p}=0.009)$. We also found association with induction therapy with basiliximab ( $\mathrm{p}=0.000)$, DILD ( $\mathrm{p}=0.025), \mathrm{IgG}+/ \mathrm{IgG}-$ donor/ recipient serostatus $(\mathrm{p}=0.004)$ and lymphopenia at the time of CMV infection and 90 days before $(\mathrm{p}=0.002)$

Summary / Conclusions: In this review of 260 LTR treated with tacrolimus/cyclosporine and dose adjustment based on therapeutic drug monitoring, no association between blood level and CMV infection was seen. Other factors related to immunity were found to be influencing, such as use of cyclosporine, lymphopenia, and basiliximab induction.

\section{Abstract session 4a \\ Horizon scanning frontiers \\ G. Lärfars ${ }^{1}$ \\ ${ }^{1}$ Ass professor at the Karolinska Institute Chair of the council for New Therapies (NT-council) Stockholm Sweden}

Introduction: In order to achieve an equal, cost-effective and appropriate use of new medicines for all patients in Sweden, all county councils, the Dental and Pharmaceutical Benefits Agency (TLV) and the pharmaceutical industry collaborate in a joint process for the introduction of new medicines.

The national process of introducing new medicines starts with horizon scanning. The aim of the work is to gather, document and validate information on new medicines or indications before these are granted marketing authorization. Horizon scanning provides the county councils and the New Therapies Council (NT-rådet) with a basis for decisions on whether a new medicine should be included in the national process for managed introduction.

Methods: The scanning process consists of several steps starting with at structured process of data collection and monitoring sources about 1-2years before expected marketing authorization and ends up in an early assessment report for those medicines that are assessed as interesting by the county council clinical experts. The early assessment report describes the current state of knowledge and is provided to the county councils about 6 months before market authorization and for the following decision in the NT-council.

Conclusion: Although the horizon scanning has become a well-defined evidence-based and structured process including a close collaboration between the working group and clinical experts there are still several challenges during this work. To create an early assessment report with limited information and just in time e.g. not to close before the expected 
marketing authorization is a challenge. The time from marketing authorization until the drug reaches the patients and health care must be held short. The difficulties for the horizon scanning working group to identify the most interesting and important drugs at an early stage often without any other data than small phase II-studies is difficult but an important task because of the requirement of a fast handling of a maybe lifesaving treatment for the further work with health economic evaluation and NTrecommendation. This orderly introduction is also an important tool for decision-making and prioritization-planning in healthcare.

\section{Invited speaker}

C. Pontes

${ }^{1}$ Catalan Health Service, Universitat Autònoma de Barcelona

\section{Targeting small populations: barriers to market access}

Introduction: Drug-development is a continuing and long lasting process that does not finish with the product commercialization. The authorization is always decided with some degree of uncertainty, based on a limited amount of data. This is particularly true for low prevalence diseases. The observation of an overwhelming effect during the clinical development poses an ethical issue, since arranging an early access to the drug may potentially offer a life-saving opportunity to subjects that would not thrive until full data is accrued. The conflict between the need for robust scientific evidence and the facilitation of early access to potentially saving treatments may result in a huge dilemma.

Objectives: To analyse pros and cons of early access to innovative drugs aimed to treat small populations.

Methods: Review of the data supporting regulatory decision for orphan drugs between 2000 and 2014 and analysis of regulatory uncertainties and risks.

Results: 38 out of 125 orphan medicinal products authorised by the EMA were supported by replicated pivotal trials; of 159 pivotal trials, 53 were single arm, 50 were not randomised, 75 were open-label, and 119 used surrogate variables as their main outcome. Data published suggests that further data is not always obtained, and reversal of conditional approvals may be largely unfeasible.

Conclusion: Early access satisfies immediate clinical needs, but at the expense of postponing the guarantees of robust decisions. Early interruption of developments may lead to overestimation of effects. Once a product is in the market, satisfying the need for additional data may be unfeasible: recruitment into ongoing clinical trials is difficult when a new drug for an unmet need is available. Price negotiation when most of the target population is already treated may be uneven, and may lead to unfair prices not reflecting benefits and risking sustainability. The regulatory requirement of replicated clinical results is intended to minimize the risk for false positives and to protect the public health from risks associated to authorization of ineffective treatments. There is need for alternative methods to conditional approval that guarantee that robust data is obtained for decision making, that early patient access to potentially life-saving interventions is possible, and that uncertainties and risks are truly shared with between companies and healthcare systems.

\section{Polypharmacy in Korea}

H. Lee ${ }^{1,2,3}$

${ }^{1}$ Seoul National University Hospital and College of Medicine, Seoul, Korea, ${ }^{2}$ Graduate School of Convergence Science and Technology, Seoul National University, Seoul, Korea, ${ }^{3}$ Center for Convergent Approaches in Drug Development, Seoul, Korea

Polypharmacy can make patients vulnerable to adverse drug reactions. Polypharmacy can undermine the financial wellness of the health insurance system. In Korea, polypharmacy, defined as the number of concomitant medications $\geq 6$, has decreased from $65.8 \%$ in early 2000 to $43.7 \%$ in 2013.[1] However, polypharmacy is still a big health issue in Korea, particularly in children aged 1-9 years and the poor. Likewise, low income is strongly associated with polypharmacy in the elderly in Korea.[2] Currently, there is no national guideline or measure implemented to curb polypharmacy in Korea.[3] Therefore, policies and nationwide efforts, along with an educational campaign led by clinical pharmacology professionals, are to be put into practice to keep the Korean patients from unwanted health problems caused by polypharmacy and to promote optimal drug therapy.

\section{References}

1. Baek YH, Shin JY: Trends in polypharmacy over 12 years and changes in its social gradients in South Korea. PloS one 2018, 13(9):e0204018.

2. Kim HA, Shin JY, Kim MH, Park BJ: Prevalence and predictors of polypharmacy among Korean elderly. PloS one 2014, 9(6):e98043.

3. Park H-Y, Sohn HS, Kwon J-W: Reviews on the Current Status and Appropriate Management of Polypharmacy in South Korea. Korean Journal of Clinical Pharmacy 2018, 28(1):1-9.

Publisher's note Springer Nature remains neutral with regard to jurisdictional claims in published maps and institutional affiliations. 